\title{
Illuminating Light
}

Neutral meson production and direct photon correlations from small to large collision systems at the LHC

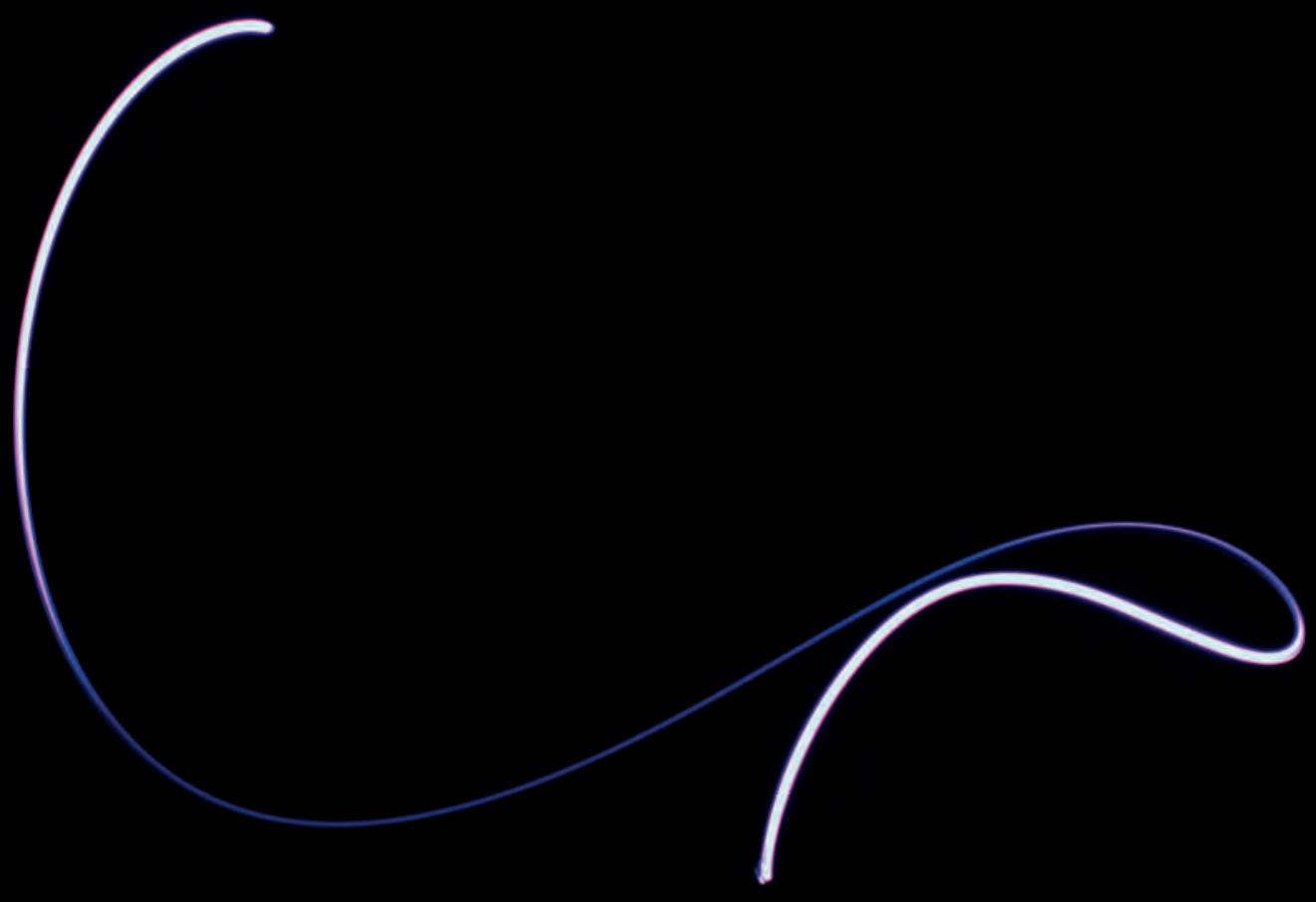




\section{Illuminating Light}

Neutral meson production and direct photon correlations from small to large collision systems at the LHC

Introductory video:

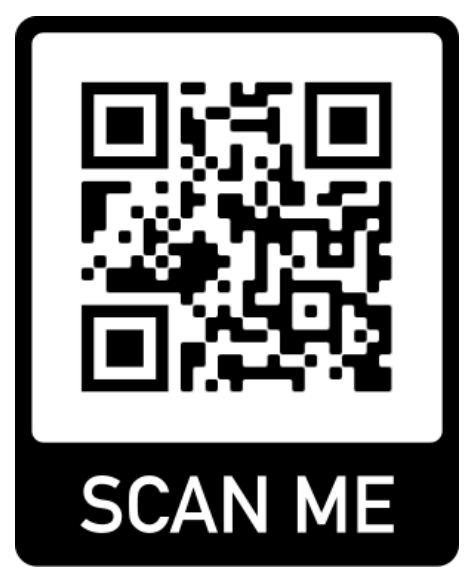

link

Mike Sas 
A catalogue record is available from the Utrecht University Library.

Copyright (C)2021 Mike Sas. All rights reserved. No part of this book may be reproduced, stored in a database or retrieval system, or published in any form or in any way, electronically, mechanically, by print, photoprint, microfilm or any other means, without prior written permission of the author.

Cover photo by Mike Sas. The chaotic nature of the double pendulum revealed by attaching a light source to its end point, captured using a DSLR with a one second exposure.

Printed by: ProefschiftMaken 


\title{
Illuminating Light \\ Neutral meson production and direct photon correlations from small to large collision systems at the LHC
}

\author{
Verhelderend licht \\ Neutralemesonproductie en directefotoncorrelaties \\ van kleine naar grote botsingen in de LHC \\ (met een samenvatting in het Nederlands)
}

Proefschrift

ter verkrijging van de graad van doctor aan de

Universiteit Utrecht

op gezag van de

rector magnificus, prof.dr. H.R.B.M. Kummeling, ingevolge het besluit van het college voor promoties

in het openbaar te verdedigen op

maandag 21 juni 2021 des middags te 2.15 uur

door

\section{Mike Henry Petrus Alphons Sas}

geboren op 31 maart 1990

te Oss

Promotor: Prof. dr. T. Peitzmann 

"Not all those who wander are lost."

J. R. R. Tolkien 



\section{Contents}

I Introduction and Theory $\quad 1$

1 General introduction 3

1.1 The Standard Model . . . . . . . . . . . . . . . . . . . . . . . . . . . 4

1.2 Quantum electrodynamics . . . . . . . . . . . . . . . . . . . . 6

1.3 Quantum chromodynamics ........................... 7

2 Heavy-ion physics $\quad 9$

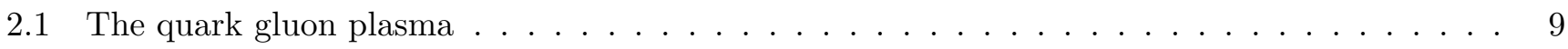

2.2 Proton proton collisions $\ldots \ldots \ldots \ldots \ldots \ldots \ldots \ldots \ldots$

2.3 Heavy-ion collisions . . . . . . . . . . . . . . . . . . . . . . . . 12

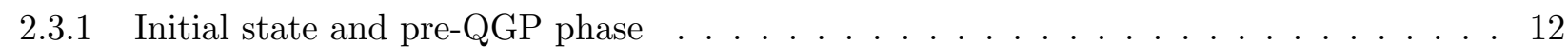

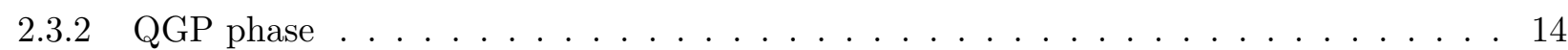

2.3 .3 Hadronization . . . . . . . . . . . . . . . . . . . . . . . . 14

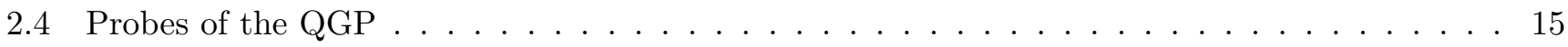

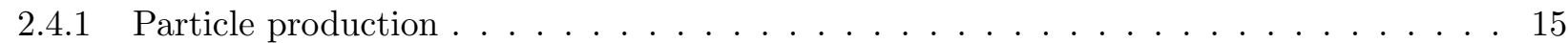

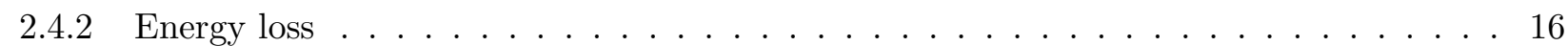

2.4 .3 Anisotropic flow . . . . . . . . . . . . . . . . . . . 17

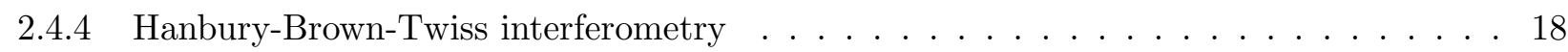

3 Probing the QGP using photons 21

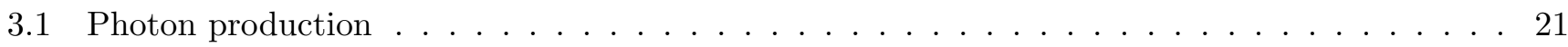

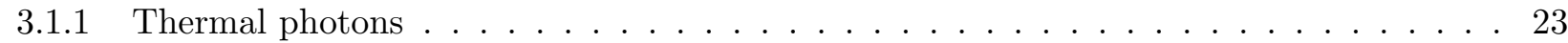

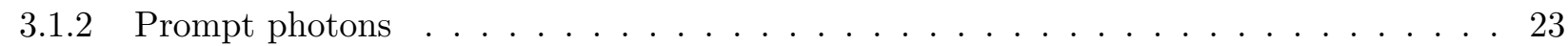

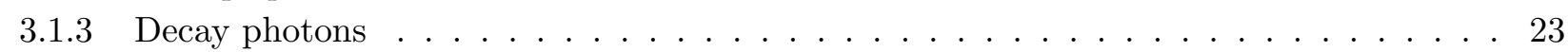

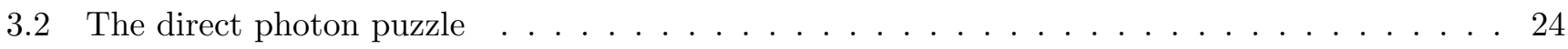

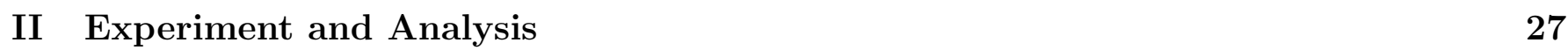

4 Experimental setup $\quad 29$

4.1 The Large Hadron Collider . . . . . . . . . . . . . . . . . . . . . . . . . . 29

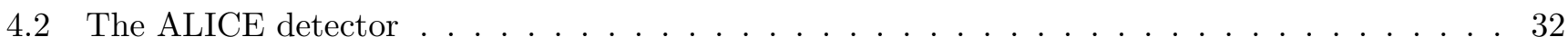

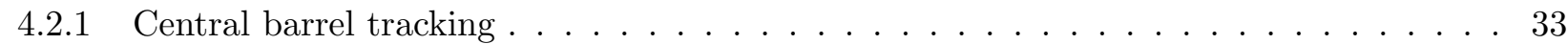

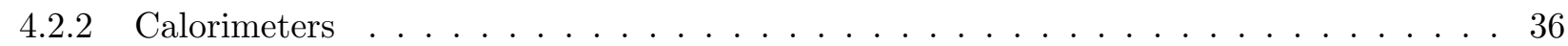

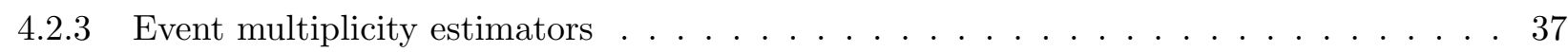

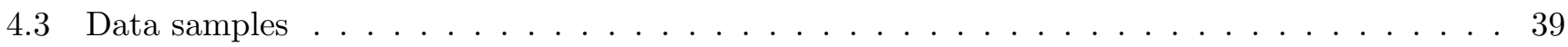

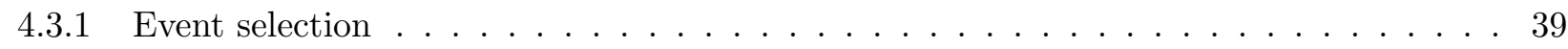

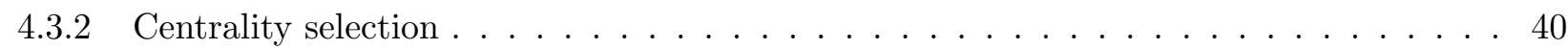




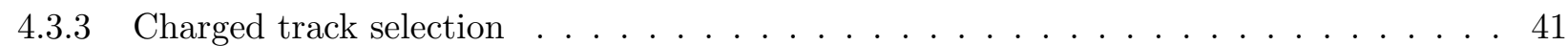

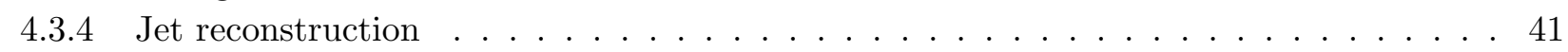

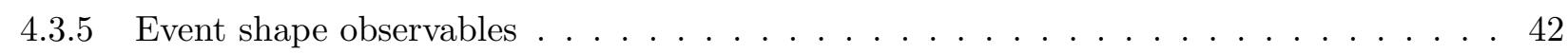

5 Photon reconstruction $\quad \mathbf{4 5}$

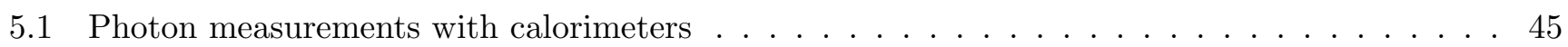

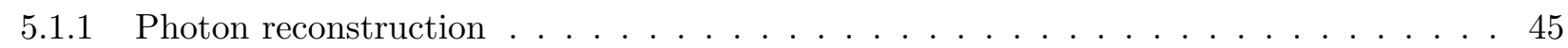

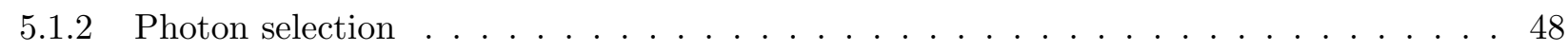

5.1 .3 Systematic uncertainties . . . . . . . . . . . . . . . . . . . 52

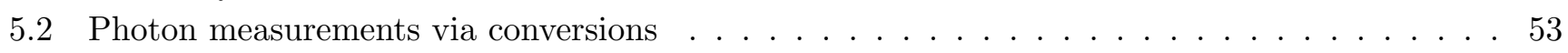

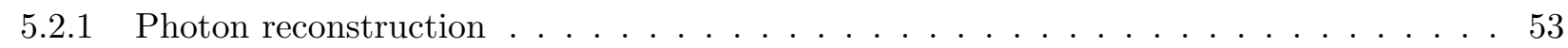

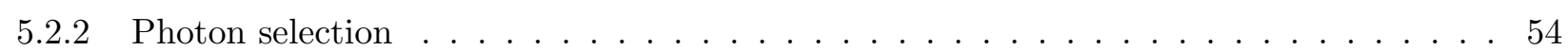

5.2 .3 Systematic uncertainties . . . . . . . . . . . . . . . . 57

$\begin{array}{ll}\text { III Analysis Results } & 59\end{array}$

6 Neutral meson production $\quad 61$

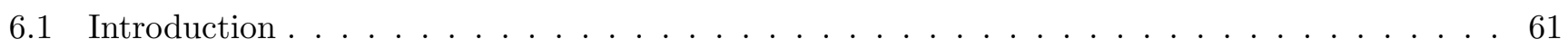

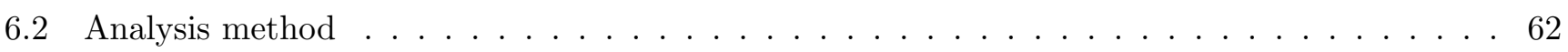

6.2 .1 The invariant mass distribution . . . . . . . . . . . . . . . 62

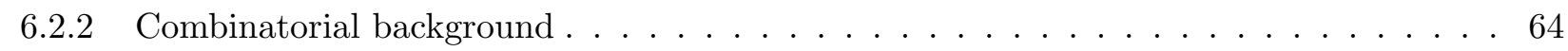

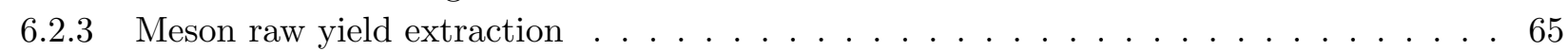

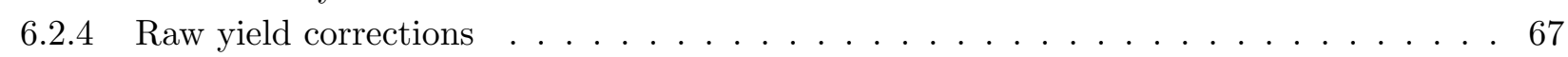

6.2.4.1 Correction for secondary neutral pions . . . . . . . . . . . . . 67

6.2.4.2 Acceptance and efficiency correction . . . . . . . . . . . . 67

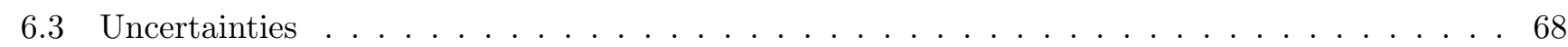

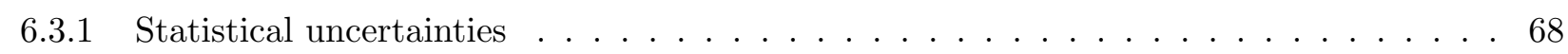

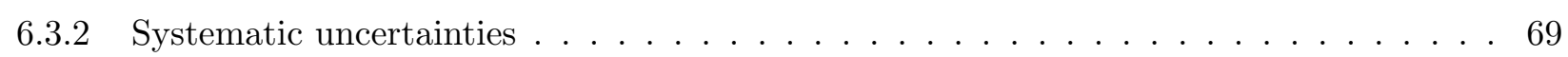

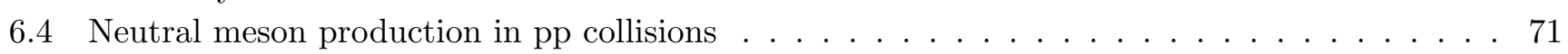

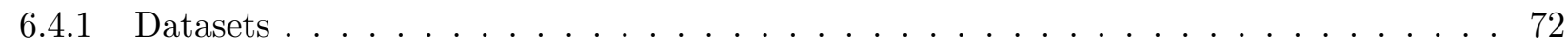

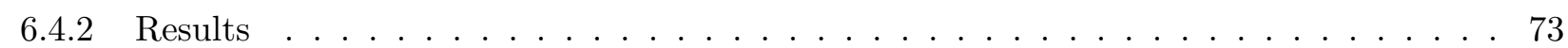

6.5 Neutral meson production in $\mathrm{p}-\mathrm{Pb}$ collisions . . . . . . . . . . . . . . . . . . . . . . . . . . . . .

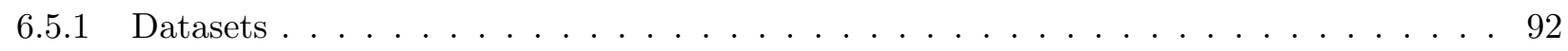

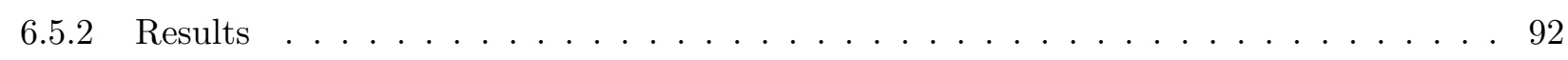

6.6 Neutral meson production in $\mathrm{Pb}-\mathrm{Pb}$ collisions . . . . . . . . . . . . . . . . 98

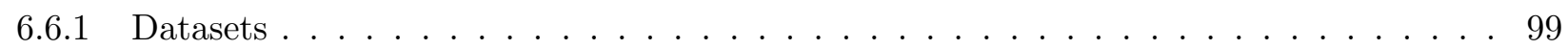

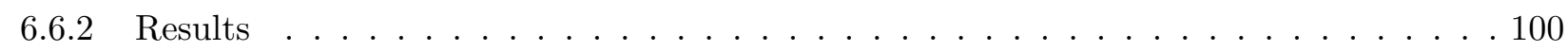

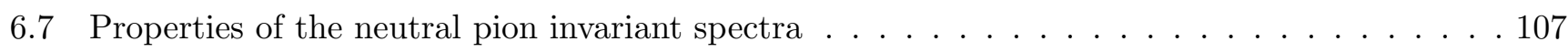

6.8 Discussion . . . . . . . . . . . . . . . . . . . . . . . . 109

7 Event-mixing studies $\quad 111$

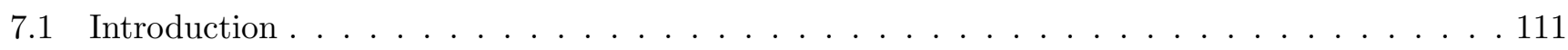

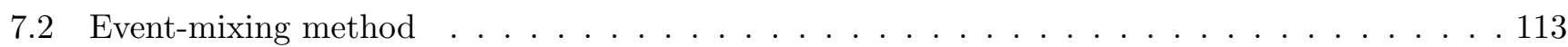

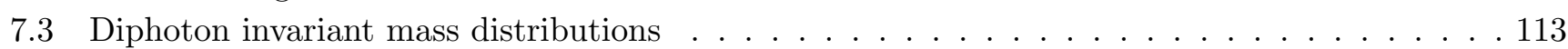

7.4 Pseudorapidity and azimuthal angle restrictions . . . . . . . . . . . . . . . 116

7.5 Diphoton pairs from same and different ancestors . . . . . . . . . . . . . . . 117

7.6 Modified diphoton event-mixing . . . . . . . . . . . . . . . . . 118 
7.7 Closer look at higher invariant mass f . . . . . . . . . . . . . . . . . . . . 119

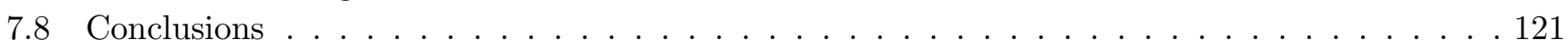

8 On event shapes and jets 123

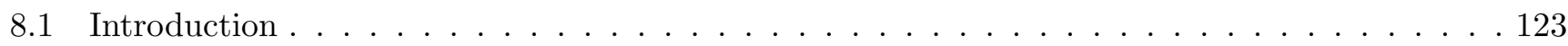

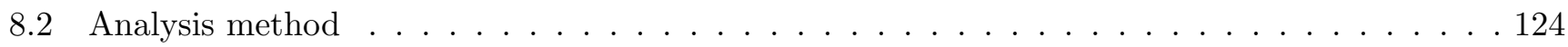

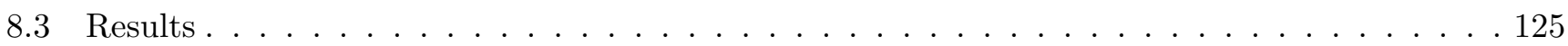

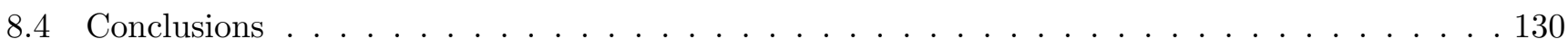

9 Direct photon flow $r 31$

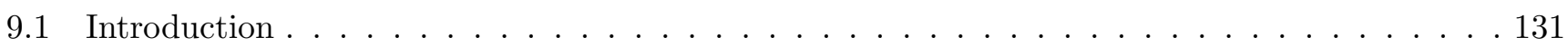

9.2 Analysis method . . . . . . . . . . . . . . . . . . . . . 132

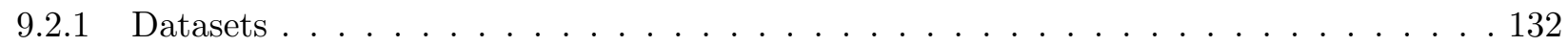

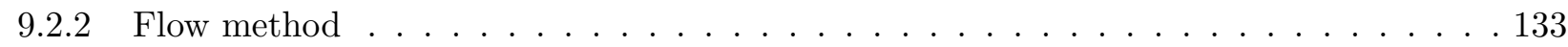

9.2 .3 Inclusive photon purity correction . . . . . . . . . . . . . . . . . 134

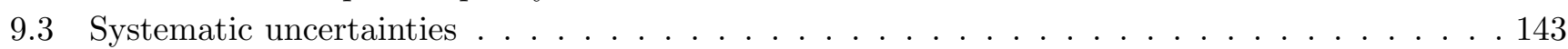

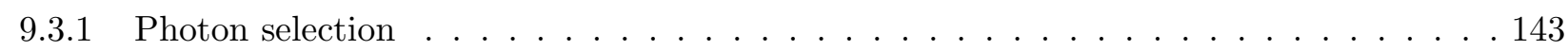

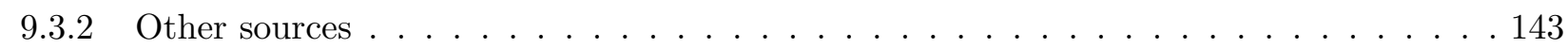

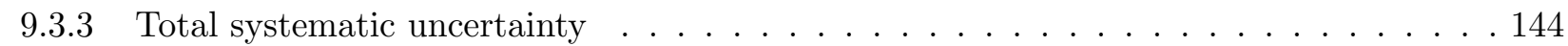

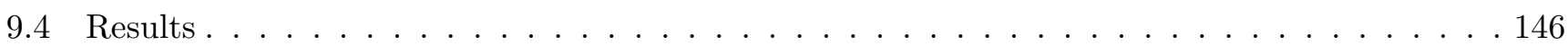

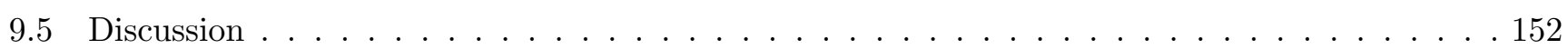

10 Direct photon HBT $\quad 153$

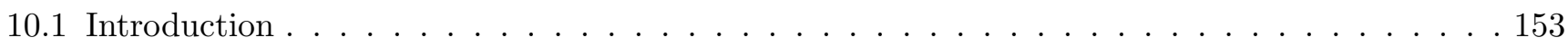

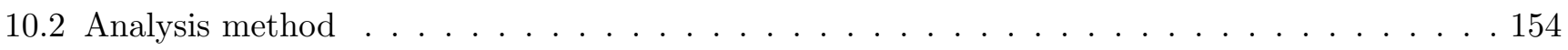

10.3 Uncertainties . . . . . . . . . . . . . . . . . . . . . 155

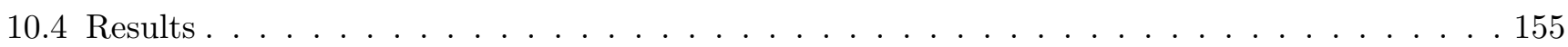

10.4.1 Photon correlations in pp collisions . . . . . . . . . . . . . . . 155

10.4.2 Photon correlations in $\mathrm{Pb}-\mathrm{Pb}$ collisions . . . . . . . . . . . . . 157

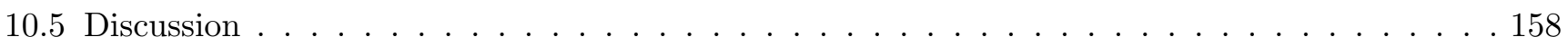

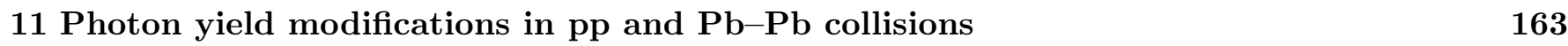

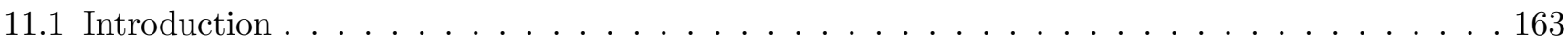

11.2 Analysis method . . . . . . . . . . . . . . . . . . 164

11.3 Results . . . . . . . . . . . . . . . . . . . . . . . 164

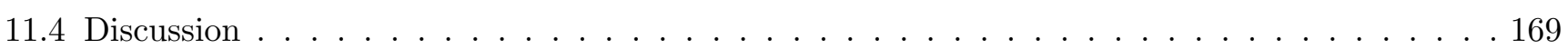

$\begin{array}{ll}\text { IV Summary and Outlook } & 171\end{array}$

12 Summary $\quad 173$

$\begin{array}{lr}13 \text { Outlook } & 177\end{array}$

14 Ideas for future measurements $\quad 179$

$\begin{array}{lr}\text { A Additional neutral meson plots } & 185\end{array}$ 
A.1 Invariant mass distributions of the neutral mesons in pp collisions . . . . . . . . . . . . 185

A.2 Neutral meson raw yield correction in $\mathrm{Pb}-\mathrm{Pb}$ collisions . . . . . . . . . . . . . . . . 194

A.3 Systematic uncertainties of the neutral meson invariant yield . . . . . . . . . . 195

$\begin{array}{lr}\text { Bibliography } & 207\end{array}$

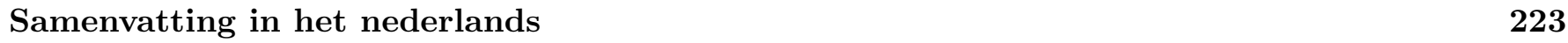

$\begin{array}{lr}\text { List of publications } & 227\end{array}$

$\begin{array}{lr}\text { Acknowledgements } & 229\end{array}$ 


\section{Part I}

\section{Introduction and Theory}





\section{Chapter 1}

\section{General introduction}

Trying to understand the universe is arguably the most beautiful endeavour of humankind. Ever since the beginning of science we have been pushing the boundary of knowledge outward, slowly but steadily gaining more ground into the infinite unknown. Each step has given us new ways to survive, develop, and shape the environment to our will. It is our curiosity that has been one of the driving forces of scientific progress, radically altering the way we view the world. From the ancient Greeks reasoning that nature consists out of atoms, to Copernicus theorizing that the solar system revolves around the sun, we have been chasing reality to build a more and more complete picture of "what makes everything tick".

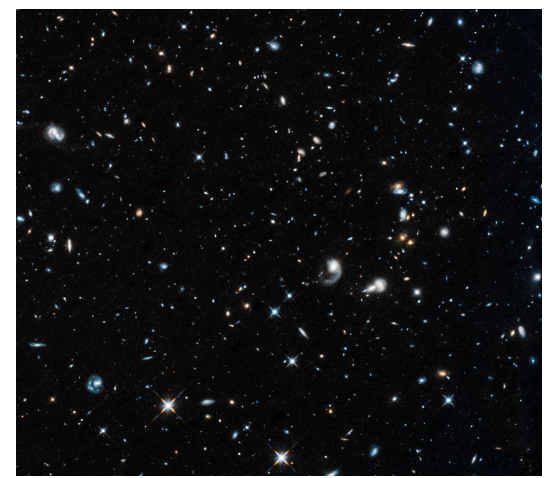

Figure 1.1: Picture taken with the Hubble space telescope.

In order to build such a comprehensive picture, we have to ask ques-

tions of why things behave or work the way they do, think of a possible explanation, and try to verify if this explanation correctly matches the phenomena. This is the very essence of the scientific method, where the process of observation, reason, and experiment is used to create knowledge. As it turns out, the natural world lets itself be described by mathematics, often in the form of elegant theories and scientific laws. The beauty of these theories is that they employ the scientific method, which gives them explanatory and predictive power. First, predictions generate hypotheses which can be tested by experiment. Then, the conclusions of the experiment are able to refine the theory and generate new hypotheses, which are followed up by new experiments. Maybe even more interestingly, this cycle of science can also go the other way around, i.e. by first stumbling upon an observation and only then developing a theoretical description. Importantly, while we embark on the journey of building a scientific theory of the universe, which is interesting in itself, it is society that benefits greatly from all the gathered knowledge.

According to the most accepted theory on the history of our universe, the big bang theory, the universe came into existence around 13.8 billion years ago [1]. During the first instances, it is space-time itself that 
underwent rapid expansion and was filled with extremely hot and dense matter. About 370.000 years after the big bang, the first neutral hydrogen atoms formed and the universe cooled down enough to become transparent for photons, leading to the cosmic microwave background we observe today. A few hundreds of millions of years later the first stars and galaxies began to form under the influence of gravity, creating the first large large structures in the universe $[2,3]$. In addition, the death of these first generation of stars created most of the heavier elements we find around us on earth. From around 1 billion years onwards, the universe gradually changed into the current state, forming over 100 billion galaxies containing billions of stars (Fig. 1.1). It is only for the last $0.0015 \%$ of the age of the universe that humans began to walk the earth and try to interpret our place in it all. The knowledge we gained over literally thousands of years accumulated in a well-tested theory which describes matter and the fundamental forces between them at the smallest possible scales.

\subsection{The Standard Model}

To our current best knowledge, the matter around us at the most fundamental level is made from indivisible constituents. These building blocks of nature and the forces that govern them are described by the Standard Model of particle physics. The Standard Model includes elementary fermions; the quarks and leptons, and elementary bosons; the gauge and scalar bosons $[4,5]$.

Figure 1.2 shows an overview of all the particles included in the Standard Model. The quarks and leptons come in three generations, where each generation is heavier than the previous and only the first one is stable. The neutrinos are an exception since they do not decay but oscillate between the different flavors. The gauge bosons are the force carriers that mediate the electromagnetic (photon), strong (gluon), and weak force ( $W^{ \pm}$and $Z$ boson). The Higgs boson, an example of the predictive power of the Standard Model, as it was theorized in $1964[6,7]$ and the existence confirmed in 2012 [8,9], explains why particles can acquire mass via the Higgs mechanism. The exact interplay between all these fundamental constituents is able to explain the matter we see and observe around us.

The mathematical framework of the Standard Model is provided by quantum field theory, and is a collection of quantum fields defined in all of space-time, where the particles are quantum excitations of the corresponding fields. It is constructed by finding the most general Lagrangian that respects the symmetries of the system. Global Poincaré symmetry is postulated to ensure translational and rotational symmetry, as well as boost invariance. More technically, it is the local $\mathrm{SU}(3) \times \mathrm{SU}(2) \times \mathrm{U}(1)$ gauge symmetry which defines the mathematical structure of the strong and electroweak interactions. In a short elegant form, the Standard Model Lagrangian reads

$$
\mathcal{L}_{\mathrm{SM}}=-\frac{1}{4} F_{\mu \nu} F^{\mu \nu} \quad+i \bar{\psi} \not \supset \psi+\text { h.c. } \quad+\bar{\psi}_{i} y_{i j} \psi_{j} \phi+\text { h.c. } \quad+\left|D_{\mu} \phi\right|^{2}-V(\phi) .
$$




\section{Standard Model of Elementary Particles}

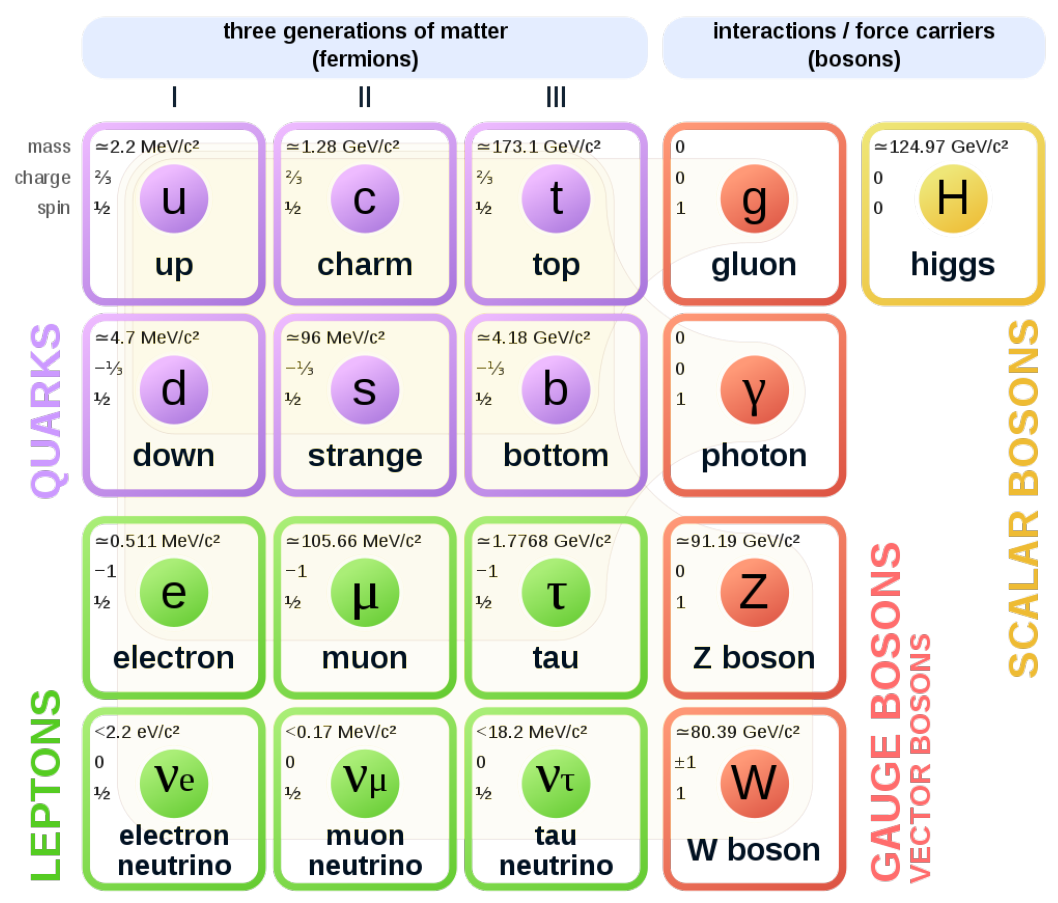

Figure 1.2: Elementary particles of the Standard Model of particle physics, the building blocks of matter at the most fundamental level.

The term $F_{\mu \nu} F^{\mu \nu}$ is the scalar product of the field strength tensor, describing the gauge bosons. It includes a kinetic term for the gluon, photon, $Z$, and $W^{ \pm}$bosons, as well as additional interaction terms describing the interactions between the gauge bosons. $\bar{\psi} \not D \psi$ describes the interactions between the gauge bosons and the quark and leptons. $\bar{\psi}_{i} y_{i j} \psi_{j} \phi$ describes the interactions of the quarks and leptons with the Higgs field $\phi$, giving rise to mass terms for the respective particles. $\left|D_{\mu} \phi\right|^{2}$ then describes the interactions between the gauge bosons, more specifically the $Z$ and $W^{ \pm}$, and the Higgs field. Finally, the $V(\phi)$ is the potential of the Higgs field.

It is important to note that this model is not a complete picture of the universe. First of all, the gravitational force is not incorporated because it turns out that it is notoriously difficult to find a quantum mechanical description of gravity that unifies with the rest of the standard model. Secondly, it only describes ordinary matter that makes up around $4 \%$ of the universe, whereas the unobserved dark matter and energy adds up to the rest [10]. The notion that the weak force is about $10^{24}$ times stronger than gravity and the fact that an extrapolation of the running coupling strengths does not lead to a unification of the fundamental forces at high energy, are additional hints that there is a greater theory out there. Extensions of the Standard Model do attempt to solve these issues, but have so far been unsuccessful or remained unconfirmed by experiment. There are two important subjects that are introduced further; the role of the photon in quantum electrodynamics, and the intricate properties of quantum chromodynamics. 


\subsection{Quantum electrodynamics}

In the classical theory of electromagnetism, as formulated by Maxwell in 1873, the force between two charged particles is described by the electric and magnetic fields that they themselves produce. We say that these electromagnetic fields permeate space and travel with the speed of light in vacuum. Light, in this view, is a wave of electromagnetic radiation. However, experiments on black-body radiation in the early 1900s showed that electromagnetic waves consist of discrete packets of energy, starting the development of quantum mechanics and later quantum electrodynamics (QED) [11]. QED, the relativistic quantum field theory of electrodynamics, describes the interaction between electrically charged particles by the exchange of a quantum of light; the photon $(\gamma)$. It explains processes such as pair production and electron positron annihilation, providing a framework for particle production via the electromagnetic interaction. The Feynman diagram, i.e. a graphical representation of the interactions between particles on microscopic scale, of the fundamental QED vertex is shown in Fig. 1.3. All electromagnetic processes can be represented by one or multiple of these diagrams.

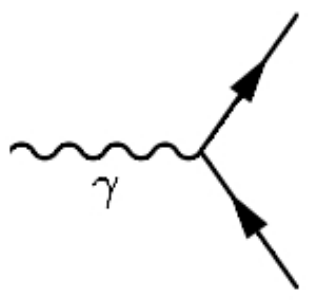

FIGURE 1.3: Feynman diagram of the fundamental QED interaction.

The QED Lagrangian obeys local U(1) symmetry and is given by:

$$
\mathcal{L}_{\mathrm{QED}}=\bar{\psi}\left(i \gamma^{\mu} D_{\mu}-m\right) \psi-\frac{1}{4} F_{\mu \nu} F^{\mu \nu}
$$

where $D_{\mu}=\partial_{\mu}+i q A_{\mu}$, such that local $U(1)$ symmetry is ensured. The QED Lagrangian contains a term for a free spin-half particle, an interaction term, and a kinetic term. It is the vector field $A_{\mu}$ which we associate to the photon. Adding a mass term would break gauge invariance and as such the photon is a massless particle moving at the speed of light in vacuum. Additionally, the classical Maxwell equations can be obtained from this Lagrangian by calculating the equations of motion with the Euler-Lagrange equation. This theory makes it possible to calculate the dynamics of the electromagnetic processes within high-energy particle collisions. 


\subsection{Quantum chromodynamics}

The strong force is responsible for the formation of hadrons such as the pions, kaons, and protons. The constituents of hadrons are the quarks, which are confined via the exchange of gluons, the force carrier of the strong force. For example, the proton has three valence quarks; two up quarks and one down quark, giving it a total electric charge of +1 . It is the theory of quantum chromodynamics(QCD) [12] that describes the interactions between quarks and gluons, with QCD interaction vertices shown in Fig. 1.4.
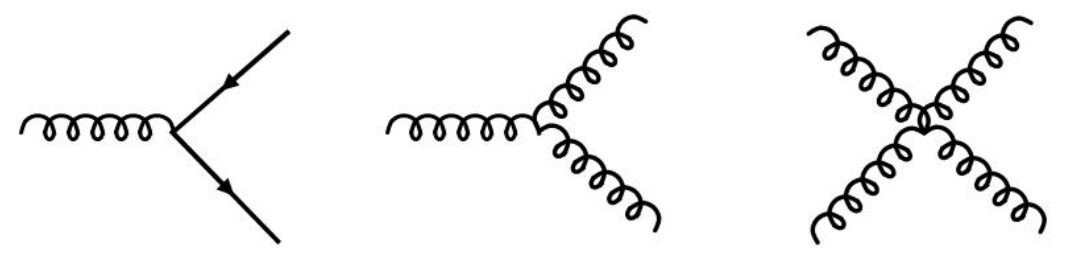

FIGURE 1.4: Feynman diagrams of the fundamental QCD interactions, where the straight and circular lines represent the quark and gluon, respectively. The additional diagrams are due to the self-coupling of the gluon.

QCD is invariant under local SU(3) transformations,

$$
\psi(x) \rightarrow \psi^{\prime}(x)=\left[i g_{s} \alpha(x) \cdot \hat{\mathbf{T}}\right] \psi(x)
$$

where $g_{s}$ is the strength of the strong interaction and $\alpha(x)$ are arbitrary functions of space-time. Furthermore, $\hat{\mathbf{T}}=\left\{T^{a}\right\}$ are the eight generators of the $\mathrm{SU}(3)$ symmetry group and are represented by $3 \times 3$ matrices. The three additional degrees of freedom are referred to as the color charges red, blue, and green. Local gauge invariance is obtained by introducing eight gluon fields $G_{\mu}^{a}(x)$, with $a=1, \ldots, 8$, corresponding to the generators of the $\mathrm{SU}(3)$ symmetry group. The Dirac equation

$$
i \gamma^{\mu}\left[\partial_{\mu}+i g_{s} G_{\mu}^{a} T^{a}\right] \psi-m \psi=0,
$$

is invariant under local SU(3) phase transformations if $G_{\mu}^{k}$ transforms as

$$
G_{\mu}^{k} \rightarrow G_{\mu}^{k \prime}=G_{\mu}^{k}-\partial_{\mu} \alpha_{k}-g_{s} f_{i j k} \alpha_{i} G_{\mu}^{j}
$$

where $f_{i j k}$ are the structure constants of the $\mathrm{SU}(3)$ group. One of the main features of this theory is that the generators of $\mathrm{SU}(3)$ do not commute, making QCD a non-Abelian gauge theory in which the gluon couples not only to quarks but also to itself. In turn this leads to anti-screening of color charge and a decreasing strong coupling constant $\alpha_{s}$ for increasing momentum scale; see Fig. 1.5. The evolution of $\alpha_{s}$ as function of momentum transfer $\mathrm{Q}$ is given by 


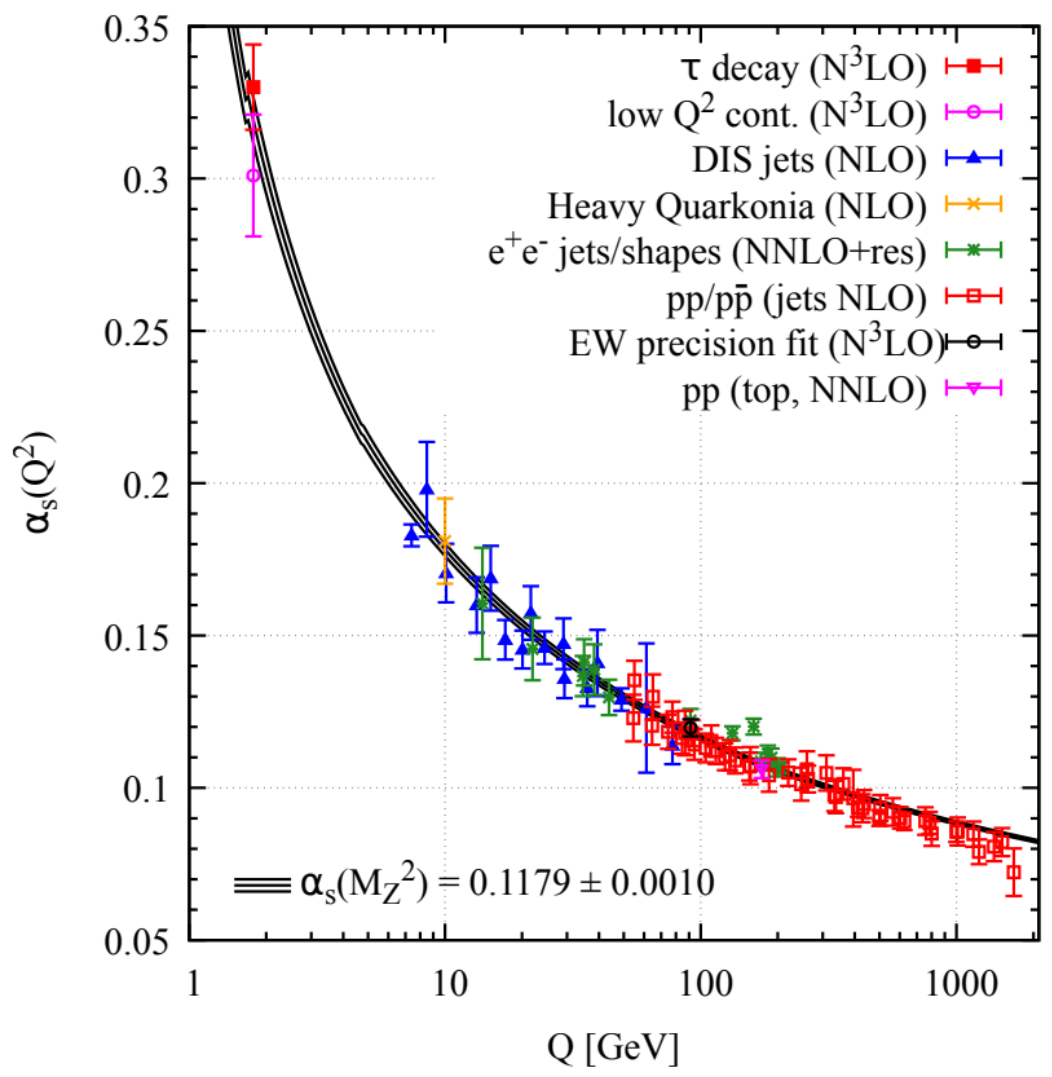

Figure 1.5: Evolution of the strong coupling constant $\alpha_{s}$ as function of momentum transfer $Q^{2}$. Figure taken from [13].

$$
\alpha_{s}\left(Q^{2}\right)=\frac{12 \pi}{\left(33-2 N_{\mathrm{f}}\right) \ln \left(Q^{2} / \Lambda_{\mathrm{QCD}}^{2}\right)},
$$

where $N_{\mathrm{f}}$ is the number of quarks and $\Lambda_{\mathrm{QCD}}$ is the QCD energy scale parameter. It can be seen that $\alpha_{s}$ decreases for increasing $Q^{2}$. This phenomenon is called asymptotic freedom, which allows for perturbation theory to be applied in this regime. For low energies, $\alpha_{s}$ becomes large and the quarks are confined in colorless objects. This is known as confinement.

As perturbation theory is applicable at large momentum transfer where the coupling constant is small, perturbative QCD (pQCD) is only able to describe phenomena involving high energy quarks and gluons. However, many processes happen at a much lower energy scale where the coupling constant is of order unity and perturbative QCD breaks down. This is where lattice QCD [14, 15] comes in, which is a tool for calculating processes in the non-perturbative energy regime. Lattice QCD is formulated on a discrete space-time grid, without introducing additional parameters besides the coupling constant and the quark masses of QCD itself. This results in a framework in which numerical Monte-Carlo simulations are able to study phenomena such as confinement and chiral symmetry breaking. It also provides an approach to perform fundamental calculations of the pressure, energy density, and entropy of QCD matter as a function of its temperature. Hot QCD matter is the main topic of study of heavy-ion physics. 


\section{Chapter 2}

\section{Heavy-ion physics}

\subsection{The quark gluon plasma}

In the early universe, just microseconds after the big bang, the universe was filled with extremely hot and dense matter. With very high temperatures, this primordial matter was too hot for any ordinary hadrons such as protons and neutrons to form. After the universe sufficiently expanded, it cooled down, and underwent a phase transition from hot QCD matter to ordinary matter. First-principle lattice QCD calculations support this picture and have shown that the phase transition proceeds via a continuous crossover rather than a first order phase transition [16]. This also has the implication that inhomogeneities of the early universe should leave no imprint in the observable universe that we can access with cosmological observations. It is only with ultra relativistic heavy-ion collisions in the laboratory, that we can create little droplets of big bang matter and study the conditions of the early universe [17].

Mathematically, we use the equation of state (EOS) to describe the hot QCD matter [18-20], which relates the temperature, pressure, and energy density. These thermodynamic quantities can be calculated on the lattice using the trace of the energy-momentum tensor $\Theta^{\mu \mu}(T)$, also called the trace anomaly, that is defined as

$$
\frac{\Theta^{\mu \mu}(T)}{T^{4}} \equiv \frac{\epsilon-3 p}{T^{4}}=T \frac{\mathrm{d}}{\mathrm{d} T}\left(\frac{p}{T^{4}}\right)
$$

where $T$ is the temperature, $\epsilon$ the energy density, and $p$ the pressure of the system. The pressure can be calculated by inverting the above equation;

$$
\frac{p}{T^{4}}=\frac{p_{0}}{T_{0}^{4}}+\int_{T_{0}}^{T} \mathrm{~d} T^{\prime} \frac{\Theta^{\mu \mu}}{T^{\prime 5}} .
$$



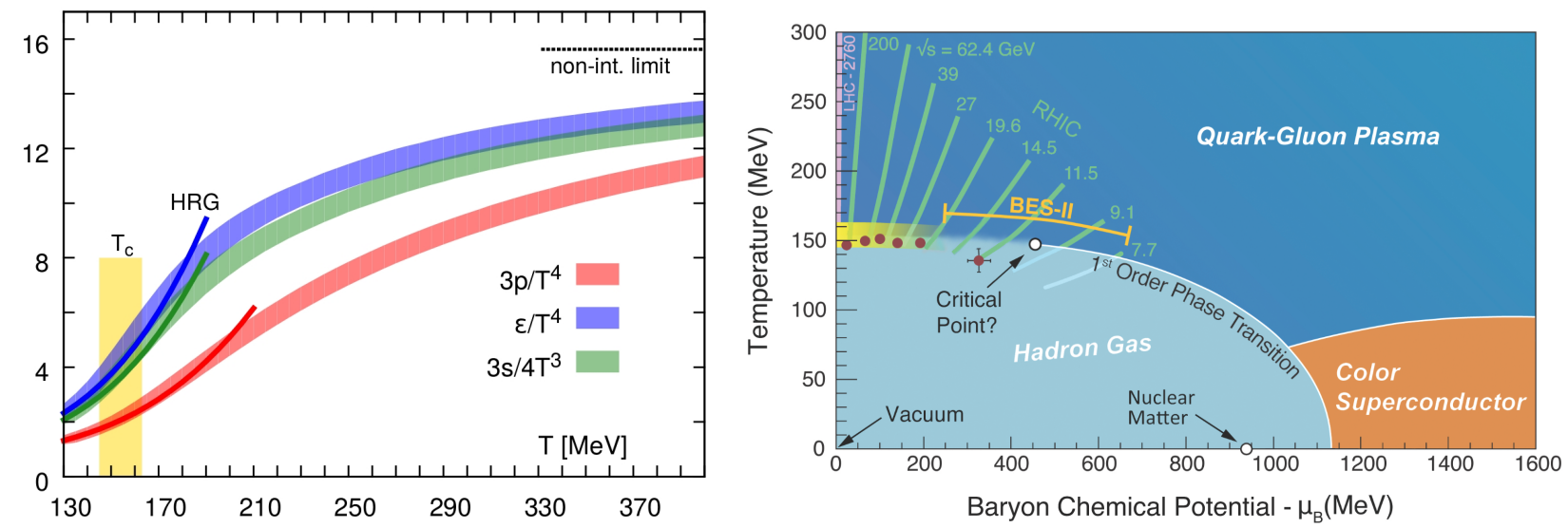

Figure 2.1: Left: The thermodynamic quantities proportional to the pressure $\left(3 p / T^{4}\right)$, energy density $\left(\epsilon / T^{4}\right)$, and entropy $\left(3 s / 4 T^{4}\right)$ of QCD matter as function of the temperature $(T)$. These quantities are calculated using the lattice QCD formalism, showing a phase transition from a hadron gas to a quark gluon plasma (QGP) at the critical temperature of $T_{C} \approx 150 \mathrm{MeV}$. Right: The phase diagram of QCD matter, indicating the phases as function of Baryon chemical potential $\left(\mu_{B}\right)$ and temperature $(T)$. Figures taken from $[17]$.

The entropy $s=(\epsilon+p) / T$ can be obtained by combining the results of $p / T^{4}$ and $(\epsilon-3 p) / T^{4}$ from equations given above. This fully defines the equation of state from lattice calculations, with all the difficulties absorbed in the trace anomaly $\Theta^{\mu \mu}(T)$.

Figure 2.1 (left) shows the result of a lattice QCD calculation of the pressure, energy density, and entropy of QCD matter at thermal equilibrium, as function of the temperature. At the Stefan-Boltzmann limit, which is reached at infinite temperature, we expect the hot QCD matter to behave like a non-interacting gas of quark and gluons. This limit is shown in the figure by the dashed line, i.e. the non-interacting limit. Between lower and higher temperatures there is a continuous crossover at $T_{c} \approx 150 \mathrm{MeV}$, from a hadron resonance gas to a deconfined system of quarks and gluons what we call the quark gluon plasma (QGP). The deconfinement of the quarks manifests itself in additional degrees of freedom, as seen in the rise of energy density and entropy, creating the "soup of quark and gluons".

Another way to look at the phase transitions of matter is via phase diagrams. These diagrams define the state of matter at a given temperature and pressure, which is then typically either a solid, liquid, or gas, like the phase diagram of water. A similar diagram can be made for QCD matter, and is shown in Fig. 2.1 (right). The state of the QCD matter is plotted as function of the temperature and net baryon density, which is related to the excess of quarks over antiquarks. Collisions between heavy nuclei at the highest LHC energies produces thousands of particles, leading to greater balance between quarks and antiquarks in the final state at mid-rapidity, such that $\mu_{B} \approx 0$. In collisions at lower energies, less particles are produced, and the amount of quarks that constitute the incoming nuclei create a larger net amount of quarks, such that $\mu_{B}>0$. This has the implication that different beam energies can access different points on the QCD diagram of Fig. 2.1, as indicated by the beam energy scan (BES) program at RHIC, and can potentially study the critical point of QCD matter [21, 22]. 


\subsection{Proton proton collisions}

Before discussing the phenomena that emerge by colliding heavy ions, it is important to discuss the physics of proton proton (pp) collisions for two main reasons. First, as the quark gluon plasma can be created in the laboratory by colliding heavy ions, which themselves consist out of many protons and neutrons, signals of the quark gluon plasma may be masked by phenomena caused by the processes that are already present in pp collisions. This means that for many measurements it is worthwhile to have a reference and attempt to disentangle the contributions of the plasma and the superimposed pp collisions. It is thus important to not only measure an observable in heavy-ion collisions, but compare it to the same observable as measured in pp collisions, accessing the modification due to the produced plasma. Secondly, even though the fundamental interactions are quite well understood, many subtleties of particle production mechanisms are not, especially for

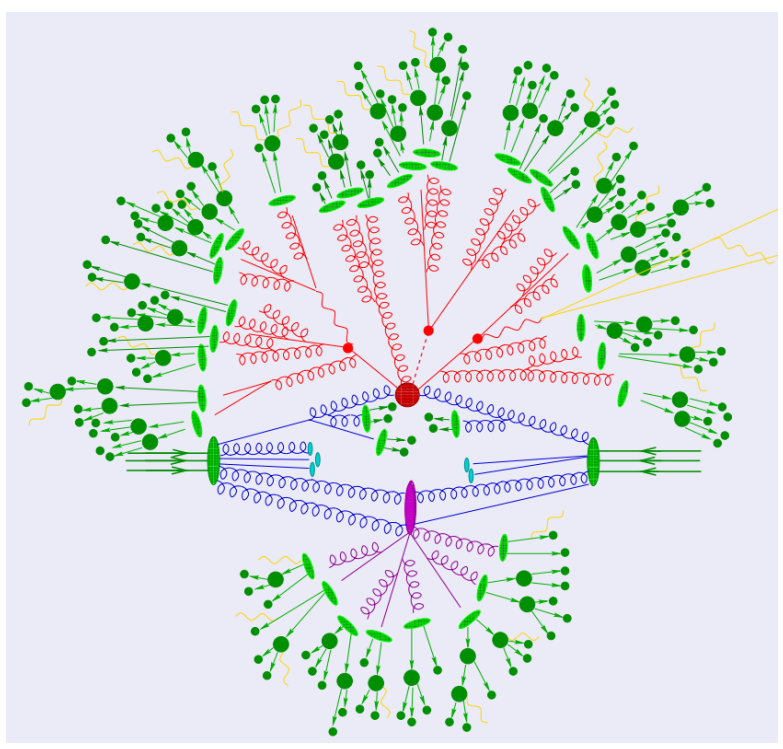

Figure 2.2: Sketch of a single proton proton collision, including the initial hard scattering, interactions from the partons, parton showers, and the hadronization into the final state particles.

particles with low to modest momenta where perturbative calculations break down. One example of the recent findings is the increasing enhancement of strange particle production relative to non-strange particle production for collisions producing an increasing amount of particles [23], which in short is referred to as strangeness enhancement. Another example is the observation of long range correlations, or flow, in $\mathrm{pp}$ collisions [24]. Both of these examples were thought to be phenomena of heavy-ion collisions.

The difficulty in understanding the processes involved in pp collisions comes from the complexity of the inner structure of the proton [25]. The proton itself consists of three valence quarks (uud), as well as a sea of quarks, antiquarks, and gluons. In a simplified view, for a proton with a large longitudinal momentum, the valence quarks each carry a third of the momentum of the proton, but in reality the momentum is distributed among all the constituents of the proton including the sea of virtual particles. The constituents of the proton are commonly referred to as partons, dating back to the parton model proposed by Richard Feynman in 1969. In a collision between two protons, any of the partons of the first proton can interact with the partons of the other proton, producing additional particles. This implicates that in a collision between two protons there can be multiple subsequent processes apart from the hardest process, and is referred to as multi-parton interactions. The particles produced in these interactions are able to radiate even more particles that produce parton showers, after finally hadronizing into the final state particles. This collection of processes is depicted in Fig. 2.2, which indicates that a single proton proton collision is highly non-trivial, which is important to keep in mind when discussing the phenomena of heavy-ion collisions. 


\subsection{Heavy-ion collisions}

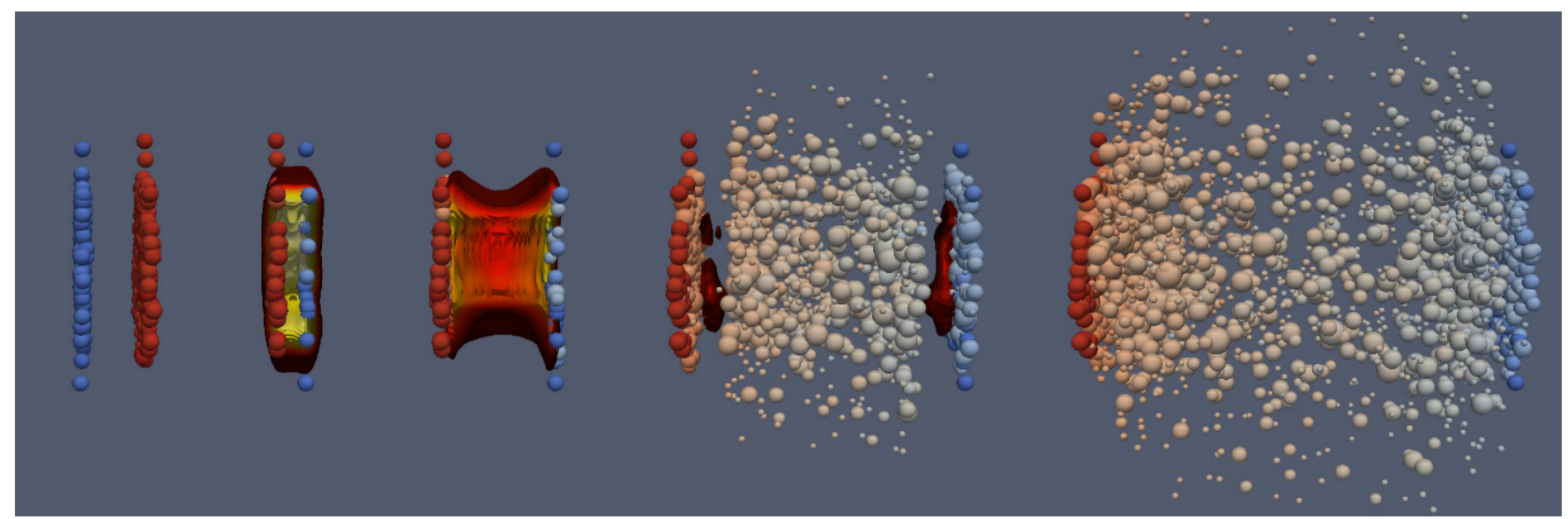

Figure 2.3: Simulation of the different stages of a heavy-ion collision, indicated from left to right for increasing time [26].

We can create a quark gluon plasma in the laboratory using ultra relativistic heavy-ion collisions. This process is shown in Fig. 2.3, where the five different snapshots each are at a subsequent later time and total about $10 \mathrm{fm} / c$ or $10^{-23} \mathrm{~s}$. The ions are Lorentz contracted due to their relativistic velocity relative to the lab frame. The overlap region of the two discs is where the QGP forms, while the rest of the nucleus disintegrates and continues in the longitudinal direction. The nucleons in the overlap regions are defined as the participants, and the other nucleons are defined as the spectators. With more participants, there are more nucleons colliding, creating a larger overlap region and increasing the size of the QGP. Similarly, the more participants, the more collision energy is available for the production of particles. In a simplified view, the quark gluon plasma is created, expands, cools down, and finally hadronizes back into ordinary matter. The different stages of the collision and our attempts to model them will be discussed in more detail in the next subsections.

\subsubsection{Initial state and pre-QGP phase}

First of all, it is important to note that there are two subsequent stages of the collisions that are both sometimes referred to as the the initial state; the state of the system before the actual collision, and the state of the matter right after first contact, where the latter is also referred to as the early state [27-29]. The term initial state will be used for the former, and the term early state for the latter. The initial state is described by the position and momentum distributions of the partons inside the incoming nuclei, whereas the early state, is described by dense color fields. With conditions leading to the formation of the QGP, the early state is also the pre-QGP phase.

In a simplified picture, each nucleon contains three valence quarks, dressed up by gluons and quark-antiquark pairs continuously being created and annihilated. As such, the interactions during the collision are predominantly involving low $-x$ gluons, where $x$ is the longitudinal momentum fraction of the gluon with respect to 
the particle [30]. Most of these individual interactions are relatively soft with little momentum transfer and thus only a small amount of transverse momentum is generated. At first contact, the distance between the centers of the incoming nuclei is defined as the impact parameter $b$. As the two colliding discs just passed each other, the two are connected by a very high density longitudinal color field, continuously creating quark antiquark pairs and creating a system with densities over twenty times that of a ordinary hadron. There are different models of the initial and early state that generate energy and entropy profiles of the collision zone, and are able to estimates the collision zone eccentricities that are used in full QGP model calculations which are able to explain the momentum anisotropies of the final state particles. Two of these models are now discussed briefly.

The Monte-Carlo Glauber model [31] is a non-dynamical model that relates the impact parameter (b), to the number of participating nucleons $N_{\text {part }}$, the number of spectator nucleons $N_{\text {spec }}$, and the amount of binary nucleon-nucleon collisions $N_{\text {coll }}$. It works by superimposing the geometry of two colliding disks with impact parameter $b$, and calculating $N_{\text {coll }}, N_{\text {part }}$, and $N_{\text {spec }}$ from the distribution of nucleons inside the nuclei. Due to the Monte Carlo nature of this model, it contains fluctuations in the nucleon distribution in the nucleus, in contrast with the analytical Glauber models. It describes the measured charged particle multiplicity distributions over a wide range in pseudorapidity when taking larger intervals of collision centrality, and confirms that $N_{\text {ch }}$ increases monotonically for decreasing $b$.

IP-Glasma is a dynamical model using color-glass condensate (CGC) effective field theory [32], that describes the initial state before and after first contact by colliding sheets of gluon fields. This model can include both the fluctuations in the nucleon distribution in the nucleus, as well as fluctuations of the color charge distributions of the nucleons, which are both subject to event-by-event fluctuations. In comparison to the Glauber model, full QGP model calculations using the collision zone eccentricities and energy density profiles calculated with the IP-Glasma model are better describing the momentum anisotropies found in data for smaller intervals of collision centrality, because it can generate long range rapidity correlations from the initial state wave-functions [33]. There is still a lot of debate ongoing that discusses the validity and the
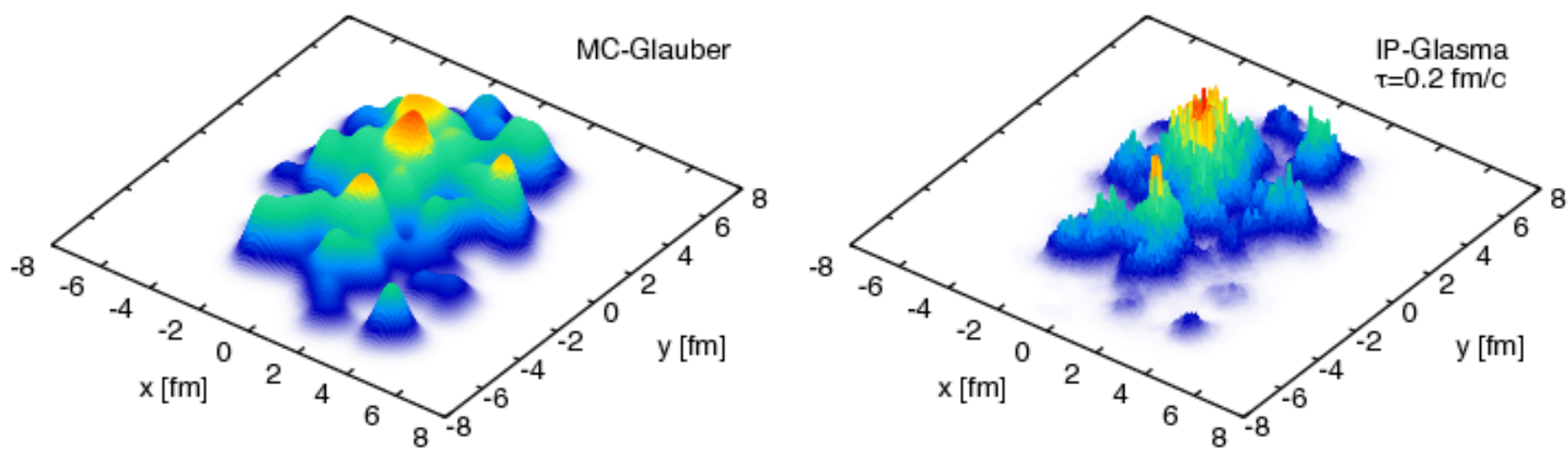

Figure 2.4: Example energy density profiles of the initial state just after first contact of the incoming nuclei, for the Glauber Monte Carlo model (left) and IP-Glasma (right). 
range of applicability of these initial state and pre-QGP phase models. Examples energy density profiles of the initial state just after the impact of the incoming nuclei is shown in Fig. 2.4.

\subsubsection{QGP phase}

The quark gluon plasma is a deconfined system of quarks and gluons, arising from the high temperature and energy density of the early state after the system thermalizes. In the QGP phase, the temperature is above the critical temperature of the phase transition $\left(T>T_{c} \sim 150 \mathrm{MeV}\right)$, and in its evolution the QGP will expand, cool down, and reach the temperature at which the system hadronizes. Understanding exactly what drives this thermal system is at the core heavy-ion physics.

Relativistic hydrodynamics is used to model this space-time evolution of the QGP by approximating it as a relativistic fluid with low specific shear viscosity [34]. It relies on a continuity equation, ensuring the conservation of energy, momentum and baryon number, which is given by:

$$
\partial_{\mu} T^{\mu \nu}=\partial_{\mu}\left((\epsilon+P) u^{\mu} u^{\nu}-P g^{\mu \nu}\right)=0,
$$

where $T^{\mu \nu}$ is the energy-momentum tensor, $\epsilon$ the energy density, $P$ the pressure, $u^{\mu}$ the four-velocity of the fluid element, and $g^{\mu \nu}$ the Minkovski metric tensor. This equation, together with the initial conditions, equation of state of the QGP, and transport coefficients, determines how the system evolves over time. Measurements have shown that the QGP has an extremely low specific shear viscosity; it is a near perfect liquid $[35,36]$. The low viscosity implies that the system is strongly coupled. Furthermore, it is interesting to note that in the transverse direction the expansion is mainly driven by the energy density distributions of the initial state, implying a relationship between the initial geometry of the system and the final velocity profiles of the fluid. Here, relativistic hydrodynamics translates spatial anisotropies into momentum anisotropies.

\subsubsection{Hadronization}

After sufficient expansion and cooling down of the quark gluon plasma (after a time of the order of $10 \mathrm{fm} / c$ ) [37], the temperature will go below the critical temperate and confinement will set in. It is important to note that even after the phase transition, the state of the system is described as a hadron gas, which is still a thermal system continuing to contribute to thermal observables. The confinement of the QGP constituents proceeds locally throughout the plasma and it is expected that this process also determines most particle species. The point in time where all particle species are frozen is called the chemical freeze-out. Although the particles are again confined in hadrons, the density is still so large that interactions continue to happen. At a later time the system expanded so much that the particles are far enough apart to stream freely, this is called the kinetic freeze-out. From this time onwards the particles continue their trajectory. 
There is still an active debate on the exact mechanisms of hadronization in both small and large collision systems. Measuring in detail the production of different type of particles and their correlations addresses various open questions. In the more simple case of pp collisions, the process of hadronization is described by fragmentation, but is not fully understood and requires ad hoc assumptions [38-40]. For heavy-ion collisions popular ways to describe hadronization is either via fragmentation-like processes as in pp collisions, or via a coalescence picture. In the coalescence model [41, 42], the basic idea is that co-moving partons in the QGP will combine their transverse momentum to form a hadron with higher transverse momentum.

\subsection{Probes of the QGP}

\subsubsection{Particle production}

Measuring as precisely as possible how many and which particles are produced in high-energy collisions greatly helps in understanding what happens during the collision. Studying particle production tries to answers questions like: how many charged particles does a collision produce on average, what is the invariant yield of specific mesons and baryons, and how does this relate to the characteristics and underlying mechanisms of the collision [43]. It is common to perform these measurements as function of the transverse momentum $\left(p_{\mathrm{T}}\right)$ of an identified particle, which covers a range from the order of $100 \mathrm{MeV} / c$ where particle production is dominated by soft QCD processes, all the way up to tens or hundreds of $\mathrm{GeV} / c$ where particle production is dominated by hard QCD interactions [44, 45].

Particle production mechanisms in pp collisions are far from understood. While perturbative QCD is able to calculate processes involving large momentum transfers leading to particles with high $p_{\mathrm{T}}$, most of the particles produced in the collisions have a momentum for which the perturbative approach breaks down. Nevertheless we can measure the $p_{\mathrm{T}}$ spectra of many different particle species and come up with phenomenological models that attempt to describe their production.

The particle production mechanisms in collisions between two heavy-ions present us with additional difficulties. First, particles produced in hard processes, thus from parton fragmentation, are affected by the presence of the quark gluon plasma. This includes effects like energy loss of the particle while traversing the medium, as well as receiving additional momentum due to medium expansion. Secondly, the extreme temperatures of the QGP make it possible to thermally produce additional quarks and gluons, which hadronize into additional final state particles.

The cross section of $\sigma_{h_{1} h_{2} \rightarrow a}$ represents the likelihood of colliding hadrons $h_{1}$ and $h_{2}$, and producing a particle $a$. Assuming that the scattering processes are independent from the fragmentation of the produced partons, the cross section is factorisable $[44,45]$ and can be calculated as:

$$
\sigma_{h_{1} h_{2} \rightarrow a}=f_{i}^{h_{1}}\left(x_{1}, Q^{2}\right) f_{j}^{h_{2}}\left(x_{2}, Q^{2}\right) \otimes \sigma^{i j \rightarrow k}\left(x_{1} p_{1}, x_{2} p_{2}, Q^{2}\right) \otimes D_{k \rightarrow a}\left(z, Q^{2}\right),
$$


where $f_{i}^{h_{1}}$ and $f_{i}^{h_{2}}$ are the parton distribution functions (PDFs) of the colliding particles, $\sigma^{i j \rightarrow k}$ the partonic cross sections., i.e. the chance that parton $i$ and $j$ produce the parton $k$, and $D_{k \rightarrow a}$ is the fragmentation function describing the production of particle $a$ from parton $k$. The PDFs for protons and the PDFs for nuclei (nPDFs) give the average quark and gluon content of the nucleon (either free or bound in a nucleus), as function of the longitudinal momentum fraction $x$ and momentum transfer $Q^{2}$.

\subsubsection{Energy loss}

As described above, particles traversing the QGP will lose energy via elastic scatterings and gluon radiation within the medium, modifying their momenta [46, 47]. A proper way to study this effect is by measuring their invariant yield in $\mathrm{Pb}-\mathrm{Pb}$ collisions and compare it to the invariant yield obtained in pp collisions. It is expected that the spectra in $\mathrm{Pb}-\mathrm{Pb}$ collisions are modified with respect to an incoherent superposition of the spectra in pp collisions. At low particle $p_{\mathrm{T}}$, the limit of incoherent superposition is not assumed to be valid, leading to a modification. At higher $p_{\mathrm{T}}$, the main production mechanism of hadrons is through hard-scatterings of quarks and gluons, processes which should allow to apply an incoherent superposition. Here, however, a modification can happen because the final state partons lose energy when traversing the medium.

In addition to such modifications due to final state interactions, which probe the produced medium, there may also be modifications of the initial state (e.g. the PDFs) in a nucleus compared to a proton. While in $\mathrm{Pb}-\mathrm{Pb}$ collisions one expects thus both final state and initial state modifications, it is expected that in $\mathrm{p}-\mathrm{Pb}$ collisions no significant medium is produced, such that final state effects should be absent. Comparing $p_{\mathrm{T}}$ spectra from $\mathrm{p}-\mathrm{Pb}$ to $\mathrm{pp}$ thus probes these so called cold nuclear matter effects, such as gluon saturation [48], nuclear shadowing [49], and Cronin enhancement [50]. The modification compared to pp is quantified by the two nuclear modification factors:

$$
R_{\mathrm{AA}}\left(p_{\mathrm{T}}\right)=\frac{\mathrm{d}^{2} N /\left.\mathrm{d} p_{\mathrm{T}} \mathrm{d} y\right|_{\mathrm{AA}}}{\left\langle T_{\mathrm{AA}}\right\rangle \mathrm{d}^{2} \sigma /\left.\mathrm{d} p_{\mathrm{T}} \mathrm{d} y\right|_{\mathrm{pp}}}, \quad R_{\mathrm{pA}}\left(p_{\mathrm{T}}\right)=\frac{\mathrm{d}^{2} N /\left.\mathrm{d} p_{\mathrm{T}} \mathrm{d} y\right|_{\mathrm{pA}}}{\left\langle T_{\mathrm{pA}}\right\rangle \mathrm{d}^{2} \sigma /\left.\mathrm{d} p_{\mathrm{T}} \mathrm{d} y\right|_{\mathrm{pp}}},
$$

with the nuclear overlap functions $\left\langle T_{\mathrm{AA}}\right\rangle$ and $\left\langle T_{\mathrm{pA}}\right\rangle$ defined as:

$$
\left\langle T_{\mathrm{AA}}\right\rangle=\frac{\left\langle N_{\text {coll }}\right\rangle}{\sigma_{\text {inel }}^{\mathrm{pp}}}, \quad\left\langle T_{\mathrm{pA}}\right\rangle=\frac{\left\langle N_{\text {coll }}\right\rangle}{\sigma_{\text {inel }}^{\mathrm{pp}}} .
$$

In a naive view, $T_{\mathrm{AA}}$ scales up the spectra of pp collisions to $\mathrm{A}-\mathrm{A}$ collisions by the assumption that a $\mathrm{A}-\mathrm{A}$ collisions are an incoherent superposition of pp collisions. A clear modification of the particle spectra in $\mathrm{Pb}-\mathrm{Pb}$ or $\mathrm{p}-\mathrm{Pb}$ collisions with respect to $\mathrm{pp}$ collisions can probe the hot and cold nuclear matter effects, respectively. More broadly, the $R_{\mathrm{AA}}$ observable can be measured experimentally for specific identified particles, probing possible effects due to the properties of the particle, such as mass, charge, and quark content in case of mesons and baryons [51]. 


\subsubsection{Anisotropic flow}

Heavy ions are length contracted in the longitudinal direction when approaching the speed of light, making them Lorentz boosted "disks" in the laboratory frame. In the collisions between these ions, the geometry of the overlap region is mostly defined by the impact parameter $b$, which is the distance between the two centers of the colliding nuclei projected on the transverse plane. The impact parameter is a measure for the centrality of the collision, and is commonly estimated by the event multiplicity. For an impact parameter $b \approx 0$, the collision geometry becomes azimuthally symmetric, while for larger impact parameters it becomes azimuthally asymmetric, as shown in Fig. 2.5. This asymmetry, or spatial anisotropy, of the system translates into a momentum anisotropy driven by the collective expansion of the medium. It is more commonly called "anisotropic flow" or just "flow" [52]. Measuring flow gives insight into the dynamics of the QGP, as this observable can also be calculated from various models. Particle distributions as function of the azimuthal angle can be described by the Fourier expansion

$$
E \frac{\mathrm{d}^{3} N}{\mathrm{~d}^{3} p}=\frac{1}{2 \pi} \frac{\mathrm{d}^{2} N}{p_{\mathrm{T}} \mathrm{d} p_{\mathrm{T}} \mathrm{d} y}\left(1+\sum_{n=1}^{\infty} 2 v_{n} \cos \left(n\left(\varphi-\Psi_{R}\right)\right)\right)
$$

where $\varphi$ is the azimuthal angle of the particle and $\Psi_{R}$ the angle between the reaction plane and the laboratory frame [53]. The reaction plane is the plane spanned by the orientation of the impact parameter $b$ and the $z$-axis. The Fourier coefficients $v_{n}$ are given by:

$$
v_{n}=\left\langle\cos \left(n\left(\varphi-\Psi_{R}\right)\right)\right\rangle
$$

where $\langle\ldots\rangle$ denotes the average over particles and events. The lower order Fourier coefficients $v_{n}$ are more commonly called directed flow $\left(v_{1}\right)$, elliptic flow $\left(v_{2}\right)$ and triangular flow $\left(v_{3}\right)$ [54-56].

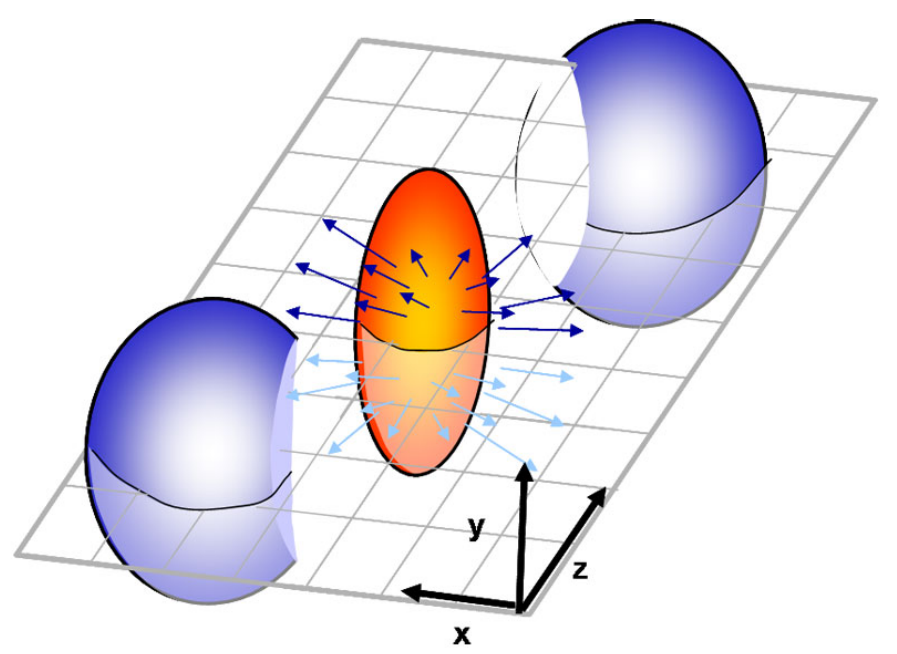

FiguRE 2.5: The collision geometry of a heavy-ion collision where the two nuclei are displaced, creating a azimuthally asymmetric medium. 


\subsubsection{Hanbury-Brown-Twiss interferometry}

Hanbury-Brown-Twiss (HBT) interferometry, was introduced as a way to measure the radii of stars using correlation effects of the intensity received in two independent detectors [57]. From quantum mechanics it turns out that there is an enhancement in the coincidence count of identical bosons with small momentum differences. This is due to quantum statistical effects described formally by the symmetrization of the multiparticle wave function for bosons which are close in phase-space. Furthermore, as for larger momentum differences this effect disappears, the width of the enhancement can be used to infer the size and sometimes even the shape of the emitting source.

Analogously in heavy-ion physics, the HBT correlations of identical particles provide information of their emission source, in this case the QGP. These HBT correlations, also called femtoscopy in this context, probe the space-time evolution of the system, and it is sensitive to the freeze out radii and thus the emission time of particles produced by the QGP (or in general the so-called lengths of homogeneity) [58-62]. The observed enhancement in the coincidence count carries information about the source from the moment these particles cease to interact, as most hadron are produced rather late in the evolution of the system. This moment in time is referred to as the kinetic freeze-out, as discussed earlier in this chapter. Furthermore, measuring the HBT correlations for identified particles with different masses and production mechanisms, such as pion-pion, kaon-kaon, proton-proton, and even photon-photon correlations, it is possible to access rich dynamical information about the times that these particles are emitted by the plasma, leading to a quantitative characterization of the emitting source at freeze-out. Photons are of particular interest, since they are emitted continuously by the hot plasma and do not have any further interactions with the plasma as their mean free path is much larger than the size of the system, providing additional sensitivity to the early part of the QGP evolution.

Quantitatively, the correlation function $C(k)$ measures short-range two-particle correlations as a function of their relative momentum. The shape and magnitude of the correlation function $C(k)$ depends on the source function $S(r)$ via the Koonin-Pratt equation [63, 64]:

$$
C(k)=\int S(r)\left|\Psi_{k}(r)\right| d^{3} r
$$

where $\Psi_{k}(r)$ is the two-particle interaction kernel and $r$ is the relative distance between the correlated particles. It is often assumed that the source function $S(r)$ is Gaussian, such that $S(r) \sim e^{-r^{2} / 4 R^{2}}$. Thus, by measuring $C(k)$ and theoretically constraining $S(r)$ and $\Psi_{k}(r)$, the emission source can be studied. In the case of plane waves (non-interacting particles) and a stationary source, one gets the Fourier transform of the source distribution. Experimentally, $C(k)$ gets denoted by $C\left(Q_{\text {inv }}\right)$, and can be obtained by

$$
C\left(Q_{\text {inv }}\right)=\frac{A\left(Q_{\text {inv }}\right)}{B\left(Q_{\text {inv }}\right)}
$$


where $Q_{\text {inv }}$ is again the relative momentum of the considered particle pair, $A\left(Q_{\mathrm{inv}}\right)$ is the same-event twoparticle distribution, and $B\left(Q_{\text {inv }}\right)$ is the two-particle distribution obtained with the event-mixing technique. The term $B\left(Q_{\text {inv }}\right)$ is needed to normalize the correlations found in the same-event pair distributions, and divides out the background without HBT enhancement. For the analyses that use charged particles to measure the pair distributions there is an addition correction needed to account for Coulomb interactions between the pairs. There is no such correction needed when measuring photon correlations.

The measurements have shown that the source size parameter $R_{\mathrm{inv}} \approx 1.6 \mathrm{fm}$ for pp collisions and $R_{\mathrm{inv}} \approx$ $2.5 \mathrm{fm}$ for $\mathrm{p}-\mathrm{Pb}$ collisions, where the latter has an additional dependence on the particle multiplicity of the collision [65]. The measurement in $\mathrm{p}-\mathrm{Pb}$ indicates a smaller emission source compared to the radius of a cold $\mathrm{Pb}$ nucleus, which is of the order of $3.2 \mathrm{fm}$. Furthermore, it is confirmed that the source size of the system increases for more central $\mathrm{Pb}-\mathrm{Pb}$ collisions, relative to source size found for peripheral collisions. Model calculation are consistent with a time delay of $2.1 \mathrm{fm} / c$ for kaons relative to pions, and have different freeze-out radii [66]. This shows that the HBT correlations of an identified particle can pose additional theoretical constraints on the evolution of the QGP. Furthermore, the invariant yield and the emission source size of direct photons can be simultaneously constrained measuring their HBT correlations, and will be explored in a later chapter. 



\section{Chapter 3}

\section{Probing the QGP using photons}

\subsection{Photon production}

Electromagnetic probes are a powerful tool to study the quark gluon plasma [67-71]. It turns out that, due to the electromagnetic force being much weaker than the strong force, the mean free path of a photon in a QGP $\left(\lambda_{\gamma}\right)$ is much larger than the size of the QGP. This can be estimated by calculating the photon equilibration time

$$
\tau_{\gamma}=\frac{9}{10 \pi \alpha_{\mathrm{em}} \alpha_{s}} \frac{E_{\gamma}}{T^{2}} \frac{e^{E_{\gamma} / T+1}}{e^{E_{\gamma} / T-1}} \frac{1}{\ln \left(3.7388 E_{\gamma} / 4 \pi \alpha_{s} T\right)},
$$

where $\alpha_{\mathrm{em}}$ and $\alpha_{s}$ are the electromagnetic and strong coupling constants, respectively [72]. Furthermore, $E_{\gamma}$ is the energy of the penetrating photon and $T$ the temperature of the plasma. For reasonable values of $\alpha_{s}=0.4$ and $T=200 \mathrm{MeV}$, and using a photon energy of $E_{\gamma}=2 \mathrm{GeV} / c$, the photon equilibration time $\tau_{\gamma} \approx 480 \mathrm{fm} / c$, which is much larger compared to the lifetime of the plasma (order $10 \mathrm{fm}$ ). From this it follows that photons that are produced throughout the evolution of the QGP escape the medium unaffected, making them uniquely suited for providing information on the earlier stages of the deconfined plasma.

As photons are created via different production mechanisms, it is customary to make a distinction based on their source. The total number of photons, $N_{\gamma, \text { inclusive, is defined as }}$

$$
N_{\gamma, \text { inclusive }}=N_{\gamma, \text { decay }}+N_{\gamma, \text { direct }}
$$

where $\gamma_{\text {decay }}$ are the photons coming from hadronic decays, and $\gamma_{\text {direct }}$ are defined as the photons not coming from hadronic decays. The total number of direct photons are in turn defined as

$$
N_{\gamma, \text { direct }}=N_{\gamma, \text { prompt }}+N_{\gamma, \text { thermal }}+N_{\gamma, \text { other }}
$$




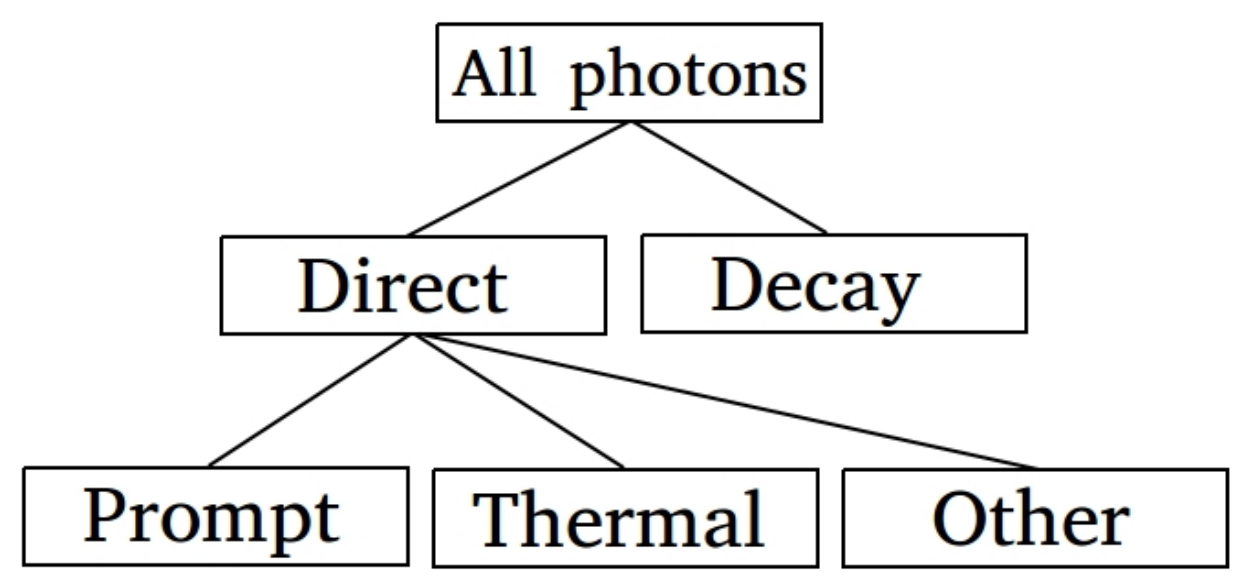

FiguRE 3.1: Overview of the different sources of photon produced in heavy-ion collisions.

where $\gamma_{\text {prompt }}$ are the photons from initial hard scattering processes, $\gamma_{\text {thermal }}$ the thermally produced photons from the hot plasma, and $\gamma_{\text {other }}$ the remaining photons from for example jet-medium interactions. These distinctions are shown schematically in Figure 3.1. It is important to note that the measurement of a single photon does not trivially reveal its source, leading to challenging experimental analysis methods.
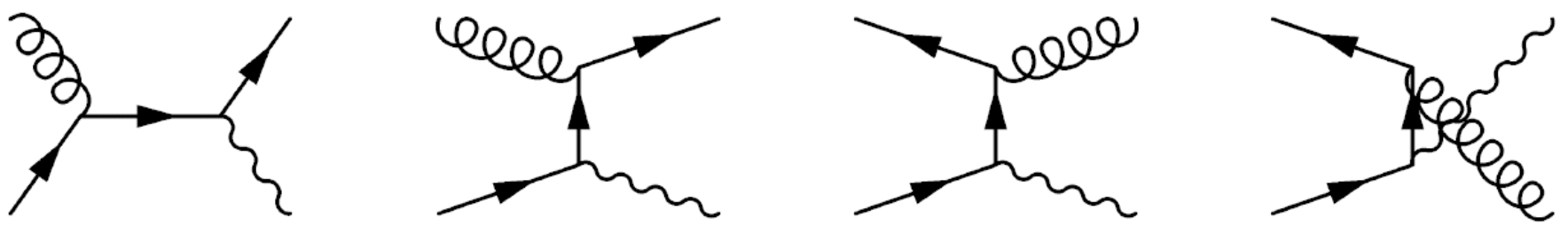

Figure 3.2: Leading order Feynman diagrams for photon production, excluding the photons that are produced in the decay of unstable hadrons.

For all these sources of photons, except the decay photons, the leading order Feynman diagrams are shown in Figure 3.2. The first two diagrams show the s- and t-channel quark-gluon Compton scattering diagrams producing a quark and photon in the final state, and the two last diagrams show the quark antiquark annihilation diagrams producing a photon and a gluon in the final state. It is via these processes that both the thermal, prompt, and photons from other sources apart from hadronic decays are produced. The calculations of these diagrams yield the amplitudes for photon emission, which have to then be folded with the quark and gluon momentum distributions. More details about thermal, prompt, and decay photons will now be discussed. 


\subsubsection{Thermal photons}

Like all thermal systems containing charged constituents, a QGP, and also a hadron gas (e.g. the Compton equivalent $\pi+\rho \rightarrow \pi+\gamma$ ), will radiate photons during its evolution [73-75]. These "thermal" photons dominate the direct photon spectrum at low transverse momentum, and the yield can be calculated by integrating the production rate over the space-time volume of the reaction zone:

$$
\frac{\mathrm{d}^{4} N_{\gamma, \text { direct }}}{\mathrm{d}^{4} k}=\int \mathrm{d}^{4} X \frac{\mathrm{d}^{4} \Gamma_{\gamma, \text { direct }}}{\mathrm{d}^{4} k}\left(K^{\mu}, u^{\mu}(X), T(X)\right),
$$

where $\Gamma_{\gamma \text {,direct }}$ is the thermal photon production rate, which depends on its four-vector $K^{\mu}$, flow velocity $u^{\mu}(X)$, and temperature of the thermal system $T(X)[76]$. The two most important factors for the total amount of thermal photons are the temperature and size of the emitting source. As the temperature goes down, the photon emission rate decreases. At the same time, a much smaller but yet significant effect is the increase of thermal photon yield due to the expansion of the spatial volume [75], which partially compensates the decrease in temperature. In addition, as the flow velocity of the plasma increases with time, the later the photon is emitted, the more blue-shifted it becomes. As a result, the measurement of the thermal photon spectrum is able to constrain the temperature and space-time evolution of the QGP, and provide valuable input for the QGP equation of state [77-80].

\subsubsection{Prompt photons}

Prompt photons are defined as the photons coming from hard parton collisions and parton fragmentation [81]. At $E_{\gamma, \text { prompt }} \gtrsim 4 \mathrm{GeV}$, the prompt photons are produced via processes such as quark-gluon Compton scattering and quark antiquark annihilation, where the corresponding amplitudes can be calculated by perturbative QCD, and the total cross section is calculated with Eq.2.4 much like the hadronic cross sections. At $E_{\gamma, \text { prompt }} \lesssim 4 \mathrm{GeV}$ the prompt photons are mainly produced as soft photon radiation coming from final state partons or the parton fragmentation. In principle these processes can be calculated in NLO (perturbative) QCD, which, however, is not applicable for lower $E_{\gamma, \text { prompt }}$. As such, there are currently no model calculations existent for this $p_{\mathrm{T}}$ region. A collection of several prompt photon measurements can be found in Ref. [82-85].

\subsubsection{Decay photons}

The largest fraction of photons is coming from the electromagnetic decay of unstable hadrons such as the $\pi^{0}$, $\eta, \omega$ mesons, and $\Sigma^{0}$ baryons. Understanding decay photons is relatively straightforward and relies on two aspects; the production of the hadrons that have a decay channel into photons and the decay kinematics. This means that if the $p_{\mathrm{T}}$ spectrum of the hadrons and decay channels are measured experimentally, the $p_{\mathrm{T}}$ spectrum of the decay photons are calculable. However, it is important to emphasize that even if the 
decay photons are theoretically straightforward to calculate, they are one of the largest sources of uncertainty for the extraction of direct photons, as the abundance of decay photons is so much larger. To illustrate this, imagine an inclusive photon sample consisting of $95 \%$ decay photons and $5 \%$ direct photons. Then, an uncertainty of only $1 \%$ on the amount of decay photons already implies a $20 \%$ uncertainty on the amount of direct photons.

\begin{tabular}{lll} 
Decay channel & Branching ratio & $\gamma_{\text {source }} / \gamma_{\text {decay }}$ \\
\hline$\pi^{0} \rightarrow \gamma \gamma$ & $98.8 \%$ & $\sim 80 \%$ \\
\hline$\eta \rightarrow \gamma \gamma$ & $39.4 \%$ & $\sim 15 \%$ \\
\hline$\omega \rightarrow \pi^{0} \gamma$ & $8.28 \%$ & $\sim 2 \%$ \\
\hline$\eta^{\prime} \rightarrow \rho^{0} \gamma$ & $28.9 \%$ & $\sim 1 \%$ \\
\hline$\phi \rightarrow \eta \gamma$ & $1.3 \%$ & $<1 \%$ \\
\hline$\rho^{0} \rightarrow \pi^{+} \pi^{-} \gamma$ & $9.9 \times 10^{-3} \%$ & $<1 \%$ \\
\hline$\Sigma^{0} \rightarrow \Lambda^{0} \gamma$ & $100 \%$ & $<1 \%$ \\
\hline
\end{tabular}

TABLE 3.1: Overview of hadronic decays that produce photons. Each hadron lists its most dominant decay channel into photons.

Table 3.1 shows the most relevant hadrons producing photons, with their corresponding branching ratios and an indicative fraction to the total amount of decay photons $\left(\gamma_{\text {source }} / \gamma_{\text {decay }}\right)$ that holds roughly for all collision systems. Since the $\pi^{0}\left(134.98 \mathrm{MeV} / c^{2}\right)$ is among the most abundant mesons and has a branching ratio of $98.8 \%$ into two photons, it is by far the largest source of decay photons. The $\eta$ meson $\left(547.86 \mathrm{MeV} / c^{2}\right)$ is the second biggest source of photons, producing about $15 \%$ of the decay photons. Then the next most dominant sources of decay photons contribute much less: $\omega$ with $\sim 2 \%, \eta^{\prime}$ with $\sim 1 \%$, and the remaining ones are below $1 \%$.

Measuring decay photons provides us with information on hadron production mechanisms for identified particles such as the $\pi^{0}$ and $\eta$, and are also the largest background for direct photons. So the decay photons are an interesting observable in itself, and essential for the study of electromagnetic probes, especially when characterizing the QGP.

\subsection{The direct photon puzzle}

From a theoretical model point of view, the yield of direct photons can be enhanced by increasing the temperature of the QGP, which enhances the production at early times. On the other hand, the anisotropic flow of direct photons is increased by later emission of the photons, when the flow of the system is more developed. As a consequence, models that increase the direct photon yields are decreasing the anisotropic flow of direct photons, and vice versa. This means that measuring both a large excess of direct photons and a sizable $v_{2}$ is difficult to reconcile theoretically [86]. However, this is exactly what was measured at RHIC by the PHENIX collaboration and posed a puzzle [77-80, 87]. 

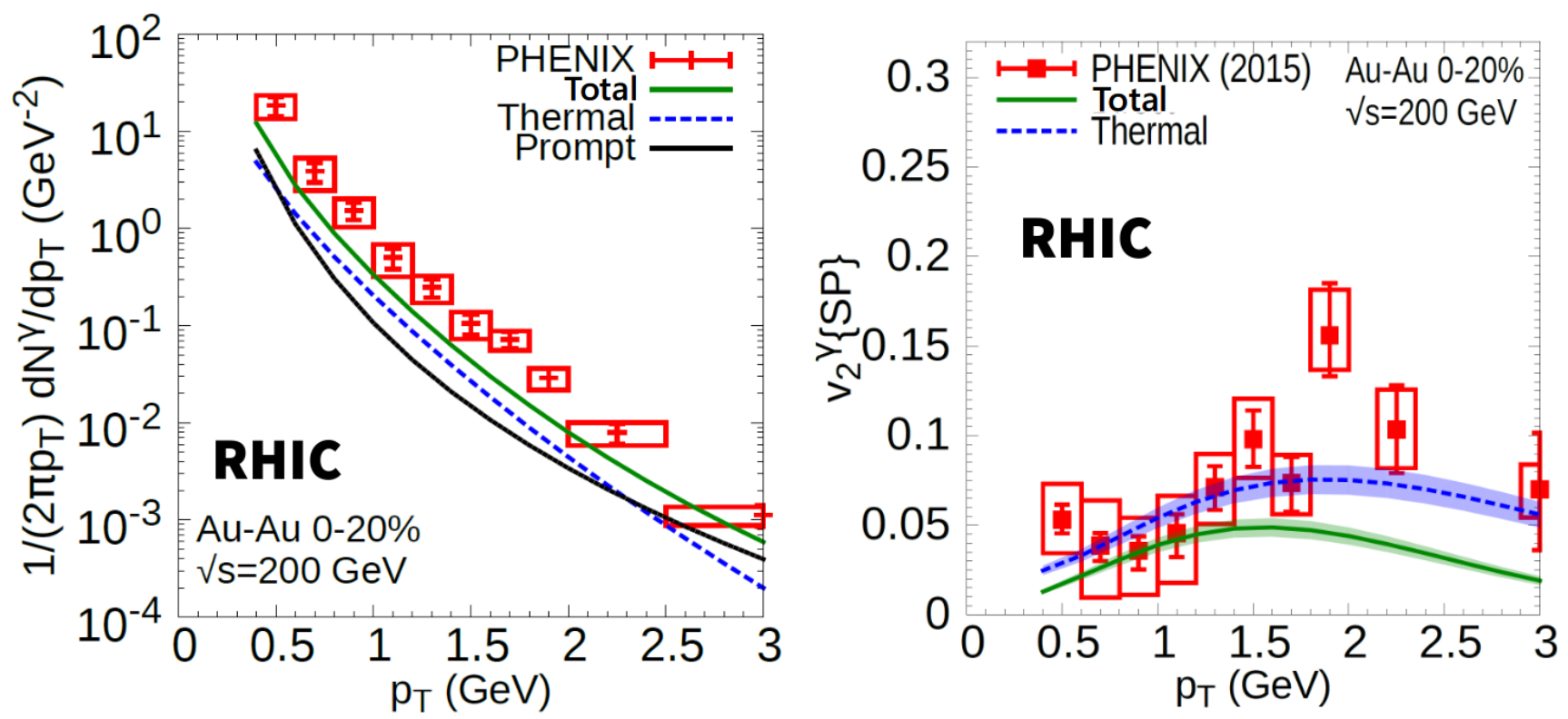

FIGURE 3.3: Direct photon invariant yield and elliptic flow as measured by the PHENIX collaboration at RHIC, and compared to theoretical calculations [77-79].

The PHENIX collaboration at RHIC measured both the invariant yield and elliptic flow of direct photons in $\mathrm{Au}-\mathrm{Au}$ collisions at $\sqrt{s_{\mathrm{NN}}}=200 \mathrm{GeV}$ for $0-20 \%$ collision centrality, and is shown in Fig. 3.3 [78, 79]. There are a few important observations to make. First, a large direct photon yield is underestimated by the model prediction. Second, the $v_{2}$ of direct photons is similar in magnitude to that of charged hadrons [88], and the measurement is again higher than the model. Since higher yields lead to lower $v_{2}$, it turns out that theoretical calculations are unable to reconcile these measurements. This is commonly referred to as the direct photon puzzle.

There are several explanations and theoretical advances that could potentially "solve" the puzzle [89, 90]. An important point to mention is that an independent measurement with either increased statistics or a correction of a possible bias could decrease the difference between theory and experiment, indicating there was no puzzle to begin with. Another solution would be that the the models are able to increase the direct photon flow components relative to the direct photon yield, for example by increasing the relative contribution of anisotropy of the initial state to the flow developed in the QGP phase, from which the puzzle would be resolved by theoretical developments [89, 91-93]. Lastly, it could be a radical different effect that is simply unknown. Whatever the solution might be, it is apparent that finding out if this puzzle remains at LHC energies will give vital input. 



\section{Part II}

\section{Experiment and Analysis}





\section{Chapter 4}

\section{Experimental setup}

\subsection{The Large Hadron Collider}

CERN is one of the world leading scientific laboratories and is based on the border of Switzerland and France, close to Geneva. The research organisation got founded in 1954 with the main purpose to advance the field of particle physics, hosting now thousands of scientists from international collaborations. The organization built multiple accelerators and facilities with ever improving technology to support a rich experimental physics program. Scientific achievements contributing to the Standard Model include the discovery of the

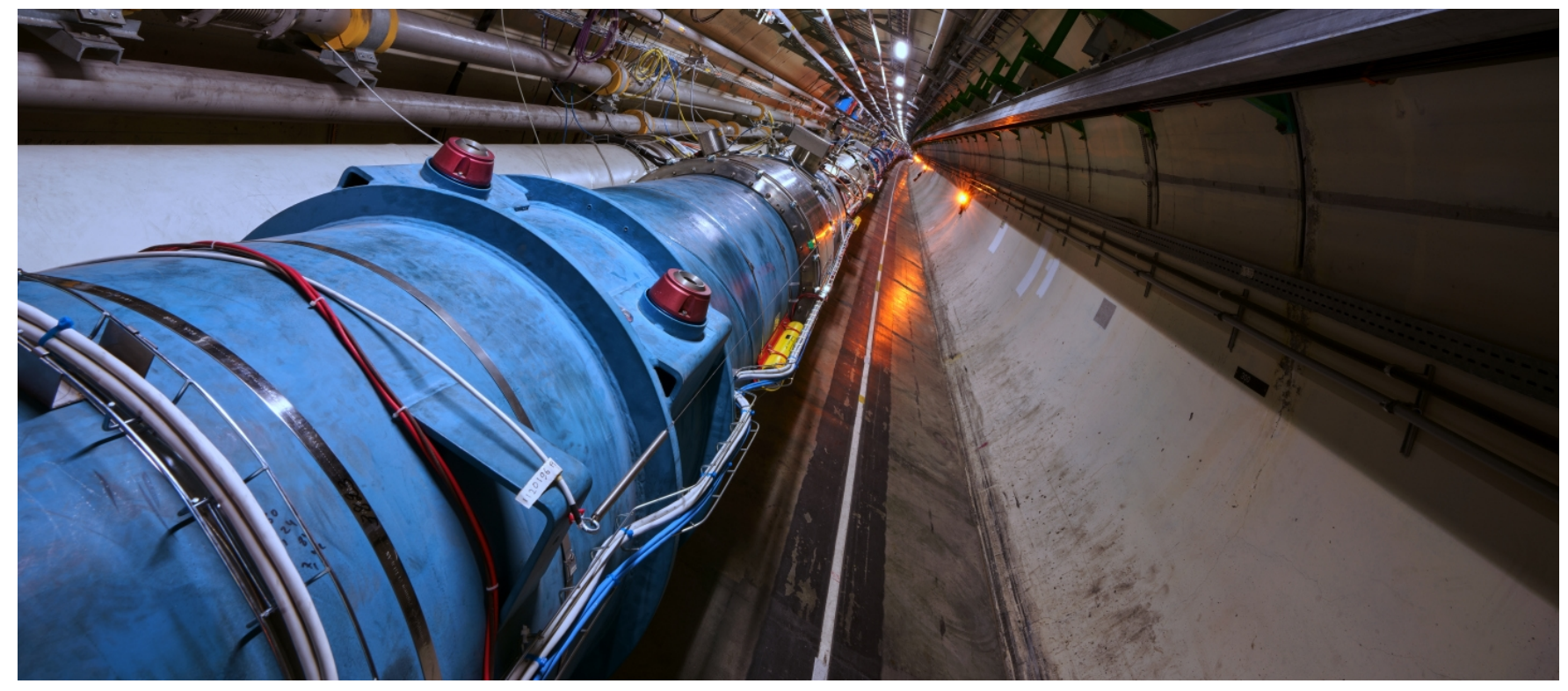

FIgURE 4.1: Picture taken inside the tunnel of the Large Hadron Collider, showing a small segment of the $27 \mathrm{~km}$ long circular accelerator [94]. The two parallel beam-pipes are enclosed in a vacuum system for thermal shielding and maintaining high beam quality. The particle beams are collided at four interaction points to produce high energy particle collisions. 
$\mathrm{W}$ and $\mathrm{Z}$ boson in 1984 [95, 96], the Higgs boson in 2012 [8, 9], and CERN is continuing to answer open questions.

The Large Hadron Collider (LHC) is the largest particle accelerator currently in operation and is part of the bigger accelerator chain at CERN [97]. The LHC is a synchrotron-type accelerator with two parallel beam pipes that have a circumference of $27 \mathrm{~km}$. Synchrotrons accelerate a beam of particles to higher and higher energies by increasing their kinetic energy, while maintaining the same curvature using magnetic fields of increasing strength. In the LHC, the beams are bend by 1232 superconducting dipole magnets to keep the particles on their circular trajectory. The proton beams injected into the LHC have an energy of $450 \mathrm{GeV}$ and get accelerated to maximum energies of $6.5 \mathrm{TeV}$ in LHC Run 2. Figure 4.1 shows a picture taken inside the accelerator tunnel and clearly indicates the scale of the machine.

Collisions are produced by having the beams intersect at four different locations within the accelerator. At these four interaction points there are the large experiments; ATLAS [98], CMS [99], ALICE [100], and LHCb [101]. These experiments each have their own well-defined physics program and are operated by collaborations consisting of thousands of scientists from all over the world. For the larger part of the running schedule from 2016 onwards, the LHC accelerates beams of protons and collides them at a center of mass energy of $13 \mathrm{TeV}$. A smaller part of the time, usually for 3 to 4 weeks per year, the LHC goes into heavy-ion mode and accelerates fully ionized heavy ions to produce collisions with either a proton or other heavy ions. It is the experimental data that is collected during this period from which the heavy-ion physics community benefits most, and it is the ALICE detector that is specifically designed to study heavy-ion collisions. 


\section{CERN Accelerators} (not to scale)

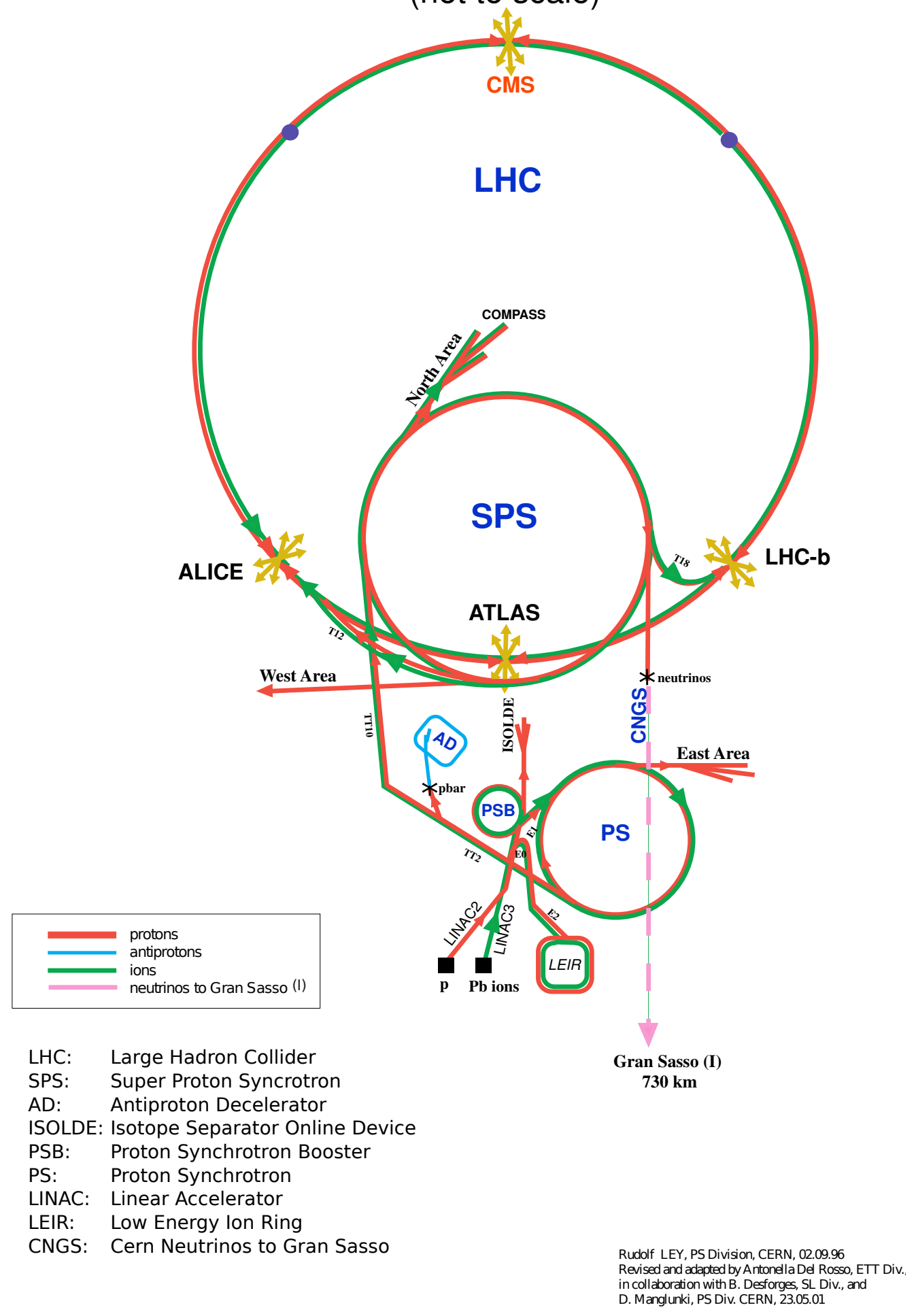

Figure 4.2: The CERN accelerator complex located near Geneva, Switzerland. The main purpose of the accelerator chain is to supply highly energetic particle collisions at the main interaction points of the Large Hadron Collider, the worlds largest particle accelerator with a circumference of $27 \mathrm{~km}$. In Run 2 it operates at a center of mass energy of $13 \mathrm{TeV}$ for proton proton collisions and $5.02 \mathrm{TeV}$ for heavy-ion collisions. The main detectors ATLAS, CMS, ALICE, and LHCb collect data to answer a wide range of fundamental scientific questions. 


\subsection{The ALICE detector}

The ALICE detector [100] is located at interaction point 2 of the LHC at CERN, and is approximately 16 meters high, 16 meters wide, 26 meters long, and weighs about 10.000 metric tons. It is optimized for studying heavy-ion collisions, capable of measuring collisions going up to high particle multiplicities. Figure 4.3 presents an overview of the detector, showing all 19 detector systems that make up the full ALICE detector. A full overview of the ALICE performance can be found in Refs. [102-104].

The various systems work together to provide information about the production point, energy, momentum, and identity of each particle produced in the collisions. For charged particles, the basic principle is to use tracking detectors along the charged particle's trajectory that disturb as little as possible. The magnetic field induced by the ALICE magnet bends its trajectory, such that the momentum of the particle can be obtained from its curvature. In addition, the charged particles that traverse the detector without decaying can be identified by their response in specific sub-detectors such as the Inner Tracking System (ITS), Time Projection Chamber (TPC), Transition Radiation Detector (TRD), and the Time Of Flight (TOF) detector. Since neutral particles leave no signal in the tracking detectors and aren't bent by the magnetic field, the overall strategy to measure these is by a destructive measurement that absorbs their entire energy in a well located detector.

The convention of ALICE is to use a right-handed coordinate system with the beam axis as z-axis, the $\mathrm{x}$-axis pointing to the center of the LHC, and the y-axis pointing upwards. The particles are described by their four-vectors $p^{\mu}=\left(E, p_{x}, p_{y}, p_{z}\right)$, from which the commonly used transverse momentum $p_{\mathrm{T}}$, rapidity $y$, and pseudorapidity $\eta$ can be calculated. These quantities are defined by

$$
p_{\mathrm{T}}=\sqrt{p_{x}^{2}+p_{y}^{2}}, \quad y=\frac{1}{2} \ln \frac{E+p_{z}}{E-p_{z}}, \quad \eta=-\ln \left(\tan \frac{\theta}{2}\right)
$$

where $\theta$ is the angle between the direction of the particle and the y-axis. The rapidity $y$, and likewise pseudorapidity $\eta$, of a particle is a convenient quantity because particle production is relatively constant as function of this quantity. In the limit of massless particles it is equivalent to the rapidity of the particle, which is additive under Lorentz transformation along the longitudinal direction.

The detector systems that are relevant to the analyses presented in this thesis will now be described in more detail. 


\subsubsection{Central barrel tracking}

The central barrel of the ALICE detector, as shown in Fig. 4.3, consists of multiple detector systems. The most relevant detectors for charged particle tracking are the Inner Tracking System (ITS) and the Time Projection Chamber (TPC), which are shown in Fig. 4.4. Their details will now be discussed.

The ITS [105] is a six-layer silicon detector in the center of ALICE directly around the collision point, with the purpose of measuring charged particle trajectories. The ITS is based on silicon semiconductor technology and registers hits that are caused by traversing charged particles. Combining hits in multiple layers of the ITS provides the track information. From the inner to outer layers it consists of two layers of Silicon Pixel Detectors (SPD), Silicon Drift Detectors (SDD), and Silicon Strip Detectors (SSD). These layers differ slightly in technologies and tracking precision. The SPD, having a pseudorapidity coverage of $|\eta|<1.95$, is able to achieve a track impact parameter resolution better than $50 \mu \mathrm{m}$ for $p_{\mathrm{T}}>1.3 \mathrm{GeV} / \mathrm{c}$, where the impact parameter is the closest distance to the origin of the detector or primary vertex, and not to be confused with collision impact parameter $b$. The ITS can cope with charged particle multiplicities of $\sim 8000$ per unit rapidity due to its high granularity and resolution.

The TPC [106] is a cylindrical detector with full coverage of the azimuthal angle and a pseudorapidity range of $|\eta|<0.9$. The chamber is filled with a gas mixture which locally can get ionized by traversing charged particles. The liberated electrons from the ionized gas molecules drift to the end-caps due to an applied electric field of $100 \mathrm{kV}$ and provide precise tracking information. The hit location on the end-caps is combined with the drift time to reconstruct the three dimensional tracks. Furthermore, the TPC provides particle identification using the specific energy loss $(\mathrm{d} E / \mathrm{d} x)$ of the charged particles traversing the detector, which is different for different type of hadrons due to their mass and total charge. Figure 4.5 shows the $\mathrm{d} E / \mathrm{d} x$ as function of track momentum $p$ for $\mathrm{Pb}-\mathrm{Pb}$ collisions at $\sqrt{s_{N N}}=2.76 \mathrm{TeV}$. It is important to note that while the different particle species have a distinct momentum dependence, the measured $\mathrm{d} E / \mathrm{d} x$ can still be ambiguous, as the bands merge for increasing $p$.

The combined TPC-ITS track resolution $\left(\sigma\left(p_{\mathrm{T}}\right) / p_{\mathrm{T}}\right)$, as measured for $\mathrm{Pb}-\mathrm{Pb}$ collisions at $\sqrt{s_{N N}}=2.76 \mathrm{TeV}$, is shown in Fig. 4.6. For increasing $p_{\mathrm{T}}$ above $1 \mathrm{GeV} / c$ the momentum resolution increases due to the fact that higher momentum tracks are bent less by the magnetic field, leading to a less constrained $p_{\mathrm{T}}$. For decreasing $p_{\mathrm{T}}$ below $1 \mathrm{GeV} / c$, the resolution increases due to the higher tracking uncertainties, driven by multiple scattering and because low momentum tracks produce less hits in the ITS and TPC detectors. For the majority of all charged tracks the resolution is below $2 \%$, which is extremely good for a particle detector. 


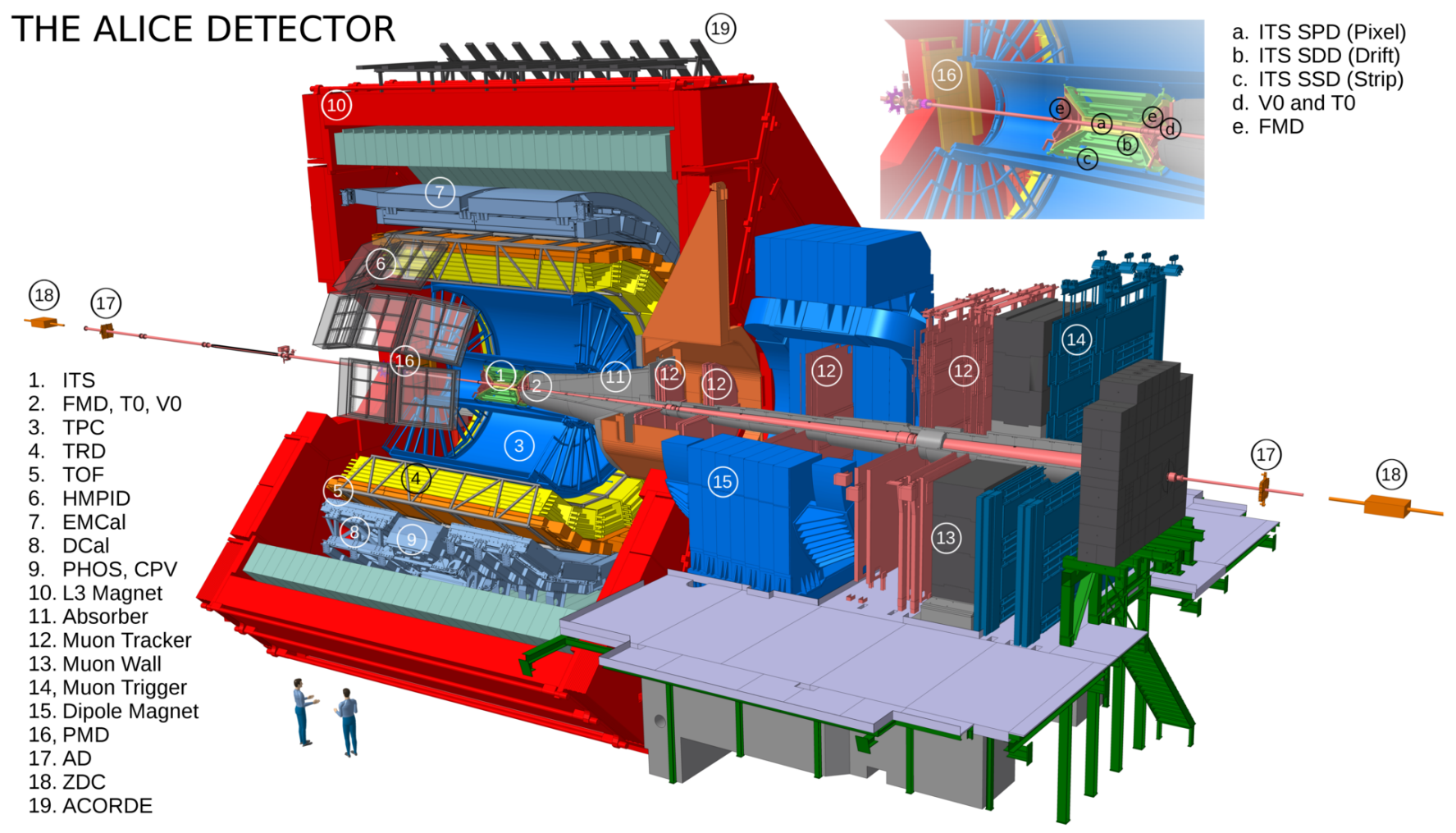

FIgURE 4.3: Schematic overview of the ALICE detector, with all the essential detector systems labeled and shown in the legend. The inset shows an enlarged view of the inner part.

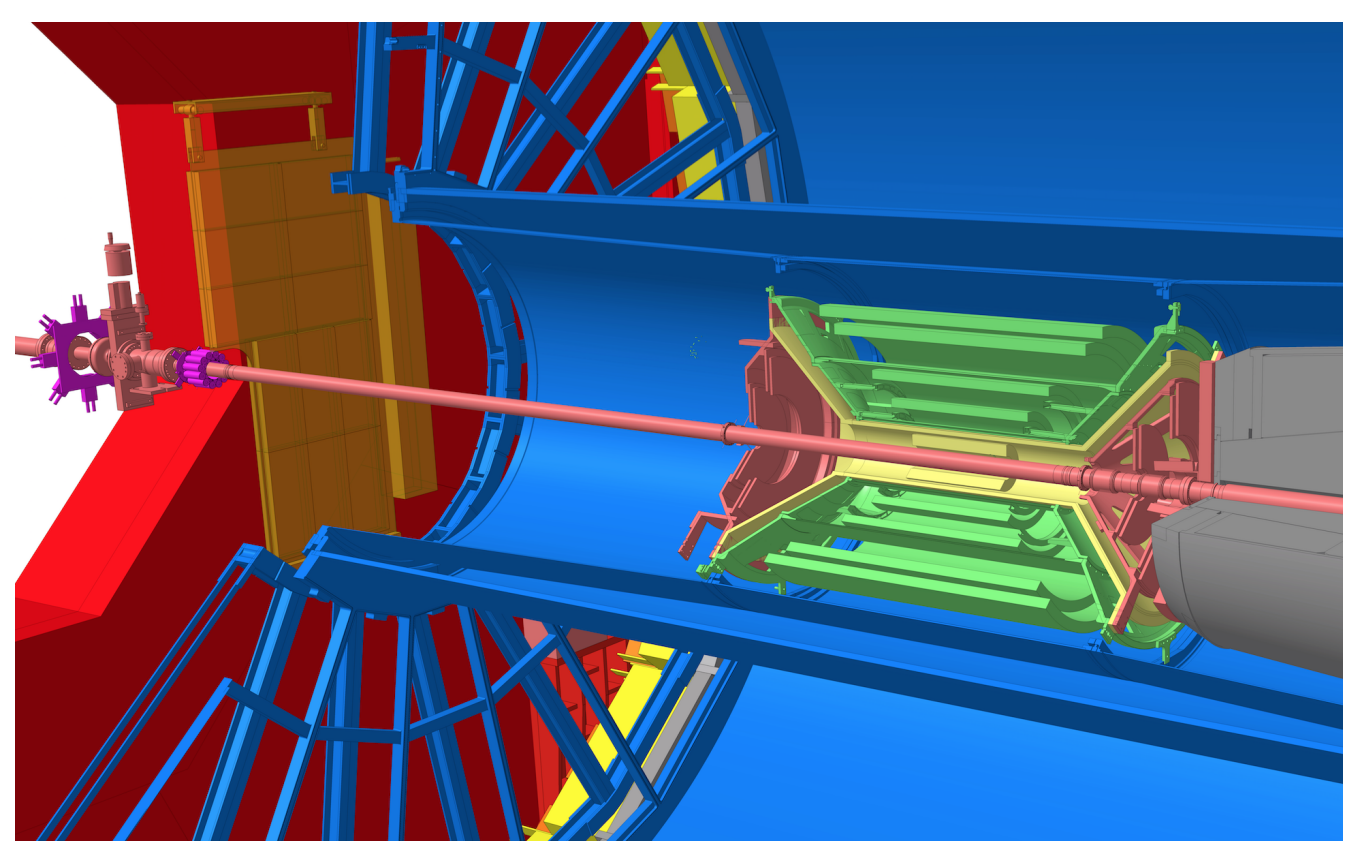

FIgURE 4.4: Enlarged view of the Inner Tracking System and of part of the Time Projection Chamber of the ALICE detector. 


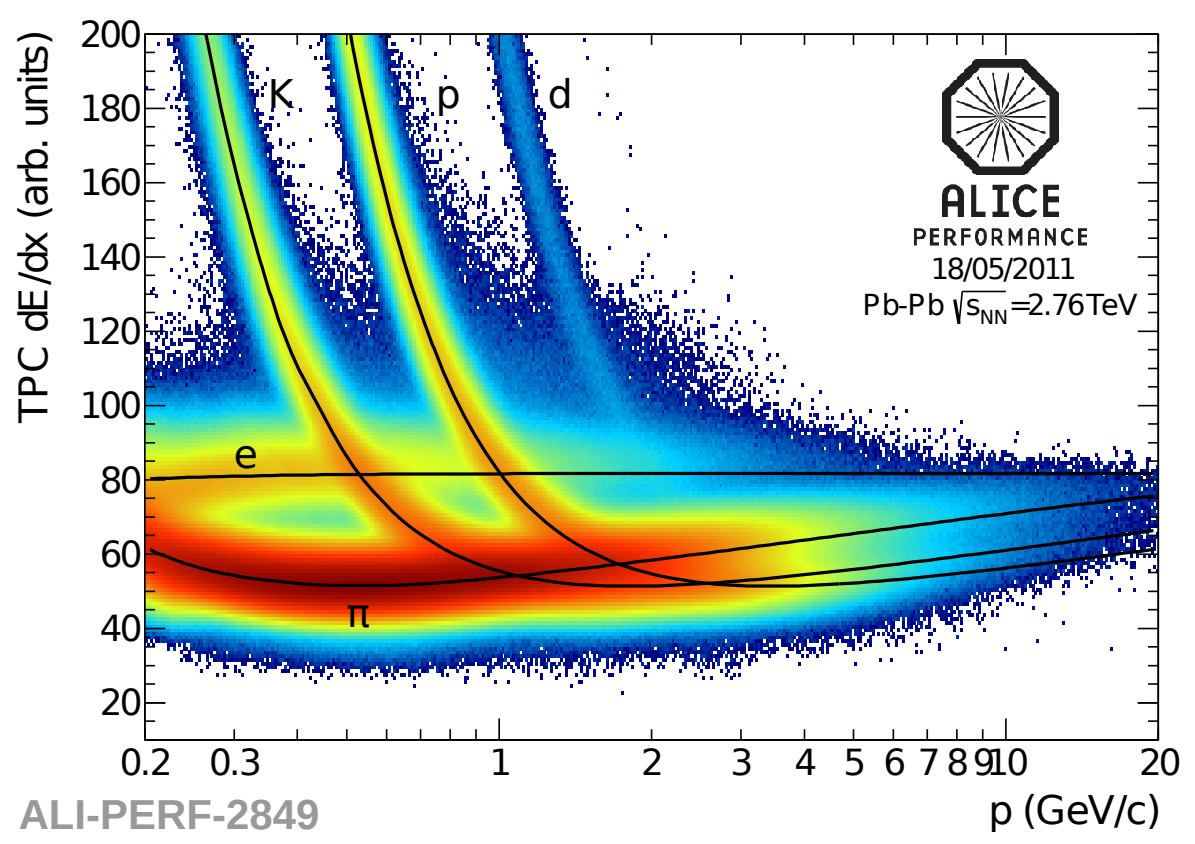

Figure 4.5: Specific energy loss $\mathrm{d} E / \mathrm{d} x$ of charged particles traversing the gas of the TPC, as function of the track momentum. As the energy loss depends on the charge, mass, and momentum of the particle, the measured value of $\mathrm{d} E / \mathrm{d} x$ can be used for particle identification.

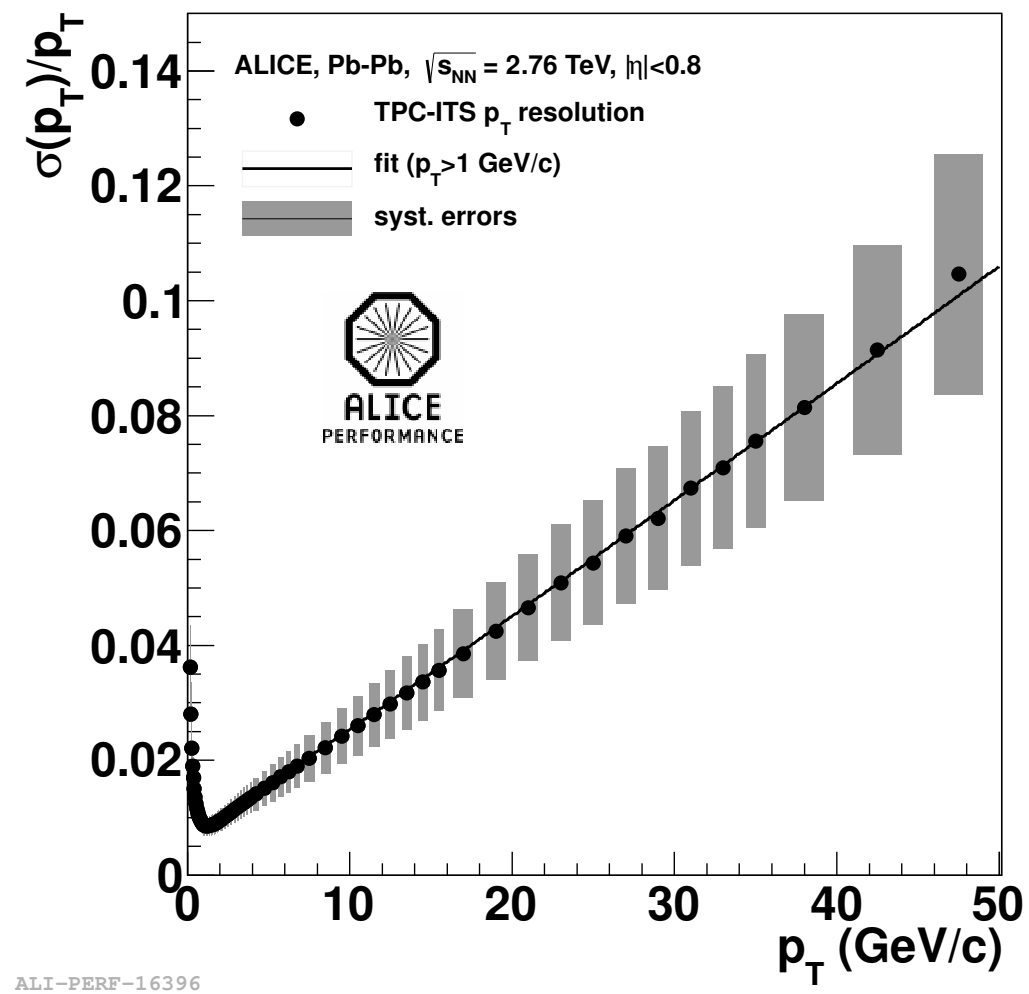

Figure 4.6: The TPC-ITS resolution $\left(\sigma\left(p_{\mathrm{T}}\right) / p_{\mathrm{T}}\right)$ of charged tracks as function of the $p_{\mathrm{T}}$ of the track, as measured for $\mathrm{Pb}-\mathrm{Pb}$ collisions at $\sqrt{s_{N N}}=2.76 \mathrm{TeV}$. 


\subsubsection{Calorimeters}

In this section, the general concept of measuring neutral particles with calorimeters will be explained. Photons and electrons produce electromagnetic showers when encountering high density material, depositing their full energy in a finite volume. The position and energy of these showers are reconstructed by using clusterizer algorithms, making calorimeters suitable for photon measurements in a wide transverse momentum range. The energy resolution of a calorimeter is given by

$$
\frac{\sigma_{E}}{E}=\frac{a}{E} \oplus \frac{b}{\sqrt{E}} \oplus c=\sqrt{\left(\frac{a}{E}\right)^{2}+\left(\frac{b}{\sqrt{E}}\right)^{2}+c^{2}},
$$

where $a$ comes from the effect of electronic noise on the electromagnetic shower reconstruction, $b$ captures the detector effects related to stochastic fluctuations, and $c$ arises from systematic effects such as imperfect calibrations, shower leakage, and detector non-uniformity. The relative energy resolution of the calorimeter is the quadratic sum of each individual contribution, which improves for increasing energies of the electromagnetic showers and converges to the constant term $c$. Note that while the relative uncertainty goes down for increasing energies, the absolute uncertainty increases.

The ALICE detector has three electromagnetic calorimeters; the EMCal [100, 107], DCal, and PHOS [108]. The EMCal and DCal are very similar detectors, as they use the same detector technology. The DCal was installed later in long shutdown 1, which is between LHC Run 1 and Run 2. The EMCal and DCal towers (cells) consist of alternating layers of absorber and scintillating material, while the PHOS towers consist of a single material that is both an absorber and scintillator. The properties of these calorimeters will now be discussed in more detail.

The EMCal and DCal [100, 107] are electromagnetic calorimeters that are build up from stacks of 77 alternating layers of absorber $(\mathrm{Pb})$ and scintillating material (polystyrene based). Wavelength-shifting fibers that penetrate these stacks extract the light which is generated by the electromagnetic showers and guide it to the avalanche photodiodes (APD) that convert the light to a measurable charge. Each of these stacks are $6 \mathrm{~cm} \times 6 \mathrm{~cm}$, and are referred to as a tower. The EMCal and DCal are made up of 12,288 and 5, 376 towers, respectively, and are grouped in arrays of super modules which are approximately projective with respect to the nominal ALICE interaction point. The coverage of the EMCal is $80^{\circ}<\varphi<187^{\circ}$ in azimuthal angle with a pseudorapidity range of $|\eta|<0.7$. The coverage of the DCal is $260^{\circ}<\varphi<320^{\circ}$ with $0.22<|\eta|<0.7$, and $320^{\circ}<\varphi<327^{\circ}$ with $|\eta|<0.7$. The energy resolution of this detector is $\sigma_{E} / E=(4.8 \% / E) \oplus(11.3 \% / \sqrt{E}) \oplus 1.7 \%$, where $E$ is in units of $\mathrm{GeV}$, as measured with the test beam setup.

The PHOS [108] is an electromagnetic calorimeter which consists of four modules installed at a radial distance of $4.6 \mathrm{~m}$ from the interaction point, covering $250^{\circ}<\varphi<320^{\circ}$ in azimuthal angle and $|\eta|<0.12$ in pseudorapidity. Each module consists of 3584 detector lead tungstate crystals $\left(\mathrm{PbWO}_{4}\right)$ cells with a of size $2.2 \times 2.2 \times 18 \mathrm{~cm}^{3}$, arranged in a matrix of $64 \times 56$. The signal from each cell is measured by an APD 


\begin{tabular}{l|ll}
\hline Calorimeter coverage & Pseudorapidity $(\eta)$ & Azimuth $(\varphi)$ \\
\hline EMCal & $|\eta|<0.7$ & $80 \operatorname{deg}<\varphi<187 \mathrm{deg}$ \\
\hline DCal & $0.22<|\eta|<0.7$ & $260 \mathrm{deg}<\varphi<320 \mathrm{deg}$ \\
& $|\eta|<0.7$ & $320 \mathrm{deg}<\varphi<327 \mathrm{deg}$ \\
\hline PHOS & $|\eta|<0.12$ & $250 \mathrm{deg}<\varphi<320 \mathrm{deg}$ \\
\hline
\end{tabular}

TABLE 4.1: Coverage in pseudorapidity $(\eta)$ and azimuthal angle $(\varphi)$ of the ALICE electromagnetic calorimeters EMCal, DCal, and PHOS.

connected to a low-noise charge-sensitive preamplifier. To increase the light yield, reduce electronic noise, and improve energy resolution, the APDs, preamplifiers, and crystals are cooled to a temperature of $-25^{\circ} \mathrm{C}$. The energy resolution is $\sigma_{E} / E=(1.3 \% / E) \oplus(3.6 \% / \sqrt{E}) \oplus 1.1 \%$, where $E$ is in units of $\mathrm{GeV}$, as measured by the test beam setup. The energy deposition in each PHOS cell is calibrated in pp collisions by tuning the $\pi^{0}$ peak position in the two-photon invariant mass distribution to the nominal value, on a cell-by-cell basis.

\subsubsection{Event multiplicity estimators}

There are several detector systems installed in ALICE that are used to estimate the total amount of particles produced in the collision, which is also referred to as the event multiplicity. The particle multiplicities at midrapidity is commonly measured using the amount of hits in the SPD, as well as the charged track multiplicity in the TPC. In addition, it is possible to remove autocorrelations between the particle multiplicity at midrapidity and the measured quantity that also uses tracks at mid-rapidity by estimating the event multiplicity with detectors at very forward and backward rapidities. This is done by the V0-A and V0-C detectors [109, 110], which are two arrays of scintillator detectors that span $2.8<\eta<5.1$ and $-3.7<\eta<-1.7$, respectively. Each of the V0 arrays has 32 channels and is segmented in four rings in the radial direction, where each ring is divided into eight sectors in the azimuthal direction. The sum of the signal amplitudes of the V0-A and V0-C detectors serves as a measure of event activity in the collisions. Furthermore, in $\mathrm{Pb}-$ $\mathrm{Pb}$ collisions these signal amplitudes are also used to select on collision centrality, which will be explained in more detail later in this chapter. 

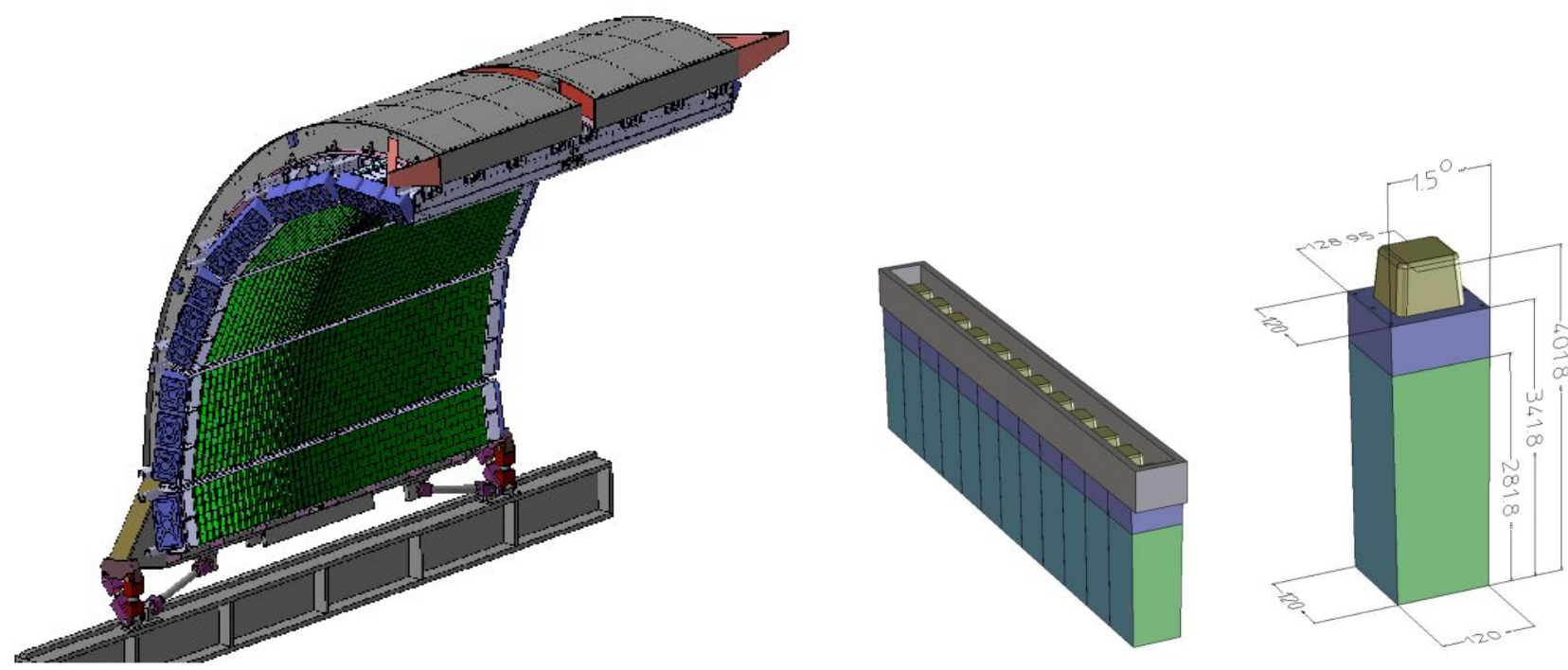

Figure 4.7: Design drawing of the EMCal (left). The detector consists of 12 large size modules, totaling 12, 000 readout channels (right). The main purpose is to measure photons and electrons originating from the collisions inside the ALICE detector, by fully absorbing their energy in one or multiple readout channels.
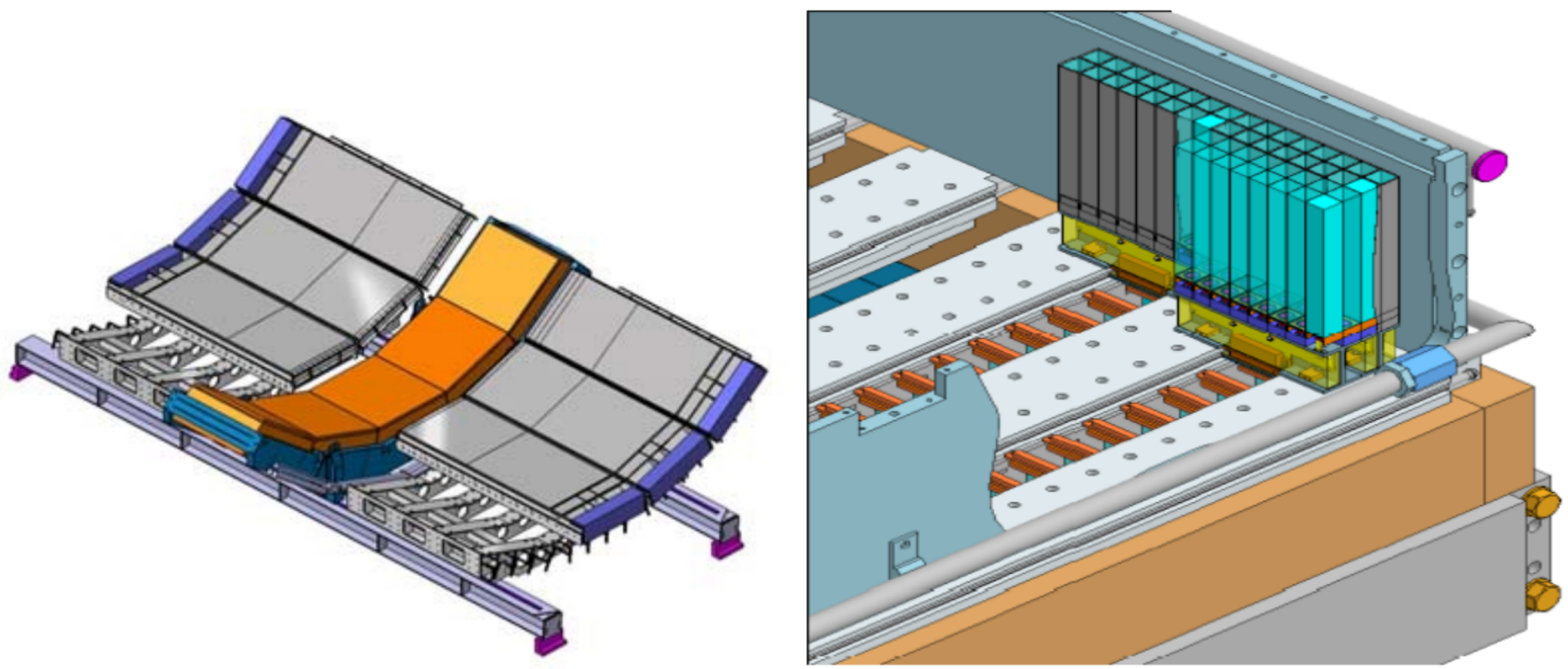

Figure 4.8: Design drawing of the PHOS and DCal calorimeters (left), and a zoom on the installation of PHOS cells (right). The DCal consists of an additional 12 large size modules with exactly the same technology as the EMCal. PHOS consists of 4 modules with a total of 12544 channels. 


\subsection{Data samples}

The analyses presented in this thesis use data from $\mathrm{pp}, \mathrm{p}-\mathrm{Pb}$, and $\mathrm{Pb}-\mathrm{Pb}$ collisions, which have been collected by the ALICE Collaboration over the course of many years during LHC Run 1 and 2. Table 4.2 shows some of the facts of the used datasets; namely collision energy, collision system, year of data taking, and the number of events used in the analyses. Most notably, the number of events for the datasets with the same collision system at the same energy increased tenfold between the first and second data taking period. This is mainly due to the increased luminosity of the accelerator and the increased data taking rate of the detector. The vast increase in statistics allows for more differential studies based on event characteristics.

\begin{tabular}{lllr}
\hline Collision energy $(\mathrm{TeV})$ & Collision system & Year of data-taking & \# events \\
\hline$\sqrt{s}=5$ & $\mathrm{pp}$ & 2015 & $0.1 \times 10^{9}$ \\
$\sqrt{s}=5$ & $\mathrm{pp}$ & 2017 & $1.0 \times 10^{9}$ \\
\hline$\sqrt{s_{\mathrm{NN}}}=5.02$ & $\mathrm{p}-\mathrm{Pb}$ & 2013 & $0.1 \times 10^{9}$ \\
$\sqrt{s_{\mathrm{NN}}}=5.02$ & $\mathrm{p}-\mathrm{Pb}$ & 2016 & $0.7 \times 10^{9}$ \\
\hline$\sqrt{s_{\mathrm{NN}}}=2.76$ & $\mathrm{~Pb}-\mathrm{Pb}$ & 2010 & $0.1 \times 10^{9}$ \\
$\sqrt{s_{\mathrm{NN}}}=5.02$ & $\mathrm{~Pb}-\mathrm{Pb}$ & 2015 & $0.04 \times 10^{9}$ \\
$\sqrt{s_{\mathrm{NN}}}=5.02$ & $\mathrm{~Pb}-\mathrm{Pb}$ & 2018 & $0.4 \times 10^{9}$ \\
\hline
\end{tabular}

TABLE 4.2: Overview of datasets used in the analyses presented in this thesis.

\subsubsection{Event selection}

The goal for the ALICE data acquisition (DAQ) is to record and archive the data of all its sub-detector systems on tape, where the data contains the information related to the particle collisions. Since the nominal bunch crossing frequency is about $40 \mathrm{MHz}$, and only up to $1-4.5 \mathrm{kHz}$ can be handled by the DAQ, a choice has to be made on what to store. This is done by the trigger system, which fires whenever the trigger conditions are satisfied and decides whether to store the event. The most common triggers used in the ALICE data taking of the LHC run 1 and 2 is the minimum bias trigger and the calorimeter triggers. The minimum bias trigger INT7 requires a signal in both V0 detectors. This coincidence signal in both the V0A and $\mathrm{VOC}$ is used to reduce the background from single diffractive interactions. The calorimeter triggers of both the EMCal and the PHOS require an energy deposit above threshold in an $2 \times 2$ patch in the calorimeter, and are able to enhance the amount of events containing high transverse momentum photons. The EMCal has in addition also triggers for $4 \times 4$ and $8 \times 8$ patch sizes, to trigger on high energy jets impending on the calorimeter surface.

In the offline reconstruction, the primary vertex of the collision is calculated for each event by extrapolating charged tracks that are reconstructed in the ITS and TPC towards the center of the detector. Only the events which contain a primary vertex within $10 \mathrm{~cm}$ of the nominal interaction point are considered for analysis. Furthermore, events which contain more than a single primary vertex are rejected with the SPD 
detector, such that events containing more than a single primary vertex, also known as pile-up events, are suppressed.

\subsubsection{Centrality selection}

Observables measured in heavy-ion collisions are interesting to study as function of the impact parameter $b$, since it naively defines the shape of the interaction zone and the number of participating partons in the collision $\left(\left\langle N_{\text {part }}\right\rangle\right)$. It is experimentally impossible to directly measure $b$, but it turns out that the charged particle multiplicity $N_{\text {ch }}$ of the final state depends monotonically on both $\left\langle N_{\text {part }}\right\rangle$ and $b$, as shown in Fig. 4.9 [31, 111-113]. Centrality classes are defined as a percentage of the total cross-section, such that $0-5 \%$ centrality corresponds to the $5 \%$ events with the highest $N_{\mathrm{ch}}$, and $5-10 \%$ centrality corresponds to the $5 \%$ events with the next highest $N_{\mathrm{ch}}$, and so on. These centrality classes are used to differentiate between central, semi-central, semi-peripheral, and peripheral collisions. It is important to note that for larger (> 5\%) intervals of collision centrality, the proportionality between $\left(N_{\mathrm{ch}}\right)$ and $b$ holds better than for smaller intervals $(\sim 1 \%)$, since the relative $N_{\mathrm{ch}}$ fluctuations for a fixed impact parameter $b$ increases.

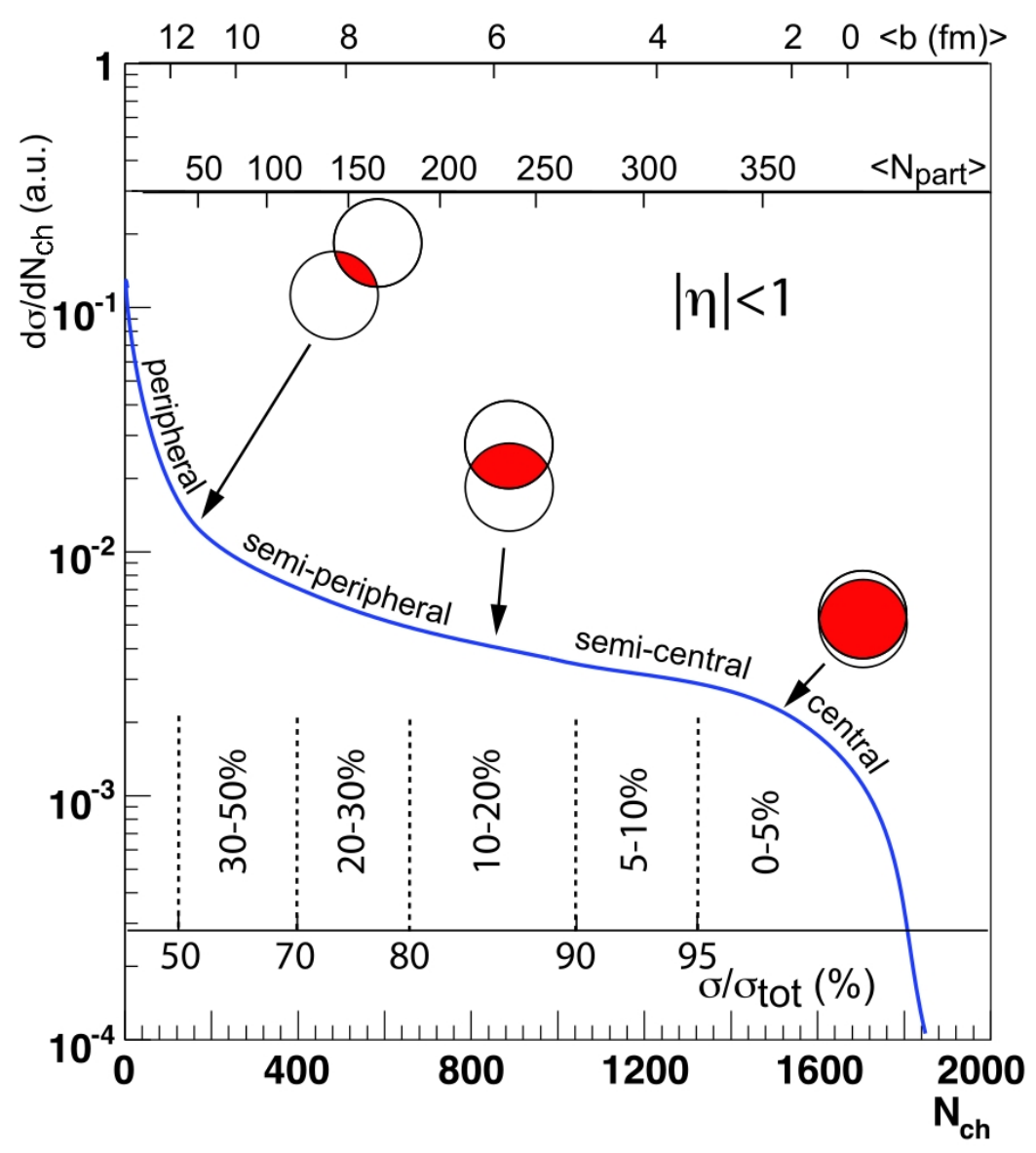

FiguRE 4.9: Distribution of charged particle multiplicity $\left(N_{\mathrm{ch}}\right)$ for heavy-ion collisions, showing the proportionality between $N_{\mathrm{ch}}, N_{\text {part }}$, and $b$. Intervals in this distribution (centrality class) are used to differentiate between central, semi-central, semi-peripheral, and peripheral collisions. 


\subsubsection{Charged track selection}

Charged tracks are measured by the inner barrel of the ALICE detector, more specifically by the ITS and TPC detectors. The charged tracks are divided into primary and secondary charged tracks [114], where primary tracks originate from the primary vertex and secondary tracks originate from secondary vertices, which are then mostly daughter tracks from a weak hadronic decay or conversion.

The overall reconstruction of the vertices and charged tracks is done as follows. First, the primary vertex itself is reconstructed by projecting the charged clusters found with the SPD layers of the ITS. Then, the most probably trajectories of the charged tracks are found by a Kalman filtering algorithm [115]. The fitting procedure starts by extrapolating the TPC clusters inwards, from the outer to inner field cage of the TPC. Then, the TPC tracks are matched to the ITS clusters in the outer layer, and again extrapolated inwards to the primary vertex. The next fitting procedure is done similarly, but starts from the inner layer of the ITS and is extrapolated outwards towards the outer field cage of the TPC. The distance of closest approach (DCA) to the primary vertex determines whether a track is primary or secondary, with the exact definition in [114]. For primary charged tracks, several selection criteria are applied to ensure the quality of the charged tracks used for analysis. First, a selection on the amount of TPC clusters of $N_{\mathrm{cl}, \mathrm{TPC}}>60$ is made to use tracks with a reliable amount of measurements along the track. Secondly, a selection of transverse momentum $p_{\mathrm{T}}>15 \mathrm{MeV} / c$ is applied. Lastly, it is required that the full track is within the acceptance of detector, i.e. $\eta<0.9$.

For the analyses presented in this thesis the primary charged tracks are mainly used for rejecting charged clusters in the calorimeters, but also for jet reconstruction, and estimating event characteristics such as the charged particle multiplicity $N_{\mathrm{ch}}$ and event shape. The secondary tracks are used to reconstruct photons converting to electron positron pairs in the detector material, which will be discussed in a later chapter.

\subsubsection{Jet reconstruction}

High momentum quarks and gluons produced in high energy collisions fragment [116] into multiple particles due to the nature of QCD. These particles are emitted in a similar direction as the original parton, i.e. concentrated in a cone since the originating parton typically has a large momentum. The group of correlated particles is referred to as a jet. Jets can be used to characterize events based on the amount and the energy of the jets, and are excellent to learn about particle production mechanisms. In heavy-ion collisions, jets are used to probe parton energy loss of the QGP, since the partons lose energy by collisional and radiative energy loss mechanisms when traversing the medium. This phenomenon is called jet quenching [117, 118].

Although the theoretical definition of a jet is simply the collimated spray of particles from a single parton, the experimental definition of a jet is not unique and depends on the implementation of the jet-finder algorithm [119], as there is no way to know for sure that a group of final state particles originate from the same parton. It is thus important that the jet-finder algorithms are optimized such that the final state 
particles relate back to the original parton, and that the algorithm is used consistently in both theory and experiment for comparisons. Furthermore, it is important that the jet-finder algorithm is safe from collinear and infrared divergences, i.e. that the definition of the jet is not changed by a parton emitting a gluon that is either very soft or collinear to the parton trajectory.

The anti- $k_{t}$ jet-finder algorithm [120] from the FastJet package [121] is the most commonly used jet-finder within the particle physics community, and is defined as follows:

1. For all possible particle pairs in the event calculate the distance

$$
d_{i j}=\min \left(p_{T, i}^{-2}, p_{T, j}^{-2}\right) \Delta R_{i j}^{2} / R^{2}
$$

where the particles are labeled with $i$ and $j, p_{T i(j)}$ is the transverse momentum of particle $i(j)$, $\Delta R_{i j}^{2}=\left(\eta_{i}-\eta_{j}\right)^{2}+\left(\varphi_{i}-\varphi_{j}\right)^{2}$, and $\eta_{i(j)}$ and $\varphi_{i(j)}$ are the pseudorapidity and azimuthal angle of particle $i(j)$. Additionally, for each particle the distance $d_{i B}$ is calculated by

$$
d_{i B}=\left(p_{T, i}\right)^{-2}
$$

2. If $d_{i j}<d_{i B}$, the particles $i$ and $j$ are grouped together using

$$
\begin{aligned}
p_{T, k} & =p_{T, i}+p_{T, j}, \\
\varphi_{k} & =\left(p_{T, i} \varphi_{i}+p_{T, j} \varphi_{j}\right) / p_{T, k}, \\
\eta_{k} & =\left(p_{T, i} \eta_{i}+p_{T, j} \eta_{j}\right) / p_{T, k} .
\end{aligned}
$$

If $d_{i j}>d_{i B}$, then particle $i$ is a jet.

3. The above steps are repeated until all particles of the event are grouped into jet objects.

The strength of the anti- $k_{t}$ algorithm is that it groups particles with the highest energy first, creating stable cone-like jets [120].

\subsubsection{Event shape observables}

The configuration of the momentum vectors of the final state particles can be used to characterize the shape or topology of the final state particle distribution, as indicated in Fig. 4.10. It is generally assumed that collisions with very large momentum transfers (hard events) that produce a di-jet structure will appear to be pencil-like, while events with smaller momentum transfers (soft events) produce a more isotropic distribution of particles and appears to be sphere-like [122-124]. This implies a correlation between the shape of the event and its dominant particle production mechanism. As a consequence, particle production spectra should be modified when measured as function of the event shape. A commonly used observable to 

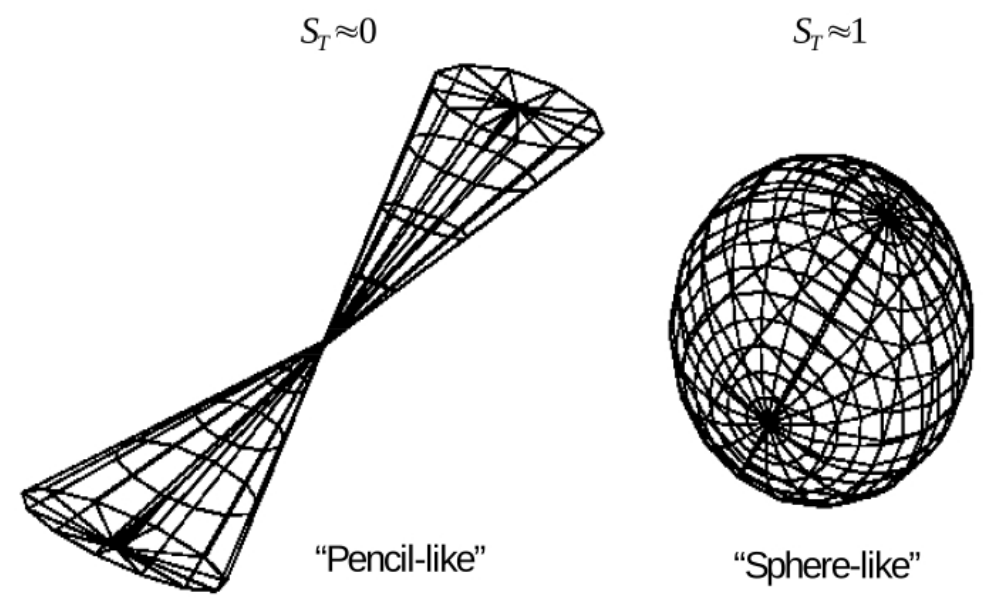

FIGURE 4.10: Sketch of event shapes formed by the final state particles, for the two extremes of a penciland sphere-like geometry.

classify the event as either pencil- or sphere-like is the transverse sphericity $S_{\mathrm{T}}$, which is calculated using the transverse momentum matrix of the event

$$
S_{x y}^{L}=\frac{1}{\sum_{i} p_{T, i}} \sum_{i} \frac{1}{p_{T, i}}\left[\begin{array}{cc}
p_{x, i}^{2} & p_{x, i} p_{y, i} \\
p_{y, i} p_{x, i} & p_{y, i}^{2}
\end{array}\right],
$$

where $p_{T, i}, p_{x, i}$, and $p_{y, i}$, are the momentum vectors of particle $i$ in transverse, $x$, and $y$ direction, respectively. The transverse sphericity is defined by

$$
S_{\mathrm{T}}=\frac{2 \lambda_{2}}{\lambda_{2}+\lambda_{1}}
$$

where $\lambda_{1}$ and $\lambda_{2}$ are the two eigenvalues of the matrix $S_{x y}^{L}$, with $\lambda_{1}>\lambda_{2}$. These equations essentially project all the particles of the event onto the $x-y$ plane and determine the eigenbasis of the transverse momentum matrix. As such, $S_{\mathrm{T}}$ is sensitive to the relative orientation of the momentum vectors, where for $\lambda_{2}=0$ the event is pencil-like, and for $\lambda_{1} \sim \lambda_{2}$ the event is sphere-like. The classification of the event-shape using the sphericity mostly applies to proton-proton collision, as the larger collision systems more quickly approximate sphere-like event shapes due to the larger particle multiplicities that are more evenly distributed in $\eta$ and $\varphi$.

In addition to transverse sphericity there are other observables that characterize the shape of the event, such as the three dimensional sphericity $\left(S_{3 \mathrm{D}}\right)$, and thrust $\left(S_{o}\right)$. Both these alternative observables highly correlate with $S_{\mathrm{T}}$. The $\left(S_{3 \mathrm{D}}\right)$ uses the three momentum vectors similarly to how $S_{\mathrm{T}}$ uses their projection on the transverse plane, which are approximately the same for detectors with relatively small $\eta$ acceptance. Thrust is calculated in a different way, namely by finding an axis which maximizes the sum of the inner products of all final state particle vectors with this axis. This axis is an approximation of the eigenvectors found by calculating the sphericity. 



\section{Chapter 5}

\section{Photon reconstruction}

\subsection{Photon measurements with calorimeters}

Calorimeters are capable of measuring single photons by absorbing their full energy within a block of material and converting the deposited energy into a measurable signal. The electromagnetic showers produced by photons spread out in longitudinal and transverse direction in the calorimeter, and as a consequence, signals related to a single photon are often registered in several detector cells, which are mostly adjacent. From the distribution of signals in the detector the photons have to be identified, and their properties have to reconstructed using specialized algorithms and selection criteria. This will now be discussed in detail.

\subsubsection{Photon reconstruction}

Photons that are incident on a calorimeter create electromagnetic showers via consecutive processes of pair production and bremsstrahlung. The particles in the shower induce a signal in one or multiple calorimeter cells. The overall strategy to measure the energy and position of these photons is to group all nearby cells into a so called cluster. The procedure of clusterizing all the energy deposited in the calorimeter is handled by a well defined clusterizer algorithm, and goes as follows:

1. Consider all cells and sort them according to their energy.

2. Start building a new cluster using the highest energy unclustered cell as seed, requiring $E>E_{\text {seed }}$.

3. Aggregate all cells that have a common edge to the seed, requiring $E>E_{\text {cell }}$.

4. Aggregate all cells that have a common edge to the newly added cells, referred to as the reference cells, again requiring $E>E_{\text {cell }}$.

5. Repeat step 4 until there are no more adjacent cells with $E>E_{\text {cell }}$. 
6. Repeat steps 2-5 until there are no more cells with $E>E_{\text {seed }}$.

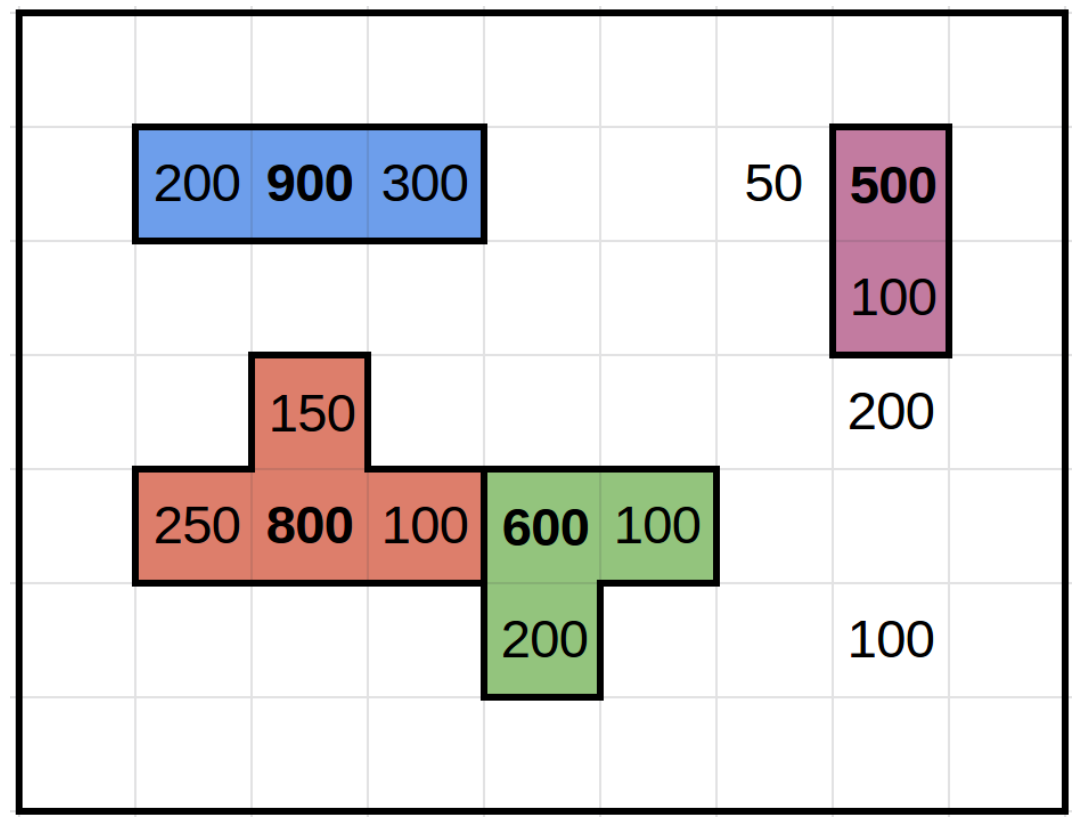

Figure 5.1: Cartoon of the clusterization process for a hypothetical event where five photons deposit their energy in the calorimeter. The energy deposited per cell is given in $\mathrm{MeV}$, and the colors indicate which cells are grouped in the same cluster by the clustering algorithm.

As an example for a hypothetical event, Fig. 5.1 shows a cartoon of the energy deposited by five photons, and indicates which clusters are found by the clustering algorithm given typical values of $E_{\text {seed }}=500$ $\mathrm{MeV}$ and $E_{\text {cell }}=100 \mathrm{MeV}$. There are two main subtleties illustrated with this example; not all the energy deposited in the calorimeter is by definition clusterized, and the highest energy seed has preference in the aggregation of additional cells. This has as a consequence that, even in a background free environment, the found clusters and the electromagnetic showers produced by the impending particles are not always a one-to-one map. Of course, the finer the granularity and the better the resolution of the detector, the more the cluster corresponds to the shower.

Multiple properties are calculated from the reconstructed cluster. First, the energy and position of the cluster is calculated using

$$
E_{\text {cluster }}=\sum_{i}^{N} E_{\text {cell }, i} \quad \text { and } \quad \vec{x}_{\text {cluster }}=\frac{\sum_{i}^{N} w_{i} \cdot \vec{x}_{\text {cell }, i}}{\sum_{i}^{N} w_{i}}
$$

where the sum is taken over all cells that are part of the cluster, with $N$ the total amount of cells of the cluster. Furthermore, $E_{\text {cell, } i}$ and $\vec{x}_{\text {cell, } i}=\left(x_{1}, x_{2}, x_{3}\right)$ are the energy and position of the $i$-th cell of the cluster, respectively. The position vector $\vec{x}_{\text {cluster }}$ is the average position of all cells that make up the cluster, weighted by the factor $w_{i}$, which is by default the logarithm of the energy of the cell. Secondly, the shape of the cluster is characterized by the shower shape parameters $M_{02}$ and $M_{20}$, which are calculated by 


$$
M_{m n}=\sum_{i}\left(x_{1}^{(i)}\right)^{m} \cdot\left(x_{2}^{(i)}\right)^{n} E_{\mathrm{cell}, i} \quad \text { with } \quad(m, n)=(0,2),(2,0)
$$

where $i$ runs again over all cells in the cluster and $E_{\text {cell, } i}$ is the cell energy. These so called shower shape parameters $M_{02}$ and $M_{20}$ characterize the major and minor width of the cluster, respectively. In general, electromagnetic showers initiated by a photon create a cluster shape that is indistinguishable from a shower initiated by an electron, as the only difference is the very first interaction of the photon or electron with the detector material. The main use of the shower shape parameters is therefor to discriminate between electromagnetic, hadronic, and overlapping showers.

For both the EMCal [107] and the PHOS [108] there are selection criteria applied on the cells before the clustering process. First of all, both calorimeters are calibrated cell by cell for their energy and timing signals. This energy calibration is a relative energy calibration, with the absolute calibration done on cluster level that also includes a non-linearity correction, which will be discussed later. Only nominally performing cells, i.e. cells that have well behaved energy and timing distributions, are selected for the clusterizer. Other cells are masked for analysis. For the EMCal and PHOS there is a cell energy threshold of $E_{\text {cell }}=100 \mathrm{MeV}$ and $E_{\text {cell }}=15 \mathrm{MeV}$, respectively. This removes most of the noise and improves the signal to background. The EMCal has a seed energy threshold of $E_{\text {seed }}=500 \mathrm{MeV}$, while PHOS has a seed energy threshold of $E_{\text {seed }}=50 \mathrm{MeV}$. For both calorimeters there is a selection on the cell time of \pm 500 ns relative to the collision time. These selection criteria are summarized in Table 5.1 and Table 5.2.

The clusterizers used for the EMCal and PHOS work as described above, with two differences. For the EMCal clusterizer, cells are only aggregated if the energy of the newly added cell is less than the reference cell, which then by construction handles the cases where showers partly overlap. The PHOS clusterizer has no such requirement and the clusters can in principle keep on aggregating more cells as long as $E>E_{\text {cell }}$. To handle overlapping showers, PHOS employs a cluster unfolding approach. The decision as to whether the cluster is unfolded is made by using the number of local maxima of a cluster, which is calculated by counting the number of cells that are above seed threshold and are surrounded by cells with lower energies with respect to the reference cell. If it contains multiple local maxima it is considered to contain overlapping showers, and is then unfolded. The unfolding itself is done by splitting the cluster into multiple clusters, taking the local minima as new edges of the clusters. The properties such as position, total energy, and shower shape parameters of the sub-clusters are recalculated using the cells belonging to these sub-clusters.

In addition, both calorimeters correct the cluster energies for the absolute energy scale and non-linearity of the calorimeter, such that the reconstructed cluster energy corresponds to the energy of the incident particle. For the EMCal and PHOS, this correction is based on data collected with a test beam experiment, which was performed prior to the installation of the calorimeter in the ALICE central barrel. The required cluster energy correction can be calculated in a straightforward way as the energy of the particles in the beam is known. The full description of this procedure can be found in [107] and [108], for the EMCal and PHOS, respectively. 


\subsubsection{Photon selection}

Electromagnetic calorimeters are highly efficient devices for reconstructing photons, electrons, and positrons, as they all produce electromagnetic showers. It is important to note that other particles, such as the $\pi^{ \pm}$, $\mathrm{K}^{ \pm}$, and $\mathrm{p}(\overline{\mathrm{p}})$, most frequently only deposit a fraction of their energy when traversing the calorimeter. The higher energy hadronic showers can be rejected using track information of the ITS and TPC. The various photon selection criteria will now be discussed, and each have the goal to reduce the background while maintaining high signal efficiencies.

There are several selections applied on cluster level, to make sure that the clusters have no significant contribution from detector noise, have a reliable energy measurement, and such that non-photonic backgrounds are suppressed. The minimum number of cells to form a cluster is set at 2 for both calorimeters. This limits the minimum energy of the reconstructed cluster to $600 \mathrm{MeV}$ for the EMCal, and $65 \mathrm{MeV}$ for PHOS. To reduce the noise background, coming from small energy deposits of traversing hadrons and hardware noise, it is chosen to have a minimum energy selection of $700 \mathrm{MeV}$ for EMCal clusters and $300 \mathrm{MeV}$ for PHOS clusters. Furthermore, another selection is done based on the cluster time relative to the timestamp of the event, such that clusters produced from out-of-bunch pileup are rejected. The cluster time is determined by the cell time of the seed, as the uncertainty on the timing decreases for larger energy deposits. by default, a cluster time selection of $-12.5<t<13 \mathrm{~ns}$ is applied for pp collisions, and $-50<t<50 \mathrm{~ns}$ for $\mathrm{Pb}-\mathrm{Pb}$ collisions. Additionally, there is a selection applied on the shower shape parameter $M_{02}$, in order to select photon-like clusters. For the EMCal this selection is $0.1 \leq M_{02} \leq 0.5$, while for PHOS this is $M_{02} \geq 0.1$. The differences in selection criteria between EMCal and PHOS is mostly driven by the different cell sizes and higher energy resolution of PHOS, which allows for lower energy thresholds for clusters reconstructed with PHOS.

To remove clusters that are produced by $e^{ \pm}$and have potential contributions from other charged particles, clusters are rejected if a charged track is pointing to the clusters. The charged track is reconstructed using the TPC and extrapolated to the EMCal surface using the respective track parameters. The cluster is rejected if the charged track is within a certain $(\Delta \eta, \Delta \varphi)$-window relative to the cluster, which is by default dependent on the $p_{\mathrm{T}}$ of the track to improve the quality of the matching. This is done by evaluating the residuals $\Delta \eta$ and $\Delta \varphi$, and fitting the widths using the following function

$$
f_{\eta, \varphi}\left(p_{\mathrm{T}}\right)=p_{1}+\frac{1}{\left(p_{\mathrm{T}}+\left(\frac{1}{p_{0}-p_{1}}\right)^{\frac{1}{p_{2}}}\right)^{p_{2}}},
$$

where $p_{\mathrm{T}}$ is the transverse momentum of the charged track, and $p_{0}, p_{1}$, and $p_{2}$ are free parameters. This functional form contains a constant term that acts as a lower bound, as well as a term that decreases for increasing $p_{\mathrm{T}}$ of the charged track. As such, the size of the rejection window in $\Delta \eta$ and $\Delta \varphi$ is smaller for higher momentum tracks. The default parameter values for EMCal and PHOS are listed in Table 5.1 and Table 5.2, respectively. 
Furthermore, the sample of clusters that pass the selection criteria receive an energy correction to improve the absolute energy scale of the calorimeter as well as its agreement in experimental and simulated data. This procedure is done slightly different for EMCal and PHOS clusters. For EMCal, it is chosen to only apply additional corrections to the cluster energy in simulated data, such that the reconstructed neutral pion mass position matches with that of experimental data. For PHOS, the energy of the clusters is corrected such that the reconstructed neutral pion mass position matches its known value of $134.98 \mathrm{MeV}$ for experimental data, with an additional correction to simulated data to make mass peak positions agree. For both cases, the cluster energy dependent correction is calculated by reconstructing the $\pi^{0}$ mass peak position using either calorimeter clusters with similar energy, or using one calorimeter cluster and one photon reconstructed with the photon conversion method (see next section), which then results in an unambiguous correction for a given cluster energy.

The $\eta-\varphi$ distributions of clusters satisfying the selection criteria are shown in Fig. 5.2 for the EMCal and PHOS calorimeters. The distributions are scaled by dividing by the maximum of the histogram, and show the coverage and geometry of the calorimeters. 


\begin{tabular}{ll}
\hline EMCal cell \& cluster selection criteria \\
\hline$E_{\text {cell }}$ & $100 \mathrm{MeV}$ \\
\hline$E_{\text {seed }}$ & $500 \mathrm{MeV}$ \\
\hline Minimum cluster energy & $E_{\text {cluster }}>700 \mathrm{MeV}$ \\
\hline Minimum number of $N_{\text {cluster }}^{\text {cells }}$ & $\geq 2$ \\
\hline Cluster $M_{02}$ & $0.1 \leq M_{02} \leq 0.5$ \\
\hline Cluster time & $-12.5<t_{\text {cluster }}<13 \mathrm{~ns}$ \\
\hline Track-matching & $p_{\mathrm{T}}$ dependent \\
$\Delta \eta$ & $p_{0}=0.04, p_{1}=0.010, p_{2}=2.5$ \\
$\Delta \varphi$ & $p_{0}=0.09, p_{1}=0.015, p_{2}=2.5$ \\
\hline
\end{tabular}

TABLE 5.1: Standard selection criteria for the clusters reconstructed with the EMCal which are used in the analyses.

\section{PHOS cell \& cluster selection criteria}

\begin{tabular}{ll}
\hline$E_{\text {cell }}$ & $15 \mathrm{MeV}$ \\
\hline$E_{\text {seed }}$ & $50 \mathrm{MeV}$ \\
\hline Minimum cluster energy & $E_{\text {cluster }}>300 \mathrm{MeV}$ \\
\hline Minimum number of $N_{\text {cluster }}^{\text {cells }}$ & $\geq 2$ \\
\hline Cluster $M_{02}$ & $>0.1$ \\
\hline Cluster time & $-12.5<t<13 \mathrm{~ns}$ \\
\hline Trackmatching & $p_{\mathrm{T}}$ dependent \\
$\Delta \eta$ & $p_{0}=0.05, p_{1}=0.005, p_{2}=3.0$ \\
$\Delta \varphi$ & $p_{0}=0.33, p_{1}=0.005, p_{2}=2.3$ \\
\hline
\end{tabular}

TABLE 5.2: Standard selection criteria for the clusters reconstructed with the PHOS which are used in the analyses. 
$0-90 \% \mathrm{~Pb}-\mathrm{Pb}, \sqrt{s_{\mathrm{NN}}}=5.02 \mathrm{TeV}-$ LHC18q V0AND- EMCal+DCal clusters

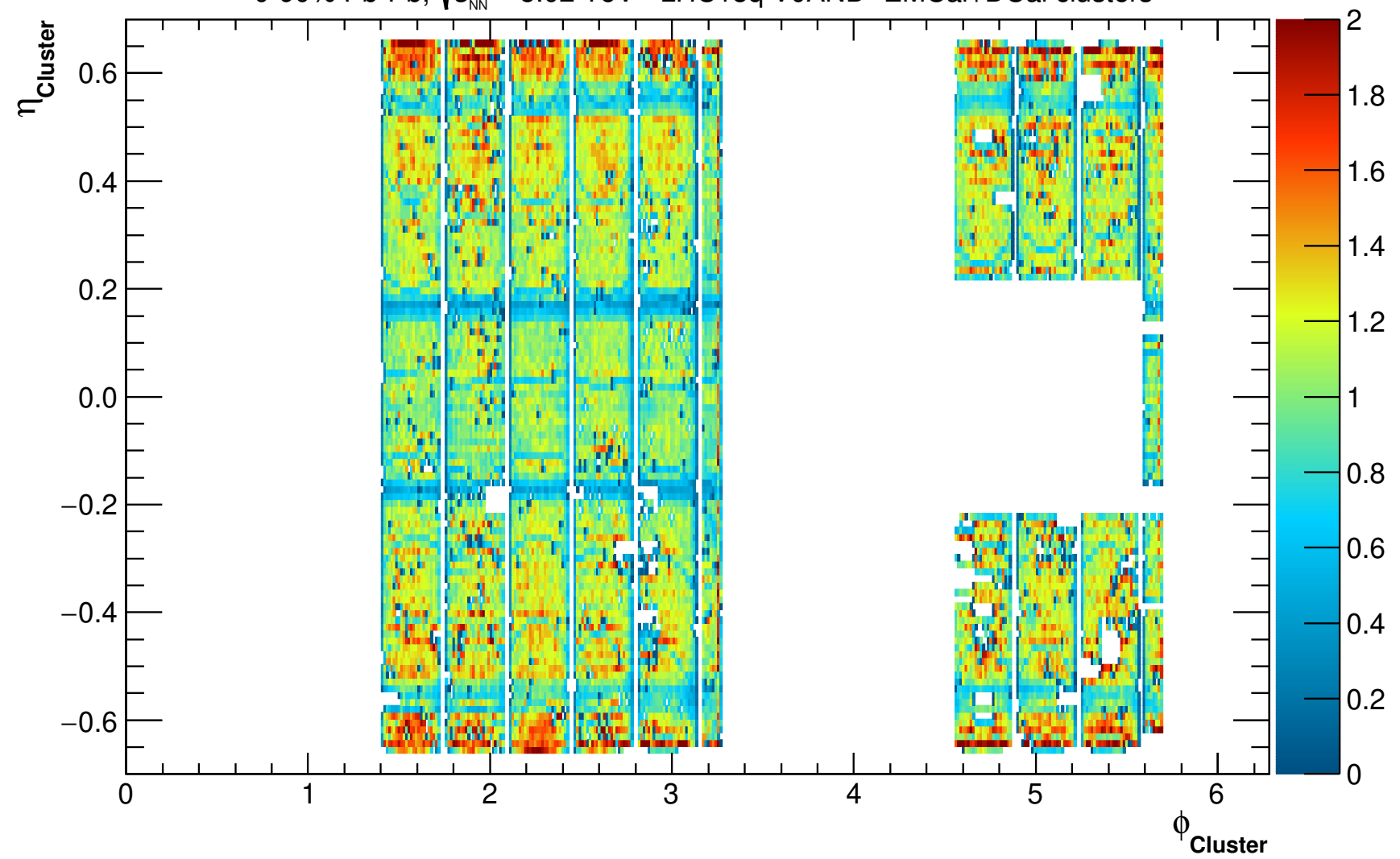

0-90\% Pb-Pb, $\sqrt{s_{N N}}=5.02 \mathrm{TeV}-$ LHC18q V0AND- PHOS clusters

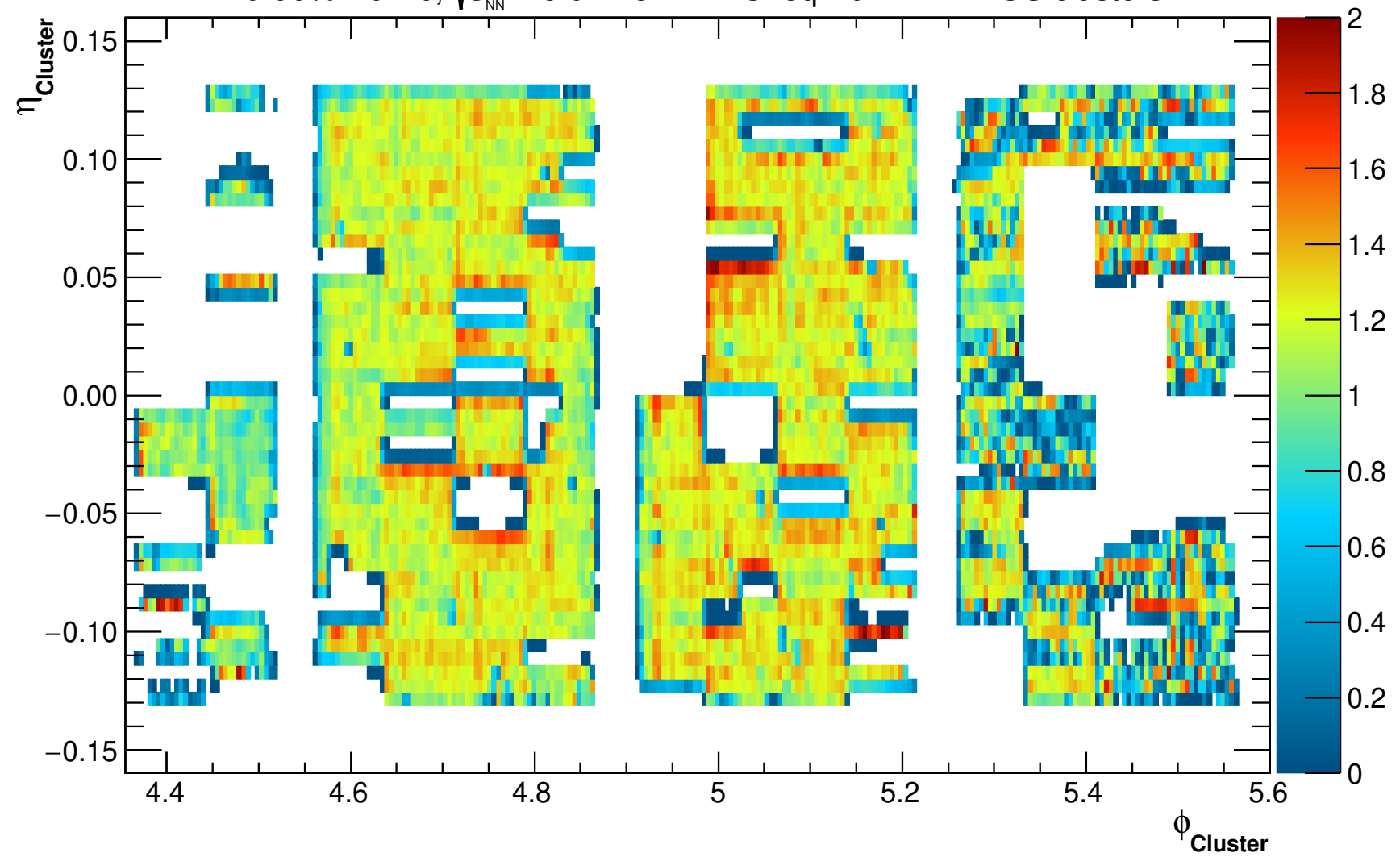

FIGURE 5.2: Relative number of clusters as a function of $\eta$ and $\varphi$ as reconstructed with the EMCal and DCal (top), and PHOS (bottom) calorimeters, for $\mathrm{Pb}-\mathrm{Pb}$ collisions at $\sqrt{s_{N N}}=5.02 \mathrm{TeV}$. Clusters are shown only if they pass the selection criteria listed in Table 5.1 and Table 5.2, and are normalized using the maximum of the distribution. It indicates the coverage, geometry, and masked readout channels of the calorimeter modules. 


\subsubsection{Systematic uncertainties}

The photons reconstructed with the calorimeters are affected by several systematic uncertainties [125], mainly due to imperfect calibrations and differences between experimental and simulated data. As described in the previous sections, the cell-by-cell and cluster calibrations are performed such that the systematic differences between the properties of the photon candidate and incoming photon is minimized as much as possible. In addition, systematic differences introduced by the photon reconstruction and selection criterion that are reproduced by the Monte Carlo simulations are included in corrections such as the acceptance and efficiency corrections. This means that the magnitude of the different sources of systematic uncertainties is mostly driven by the quality and precision of the Monte Carlo simulation. Since the simulated data is not perfect, there are several significant contributions to the total systematic uncertainty, and it is important to emphasize that the magnitude of the systematic uncertainty, even for a single selection criteria, is dependent on the measured observable. The sources and magnitude of the systematic uncertainty will now be discussed in more detail.

The clusterizer settings, i.e. the energy thresholds $E_{\text {cell }}$ and $E_{\text {seed }}$, define the minimum energy that has to be deposited in a calorimeter cell in order to be taken into account for the clustrization process. This unavoidably creates a systematic difference between the reconstructed cluster energy and the energy of the impending particle, as small energy leakage towards additional surrounding cells is not taken into account, and this is a larger effect for cluster energies closer to the threshold. Most of these effects are captured by the cluster energy corrections and are also reproduced by the simulated data. For the EMCal and PHOS measurements of the $\pi^{0}$ invariant yield, it turn out that the systematic uncertainty attributed to the clusterizer settings is about $2 \%$, which is obtained by making variations of the chosen thresholds. Similarly for the selection criteria $E_{\text {cluster }}$ and $N_{\text {cluster }}^{\text {cels }}$, it results in about a $1.5 \%$ systematic uncertainty for the neutral meson invariant yield. For $t_{\text {cluster }}$ the situation is slightly different, as the simulated data does not include timing distributions of the calorimeter cells and neither includes pile-up. The uncertainty is purely estimated from variations of the cluster time in experimental data, and the associated systematic uncertainty is estimated to be $1 \%$ for the neutral meson invariant yield. The systematic uncertainty related to the shower shape parameter $M_{02}$ is obtained by simultaneous variations of the selection criteria in both experimental and simulated data, and recalculating the resulting neutral meson invariant yield, and turns out to be a $1 \%$ systematic uncertainty. The systematic uncertainty related to the matching of charged tracks to the calorimeter clusters is obtained by again making variations of the selection criteria, i.e. the width in $\eta$ and $\varphi$ in which the charged track is considered to be matched to the cluster. As this is reproduced well by the simulated data, it results in an uncertainty of $3 \%$ for the neutral meson invariant yield. Systematic uncertainties for other observables will be discussed in the corresponding results section. 


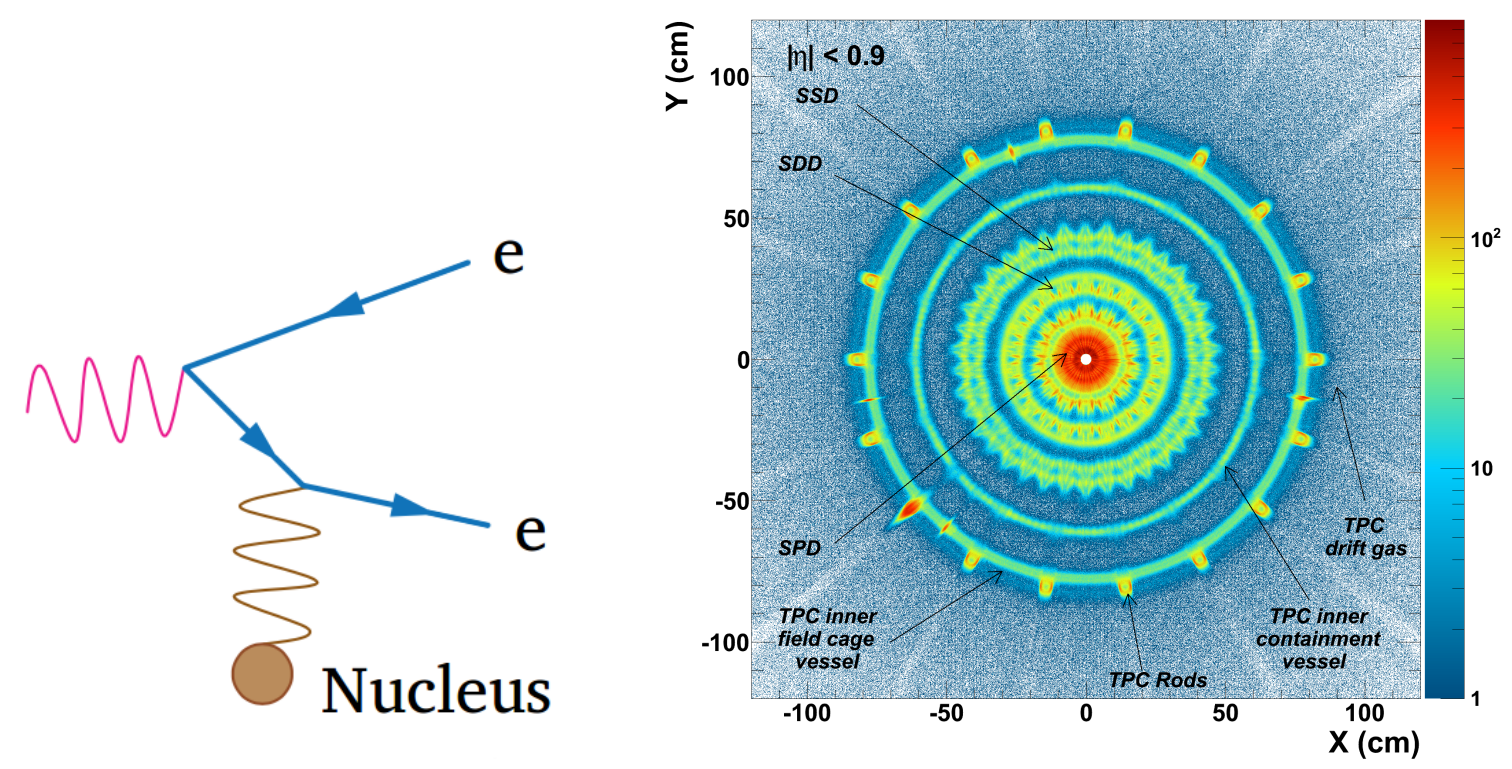

FIGURE 5.3: Left: Feynman diagram of a photon converting into an electron-positron pair via pair conversion. Right: Tomography of the ALICE detector using conversion photons.

\subsection{Photon measurements via conversions}

The second method, the photon conversion method (PCM), measures photons that convert into an electronpositron pair within the ALICE detector, and relies on reconstructing the charged tracks and their secondary vertex. As photons are stable in vacuum, this process $\left(\gamma+X \rightarrow e^{+}+e^{-}\right)$, as indicated in Fig. 5.3, is only possible in the presence of a charged scattering partner, which can e.g. be a nucleus in the detector material. From this it follows that the chance of a photon converting within the detector is proportional to the density and length of the material it traverses. The main signature of a conversion photon is a $e^{+} e^{-}$ production vertex at distance $R$ from the primary vertex, without a charged track connecting the primary and secondary vertex. The reconstruction, selection, and systematic uncertainties of converted photons will now be discussed in detail.

\subsubsection{Photon reconstruction}

The common algorithm used within the ALICE Collaboration to reconstruct neutral particles that decay into two oppositely charged particles is the on-the-fly $\mathrm{V}^{0}$-finder, where $\mathrm{V}^{0}$ is the generic name of candidates that share this same decay topology. Apart from $\gamma \rightarrow e^{+} e^{-}$, the algorithm can be used to find particles decays such as the $\Lambda^{0} \rightarrow \mathrm{p}^{+} \pi^{-}$and $\mathrm{K}_{S}^{0} \rightarrow \pi^{+} \pi^{-}$. It is also possible that the $\mathrm{V}^{0}$ is not corresponding to any particle decay at all, and can be reconstructed by combining tracks that accidentally result in a similar decay topology. This background is reduced by applying quality selection criteria on the charged tracks that are used in the $\mathrm{V}^{0}$-finder. The charged tracks are required to have at least a transverse momentum of $50 \mathrm{MeV} / c$, are within $\left|\eta_{\text {track }}\right|<0.9$, and need to have a large amount of clusters in the TPC 


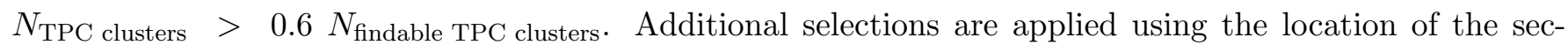
ondary vertex with respect to the nominal position of the interaction point in ALICE, which includes a selection on the conversion radius of $5 \mathrm{~cm}<R_{\mathrm{conv}}<180 \mathrm{~cm}$ and a selection on the secondary vertex location along the z-axis $\left|\mathrm{z}_{\text {conv }}\right|<240 \mathrm{~cm}$. The lower bound of $R_{\text {conv }}$ corresponds to the first layer of the ITS, and the upper bound to outer field cage of the TPC cylinder.

\subsubsection{Photon selection}

Further discrimination between signal and background is based on the identification of the electron-positron pair and the decay topology of the pair production process. Electrons and positrons are identified making use of the specific energy loss $\mathrm{d} E / \mathrm{d} x$ in the TPC that is dependent on the type and transverse momentum of the particle traversing the gas volume. Rather than using the $\mathrm{d} E / \mathrm{d} x$ of the candidate directly, it is rescaled using

$$
n \sigma_{\mathrm{e}, \mathrm{TPC}}=\frac{\left.\frac{\mathrm{d} E}{\mathrm{~d} x}\right|_{\text {candidate }}-\left\langle\left.\frac{\mathrm{d} E}{\mathrm{~d} x}\right|_{\text {electron }}\right\rangle}{\sigma_{\left\langle\left.\frac{\mathrm{d} E}{\mathrm{~d} x}\right|_{\text {electron }}\right\rangle},}
$$

where $\left\langle\left.\frac{\mathrm{d} E}{\mathrm{~d} x}\right|_{\text {electron }}\right\rangle$ is the expected value for an electron, and $\sigma_{\left\langle\left.\frac{\mathrm{d} E}{\mathrm{~d} x}\right|_{\text {electron }}\right\rangle}$ is the characteristic width of the $\mathrm{d} E / \mathrm{d} x$ distribution of the electron. More explicitly, the $\mathrm{V}^{0}$ is only selected if both daughter tracks have a response with $-4<n \sigma_{e, \mathrm{TPC}}<5$. Depending on the pair momentum, this criterion can remove about $50 \%$ of the background, without any significant loss on the signal. As shown in Fig. 5.4, there is an extended range in $p_{\mathrm{T}}$ where the response of $e^{ \pm}$and $\pi^{ \pm}$overlap. The signal to background ratio $(S / B)$ in this region is improved by rejecting $\mathrm{V}^{0}$ candidates where either of the daughter tracks have a $\mathrm{d} E / \mathrm{d} x$ value below $n \sigma_{\pi}=1$, with $n \sigma_{\pi}$ defined similarly as $n \sigma_{e, \text { TPC. }}$
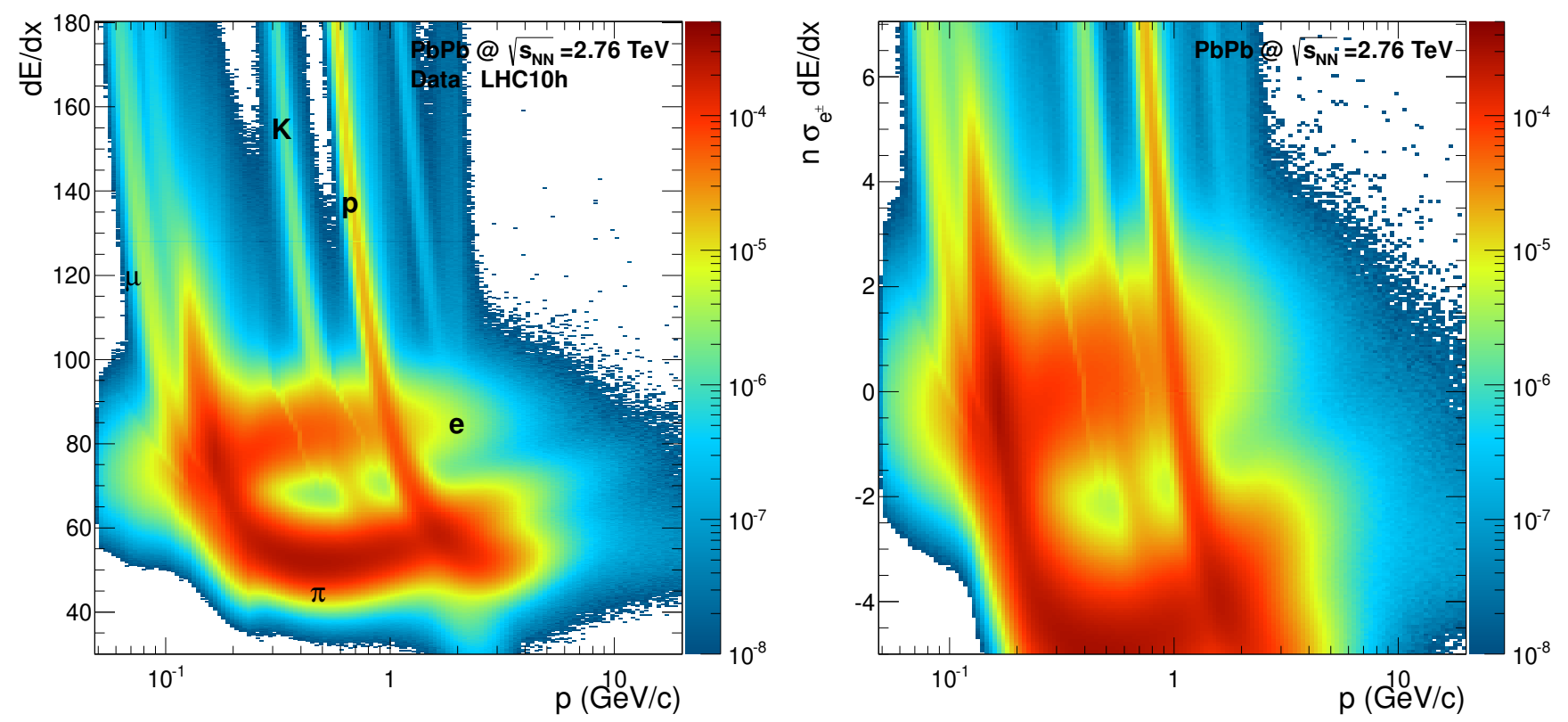

FIgURE 5.4: The specific energy loss $(d \mathrm{E} / d \mathrm{x})$ of charged particles traversing the TPC (left), and the same distribution relative to the electron hypothesis $\left(n \sigma_{e, \mathrm{TPC}}\right)$ (right). 


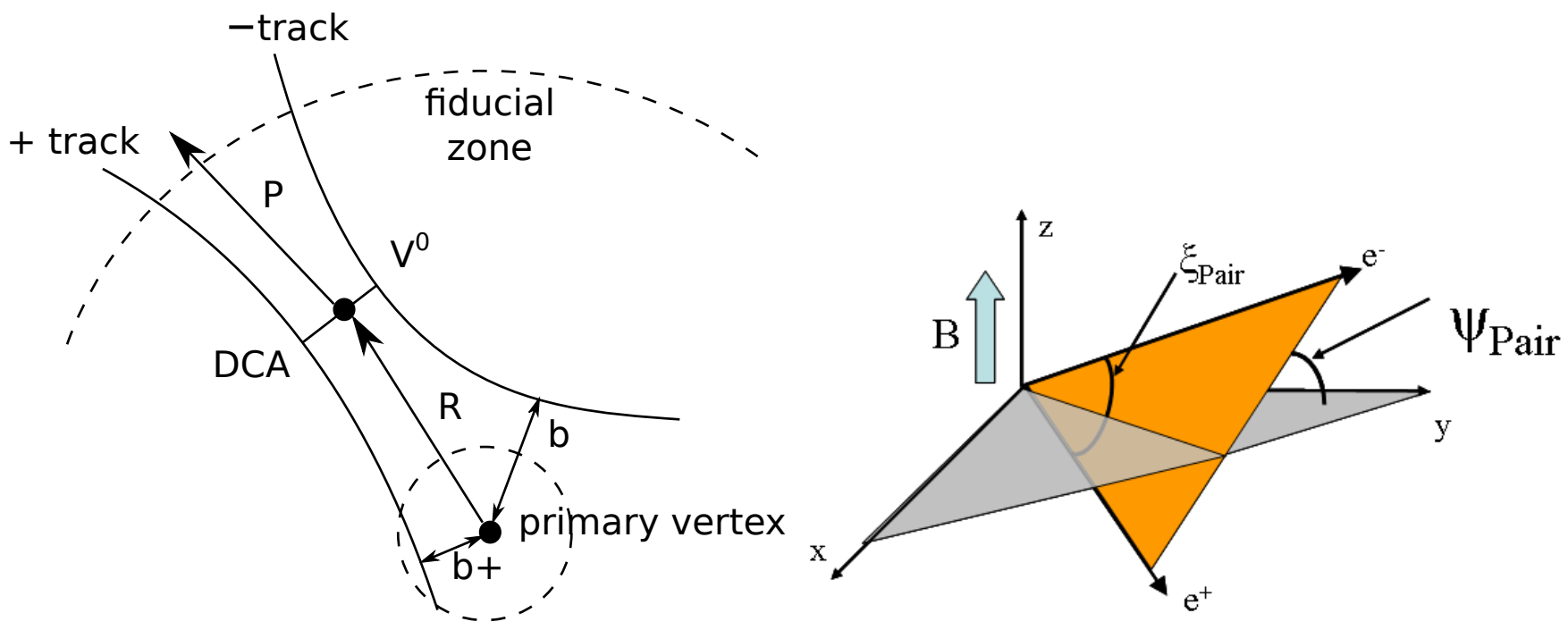

FIGURE 5.5: Topology of a photon converting into an electron-positron pair (left), and the visualization of the $\Psi_{\text {pair }}$ variable (right).

Further selection of photon conversions is done using the topology of the decay, which is shown in detail in Fig. 5.5. Here, the production point is indicated by the secondary vertex at distance $R=|\vec{R}|$ from the primary vertex, and is located by finding the distance of closest approach (DCA) of the two charged tracks. The sum of the momentum vectors of the two charged tracks is labeled with $\vec{P}$. As the $\mathrm{V}^{0}$-finder uses these vectors and geometrical quantities for the minimization process with the $\chi^{2}$ method, the $\chi^{2} /$ ndf that is calculated for each candidate can be used to select on the overall quality of the decay. By default candidates with $\chi^{2} /$ ndf $<30$ are selected.

Additionally, since the electron and positron are bent in opposite directions by the magnetic field and are produced with nearly zero relative momentum, the orientation of the decay is correlated with the direction of the magnetic field. The associated geometric variable is $\Psi_{\text {pair }}$, and is defined as the angle between the plane spanned by the two charged tracks and the plane which is perpendicular to the magnetic field. The distribution is centered around $\Psi_{\text {pair }}=0$, with a much narrower width for signal compared to $\mathrm{V}^{0}$ candidates from combinatorial background. It is advantageous to simultaneously use the two-dimensional $\Psi_{\text {pair }}$ and $\chi^{2} /$ ndf, accepting candidates if $\left|\Psi_{\text {pair }}\right|<\Psi_{\text {pair,cut }}-\frac{-\Psi_{\text {pair }}^{\text {cut }}}{\chi_{\text {cut }}^{2} \cdot \chi_{\gamma}^{2}}$, where $\Psi_{\text {pair,cut }}=0.1$ and $\chi^{2} /$ ndf $<30$ as before.

Furthermore, if the $\mathrm{V}^{0}$ candidate is from a correctly reconstructed decay or conversion, $\vec{P}$ should point in the same direction as $\vec{R}$. This fact is exploited using $\cos \left(\theta_{\text {point }}\right)$, where $\theta_{\text {point }}$ is the opening angle between $\vec{P}$ and $\vec{R}$. For the signal, the distribution peaks sharply for $\cos \left(\theta_{\text {point }}\right)=1$, while the distribution for the background is much broader.

Lastly, the identity of the $\mathrm{V}^{0}$ candidates can be further discriminated by selecting a region in the so-called Armenteros-Podolanski space [126]. This combines the two kinematic variables $q_{\mathrm{T}}$ and $\alpha$, where $q_{\mathrm{T}}$ is the relative momentum of the daughters with respect to the $\mathrm{V}^{0}$, and $\alpha$ is the longitudinal momentum asymmetry 
of the oppositely charged daughters. $\alpha$ defined as

$$
\alpha=\frac{p_{\mathrm{L}}^{+}-p_{\mathrm{L}}^{-}}{p_{\mathrm{L}}^{+}+p_{\mathrm{L}}^{-}},
$$

where $p_{\mathrm{L}}^{+}$is the longitudinal momentum of the positively charged daughter with respect to the parent particle, and $p_{\mathrm{L}}^{-}$is the longitudinal momentum of the negatively charged daughter, again with respect to the parent particle. The main discriminating power here is between $\gamma, \mathrm{K}_{\mathrm{S}}^{0}$, and $\Lambda(\bar{\Lambda})$. The asymmetry in $\alpha$ for $\Lambda$ and $\bar{\Lambda}$ is caused by the mass difference for the decay products. As shown in Figure $5.6, \gamma, \mathrm{K}_{\mathrm{S}}^{0}$ and $\Lambda(\bar{\Lambda})$ each have a characteristic band. A selection in the Armenteros-Podolanski space improves the $S / B$ for photons and is implemented by the two-dimensional selection:

$$
\left(\frac{\alpha}{0.95}\right)^{2}+\left(\frac{q_{\mathrm{T}}}{q_{\mathrm{T}, \max }}\right)^{2}<1,
$$

with $q_{\mathrm{T}, \max }=0.05 \mathrm{GeV} / \mathrm{c}$ which has been found by optimization. All the criteria for photon reconstruction via PCM and the corresponding selections are summarized in Table 5.3.
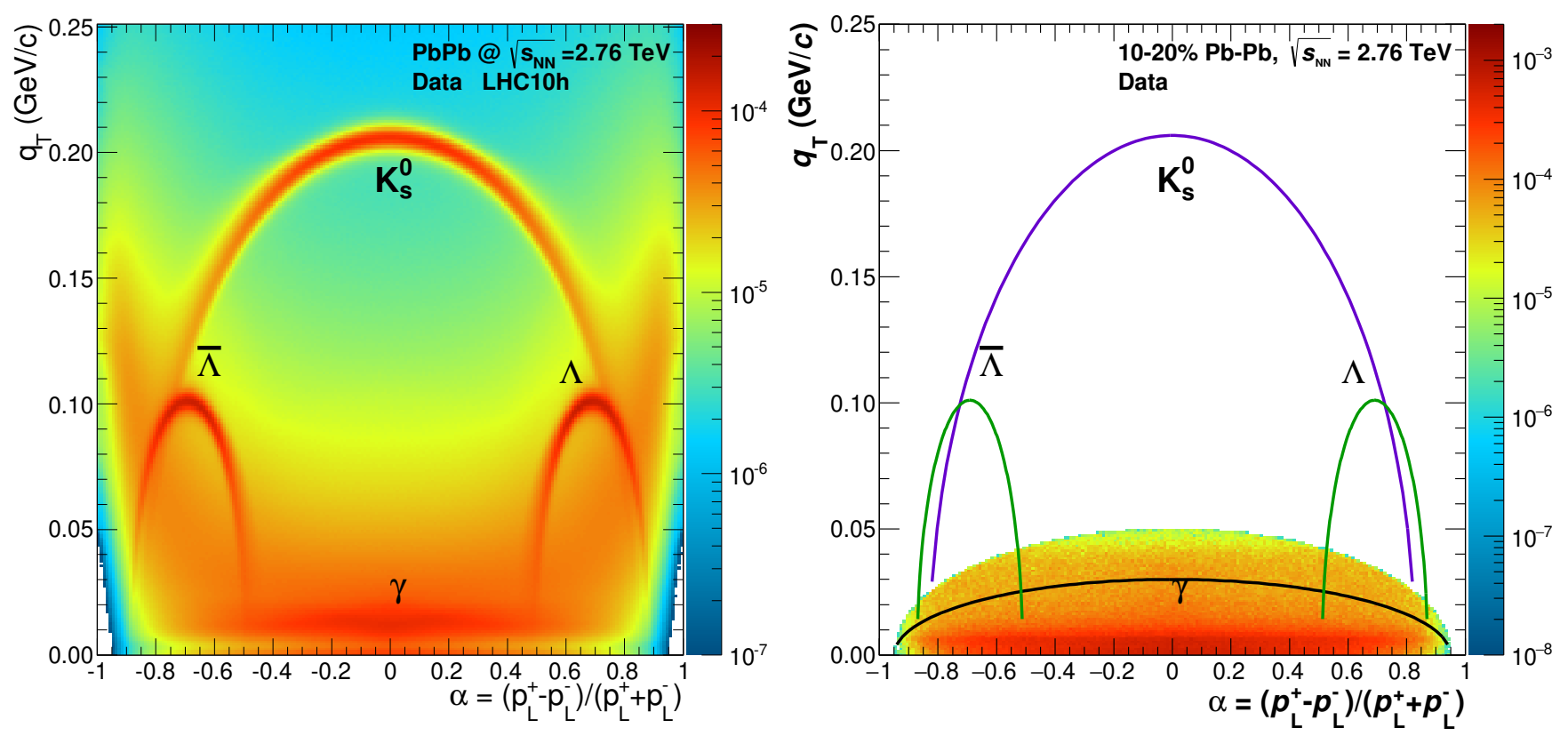

Figure 5.6: Armenteros-Podolanski distributions before (left) and after two-dimensional selection to improve the signal to background ratio of the $\gamma_{\text {conv }}$ sample (right). 


\subsubsection{Systematic uncertainties}

As explained at length in this section, the photon conversion method is based on reconstructing photons that happen to convert to an electron-positron pair in the detector material. As a result, the systematic uncertainties [125] are mainly related to the $e^{+} e^{-}$reconstruction and selection criteria, as well as the description of the detector material in the Monte Carlo simulations.

The $p_{\mathrm{T}, \text { track }}, n \sigma_{e}$, and $n \sigma_{\pi}$ selection criteria of the electron and positron tracks are found to have a significant contribution to the systematic uncertainty, and result in a $1 \%, \sim 4 \%$, and $1-6 \%$ uncertainty for the neutral meson measurements, respectively. The two latter have a larger dependence on the $p_{\mathrm{T}}$ of the photon, as the average $\mathrm{d} E / \mathrm{d} x$ for different particle species changes with track momentum, as indicated in Fig. 5.4. These uncertainties are estimated by making variations of the selection criteria, and thus again depend directly on the quality of the Monte Carlo description. Furthermore, the systematic uncertainty of the two-dimensional $\chi_{\gamma}^{2} /$ ndf and $\Psi_{\text {pair }}$ selection decreases from $\sim 7 \%$ at low neutral meson $p_{\mathrm{T}}$ to $\sim 2 \%$ at higher neutral meson $p_{\mathrm{T}}$. The two-dimensional $q_{\mathrm{T}, \max }$ and $\alpha$ photon selection results in a $\sim 2 \%$ systematic uncertainty.

The largest contribution to the systematic uncertainty comes from the difference in the amount of detector material present in the experimental and simulated data. If the material budget of the detector is overestimated in simulated data, the calculated conversion probability is too high, and vice versa. As a result, the invariant yield of photons, or neutral mesons reconstructed with PCM, can be systematically different from the true value. Studies have resulted in an estimate of the material budget uncertainty of $4.5 \%$ per photon and $9 \%$ for neutral meson measurements, which has been calculated by comparing the conversion point distributions in data and MC. Since this is the largest uncertainty in PCM, there is an ongoing investigation to reduce this uncertainty further, mainly by improving the MC description. 


\begin{tabular}{l|l}
\hline Charged track \& $\mathbf{V}^{0}$ selection & \\
\hline $\mathrm{V}^{0}$-finder & on-the-fly \\
\hline minimum track $p_{\mathrm{T}}$ & $p_{\mathrm{T}, \text { track }}>0.05 \mathrm{GeV} / c$ \\
\hline$\eta$, tracks \& $\mathrm{V}^{0} \mathrm{~s}$ & $\left|\eta_{\text {track }, \mathrm{V} 0}\right|<0.9$ \\
\hline$N_{\mathrm{TPC} \text { clusters }} / N_{\text {findable TPC clusters }}$ & $>60 \%$ \\
\hline$R_{\text {conv }}$ & $5<R_{\text {conv }}<180 \mathrm{~cm}$ \\
\hline $\mathrm{Z}_{\text {conv }}$ & $\left|\mathrm{z}_{\text {conv }}\right|<240 \mathrm{~cm}$ \\
\hline$\varphi_{\text {conv }}$ & $0 \leq\left|\varphi_{\text {conv }}\right| \leq 2 \pi$ \\
\hline Electron-positron identification & $-4<n \sigma_{e}<5$ \\
\hline$n \sigma_{e}$ TPC $\mathrm{d} E / \mathrm{d} x$ & $0.4<p<100 \mathrm{GeV} / c$ \\
\hline$n \sigma_{\pi} \mathrm{TPC} \mathrm{d} E / \mathrm{d} x$ & $n \sigma_{\pi}>1$ \\
\hline Photon selection & $<0.05 \mathrm{GeV} / c$ \\
\hline$q_{\mathrm{T}, \text { max }}\left(2 \mathrm{D}\right.$ cut,$\left.\left(\frac{\alpha}{0.95}\right)^{2}+\left(\frac{q_{\mathrm{T}}}{q_{\mathrm{T}, \text { max }}}\right)^{2}<1\right)$ & $<30$ \\
\hline$\chi_{\gamma}^{2} /$ ndf & $<0.1$ \\
\hline$\Psi_{\text {pair }}\left(2 \mathrm{D}\right.$ cut, $\left.\left|\Psi_{\text {pair }}\right|<\frac{-\Psi_{\text {pair }}^{\text {cut }}}{\chi_{\gamma, \text { cut }}^{2} \cdot \chi_{\gamma}^{2}}+\Psi_{\text {pair,cut }}\right)$ & $<0.85$ \\
\hline $\cos \left(\theta_{\mathrm{P} . \mathrm{A} .}\right)$ cut & $>$ \\
\hline
\end{tabular}

TABLE 5.3: Reconstruction and selection criteria of photon conversions, as done for the charged track and $\mathrm{V}^{0}$ selection, electron-positron identification of the $\mathrm{V}^{0}$ daughter tracks, and photon selection. 


\section{Part III}

Analysis Results 



\section{Chapter 6}

\section{Neutral meson production}

\subsection{Introduction}

Measuring the production of identified particles is undeniably one of the most fruitful methods to gain knowledge about the nature of high energy pp, pA, and AA collisions. Although QCD provides us with a framework to accurately calculate high momentum transfer processes in pp collisions, at lower momentum and as well for larger collision systems the production mechanisms are far from understood. Here, the main idea is to measure the invariant yield of neutral mesons [127] and their nuclear modification [128] for all collision systems and compare them to different model calculations that have varying underlying assumptions.

While the mechanisms behind light neutral meson production [129-133] are like the ones of charged light mesons [134-137], their experimental reconstruction technique is completely different, as they are measured by their decay channel into two photons. This offers both an independent measurement, as well as other advantages such as exploiting the specialized photon triggers of the calorimeters, enhancing greatly the statistics and reach at higher momentum. Furthermore, precise neutral meson measurements improve the extraction of direct photons, as the photons coming from neutral meson decays are by far the largest background that has to be known and understood.

In this chapter the analysis method of reconstructing neutral mesons and obtaining their invariant yield including all necessary corrections and sources of systematic uncertainty will be explained in detail. The result section will present all measurements relevant for this thesis, and will cover both pp, $\mathrm{p}-\mathrm{Pb}$, and $\mathrm{Pb}-\mathrm{Pb}$ collisions. The chapter is concluded with an overall discussion of neutral meson production. 


\subsection{Analysis method}

The production of neutral mesons is measured as the transverse-momentum-dependent invariant yield, and is given by

$$
E \frac{\mathrm{d}^{3} N}{d p^{3}}=\frac{\mathrm{d}^{3} N}{p_{\mathrm{T}} \mathrm{d} p_{\mathrm{T}} \mathrm{d} y d \varphi}=\frac{1}{2 \pi} \frac{1}{p_{\mathrm{T}}} \frac{\mathrm{d}^{2} N}{\mathrm{~d} y \mathrm{~d} p_{\mathrm{T}}}=\frac{1}{2 \pi} \frac{1}{N_{\text {ev }}} \frac{1}{p_{\mathrm{T}}} \frac{1}{\epsilon_{\text {reco }}} \frac{1}{A} \frac{1}{\mathrm{BR}} \frac{N}{\Delta y \Delta p_{\mathrm{T}}},
$$

where $N_{\mathrm{ev}}$ is the number of events that pass the event selection criteria, and are used to calculate the meson candidates. Then, $p_{\mathrm{T}}$ is the transverse momentum of the meson candidate which is calculated as the vector sum of the $p_{\mathrm{T}}$ of the two photons, and $\epsilon_{\text {reco }}$ and $A$ are respectively the reconstruction efficiency and geometrical acceptance of the meson candidate and are largely driven by the single photon efficiency and acceptance of the detector. The BR is the branching ratio of the decay, and corrects for the fact that not all neutral mesons decay via the chosen channel. In this case we have $\operatorname{BR}\left(\pi^{0} \rightarrow \gamma \gamma\right)=98.8 \%$ and $\mathrm{BR}(\eta \rightarrow \gamma \gamma)=39.4 \%$ [138, 139]. Furthermore, $N$ is the raw yield of the meson candidate obtained by integrating the invariant mass distribution around its reconstructed mass position. Lastly, the invariant yield is calculated for a specific interval in rapidity $(\Delta y)$ and transverse momentum $\left(\Delta p_{\mathrm{T}}\right)$, resulting in a collection of data points that together form the a spectrum that spans a range in $p_{\mathrm{T}}$.

\subsubsection{The invariant mass distribution}

The mass of a particle is a Lorentz invariant quantity, and is given by $M_{\mathrm{inv}}^{2}=E^{2}-p^{2}$, using the convention $c=1$. Since the energy and momentum are conserved quantities for particle decays, the invariant mass of a particle decaying into two daughters, $C \rightarrow A+B$, is given by

$$
M_{C}^{2}=\left(E_{A}+E_{B}\right)^{2}-\left(p_{A}+p_{B}\right)^{2},
$$

which for massless particles reduces to

$$
M_{A B}=\sqrt{2 E_{A} E_{B}\left(1-\cos \theta_{A B}\right)}
$$

Here, $E_{A}$ and $E_{B}$ represent the energies of the two massless particles and $\theta_{A B}$ is the angle between their respective momentum vectors. Experimentally this can be utilized to measure particles that otherwise escape detection, such as the $\pi^{0}$ and $\eta$ meson that have a high branching ratio into two photons. These mesons can thus be reconstructed from "just" measuring the four-momentum vector of the decay products. So, if the two photons coming from a $\pi^{0}(\eta)$ decay are measured accurately, then $M_{\gamma \gamma}=134.98$ (547.86) $\mathrm{MeV}[138,139]$. The procedure for reconstructing neutral meson candidates is to calculate the $M_{\text {inv }}$ of all possible photon pairs that originate from the same collision and counting how many photon pairs have an 
invariant mass of the respective meson. However, there are two main complications. First, two photons that are not coming from the same hadronic decay can still by chance give an invariant mass that is equal or indistinguishable to that of the respective neutral meson. Secondly, the energy and opening angle of the two photons are measured with finite precision, which broadens the calculated $M_{\text {inv }}$. These complications lead to the fact that neutral mesons are reconstructed as an excess yield on top of a combinatorial background, which has to be removed in order to give a correct and reliable neutral meson count.

The signal distribution of the neutral meson has a large Gaussian component, attributed mostly to the energy resolution of the detector. The background distribution has two main components; correlated and uncorrelated combinatorial background. The correlated part is driven by photon pairs that do not come from the same hadronic decay, but are created in a common physics process like a parton shower. The uncorrelated part of the background comes from photon pairs that do not share a common production process and the shape of the distribution is driven by the geometry and characteristics of the detectors, which impact the energy and opening angle distributions of the photons. The sum of the signal and background photon-pair invariant mass distributions is shown in Fig. 6.1 and Fig. 6.2 for pp at $\sqrt{s}=5 \mathrm{TeV}$ and $\mathrm{Pb}-\mathrm{Pb}$ collisions at $\sqrt{s_{N N}}=5 \mathrm{TeV}$, respectively. The signal-to-background ratio depends on the collision system, reconstruction method of the photons, and the $p_{\mathrm{T}}$ of the neutral meson.
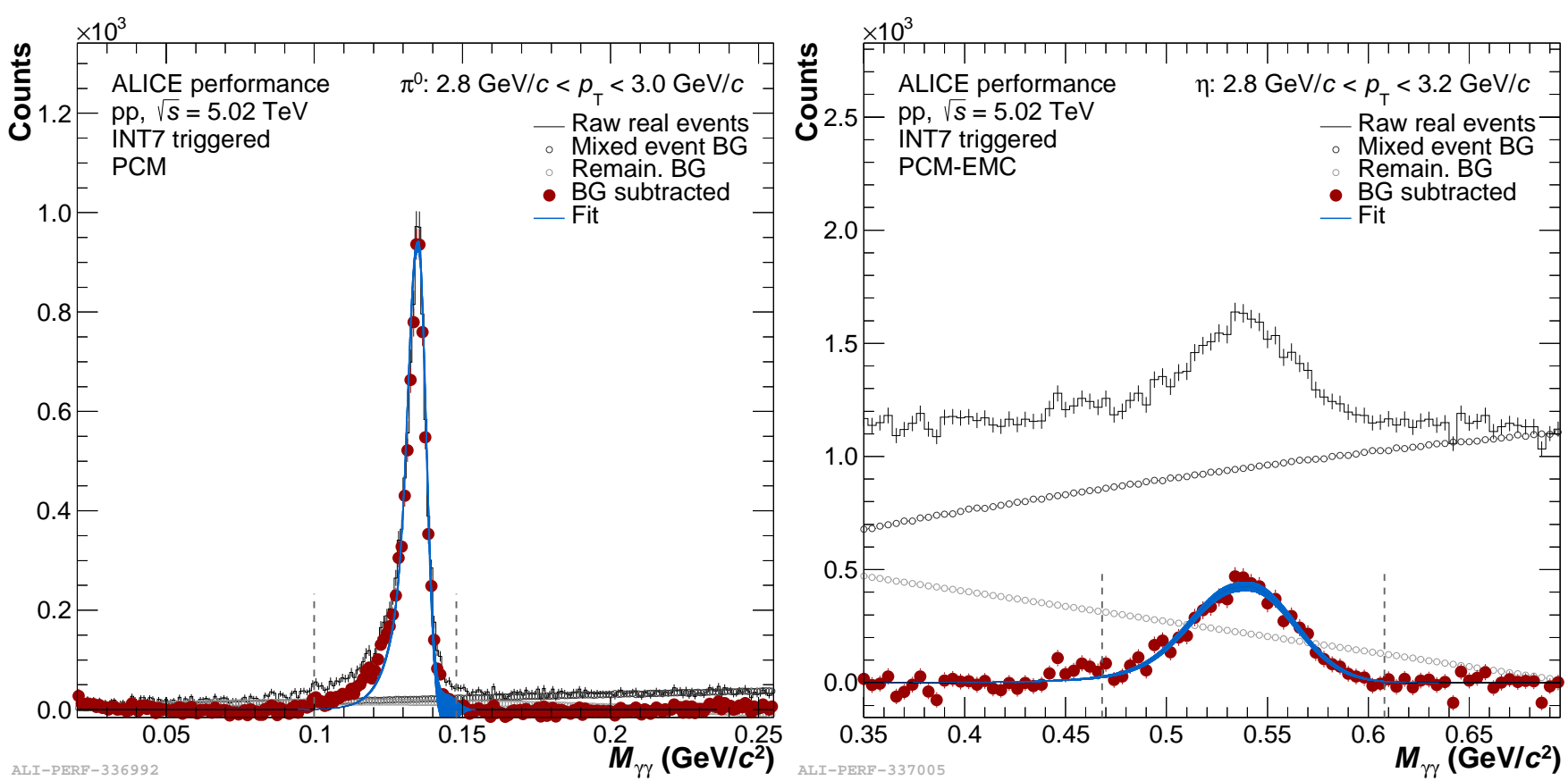

Figure 6.1: The photon-pair invariant mass distributions around the $\pi^{0}$ mass (left) and $\eta$ mass (right), for pp collisions at $\sqrt{s}=5.02 \mathrm{TeV}$. In this example the $\pi^{0}$ is obtained using PCM for a photon-pair $p_{\mathrm{T}}$ of $2.8 \mathrm{GeV} / c<p_{\mathrm{T}}<3.0 \mathrm{GeV} / c$, and the $\eta$ meson is obtained using PCM-EMC for a photon-pair $p_{\mathrm{T}}$ of $2.8 \mathrm{GeV} / c<p_{\mathrm{T}}<3.2 \mathrm{GeV} / c$. 

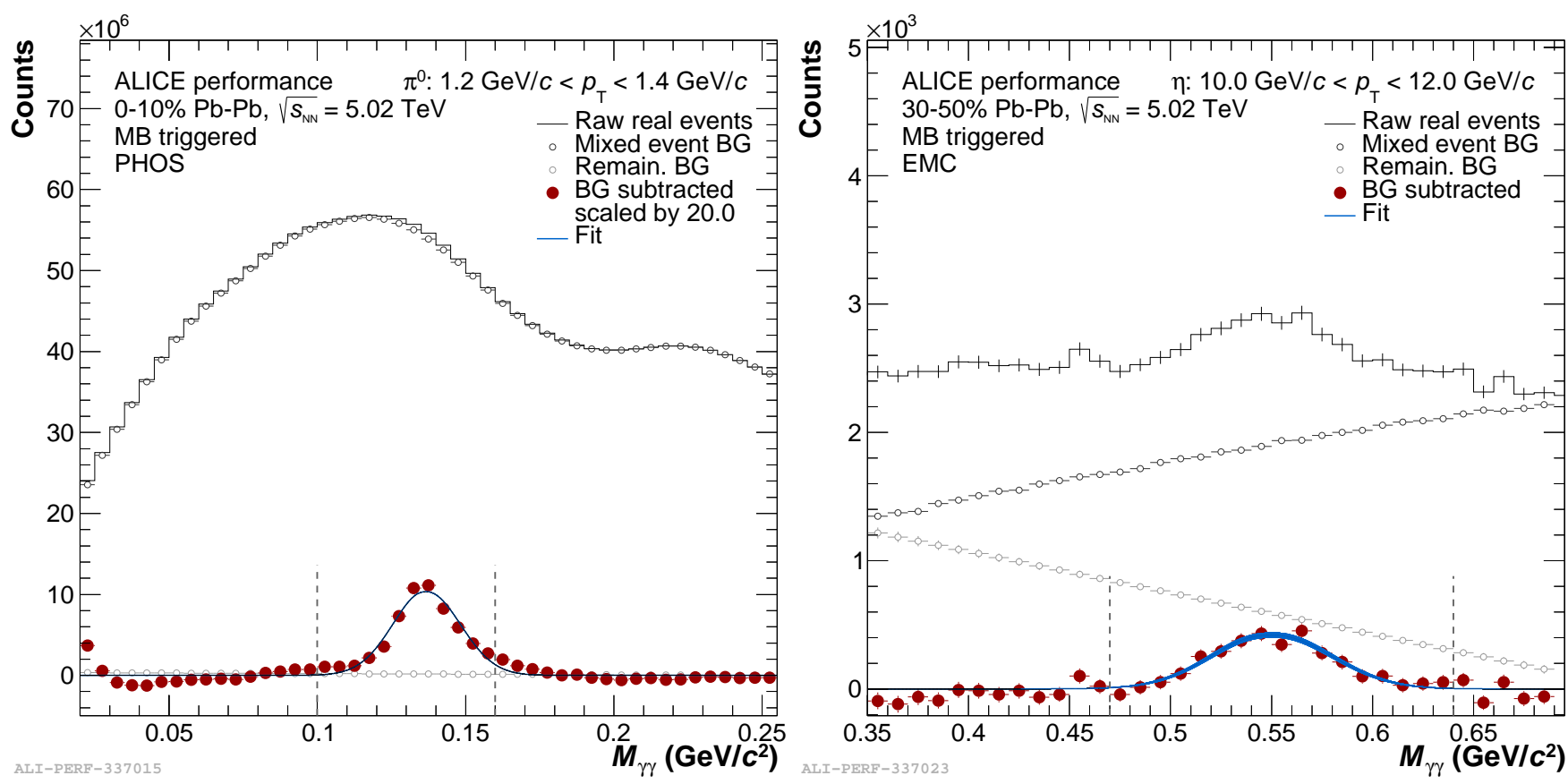

Figure 6.2: The photon-pair invariant mass distributions around the $\pi^{0}$ mass (left) and $\eta$ mass (right), for $\mathrm{Pb}-\mathrm{Pb}$ collisions at $\sqrt{s}=5.02 \mathrm{TeV}$. In this example the $\pi^{0}$ is obtained using the PHOS for a photonpair $p_{\mathrm{T}}$ of $1.2 \mathrm{GeV} / c<p_{\mathrm{T}}<1.4 \mathrm{GeV} / c$, and the $\eta$ meson is obtained using EMC for a photon-pair $p_{\mathrm{T}}$ of $10.0 \mathrm{GeV} / c<p_{\mathrm{T}}<12.0 \mathrm{GeV} / c$.

\subsubsection{Combinatorial background}

The combinatorial background can be approximated using the event-mixing method. With this method, photons are paired exclusively from different events. This ensures that the photons can never originate from the same hadronic decay, and hence no signal peak can be present.

The invariant mass distribution obtained from the event mixing method approximates the combinatorial background from same-event pairs if the energy and opening angle distributions of the photons in the same event equals that of the paired event. Experimentally this is done by performing the mixing for events with similar primary vertex $(z)$ locations and photon multiplicities. The uncorrelated part of the combinatorial background is very effectively modeled with this method. The correlated part is not captured by this method; this would require mixing events with exactly the same physics processes orientated in exactly the same direction in the detector, and is experimentally near impossible. Attempts to improve event mixing to also capture the correlated part of the background have been made, but none of them turned out to be successful.

The technical implementation of the event-mixing method is done as follows. After an event has been analysed, the energy and the momentum vectors of the reconstructed photons are stored in a first-in-firstout (FIFO) buffer with a standard pool size of 80 , meaning the buffer stores the information of the last 80 photons. For each event the photons of the current event are paired one by one with all the photons in the FIFO buffer and the invariant mass of the pair is calculated. In addition, there are multiple FIFO buffers 


\begin{tabular}{lrr}
\hline Class & Bin & \\
\hline Photon multiplicity $\left(N_{\gamma}\right)$ & 1 & 2 \\
& 2 & 3 \\
& 3 & 4 \\
& 4 & $>4$ \\
\hline$z$-vertex coordinate $(\mathrm{cm})$ & 1 & $-50.0--3.38$ \\
& 2 & $-3.38--1.61$ \\
& 3 & $-1.61--0.23$ \\
& 4 & $-0.23-1.07$ \\
& 5 & $1.07-2.45$ \\
& 6 & $2.45-4.25$ \\
& 7 & $4.25-50.0$ \\
\hline
\end{tabular}

TABLE 6.1: Class definition for event mixing classes.

to enable the functionality to mix events with similar characteristics, as defined in Table 6.1. The speed at which the FIFO buffers are filled depends on the overall photon multiplicities and characteristics of the dataset, as well as the amount of FIFO buffers. Overall, this method is able to generate large amounts of statistics for the combinatorial background distributions.

The invariant mass distributions obtained with the event mixing are normalized to the invariant mass distributions of the same-event pairs. This normalization is done by calculating the normalization factors on the right side of the neutral meson signal peak. The normalization factors $C$ are determined by

$$
C=\int_{c_{1}}^{c_{2}} N_{\gamma \gamma} \mathrm{d} M_{\gamma \gamma} / \int_{c_{1}}^{c_{2}} N^{\text {comb. } \mathrm{BG}} \mathrm{d} M_{\gamma \gamma}
$$

where by default $c_{1}=0.2 \mathrm{GeV} / c^{2}$ and $c_{2}=0.3 \mathrm{GeV} / c^{2}$ for the $\pi^{0}$ meson, and $c_{1}=0.65 \mathrm{GeV} / c^{2}$ and $c_{2}=0.8 \mathrm{GeV} / c^{2}$ for the $\eta$ meson. Using an integral over a larger range in invariant mass rather than a single point in the distribution stabilizes the normalization procedure, as calculating the factor on a single point is subject to much larger fluctuations. In Fig. 6.1 and Fig. 6.2 the results of the event mixing method for a few example bins are shown. The results of the method agree reasonably well with the combinatorial background present in the invariant mass distribution of same-event pairs of the $\pi^{0}$, but for the $\eta$ there is a large amount of background remaining after subtracting the combinatorial background obtained with the event-mixing technique.

\subsubsection{Meson raw yield extraction}

The meson raw yield is extracted from the invariant mass distributions after subtracting the event-mixed background, for intervals in meson $p_{\mathrm{T}}$. The covered range in $p_{\mathrm{T}}$ is carefully optimized and depends on the reconstruction methods of the photons, the available statistics in the dataset $\left(N_{\mathrm{ev}}\right)$, and the quality of the background description [140]. The mass distributions after subtraction are fitted with 


$$
\begin{gathered}
f\left(M_{\gamma \gamma}\right)=A \cdot\left(G\left(M_{\gamma \gamma}\right)+e^{\left(\frac{M_{\gamma \gamma}-M_{\pi^{0}(\eta)}}{\lambda}\right)} \cdot\left(1-G\left(M_{\gamma \gamma}\right)\right) \cdot \theta\left(M_{\pi^{0}(\eta)}-M_{\gamma \gamma}\right)\right)+B+C \cdot M_{\gamma \gamma} \\
, \text { with } G=e^{\left(-0.5\left(\frac{M_{\gamma \gamma}-M_{\pi^{0}, \eta}}{\sigma_{M_{\gamma \gamma}}}\right)^{2}\right)} .
\end{gathered}
$$

Here, $G$ is a Gaussian function with width $\sigma$, amplitude $A$, and mean position $M_{\pi^{0}(\eta)}$, which can be identified with the reconstructed mass position of the corresponding meson. The parameter $\lambda$ is a free parameter of the exponential function. The contribution of the exponential function above $M_{\pi^{0}(\eta)}$ is switched off by the Heaviside function $\theta\left(M_{\pi^{0}, \eta}-M_{\gamma \gamma}\right)$. Furthermore, $B$ and $C$ are the parameters of the linear function. The Gaussian describes the majority of the signal, and the exponential tail on the left side is implemented to account for an incomplete energy measurement of either of the two photons. In the case where the photon is reconstructed with a calorimeter, the reconstructed energy of this photon can be incomplete due to the fact that the photon converted in the material just in front of the calorimeter, and the cluster is one of the electron daughters. In the case where the photon is measured using the photon conversion method, the electron daughters emit photons, i.e. electron bremsstrahlung, that reduce their energy and as a result that of the reconstructed photon. The linear part is used to account for remaining combinatorial background that is left in case the background obtained by the event-mixing method does not fully describe the background in the signal region.

The raw meson yield is obtained by integrating invariant-mass distributions after the combinatorial background has been subtracted. More specifically, it is obtained by counting the histogram values in a mass range around the fitted meson mass $M_{\pi^{0}(\eta)}$ using

$$
N_{\text {raw }}^{\pi^{0}}=\int_{M_{\pi^{0}}-0.032 \mathrm{GeV} / c^{2}}^{M_{\pi^{0}}+0.022 \mathrm{GeV} / c^{2}}\left(N_{\gamma \gamma}-N^{\text {comb. BG }}\right) \mathrm{d} M_{\gamma \gamma}-\int_{M_{\pi^{0}}-0.032 \mathrm{GeV} / c^{2}}^{M_{\pi^{0}}+0.022 \mathrm{GeV} / c^{2}}\left(B+C \cdot M_{\gamma \gamma}\right) \mathrm{d} M_{\gamma \gamma},
$$

where the remaining background is subtracted using the integral of the linear part of Eq. 6.5 and Eq. 6.6. The standard integration range for the $\pi^{0}$ meson is $\left(M_{\pi^{0}}-0.05 \mathrm{GeV} / c^{2}, M_{\pi^{0}}+0.04 \mathrm{GeV} / c^{2}\right)$, and for the $\eta$ meson the integration is performed in the interval $\left(M_{\eta}-0.08 \mathrm{GeV} / c^{2}, M_{\eta}+0.08 \mathrm{GeV} / c^{2}\right)$, taking into account the larger reconstructed width of the $\eta$ meson. This range is varied for both the $\pi^{0}$ and $\eta$ meson as a method to estimate the systematic uncertainty. The integration window is asymmetric to account for an incomplete energy measurement of either of the two photons as explained in more detail above. 


\subsubsection{Raw yield corrections}

The invariant yield is a universal quantity and should be independent of the detector that is used to obtain the results. The raw yields are corrected for feed-down from secondary neutral meson decays and all detector related effects. These correction factors are dependent on the detector technology, geometry, and running conditions. They are obtained by studying simulated data that include the full detector response. More specifically, realistic events are generated by state-of-the-art event generators, after which the produced particles are propagated through the detector material. The corrections can then be obtained by calculating the difference between the amount of generated and reconstructed particles.

\subsubsection{Correction for secondary neutral pions}

It is a convention to exclude the contributions from neutral mesons produced in weak decays or from hadronic interactions in the detector material. The largest source of secondary neutral mesons is the weak decay $\mathrm{K}_{\mathrm{S}}^{0} \rightarrow \pi^{0} \pi^{0}(\mathrm{BR}=30.7 \%)$, which can result in up to $\sim 10 \%$ of the total $\pi^{0}$ yield, depending on the reconstruction method and $p_{\mathrm{T}}$ of the meson. After that, the dominant contribution is from hadronic interactions, with a maximum contribution to the total $\pi^{0}$ invariant yield of $\sim 1 \%$ at low $p_{\mathrm{T}}$. The feeddown from $\mathrm{K}_{\mathrm{L}}^{0}$ and $\Lambda$ is $<0.1 \%$, and is not significant. Additionally, there is no significant contribution of secondary $\eta$ mesons from weak decays to the total $\eta$ yield, so the correction is only done for the $\pi^{0}$.

The correction of the $\pi^{0}$ raw yield for the secondary $\pi^{0}$ mesons is done by calculating

$$
r_{X}\left(p_{\mathrm{T}}\right)=\frac{N^{\pi^{0} \text { from } X}\left(p_{\mathrm{T}}\right)}{N^{\pi^{0}}\left(p_{\mathrm{T}}\right)},
$$

where $r_{X}$ is the fraction of secondary $\pi^{0}$ mesons as a decay product of particle $X$, which is obtained for $X=\mathrm{K}_{\mathrm{S}}^{0}, \mathrm{~K}_{\mathrm{L}}^{0}, \Lambda$, and hadronic interactions (rest) using simulated data. This strategy is improved for the cases where the $\mathrm{K}_{\mathrm{S}}^{0}$ spectrum is published for the same dataset and event selection criteria. In this case the measured $\pi^{0}$ and $\mathrm{K}_{\mathrm{S}}^{0}$ spectrum is parametrized and used as input for a dedicated "cocktail" simulation.

\subsubsection{Acceptance and efficiency correction}

The detector technology, geometry, and running conditions affect the likelihood that a particle produced in the collision is actually measured. For instance, the coverage of the detector in $\eta$ and $\varphi$ around the collision zone is directly proportional to the chance that a particle is within the detector acceptance. Even if the particle is within the acceptance of the detector, there are many factors that can alter the outcome of the measurement; tracking and cluster algorithms, signal selection criteria such as energy thresholds, broken or noisy readout channels, overlapping signals, and so on. The amount of measured neutral mesons are corrected to equal the number of mesons produced in the collision. It is important to note that, although the neutral mesons are reconstructed from two photons, their corrections are not simply the 
product of the single photon correction factors, as there are additional non-trivial effects like for example the correlation between $p_{\mathrm{T}}$ of the meson and the opening angle of the daughter photons. To correct for all these effects it is customary to define two correction factors, the geometrical acceptance $A\left(p_{\mathrm{T}}\right)$, and the reconstruction efficiency $\epsilon_{\text {reco }}\left(p_{\mathrm{T}}\right)$. These quantities are calculated using Monte Carlo simulations that are explicitly reflecting the running conditions of the respective dataset.

The geometrical acceptance $A\left(p_{\mathrm{T}}\right)$ is defined as the ratio of $\pi^{0}(\eta)$ mesons where both of the daughter photons are within the acceptance of the detector, over all the $\pi^{0}(\eta)$ mesons generated in the same rapidity window

$$
A\left(p_{\mathrm{T}}\right)=\frac{N_{\pi^{0}(\eta),|y|<y_{\max }, \gamma \gamma \text { in acceptance }}}{N_{\pi^{0}(\eta),|y|<y_{\max }}},
$$

where in both cases the mesons are only counted if they are within $|y|<y_{\max }$, with $y_{\max }$ the maximum rapidity of the mesons in the analysis. The reconstruction efficiency $\epsilon_{\text {reco }}$ is defined as the ratio of $\pi^{0}(\eta)$ mesons that are reconstructed in the MC, over all the $\pi^{0}(\eta)$ mesons generated within the acceptance of the detector

$$
\epsilon_{\text {reco }}\left(p_{\mathrm{T}}\right)=\frac{N_{\pi^{0}(\eta), \mathrm{MC} \text { rec }}}{N_{\pi^{0}(\eta),|y|<y_{\max ,}, \gamma \gamma \text { in acceptance }}} .
$$

The $A\left(p_{\mathrm{T}}\right)$ increases monotonously with meson $p_{\mathrm{T}}$, as the opening angle between the photons decrease for increasing meson $p_{\mathrm{T}}$, and as a result increases the probability that both photons are within the same rapidity range as the meson. To first order, the reconstruction efficiency also increases for increasing meson $p_{\mathrm{T}}$, as the daughter photons with higher energies are less affected by energy thresholds of the calorimeters or tracking inefficiencies in the central barrel. However, at higher meson $p_{\mathrm{T}}$, the opening angle $\theta_{A B}$ decreases, creating overlaps of tracks and clusters that lead to indistinguishable photons and hence lowering the reconstruction efficiency $\epsilon_{\text {reco }}$.

\subsection{Uncertainties}

\subsubsection{Statistical uncertainties}

The statistical uncertainty of the neutral meson invariant yield for a given interval in $p_{\mathrm{T}}$ is calculated from the raw yield of the meson. More specifically, the statistical uncertainty of the raw yield is given by $\sigma_{\text {stat }}\left(p_{\mathrm{T}}\right)=\sqrt{N_{\text {meson }}}\left(p_{\mathrm{T}}\right)$, where $N_{\text {meson }}$ is obtained by integrating the invariant mass distribution as previously explained. The other terms in Eq. 6.1 are assumed to have no statistical uncertainty and scale $\sigma_{\text {stat }}\left(p_{\mathrm{T}}\right)$ in a straightforward way. The uncertainties related to these terms are taken into account in the systematic uncertainty. It is important to note that the size of $\sigma_{\text {stat }}\left(p_{\mathrm{T}}\right)$ drives the choice of the intervals in $p_{\mathrm{T}}$ that are used in the analyses, which are chosen such that the $\sigma_{\mathrm{stat}}\left(p_{\mathrm{T}}\right)$ is in general smaller than the systematic uncertainty. 


\subsubsection{Systematic uncertainties}

The systematic uncertainties $\left(\sigma_{\text {syst }}\left(p_{\mathrm{T}}\right)\right)$ of the neutral meson invariant yield have contributions from multiple sources. The largest source comes from imperfections of the Monte Carlo simulations, leading to a possible bias in the meson acceptance and efficiency calculation. The second largest source comes from the raw yield extraction. The $\sigma_{\text {syst }}\left(p_{\mathrm{T}}\right)$ for the $\pi^{0}$ and $\eta$ meson is estimated by varying the selection criteria of the photons and mesons, as well as varying the method of the raw yield extraction, and recalculating the fully corrected invariant yield. Each of the variations is done independently, as well as to multiple different values. This procedure results in a large number of spectra that deviate accordingly, and the magnitude of these deviations are used to estimate $\sigma_{\text {syst }}\left(p_{\mathrm{T}}\right)$. More specifically, for selection criterion $X$ with value $x$, one or multiple variations $(x+a, x-a, x+b, x-b, \ldots)$ are done. Then, the invariant yield is recalculated, and the systematic uncertainty $\sigma_{\mathrm{syst}}\left(p_{\mathrm{T}}\right)$ related to $X$ is estimated from the point-to-point differences in $p_{\mathrm{T}}$

$$
\Delta_{X}\left(p_{\mathrm{T}}\right)=\left(\frac{\mathrm{d}^{2} N}{\mathrm{~d} y \mathrm{~d} p_{\mathrm{T}}}\right)_{\text {modified }}\left(p_{\mathrm{T}}\right)-\left(\frac{\mathrm{d}^{2} N}{\mathrm{~d} y \mathrm{~d} p_{\mathrm{T}}}\right)_{\text {standard }}\left(p_{\mathrm{T}}\right)
$$

Then the systematic uncertainty belonging to selection criterion $X$ is calculated using the RMS

$$
\sigma_{\mathrm{syst}, X}\left(p_{\mathrm{T}}\right)=\sqrt{\frac{1}{n} \sum \Delta_{X}\left(p_{\mathrm{T}}\right)^{2}}
$$

where $n$ is the number of variations done for selection criterion $X$. In addition, for variations where $\sigma_{\text {syst }, X}\left(p_{\mathrm{T}}\right)$ is expected to be correlated in $p_{\mathrm{T}}$, the point-to-point fluctuations in $\sigma_{\mathrm{syst}, X}\left(p_{\mathrm{T}}\right)$ are reduced by applying a smoothing procedure. This is done by fitting the $\sigma_{\mathrm{syst}, X}\left(p_{\mathrm{T}}\right)$ distribution with $n$-order polynomials, where a smaller $\mathrm{n}$ is favored as long as the distribution is well described. It turns out that a 0th- or 1st-order polynomial describes most of the systematic uncertainties that are correlated in $p_{\mathrm{T}}$.

Finally, the total systematic uncertainty on the invariant yield is calculated by

$$
\sigma_{\mathrm{syst}}\left(p_{\mathrm{T}}\right)=\sqrt{\sum \sigma_{\mathrm{syst}, X}\left(p_{\mathrm{T}}\right)^{2}}
$$

In previous sections the photon reconstruction with PCM and the calorimeters EMCal and PHOS was explained in detail, including discussions on the their systematic uncertainties. This discussion will now be continued for the neutral meson selection criteria and raw yield extraction.

For the meson reconstruction techniques there are three sources of systematic uncertainty; the meson reconstruction efficiency, the selection on the minimum opening angle between the two photons, and the selection on the energy asymmetry $\alpha=\left(E_{\gamma, 1}-E_{\gamma, 2}\right) /\left(E_{\gamma, 1}+E_{\gamma, 2}\right)$. The meson reconstruction efficiency is dependent on the overall quality of the generator and the available statistics in the respective dataset. The uncertainty 
introduced due to this dependency is estimated by calculating the invariant yields using the efficiencies from a different generator. The minimum opening angle of a photon pair is driven by detector constraints, as photon pairs with $\theta_{A B}=0$ are nearly impossible to distinguish. The uncertainty introduced due to $\theta_{A B \text {,min }}$ is estimated by varying the criterion. This systematic uncertainty is mostly independent on $p_{\mathrm{T}}$. The energy asymmetry $\alpha$, which is in some cases is used to increase the $S / B$ of meson candidates, is also estimated by varying the criterion. All these uncertainties turn out to be relatively small with respect to the total systematic uncertainty.

For the raw yield extraction there are multiple sources of systematic uncertainty; the normalization and subtraction of the combinatorial background, the choice of integration range of the raw yield, and the shape of the remaining background. The first two sources are estimated by the variation method, which are all listed in Table 6.2 for both the $\pi^{0}$ and $\eta$ mesons. The error on the raw yield due to the remaining background is estimated by changing the fit function of the background. The RMS of the spread in the raw yield extraction is used as systematic uncertainty. The yield extraction error is one of the dominating sources of systematic uncertainty. All the results of the systematic uncertainties are listed in Appendix A.3.

\begin{tabular}{lll}
\hline & $\pi^{0}$ & $\eta$ \\
\hline $\begin{array}{l}\text { Normalization window } \\
\text { Right side (standard) }\end{array}$ & $(0.19,0.3) \mathrm{GeV} / c^{2}$ & $(0.65,0.75) \mathrm{GeV} / c^{2}$ \\
Left side (variation) & $(0.03,0.05) \mathrm{GeV} / c^{2}$ & $(0.35,0.42) \mathrm{GeV} / c^{2}$ \\
\hline Integration range & & \\
standard & $\left(M_{\pi^{0}}-0.05, M_{\pi^{0}}+0.04\right) \mathrm{GeV} / c^{2}$ & $\left(M_{\eta}-0.080, M_{\eta}+0.080\right) \mathrm{GeV} / c^{2}$ \\
narrow & $\left(M_{\pi^{0}}-0.03, M_{\pi^{0}}+0.024\right) \mathrm{GeV} / c^{2}$ & $\left(M_{\eta}-0.060, M_{\eta}+0.060\right) \mathrm{GeV} / c^{2}$ \\
wide & $\left(M_{\pi^{0}}-0.07, M_{\pi^{0}}+0.056\right) \mathrm{GeV} / c^{2}$ & $\left(M_{\eta}-0.10, M_{\eta}+0.10\right) \mathrm{GeV} / c^{2}$ \\
\hline
\end{tabular}

TABLE 6.2: Variations of the integration and normalization windows to estimate the systematic error from the raw yield extraction. 


\subsection{Neutral meson production in pp collisions}

In collaboration with Nicolas Schmidt.

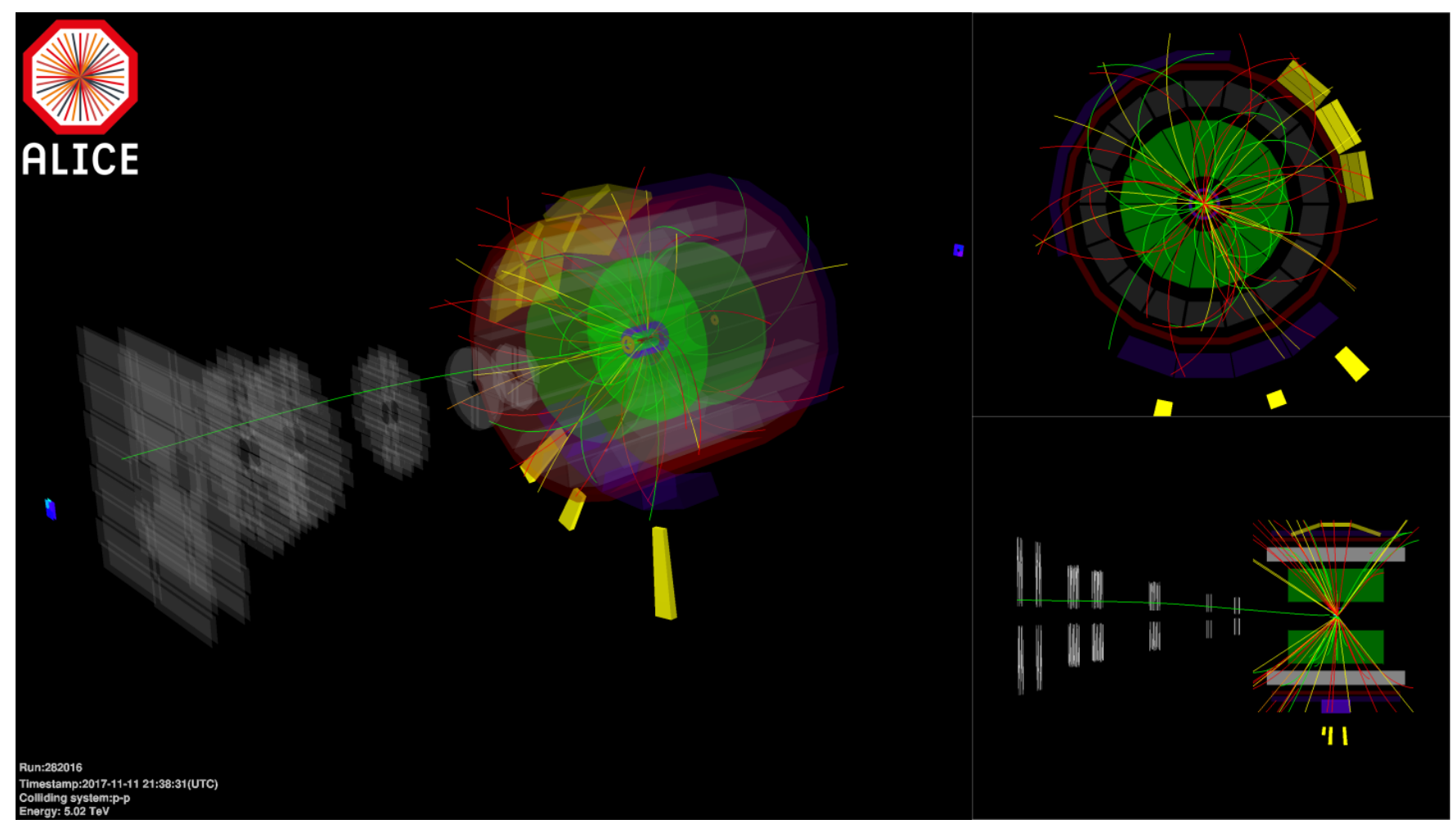

Figure 6.3: An example of a typical pp collision, recorded during the 2017 pp run at $\sqrt{s}=5.02 \mathrm{TeV}$.

An example of a typical pp collision is shown in Fig. 6.3, which was recorded during the 2017 pp run at $\sqrt{s}=5.02 \mathrm{TeV}$. The event display shows few tens of particles flying through the detector, being curved by the presence of the magnetic field generated by the ALICE magnet. The average amount of particles produced per unit rapidity in pp collisions $\left\langle\mathrm{d} N_{\mathrm{ch}} / \mathrm{d} \eta\right\rangle \sim 3.4$, meaning that the ALICE detector is operating much below its design occupancy capabilities when collecting data from pp collisions [141].

This section will present the neutral meson measurements in pp collisions at a center of mass energy of $\sqrt{s}=5.02 \mathrm{TeV}$, where the $p_{\mathrm{T}}$ dependent invariant yield of the $\pi^{0}$ and $\eta$ meson is measured for minimum bias data, different multiplicity and sphericity event classes, as well as in-jet production. The results will be compared to theoretical model calculations, including a larger reflection and discussion at the end of the chapter. 


\subsubsection{Datasets}

The datasets used in the neutral meson measurements for pp collisions at $\sqrt{s}=5.02 \mathrm{TeV}$ is given in Table 6.3. It utilizes all the minimum bias data collected by ALICE at this collision energy, as well as the calorimeter triggers for EMCal and PHOS. The data has been carefully inspected and run periods are only included if the required detectors are operating nominally. After applying the event selection criteria, which are described in section 4.3.1, there are 940 million minimum bias and over 13 million triggered events used for the analysis. Multiple Monte Carlo simulations are used to study detector effects and to calculate the various correction factors. The MC sampling is close to $100 \%$, meaning that the total amount of events in the simulated data is close to that of the experimental data. Furthermore, so called jet-jet simulations are used to enhance the amount of high $p_{\mathrm{T}}$ particles, which are used for the events triggered with the calorimeters. These simulations include a procedure that mimics the different hardware triggers of the calorimeters.

Calibration maps are used for the TPC to ensure good particle identification by the central barrel, and are dependent on $\eta$ and $\phi$. Furthermore, the temperature, time, and energy of the EMCal, DCal, and PHOS calorimeters cells have been calibrated channel by channel for all datasets, again to ensure good detector performance and good quality photon candidates.

\begin{tabular}{cllrr}
\hline$\sqrt{s}(\mathrm{TeV})$ & Data Set & & Used triggers & \# events \\
\hline 5.02 & Data & LHC15n pass 4 & INT7 & $100 \cdot 10^{6}$ \\
& Data & LHC15n pass 4 & CF-EMC7 & $1.17 \cdot 10^{6}$ \\
& PYTHIA8 (MC) & LHC17e2 + LHC18j3 & INT7 & $90 \cdot 10^{6}$ \\
& PYTHIA8 JJ (MC) & LHC16h3 & simulated EMC7 & $145 \cdot 10^{6}$ \\
\hline \multirow{2}{5}{5.02} & Data & LHC17p + LHC17q pass 1 & & \\
& Data & FAST \& CENT(woSDD) & INT7 & $830 \cdot 10^{6}$ \\
& Data & LHC17p + LHC17q pass 1 & $\mathrm{kPHI7}$ & $1.2 \cdot 10^{6}$ \\
& PYTHIA8 (MC) & LHC17p + LHC17q pass 1 & CF-EG2 & $10.7 \cdot 10^{6}$ \\
& LHC1713b + LHC18j2 & & \\
& PYTHIA8 JJ (MC) & LAST \& CENT(woSDD) & INT7 & $720 \cdot 10^{6}$ \\
& & sHCb8 & &
\end{tabular}

TABlE 6.3: Datasets used for the neutral meson measurements in pp collisions at a center of mass energy of $\sqrt{s}=5.02 \mathrm{TeV}$. 


\subsubsection{Results}

\section{Invariant mass distributions}

As explained in detail in the previous sections, the transverse momentum $\left(p_{\mathrm{T}}\right)$ dependent invariant yield of the $\pi^{0}$ and $\eta$ mesons is obtained by correcting the respective raw yields. For this thesis, the photons are reconstructed with the Photon Conversion Method (PCM) as well as the calorimeters EMCal and PHOS. Furthermore, the invariant mass $\left(M_{\mathrm{inv}}\right)$ and $p_{\mathrm{T}}$ of the meson candidate are calculated using the methods; PCM, EMCal, PHOS, PCM-EMCal, and PCM-PHOS. For the first three methods both photons are reconstructed using the same respective method. For the latter two methods the converted photon is paired with a photon reconstructed by the EMCal or PHOS calorimeter.

In Fig. 6.4 (top), the invariant mass distributions of photon pairs around the $\pi^{0}$ mass are shown for 27 intervals of meson $p_{\mathrm{T}}$, using the PCM-EMC reconstruction method. There is a clear $\pi^{0}$ signal on top of the combinatorial background. The background is estimated using the event-mixing method, and scaled at the right side of the $\pi^{0}$ peak to match the same-event pair distribution. The shape of the combinatorial background is changing as function of $p_{\mathrm{T}}$, and is within uncertainties well described by the event-mixing method. Figure 6.4 (bottom) shows the $M_{\text {inv }}$ distribution of the meson candidates after subtracting the combinatorial background. Each distribution is fitted using Eq. 6.5, such that the mass peak positions and widths are calculated. The mass peak position is used to determine the integration range of the raw yield, and is indicated by the range between the vertical lines.

The $p_{\mathrm{T}}$-dependent invariant mass distributions of photon pairs around the nominal value of the $\eta$ mass are shown in Fig. 6.5 (top) for 14 intervals in meson $p_{\mathrm{T}}$. Since the $\eta$ is less abundant than the $\pi^{0}$, and the combinatorial background increases with $M_{\mathrm{inv}}$, the signal stands out less. Also, the shape of the combinatorial background obtained with the event-mixing method does not fully describe the background of the same-event pairs. This is attributed to the increase of correlated background for higher $M_{\text {inv }}$. The subtraction is done to remove the uncorrelated part of the background. Figure 6.5 (bottom) shows the $M_{\mathrm{inv}}$ distributions after background subtraction, and is again fitted to obtain the mass peak positions and widths. It is apparent that there is a large amount of remaining background for the $\eta$ meson, which introduce larger yield extraction uncertainties compared to the $\pi^{0}$. The $M_{\mathrm{inv}}$ distributions around the $\pi^{0}$ and $\eta$ meson mass of all the reconstruction methods are given in appendix A.1. For all methods the neutral meson peaks are well distinguishable from the combinatorial background. Differences in the shape of the combinatorial background are mainly driven by the minimum energy thresholds of the respective photons and the geometry of the detectors. The reach in $p_{\mathrm{T}}$ of each method is largely determined by the reconstruction method of the photon and the available statistics. For example, as a PCM photon has a much lower energy threshold $(50 \mathrm{MeV})$ compared to an EMCal photon $(700 \mathrm{MeV})$, the neutral mesons reconstructed with PCM can go lower in meson $p_{\mathrm{T}}$. On the other hand, since the EMCal photons do not suffer from the conversion probability of the photon conversion method, the neutral mesons reconstructed with the EMCal are able to go much higher in $p_{\mathrm{T}}$. 


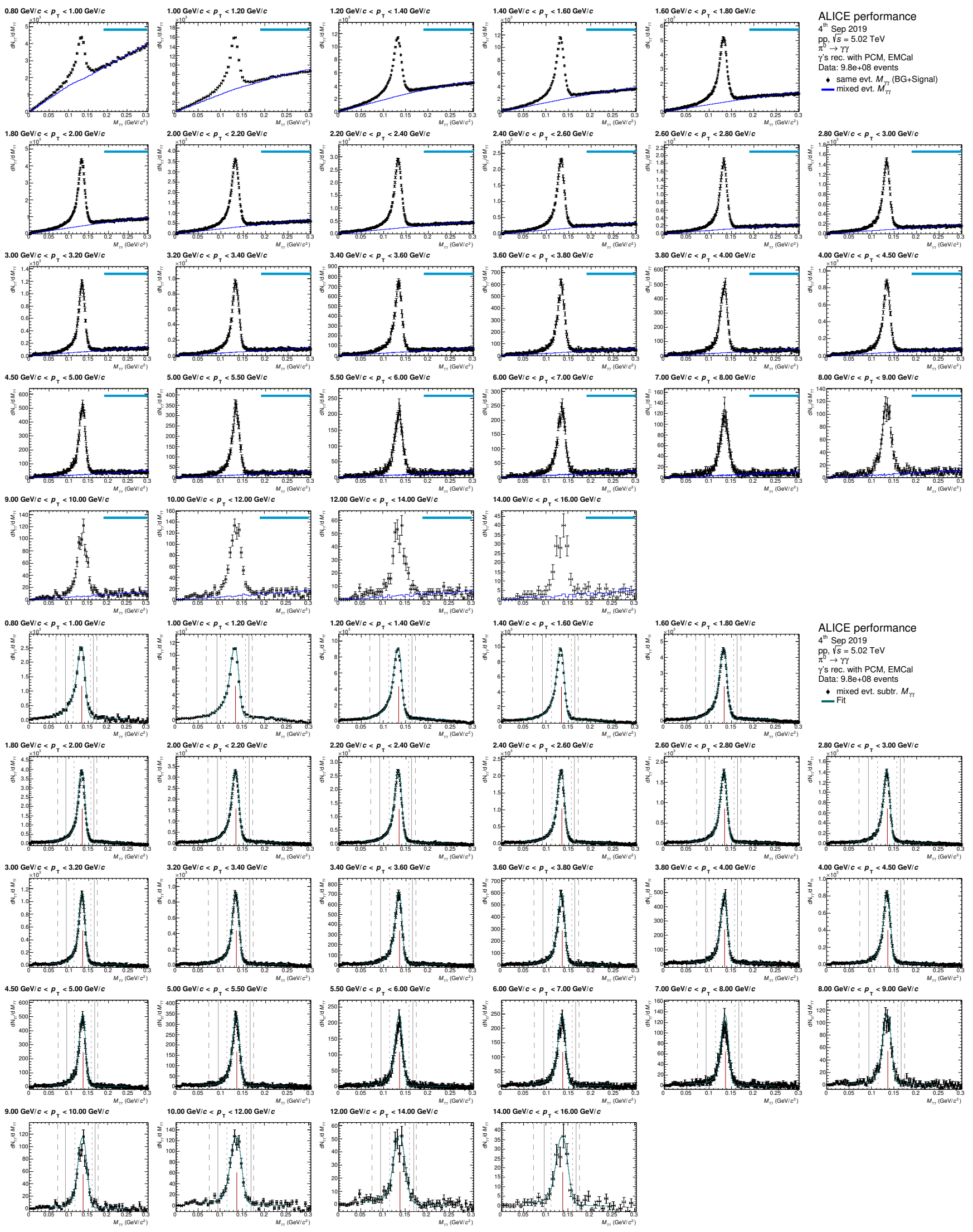

FIGURE 6.4: Invariant mass distribution of photon pairs around the nominal value of the $\pi^{0}$ mass before(top) and after(bottom) subtraction of the combinatorial background, as measured with the ALICE detector in pp collisions at a center-of-mass energy of $\sqrt{s}=5.02 \mathrm{TeV}$. The meson candidates are reconstructed using PCM-EMCal, covering $0.8<p_{\mathrm{T}}<16.0 \mathrm{GeV} / c$. 

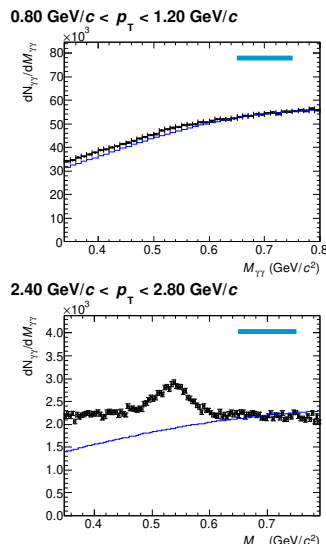

$4.50 \mathrm{GeV} / c<p_{T}<5.00 \mathrm{GeV} / c$

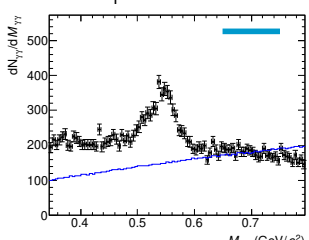

$10.00 \mathrm{GeV} / c<p_{T}<12.00 \mathrm{GeV} / c$
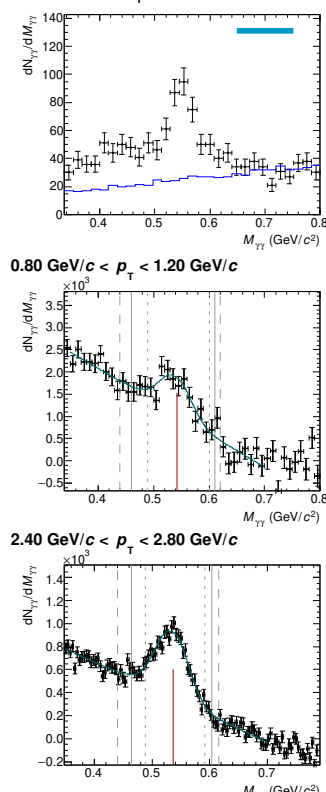

$4.50 \mathrm{GeV} / c<p_{\mathrm{T}}<5.00 \mathrm{GeV} / c$

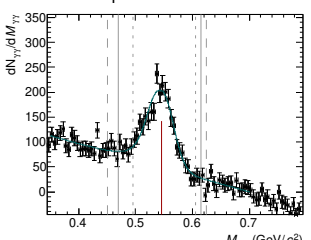

$10.00 \mathrm{GeV} / c<p_{\mathrm{T}}<12.00 \mathrm{GeV} / c$

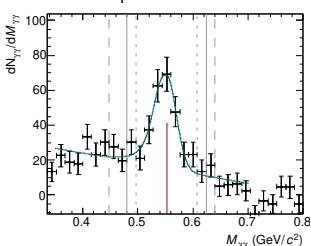

$2.80 \mathrm{GeV} / c<p_{\mathrm{T}}<3.20 \mathrm{GeV} / c$

$5.00 \mathrm{GeV} / c<p_{T}<6.00 \mathrm{GeV} / c$
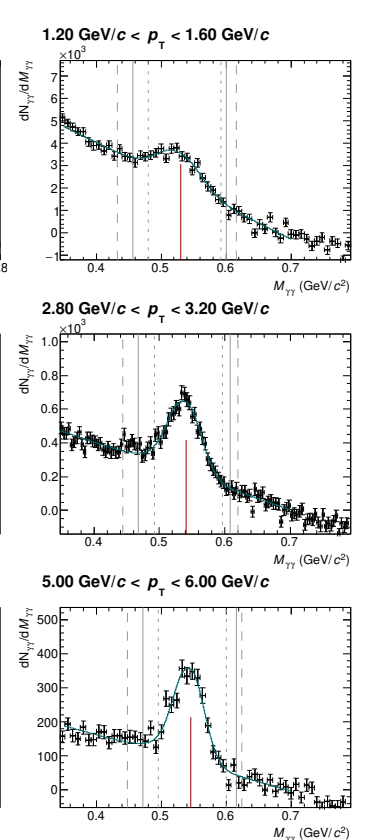
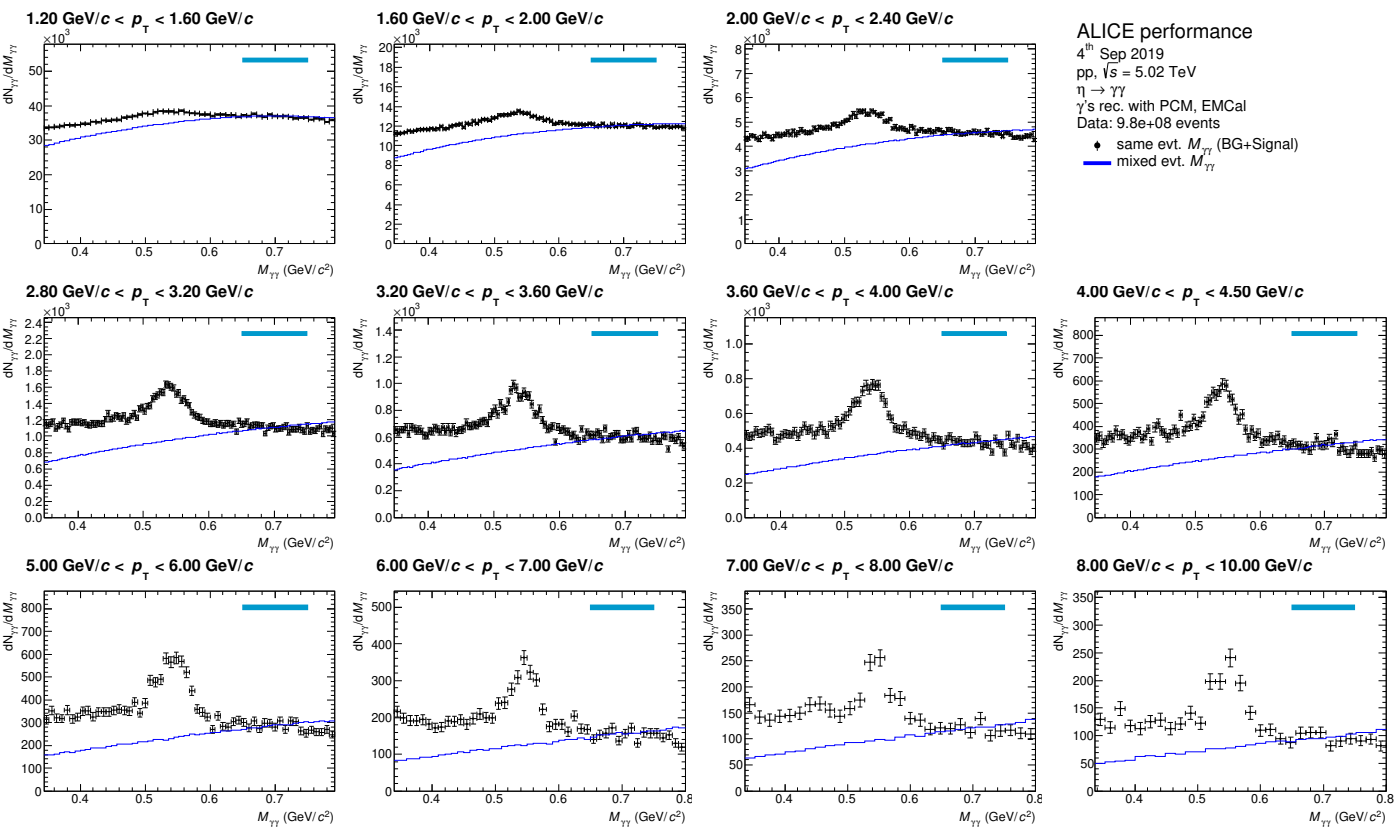

ALICE performance

$4^{4 h}$ Sep 2019
$\mathrm{pp}, \sqrt{s}=5.02 \mathrm{TeV}$

$\eta \rightarrow \gamma \gamma$, sec. with PCM, EMC

$\gamma$ 's rec. with PCM, EMC
Data: $9.8 \mathrm{e}+08$ events

- same evt. $M_{r y}$ (BG+Signal)

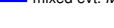

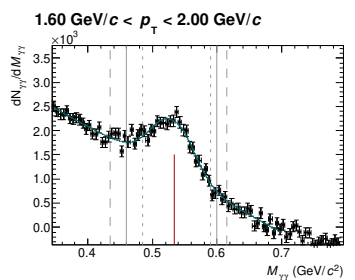

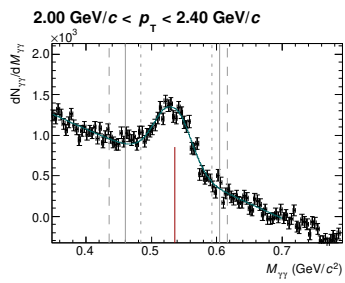

ALICE performance

$4^{\text {th }}$ Sep 2019
$\mathrm{pp}, \sqrt{s}=5.02 \mathrm{TeV}$

$\mathrm{pp}, \sqrt{s}=5.02 \mathrm{TeV}$
$\eta \rightarrow \gamma \gamma$
D

$\eta \rightarrow \gamma \gamma$
$\gamma$ 's rec. with PCM, EMCa
Data: $9.8 \mathrm{e}+08$ events

- mixed evt. subtr. $M_{v y}$

$3.20 \mathrm{GeV} / c<p_{T}<3.60 \mathrm{GeV} / c^{M_{T r}(\mathrm{GeV} / \mathrm{c})}$

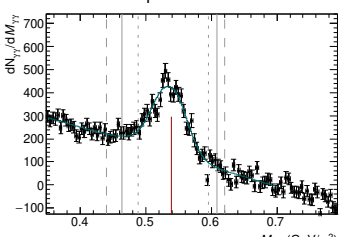

$3.60 \mathrm{GeV} / c<p_{\mathrm{T}}<4.00 \mathrm{GeV} / c$

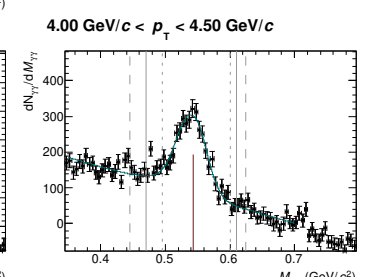

$6.00 \mathrm{GeV} / c<p_{\mathrm{T}}<7.00 \mathrm{GeV} / c$
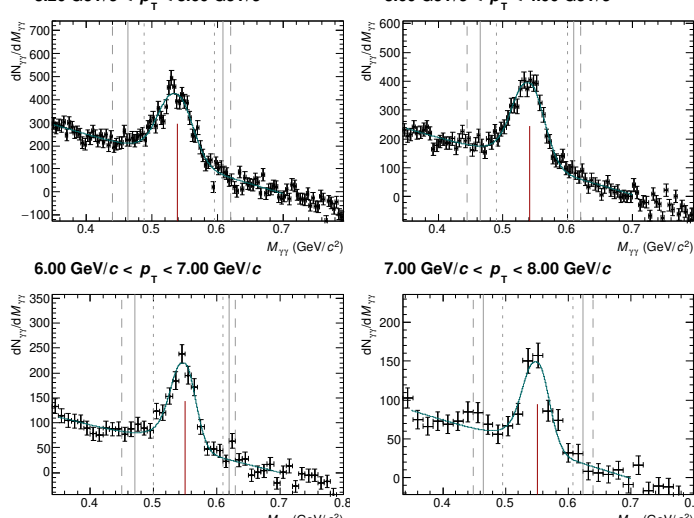

$7.00 \mathrm{GeV} / c<p<8.00 \mathrm{GeV} / c$

$8.00 \mathrm{GeV} / c<p_{\tau}<10.00 \mathrm{GeV} / c$
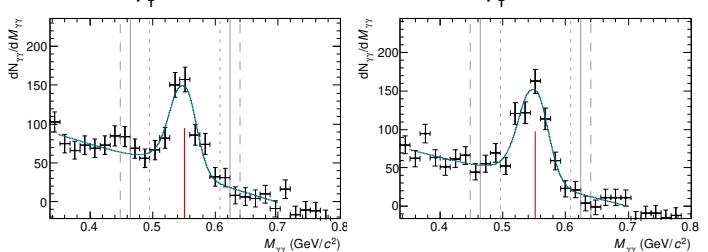

Figure 6.5: Invariant mass distribution of photon pairs around the nominal value of the $\eta$ mass before(top) and after(bottom) subtraction of the combinatorial background, as measured with the ALICE detector in pp collisions at a center-of-mass energy of $\sqrt{s}=5.02 \mathrm{TeV}$. The meson candidates are reconstructed using PCM-EMCal, covering $0.8<p_{\mathrm{T}}<12.0 \mathrm{GeV} / c$. 


\section{Mass peak positions and widths}

The reconstructed mass peak positions and widths of the $\pi^{0}$ and $\eta$ meson are obtained from fitting the respective background subtracted $M_{\mathrm{inv}}$ distributions. The mean values $(\mu)$ and widths $(\sigma)$ of the fits of Eq. 6.5 and Eq. 6.6 are shown in Fig. 6.6 for the $\pi^{0}$ (left) and $\eta$ meson (right), for each reconstruction method. In general, the reconstructed mass peak position should be as close as possible to the nominal meson mass, which is $134.98 \mathrm{MeV}$ for the $\pi^{0}$, and $547.86 \mathrm{MeV}$ for the $\eta$ meson $[138,139]$. The meson widths are driven mainly by the energy and opening angle resolution of the meson reconstruction methods, as the true meson widths without detector effects are effectively zero for both the $\pi^{0}$ and $\eta$ meson.
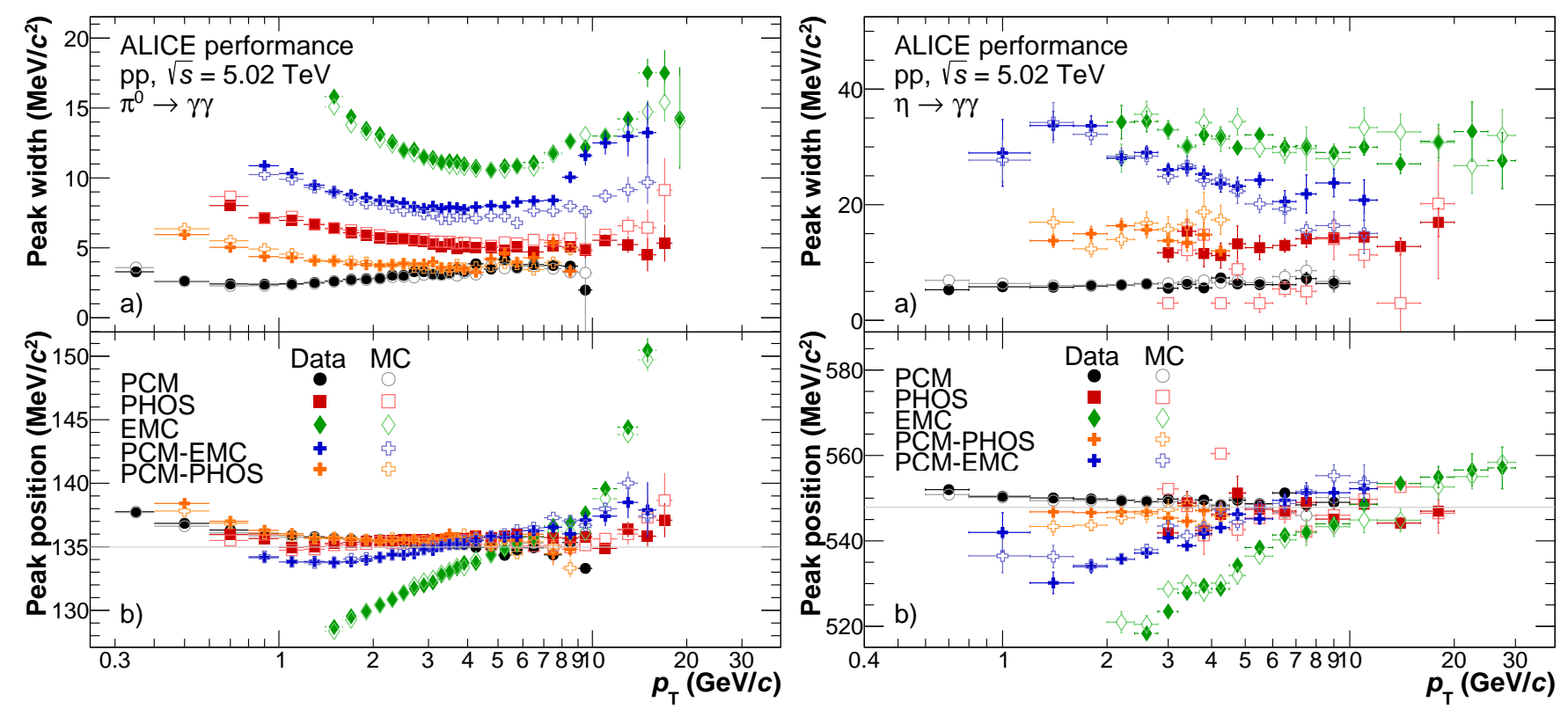

FiguRE 6.6: The reconstructed mass peak positions and widths for the different reconstruction methods of the $\pi^{0}$ (left) and $\eta$ meson (right), as measured with the ALICE detector in pp collisions at a center-of-mass energy of $\sqrt{s}=5.02 \mathrm{TeV}$. The values are obtained by fitting the transverse momentum dependent invariant mass distributions with Eq. 6.5. For all reconstruction methods and $p_{\mathrm{T}}$ bins the values are well reproduced by the MC.

For PCM, the reconstructed $\pi^{0}$ mass relies on the momentum measurement of the electron-positron pairs in the TPC. Due to the high track $p_{\mathrm{T}}$ resolution of the TPC, PCM is able to reconstruct the $\pi^{0}$ mass peak position within $2 \%$ of the nominal mass for the full $p_{\mathrm{T}}$ range and within $0.2 \%$ of the reconstructed mass peak position in MC. For EMCal, it is chosen to calibrate the MC such that the mass peak positions correspond to the respective positions in data, without explicitly calibrating the energy of the photons in data to the nominal $\pi^{0}$ mass, as explained in section 5.1. This calibration procedure does lead to a small bias in the photon energies, but the invariant yield of the neutral mesons get implicitly corrected for this effect by the efficiency. After the calibration procedure, the EMCal is able to measure the reconstructed mass peak position in data and Monte Carlo simulations with $\left|M_{\pi^{0}, \text { data }}-M_{\pi^{0}, \mathrm{MC}}\right| / M_{\pi^{0}, \mathrm{MC}}<0.4 \%$. For PHOS, the reconstructed mass peak positions are calibrated to the nominal $\pi^{0}$ mass by correcting the energy of the clusters. Furthermore, it uses an additional correction to the cluster energies in MC, resulting in a ratio of $\left|M_{\pi^{0}, \mathrm{data}}-M_{\pi^{0}, \mathrm{MC}}\right| / M_{\pi^{0}, \mathrm{MC}}<0.3 \%$. For the hybrid methods PCM-EMC and PCM-PHOS, the mass peak 
positions inherit their properties from the calorimeter photon in terms of calibration procedure, and in both cases benefit from the high track resolution of the TPC.

\section{Neutral meson raw yields}

The raw yield of the neutral mesons is extracted by integrating the background subtracted $M_{\mathrm{inv}}$ distributions, as explained in previous sections. As an example, Fig. 6.7 shows the raw yield for $\pi^{0}$ (left) and $\eta$ mesons (right) as reconstructed with the EMC method, for all detector triggers used in the analysis. The raw yield for lower meson $p_{\mathrm{T}}$ is orders of magnitude more than for higher $p_{\mathrm{T}}$, as it is increasingly unlikely to produce particles with higher momentum. For the INT7 (MB) trigger, at $p_{\mathrm{T}} \sim 2-3 \mathrm{GeV} / c$, there is a maximum in the meson raw yield for both the $\pi^{0}$ and the $\eta$ meson. The spectrum decreases for lower meson $p_{\mathrm{T}}$, and this is partly due to the increasing difficulty to reconstruct lower energy photons and larger opening angles of the meson, and as such is an effect of decreasing photon efficiency and acceptance of the meson. The other triggers EMC7 and EG2 show an enhanced raw yield of mesons by about three orders of magnitude, showcasing the strength of utilizing hardware triggers with a high energy photon threshold. These raw yields are scaled to the INT7 raw yields by using the trigger rejection factor, which is calculated by a fit for higher cluster energies as shown in Fig. 6.8. The distributions are obtained by dividing the raw cluster yield spectra of the specialized triggers by the INT7 (MB) trigger.

For all reconstruction methods the raw yields are also obtained for variations of the integration window and functional form of the remaining background fit. The RMS of these variations is taken as the systematic uncertainty for the raw yield extraction, and varies between $2-8 \%$ for the $\pi^{0}$ and between $3-20 \%$ for the $\eta$ meson, depending on the meson $p_{\mathrm{T}}$ and reconstruction method. The meson raw yields for the other reconstruction methods show qualitatively the same behavior. 

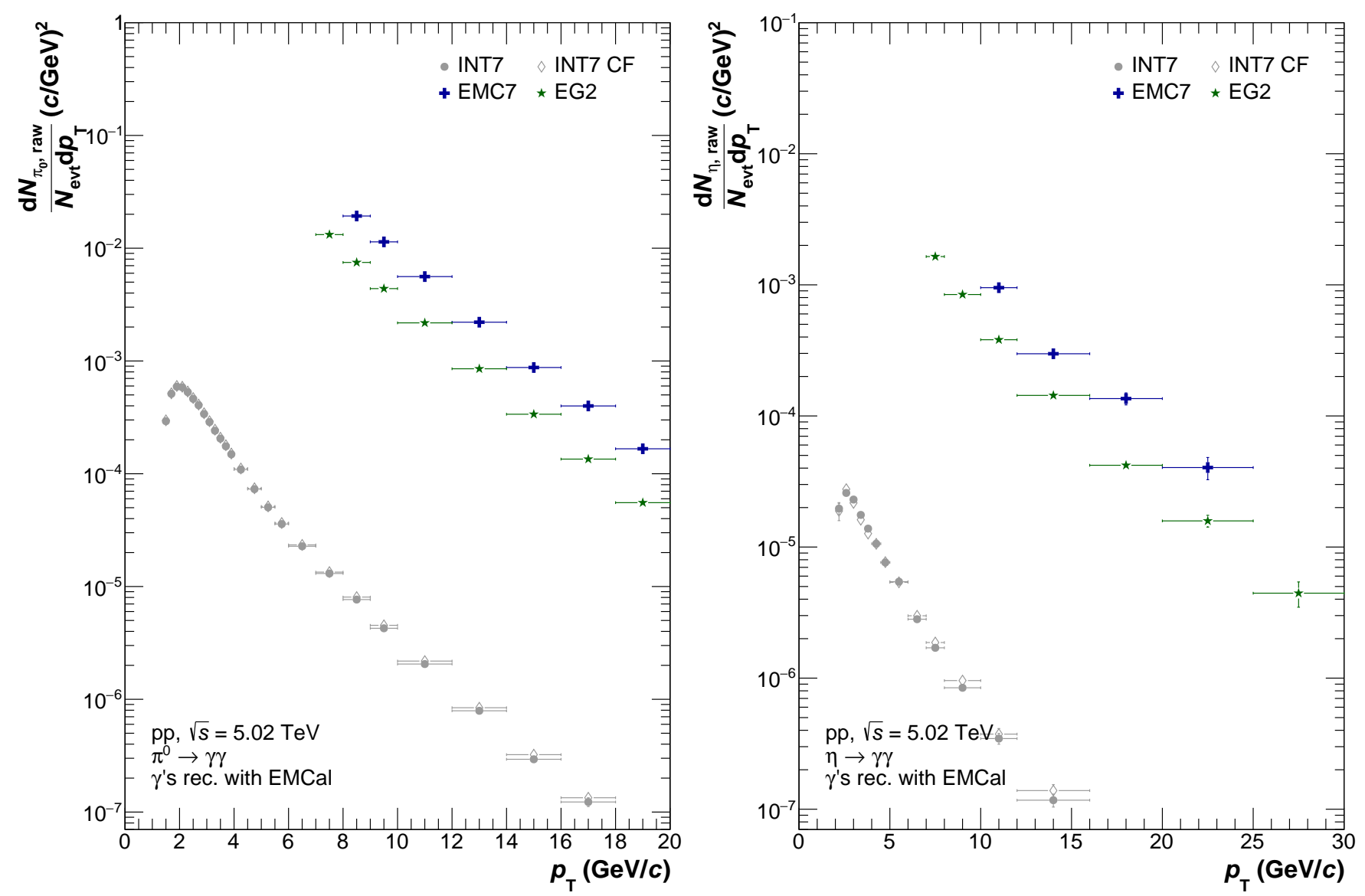

FIgURE 6.7: The raw yield of $\pi^{0}$ (left) and $\eta$ mesons (right), as measured with the ALICE detector in pp collisions at a center-of-mass energy of $\sqrt{s}=5.02 \mathrm{TeV}$, using the EMCal reconstruction method. The raw yields are obtained by integrating the background subtracted $M_{\text {inv }}$ distributions shown in Fig. 6.4 and Fig. 6.5, covering $0.8<p_{\mathrm{T}}<16.0 \mathrm{GeV} / c$, and $0.8<p_{\mathrm{T}}<12.0 \mathrm{GeV} / c$, respectively.
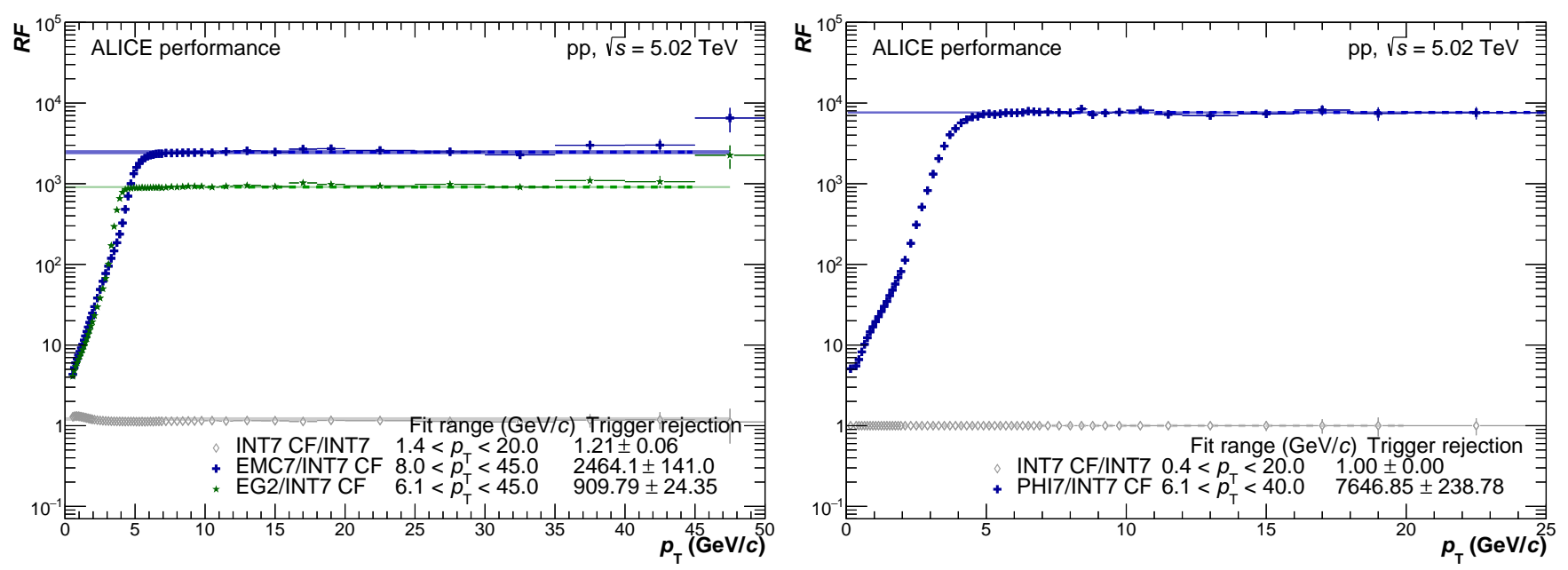

FIGURE 6.8: The trigger rejection factors for EMCal (left) and PHOS (right), as measured with the ALICE detector in pp collisions at a center-of-mass energy of $\sqrt{s}=5.02 \mathrm{TeV}$. The distributions are obtained by dividing the raw cluster yield spectra of the specialized triggers by the INT7 (MB) trigger. 


\section{Correction for secondary $\pi^{0}$ yield}

As explained in section 6.2.4, the raw yield of the $\pi^{0}$ meson needs to be corrected for the contribution from weak decays and the hadronic interactions in the detector material. The secondary $\pi^{0}$ yield in pp collisions is estimated using the decay photon cocktail approach. As an example, Fig. 6.9 (left) shows the ratio $r_{\mathrm{sec} \pi^{0}}$ from $X$ for the PCM reconstruction method. The largest contribution is the feed-down from the $\mathrm{K}_{\mathrm{S}}^{0}$ meson, with a $3 \%$ correction at low $p_{\mathrm{T}}$, decreasing to $\sim 1 \%$ for higher meson $p_{\mathrm{T}}$. The second largest contribution are the $\pi^{0}$ from hadronic interactions with the material, contributing to up to $1 \%$ for low meson $p_{\mathrm{T}}$. The feed-down from the $\mathrm{K}_{\mathrm{L}}^{0}$ and $\Lambda$ is negligible within experimental uncertainties. The secondary $\pi^{0}$ yield is subtracted from the total $\pi^{0}$ yield before any of the remaining corrections are applied. The reconstruction efficiency of the decaying hadron is taken into account, which is shown in Fig. 6.9 (right).

The same procedure is done for all other reconstruction methods, and result in similar corrections.
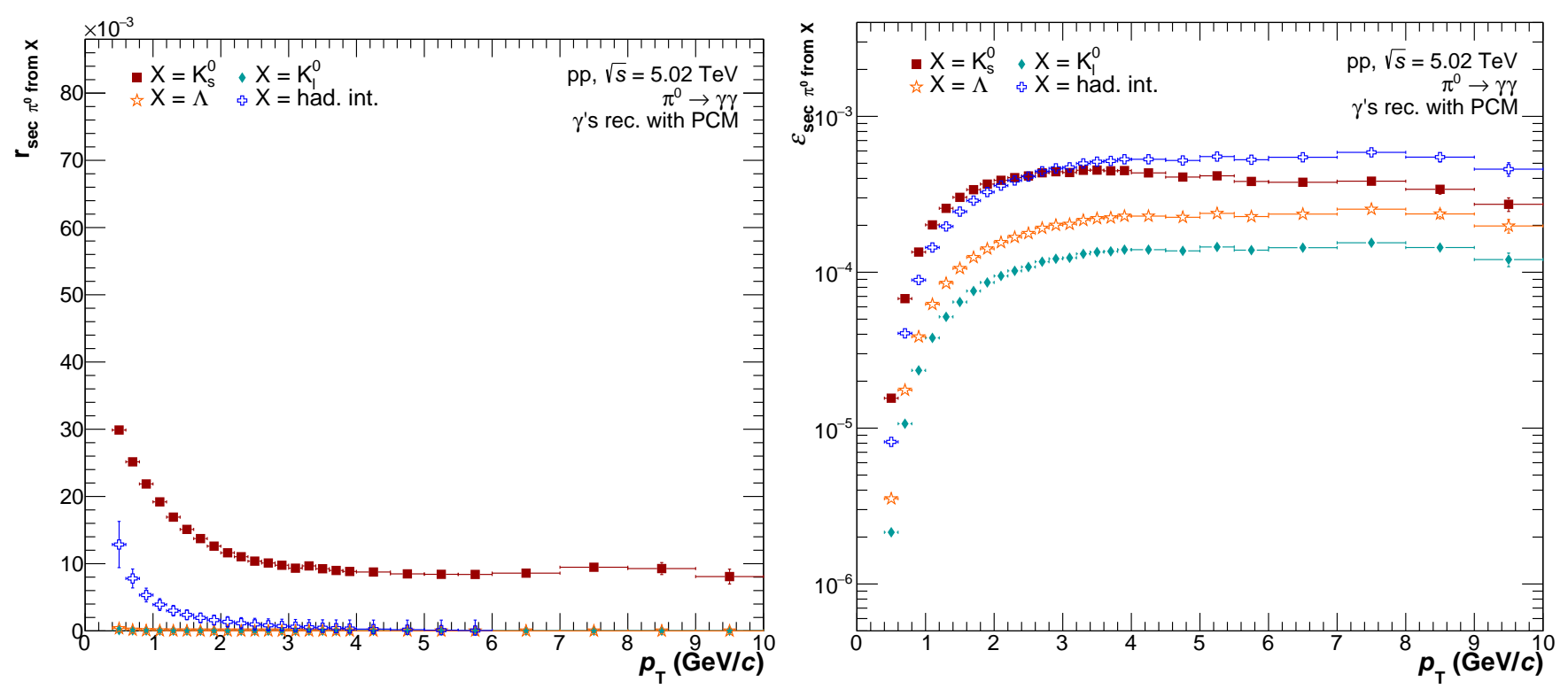

FIGURE 6.9: Ratio of the secondary $\pi^{0}$ yield compared to the total yield of the $\pi^{0}$ (left) and the reconstruction efficiency of the $\pi^{0}$ from these sources, as measured with PCM (right). The ratio with the corresponding reconstruction efficiencies are used to correct the raw yield of the $\pi^{0}$ for feed-down from weak decays and hadronic interactions. 


\section{Acceptance and efficiency correction}

The corrections for detector acceptance and efficiency, denoted by $A$ and $\epsilon_{\text {eff }}$, respectively, are performed to calculate the invariant yield of the neutral meson production, which is independent of all detector effects. It is the largest correction to the meson raw yield, as there is a sizable chance that one of the photons escapes the geometry of the detector or is not reconstructed due to the energy thresholds of the photon. The procedure to obtain this correction is described in section 6.2.4.

The acceptance times efficiency for the different reconstruction methods of the $\pi^{0}$ and $\eta$ meson is shown in Fig. 6.10. The statistical uncertainty on $\epsilon$ is proportional to the available statistics in the MC dataset. Furthermore, the widths of the meson $p_{\mathrm{T}}$ intervals is the same for the different reconstruction methods, such that they can be combined to a single result. The characteristics of the methods will now be discussed.
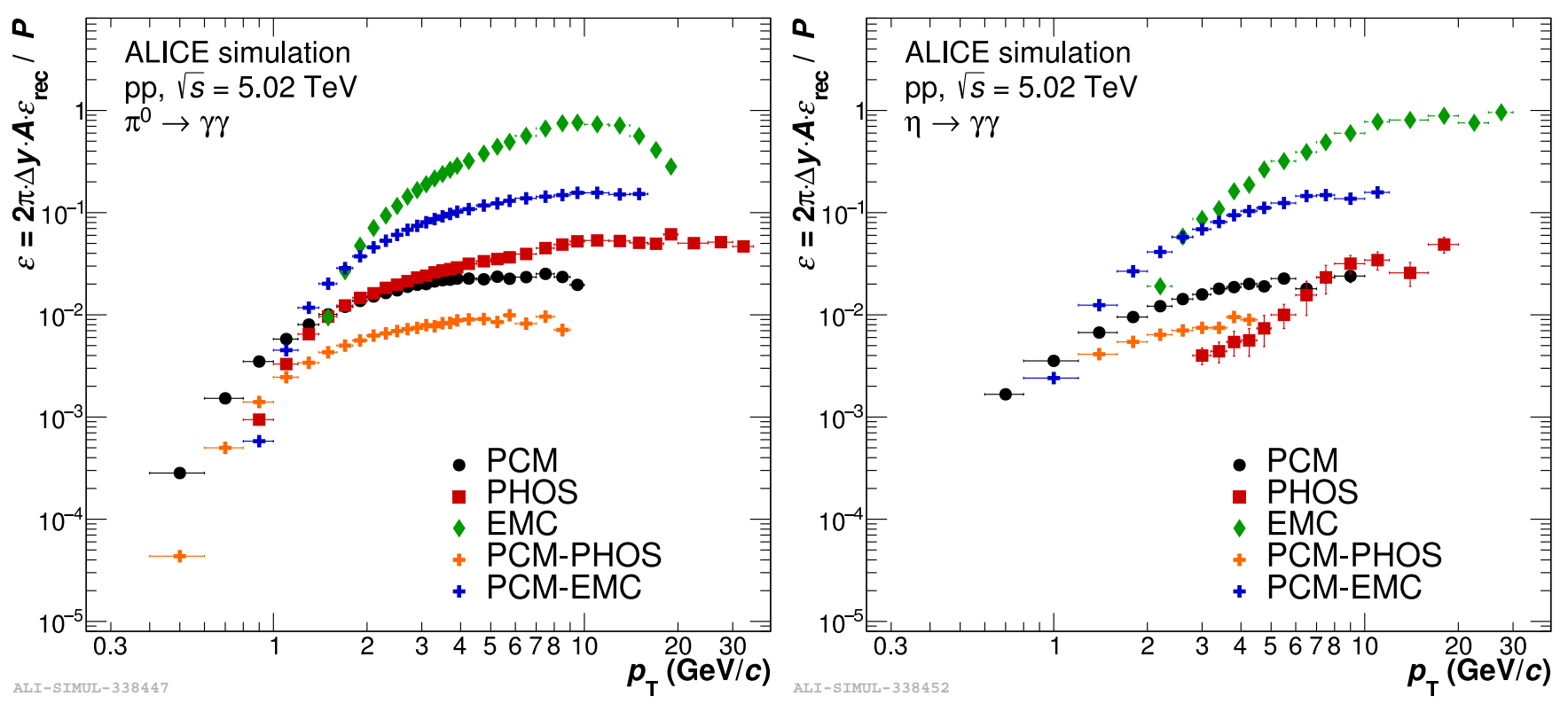

FIGURE 6.10: Acceptance times efficiency for the different reconstruction methods of the $\pi^{0}$ (left) and $\eta$ meson (right), as measured with the ALICE detector in pp collisions at a center of mass energy of $\sqrt{s}=$ $5.02 \mathrm{TeV}$. The correction factors are obtained using simulated data, and are dependent on the meson $p_{\mathrm{T}}$. The calorimetric methods are very strong at higher meson $p_{\mathrm{T}}$, while PCM is able to extend to much lower $p_{\mathrm{T}}$, clearly indicating the complementarity of the methods.

The photons reconstructed with the photon conversion method have the lowest energy thresholds, and as such the measurement starts at a $p_{\mathrm{T}}>400 \mathrm{MeV} / c$ for the $\pi^{0}$, and $p_{\mathrm{T}}>600 \mathrm{MeV} / c$ for the $\eta$ meson. $\epsilon$ increases with $p_{\mathrm{T}}$, and is limited by the statistical uncertainty of the MC simulation. The EMCal photons have a minimum energy of $700 \mathrm{MeV}$, enabling the $\pi^{0}$ to be measured for $p_{\mathrm{T}}>1.4 \mathrm{GeV} / c$, and the $\eta$ meson for $p_{\mathrm{T}}>2.0 \mathrm{GeV} / c$. For increasing meson $p_{\mathrm{T}}$, the opening angle between the two photons decreases in the lab frame due to the Lorentz boost of the two-particle system, leading to a decrease in $\epsilon$, as overlapping photons are no longer easy to distinguish. Since the $\eta$ meson is heavier, this effect does not occur for $p_{\mathrm{T}}<35 \mathrm{GeV} / c$, while for the $\pi^{0}$ this effect starts to play a role already for $p_{\mathrm{T}}>14 \mathrm{GeV} / c$. The minimum energy of photons reconstructed with PHOS is $300 \mathrm{MeV}$, and as a consequence the $\pi^{0}$ can be measured 
from $0.8 \mathrm{GeV} / c$ onwards. As the granularity of the PHOS is much higher than the EMCal, the clusters corresponding to the photons of the $\pi^{0}$ decay start to merge much later, leading to $\pi^{0}$ measurement up to $35 \mathrm{GeV} / c$. The $\eta$ is measured for $p_{\mathrm{T}}>2.8 \mathrm{GeV} / c$, due to the small acceptance and larger opening angle of the $\eta$ meson. At higher $p_{\mathrm{T}}$ this measurement is limited by statistics, similarly to the $\pi^{0}$. The hybrid methods benefit on one hand from the high energy resolution of the PCM photon at low $p_{\mathrm{T}}$, and on the other hand from the high reconstruction efficiency of calorimeter photons at high meson $p_{\mathrm{T}}$. Despite the fact that the hybrid methods do not have an exclusive range in meson $p_{\mathrm{T}}$, they do increase the precision of the measurement in the range where they are measuring the $\pi^{0}$ and $\eta$. Their performance in terms of $\epsilon$ is naively in between the corresponding non-hybrid methods, as the acceptance and efficiency of each photon roughly factorizes, but there is a non-trivial interplay of the acceptance for both hybrid methods. For PCM-EMC and PCM-PHOS, the calorimeter photon is restricted to either the EMCal of PHOS acceptance. Since the neutral meson has an opening angle which is correlated with its $p_{\mathrm{T}}$, the acceptance of the PCM photon reduces with increasing meson $p_{\mathrm{T}}$. The PCM-PHOS method is impacted more than PCM-EMC for this effect, due to its smaller acceptance. In summary, it is clear that each reconstruction method has its relative strengths and weaknesses, and complements each other in different regions of meson $p_{\mathrm{T}}$. 


\section{Consistency of the reconstruction methods}

The invariant yields of the different reconstruction methods are combined bin-by-bin into a single measurement such that the highest possible precision is reached. The value of the invariant yield of the combined result is the weighted average of the invariant yield of the individual methods. The weights are proportional to the statistical and systematic uncertainty, where the highest precision measurement has the larger weight. The weights are calculated using the Best Linear Unbiased Estimate (BLUE) method [142, 143], such that the correlations between the systematical uncertainties of the meson reconstruction methods are taken into account.

To test the consistency between the reconstruction methods, the invariant yield of both the $\pi^{0}$ and $\eta$ meson for each reconstruction method is compared with the combined result. This is done by first fitting the combined results with the so called two-component model (TCM) [144, 145], which is given by

$$
\frac{1}{2 \pi} \frac{1}{N_{\mathrm{ev}}} \frac{\mathrm{d}^{2} N}{p_{\mathrm{T}} \mathrm{d} p_{\mathrm{T}} \mathrm{d} y}=A_{e} \exp \left(\frac{\sqrt{p_{\mathrm{T}}^{2}+M^{2}}-M}{T_{e}}\right)+A\left(1+\frac{p_{\mathrm{T}}^{2}}{T^{2} n}\right)^{-n}
$$

where $M$ is the mass is the meson, $A_{e}$ and $A$ are normalization factors, and $T_{e}, T$, and $n$ are free parameters of the fit. The last term in this fit is a power law-like component, mainly describing the high $p_{\mathrm{T}}-$ part of the spectrum, while the first term dominates the fit at low meson $p_{\mathrm{T}}$. The ratio of the invariant yield of the reconstruction methods to the fit through the combined result is shown in Fig. 6.11, and is used to test the consistency of the methods. The large majority of the measured values are within one standard deviation of both the statistical and systematic uncertainties, and show a good agreement. For the $\eta$ meson there are larger fluctuations at higher meson $p_{\mathrm{T}}$, which is expected due to the larger statistical uncertainties. This procedure of combining the meson reconstruction methods to a single result is used for all invariant yield measurements presented in this chapter.
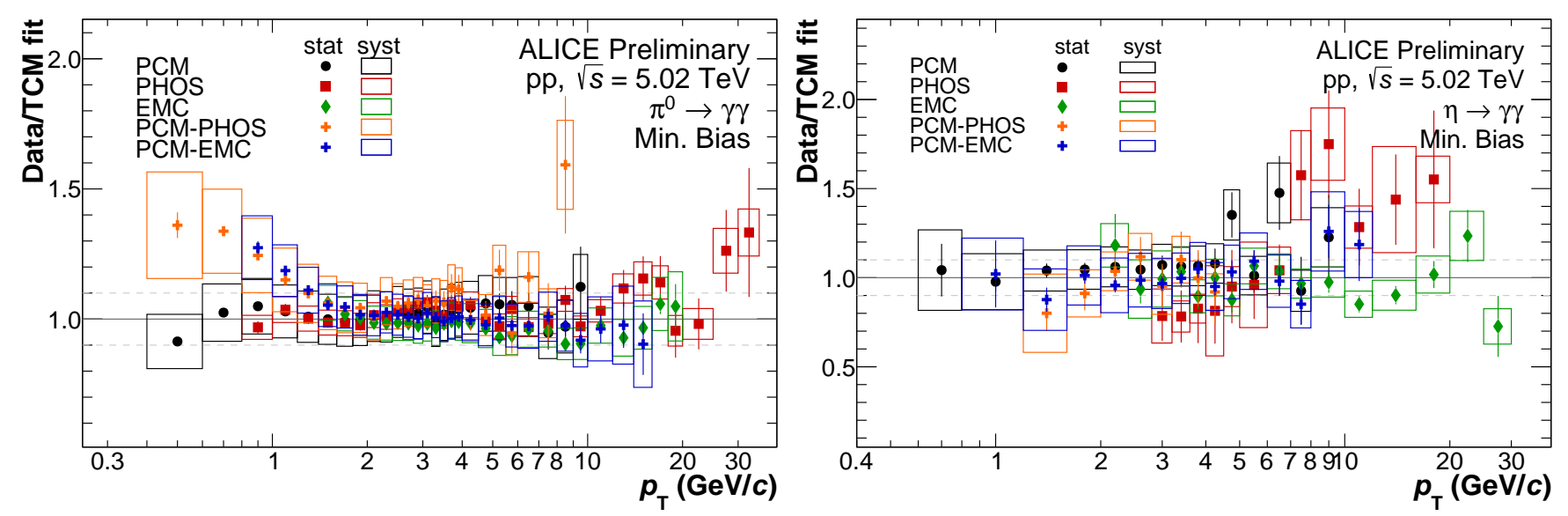

FIGURE 6.11: The ratio of the invariant yield of a single reconstruction method to the fit through the combined spectrum, for the $\pi^{0}$ (left), and the $\eta$ meson (right). All measured values are found to be consistent within uncertainties. 


\section{Invariant yield in MB collisions}

The transverse momentum dependent invariant yield of the $\pi^{0}$ and $\eta$ meson, as measured with the ALICE detector in minimum bias pp collisions at $\sqrt{s}=5.02 \mathrm{TeV}$, is shown in Fig. 6.12. The mesons are measured over a wide range in $p_{\mathrm{T}}$, with the $\pi^{0}$ covering $0.4<p_{\mathrm{T}}<35 \mathrm{GeV} / c$, and the $\eta$ covering $0.6<p_{\mathrm{T}}<30 \mathrm{GeV} / c$. The spectra are fitted using a TCM and a Levy-Tsallis fit, which are both able to describe the data. Additionally, both mesons are compared to prediction from the PYTHIA event generator [146], as well as NLO pQCD calculations. For both mesons the NLO calculations overestimate the invariant yield, by $10-45 \%$ for the $\pi^{0}$ and $40-90 \%$ for the $\eta$ meson. These calculations would benefit from updated PDFs and FFs. Similarly, PYTHIA 8.2 with the Monash 2013 tune overestimates the $\pi^{0}$ invariant yield by $\sim 10 \%$, mostly independent of meson $p_{\mathrm{T}}$. For $p_{\mathrm{T}}>2 \mathrm{GeV} / c$, the model does describe the $\eta$ meson quite well, but is off by $\sim 20-100 \%$ for $p_{\mathrm{T}}<2 \mathrm{GeV} / c$.
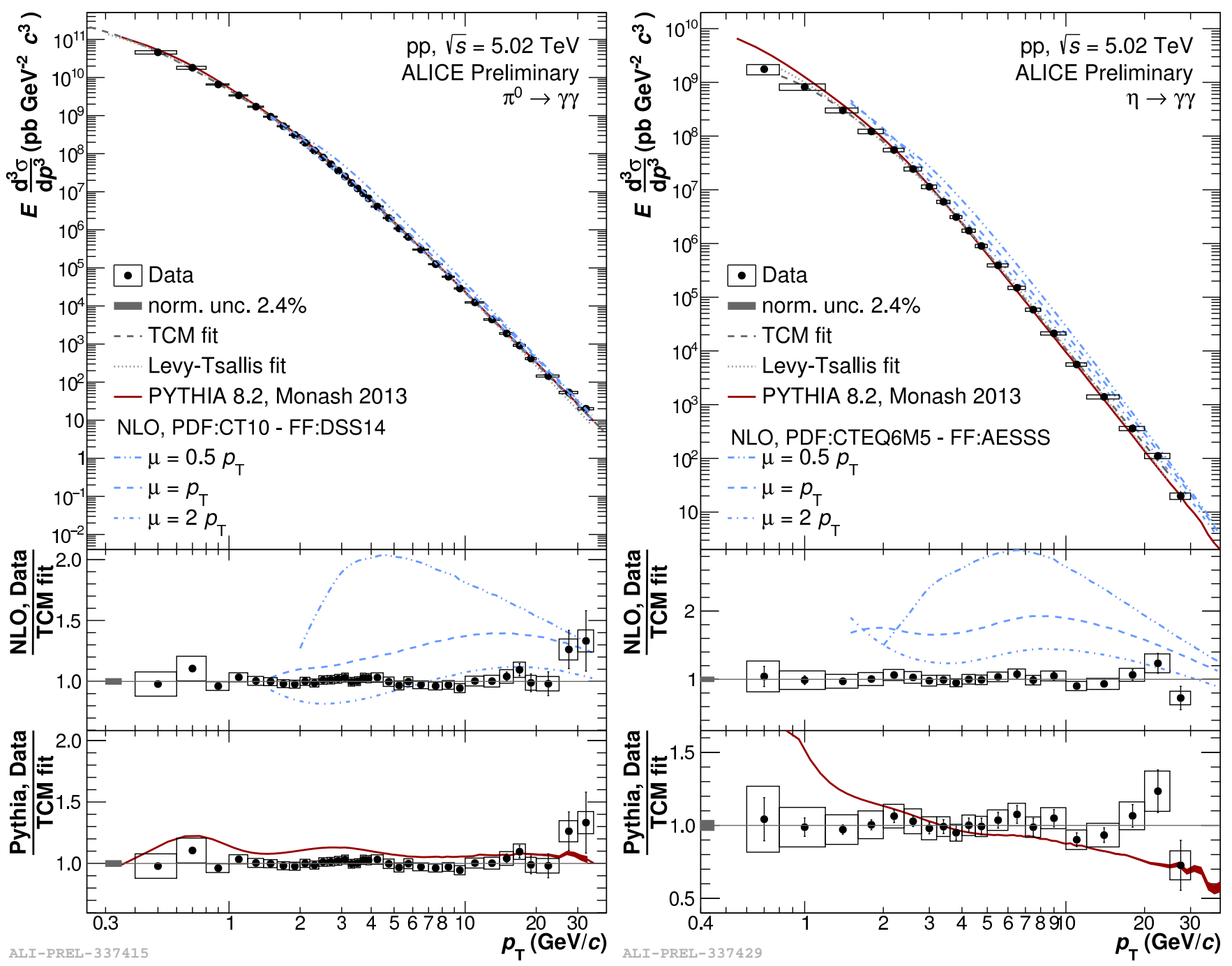

FIGURE 6.12: The invariant yield of the $\pi^{0}$ (left) and $\eta$ meson (right), as measured with the ALICE detector in pp collisions at $\sqrt{s}=5.02 \mathrm{TeV}$. The meson reconstruction methods are combined to a single result, and are compared to PYTHIA8 and NLO calculations. The lower panels show the ratio of the invariant yield to the fit. 


\section{Selection on multiplicity}

In a naive expectation, the invariant yield of neutral mesons is proportional to the total charged particle multiplicity of the event, as the neutral mesons are among the most abundantly produced particles. The charged particle multiplicity is estimated by measuring the energy deposited in the V0M detectors, which are placed in the forward regions of the ALICE detector. The invariant yield of the $\pi^{0}$ and $\eta$ is measured in different selection intervals of V0M multiplicity. Table 6.4 gives the average $N_{\text {ch }}$ per rapidity for the different multiplicity event classes. It shows that the collisions selected with the $0-1 \%$ event class produce on average over 3 times more particles on average compared to MB triggered events, which has a $\left\langle\mathrm{d} N_{\mathrm{ch}} / \mathrm{d} \eta\right\rangle=3.4$.

\begin{tabular}{l|r}
\hline V0M multiplicity class & $\left\langle d N_{\mathrm{ch}} / d \eta\right\rangle$ \\
\hline Inclusive (MB) & 3.4 \\
$0-1 \%$ & 11.5 \\
$1-5 \%$ & 9.0 \\
$5-20 \%$ & 6.4 \\
$20-40 \%$ & 4.4 \\
$40-70 \%$ & 2.7 \\
$70-100 \%$ & 1.6 \\
\hline
\end{tabular}

TABLE 6.4: Average number of charged particle multiplicity in the forward V0M detectors.

The invariant yield of the $\pi^{0}$ and $\eta$ mesons has been measured for the V0M event classes defined in Table 6.4, where again each measurement utilizes all the meson reconstruction methods which have been combined to a single result. The results of the invariant yields are shown in Fig. 6.13. The ratio of the invariant yield of the $\pi^{0}$ and $\eta$ meson for the different V0M event classes to the inclusive invariant yield is shown in Fig. 6.14. As expected, the first four V0M event classes show an increased invariant yield for both the $\pi^{0}$ and $\eta$ meson, as their ratio to the inclusive is above unity. Apart from this, the shape of the distribution also changes significantly, showing an increasingly flatter spectrum for increasing V0M multiplicities. This indicates that those events contain not only more particles, but relatively even more high $p_{\mathrm{T}}$ particles. In addition, the results are again compared to prediction from the PYTHIA8 event generator using the Monash 2013 tune, and shows large deviations for the $\pi^{0}$ result in $0-1 \%$ and $70-100 \%$. It overestimates the measurement for the $20-40 \%$ V0M event class, as has been the case for the inclusive measurement with similar event multiplicities. The $\eta$ measurement is showing qualitatively the same behavior, with larger fluctuations due to the increased uncertainties. 

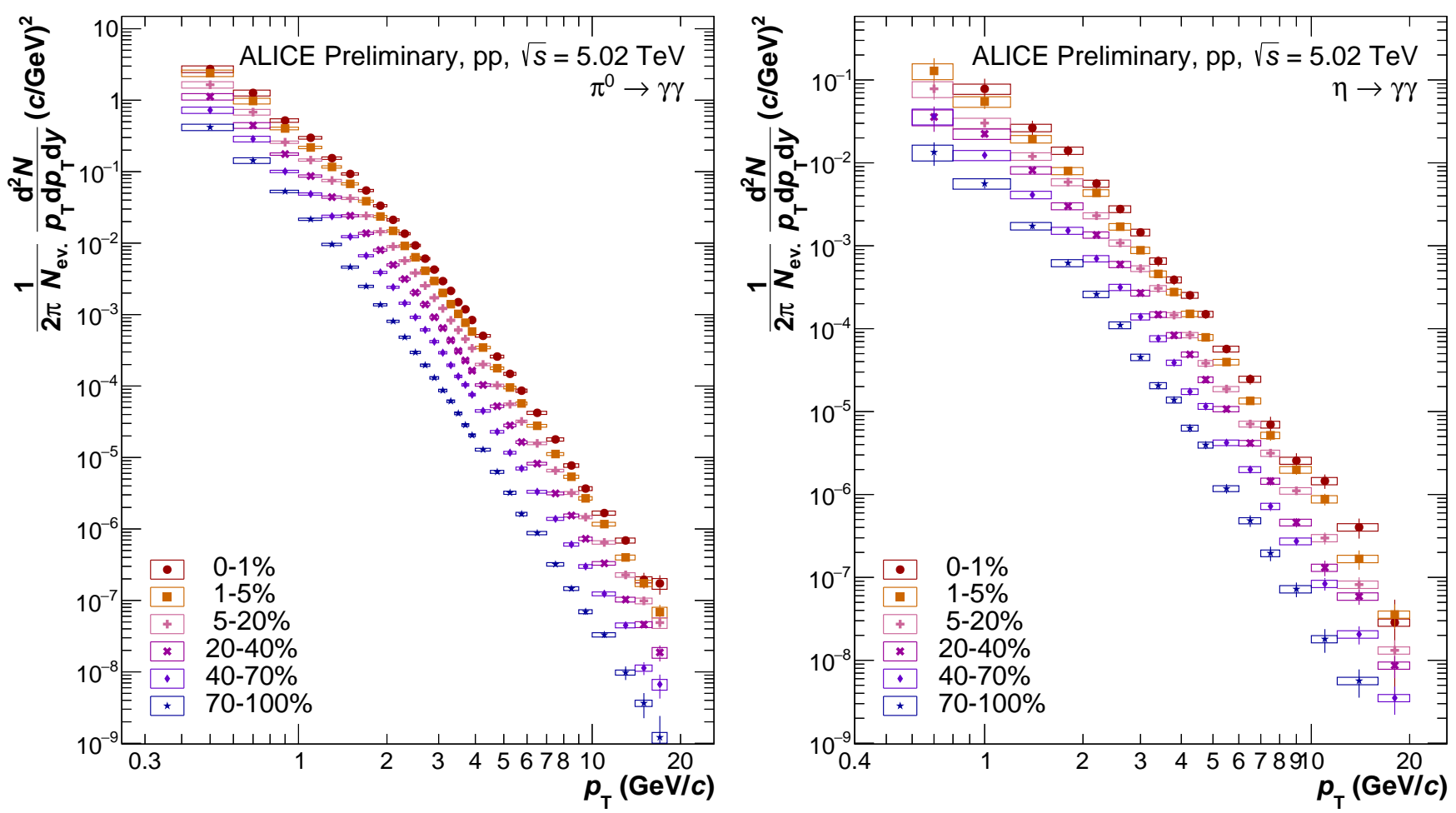

Figure 6.13: The invariant yield of the $\pi^{0}$ (left) and $\eta$ meson (right) for different multiplicity classes, as measured with the ALICE detector in pp collisions at $\sqrt{s}=5.02 \mathrm{TeV}$. Each measurement utilizes all the meson reconstruction methods which have been combined to a single result.
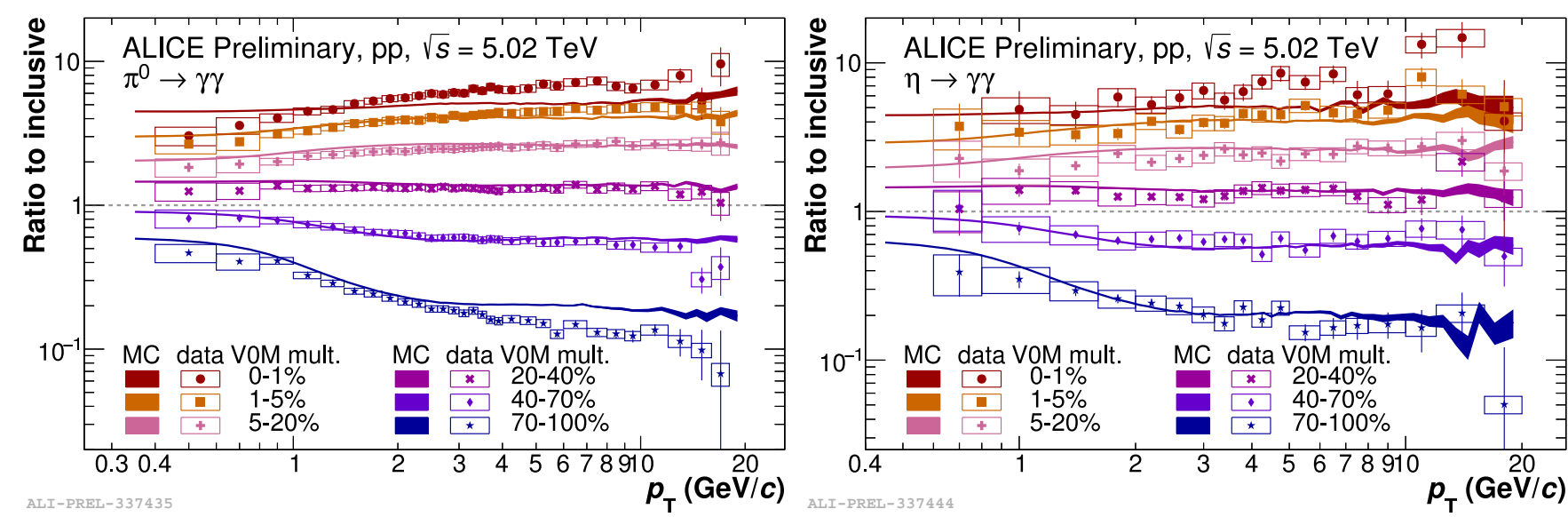

FIGURE 6.14: The ratio of the invariant yield of the $\pi^{0}$ (left) and $\eta$ meson (right) for different multiplicity classes to the invariant yield in minimum bias data, as measured with the ALICE detector in pp collisions at $\sqrt{s}=5.02 \mathrm{TeV}$. The results are compared to model predictions from the PYTHIA 8 event generator, and show to be compatible over a large transverse momentum range, except for the lowest and highest multiplicity class of the $\pi^{0}$. 


\section{Selection on sphericity}

The shape of the distribution of the final state particles, or event shape, of a collision can be approximated by calculating the transverse sphericity $\left(S_{\mathrm{T}}\right)$, as previously described in section 4.3.5. Pencil-like configurations give values that tend to $S_{\mathrm{T}} \sim 0$, while sphere-like configurations give values that tend to $S_{\mathrm{T}} \sim 1$, with $0<S_{\mathrm{T}}<1$. It is expected that the sphericity correlates with different characteristics of the event, such as the multiplicity, mean $p_{T}$ of the tracks in the event, and presence of jets. This means that it is expected that also the neutral meson spectra will change for different values of $S_{\mathrm{T}}$. First, the sphericity distributions for data and MC simulations is shown in Fig. 6.15 (left), with a maximum around $S_{\mathrm{T}}=0.6$. The dependence of $S_{T}$ on the number of charged tracks in the event $\left(N_{\text {tracks }}\right)$ is given in Fig. 6.15 (right), showing that events with increasing multiplicity also have increasing $S_{\mathrm{T}}$, and that for $N_{\text {tracks }}>20$ the average value stabilizes around $S_{\mathrm{T}}=0.7$. Moreover, both results are relatively well reproduced by the MC simulations.
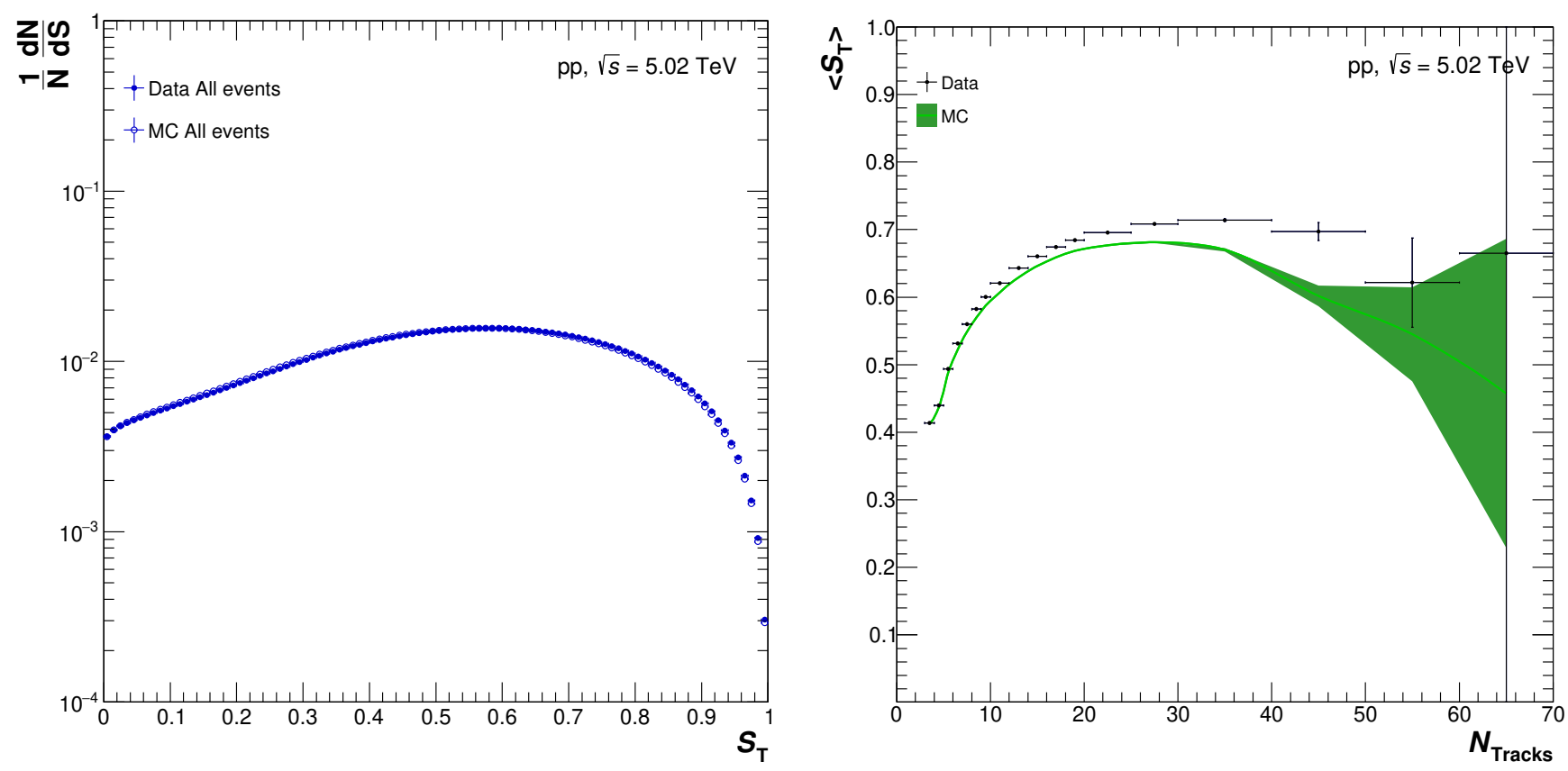

Figure 6.15: The sphericity distributions of data and MC simulations (left), and the mean sphericity $\left(S_{\mathrm{T}}\right)$ as function of $N_{\text {tracks }}$ (right).

The invariant yields of the $\pi^{0}$ and $\eta$ mesons are measured for the $S_{\mathrm{T}}$ integrated distribution as well as for different selections of the $S_{\mathrm{T}}$ distribution. The result for the $S_{\mathrm{T}}$ integrated distribution differs from the result measured in $\mathrm{MB}$ collisions, as the $S_{\mathrm{T}}$ calculation requires at least 3 charged tracks within the event, which is effectively only a multiplicity requirement. The results for the invariant yields are shown in Fig. 6.16, and the ratio of the two most extreme selections in $S_{\mathrm{T}}$ to the $S_{\mathrm{T}}$ integrated distribution in Fig. 6.17. The ratio clearly shows that the results change significantly for both the $\pi^{0}$ and $\eta$ meson, with a cross-over at $p_{\mathrm{T}} \sim 5 \mathrm{GeV} / c$. For low meson $p_{\mathrm{T}}$, the invariant yield of both mesons is suppressed for pencil-like events, while it is enhanced for sphere-like events, and vice-versa for $p_{\mathrm{T}}>5 \mathrm{GeV} / c$. This indicates that a selection on $S_{\mathrm{T}}$ is different from selecting on event multiplicity, which was shown in Fig. 6.14. Furthermore, the comparison to the PYTHIA event generator shows a significant difference for the $\pi^{0}$ prediction after the 
cross-over point. The model overestimates the amount of high $p_{\mathrm{T}} \pi^{0}$ mesons for pencil-like events, and underestimates the amount of high $p_{\mathrm{T}} \pi^{0}$ mesons for sphere-like events.
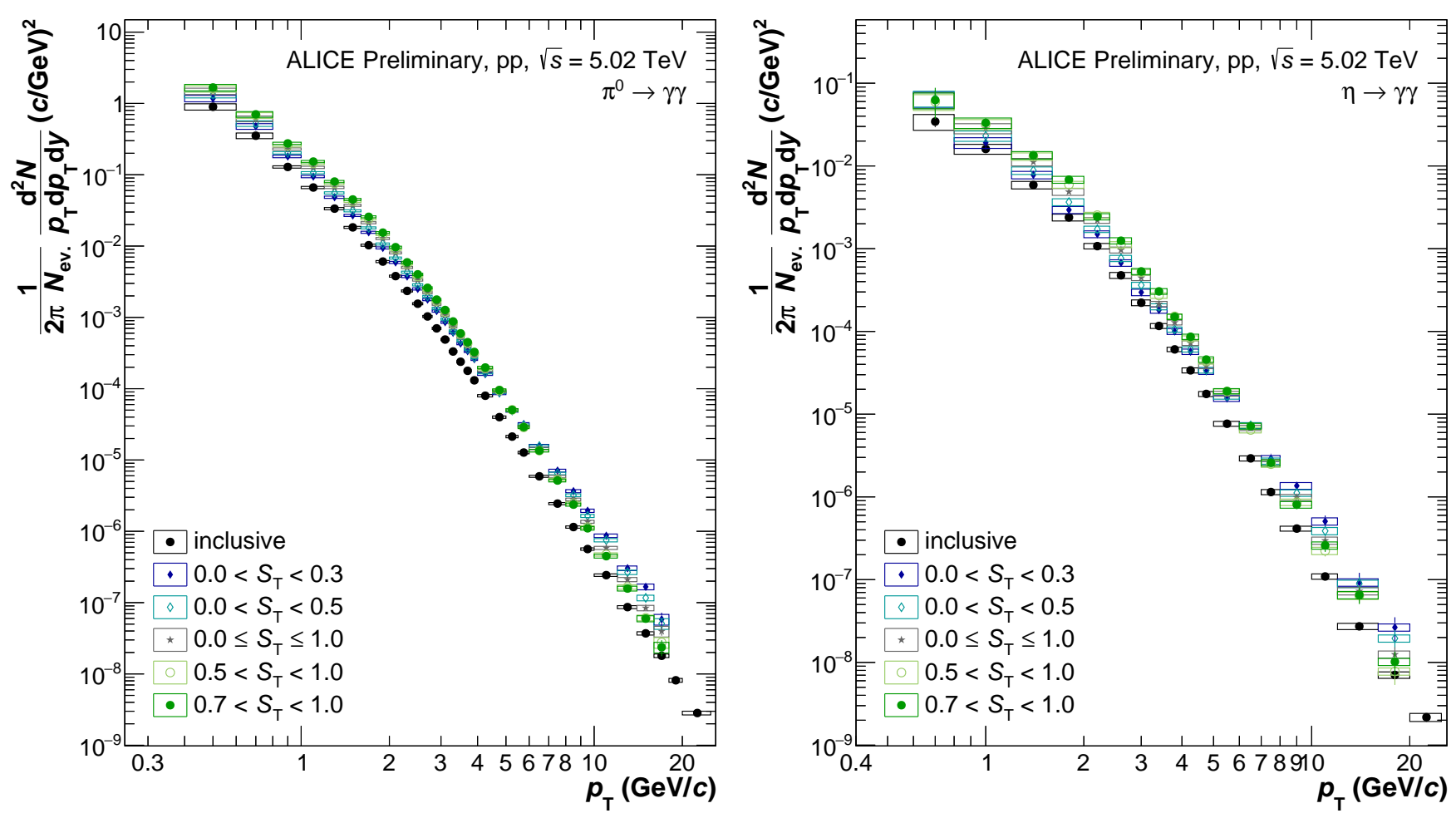

FIGURE 6.16: The invariant yield of the $\pi^{0}$ (left) and $\eta$ meson (right) for different sphericity classes, as measured with the ALICE detector in pp collisions at $\sqrt{s}=5.02 \mathrm{TeV}$.
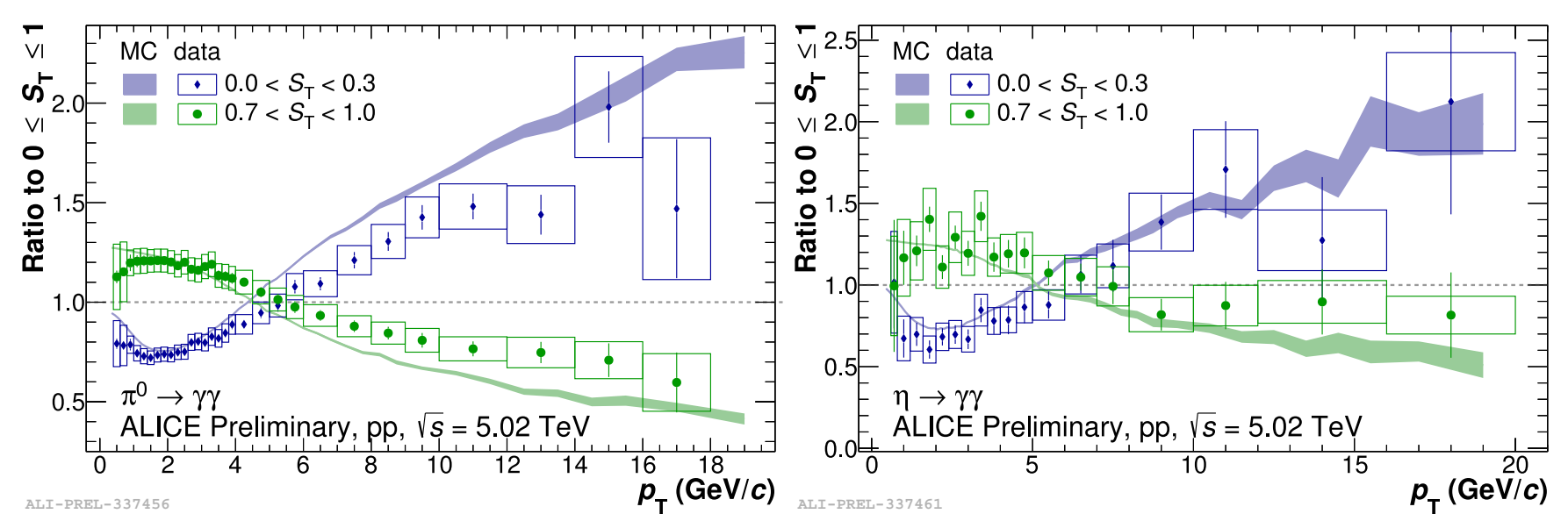

FiguRE 6.17: The ratio of the invariant yield of the $\pi^{0}$ (left) and $\eta$ meson (right) for different sphericity classes to the sphericity integrated invariant yield, as measured with the ALICE detector in pp collisions at $\sqrt{s}=5.02 \mathrm{TeV}$. The results are compared to model predictions from the PYTHIA 8 event generator, and show to be compatible over a large transverse momentum range, except for higher transfer momentum $\left(p_{\mathrm{T}}>8 \mathrm{GeV} / c\right)$. Pencil-like events show to have steeper spectra at high $p_{\mathrm{T}}$, and the spectra are softer for sphere-like events at high $p_{\mathrm{T}}$. 


\section{Neutral meson production inside charged jets}

To test the production of neutral mesons further, the invariant yield of $\pi^{0}$ and $\eta$ mesons is measured for the case when they are within the cone of a charged jet. This is particularly interesting, because jet reconstruction attempts to capture the hard part of the collision, in contrast to an inclusive measurement which captures mostly the softer particle production of the underlying event. Also, as the neutral meson is reconstructed using its neutral daughter photons, there is no autocorrelation between the constituents of the jet and the meson.

First, the jets are reconstructed from charged particles using the anti- $k_{t}$ algorithm (section 4.3.4), with a radius $R=0.4$ and a minimum energy requirement of $10 \mathrm{GeV}$. The momentum vector of the neutral meson candidate is required to have $\Delta R<R$ as measured from the jet-axis, and the photons are allowed to be outside the cone of the jet. The extraction of the raw yields is done using the same procedure as for the other results, and is obtained for the PCM, PCM-EMC, EMC, and PHOS meson reconstruction methods. Since the amount of events containing a jet with $p_{\mathrm{T}}>10 \mathrm{GeV} / c$ within the acceptance of the detector is $\sim 1 \%$, the statistics of the mesons are limited. Larger intervals in meson $p_{\mathrm{T}}$ are used to still have significant meson peaks in both the experimental data and MC simulations. Furthermore, the raw yield has been corrected for the resolution of the jet energy $[147,148]$, by explicitly calculating the raw yields corresponding to the cases where the true energy satisfies the jet energy threshold, using simulated data. This procedure is needed due to detector effects such as charged track efficiency and track $p_{\mathrm{T}}$ resolution, and results in a $25-30 \%$ correction on the meson raw yields which depends only slightly on meson $p_{\mathrm{T}}$ and reconstruction method.

The invariant yield of the $\pi^{0}$ and $\eta$ meson for in-jet production is shown in Fig. 6.18. It clearly shows that for lower meson $p_{\mathrm{T}}$ the $\pi^{0}$ and $\eta$ are less associated with a charged jet than for higher meson $p_{\mathrm{T}}$, indicating that high $p_{\mathrm{T}}$ mesons are mostly produced in jets. Also, the $\eta$ meson is more suppressed at low $p_{\mathrm{T}}$ compared to the $\pi^{0}$, which will be discussed in more detail in the next section. 

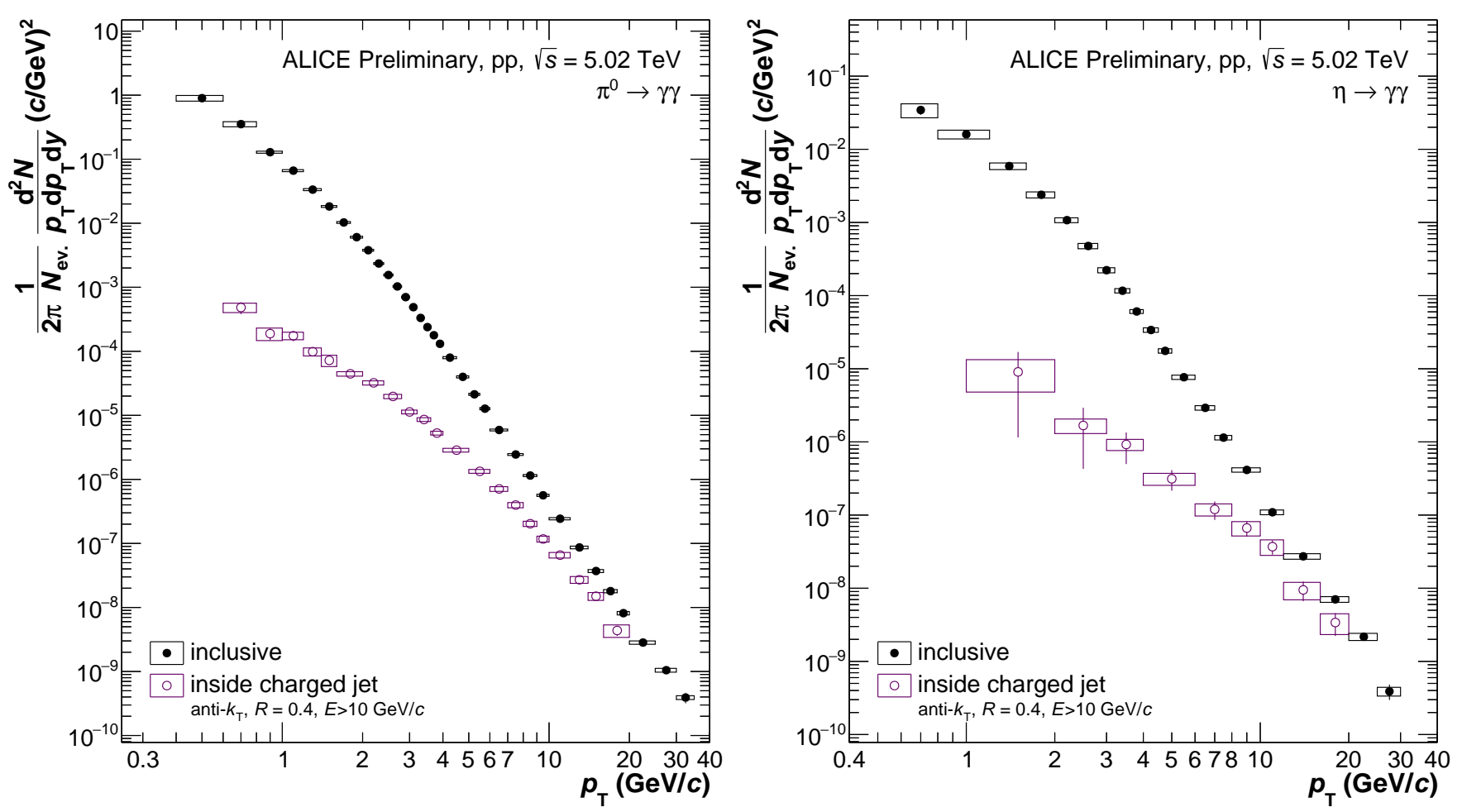

Figure 6.18: The invariant yield of the $\pi^{0}$ (left) and $\eta$ meson (right) for in-jet production, as measured with the ALICE detector in pp collisions at $\sqrt{s}=5.02 \mathrm{TeV}$. Each measurement utilizes all the meson reconstruction methods except PCM-PHOS, and have been combined to a single result. 
$\eta / \pi^{0}$ ratio

Additionally to the results presented in the previous sections, the invariant yield of the $\eta$ can be compared to the $\pi^{0}$ by calculating the $\eta / \pi^{0}$ ratio. This is done by measuring the invariant yield of the $\pi^{0}$ in the same meson $p_{\mathrm{T}}$ intervals as the $\eta$ meson, such that the ratio is calculated by simply taking the point-by-point ratio. The systematic uncertainty in this ratio is reduced since most of this uncertainty is related to the photon reconstruction methods and is present in both the nominator and denominator. The $\eta / \pi^{0}$ ratio is shown in Fig. 6.19 for minimum bias collisions (top left), selection of event multiplicity classes (top right), selection of sphericity classes (lower left), and the in-jet production (bottom right). The high $p_{\mathrm{T}}$ average of the minimum bias result gives $0.459 \pm 0.006^{\text {stat }} \pm 0.011^{\text {sys }}$, which is compatible with the results obtained at other center-of-mass energies. The $\eta / \pi^{0}$ ratio shows no significant dependence on the event multiplicity or sphericity. The most significant difference is for the in-jet measurement, where for $p_{\mathrm{T}}<8 \mathrm{GeV} / c$ the $\eta / \pi^{0}$ ratio is suppressed by a factor $2-4$, and this is mainly attributed to the reduction of the $\eta$ meson. Additionally, the results are compared with the prediction from the PYTHIA event generator, where for the minimum bias result the prediction from PYTHIA overestimates the ratio for $p_{\mathrm{T}}<2 \mathrm{GeV} / c$, and underestimates it for $p_{\mathrm{T}}>2 \mathrm{GeV} / c$.
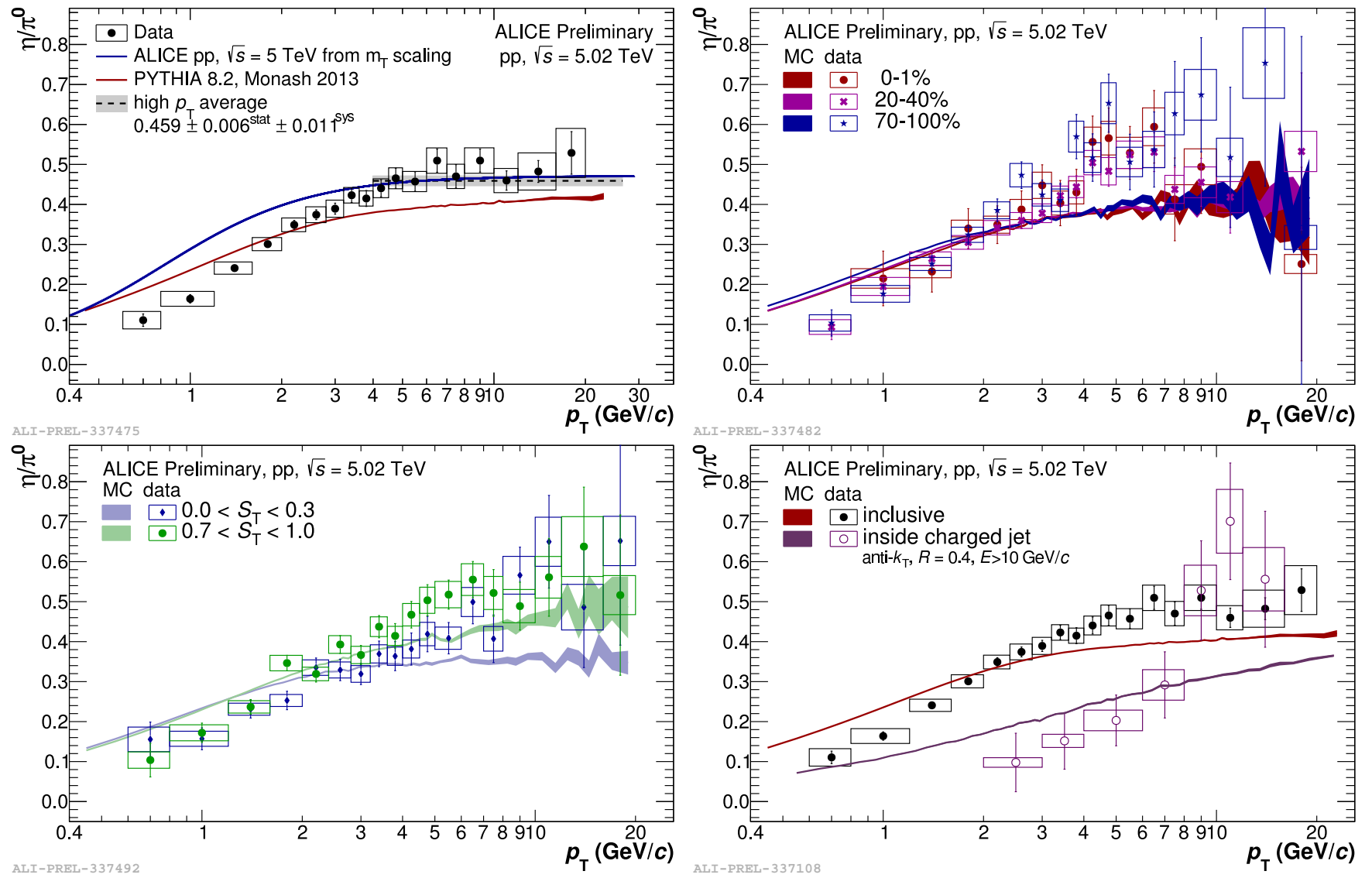

FIGURE 6.19: The $\eta / \pi^{0}$ ratio for minimum bias (top left), different multiplicity classes (top right), sphericity classes (lower left), and in-jet (bottom right), as measured with the ALICE detector in pp collisions at $\sqrt{s}=5.02 \mathrm{TeV}$. The results are all compared to the prediction from the PYTHIA8 event generator. The ratio significantly modifies for the in-jet production. 


\subsection{Neutral meson production in $\mathrm{p}-\mathrm{Pb}$ collisions}

In collaboration with Friederike Bock.

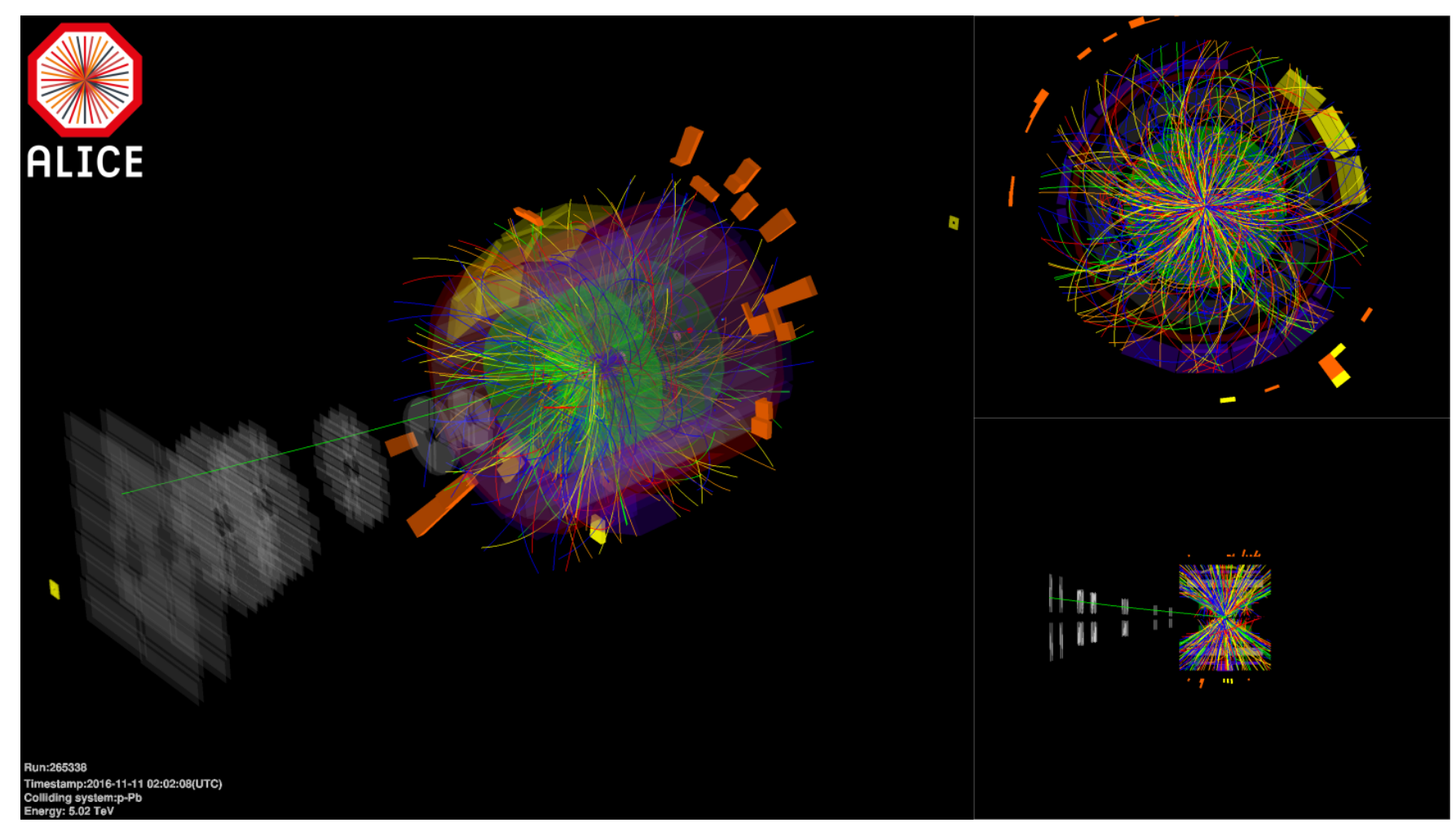

Figure 6.20: An example of a typical $\mathrm{p}-\mathrm{Pb}$ collision, recorded during the $2016 \mathrm{p}-\mathrm{Pb}$ run at $\sqrt{s_{N N}}=$ $5.02 \mathrm{TeV}$.

An example of a typical p-Pb collision is shown in Fig. 6.20, which was recorded during the $2016 \mathrm{p}-\mathrm{Pb}$ run at $\sqrt{s_{N N}}=5.02 \mathrm{TeV}$. The event display shows the larger particle multiplicities encountered in $\mathrm{p}-\mathrm{Pb}$ collisions, where the average amount of particles produced per unit rapidity $\left\langle\mathrm{d} N_{\mathrm{ch}} / \mathrm{d} \eta\right\rangle=10.8$ for a $\mathrm{p}-\mathrm{Pb}$ collision. In general, this collision system serves as an intermediate size system in between pp and $\mathrm{Pb}-\mathrm{Pb}$, where the effects of particle multiplicity and cold nuclear matter are studied.

This section will present the neutral meson measurements in $\mathrm{p}-\mathrm{Pb}$ collisions at a center-of-mass energy of $\sqrt{s_{N N}}=5.02 \mathrm{TeV}$, where the $p_{\mathrm{T}}$-dependent invariant yield of the $\pi^{0}$ and $\eta$ meson is measured for minimum bias data as well as different multiplicity classes and centrality estimators [133]. The results will be compared to theoretical model calculations, with an overall discussion at the end of this chapter. 


\subsubsection{Datasets}

The neutral meson measurements performed for $\mathrm{p}-\mathrm{Pb}$ collisions at $\sqrt{s_{N N}}=5.02 \mathrm{TeV}$ uses the datasets obtained in LHC Run 1, in year 2013. As for the analyses in pp, the data has been inspected for all data-taking runs and they are only included if the relevant detector systems operate as required. In the datasets LHC13b and LHC13c there are in total 102 million events passing the event selection criteria. The Monte Carlo simulation LHC13b2 is used to obtain the various correction factors used to correct the meson raw yields. All detectors are again fully calibrated to yield the best possible performance, which include calorimeter cell-by-cell calibrations, TPC energy loss corrections, and gain calibration of the centrality estimators.

\begin{tabular}{rllrr}
\hline$\sqrt{s_{N N}}(\mathrm{TeV})$ & Dataset & & Used triggers & \# events \\
\hline 5 & Data & LHC13bc & INT7 & $102 \cdot 10^{6}$ \\
& DPMJET $(\mathrm{MC})$ & LHC13b2_efix & INT7 & $111 \cdot 10^{6}$ \\
\hline
\end{tabular}

TABLE 6.5: Datasets used for the neutral meson measurements in $\mathrm{p}-\mathrm{Pb}$ collisions at a center-of-mass energy of $\sqrt{s_{N N}}=5.02 \mathrm{TeV}$.

\subsubsection{Results}

\section{Invariant yield in MB collisions}

The invariant yield of the $\pi^{0}$ and $\eta$ meson in minimum bias $\mathrm{p}-\mathrm{Pb}$ collisions at $\sqrt{s_{N N}}=5.02 \mathrm{TeV}$ are shown in Fig. 6.21 (left). The $\pi^{0}$ has been measured for $0.3<p_{\mathrm{T}}<20 \mathrm{GeV} / c$, and the $\eta$ meson for $0.7<p_{\mathrm{T}}<20 \mathrm{GeV} / c$. Both mesons are fitted using the Levy-Tsallis fit [149, 150], and the invariant yield with respect to the fit are shown in Fig. 6.21 (right). In addition, the results are compared to a wide variety of model calculations and event generators.

The NLO calculations in $\mathrm{p}-\mathrm{Pb}$ collisions now use a nuclear PDF (nPDF) for the Pb side of the collision, while using the proton PDF for the proton side. More specifically, the nPDFs EPPS16 [151] and the fragmentation function DSS14 $[152,153]$ are used to predict the invariant yield of the $\pi^{0}$ meson, and the $\mathrm{nPDF}$ nCTEQ [154] is used with the AESSS fragmentation function [155] for the $\eta$ meson. Since the nPDFs are not that well constrained, its uncertainties are of the order of $25-400 \%$, and overestimate the production of both mesons at higher meson $p_{\mathrm{T}}$. It is clear that these measurements can help to further improve future pQCD calculations.

The EPOS3 model $[156,157]$ includes collective radial flow in $\mathrm{p}-\mathrm{Pb}$ collisions, and describes the data for $p_{\mathrm{T}}<4 \mathrm{GeV} / c$ while significantly overestimating the yields at higher $p_{\mathrm{T}}$.

The VISHNU model $[158,159]$ is a $3+1 \mathrm{D}$ viscous hydrodynamical model coupled to a hadronic cascade model, where the fluctuations of the initial conditions are generated using a Monte Carlo Glauber approach. 
This model reproduces the $\pi^{0}$ and $\eta$ meson yields for $0.7<p_{\mathrm{T}}<1.5 \mathrm{GeV} / c$, and underestimates the yields otherwise.

The DPMJET event generator [160] is based on a Glauber approach and uses a two-component Dual Parton Model. Within this model, the soft and hard processes are implemented using Regge theory [161] and first order pQCD, respectively. DPMJet is tuned to hadron production at RHIC, for the low and moderate $p_{\mathrm{T}}$ region. For the results in Fig. 6.21, DPMJet underestimates the yield by $\sim 40 \%$ at higher $p_{\mathrm{T}}$ for both the $\pi^{0}$ and $\eta$ meson.

The HIJING model [162] is an event generator that also uses a Glauber approach for the initial state, but relies on QCD-based calculations for multiple jet production with low $p_{\mathrm{T}}$ multi-string phenomenology. This model includes mini-jet production, as well as nuclear shadowing of the parton cross-sections. For the results in Fig. 6.21, this model reproduces the data at intermediate $p_{\mathrm{T}}$, but overestimates the $\pi^{0}$ and $\eta$ meson otherwise by $60-80 \%$.

Furthermore, the Color Glass Condensate (CGC) model [32] is an effective theory for the nuclear environment at low $x$ where the gluon density is high and non-linear processes are expected to play a significant role. This model prediction for the $\pi^{0}$ describes the yield for $p_{\mathrm{T}}<5 \mathrm{GeV} / c$, and overestimates it for higher $p_{\mathrm{T}}$.
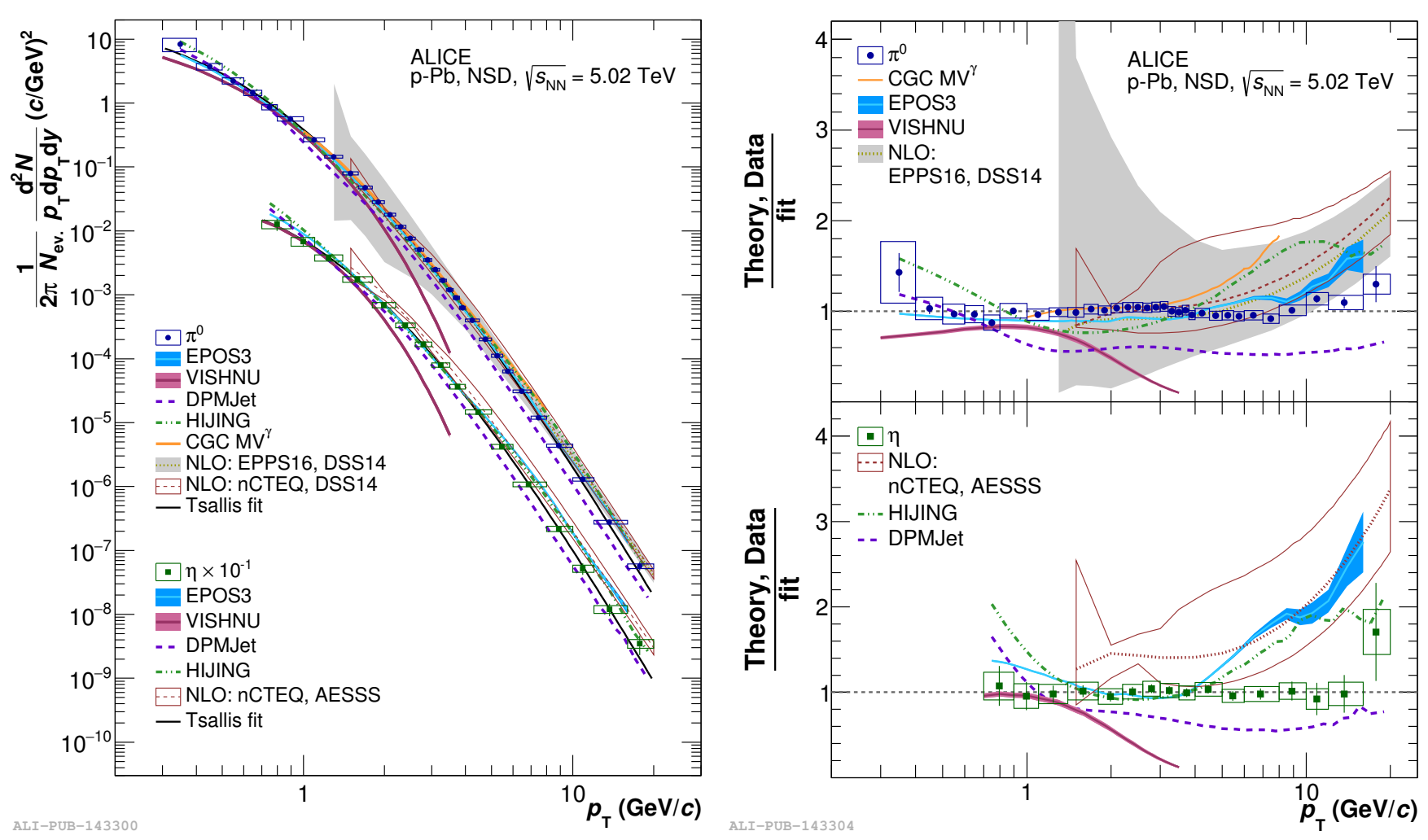

Figure 6.21: Left: The invariant yield of $\pi^{0}$ and $\eta$ meson, as measured with the ALICE detector in $\mathrm{p}-\mathrm{Pb}$ collisions at $\sqrt{s_{N N}}=5.02 \mathrm{TeV}$. The results are compared to several event generators and model calculations. Right: The ratio of the invariant yield of the $\pi^{0}$ (top) and $\eta$ (bottom) to the fit of the respective meson, and compared to the ratio of the theoretical calculations to the same fit. 


\section{Nuclear modification of neutral mesons in MB collisions}

One of the key observables in studying cold nuclear matter effects is the nuclear modification of particle production, as described in section 2.4.2. The results for the $\pi^{0}$ and $\eta$ meson $R_{\mathrm{pPb}}$ is shown in Fig. 6.22. The $R_{\mathrm{pPb}}$ is calculated using $\left\langle T_{p A}\right\rangle=0.0983 \pm 0.0035 \mathrm{mb}^{-1}$ and an interpolation between the measured neutral meson invariant yields in pp collisions of $\sqrt{s}=2.76 \mathrm{TeV}$ and $\sqrt{s}=7 \mathrm{TeV}$, because the dataset at $\sqrt{s}=5.02 \mathrm{TeV}$ was unavailable at the time of the publication. The $R_{\mathrm{pPb}}$ of both mesons show to be consistent with unity for $p_{\mathrm{T}}>2 \mathrm{GeV} / c$, indicating that high momentum mesons are unaffected by the cold nuclear matter effects. However, at lower meson $p_{\mathrm{T}}$ there is a strong suppression of meson production, which could be attributed to nuclear shadowing or non-perturbative effects. These observations are tested by comparing the results to NLO calculations which use nPDFs and the CGC model. The NLO calculations show qualitatively the same behavior, but have large uncertainties and are limited in $p_{\mathrm{T}}$ reach. The CGC model does quantitatively describe the $\pi^{0} R_{\mathrm{pPb}}$, but no model uncertainties are given.
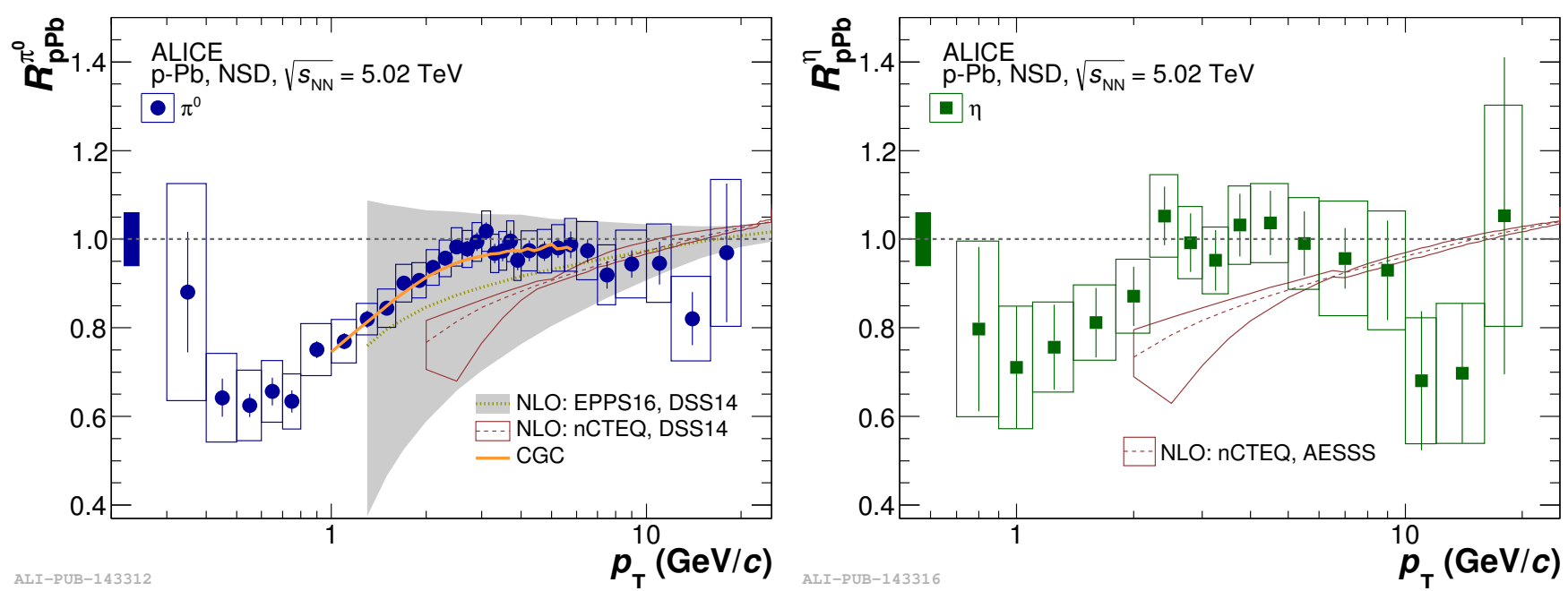

FiguRE 6.22: The nuclear modification factor $R_{\mathrm{pPb}}$ of the $\pi^{0}$ (left) and $\eta$ meson (right), as measured with the ALICE detector in $\mathrm{p}-\mathrm{Pb}$ collisions at $\sqrt{s_{N N}}=5.02 \mathrm{TeV}$. The interpolated invariant yield of neutral mesons in pp collisions at $\sqrt{s}=2.76 \mathrm{TeV}$ and $\sqrt{s}=7 \mathrm{TeV}$ is used as the reference. Comparisons are made to the predictions of the CGC model as well as NLO calculations. 


\section{Multiplicity dependent invariant yield of neutral mesons}

In addition to the invariant yields of the $\pi^{0}$ and $\eta$ meson for $\mathrm{MB} \mathrm{p}-\mathrm{Pb}$ collisions, the measurements are repeated for the different intervals $0-20 \%, 20-40 \%, 40-60 \%$, and $60-100 \%$ of V0A multiplicity. More specifically, the event class $0-20 \%$ corresponds to the $20 \%$ events with highest V0A multiplicities, and $60-100 \%$ corresponds to the $40 \%$ event with the lowest V0A multiplicities. This way, the dependence of the neutral meson spectra on the event multiplicity can be tested. In Fig. 6.23 the invariant yield of the $\pi^{0}$ and $\eta$ meson is shown for different multiplicity classes of $\mathrm{p}-\mathrm{Pb}$ collisions at $\sqrt{s_{N N}}=5.02 \mathrm{TeV}$, in addition to the $\mathrm{MB} 0-100 \%$ result. As these measurements are done on subsets of the MB dataset, the width of the $p_{\mathrm{T}}$ intervals are increased to have enough statistics for the extraction of the raw yields and calculation of the various correction factors.
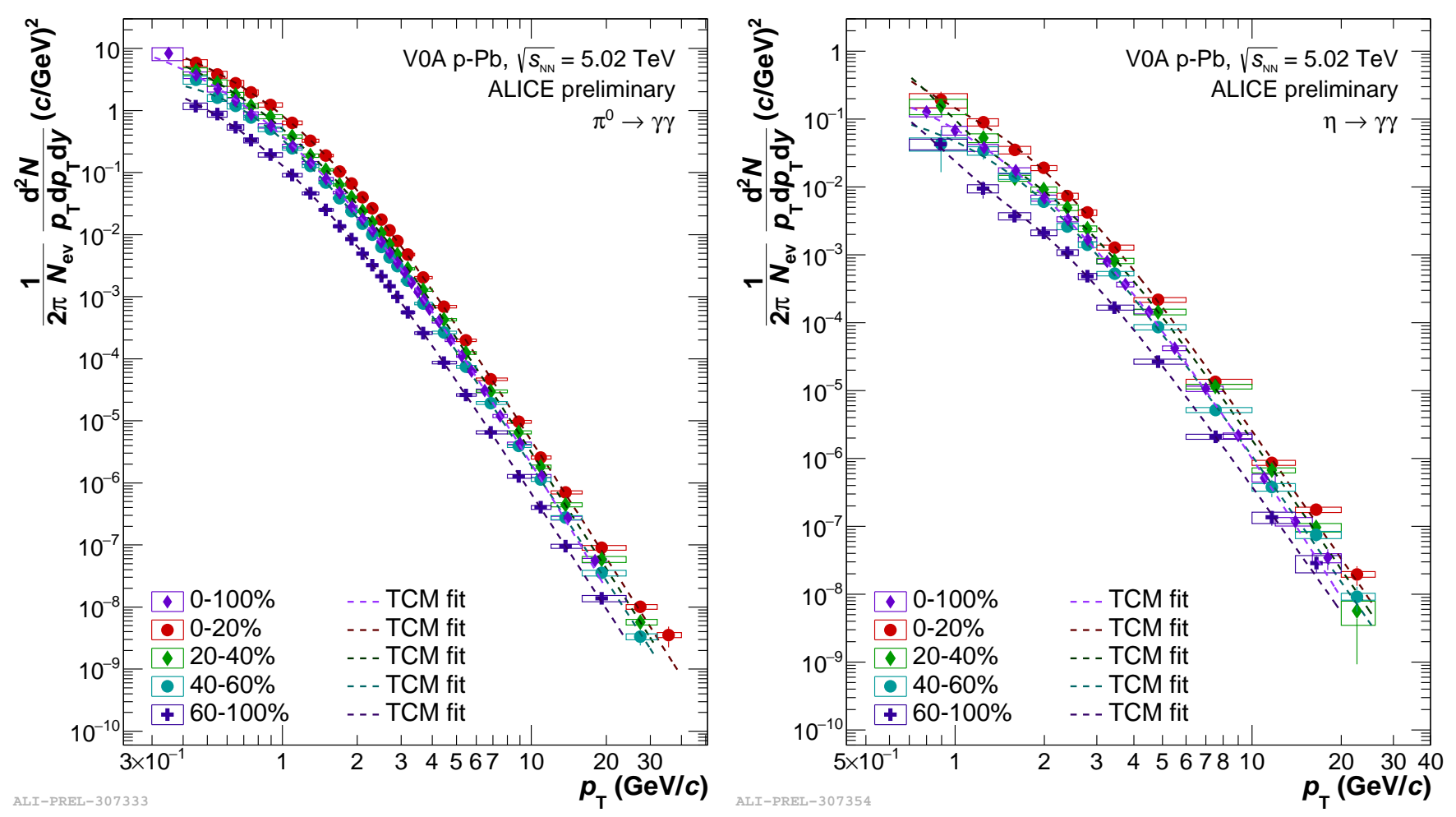

Figure 6.23: The invariant yield of $\pi^{0}$ (left) and $\eta$ meson (right) for different centrality classes of $\mathrm{p}^{-}$ $\mathrm{Pb}$ collisions at $\sqrt{s_{N N}}=5.02 \mathrm{TeV}$, as measured with the ALICE detector. 


\section{Multiplicity dependent nuclear modification of neutral mesons}

The modification of the $\pi^{0}$ and $\eta$ meson invariant yields as function of the V0A multiplicity is studied using the nuclear modification factor $Q_{\mathrm{pA}}$. This observable is explicitly labeled differently than $R_{p A}$, as the number of binary collisions may not be straightforward and the multiplicity selection can cause a bias that could be partly responsible for the enhancements and suppressions at higher $p_{\mathrm{T}}$. It is defined as

$$
Q_{\mathrm{pA}}\left(p_{\mathrm{T}}\right)=\frac{\mathrm{d}^{2} N /\left.\mathrm{d} p_{\mathrm{T}} \mathrm{d} y\right|_{\mathrm{pA}, \text { multclass }}}{\left\langle T_{\mathrm{pA}, \text { multclass }}\right\rangle \mathrm{d}^{2} \sigma /\left.\mathrm{d} p_{\mathrm{T}} \mathrm{d} y\right|_{\mathrm{pp}}},
$$

where the difference with the observable $R_{\mathrm{pA}}$ is the use of the invariant yield for a specific V0A multiplicity class in the numerator. In addition, the values for $T_{\mathrm{pA} \text {,multclass }}$ are calculated using a Glauber model and are specific to the multiplicity class. The multiplicity selection might introduce biases in the spectra, and the calculation of $T_{\mathrm{pA}}$ is potentially also biased in these cases. As was studied in detail in [112], the bias is likely small for the use of the V0A multiplicity estimator. To make these possible biases explicit, this quantity is given a different name to distinguish it from $R_{\mathrm{pA}}$.

The results for the $\pi^{0}$ and $\eta$ meson $Q_{\mathrm{pA}}\left(p_{\mathrm{T}}\right)$ for different V0A multiplicity classes are shown in Fig. 6.24, and indicate a significant difference between the $0-20 \%, 60-100 \%$, and the $R_{\mathrm{pA}}\left(p_{\mathrm{T}}\right)$. For the $60-100 \%$ event class there is no strong $p_{\mathrm{T}}$ dependence, but a strong overall suppression. For the $0-20 \%$ event class there is a significant enhancement at $p_{\mathrm{T}} \sim 2 \mathrm{GeV} / c$, and a suppression for lower $p_{\mathrm{T}}$. This implies that the V0A multiplicity classes are able to probe different magnitudes of the cold nuclear matter effects. For the $\eta$ meson the same behavior is seen, but the experimental uncertainties are too large to make concise conclusions.
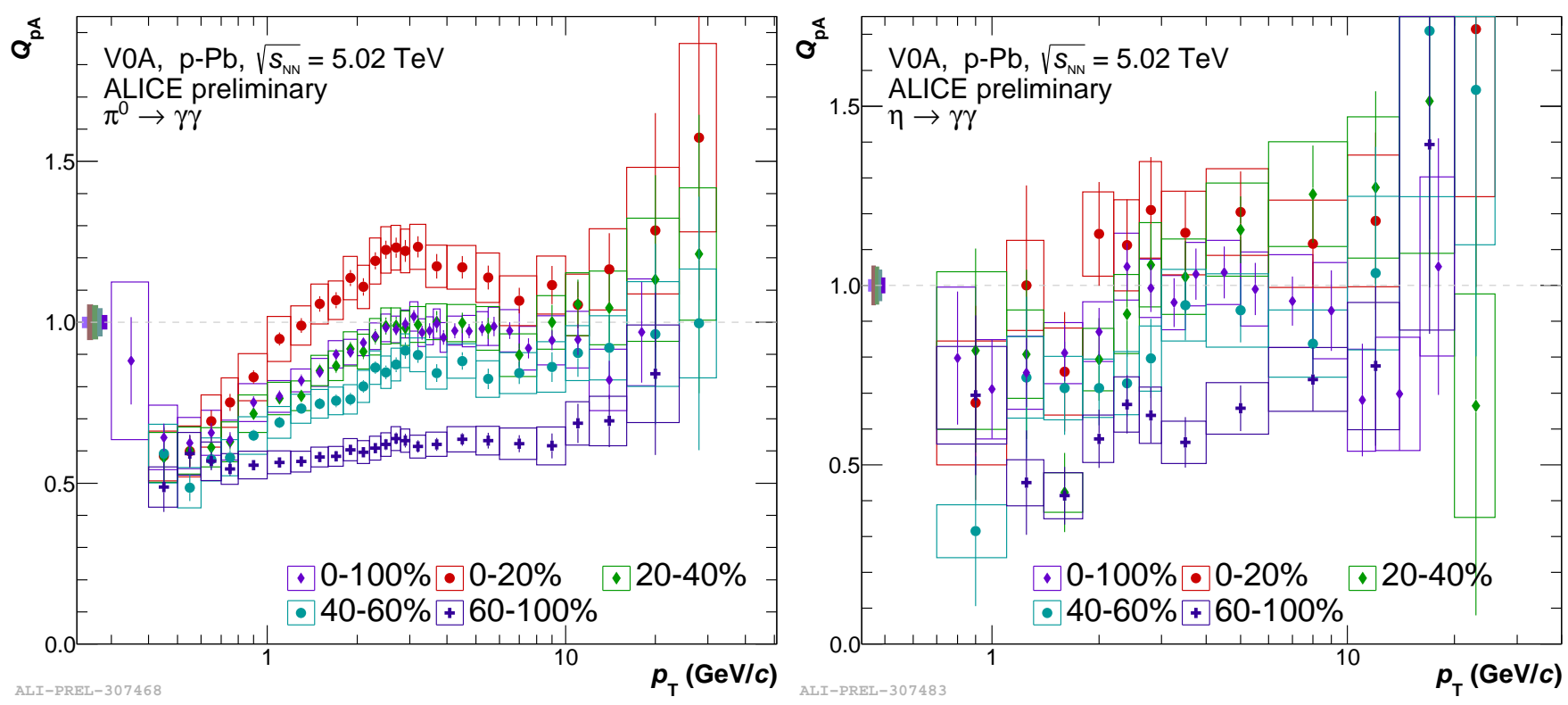

FiguRE 6.24: The nuclear modification factor $Q_{\mathrm{pPb}}$ of the $\pi^{0}$ (left) and $\eta$ meson (right), as measured with the ALICE detector in $\mathrm{p}-\mathrm{Pb}$ collisions at $\sqrt{s_{N N}}=5.02 \mathrm{TeV}$. The respective neutral meson spectrum in pp collisions at $\sqrt{s}=5.02 \mathrm{TeV}$ is used as reference. 
$\eta / \pi^{0}$ ratio

In Fig. 6.25 the $\eta / \pi^{0}$ ratio for $\mathrm{MB}$ and multiplicity dependent $\mathrm{p}-\mathrm{Pb}$ collisions at $\sqrt{s_{N N}}=5.02 \mathrm{TeV}$ is shown. Similar to the result for this ratio in pp collisions, the invariant yield of the $\pi^{0}$ has been measured for the same $p_{\mathrm{T}}$ intervals as the $\eta$ meson, such that the ratio is calculated by taking the point-by-point ratio, also canceling the relevant systematic uncertainties. The high $p_{\mathrm{T}}$ average of $0.485 \pm 0.015^{\text {stat }} \pm 0.031^{\text {sys }}$ for the $\mathrm{MB}$ result is consistent with high $p_{\mathrm{T}}$ average found in pp collisions, and also shows qualitative the same behavior for lower $p_{\mathrm{T}}$.

The DPMJet and HIJING event generators both overestimate the ratio for $p_{\mathrm{T}}<4 \mathrm{GeV} / c$ by $10-50 \%$, which, as shown in Fig. 6.21, is mostly driven by the larger underestimation of the $\pi^{0}$. For $p_{\mathrm{T}}>4 \mathrm{GeV} / c$, the generators are consistent with the experimental results. The low $p_{\mathrm{T}}$ part is better described by the EPOS3 and VISHNU models. At higher $p_{\mathrm{T}}$, the EPOS3 model predicts a ratio that is $\sim 60 \%$ higher than the data, which should be the region that is more easily described as particle masses no longer play a major role.

To summarize the results of the $\eta / \pi^{0}$ ratio in $\mathrm{p}-\mathrm{Pb}$ collisions, no significant changes are observed for the multiplicity dependent $\eta / \pi^{0}$ ratio, indicating a universal value of this ratio independent of multiplicity.
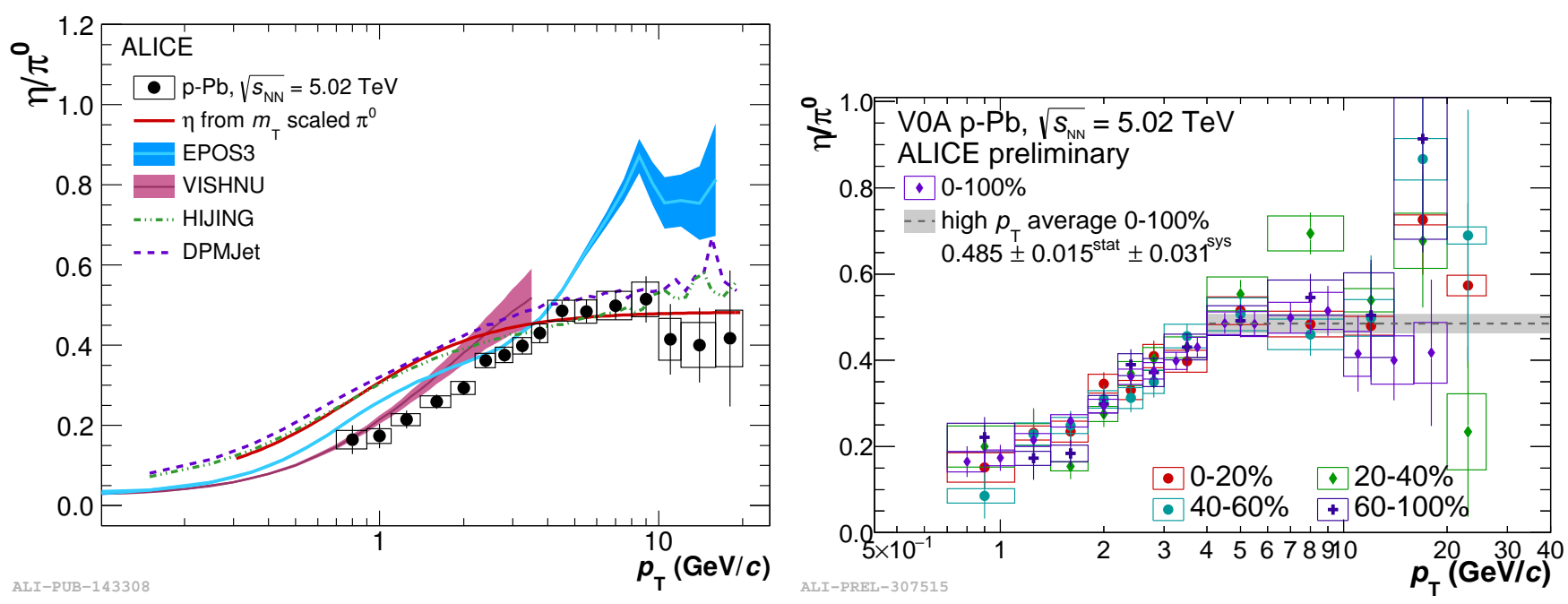

FIgURE 6.25: The $\eta / \pi^{0}$ ratio for MB and multiplicity dependent $\mathrm{p}-\mathrm{Pb}$ collisions at $\sqrt{s_{N N}}=5.02 \mathrm{TeV}$, as measured with the ALICE detector. The result for MB is compared with the prediction from the event generators DPMJet and HIJING as well as the EPOS3 and VISHNU model. 


\subsection{Neutral meson production in $\mathrm{Pb}-\mathrm{Pb}$ collisions}

In collaboration with Meike Danisch.

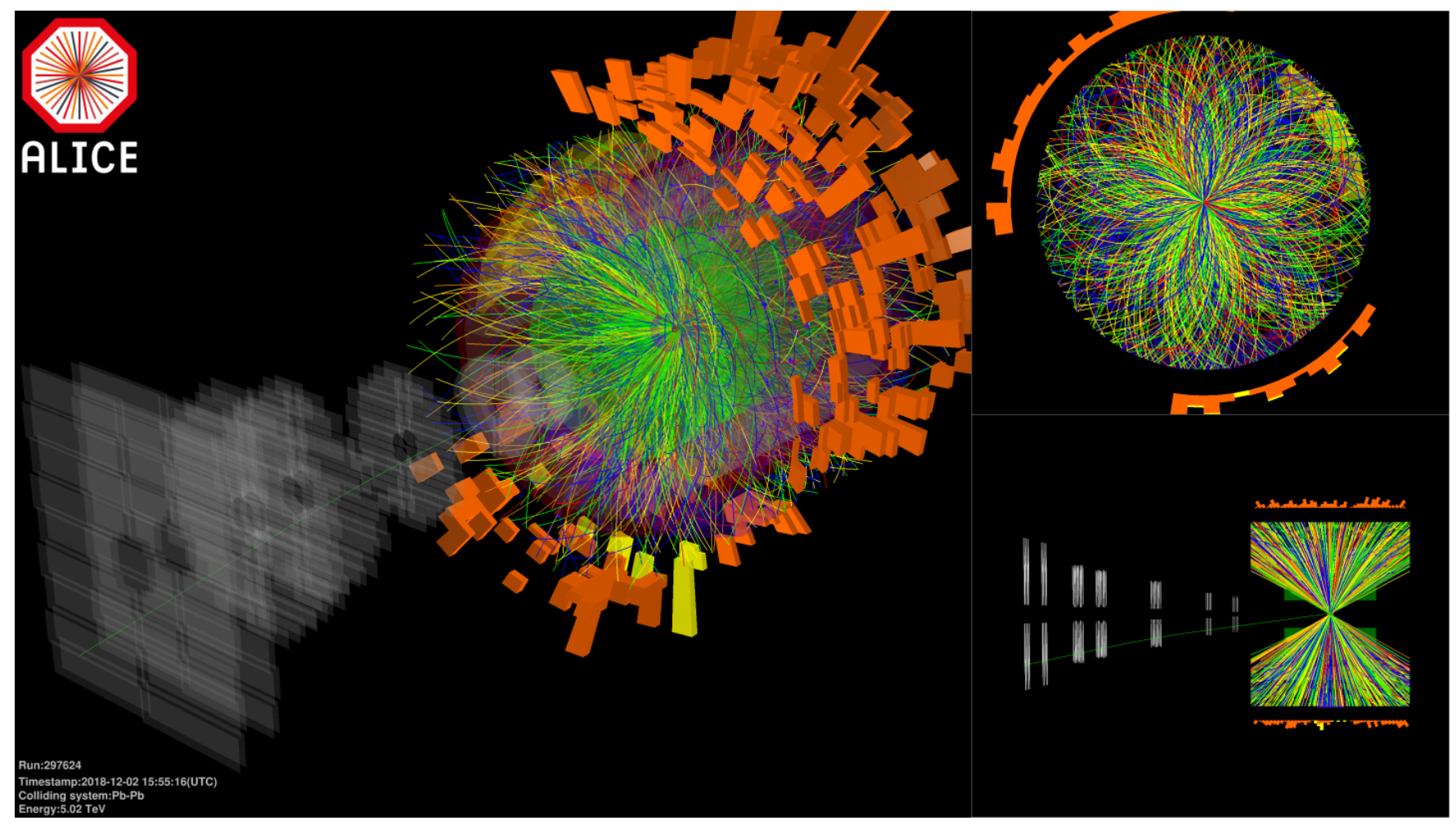

Figure 6.26: An example of a typical $\mathrm{Pb}-\mathrm{Pb}$ collision, recorded during the $2018 \mathrm{~Pb}-\mathrm{Pb}$ run at $\sqrt{s_{N N}}=$ $5.02 \mathrm{TeV}$.

An example of a typical $\mathrm{Pb}-\mathrm{Pb}$ collision is shown in Fig. 6.26, which was recorded during the $2018 \mathrm{~Pb}-$ $\mathrm{Pb}$ run at $\sqrt{s_{N N}}=5.02 \mathrm{TeV}$. The event display shows the extremely large particle multiplicities produced in $\mathrm{Pb}-\mathrm{Pb}$ collisions, which span a range of $\left\langle\mathrm{d} N_{\mathrm{ch}} / \mathrm{d} \eta\right\rangle=52.7$ for peripheral $\mathrm{Pb}-\mathrm{Pb}$ collisions all the way to $\left\langle\mathrm{d} N_{\mathrm{ch}} / \mathrm{d} \eta\right\rangle=1080$ for central $\mathrm{Pb}-\mathrm{Pb}$ collisions. The energy densities reached in this system are sufficient for QGP formation, and allows for studying hot nuclear matter effects that were broadly discussed in Chapter 2 .

This section will present the neutral meson measurements in $\mathrm{Pb}-\mathrm{Pb}$ collisions at a center-of-mass energy of $\sqrt{s_{N N}}=5.02 \mathrm{TeV}$, where the $p_{\mathrm{T}}$-dependent invariant yield of the $\pi^{0}$ and $\eta$ meson is measured for minimum bias data as well as different multiplicity classes. The results will be compared to theoretical model calculations, with an overall discussion at the end of this chapter. 


\subsubsection{Datasets}

The neutral meson invariant yield measurements in $\mathrm{Pb}-\mathrm{Pb}$ collisions at $\sqrt{s_{N N}}=5.02 \mathrm{TeV}$ uses the datasets obtained in LHC Run 2, taken in the years 2015 and 2018. It is the first analysis in ALICE that combines the two $\mathrm{Pb}-\mathrm{Pb}$ datasets to fully utilize the available statistics. Only data-taking runs with optimal detector conditions are selected for the analysis. In Table 6.6 the used datasets and MC productions are listed, showing that the INT7 trigger recorded 100 million MB collisions for both LHC15o and LHC18qr. The two dedicated triggers kCentral and kSemiCentral were running in 2018, enhancing greatly the statistics of the central and semi-central $\mathrm{Pb}-\mathrm{Pb}$ collisions. In addition, pile-up events are rejected by making use of the correlation between SPD tracklets and TPC clusters.

In $\mathrm{Pb}-\mathrm{Pb}$ collisions, the highest V0A multiplicity class $0-10 \%$ is associated with the $10 \%$ most central collisions, meaning that the impact parameter $b$ is on average the lowest. For the lowest V0A multiplicity class $50-90 \%$, the $\mathrm{Pb}$ ions are assumed to hit each other peripherally, thereby coming closer to the phenomenology of a pp collision.

\begin{tabular}{rlllrr}
\hline$\sqrt{s_{N N}}(\mathrm{TeV})$ & Data Set & & Used triggers & V0M mult. class & \# events \\
\hline 5.02 & Data & LHC15o & INT7 & $0-10 \%$ & $7.36 \cdot 10^{6}$ \\
& & & INT7 & $10-30 \%$ & $14.7 \cdot 10^{6}$ \\
& & INT7 & $30-50 \%$ & $14.8 \cdot 10^{6}$ \\
5.02 & HIJING (MC) & LHC16h4 & INT7 & $50-90 \%$ & $29.5 \cdot 10^{6}$ \\
5.02 & HIJING (MC) & LHC18e1 & INT7 & $0-90 \%$ & $1.6 \cdot 10^{6}$ \\
5.02 & HIJING (MC) & LHC18e1(a,b,c) & INT7 & $0-90 \%$ & $1.5 \cdot 10^{6}$ \\
5.02 & Data & LHC18qr & INT7 \& kCentral & $0-90 \%$ & $7.7 \cdot 10^{6}$ \\
\hline & & & INT7 & $0-10 \%$ & $148.9 \cdot 10^{6}$ \\
& & & INT7 \& kSemiCentral & $10-30 \%$ & $17.6 \cdot 10^{6}$ \\
5.02 & HIJING (MC) & LHC1818(a4,b2,c2) & INT7 & $30-50 \%$ & $86.6 \cdot 10^{6}$ \\
5.02 & HIJING (MC) & LHC19h3 & INT7 & $50-90 \%$ & $34.1 \cdot 10^{6}$ \\
5.02 & HIJING (MC) & LHC19h2(a,b,c) & INT7 & $0-90 \%$ & $5.1 \cdot 10^{6}$ \\
& & & $0-90 \%$ & $2.3 \cdot 10^{6}$ \\
& & & $0-90 \%$ & $6.3 \cdot 10^{6}$ \\
\hline
\end{tabular}

TABlE 6.6: Datasets used for the neutral meson measurements in $\mathrm{Pb}-\mathrm{Pb}$ collisions at a center-of-mass energy of $\sqrt{s_{N N}}=5.02 \mathrm{TeV}$. 


\subsubsection{Results}

\section{Raw yield corrections}

The challenge of measuring neutral mesons produced in $\mathrm{Pb}-\mathrm{Pb}$ collisions is to deal with the large particle multiplicities in the detector, which drastically increases the combinatorial background in the invariant mass distributions. Furthermore, the reconstruction efficiency of photons gets significantly modified when the density of tracks and clusters is such that the signals begin to overlap, which happens for the multiplicities encountered in $\mathrm{Pb}-\mathrm{Pb}$ collisions.

The neutral meson efficiency times acceptance $(\epsilon)$ for the different reconstruction methods are shown in Fig. 6.27 for the $0-10 \%$ and $50-90 \%$ V0A multiplicity class and in Fig. A.9 (Appendix A.2) for the $10-30 \%$ and $30-50 \%$ multiplicity class. It shows that $\epsilon$ decreases for higher event multiplicities, with an increased effect for lower meson $p_{\mathrm{T}}$. These observations hold for both the $\pi^{0}$ and the $\eta$.

\section{Consistency of the reconstruction methods}

The invariant yields as measured with the various reconstruction methods are compared to the combined result, and is shown in Fig. 6.28 for the different V0A multiplicity classes. It shows that the methods are compatible within the uncertainties for both the $\pi^{0}$ and the $\eta$ meson. The fluctuations for the $\eta$ meson measurements are larger, but this is in line with the increased statistical uncertainty. 

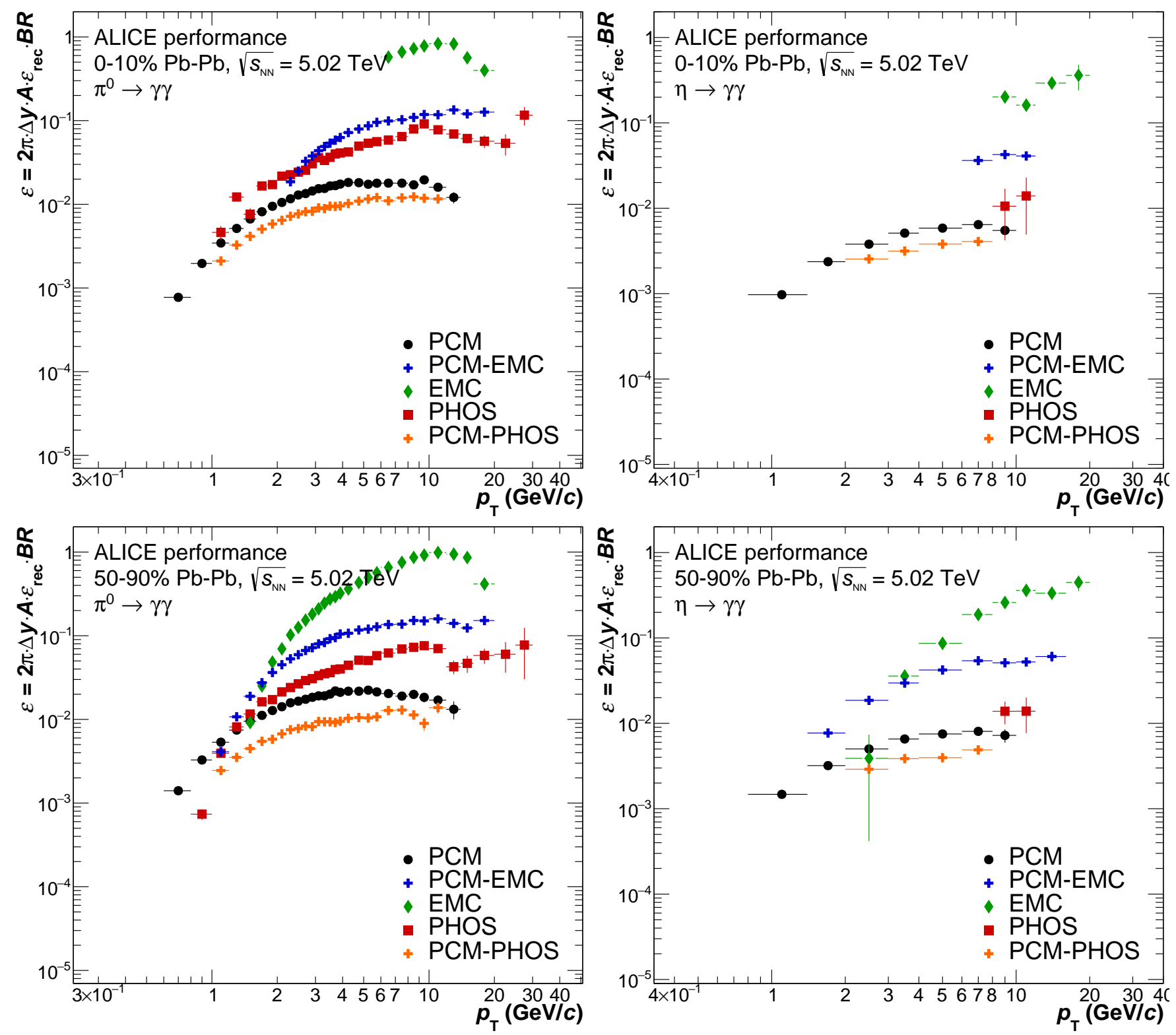

FiguRE 6.27: The acceptance times efficiency for the $\pi^{0}$ (left), and the $\eta$ meson (right), as measured for the $0-10 \%$ (top) and $50-90 \%$ (bottom) V0M multiplicity classes in $\mathrm{Pb}-\mathrm{Pb}$ collisions at $\sqrt{s_{N N}}=5.02 \mathrm{TeV}$. 

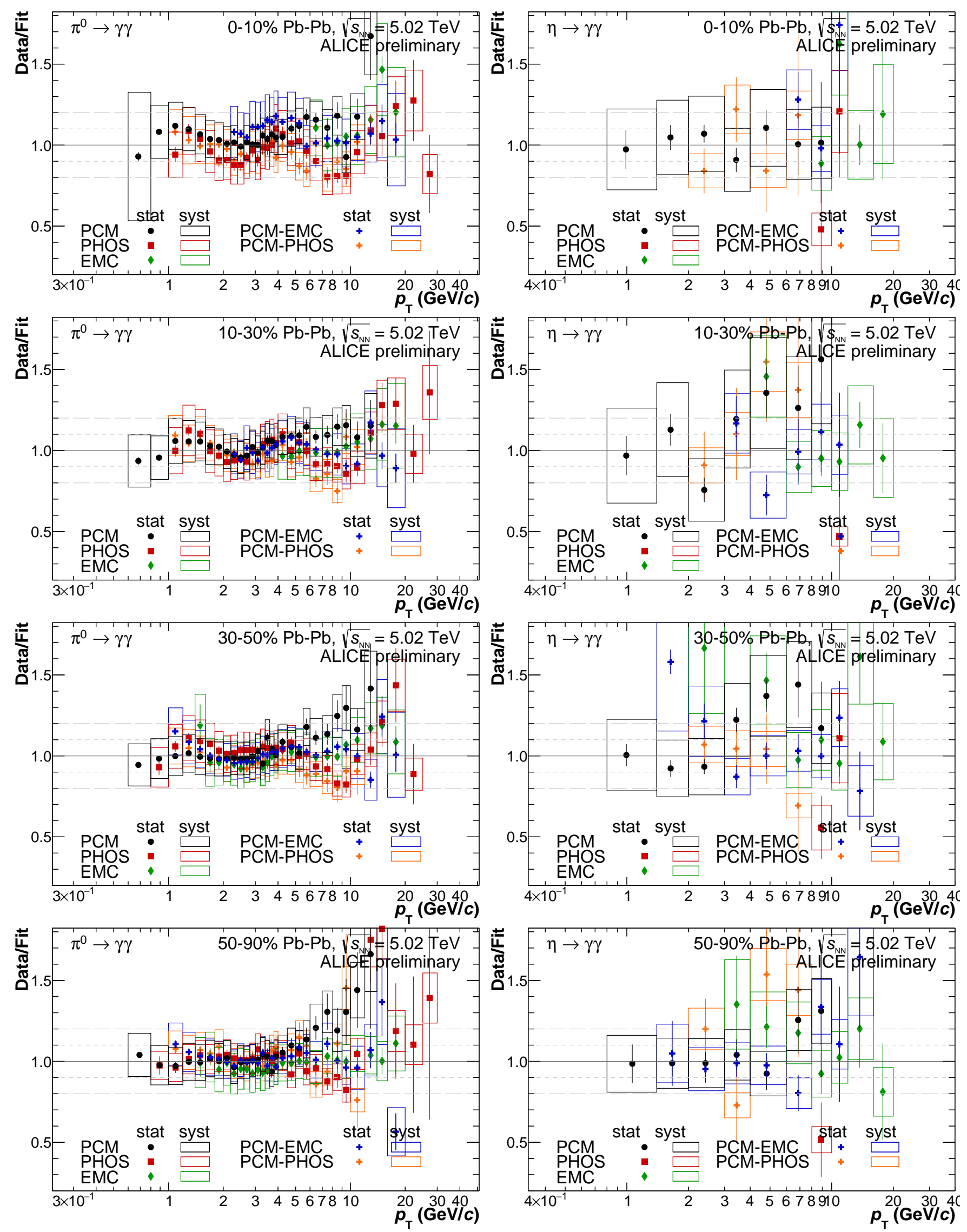

FIGURE 6.28: The ratio of the invariant yield of a single reconstruction method to the fit through the combined spectrum, for the $\pi^{0}$ (left), and the $\eta$ meson (right), as measured for the $0-10 \%, 10-30 \%$, $30-50 \%$, and $50-90 \% \mathrm{V0A}$ multiplicity classes in $\mathrm{Pb}-\mathrm{Pb}$ collisions at $\sqrt{s_{N N}}=5.02 \mathrm{TeV}$. All measured values are found to be consistent within uncertainties. 


\section{Centrality dependent invariant yield}

The invariant yield of the $\pi^{0}$ and $\eta$ meson in $\mathrm{Pb}-\mathrm{Pb}$ collisions at $\sqrt{s_{N N}}=5.02 \mathrm{TeV}$ is shown in Fig. 6.29 for the different V0A multiplicity classes, covering the most central to the most peripheral $\mathrm{Pb}-\mathrm{Pb}$ collisions. As expected, the overall production increases for event classes with larger particle multiplicities. In addition, it is evident that the shape of the $\pi^{0}$ and $\eta$ meson invariant yields in $\mathrm{Pb}-\mathrm{Pb}$ collisions are modified more significantly for the more central collisions with respect to the yields measured in pp collisions. This modification is associated to the hot nuclear matter, or in-medium effects. At low $p_{\mathrm{T}}$ the production of particles is possibly increased by thermal production of the medium, relative to intermediate meson $p_{\mathrm{T}}$. The origin of the particles at high $p_{\mathrm{T}}$ is mostly from the hard interactions of the constituents of the $\mathrm{Pb}$ ions and the particles are affected by energy loss in the QGP.
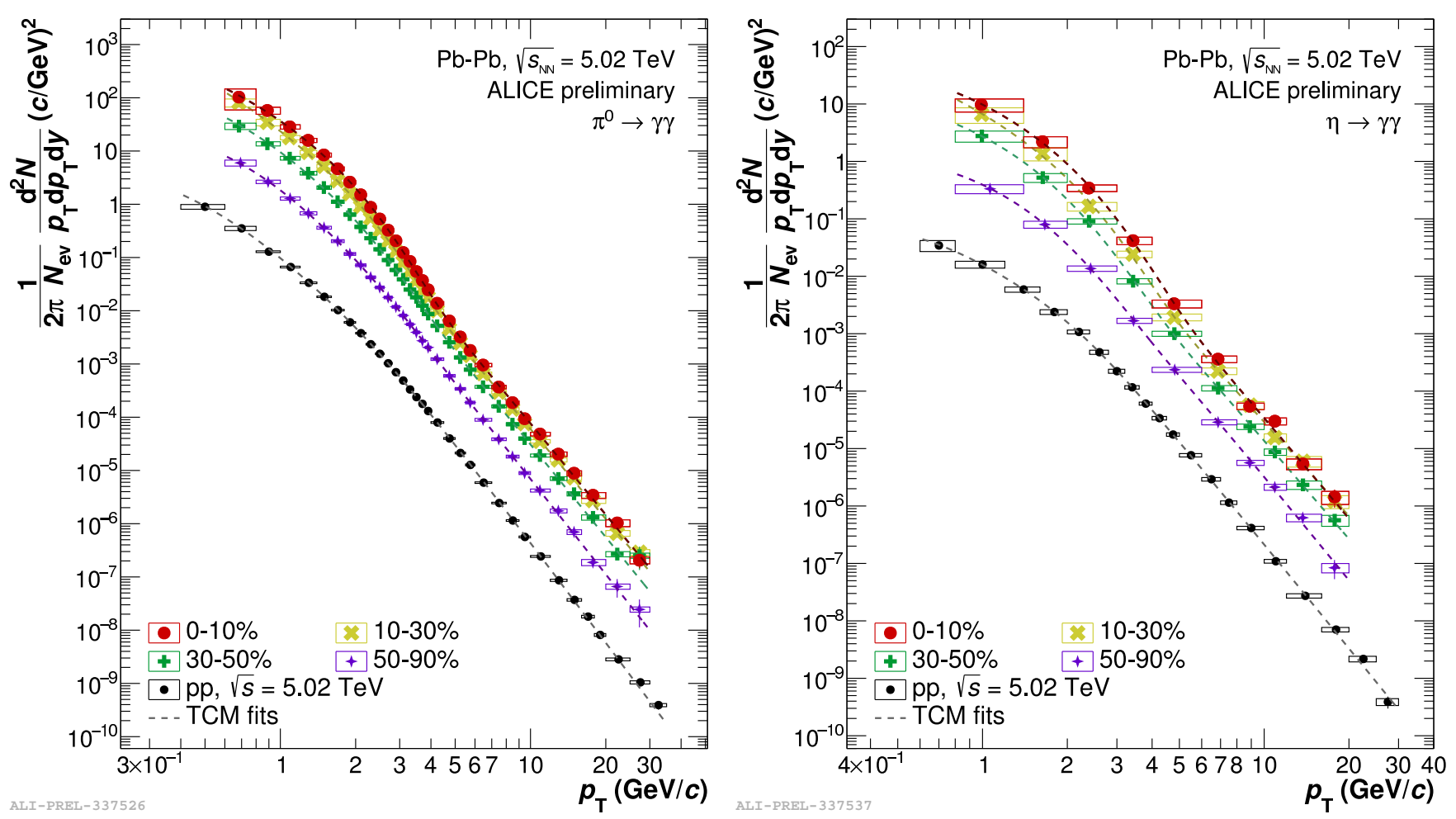

Figure 6.29: The invariant yield of $\pi^{0}$ (left) and $\eta$ meson (right) for different centrality classes in $\mathrm{Pb}-$ $\mathrm{Pb}$ collisions at $\sqrt{s_{N N}}=5.02 \mathrm{TeV}$, as measured with the ALICE detector. 


\section{Centrality dependent $\eta / \pi^{0}$ ratio}

The $\eta / \pi^{0}$ ratio for $\mathrm{Pb}-\mathrm{Pb}$ collisions at $\sqrt{s_{N N}}=5.02 \mathrm{TeV}$ is shown in Fig. 6.30 (left) for each of the V0A multiplicity classes as well as the results obtained in MB pp collisions at $\sqrt{s}=5.02 \mathrm{TeV}$. There is no significant difference between the ratio measured in $\mathrm{Pb}-\mathrm{Pb} 50-90 \%$ and in $\mathrm{MB}$ pp, indicating that there are no strong medium effects in low multiplicity $\mathrm{Pb}-\mathrm{Pb}$ events that are associated with peripherally hitting nuclei. However for larger event multiplicities, the ratio significantly higher than the ratio in MB pp between $1.4<p_{\mathrm{T}}<4 \mathrm{GeV} / c$. This behavior is could possibly be attributed to the increase in radial flow which affects heavier particles more, but has yet to be confirmed by model calculations. At higher $p_{\mathrm{T}}$ values, the ratio is again consistent with the ratio found in pp.

In Fig. 6.30 (right) the $\eta / \pi^{0}$ ratio for the $0-10 \%$ multiplicity class is shown. For $4<p_{\mathrm{T}}<10 \mathrm{GeV} / c$, the average of the ratio is $0.465 \pm 0.025^{\text {stat }} \pm 0.065^{\text {sys }}$, which is compatible with the high $p_{\mathrm{T}}$ average found in both pp and $\mathrm{p}-\mathrm{Pb}$ collisions at the same center-of-mass energy. The $m_{\mathrm{T}}-$ scaled $\eta / \pi^{0}$ ratio, i.e. the ratio where the invariant yield of the $\eta$ meson is calculated from $m_{\mathrm{T}}$-scaling the $\pi^{0}$ invariant yield, is unable to describe the measurement for $2<p_{\mathrm{T}}<6 \mathrm{GeV} / c$, which is expected since radial flow effects are not taken into account when $m_{\mathrm{T}}$-scaling the $\pi^{0}$ spectrum. Similarly, the HIJING event generator also fails to describe the data, as again no flow-like effects are included. The theory calculation from Paquet et al., which includes a full hydrodynamical description and also flow-like effects, quantitatively describes the data up to $3 \mathrm{GeV} / c$. For higher $p_{\mathrm{T}}$ this model is most likely dominated by statistical uncertainties. The SHM (statistical hadronization model), which is aimed at explaining low $-p_{\mathrm{T}}$ particle production, only predicts the ratio for $0<p_{\mathrm{T}}<3 \mathrm{GeV} / c$, and underestimates the data.
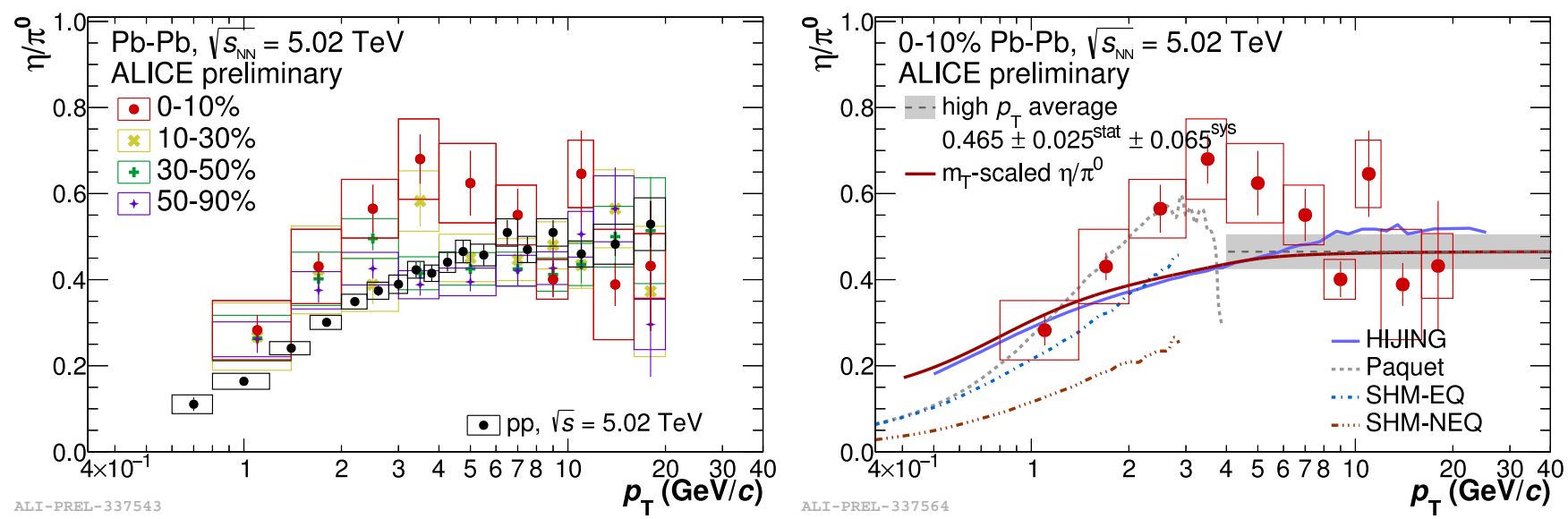

Figure 6.30: The $\eta / \pi^{0}$ ratio for different centrality classes of $\mathrm{Pb}-\mathrm{Pb}$ collisions at $\sqrt{s_{N N}}=5.02 \mathrm{TeV}$, as measured with the ALICE detector. 


\section{Centrality dependent nuclear modification factor}

The nuclear modification factors $R_{\mathrm{AA}}$ of the $\pi^{0}$ and $\eta$ meson are shown in Fig. 6.31 for the different V0A multiplicity classes in $\mathrm{Pb}-\mathrm{Pb}$ collisions at $\sqrt{s_{N N}}=5.02 \mathrm{TeV}$. The invariant yield and the corresponding $R_{\mathrm{AA}}$ of the $\pi^{0}$ for a finer selection in V0A multiplicity is shown in Fig. 6.32. These results have been calculated using the $\pi^{0}$ and $\eta$ meson invariant yields measured in $\mathrm{Pb}-\mathrm{Pb}$ and pp collisions at $\sqrt{s_{N N}}=5.02 \mathrm{TeV}$ and $\sqrt{s}=5.02 \mathrm{TeV}$, respectively, as well as the nuclear overlap factor $\left\langle T_{\mathrm{AA}}\right\rangle$. For both mesons the overall magnitude of the $R_{\mathrm{AA}}$ is much below unity, indicating a large suppression of hadron production with respect to $\left\langle T_{\mathrm{AA}}\right\rangle \times \sigma_{\mathrm{pp}}$.

The $\pi^{0}$ meson shows a significantly different $R_{\mathrm{AA}}$ for the various V0A multiplicity classes, with more suppression for the more central collisions. In addition, there is a stronger $p_{\mathrm{T}}$ dependence observed for the more central collisions. This is consistent with the picture that the size, and thus medium effects, increase for the more central collisions. For the $\eta$ meson, the same behavior is seen, although at low $p_{\mathrm{T}}$ the results are more dominated by experimental uncertainties.
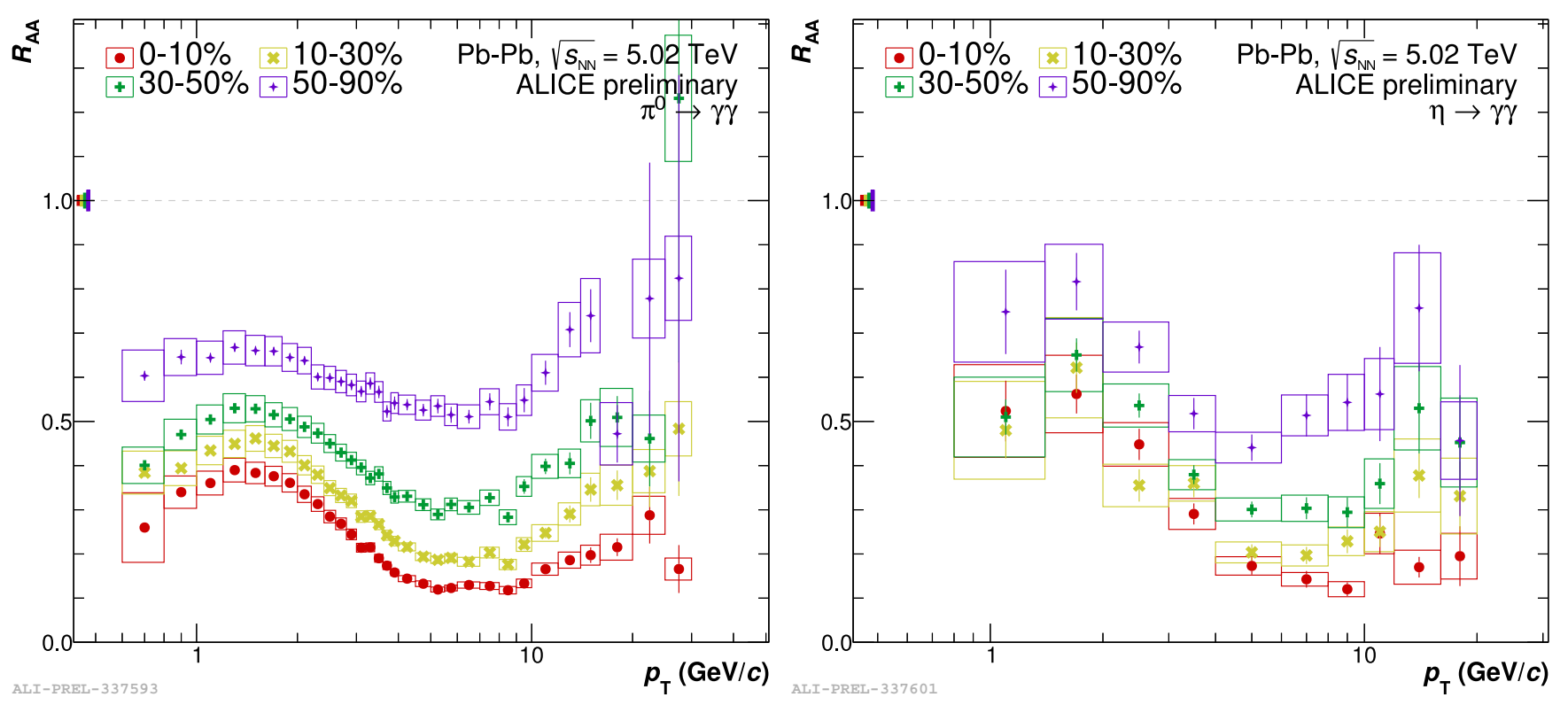

FIgURE 6.31: The nuclear modification factor $R_{\mathrm{AA}}$ of the $\pi^{0}$ (left) and $\eta$ meson (right), as measured with the ALICE detector in $\mathrm{Pb}-\mathrm{Pb}$ collisions at $\sqrt{s_{N N}}=5.02 \mathrm{TeV}$, for wide intervals of $\mathrm{VOM}$ multiplicities. The respective neutral meson spectrum in pp collisions at $\sqrt{s}=5.02 \mathrm{TeV}$ is used as reference. 

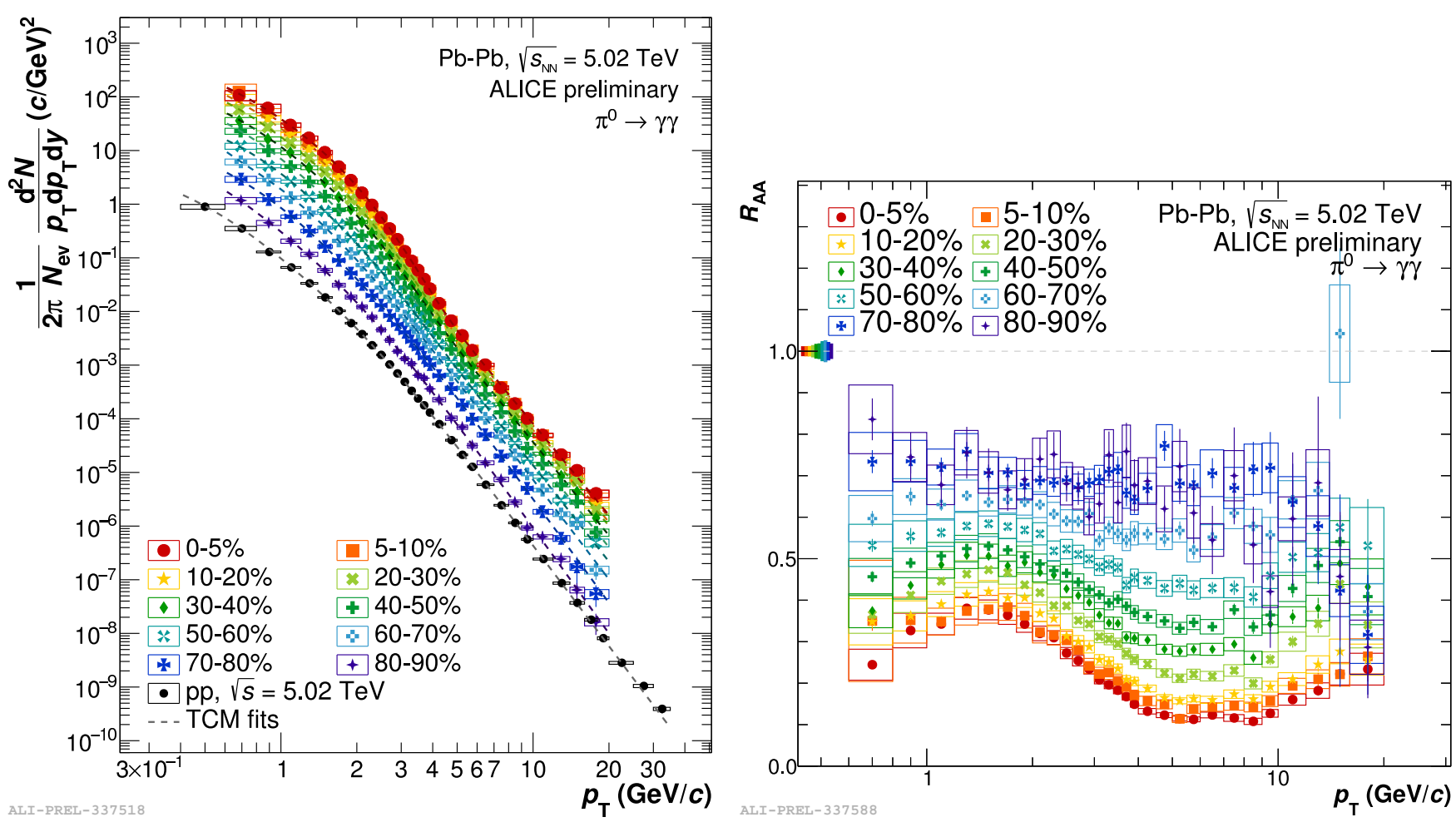

FiguRE 6.32: The invariant yield of $\pi^{0}$ (left) and its nuclear modification factor $R_{\mathrm{AA}}$ (right) in $\mathrm{Pb}-\mathrm{Pb}$ collisions at $\sqrt{s_{N N}}=5.02 \mathrm{TeV}$, for narrow intervals of V0M multiplicities. The respective neutral meson spectrum in pp collisions at $\sqrt{s}=5.02 \mathrm{TeV}$ is used as reference. 


\subsection{Properties of the neutral pion invariant spectra}

As presented in the earlier sections, the neutral pion invariant yield has been measured in $\mathrm{pp}, \mathrm{p}-\mathrm{Pb}$, and $\mathrm{Pb}-\mathrm{Pb}$ collisions, all for various intervals of event multiplicity. One of the interesting questions to pose is how the properties of the spectra change as function of particle multiplicity, i.e. with the mean number of reconstructed charged particles in the central barrel per unit of pseudorapidity $\left(\left\langle\mathrm{d} N_{\text {charged }} / \mathrm{d} \eta\right\rangle\right)$. In this section the shape of the measured neutral pion invariant yields will be quantified in three different ways, and these quantifications are then used to compare different collision systems at similar multiplicities.

First, the mean transverse momentum, $\left\langle p_{\mathrm{T}}\right\rangle$, has been calculated for the distributions shown in Fig. 6.13, Fig. 6.23, and Fig. 6.32, in the range $0.8<p_{\mathrm{T}}<20 \mathrm{GeV} / c$. The approach of using a truncated spectrum has been chosen over the more traditional method where the $\left\langle p_{\mathrm{T}}\right\rangle$ is calculated from a multi-component or Tsallis fit, because these fits introduce additional uncertainties due to the extrapolation to $p_{\mathrm{T}}=0$. The $\left\langle p_{\mathrm{T}}\right\rangle$ of a truncated spectrum does differ compared to its value for the full spectrum, but it is still useful as long as the same $p_{\mathrm{T}}$ range is used for all distributions. The result, as shown in Fig.6.33, indicates that $\left\langle p_{\mathrm{T}}\right\rangle$ is much more dependent on $\left\langle\mathrm{d} N_{\text {charged }} / \mathrm{d} \eta\right\rangle$ in pp collisions than in $\mathrm{p}-\mathrm{Pb}$ and $\mathrm{Pb}-\mathrm{Pb}$ collisions. Also, $\left\langle p_{\mathrm{T}}\right\rangle$ does not significantly increase with $\left\langle\mathrm{d} N_{\text {charged }} / \mathrm{d} \eta\right\rangle$ in $\mathrm{Pb}-\mathrm{Pb}$ collisions, and show an indication of a lower $\left\langle p_{\mathrm{T}}\right\rangle$ for the event multiplicities that are associated with the most central $\mathrm{Pb}-\mathrm{Pb}$ collisions, which is consistent with an increase in thermal pion production.

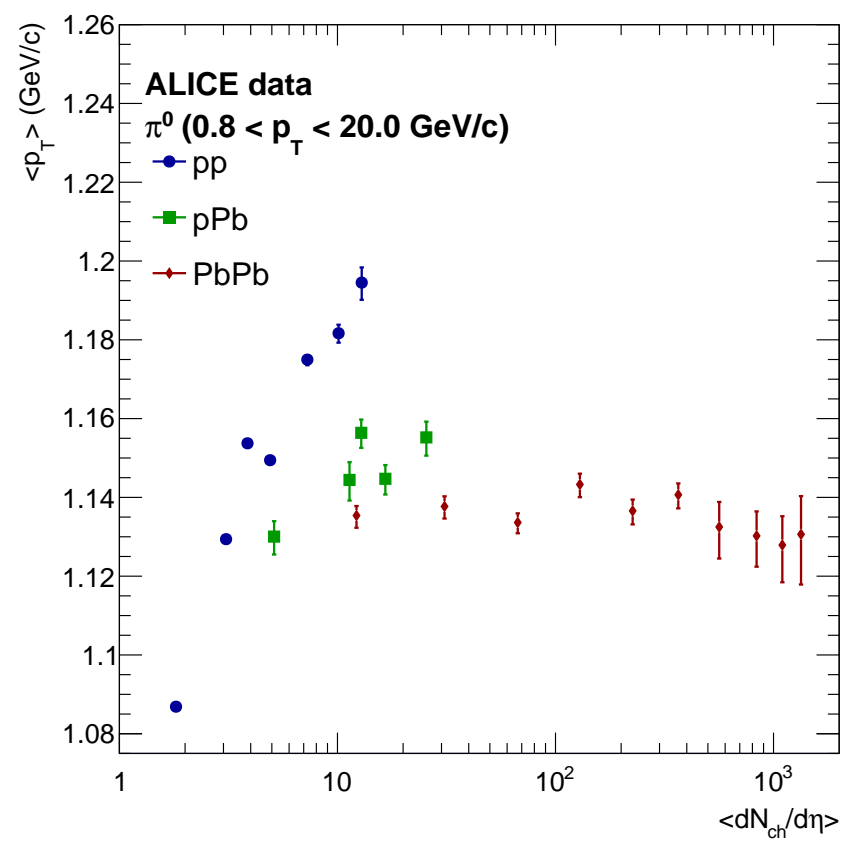

FiguRE 6.33: Mean transverse momentum $\left(\left\langle p_{\mathrm{T}}\right\rangle\right)$ of the neutral pion invariant yield distributions as function of the mean number of reconstructed charged particles in the central barrel per unit of pseudorapidity $\left(\left\langle\mathrm{d} N_{\text {charged }} / \mathrm{d} \eta\right\rangle\right)$. The $\left\langle p_{\mathrm{T}}\right\rangle$ has been calculated in the range $0.8<p_{\mathrm{T}}<20.0 \mathrm{GeV} / c$.

Secondly, the distributions at low $p_{\mathrm{T}}$ can be characterized by the slope parameter $c$, which is obtained by fitting the spectra using

$$
f(x)=a e^{\frac{-x}{c}},
$$


where in this case $x$ is the transverse momentum $p_{\mathrm{T}}$, and $a$ is an overall scaling parameter of the fit, with the fit performed in the range $0.6<p_{\mathrm{T}}<1.4 \mathrm{GeV} / c$. In this definition, the lower the slope parameter $c$, the stronger the slope at low $p_{\mathrm{T}}$, and vice versa. The results are shown in Fig. 6.34 (left), and are again given as function of $\left\langle\mathrm{d} N_{\text {charged }} / \mathrm{d} \eta\right\rangle$. It indicates that there is a gradual increase in $c$ for increasing multiplicities. Furthermore, although the uncertainties are large, it seems that different collision systems with similar final state multiplicities have similar slopes at lower $p_{\mathrm{T}}$.

Thirdly, the powerlaw exponent $n$ can be used to characterize the distributions at higher $p_{\mathrm{T}}$, and is obtained by fitting the neutral pion spectra using

$$
f(x)=a x^{-n}
$$

where again in this case $x$ is the transverse momentum $p_{\mathrm{T}}$, and $a$ is an overall scaling parameter of the fit. The powerlaw exponent $n$ then quantifies how steep the spectrum is over a given $p_{\mathrm{T}}$ range, which is taken to be $10.0<p_{\mathrm{T}}<16.0 \mathrm{GeV} / c$. The result is shown in Fig. 6.34 (right), and indicates that the slope at high $p_{\mathrm{T}}$ does not change significantly, although semi-central to central $\mathrm{Pb}-\mathrm{Pb}$ does seem to have a harder spectrum as the slope parameter is slightly lower. This observation is in line with the $p_{\mathrm{T}}$ dependence of the $R_{\mathrm{AA}}$.

These last two methods to characterize the neutral pion invariant yields show that although $\left\langle\mathrm{d} N_{\text {charged }} / \mathrm{d} \eta\right\rangle$ spans multiple orders of magnitude, that the slopes at both low and high meson $p_{\mathrm{T}}$ are relatively similar and also do not show a large dependence on the collision system. However, the $\left\langle p_{\mathrm{T}}\right\rangle$ indicates that especially high multiplicity pp collisions are fundamentally different from the rest, as the $\mathrm{p}-\mathrm{Pb}$ and $\mathrm{Pb}-\mathrm{Pb}$ collision system show a lower $\left\langle p_{\mathrm{T}}\right\rangle$ at similar multiplicities.
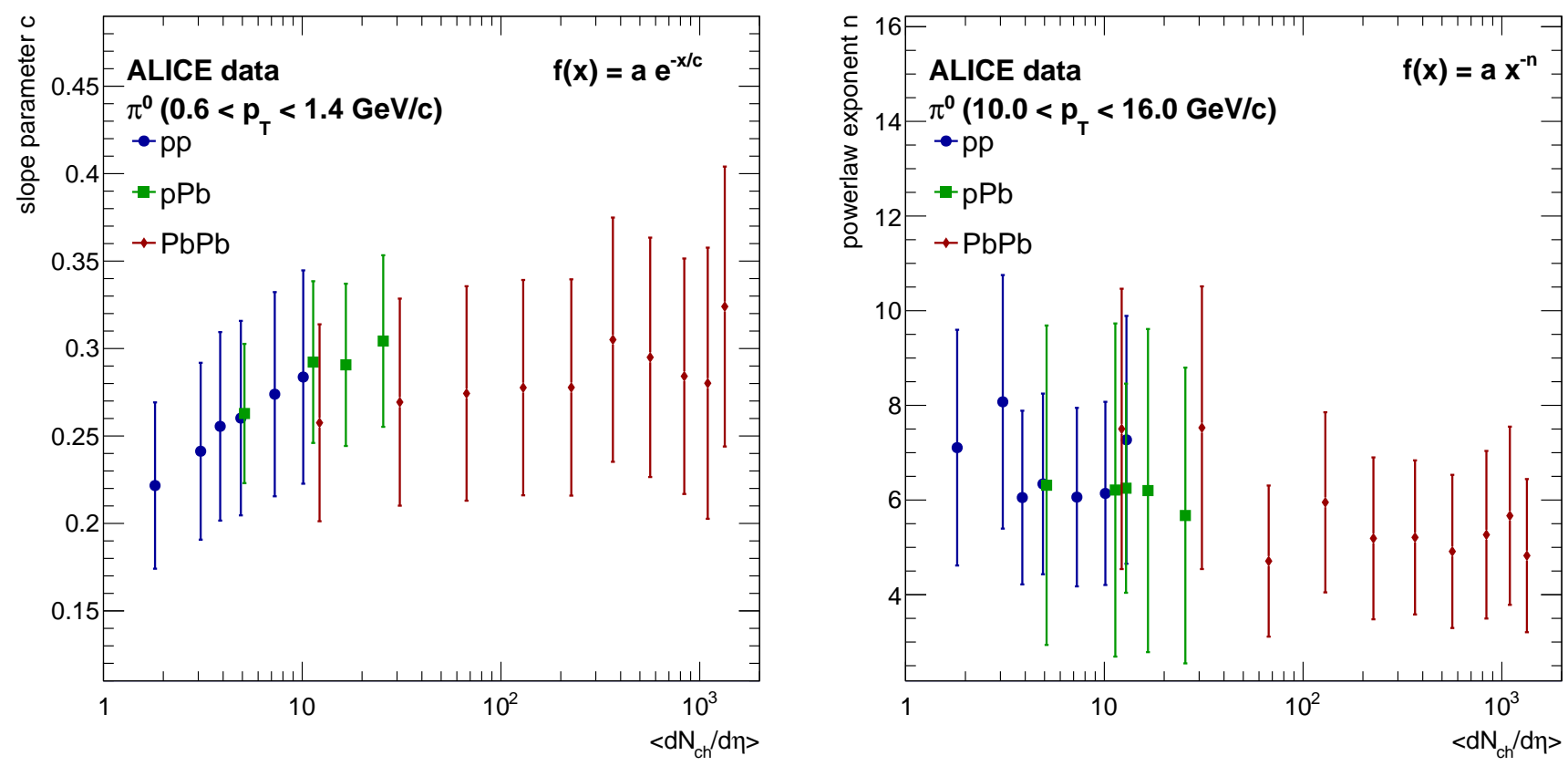

FiguRE 6.34: Slope parameter $c$ and powerlaw exponent $n$ as function of $\left\langle\mathrm{d} N_{\text {charged }} / \mathrm{d} \eta\right\rangle$, which are obtained from fitting the neutral pion invariant yield distributions. 


\subsection{Discussion}

The invariant yield of $\pi^{0}$ and $\eta$ mesons has been measured for all collisions systems at a center-of-mass energy of $5.02 \mathrm{TeV}$. The measurements are performed by calculating the invariant mass of all photons pairs in the event, and counting the excess of candidates on top of a background. The quality of the measurement mostly relies on the ability to reconstruct and select photons in a wide $p_{\mathrm{T}}$ range, as well as a good description of all the relevant distributions in the Monte Carlo simulations. The large increase in particle multiplicities make the analysis more difficult in $\mathrm{Pb}-\mathrm{Pb}$ collisions compared to $\mathrm{pp}$ and $\mathrm{p}-\mathrm{Pb}$ collisions, as is indicated by the mass peaks in Fig. 6.2 and Fig. 6.1, and the differences in meson reconstruction efficiencies. Overall, the ALICE detector is very capable in handling the high multiplicities of central $\mathrm{Pb}-\mathrm{Pb}$ collisions, although the measurements using the EMCal do benefit from increased energy thresholds for the photon candidates. Furthermore, for intermediate $-p_{\mathrm{T}}$ mesons, the available statistics in the used data sets is sufficient to have statistical uncertainties of the order of $2-5 \%$, which is smaller than the systematic uncertainty of the order of $5-10 \%$, depending on the meson, collision system, and further event selections. This makes it possible to measure the production of neutral mesons for various event characteristics with decent precision, and make precise comparisons to model calculations.

For the neutral meson measurements in pp collisions at $\sqrt{s}=5.02 \mathrm{TeV}$, the neutral meson invariant yield has been measured in minimum bias data, different event multiplicity classes, event shapes, and in-jet production. Most notably, the ratio of the multiplicity dependent to the inclusive $\pi^{0}$ and $\eta$ invariant yields show a qualitative different behavior as function of $p_{\mathrm{T}}$ compared to the ratio of the event-shape dependent to the inclusive $\pi^{0}$ and $\eta$ invariant yields, i.e. comparing Fig. 6.14 and Fig. 6.17. This means that selecting on the shape of the event is different from selecting on event multiplicity. Both trends are qualitatively reproduced by the PYTHIA model calculations. Furthermore, the $\eta / \pi^{0}$ ratio shows no large differences for these different event selection criteria, but the neutral mesons that are produced in jets do show a striking difference for this ratio, which again is captured by the model prediction. This also indicates that selecting neutral mesons that are produced in jets is not the same as selecting neutral mesons produced in pencil-like events. In literature, the pencil-like events have been associated to jet-like events, but these results clearly indicate otherwise. Also the $p_{\mathrm{T}}$ dependence shows that selecting on event multiplicity is different than selecting spherical events, while there is a clear correlation between multiplicity and $S_{\mathrm{T}}$. It is therefore interesting to perform a dedicated model study to find out which physics mechanisms drive these event characteristics, and will be discussed in chapter 8 .

The neutral meson measurements in $\mathrm{p}-\mathrm{Pb}$ are probing the cold nuclear matter effects using $R_{\mathrm{pA}}$ for $\mathrm{MB}$ collisions and $Q_{\mathrm{pA}}$ for different V0A multiplicity classes, which are shown in Fig. 6.22 and Fig. 6.24. The main conclusions are that at high meson $p_{\mathrm{T}}$, the nuclear modification factors are consistent with unity, and thus agrees with no modification. Also, there seems to be a multiplicity dependent relative enhancement at intermediate meson $p_{\mathrm{T}}$, consistent with a Cronin peak, and a large suppression at lower meson $p_{\mathrm{T}}$, consistent with nuclear shadowing, both possibly in combination with other non-perturbative effects. Interestingly, for the most central $\mathrm{p}-\mathrm{Pb}$ collisions, the Cronin peak is significantly above unity, indicating an enhancement 
of mesons with these momenta compared to the spectrum in pp collisions. However, a possible multiplicity bias of the $Q_{p A}$ could remove this Cronin enhancement, which would be the case when the high momentum mesons turn out to be significantly above unity as well. Furthermore, there is no indication of differences for the $\pi^{0}$ and $\eta$, as the minimum bias and multiplicity dependent $\eta / \pi^{0}$ ratio are consistent with one another within experimental uncertainties. Here, it would be interesting to see whether the $\pi^{0}$ and $\eta$ in-jet production is also modified like in pp, or perhaps even shows different behavior, which would indicate a dependence on the nuclear PDFs.

In $\mathrm{Pb}-\mathrm{Pb}$ collisions, the invariant yield of neutral mesons probe the hot nuclear matter effects, as we expect for a QGP to be formed. Here, the nuclear modification factor $R_{A A}$, as shown in Fig. 6.31, clearly shows an increasing suppression for increasing event multiplicities. At lower meson $p_{\mathrm{T}}$ there is a relative increase in $R_{\mathrm{AA}}$, which is attributed to the radial flow of the expanding medium. Unfortunately, the uncertainties of the $\eta$ meson are too large to see significant differences between the $R_{\mathrm{AA}}$ of the $\pi^{0}$ and $\eta$. However, the $\eta / \pi^{0}$ for the most central collisions show, for the first time, a significant enhancement for intermediate meson $p_{\mathrm{T}}$ with respect to the ratio in $\mathrm{pp}, \mathrm{p}-\mathrm{Pb}$, and non-central $\mathrm{Pb}-\mathrm{Pb}$ collisions. This is most likely the effect from radial flow being stronger in central $\mathrm{Pb}-\mathrm{Pb}$ collisions and indicates a different impact depending on the mass of the particle. For this collision system it would be interesting to see how the in-jet production is affected by the medium, and how well the latest model calculations agree with the experimental results. 


\section{Chapter 7}

\section{Event-mixing studies}

In collaboration with Lizette Lamers.

\subsection{Introduction}

As explained before in this thesis, pair correlation analysis is a standard analysis technique in particle and nuclear physics. One of the main examples is the study of two-body decays via the invariant mass spectrum of potential decay daughters, where one can extract the yield of the mother particles from a correlated peak in the pair distribution atop a broader background from random pairs, the so-called combinatorial background. Another example is that of femtoscopy, the study of (most often identical) pair correlations in momentum space, where quantum statistics and/or interactions cause a correlation, often at very small relative momentum. In all of these cases one has to find a means to describe the background. Sometimes, e.g. in invariant mass analysis, the correlation produces a prominent, distinct structure on a very smooth background, which makes the description of the latter possible with simple analytical functions. There are, however, many cases where high precision measurements require knowledge about the shape of the background. This is important, when the phase space acceptance of the detector leads to a non-monotonous shape of the background near the signal. A widely used method to estimate the shape of the combinatorial background is that of event mixing, i.e. combining particle pairs from different events, which by construction will not be correlated. Below, the example of photon-pair distributions is used, but our analysis should be relevant also for the analysis of other pair-correlations.

The yield of light neutral mesons, such as the $\pi^{0}$ and $\eta$ meson, is calculated by integrating the signal peak in the diphoton invariant mass distribution on top of a combinatorial background. The invariant mass distribution can either be fitted simultaneously with a Gaussian describing the signal peak and a polynomial describing the background, or the background can first be subtracted using the event-mixing method. Also, for direct photon HBT measurements the correlator $C\left(Q_{\text {inv }}\right)=A\left(Q_{\text {inv }}\right) / B\left(Q_{\text {inv }}\right)$ divides the same-event pair distribution by the event-mixing distribution. For such measurements it is important that the combinatorial 
background which is present in same-event photon pairs is properly described by the distributions obtained with the event-mixing method.

However, as demonstrated through an example shown in Fig. 7.1, the combinatorial background obtained with the event-mixing method fails to describe the background of the same-event pair distribution. In this case the residual background is subtracted via a linear fit, which only works well if the background underneath the peak is well behaved. In fact, a qualitatively similar correlation effect has been observed in femtoscopic analysis (see e.g. [163]).

In this chapter the photon pair distributions $\left(0<M_{\text {inv }}<5 \mathrm{GeV} / c^{2}\right)$ are investigated, for three different pair $p_{\mathrm{T}}$ intervals below $10 \mathrm{GeV} / c$ in simulated proton-proton collisions at $\sqrt{s}=13 \mathrm{TeV}$, using the PYTHIA8.2 [164] event generator, and discuss the performance of describing the background using the event-mixing method also in more critical cases.

The chapter is divided into the following sections: In Sec. 7.2 the event-mixing method used to obtain the results is defined. In Sec. 7.3 the diphoton invariant mass distributions and the estimated background using the event-mixing method is presented. In Sec. 7.4 the performance of event-mixing when applying additional detector constraints is discussed. In Sec. 7.5 the origin of the correlated background is investigated. In Sec 7.6. it is attempted to improve the conventional way of event-mixing, i.e. to also capture the correlated part of the combinatorial background. Sec 7.7. takes a closer look at higher invariant masses and Sec 7.8. concludes with a with a summary.

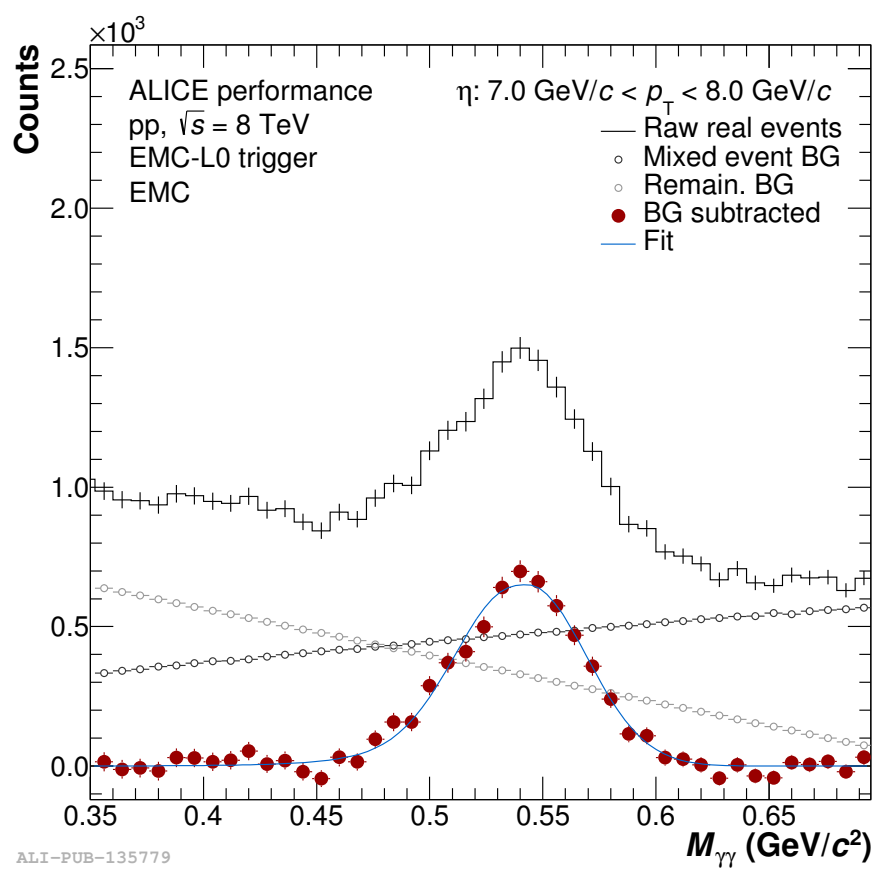

FiguRE 7.1: The diphoton $M_{\text {inv }}$ distribution around the $\eta$ meson mass for $7.0<p_{T \text {, pair }}<8.0 \mathrm{GeV} / \mathrm{c}$, as measured with the ALICE EMCal detector in pp collisions at $\sqrt{s}=8 \mathrm{TeV}$. It shows that the combinatorial background is not described by the distribution obtained from event-mixing. 


\subsection{Event-mixing method}

Using the event-mixing method, the combinatorial background is estimated by combining photons exclusively from different events. These photon pairs never share a common ancestor and thus make sure that there are no resonances. The method can also describe non-trivial shapes of the combinatorial background in the signal regions, which for a polynomial fit would be impossible. These non-trivial background shapes are usually created by detector constraints and non-homogeneous acceptance, such as calorimeter module sizes or dead calorimeter cells/modules. As can be seen in [165-168], this remains to be a challenge for especially the $\eta$ meson.

For the results presented in this chapter all current event photons are paired with all photons from all events in the mixing-pool. The mixing-pool acts as a First-In-First-Out (FIFO) buffer storing the last 200 events. After the current event is processed the event is added to the mixing-pool if it has at least two photons. Also, it is customary to define multiple mixing-pools, each having distinct event characteristics, such as charged/neutral particle multiplicity and z-vertex location. The photons from the current event are then paired with the events from the pool which shares the same event characteristics. The z-vertex location is only important to take into account for experimental data, since PYTHIA has a fixed z-vertex. In addition, it is found that mixing events with different multiplicities is not changing the distribution, as long as the buffer is large and the single particle energy spectra are the same. The latter would not be the case for heavy-ion collisions, where the nuclear matter effects have shown to radically change the shapes for different collision centralities. For this study it suffices to use a single mixing-pool of 200 events.

\subsection{Diphoton invariant mass distributions}

The presented results are based on PYTHIA-generated [164] proton-proton collisions at $\sqrt{s}=13 \mathrm{TeV}$, using two data-sets; SoftQCD, and HardQCD with a minimum $\hat{p}_{\mathrm{T}}$ of $20 \mathrm{GeV} / c$. This enables us to study the diphoton invariant mass distributions in minimum bias $(\mathrm{MB})$ and high- $p_{\mathrm{T}}$ jet type events. In the dataset with HardQCD, the minimum $\hat{p}_{\mathrm{T}}$ of $20 \mathrm{GeV} / c$ ensures that we have high- $p_{\mathrm{T}}$ partons, enhancing the correlated part of the pair distributions. The diphoton invariant mass is calculated as

$$
\begin{aligned}
M_{\mathrm{inv}} & =\sqrt{2 E_{\gamma, 1} E_{\gamma, 2}(1-\cos \psi)} \\
& =\left(E_{\gamma, 1}+E_{\gamma, 2}\right) \sqrt{\frac{1-\alpha^{2}}{2}(1-\cos \psi)}
\end{aligned}
$$

where $E_{\gamma}$ is the energy of the photon, $\psi$ is the opening angle between the two photons, and $\alpha$ is the energy asymmetry with $\alpha=\left(E_{\gamma, 1}-E_{\gamma, 2}\right) /\left(E_{\gamma, 1}+E_{\gamma, 2}\right)$. For same-event pair combinations, the $\pi^{0}$ and $\eta$ will create 
distinct peaks at $M_{\mathrm{inv}}=134.98 \mathrm{GeV} / c^{2}$ and $M_{\mathrm{inv}}=547.86 \mathrm{GeV} / c^{2}$, respectively. Due to physics effects such as jet production, the energy and opening angle distributions from the two combined photons are not necessarily qualitatively the same for same-event and event-mixing pair distributions. This will then be apparent from the comparison of the $M_{\mathrm{inv}}$ distributions.
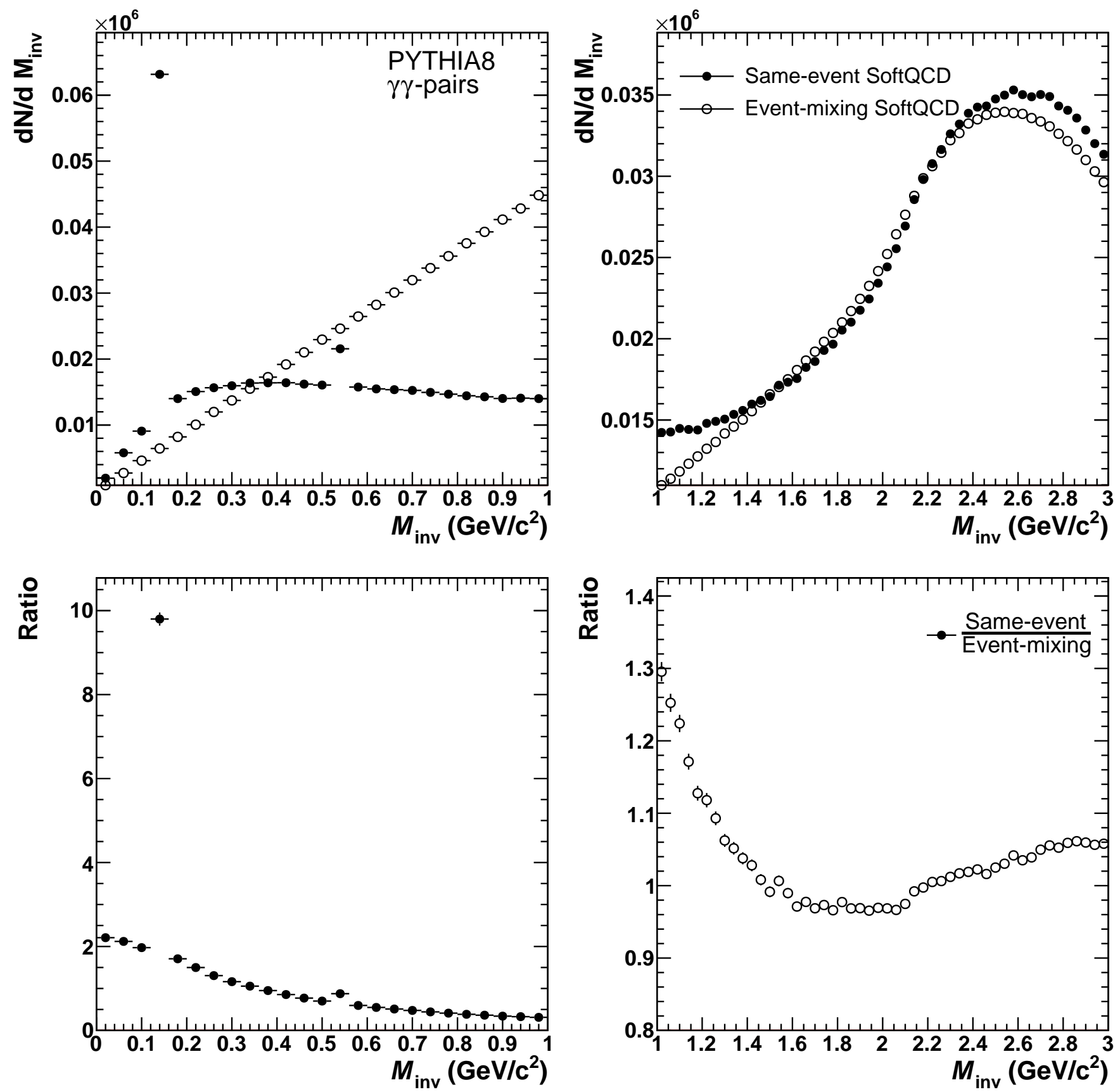

Figure 7.2: The diphoton invariant mass distribution obtained from PYTHIA (SoftQCD), for $0<M_{\mathrm{inv}}<$ 1.0 (top left) and $1.0<M_{\mathrm{inv}}<3 \mathrm{GeV} / c^{2}$ (top right) for same event pairs (closed) and event-mixing (open), for a diphoton pair momentum of $1<p_{\mathrm{T}}<2 \mathrm{GeV} / c$. The bottom panels show a ratio of the same-event pair to the event-mixing distributions.

Figure 7.2 shows the diphoton invariant mass distribution obtained from PYTHIA (SoftQCD) for $0<$ 
$M_{\text {inv }}<1.0 \mathrm{GeV} / c^{2}$ and $1.0<M_{\text {inv }}<3.0 \mathrm{GeV} / c^{2}$, for same-event pairs (filled markers) and for eventmixing (open markers). The event-mixing distribution is scaled to match the same-event pair distributions, at $0.35 \mathrm{GeV} / c^{2}$ for the upper left panel and at $1.5 \mathrm{GeV} / c^{2}$ for the upper right panel. The figure indicates that event mixing is unable to describe the shape of the $M_{\text {inv }}$ distribution around the $\pi^{0}$ and $\eta$ mass, and more generally is unable to capture the background of photon pairs for $0<M_{\text {inv }}<3.0 \mathrm{GeV} / c^{2}$. For $M_{\mathrm{inv}}<0.2 \mathrm{GeV} / c^{2}$, the same-event pairs show a much steeper distribution compared to the event-mixing distribution. Furthermore, the shape of the same-event pairs flattens for $M_{\text {inv }}>0.3 \mathrm{GeV} / c^{2}$, while the event-mixing distribution continues to rise. The simulated PYTHIA events thus reproduce the observations made from Fig. 7.1.
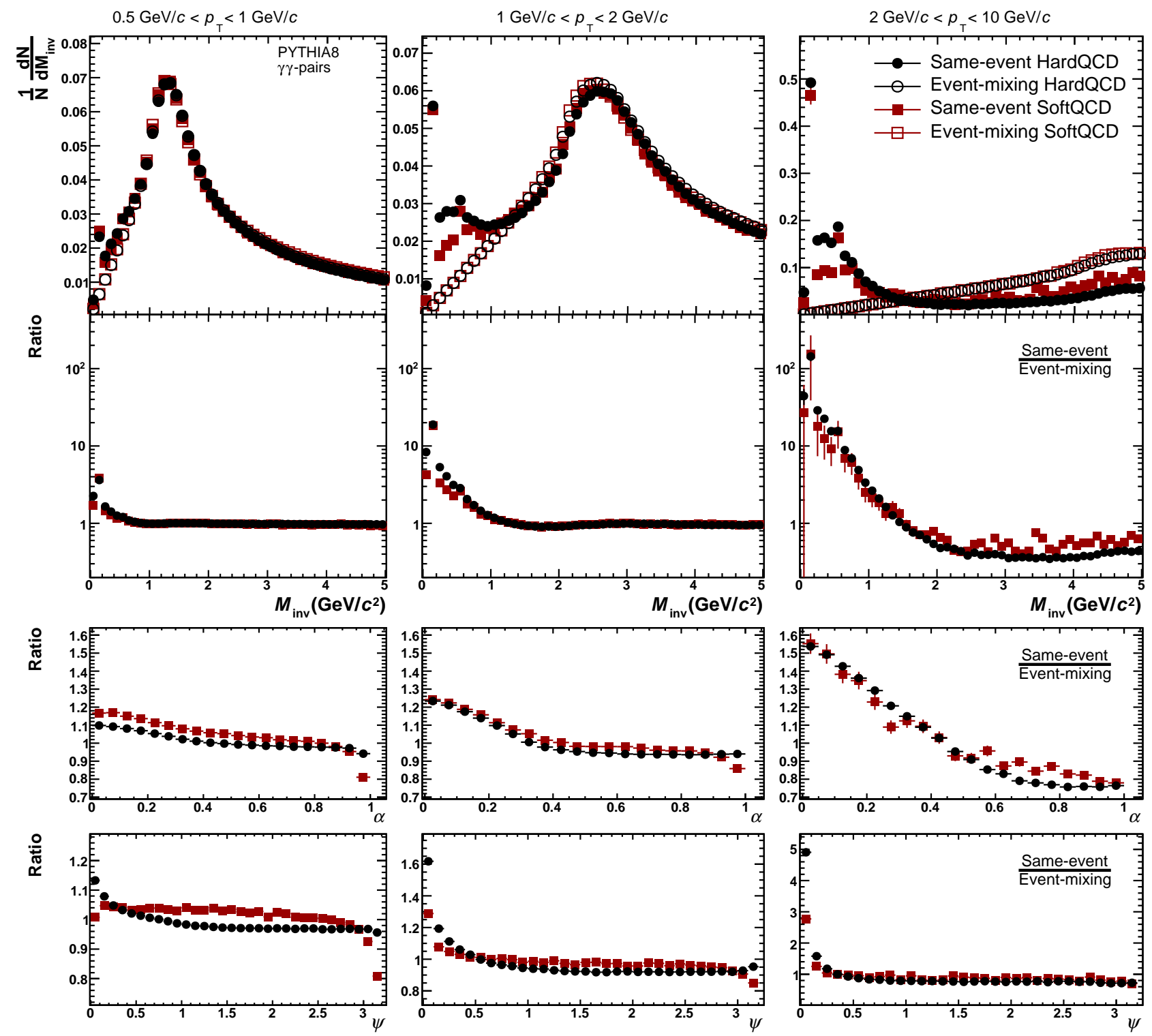

Figure 7.3: The diphoton $M_{\mathrm{inv}}$ distributions for PYTHIA HardQCD (black) and SoftQCD (red), for same-event pair distributions (filled) and event-mixing (open) symbols. The lower panels show the ratios of same-event pairs to event-mixing pairs, for the $M_{\text {inv }}$, energy asymmetry $\alpha=\left(E_{\gamma, 1}-E_{\gamma, 2}\right) /\left(E_{\gamma, 1}+E_{\gamma, 2}\right)$, and opening angle $\psi$ distributions. 
Next, the PYTHIA HardQCD is compared to PYTHIA SoftQCD. In SoftQCD the particle production is not dominated by high- $p_{\mathrm{T}}$ parton fragmentation as with HardQCD, but rather by the soft component or underlying event. The results are shown in Fig. 7.3. There is a dominant correlation effect for $M_{\mathrm{inv}}<$ $1.0 \mathrm{GeV} / c^{2}$, which shows that event-mixing does not describe the same-event pair distributions. The effect also increases with pair- $p_{\mathrm{T}}$, indicating that this is related to jet fragmentation. For $M_{\mathrm{inv}}>2.0 \mathrm{GeV} / c^{2}$, the shapes of the $M_{\mathrm{inv}}$ distributions from same-event and event-mixing photon pairs for both SoftQCD and HardQCD events match within about $\pm 2 \%, \pm 5 \%$, and $\pm 10 \%$ for pair- $p_{\mathrm{T}}$ intervals of $0.5<p_{\mathrm{T}}<1$ $\mathrm{GeV} / c, 1<p_{\mathrm{T}}<2 \mathrm{GeV} / c$, and $2<p_{\mathrm{T}}<10 \mathrm{GeV} / c$, respectively, and rapidly start to diverge for lower $M_{\text {inv }}$ (also see Appendix Fig. 7.7). As can be seen in the ratio of the $M_{\text {inv }}$ distributions, the same-event and event-mixing distributions of SoftQCD events are more in agreement compared to distributions of the HardQCD events. This is probably due to the decrease in correlated background in the same-event pair distributions. Furthermore, the event-mixing pair distribution for HardQCD and SoftQCD production is within uncertainties the same, showing that they both reproduce the combinatorial background to the same extent, and are both unable to describe the same-event pair distributions especially at lower $M_{\mathrm{inv}}$. The explicit dependence of the energy asymmetry $\alpha=\left(E_{\gamma, 1}-E_{\gamma, 2}\right) /\left(E_{\gamma, 1}+E_{\gamma, 2}\right)$ and opening angle $\psi$ on $M_{\text {inv }}$ show a deviation that increases for lower values of $\alpha$ and $\psi$ in all $p_{\mathrm{T}}$ bins.

\subsection{Pseudorapidity and azimuthal angle restrictions}

In experiments we have to deal with the actual size of the detector, and this constrains the pseudorapidity $\eta$ and/or the azimuthal angle $\varphi$ regions from which we can obtain the photons. This limits the opening angle of the diphoton pair and changes the $M_{\mathrm{inv}}$ distributions in a non-trivial way. And since the average opening angle of a $\pi^{0}$ decay is $p_{\mathrm{T}}$ dependent, detector constraints will affect low and high diphoton $p_{\mathrm{T}}$ distributions differently.

In Fig. 7.4, using the HardQCD dataset, the following detector constraints are introduced; $|\eta|<1, \varphi<\pi / 2$, and the combination of the two. The results for restricting the photons in $\eta$ show a large improvement in the $\alpha$ distributions. Jet correlations show a constraint in $\Delta \eta$, which translates into a constraint in $\alpha$ for correlated pairs, when $p_{\mathrm{T}}$ is fixed. The true pairs are thus found more closely together in $\alpha$ compared to pairs from event mixing, which can be more widespread in $\eta$. In this way, an apparent correlation in the energy difference shows up, even though the physical mechanism may introduce only a correlation in angle. The difference disappears, if an artificial constraint in $\eta$ is introduced for all pairs, and in this case the correlation as a function of $\alpha$ also disappears. The restriction in $\varphi$ shows similar results compared to the unrestricted case, because a difference in $\varphi$ does not change the relation between $p_{\mathrm{T}}$ and $E$, opposite to a difference in $\eta$. Detector constraints do change the distributions, but the combinatorial background at low $M_{\mathrm{inv}}$ is still poorly described by the event-mixing method. 

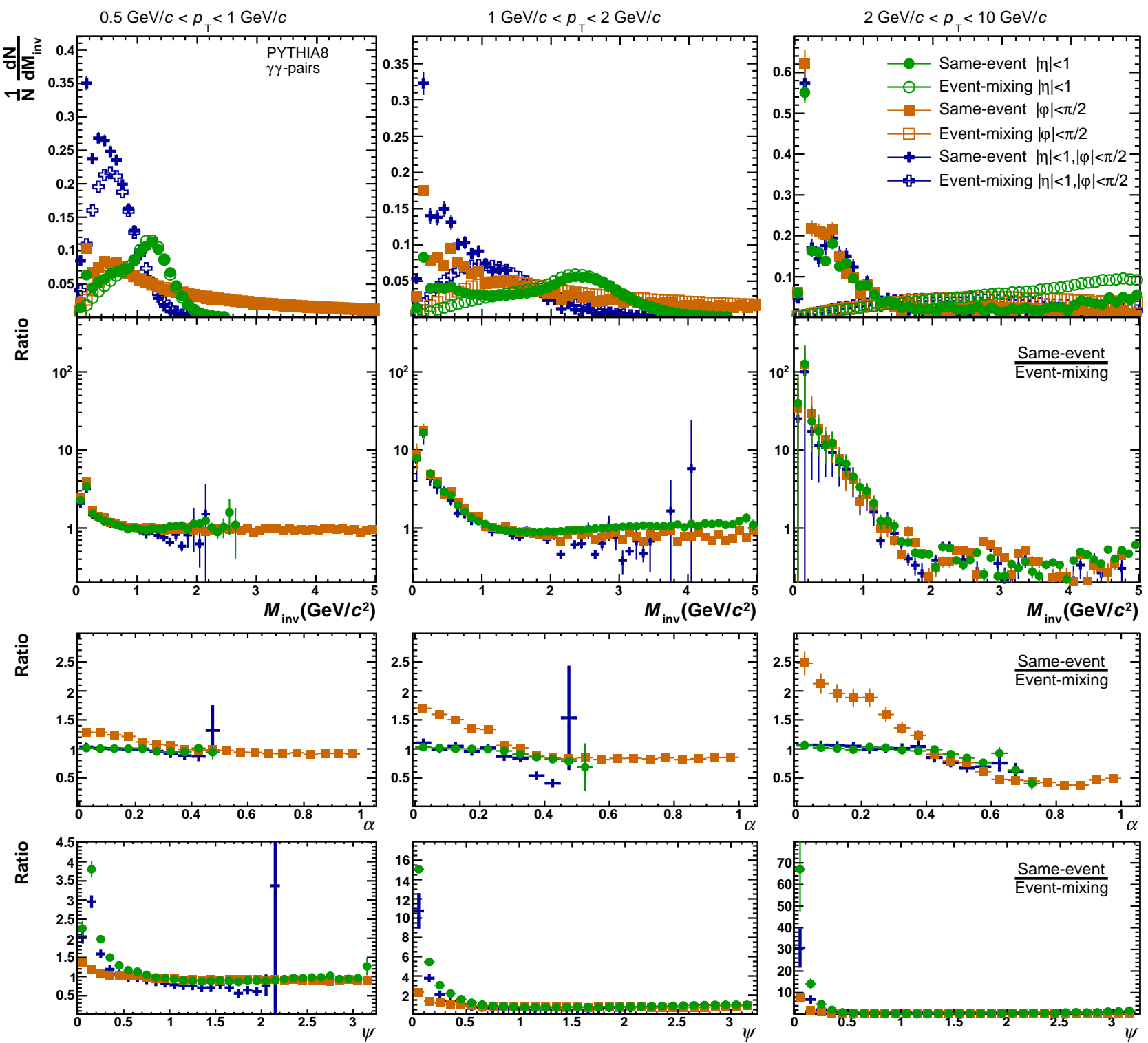

FIGURE 7.4: Diphoton $M_{\text {inv }}$ distributions for same-event and event-mixing pairs, for the detector constraints; $|\eta|<1, \varphi<\pi / 2$, and $|\eta|<1 \& \varphi<\pi / 2$. The lower panels show the ratios of same-event pairs to the eventmixing pair distributions, for the $M_{\text {inv }}$, energy asymmetry $\alpha=\left(E_{\gamma, 1}-E_{\gamma, 2}\right) /\left(E_{\gamma, 1}+E_{\gamma, 2}\right)$, and opening angle $\psi$ distributions.

\subsection{Diphoton pairs from same and different ancestors}

The initial hard scatterings simulated by PYTHIA produce partons that fragment and give rise to final state hadrons. These partons are the most dominant source for particle production and all final state particles sharing the parton as common ancestor will thus have correlations in $\Delta \eta$ and $\Delta \varphi$. To investigate whether this affects the diphoton mass for same-event pairs and event-mixing pairs, using the HardQCD dataset, the following three cases are studied; 1 . when the photon pair shares a common parton ancestor, 2 . when both photons do not share a common parton ancestor, and 3. when the photon pair does not originate from one hadronic decay, such as the $\pi^{0}$ or $\eta$. In this last case the photons can still share a common parton 
ancestor. For all the three cases the event-mixing procedure is the same, namely all photons come from different events and thus ancestors.

The results for the three different cases are shown in Fig. 7.5. First, photon pairs coming from the same parton give qualitatively the same results compared to the earlier distributions. When compared to case 3 , it is seen that except for the $\pi^{0}$ and $\eta$ peak being removed, there is no difference. For case 2, where both photons do not share a common parton ancestor, it is shown that the event-mixing is now describing the whole invariant mass distribution much better also at lower $M_{\mathrm{inv}}$ (see also Appendix Fig. 7.8). Both the observations at lower and higher $M_{\mathrm{inv}}$ are now shown to be related to the jet fragmentation, where the effects at lower and higher $M_{\text {inv }}$ are then related to the near- and away-side jet structures, respectively. A qualitatively similar effect at low $M_{\text {inv }}$ was already seen in studies at RHIC [169], but the jet correlations observed were significantly weaker than what is found at LHC energy. The ratios of the energy asymmetry $\alpha$ and opening angle $\psi$ are also individually much closer to unity. This indicates that the inability of the event-mixing method to properly describe the combinatorial background is due to the fact that the method does not conserve the particle to particle correlations of the parton shower. It should be noted, that femtoscopy measurements also show a residual pair correlation at small momentum difference, which is in that case attributed to mini-jets [163].

\subsection{Modified diphoton event-mixing}

The previous section showed that pairing photons from the same parton, even when the photons do not come from the same hadronic decay, creates a broad correlated background in the region of the $\pi^{0}$ and $\eta$ meson mass. Pairing photons that originate from different partons do destroy this correlation, but would make the neutral meson measurement practically impossible. In order to attempt to improve the eventmixing, such that it describes the correlated background in the low $M_{\text {inv }}$ region, one can choose to only pair photons if the parton ancestor from both photons are close in $\eta$ and $\varphi$. This can partly preserve the $\alpha$ and $\psi$ distributions of the same-event pairs with respect to the event-mixing pairs. Here, it is chosen to only pair the photons if the parton ancestor is within $|\eta|<0.25$ and $|\varphi|<0.25$. The result in Fig. 7.6 shows that the $M_{\text {inv }}$ distribution from the modified event-mixing has a qualitatively different shape and describes the same-event pair distributions better. However, for the first two $p_{\mathrm{T}}$ intervals, the modified event-mixing overestimates the background in real events, while for $2.0<p_{\mathrm{T}}<10 \mathrm{GeV} / c$, it underestimates the background. The behaviour at low $p_{\mathrm{T}}$ could be due to the fact that not all photons in that case come from a single jet, so the restriction on the particle from the direction of the parton in the event mixing is stronger than in real data. On the other hand, at higher $p_{\mathrm{T}}$ the correlation may be stronger in the same-event pair distributions, because the uncertainty of the parton direction in $\eta$ and $\phi$ is not negligible compared to the width of a jet, and thus leads to a dilution of the correlation in mixed events. 

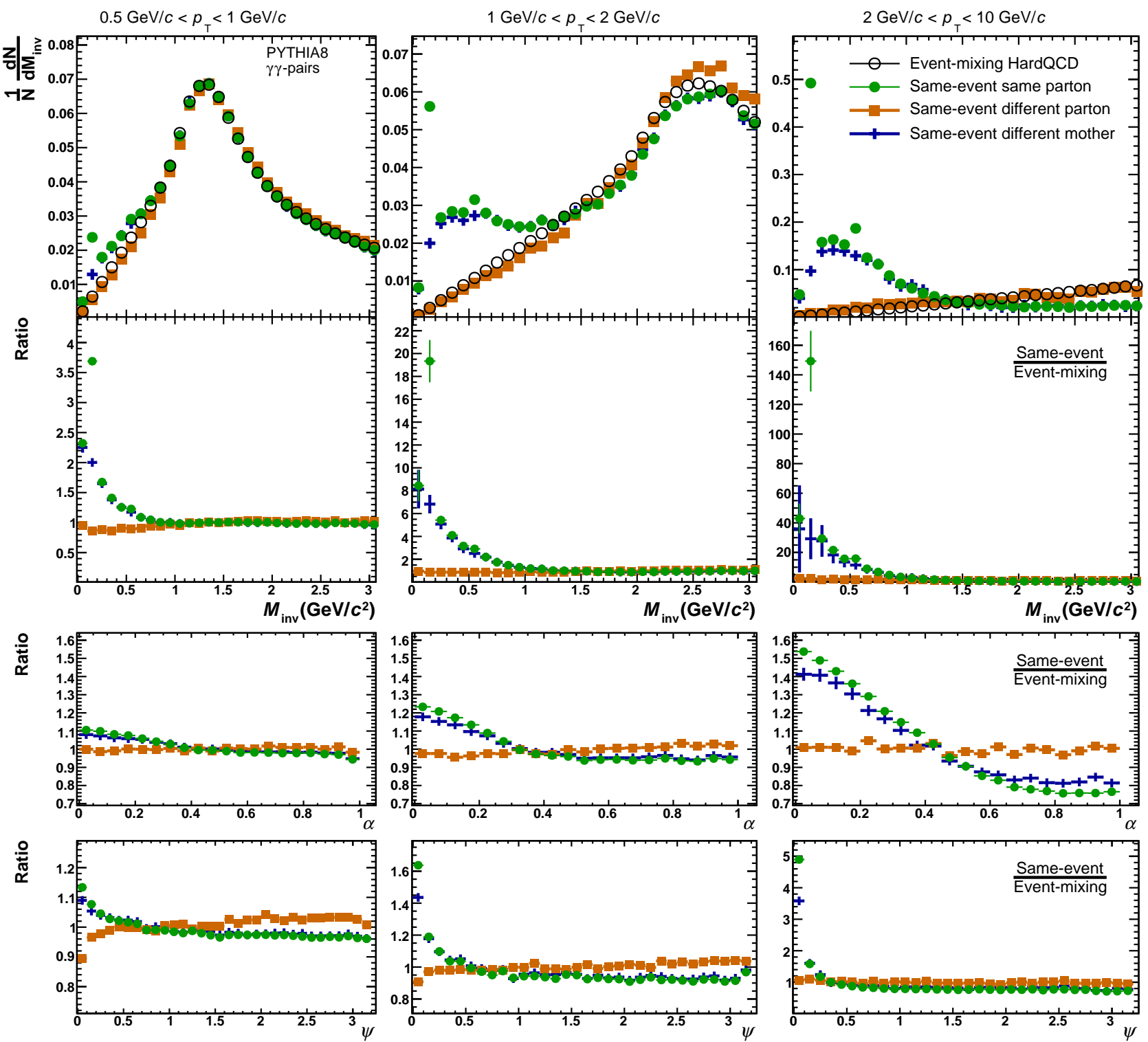

Figure 7.5: The diphoton $M_{\text {inv }}$ mass and the ratios for same-event pairs and event mixing for the following three cases; 1 . when the photon pair for same-event-mixing shares a common parton ancestor, 2 . when both photons do not share a common parton ancestor, and 3. when the photon pair do not share the same motheror grandmother particle whenever it considers a hadronic decay.

\subsection{Closer look at higher invariant mass}

This chapter discusses mainly the behaviour of the relatively strong correlation seen in the continuous background of the two-photon invariant mass spectra at low masses $\left(M_{\mathrm{inv}}<2 \mathrm{GeV} / c^{2}\right)$, which leads to a discrepancy between the background in real data and the event mixing. It was noted there that the background description is reasonably good for larger masses. However, even at larger masses discrepancies are observed, albeit on a much smaller level, which is briefly discussed here, even when they are not relevant for the main analysis. In Fig. 7.7 and Fig. 7.8 the ratio panels are shown such that the discrepancies at higher masses are visible. Figure 7.7 indicates that the shapes of the $M_{\mathrm{inv}}$ distributions from same-event and 

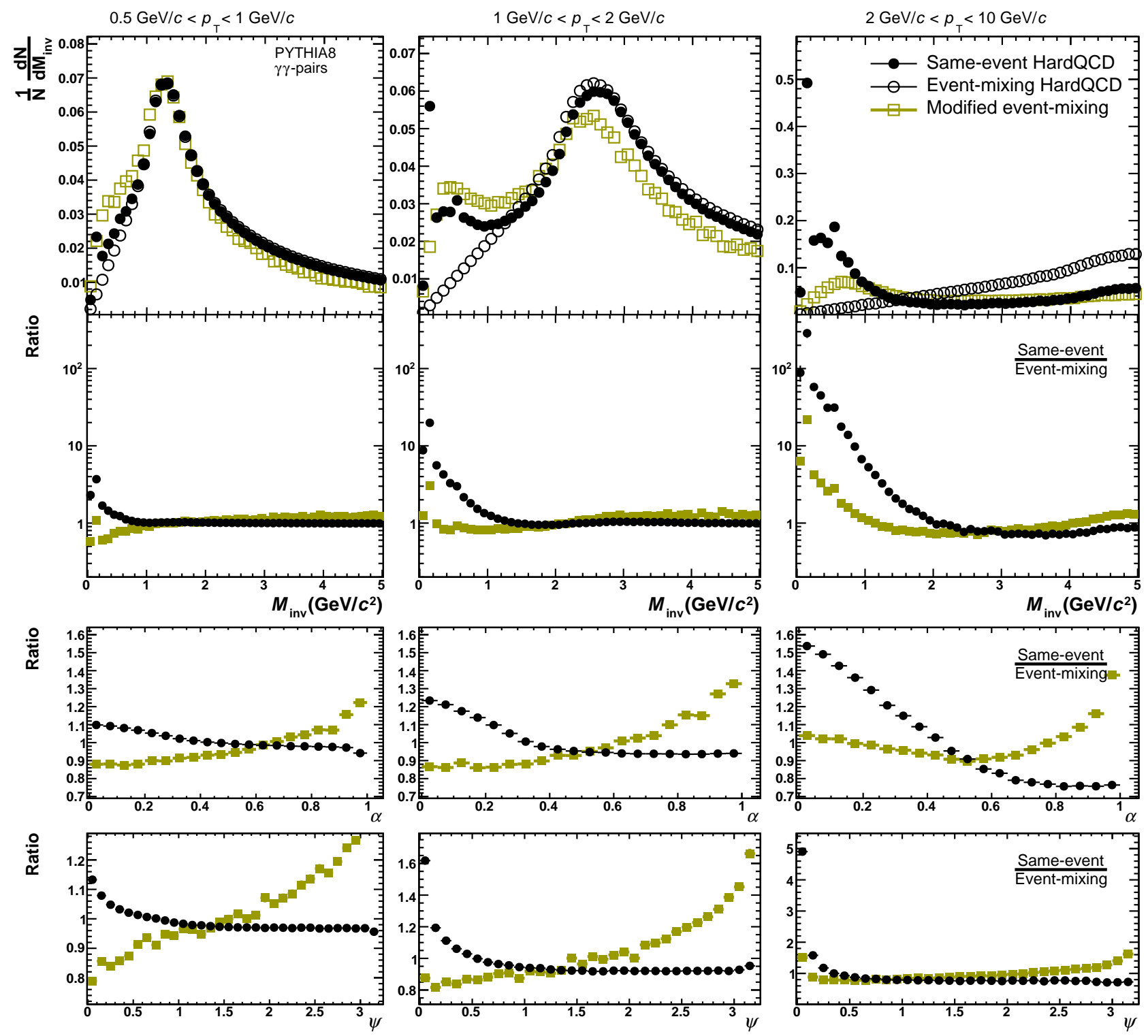

Figure 7.6: The diphoton $M_{\mathrm{inv}}$ distributions for same-event pair distributions (filled circle), event-mixing (open circle), and modified event-mixing (open square). For the modified event-mixing, photon are only paired when the parton ancestor is within $|\eta|<0.25$ and $|\varphi|<0.25$. The lower panels show the ratios of same-event pairs to event-mixing pairs, for the $M_{\text {inv }}$, energy asymmetry $\alpha=\left(E_{\gamma, 1}-E_{\gamma, 2}\right) /\left(E_{\gamma, 1}+E_{\gamma, 2}\right)$, and opening angle $\psi$ distributions.

event-mixing photon pairs for both SoftQCD and HardQCD events match within $\pm 2 \%, \pm 5 \%$, and $\pm 10 \%$ for pair- $p_{\mathrm{T}}$ intervals of $0.5<p_{\mathrm{T}}<1 \mathrm{GeV} / c, 1<p_{\mathrm{T}}<2 \mathrm{GeV} / c$, and $2<p_{\mathrm{T}}<10 \mathrm{GeV} / c$, respectively. However, within these limits one observes interesting characteristic structures: In particular for the two lower $p_{\mathrm{T}}$ bins there is a minimum in the ratio at intermediate mass, and a maximum at $M_{\mathrm{inv}} \approx 1.5$ and $\approx 3$ $\mathrm{GeV} / c$, respectively. Most likely, this structure is due to away-side jet-like correlations, as the mass values are close to twice the average energy of the two photons used. For the highest $p_{\mathrm{T}}$ bin, a similar maximum would be outside of the mass range and thus not be visible. Figure 7.8 shows that for higher masses all the different cases are described similarly well by the event-mixing method. The main feature here, as described in the main text, is the fact that the distribution obtained by pairing photons that originate from a different 
parton do not have the large enhancement found at lower $M_{\mathrm{inv}}$. In the ratio plot it shows that the sameevent distribution decreases significantly for lower $M_{\mathrm{inv}}$ for the first two $p_{\mathrm{T}}$-intervals. The combination of the two cases, the pairs from the same parton, which show the strong enhancement at low mass, and the pairs from different partons, which show a depletion, most likely leads to the characteristic structure in Fig. 7.7. A further study of this additional correlation phenomenon is beyond the scope of the chapter.
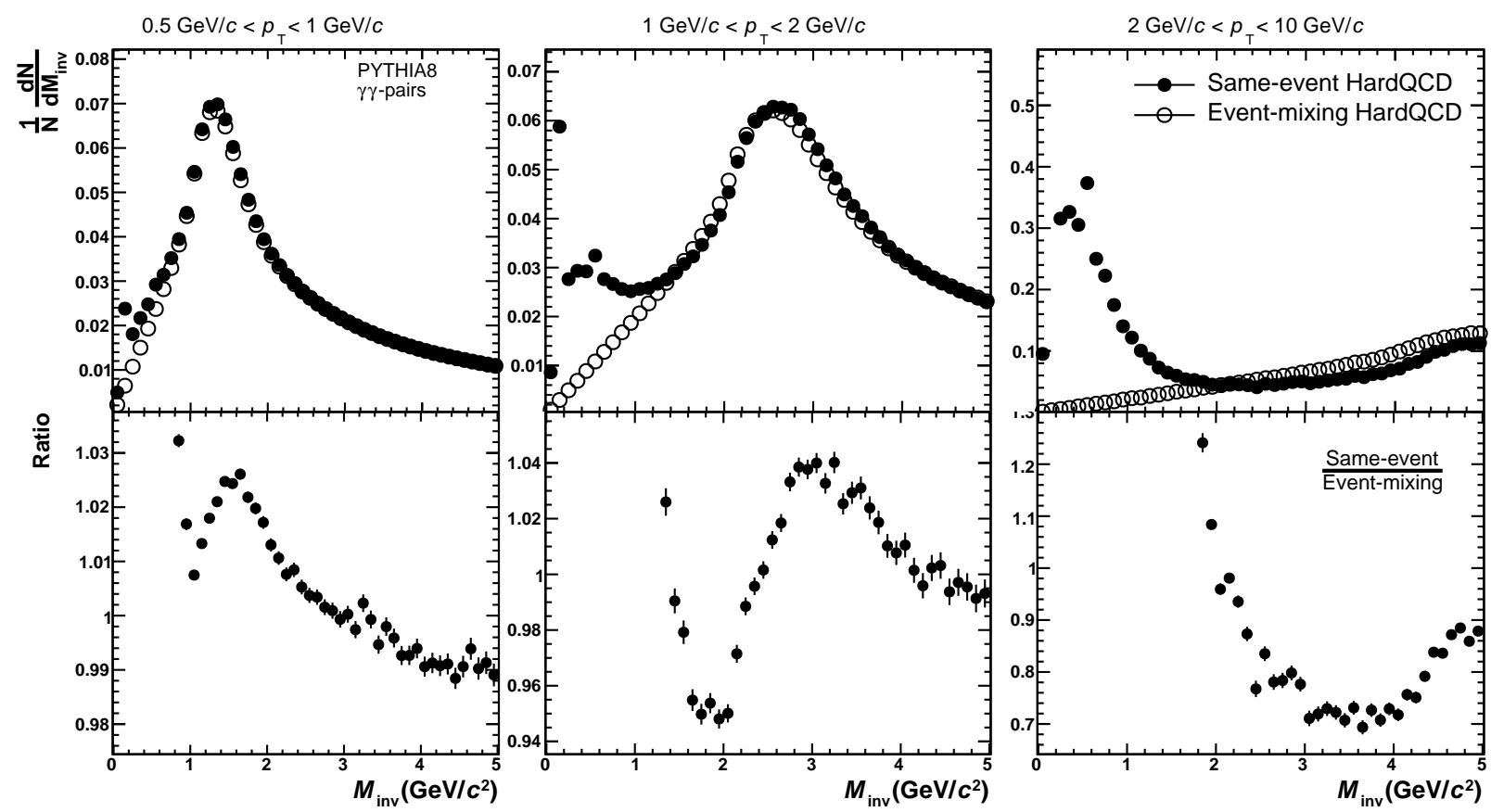

FIGURE 7.7: The diphoton $M_{\text {inv }}$ distributions for PYTHIA8 HardQCD, for same-event pair distributions (filled) and event-mixing (open) symbols. The lower panels show the ratios of same-event pairs to eventmixing pairs over a wide mass range. The distributions from the PYTHIA8 SoftQCD dataset has been omitted as it doesn't have the appropriate statistical precision.

\subsection{Conclusions}

In this chapter the diphoton invariant mass distributions are investigated for simulated PYTHIA HardQCD and SoftQCD events. It is shown that for both lower and higher $M_{\text {inv }}$, the same-event pair distributions are not fully described by the distributions obtained by the event-mixing technique. For lower invariant masses typically around the $\pi^{0}$ and $\eta$ meson mass, as shown in Fig. 7.3, the event-mixing method completely fails to describe the distribution as it gives a qualitatively different shape. In addition, detector constraints do change the $M_{\text {inv }}$ distributions, but the same-event pairs are similarly (un)described by the event-mixing, as can be seen in Fig. 7.4.

In Fig. 7.5, three cases are studied; 1. when the photon pair shares a common parton ancestor, 2. when both photons do not share a common parton ancestor, and 3. when the photon pair do not share the same mother- or grandmother particle whenever it considers an hadronic decay. From this it is concluded that the correlations at lower $M_{\mathrm{inv}}$ is not caused by decay kinematics, but rather by the parton fragmentation into final state hadrons. 

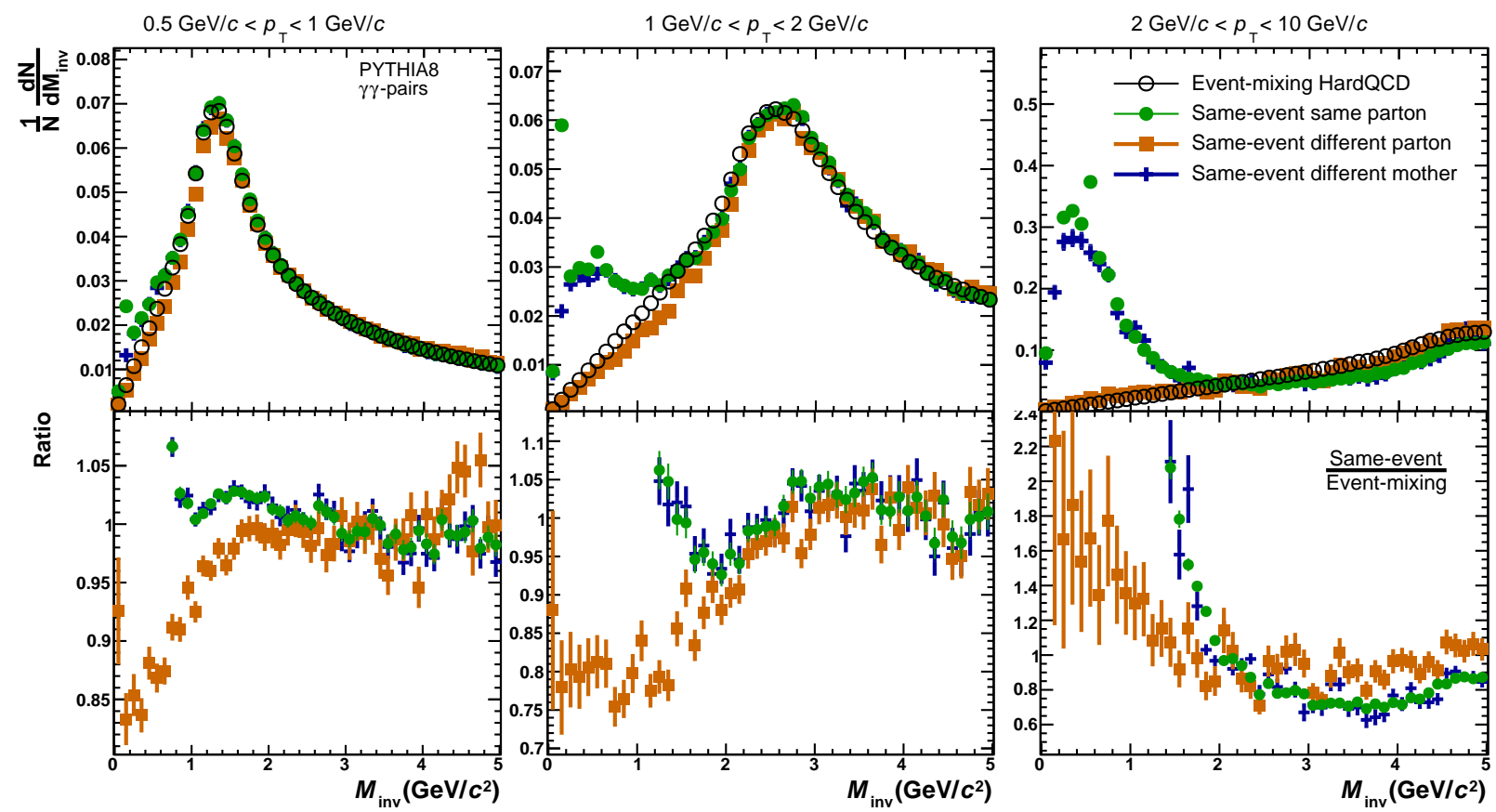

FiguRE 7.8: The diphoton $M_{\text {inv }}$ mass and the ratios for same-event pairs and event mixing over a wide mass range for the following three cases; 1 . when the photon pair for same-event-mixing shares a common parton ancestor, 2. when both photons do not share a common parton ancestor, and 3. when the photon pair do not share the same mother- or grandmother particle whenever it considers a hadronic decay.

In Fig. 7.6, it is attempted to improve the quality of event-mixing by pairing photons only when the parton ancestor is within $|\eta|<0.25$ and $|\varphi|<0.25$, preserving the $\alpha$ and $\psi$ distributions of the same-event pairs with respect to the event-mixing pairs. This improves the description of the combinatorial background by a factor of 2-10 in the region of the $\pi^{0}$ and $\eta$ meson mass. Experimentally this approach is more difficult to implement, but jet-finding algorithms with a low minimum jet $p_{\mathrm{T}}$ can approximate the direction of the parton that produces the photon. In [169] the qualitatively similar residual correlations were described with a simple jet-aligned mixing, however for the much stronger effect we see, a similarly simple approach was unfortunately not successful.

These observations have implications for the ability to describe the correlated and uncorrelated part of the diphoton invariant mass background using the event-mixing method, which is used for neutral meson as well as for direct photon HBT measurements. Both these measurements rely on either subtracting or dividing out the background from wrong-pair combinations, and it would be interesting to see whether jet-aligned mixing can improve the background description also for experiments. 


\section{Chapter 8}

\section{On event shapes and jets}

In collaboration with Jonno Schoppink.

\subsection{Introduction}

Transverse sphericity is an event shape observable that has been used throughout the particle physics community as a way to characterize the configuration of the momentum vectors of the final state particles as either pencil-, sphere-like, or anything in between. Pencil-like events are typically associated with events that have a di-jet structure, and sphere-like events with those containing mostly soft processes and an absence of jets $[170,171]$.

Historically, quantifying the shape of the event involved calculating the thrust axis, i.e. the axis which maximizes the inner product of the final-state particle momentum vectors. The thrust axis thus aligns with the average momentum of the particles and is a good approximation for the main production axis of the event, especially for $e^{+} e^{-}$collisions [172-176], where to leading order there are two partons produced with opposite momenta. For this collision system pencil-like events should correspond to a di-jet structure, and spherical events to multi-jet topologies [177-180].

This paper addresses whether this holds for pp collisions at RHIC and top LHC energies, where there are more sub-leading processes in addition to the initial hard scattering [181, 182]. This paper presents the results of an investigation on how the transverse sphericity $\left(S_{\mathrm{T}}\right)$ correlates with jet production, using simulated $e^{+} e^{-}$and pp collisions. In addition, correlations between $\left(S_{\mathrm{T}}\right)$ and other event characteristics, such as leading parton $p_{\mathrm{T}}$, particle multiplicity, and number of multi-parton interactions are studied. 


\subsection{Analysis method}

The analysis presented in this paper is performed using simulated $e^{+} e^{-}$and pp collisions, using the PYTHIA8.1 event generator [164], with the settings listed in Table 8.1. The center-of-mass energy of the $e^{+} e^{-}$dataset corresponds to the mass of the $Z$ boson, which is also the energy at which the LEP collider operated. For the pp datasets the beam energies are chosen to be the same as the nominal operating energy at the RHIC and the LHC in Run 2, both using the Monash 2013 tune. Furthermore, the pp datasets are generated twice, once with multi-parton interactions (MPI) turned on, and once with MPI turned off. This will be used to study the sphericity distributions with and without MPI in pp collisions. Tracks with $p_{\mathrm{T}}>100 \mathrm{MeV} / c$ and $|\eta|<1$ are selected for the analysis, and in the case of the $e^{+} e^{-}$dataset only the hadronic final states are considered.

The transverse sphericity is calculated, as also discussed in section 4.3.5, using

$$
S_{\mathrm{T}}=\frac{2 \lambda_{2}}{\lambda_{2}+\lambda_{1}}
$$

where $\lambda_{1}$ and $\lambda_{2}$, with $\lambda_{1}>\lambda_{2}$, are the two eigenvalues of the transverse momentum matrix $S_{x y}^{L}$, which is given by

$$
S_{x y}^{L}=\frac{1}{\sum_{i} p_{T, i}} \sum_{i} \frac{1}{p_{T, i}}\left[\begin{array}{cc}
p_{x, i}^{2} & p_{x, i} p_{y, i} \\
p_{y, i} p_{x, i} & p_{y, i}^{2}
\end{array}\right],
$$

where $p_{T, i}, p_{x, i}$, and $p_{y, i}$, are the components of the momentum vectors of particle $i$ in transverse, $x$, and $y$ direction, respectively.

These equations essentially project all the particles of the event onto the $x-y$ plane and determine the eigenbasis of the transverse momentum matrix. As such, $S_{\mathrm{T}}$ is sensitive to the relative orientation of the momentum vectors, where $\lambda_{2}=0$ is for pencil-like events, i.e. $S_{\mathrm{T}} \sim 0$, and $\lambda_{1} \sim \lambda_{2}$ for sphere-like events, i.e. $S_{\mathrm{T}} \sim 1$.

Furthermore, the number of jets contained in each event is obtained by employing the FastJet package [121]. We chose to use the anti- $k_{t}$ algorithm with a jet radius of $R=0.4$ and minimum jet energy of $E=10 \mathrm{GeV}$. As this study is purely based on model calculations, it is chosen to only include statistical uncertainties that are proportional to the number of events as generated in the respective dataset. In all cases the datasets are large enough to lead to statistically significant conclusions.

\begin{tabular}{l|l|l}
\hline System & $\sqrt{s}$ & PYTHIA8.1 settings \\
\hline$e^{+} e^{-}$ & $91 \mathrm{GeV}$ & $Z$ decay to quarks \\
\hline $\mathrm{pp}$ & $200 \mathrm{GeV}$ & Monash tune, MPI-ON \\
\hline $\mathrm{pp}$ & $200 \mathrm{GeV}$ & Monash tune, MPI-OFF \\
\hline $\mathrm{pp}$ & $13 \mathrm{TeV}$ & Monash tune, MPI-ON \\
\hline $\mathrm{pp}$ & $13 \mathrm{TeV}$ & Monash tune, MPI-OFF \\
\hline \hline
\end{tabular}

TABLE 8.1: Data sets used. 


\subsection{Results}

The distributions of the transverse sphericity $S_{\mathrm{T}}$ for the respective datasets as well as for events with different numbers of reconstructed mid-rapidity jets are shown in Fig. 8.1. The top left figure shows first of all that the $S_{\mathrm{T}}$ distribution for $e^{+} e^{-}$collisions has a maximum around $S_{\mathrm{T}} \sim 0.1$, with a long tail towards higher values. This is consistent with the picture that lepton collisions, to leading order, produce two outgoing quarks that fragment into final state particles. The $S_{\mathrm{T}}$ distributions for the pp datasets have a mean around $S_{\mathrm{T}} \sim 0.75$ for MPI-ON, and $S_{\mathrm{T}} \sim 0.6$ for MPI-OFF. Clearly, pp collisions produce a much broader distribution with a larger mean. There is no abundance of events with a pencil-like configuration, which is consistent with the idea that pp collisions involve many more processes compared to the "cleaner" $e^{+} e^{-}$collisions, tending to be more spherical due to the overall higher particle multiplicities produced by a larger number of independent processes.

Figure 8.1 shows the $S_{\mathrm{T}}$ distributions for the number of mid-rapidity jets reconstructed in the events, for $N_{\text {jets }}=0,1,2,3+$. In the top right figure, the simulated $e^{+} e^{-}$collisions are dominated by 2 -jet events at lower $S_{\mathrm{T}}$. For slightly higher values, $N_{\text {jets }}=3+$ contributes the most, after which for $S_{\mathrm{T}}>0.5$ the events with $N_{\text {jets }}=0$ take over and dominate the contribution to the inclusive sample. Single jet events exhibit a broad distribution that have a maximum at lower values of $S_{\mathrm{T}}$. For pp collisions, strikingly, the entire sample is dominated by events that do not have a jet that passes the selection criteria. This is consistent with the notion that, even though the collision energy is much higher, pp collisions have on average significantly fewer high momentum jets in the final state compared to $e^{+} e^{-}$collisions, as the rapidity distribution is wider and tracks with $|\eta|<1$ are selected. For the pp dataset with MPI-ON at $\sqrt{s}=13 \mathrm{TeV}$, contrary to the same distributions found at $\sqrt{s}=200 \mathrm{GeV}$, the single and multi-jet events are more pronounced for higher values of $S_{\mathrm{T}}$, while for the datasets with MPI-OFF the events containing a jet have a lower sphericity value. This indicates that multi-parton interactions do not play a major role at RHIC energies.

To better quantify the effect of making a selection in $S_{\mathrm{T}}$ on the number of jets in the sample, the respective distributions for the highest $10 \%$ of the most pencil- and sphere-like events are integrated and the fraction of each to the inclusive distribution is calculated. The results of this calculation are given in Table 8.2. First, it shows that $e^{+} e^{-}$collisions have jets in all but $16.1 \%$ of the events, while this fraction of non-jet events rises to $85.5 \%$ and $93.3 \%$ for pp collisions at $\sqrt{s}=13 \mathrm{TeV}$ with MPI-ON and MPI-OFF, respectively. Most of the $e^{+} e^{-}$collisions contain 2 jets, followed by $3+$ jets. For pp collisions the majority of events do not contain a jet, followed by single-jet events. These events are most likely events where one of the two jets is outside of the detector acceptance, i.e. the majority of the higher $p_{\mathrm{T}}$ constituent tracks have $|\eta|>1$. In $e^{+} e^{-}$collisions the fraction of events containing $N_{\text {jets }}=2$ increases from $44.9 \%$ for all events to $83.3 \%$ for the $10 \%$ most pencil-like events. It decreases from $3.2 \%$ to $1.3 \%$ for pp collisions at $\sqrt{s}=13 \mathrm{TeV}$ with MPI-ON and slightly increases for the case with MPI-OFF. For the $10 \%$ most sphere-like events, the fraction of events without a jet in $e^{+} e^{-}$collisions increases from $16.1 \%$ for all events to $58.8 \%$ in the most spherical-like events, followed by a sizeable contribution from $N_{\text {jets }}=3+$. Selecting on spherical events in pp collisions doesn't change the fractions of events that have a jet like it does for $e^{+} e^{-}$collisions, but shows 
an increase in $N_{\text {jets }}=2$ from $3.2 \%$ to $4.4 \%$ for pp collisions at $\sqrt{s}=13 \mathrm{TeV}$ with MPI-ON. As jets are so rare in pp collisions at $\sqrt{s}=200 \mathrm{GeV}$, none of the selections have a significant effect on the $N_{\text {jets }}$ fractions. These results indicate that for $e^{+} e^{-}$collisions a selection on the sphericity is able to enhance a specific amount of jets within that sample, while for pp collisions there is no such strong correlation.

\begin{tabular}{|c|c|c|c|c|c|c|c|c|c|c|c|c|c|c|}
\hline \multirow[b]{2}{*}{ Data Set } & \multirow[b]{2}{*}{ energy } & \multirow[b]{2}{*}{ MPI } & \multicolumn{4}{|c|}{$N_{\text {jets }}(\%)$, All } & \multicolumn{4}{|c|}{$N_{\text {jets }}(\%)$, pencil-like } & \multicolumn{4}{|c|}{$N_{\text {jets }}(\%)$, sphere-like } \\
\hline & & & 0 & 1 & 2 & $3+$ & 0 & 1 & 2 & $3+$ & 0 & 1 & 2 & $3+$ \\
\hline$e^{+} e^{-}$ & $91 \mathrm{GeV}$ & - & 16.1 & 11.8 & 44.9 & 27.2 & 1.9 & 5.9 & 83.8 & 8.4 & 58.8 & 11.0 & 10.4 & 19.8 \\
\hline pp & $200 \mathrm{GeV}$ & $\mathrm{ON}$ & $>99.9$ & $<0.01$ & $<0.01$ & $<0.01$ & $>99.9$ & $<0.01$ & $<0.01$ & $<0.01$ & $>99.9$ & $<0.01$ & $<0.01$ & $<0.01$ \\
\hline pp & $200 \mathrm{GeV}$ & OFF & $>99.9$ & $<0.01$ & $<0.01$ & $<0.01$ & $>99.9$ & 0.02 & $<0.01$ & $<0.01$ & $>99.9$ & $<0.01$ & $<0.01$ & $<0.01$ \\
\hline pp & $13 \mathrm{TeV}$ & $\mathrm{ON}$ & 85.5 & 9.2 & 3.2 & 2.1 & 93.1 & 5.5 & 1.3 & 0.2 & 83.9 & 8.7 & 4.4 & 3.0 \\
\hline pp & $13 \mathrm{TeV}$ & $\mathrm{OFF}$ & 93.9 & 5.2 & 0.8 & 0.1 & 83.6 & 13.9 & 2.2 & 0.3 & 99.2 & 0.6 & 0.1 & 0.1 \\
\hline
\end{tabular}

TABle 8.2: Percentage of events containing $N_{\text {jets }}=0,1,2,3+$ jets with $E>10 \mathrm{GeV}$, for ensembles of all events, the $10 \%$ most pencil-like events, and $10 \%$ most sphere-like events as calculated using the transverse sphericity $S_{\mathrm{T}}$.

Now, since the correlation between the shape of the event and the number of reconstructed jets in pp collisions is rather weak, it would be interesting to establish if there is another event characteristic that correlates well with the event shape. The distributions of the $p_{\mathrm{T}}$ of the leading parton within the event for pencil-like and sphere-like events for $e^{+} e^{-}$, pp (MPI-OFF), and pp (MPI-ON) collisions are shown in Fig. 8.2. Similar to the previous results, the $10 \%$ most pencil-like events (low $S_{\mathrm{T}}$ ) and $10 \%$ most sphere-like events (high $\left.S_{\mathrm{T}}\right)$ are used to obtain the results. It indicates that selecting pencil- rather than sphere-like events leads to an increase of the mean $p_{\mathrm{T}}$ of the leading parton of $\sim 100 \%$ for $e^{+} e^{-}$collisions. This is consistent with the previous results presented in Fig. 8.1, as multi-jet topologies correlate with higher values of $S_{\mathrm{T}}$. Interestingly, for pp collisions, the mean $p_{\mathrm{T}}$ of the leading parton is larger for sphere-like events compared to pencil-like events for the case with MPI-ON, while it is not the case with MPI-OFF. This observation is consistent with the conclusions taken from Table 8.2.

The distributions for the number of multi-parton interactions for pp collisions (MPI-ON) are shown in Fig. 8.3, for pencil- and sphere-like events (left), as well as for the events with the $10 \%$ lowest and highest final state particle multiplicities (right). It shows that events with higher sphericity and higher multiplicity both increase the mean number of multi-parton interactions, which can be understood from the idea that sphericity correlates with multiplicity. Moreover, $N_{\text {MPI }}$ increases more when selecting the events with highest multiplicity instead of the most sphere-like configuration, and also more strongly excludes lower $N_{\text {MPI }}$. Thus, the multiplicity of the event is found to be a better observable to select events with a larger number of multi-parton interactions in pp collisions. 

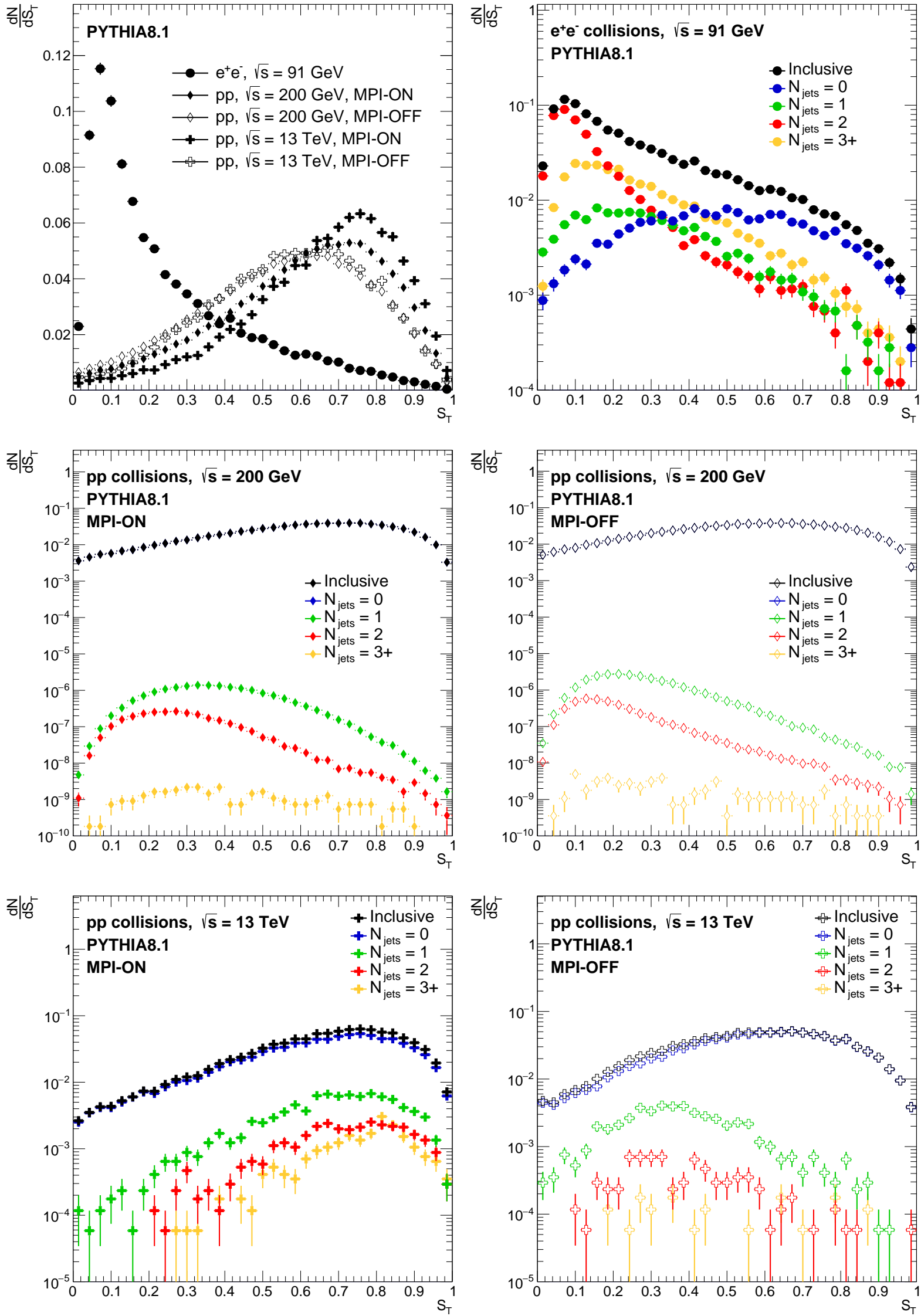

Figure 8.1: Transverse sphericity distributions $\left(S_{\mathrm{T}}\right)$ for simulated $e^{+} e^{-}$and pp collisions (top left), and differentiated for the amount of jets reconstructed in the events $\left(N_{\text {jets }}=0,1,2,3+\right)$ for each respective dataset. The inclusive distributions are normalized to unity. The inclusive and $N_{\text {jets }}=0$ distributions for the pp collisions dataset at $\sqrt{s}=200 \mathrm{GeV}$ are indistinguishably close. 

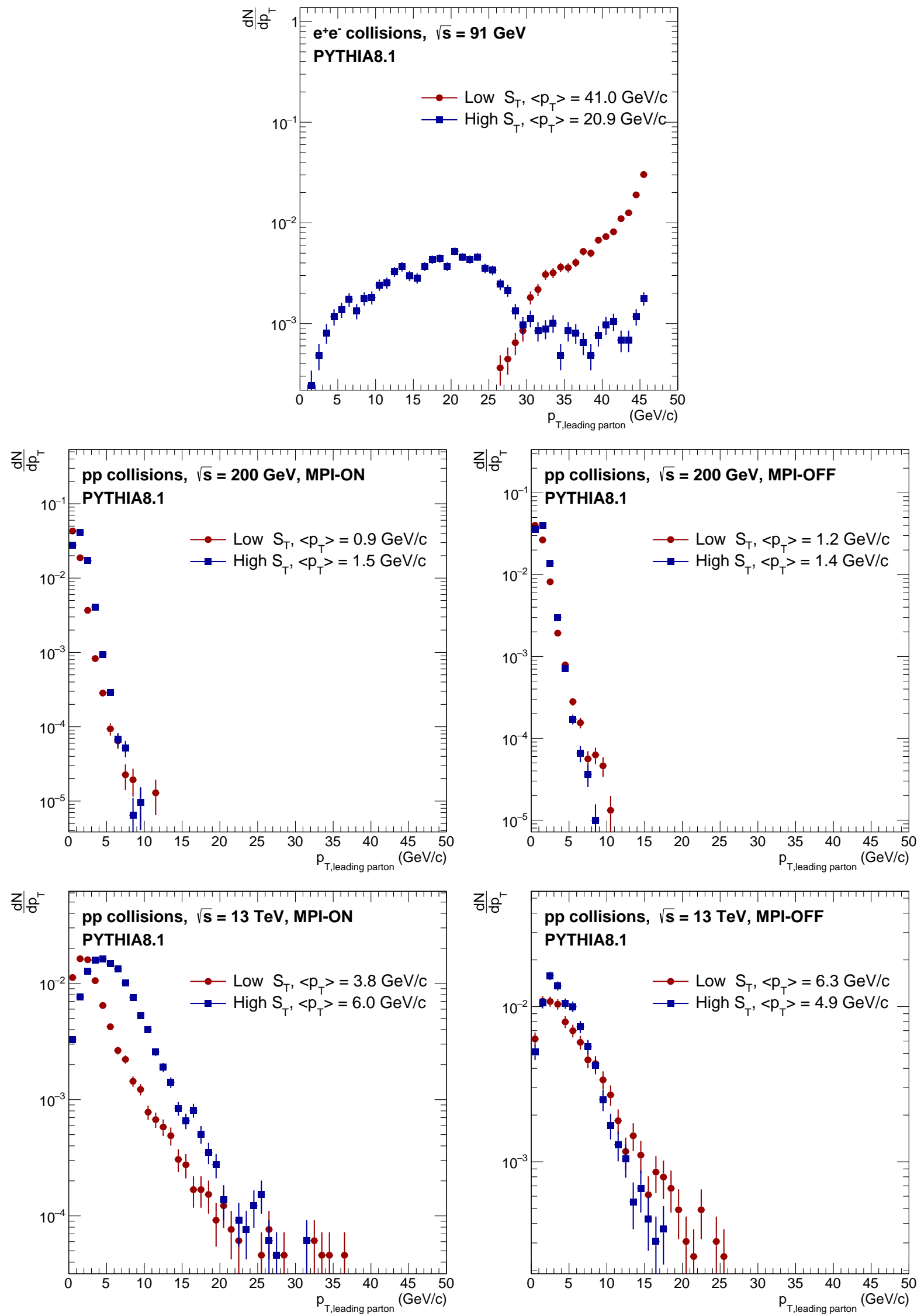

Figure 8.2: The distributions for the $p_{\mathrm{T}}$ of the leading parton for pencil-like (low $S_{\mathrm{T}}$ ) and sphere-like (high $\left.S_{\mathrm{T}}\right)$ events, as calculated for each respective dataset. The mean of each distribution is given in the figure. 

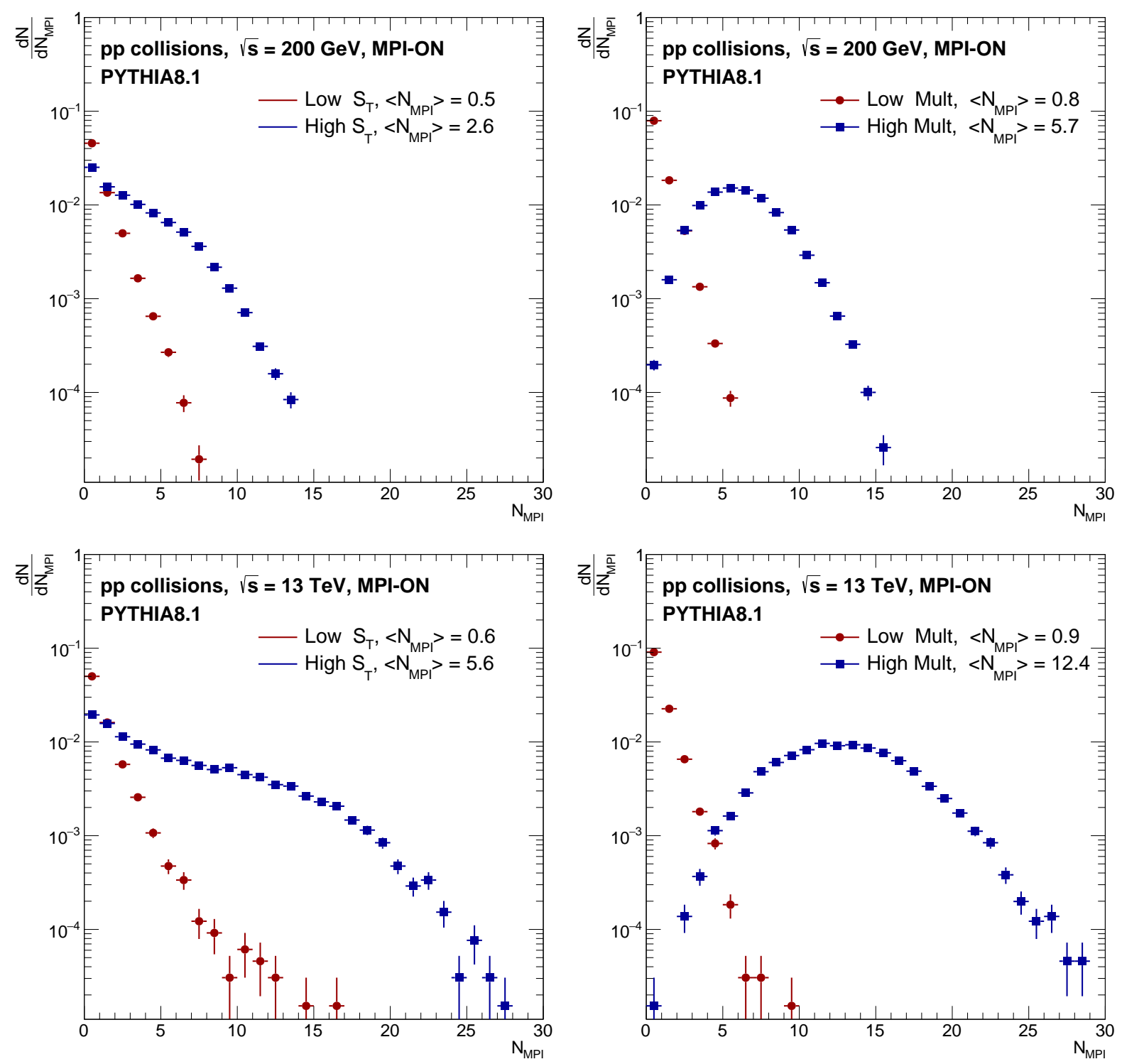

Figure 8.3: The distributions of the number of multi-parton interactions for pencil- and sphere-like events (left), as well as events with low and high multiplicity (right), as calculated for simulated pp (MPI-ON) collisions at $\sqrt{s}=200 \mathrm{GeV}$ (top) and $\sqrt{s}=13 \mathrm{TeV}$ (bottom), respectively. The mean of each distribution is given in the figure. 


\subsection{Conclusions}

The analysis presented in this paper investigates the relationship between the shape of the event, the number of reconstructed jets, and other event characteristics such as the particle multiplicity, $p_{\mathrm{T}}$ of the leading parton, and the number of multi-parton interactions. In Fig. 8.1 it is shown that the $S_{\mathrm{T}}$ distributions are qualitatively different for $e^{+} e^{-}$and pp collisions, as most $e^{+} e^{-}$collisions produce a pencil-like shape, while the particles produced in a pp collisions are on average in a more sphere-like configuration. Also, it turns out that a large percentage of $e^{+} e^{-}$collisions contain a reconstructed jet, while this is not the case for pp collisions. The correlation between the transverse sphericity $S_{\mathrm{T}}$ and the number of reconstructed jets in $e^{+} e^{-}$collisions enables the use of this observable to discriminate between di-jet and multi-jet topologies. Surprisingly, it turns out that in pp collisions (MPI-ON) $S_{\mathrm{T}}$ is not strongly correlated to $N_{\text {jets }}$, as any selection in $S_{\mathrm{T}}$ results in a sample dominated by $N_{\text {jets }}=0$. Thus, these results suggest that pp collisions with a pencil-like shape are by no means more jet-like compared to events without a selection on their shape.

Furthermore, the results shown in Fig. 8.3 indicate a strong correlation between $S_{\mathrm{T}}$ and the number of multiparton interactions. The average number of these interactions increases relatively more for pp collisions at $\sqrt{s}=13 \mathrm{TeV}$ compared to pp collisions at $\sqrt{s}=200 \mathrm{GeV}$. However, as Fig. 8.3 (right) shows, the mean number of multi-parton interactions increase even more between events with low and high multiplicities. These observations are consistent with the idea that $S_{\mathrm{T}}$ correlates with the particle multiplicity, i.e. a large number of multi-parton interactions lead to a more sphere-like configuration with increased multiplicity. As it is experimentally impossible to directly measure the $N_{\mathrm{MPI}}$, it would be very interesting to use $S_{\mathrm{T}}$, as well as the event multiplicity, to explore pp collisions with small and large amounts of multi-parton interactions and compare them to model predictions. This study cannot fully explain the different features observed in the neutral meson yield for selected sphericity and multiplicity intervals. 


\section{Chapter 9}

\section{Direct photon flow}

In collaboration with Dmitri Peressounko and Klaus Reygers.

\subsection{Introduction}

Unique insights into the properties of the quark gluon plasma that is created in collisions of heavy-ions can be obtained by studying direct photons. As explained in detail in chapter 3, direct photons are the photons not coming from hadronic decays, and as such originate either from interactions between the constituents of the colliding nuclei, or by interactions between the constituents of the QGP. Since the mean free path of photons in a QGP is much larger than its typical size, they escape unaffected and carry information on the temperature and space-time evolution of the system. Experimentally this information is obtained by measuring the invariant yield and anisotropic flow of direct photons.

The invariant yield of direct photons has been measured by the ALICE collaboration [183] and got published in 2015. It uses the $\mathrm{Pb}-\mathrm{Pb}$ dataset at $2.76 \mathrm{TeV}$ obtained in the fall of 2010 , which is analysed for three classes of collision centrality; central, semi-central, and peripheral collisions. The photons are reconstructed with the photon conversion method as well as the PHOS calorimeter, and are combined into a single result. The main results of this paper, which are the excess and invariant yield of direct photons as function of transverse momentum, are shown in Fig. 9.1. It shows that the $R_{\gamma}$ is either consistent or larger than unity for all the measured collision centralities and $p_{\mathrm{T}}$ intervals, and agrees rather well with various model calculations.

The PHENIX collaboration at RHIC measured both a large direct photon yield and elliptic flow in 2012 [79], leading to the direct photon puzzle. The main point of the puzzle is the inability of theoretical calculations to describe both measurements with the same medium properties, as explained in more detail in section 3.2. It still remains to be one of the larger puzzles of heavy-ion physics to this day. This thesis will present the analysis and results of the direct photon elliptic flow measurement done with the ALICE experiment at 

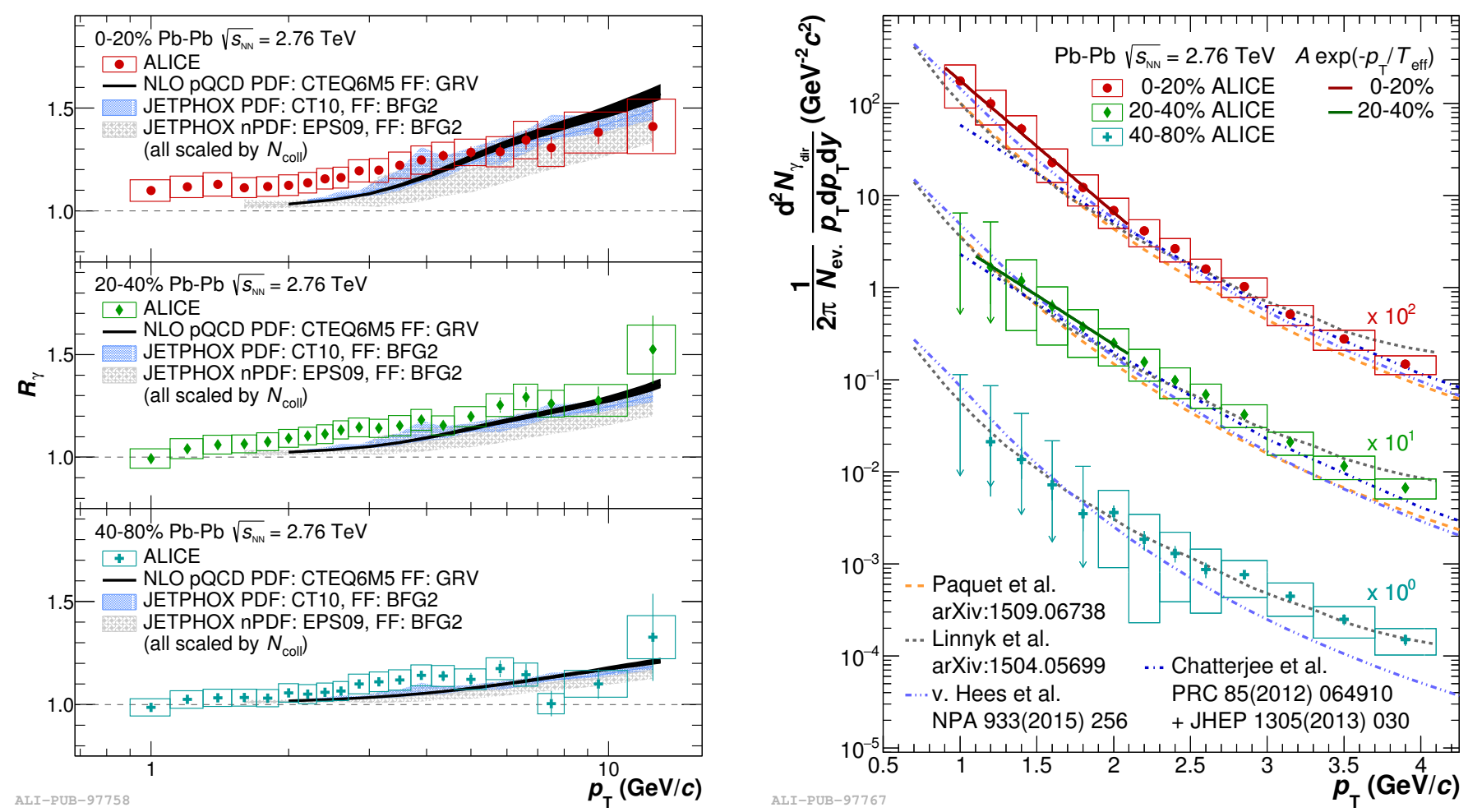

FiguRE 9.1: Direct photon invariant yield and $R_{\gamma}$, as measured in 2015 by the ALICE collaboration.

LHC energies, and can potentially find out if the same puzzle exists at the LHC. The findings are published in $[87]$.

\subsection{Analysis method}

\subsubsection{Datasets}

The analysis is performed for $\mathrm{Pb}-\mathrm{Pb}$ collisions with a center-of-mass energy of $2.76 \mathrm{TeV}$, using the ALICE data collected in the heavy-ion run of 2010. The minimum bias interaction trigger is used to activate the detector readout. This trigger requires a simultaneous signal in the V0-A and V0- , as well as at least one hit in the SPD detectors, resulting in an efficiency to trigger on a $\mathrm{Pb}-\mathrm{Pb}$ hadronic interaction between $98.4 \%$ and $99.7 \%$. The events are divided into the central and semi-central centrality classes 0-20\% and 20-40\%, respectively, according to the V0-A and V0-C summed amplitudes [111]. To study various detector related effects, such as efficiency and purity of the inclusive photon sample, a Monte Carlo simulation is used. The simulated $\mathrm{Pb}-\mathrm{Pb}$ collisions are produced by the HIJING event generator, and the particles in each event are propagated through the ALICE detector using the GEANT3 [184, 185] framework to simulate the full detector response.

Careful run by run quality assurance (QA) has been performed to only include data taking runs where the central barrel and calorimeters have nominal running conditions. Furthermore, events are accepted only if 
a primary vertex is reconstructed within $\pm 10 \mathrm{~cm}$ from the nominal interaction point along the beam line ( $z$-direction). This ensures a uniform pseudorapidity $(\eta)$ distribution of tracks within the acceptance of the detector. These event selection criteria result in $13.6 \times 10^{6}$ events that are available for the analysis using the central barrel, and $18.8 \times 10^{6}$ events for the analysis using the PHOS calorimeter.

\begin{tabular}{l|l|l|l|l}
\hline System & $\sqrt{s_{N N}}$ TeV & Data set & Centrality & $\mathbf{N}_{\text {events }}$ \\
\hline $\mathrm{Pb}-\mathrm{Pb}$ & 2.76 & LHC10h & $0-20 \%$ & $3.29 \cdot 10^{6}$ \\
& & & $20-40 \%$ & $3.30 \cdot 10^{6}$ \\
\hline $\mathrm{Pb}-\mathrm{Pb}$ & 2.76 & LHC13d2 & $0-20 \%$ & $1.17 \cdot 10^{6}$ \\
& & & $20-40 \%$ & $6.28 \cdot 10^{5}$ \\
\hline \hline
\end{tabular}

TABLE 9.1: Used data sets.

\subsubsection{Flow method}

The inclusive photon yield is defined as the sum of direct and decay photons $\left(N_{\gamma, \text { inc }}=N_{\gamma, \text { dir }}+N_{\gamma, \text { dec }}\right)$, such that the direct photon yield is obtained by measuring the yield of inclusive and decay photons and computing the difference. The direct-photon elliptic flow is obtained in a similar way; the direct-photon elliptic flow is extracted by subtracting the elliptic flow of photons from hadron decays from the inclusive photon elliptic flow. Since the value of the anisotropic flow coefficients is the weighted average of the measured particles, the inclusive photon flow can be decomposed as

$$
v_{2}^{\gamma, \text { inc }}=\frac{N_{\gamma, \text { dir }}}{N_{\gamma, \text { inc }}} v_{2}^{\gamma, \text { dir }}+\frac{N_{\gamma, \text { dec }}}{N_{\gamma, \text { inc }}} v_{2}^{\gamma, \text { dec }}
$$

where again the inclusive photon yield is decomposed into the contributions of direct and decay photons. The $v_{2}^{\gamma, \text { inc }}, v_{2}^{\gamma, \text { dir }}$ and $v_{2}^{\gamma, \text { dec }}$ are the corresponding elliptic flow coefficients. Using the direct photon excess ratio $R_{\gamma}=N_{\gamma, \text { inc }} / N_{\gamma, \text { dec }}$, the direct photon flow can be written as:

$$
v_{2}^{\gamma, \text { dir }}=\frac{v_{2}^{\gamma, \text { inc }} R_{\gamma}-v_{2}^{\gamma, \text { dec }}}{R_{\gamma}-1}
$$

The ratio $R_{\gamma}$ was previously measured in the same dataset [183], and is used as input for this analysis. The $v_{2}^{\gamma, \text { inc }}$ is measured using all the events that pass the event selection criteria, and the $v_{2}^{\gamma, \text { dec }}$ is calculated with a simulation of photons from hadronic decays.

The photon elliptic flow $v_{2}$ is calculated with a two-particle correlation method [186], more specifically the Scalar Product (SP) method. The main characteristic and advantage of this method is the usage of a pseudorapidity gap of $|\Delta \eta|>0.9$ between the particle of interest, in this case the inclusive photon, and the reference flow particles. The applied gap reduces the so called non-flow contributions, which are the correlations arising from resonance decays and jets. The SP method uses the reference flow particles (RFP) to calculate the $Q$-vector that is valid for every event as well as sub-events within the event, which is defined 
by:

$$
\vec{Q}_{n}=\sum_{i \in \mathrm{RFP}} w_{i} e^{i n \varphi_{i}}
$$

where $\varphi_{i}$ is the azimuthal angle of the $i$-th RFP, $n$ is the order of the harmonic and $w_{i}$ is a weight applied for every RFP. The RFPs are taken from the V0-A and V0-C detectors. Since these detectors do not provide tracking information, the RFP particles are assumed to point to the centers of V0A/V0C cells, while the amplitudes of the signal from each cell, which are proportional to the number of particles that cause a hit, are used as a weight $w_{i}$. The non-uniformity of the detector is taken into account in the SP method by applying the inverse of the event-averaged amplitudes as a weight for each of the V0 segments, together with a recentering procedure to correct for variations of the beam crossing position and vertex location [104, 110]. Furthermore, the unit flow vector for the particles of interest, the inclusive photons, is defined by:

$$
\overrightarrow{\mathrm{u}}_{2}=e^{i 2 \varphi}
$$

The elliptic flow $v_{2}$ is then calculated by computing the inner product between the unit flow vectors of all particles of interest with the two $Q$-vectors of the sub-events:

$$
v_{2}=\sqrt{\frac{\left\langle\left\langle\overrightarrow{\mathrm{u}}_{2} \cdot \frac{\vec{Q}_{2}^{\mathrm{A} *}}{M_{\mathrm{A}}}\right\rangle\right\rangle\left\langle\left\langle\overrightarrow{\mathrm{u}}_{2} \cdot \frac{\vec{Q}_{2}^{\mathrm{C} *}}{M_{\mathrm{C}}}\right\rangle\right\rangle}{\left\langle\frac{\vec{Q}_{2}^{\mathrm{A}}}{M_{\mathrm{A}}} \cdot \frac{\vec{Q}_{2}^{\mathrm{C} *}}{M_{\mathrm{C}}}\right\rangle}},
$$

where the two pairs of brackets in the numerator indicate an average over all photons and over all events. $M_{\mathrm{A}}$ and $M_{\mathrm{C}}$ are the estimates of multiplicity from the V0-A and V0-C detectors, respectively; and $\vec{Q}_{2}^{\mathrm{A} *}$, $\vec{Q}_{2}^{\mathrm{C} *}$ are the complex conjugates of the flow vector calculated in sub-event A and C, respectively.

\subsubsection{Inclusive photon purity correction}

The photons used in this analysis are reconstructed using the Photon Conversion Method (PCM) and the PHOS calorimeter. The inclusive photons measured with PCM are relevant for this thesis, as the PHOS measurement has been carried out by a collaborator. The overall reconstruction and selection of conversion

photons is described in chapter 5 and is identical for this analysis. However, $v_{2}^{\gamma, \text { inc }}$ is sensitive to impurities of the inclusive photon sample, and an additional correction is required.

Photons candidates that are reconstructed with the photon conversion method are not exclusively converted photons from the process $\gamma \rightarrow e^{+} e^{-}$, mainly because the selection criteria for electrons and positrons are not perfect. It is possible that, for example, a $\pi^{ \pm}$is identified as $e^{ \pm}$and still pass all other photon selection criteria. This results in a fake photon in the inclusive photon sample. More specifically, for photons reconstructed from conversions into electron-positron pairs one expects a combinatorial background from misidentified pairs $\left(e^{+} e^{-}, \pi^{\mp} e^{ \pm}, K^{\mp} e^{ \pm}, p^{\mp} e^{ \pm}, \ldots\right)$. These background sources potentially carry elliptic flow 
and thus may affect the measured $v_{2}^{\gamma, \text { inc }}$. To be able to correct for the impurities one needs to know the size and the $v_{2}$ of the various background sources.

A way to investigate the purity of the inclusive photon sample is by using the energy loss of the daughter particles in the TPC. Charged particles traversing the TPC have a characteristic energy loss dependent on momentum and type of particle, as shown in figure 9.2 (left). This quantity can be related to the average response of the electron in the TPC, by calculating the number of standard deviations the response of the candidate is separated from the fitted electron response. This is labeled as $n \sigma_{e^{ \pm}}$. The $n \sigma_{e^{ \pm}}$for secondary particles is shown in figure 9.2 (right). For certain momentum ranges the energy loss can become similar for different particles, which shows that there are background sources in the electron sample.
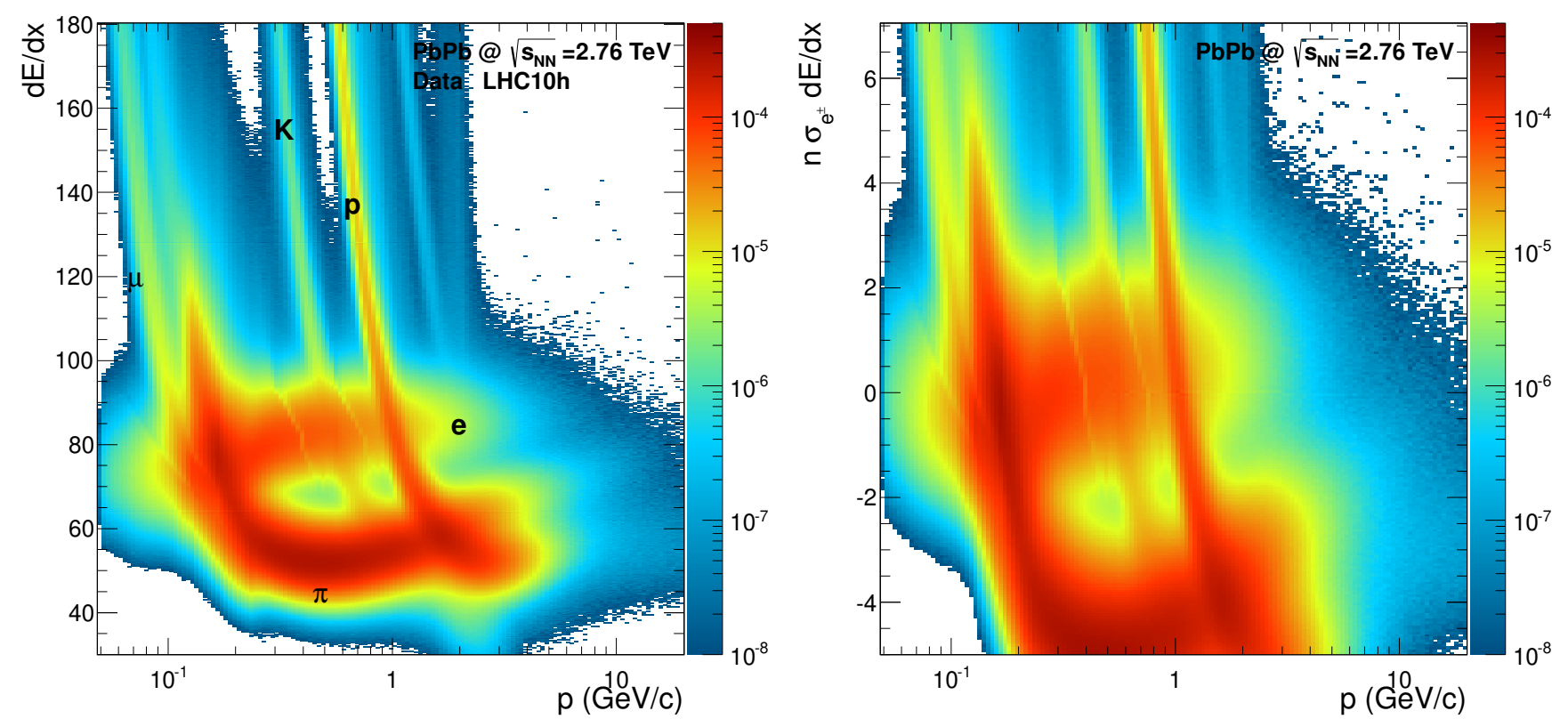

FIGURE 9.2: The energy loss per unit distance (left) and the number of standard deviations from the average electron response (right) of a particle traversing the TPC as function of transverse momentum.

To identify an $e^{+}$and $e^{-}$pair, the specific energy loss in the TPC [104] of both daughters is combined to calculate the dimensionless quantity $\mathrm{K}$, which is defined as

$$
K=\frac{\left|\kappa^{+}\right|+\left|\kappa^{-}\right|}{2}+2\left(\kappa^{+}+\kappa^{-}\right)
$$

where

$$
\kappa^{ \pm}=\frac{\left.\frac{d E}{d x}\right|_{\text {candidate }}-\left\langle\left.\frac{d E}{d x}\right|_{\text {electron }}\right\rangle}{\sigma_{\left\langle\left.\frac{d E}{d x}\right|_{\text {electron }}\right\rangle}} .
$$

True electrons and positrons will be centered around $\kappa^{ \pm} \simeq 0$ with a width equal to the spread in $\left.\frac{d E}{d x}\right|_{T P C}$. Fake photons reconstructed using a real pion and electron will have displaced peaks since the response of a pion in the TPC is different, on average. As can be seen in figure 9.3 and figure 9.4, each background source has a response that can be discriminated from the others. There are two main background sources; $\gamma_{f a k e} \rightarrow \pi^{ \pm}+\pi^{\mp}$ and $\gamma_{f a k e} \rightarrow \pi^{ \pm}+e^{\mp}$. Furthermore, it has been chosen to combine all the other background 
sources into $\gamma_{f a k e} \rightarrow$ remaining, which results in a total of nine background sources for Fig. 9.3 and three background sources for 9.4, where the latter is done to stabilize the template fitting procedure in both experimental and simulated data. Real $e^{+} e^{-}$pairs are centered around $K \simeq 0$ with a slightly longer tail to positive values of $K \cdot \gamma_{f a k e} \rightarrow \pi^{ \pm}+e^{\mp}$ are centered around $K \simeq-8$ because of the average response of the pion. $\gamma_{\text {fake }} \rightarrow \pi^{ \pm}+\pi^{\mp}$ are centered around $K \simeq-16$ because of the response of two pions. $\gamma_{\text {fake }} \rightarrow$ remaining is reasonably flat in $K$ because it consists of many different particles with different TPC responses.

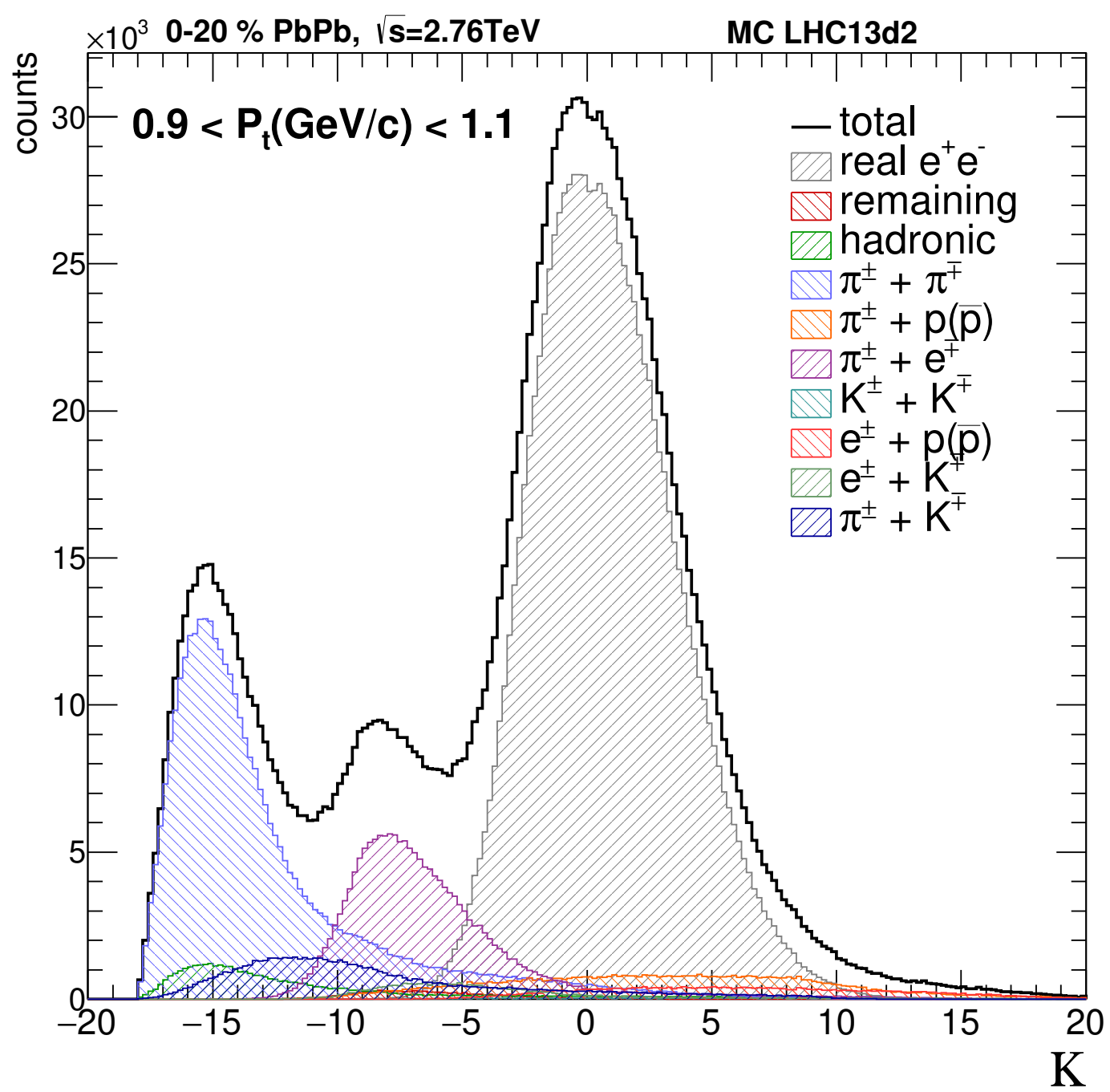

Figure 9.3: K distributions conversion photon candidates where MC information is used to classify the conversion daughters.

The $v_{2}$ of the different contributions in the $(\mathrm{d} E / \mathrm{d} x)$ distributions is measured and subtracted from the main signal region using the purity, which is obtained by fitting using Monte Carlo templates to data. The purity of the inclusive photon sample is estimated by fitting the four MC templates(figure 9.3) to the total response of data and calculating:

$$
p=\frac{N_{\text {signal }}}{N_{\text {signal }}+N_{\text {background }}}
$$



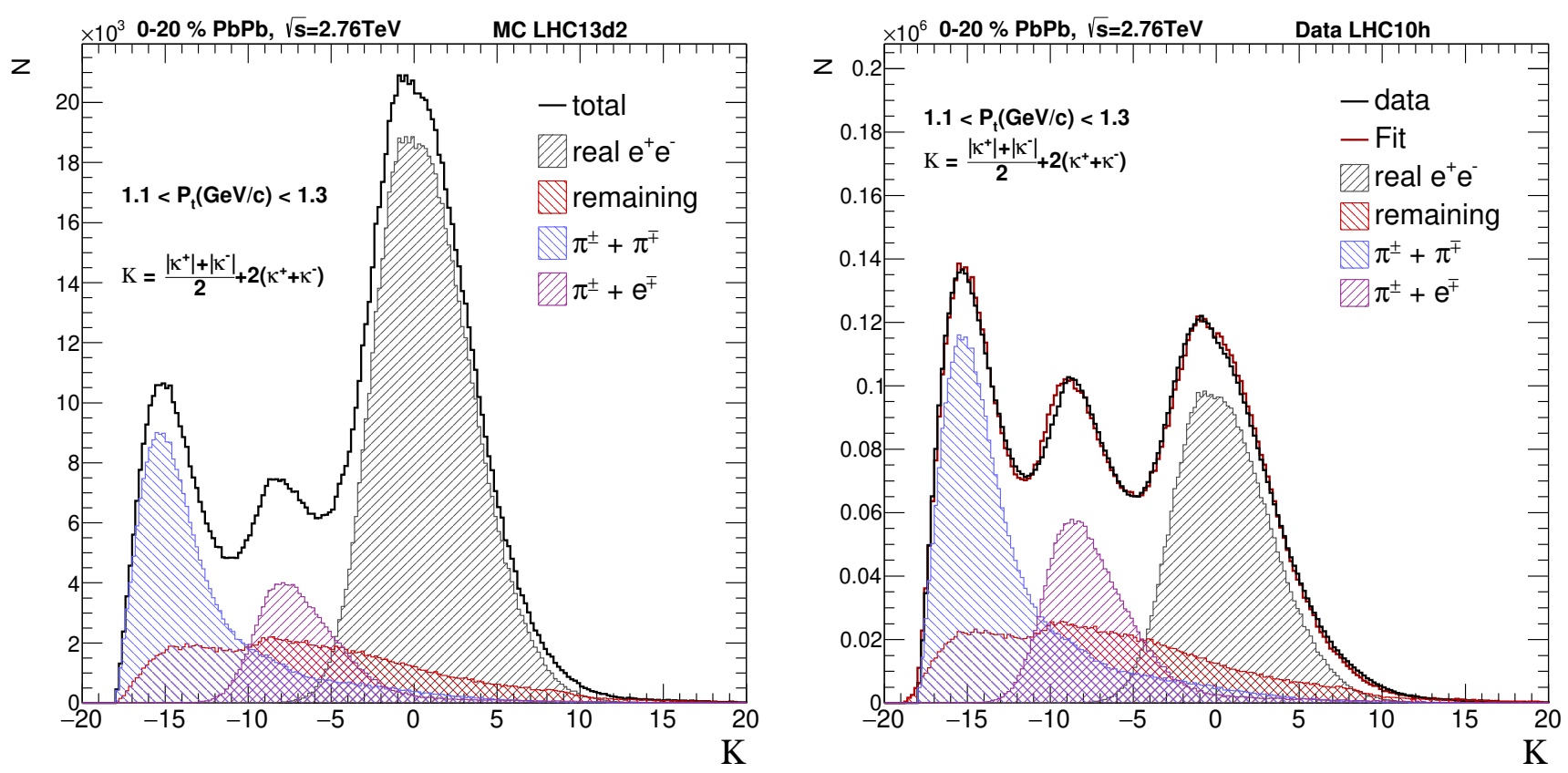

FiguRE 9.4: K distributions for signal and background sources for conversion photon candidates using 4 MC templates. The distributions in the left plot are obtained by using MC information to classify the source of the reconstructed photon. These distributions are the MC templates used for the fit on data and are different for each $p_{\mathrm{T}}$ bin. The distributions on the right are obtained by fitting the MC templates on data in which all templates are scaled to best fit the data.

where $N_{i}$ is obtained by counting bins in a region in K. The purity can be enhanced by selecting regions in $\left.\frac{d E}{d x}\right|_{T P C} .-3<K<5$ is optimal for selecting signal and has a purity of $p>86 \%$ for all centrality classes. The amount of contamination in the inclusive photon sample is then obtained with $c=1-p$. The results for the purity of the inclusive photon sample are shown in figure 9.5 , for centralities $0-20 \%$ and $20-40 \%$. The template fits for higher $p_{\mathrm{T}}$ have been constrained to be within 1.2 and 1.3 of the MC prediction for centralities $0-20 \%$ and $20-40 \%$, respectively. This is done to stabilize the fitting process.

The effect of background contaminating $v_{2}^{\gamma, \text { inc }}$ depends on the purity of the inclusive photon sample and the strength of the background flow $v_{2}^{\gamma, \text { bck }}$. The measured $v_{2}$ contains contributions from both signal and background sources and is given by:

$$
N_{\gamma}^{\text {candidate }} v_{2}^{\gamma, \text { candidate }}=N_{\gamma}^{\text {inc }} v_{2}^{\gamma, \text { inc }}+N_{\gamma}^{b c k} v_{2}^{\gamma, b c k}
$$

There is no direct way of measuring $v_{2}^{\gamma, \text { bck }}$ in the main signal region $(-3<K<5)$. However, as shown in figure 9.3, the background sources extent further in $\mathrm{K}$ and each dominate in a certain region. It is therefore possible to measure $v_{2}^{\gamma, \text { bck }}$ in different regions and calculate $v_{2}^{r e m}, v_{2}^{\pi \pi}$ and $v_{2}^{\pi e}$, from which the $v_{2}^{\gamma, \text { bck }}$ can be reconstructed. This is under the assumption that $v_{2}$ is not $\mathrm{K}$ dependent for one given component. The three background regions are defined as; Region 1: $-18<K<-13$, Region 2: $-11<K<-6$, Region 3 : $11<K<20$, which, as shown in figure 9.6 with the ratio $N_{\mathrm{bkg}, \mathrm{i}} / N_{\mathrm{bkg}, \text { tot }}$, are each dominated by a different background source. Measurements of $v_{2}$ for region 1, 2 and 3 can be related to $v_{2}^{r e m}, v_{2}^{\pi \pi}$ and $v_{2}^{\pi e}$ by knowing 

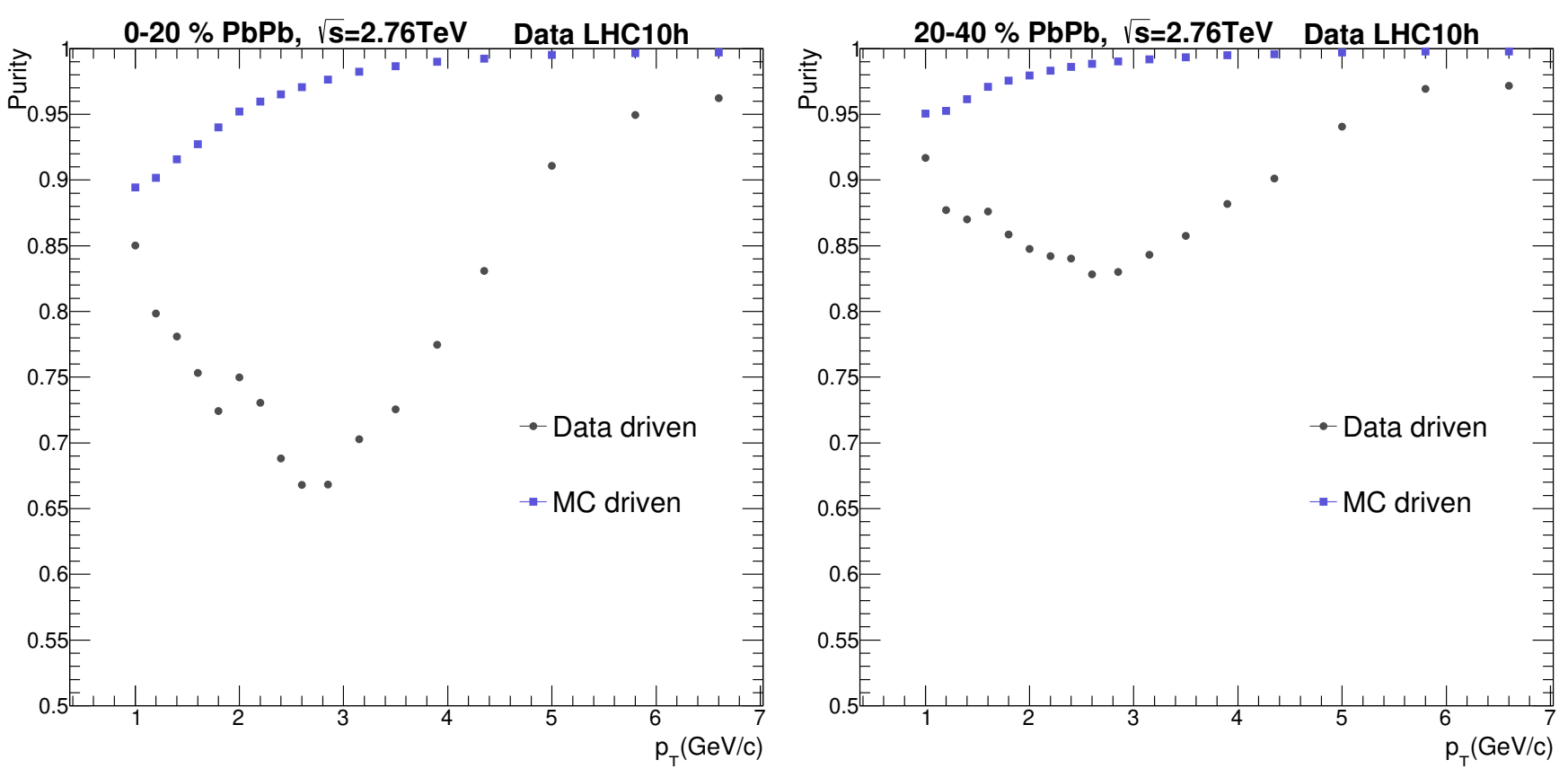

Figure 9.5: Purity of the inclusive photon sample for centralities $0-20 \%$ and $20-40 \%$. The purity is obtained by fitting MC templates of the $\mathrm{K}$ distributions on data and integrating the resulting distribution in the region $-3<K<5$.

the relative contributions of all background sources in each region. The relative contribution of a background source to the total background in a specific region is defined by $n_{i}=\frac{N_{i}}{\sum_{j} N_{j}}$, with $N_{i}=\left\{N_{r e m}, N_{\pi \pi}, N_{\pi e}\right\}$. This also implies $\sum_{i} n_{i}=1$ as required. The results for $v_{2}^{\text {region } 1}, v_{2}^{\text {region } 2}$ and $v_{2}^{\text {region } 3}$ are shown in figure 9.7 for centralities $0-20 \%$ and $20-40 \%$.
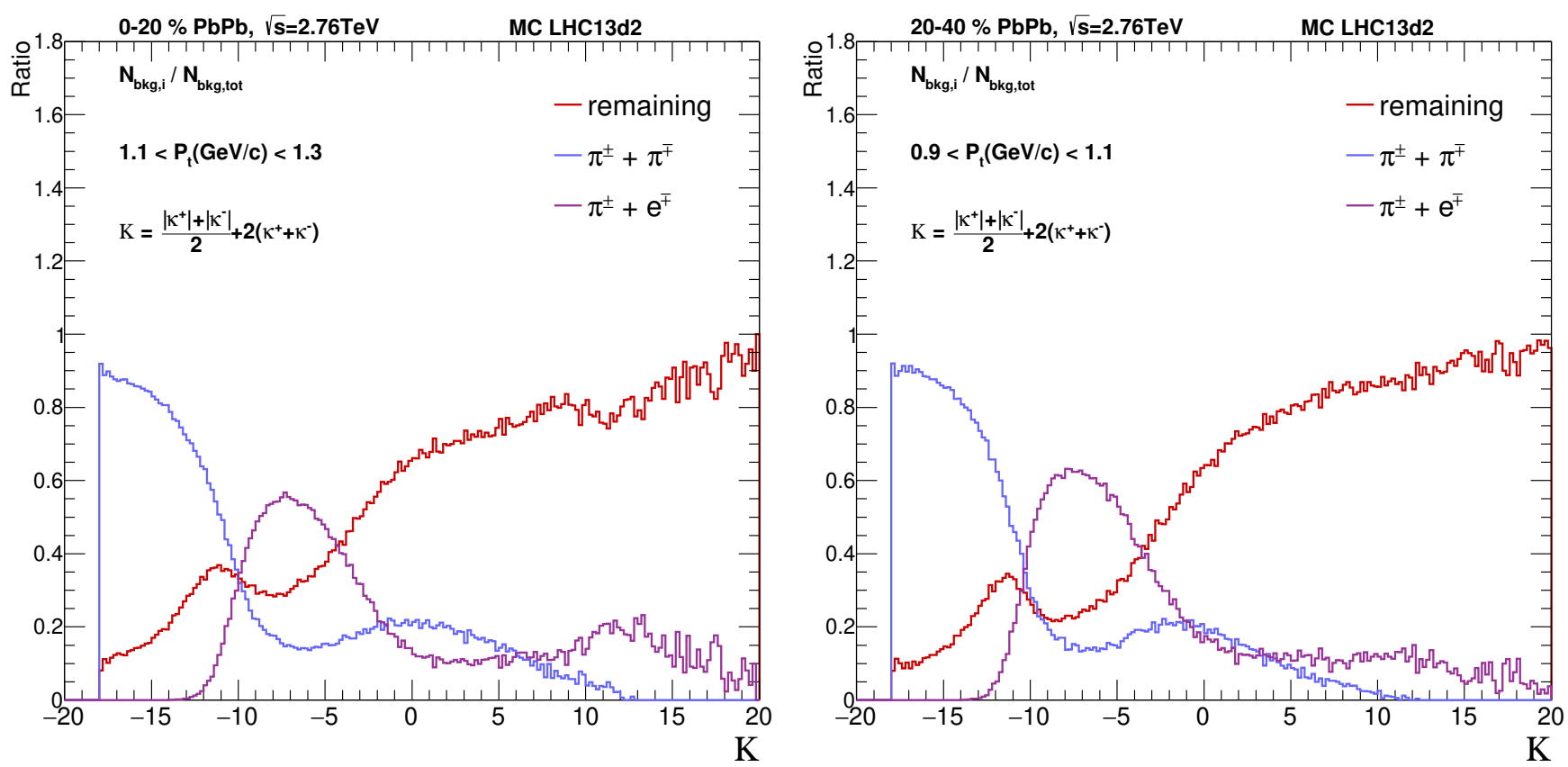

Figure 9.6: Ratio of background sources to the total background as function of $\mathrm{K}$ for centralities $0-20 \%$ (left) and $20-40 \%$ (right) for two selected $p_{\mathrm{T}}$ intervals. 

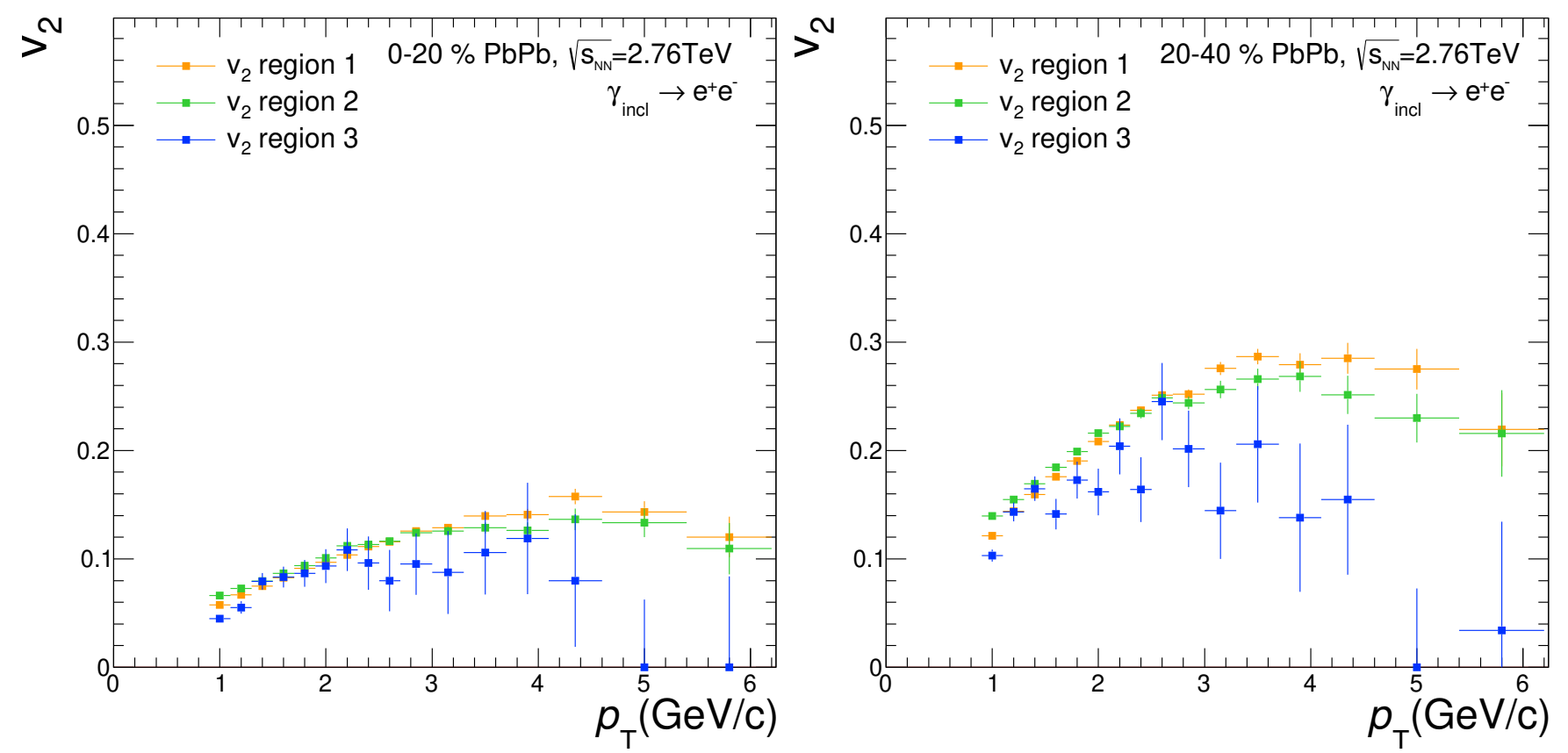

FIGURE 9.7: $v_{2}^{\text {region } 1}, v_{2}^{\text {region } 2}$ and $v_{2}^{\text {region } 3}$ as function of $p_{\mathrm{T}}$ for centralities $0-20 \%$ (left) and $20-40 \%$ (right).

Region 1 is mostly dominated by $\gamma_{f a k e} \rightarrow \pi^{ \pm} \pi^{\mp}$, and there is an additional background contribution from $\gamma_{\text {fake }} \rightarrow$ remaining. Therefore, the measured $v_{2}^{\text {region } 1}$ consists of:

$$
v_{2}^{\text {region } 1}=n_{\pi \pi} v_{2}^{\pi \pi}+n_{r e m} v_{2}^{r e m}
$$

Region 2 contains contributions from all three background sources. Therefore, the measured $v_{2}^{\text {region } 2}$ consists of:

$$
v_{2}^{r e g i o n} 2=n_{\pi \pi} v_{2}^{\pi \pi}+n_{\pi e} v_{2}^{\pi e}+n_{r e m} v_{2}^{r e m}
$$

Region 3 is completely dominated by $\gamma_{f a k e} \rightarrow$ remaining. As shown in figure 9.6 , it is reasonable to assume that:

$$
v_{2}^{\text {region } 3}=v_{2}^{\text {rem }}
$$

This set of equations can be solved to calculate $v_{2}^{\gamma, \text { bck }}$ :

$$
v_{2}^{\gamma, \mathrm{bck}}=n_{\pi \pi} v_{2}^{\pi \pi}+n_{\pi e} v_{2}^{\pi e}+n_{r e m} v_{2}^{r e m}
$$

The results for $v_{2}^{\pi \pi}, v_{2}^{\pi e}, v_{2}^{r e m}$, and $v_{2}^{\gamma, \text { bck }}$ are shown in figure 9.8 , for centralities $0-20 \%$ and $20-40 \%$. The $v_{2}^{\gamma, \text { bck }}$ and the purity obtained with the template fits are used to correct the measured inclusive photon flow, and the results are shown in figure 9.9 and figure 9.10. For both centrality intervals the uncorrected and background $v_{2}$ are significantly different, especially for higher photon $p_{\mathrm{T}}$. The impact on the corrected 
inclusive photon flow depends on the purity which has a minimum at intermediate $p_{\mathrm{T}}$. The corrected $v_{2}^{\gamma, \text { inc }}$, with respect to the uncorrected $v_{2}^{\gamma, \text { inc }}$, increases for low $p_{\mathrm{T}}$, and decreases for high $p_{\mathrm{T}}$. A contribution to the systematic uncertainty due to this correction has been estimated to be $1.6 \%$ and $1.3 \%$ for centralities $0-20 \%$ and $20-40 \%$, respectively, and is determined from the difference in purity between the data and MC driven approaches at high photon $p_{\mathrm{T}}$.
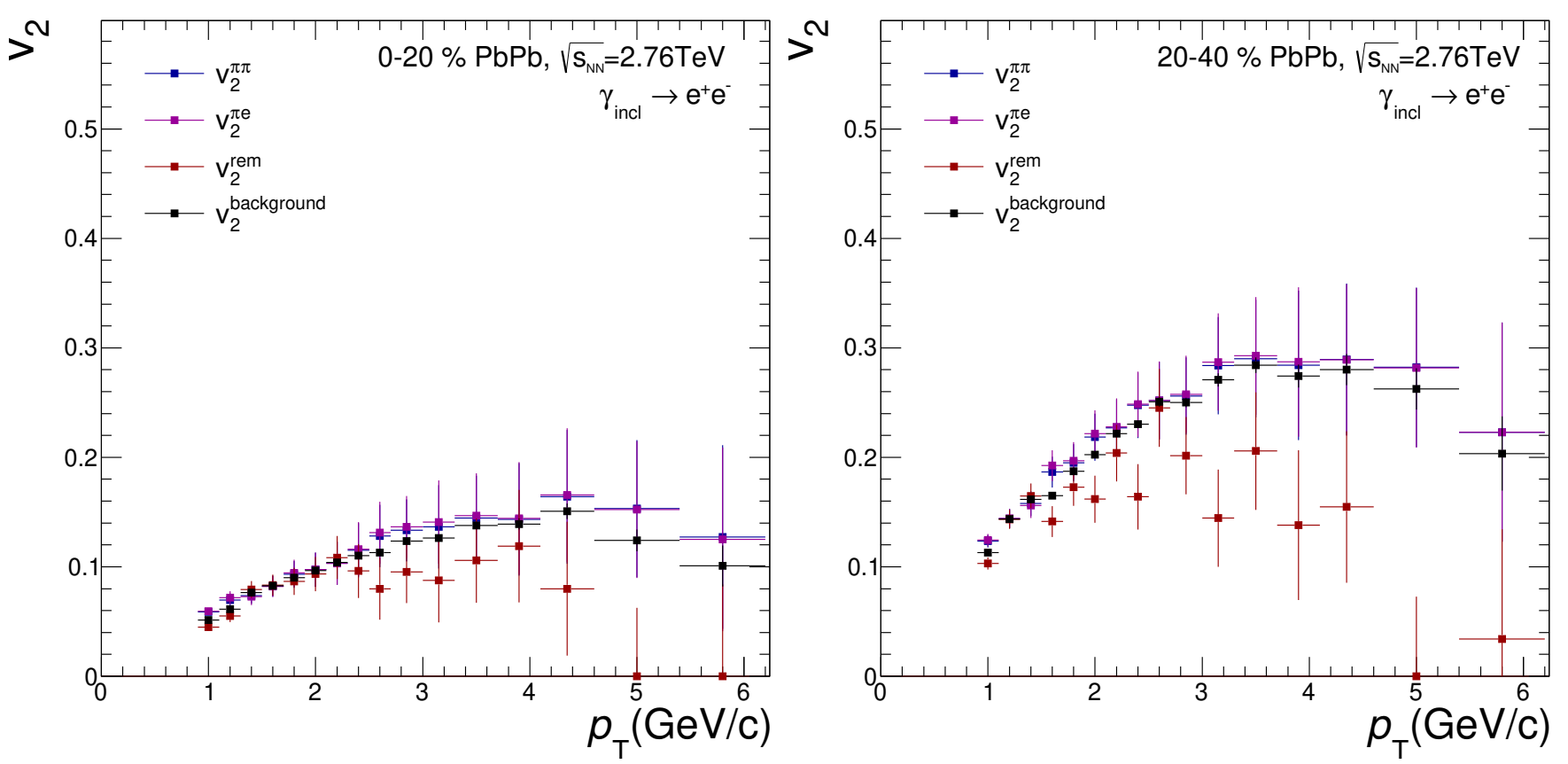

FIGURE 9.8: The $v_{2}$ of the different background sources with the resulting $v_{2}^{\gamma, \text { bck }}$ in the signal region for the centrality classes $0-20 \%$ (left) and $20-40 \%$ (right). 

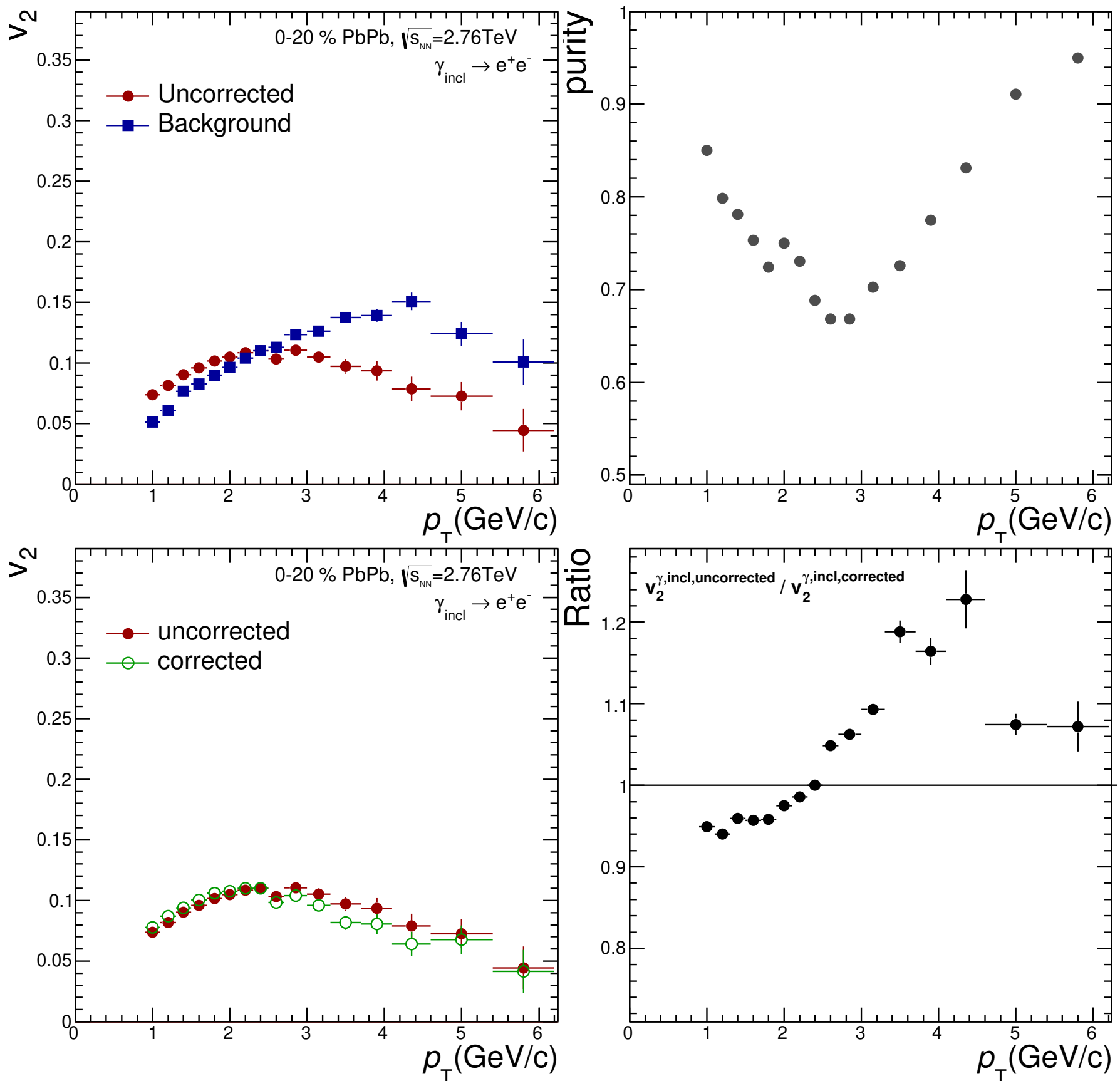

FIGURE 9.9: Overview of the magnitude of the background flow correction that is applied on the inclusive photon sample for collision centrality $0-20 \%$. It shows the uncorrected $v_{2}^{\gamma, \text { inc }}$ and $v_{2}^{\gamma, \text { bck }}$ (top left), the purity of the photon sample (top right), the uncorrected and corrected $v_{2}^{\gamma, \text { inc }}$ (bottom left), and the ratio between the latter two (bottom right). 

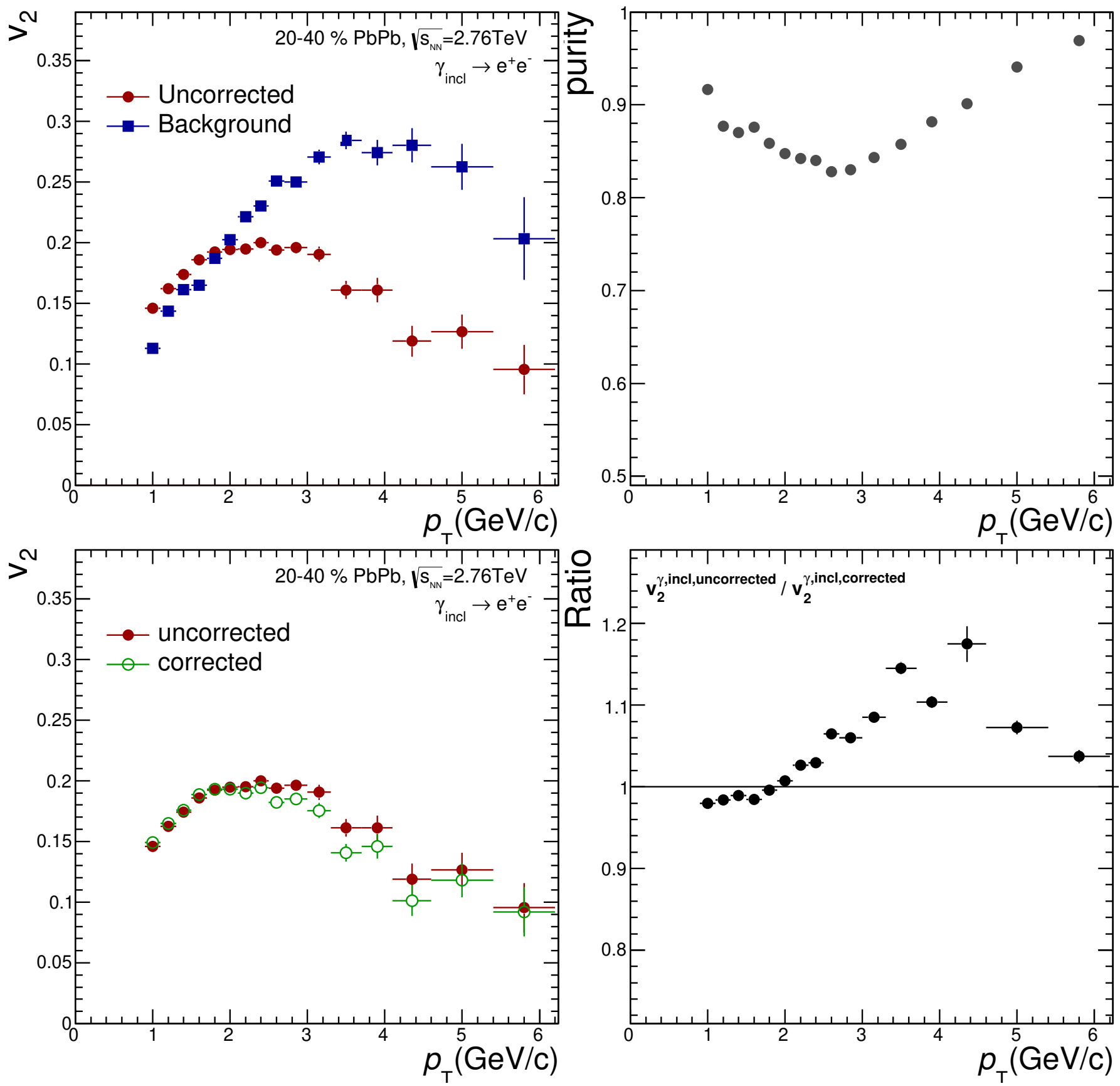

FigURE 9.10: Overview of the magnitude of the background flow correction that is applied on the inclusive photon sample for collision centrality $20-40 \%$. It shows the uncorrected $v_{2}^{\gamma, \text { inc }}$ and $v_{2}^{\gamma, \text { bck }}$ (top left), the purity of the photon sample (top right), the uncorrected and corrected $v_{2}^{\gamma, \text { inc }}$ (bottom left), and the ratio between the latter two (bottom right). 


\subsection{Systematic uncertainties}

\subsubsection{Photon selection}

The conversion photon selection cuts are varied to study the effect on the inclusive photon flow. All variations are chosen such that a reasonable deviation is obtained. The variations are done one at a time with respect to the standard selection cuts. For each variation the following quantity is calculated:

$$
\Delta\left(p_{T}\right)=\left(v_{2}^{\gamma, \text { inc }}\right)_{\text {modified }}\left(p_{T}\right)-\left(v_{2}^{\gamma, \text { inc }}\right)_{\text {standard }}\left(p_{T}\right)
$$

Which quantifies the deviation from $v_{2}^{\gamma, \text { inc }}$ with standard selection cuts for each $p_{\mathrm{T}}$ bin. If the photon selection cut is varied more than once, the assigned systematic is taken from the variation with the largest $\Delta\left(p_{\mathrm{T}}\right)$. The total systematic error on $v_{2}^{\gamma, \text { inc }}$ is assumed to be symmetrically distributed and is defined as:

$$
\sigma_{\text {syst }}\left(p_{T}\right)=\sqrt{\sum_{i}\left(\Delta_{i}\left(p_{T}\right)\right)^{2}},
$$

with $i$ representing all quantities that are varied. Table 9.2 shows the default cut values and the corresponding variations.

\begin{tabular}{l|l|l|l}
\hline Quantity & Standard & Cut variation 1 & Cut variation 2 \\
\hline$|\eta|$ & $<0.9$ & $<0.75$ & \\
$\left(R_{\min }, R_{\max }\right)$ & $(5,180) \mathrm{cm}$ & $(5,70) \mathrm{cm}$ & $(10,180) \mathrm{cm}$ \\
$\min \mathrm{p}_{T}$ & $50 \mathrm{MeV} / \mathrm{c}$ & $40 \mathrm{MeV} / \mathrm{c}$ & $100 \mathrm{MeV} / \mathrm{c}$ \\
$q_{T, \max }$ & $0.05 \mathrm{GeV} / \mathrm{c}$ & $0.03 \mathrm{GeV} / \mathrm{c}$ & $0.1 \mathrm{GeV} / \mathrm{c}$ \\
$\chi^{2} /$ ndf & $<30$ & $<20$ & $<50$ \\
$\Psi_{\text {pair }}$ & $<0.1$ & $<0.05$ & $<0.2$ \\
$\cos \left(\Theta_{P . A .}\right)$ & $>0.85$ & $>0.9$ & $>0.75$ \\
\hline \hline
\end{tabular}

TABLE 9.2: Variations of the inclusive photon selection cuts to evaluate the systematic uncertainty of $v_{2}^{\gamma, \text { inc }}$.

\subsubsection{Other sources}

Apart from the systematic uncertainties arising from photon selection criteria there are contributions from three other sources; the purity of the inclusive photon sample, smearing of the photon energy due to detector effects, and changes in photon reconstruction efficiency in and out of plane. The contribution from the purity of the photons sample has been discussed in the preceding section. The smearing of the photon energy is due to fact that the conversion daughters lose energy with bremsstrahlung, which is lost in reconstruction. A toy model approach has been used to estimate this effect, by unfolding the reconstructed to the true $v_{2}^{\gamma, \text { inc }}$. Because of the high energy resolution of the ITS and TPC, the change to $v_{2}^{\gamma, \text { inc }}$ due to energy smearing is not statistically significant, and it is chosen to apply a relative systematic error of $1 \%$ to the measured $v_{2}^{\gamma, \text { inc }}$. 
In a $v_{2}$ measurement one does not correct for reconstruction efficiency $(\epsilon)$, as it doesn't matter if you reconstruct half or all of your particles of interest, as long as it is not $\varphi$ dependent. However, there can be a small effect on the measured $v_{2}$ if the reconstruction efficiency is different in and out of plane, i.e. for $\epsilon_{\text {in }} \neq \epsilon_{\text {out }}$. This can be the case if the particle density is different in and out of plane. More specifically, the measured $v_{2}$ is split in the contribution $v_{2, \text { true }}$ and $w_{2}$;

$$
v_{2, \text { measured }}=v_{2, \text { true }}-w_{2},
$$

where $w_{2}$ is the modification due to $\epsilon_{\text {in }}-\epsilon_{\text {out }}$. The reconstruction efficiency $\epsilon$ is defined as:

$$
\epsilon=<\epsilon>\left(1-2 w_{2} \cos (2 \varphi)\right)
$$

with the average reconstruction efficiency $\langle\epsilon\rangle$. Considering the equation above for the cases of in and out plane we get the expression

$$
w_{2}=\frac{\left|\epsilon_{\text {in }}-\epsilon_{\text {out }}\right|}{4<\epsilon>}
$$

With a $v_{2} \sim 0.1$, the photon multiplicity in and out of plane is modified by $\pm 10 \%$. Then, $\left|\epsilon_{\text {in }}-\epsilon_{\text {out }}\right|$ is calculated by using $\langle\epsilon\rangle=0.55$ at $0-20 \%$ centrality and $\langle\epsilon\rangle=0.58$ at $40-80 \%$ centrality, and using linear interpolation with the corresponding track multiplicities. For $0-20 \%$ this results in $\left|\epsilon_{\text {in }}-\epsilon_{\text {out }}\right|=0.007$ and as such $w_{2}=0.003$. For $20-40 \%$ this results in $\left|\epsilon_{\text {in }}-\epsilon_{\text {out }}\right|=0.0043$ and as such $w_{2}=0.0019$. The magnitude of $w_{2}$ is rather small compared to the typical $v_{2}^{\gamma, \text { inc }}$ values, and the relative systematic uncertainty to the measured $v_{2}^{\gamma, \text { inc }}$ has been determined to be $3 \%$ and $1.9 \%$, for $0-20 \%$ and $20-40 \%$ collision centrality, respectively.

\subsubsection{Total systematic uncertainty}

The total systematic error with its different contributions are shown in figure 9.11 for the centrality classes $0-20 \%$ and $20-40 \%$. The systematic error increases for increasing $p_{\mathrm{T}}$ and is not very dependent on the centrality class. This is expected since the systematic error is not driven by the multiplicity of the event. 

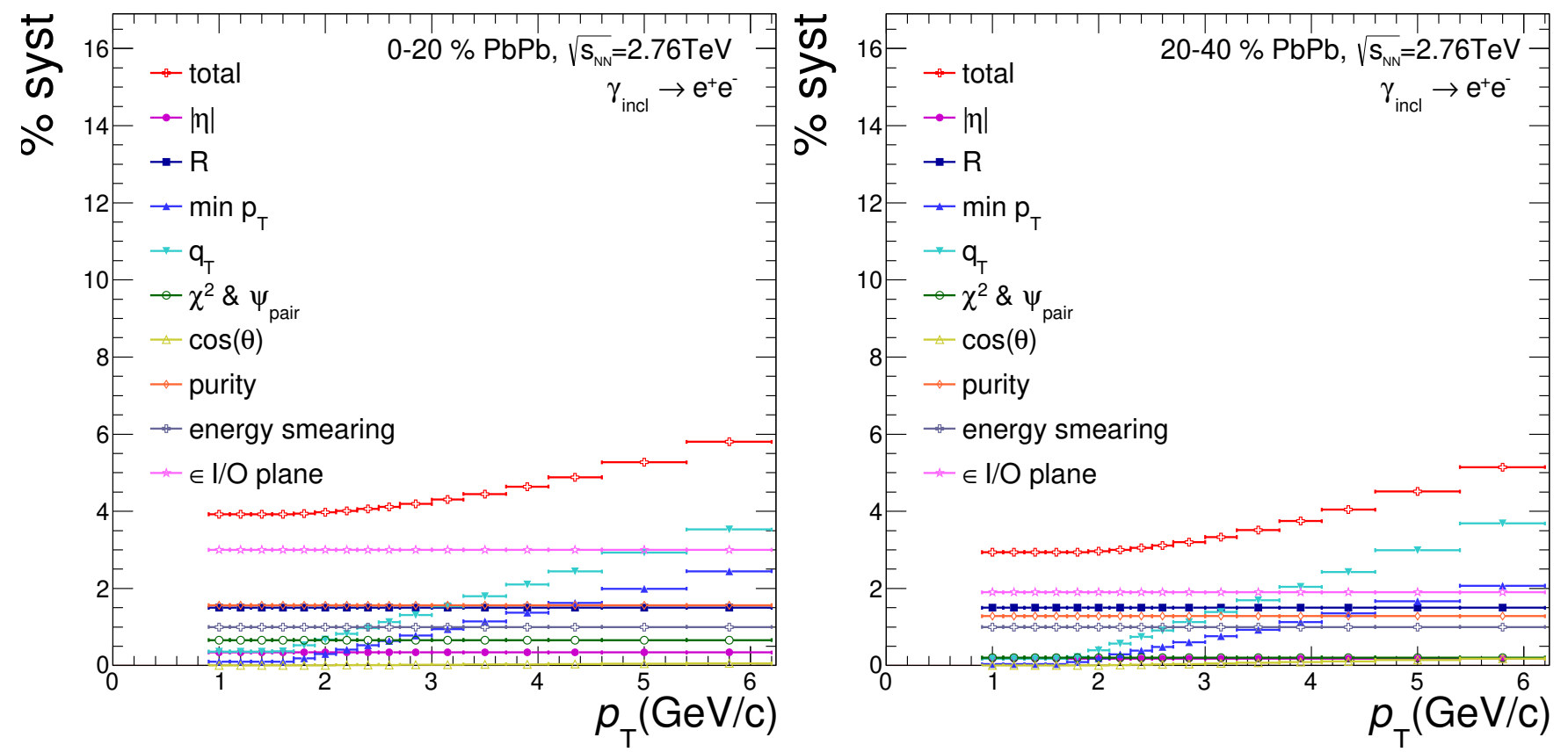

FIGURE 9.11: The total systematic error and the different contributions for the centrality classes $0-20 \%$ (left) and $20-40 \%$ (right), given in relative $\%$. 


\subsection{Results}

In the combination of the inclusive photon $v_{2}$ results from PCM and PHOS, both measurements are treated as independent. Possible correlations due to the use of the same V0A and V0C event plane vectors are found to be negligible. To take into account correlations of the individual measurements in bins of transverse momentum, we describe the measured inclusive photon flows as vectors $\vec{v}_{2}^{\gamma, \text { inc,PCM }}, \vec{v}_{2}^{\gamma, \text { inc, PHOS }}$, where the vector components correspond to the measured $p_{\mathrm{T}}$ bins, and the correlations of the total uncertainties are described by covariance matrices $V_{v_{2}}$, PCM and $V_{v_{2}}$, PHOS, respectively. The elements of the covariance matrix are calculated assuming uncorrelated statistical uncertainties and fully correlated $(\rho=1)$ systematic uncertainties; $V_{i j}=V_{\text {stat }, i j}+V_{\text {syst }, i j}$, where $V_{\text {syst }, i j}=\rho \sigma_{\text {syst }, i} \sigma_{\text {syst }, j}$, for $p_{\mathrm{T}}$ bin $i$ and $j$. Then, the combined inclusive photon flow is the vector

$$
\vec{v}_{2}^{\gamma, \text { inc }}=\left(V_{v_{2}, \mathrm{PCM}}^{-1}+V_{v_{2}, \mathrm{PHOS}}^{-1}\right)^{-1}\left(V_{v_{2}, \mathrm{PCM}}^{-1} \vec{v}_{2}^{\gamma, \text { inc,PCM }}+V_{v_{2}, \mathrm{PHOS}}^{-1} \vec{v}_{2}^{\gamma, \text { inc,PHOS }}\right) .
$$

The inclusive photon $v_{2}$ measured with PCM and PHOS are compared in Fig. 9.12, which shows the ratio of the individual values to the combined flow. The PCM and PHOS measurements are found to be consistent with each other with $p$-values of 0.93 and 0.43 for the centrality classes $0-20 \%$ and $20-40 \%$, respectively.
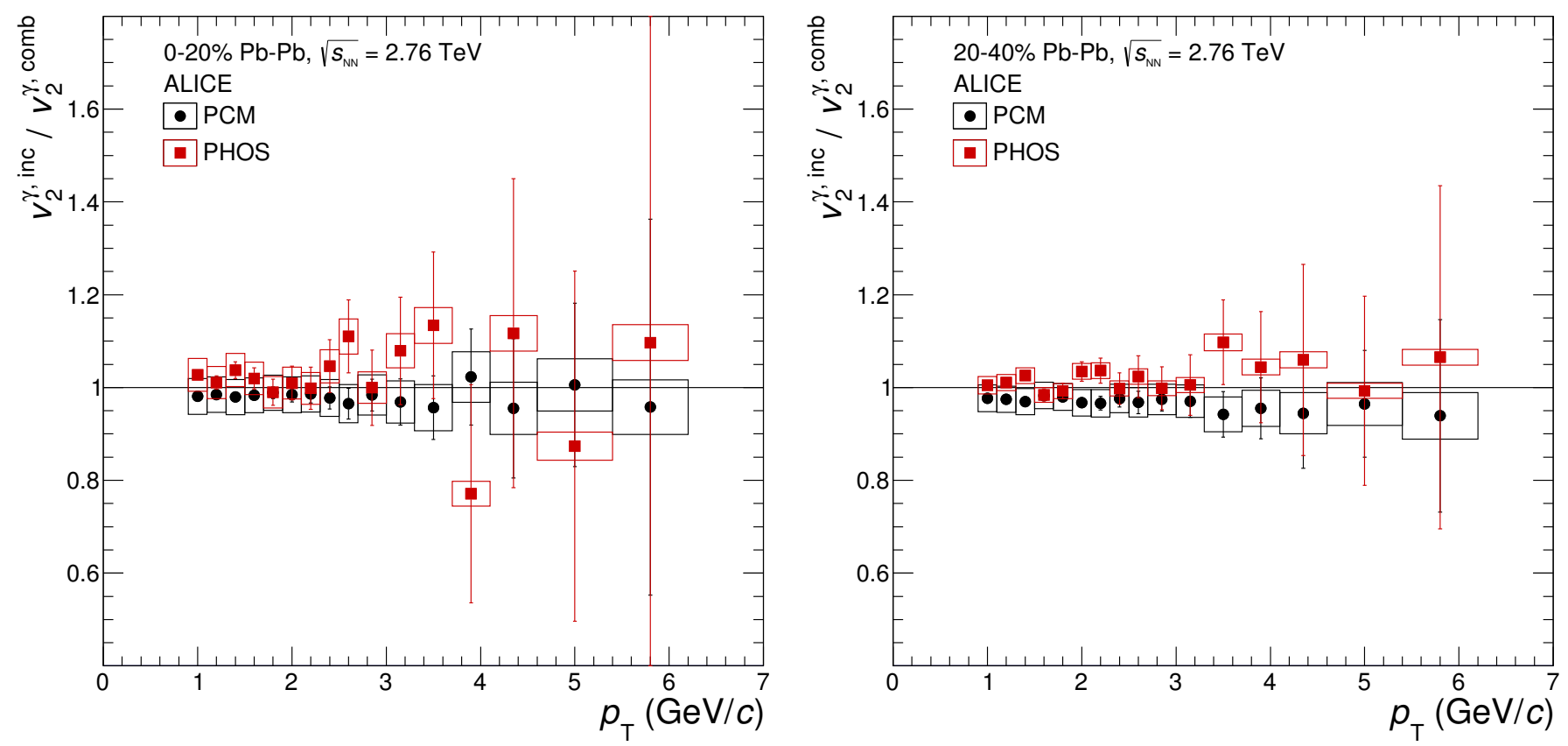

Figure 9.12: (Color online) Comparison of the measured inclusive photon flow $\left(v_{2}^{\gamma, \text { inc }}\right)$ to the individual PCM and PHOS measurements $\left(v_{2}^{\gamma, \text { ind }}\right)$ in the $0-20 \%$ (left) and $20-40 \%$ (right) centrality classes. The individual results are divided by the combined $v_{2}^{\gamma, \text { inc }}$. The vertical bars on each data point indicate the statistical uncertainties and the boxes indicate the systematic uncertainties.

The decay photon flow is estimated using a cocktail simulation. Decays that contribute more than $1 \%$ of the total decay photon yield are taken into account: $\pi^{0} \rightarrow 2 \gamma, \eta \rightarrow 2 \gamma, \omega \rightarrow \gamma \pi^{0}, K_{s}^{0} \rightarrow 2 \pi^{0} \rightarrow 4 \gamma$. Other contributions are negligible compared to the systematic uncertainties of the cocktail. In decays of $\eta$ and $\omega$ mesons only photons produced directly in decays are accounted, while those coming from daughter $\pi^{0}$ 

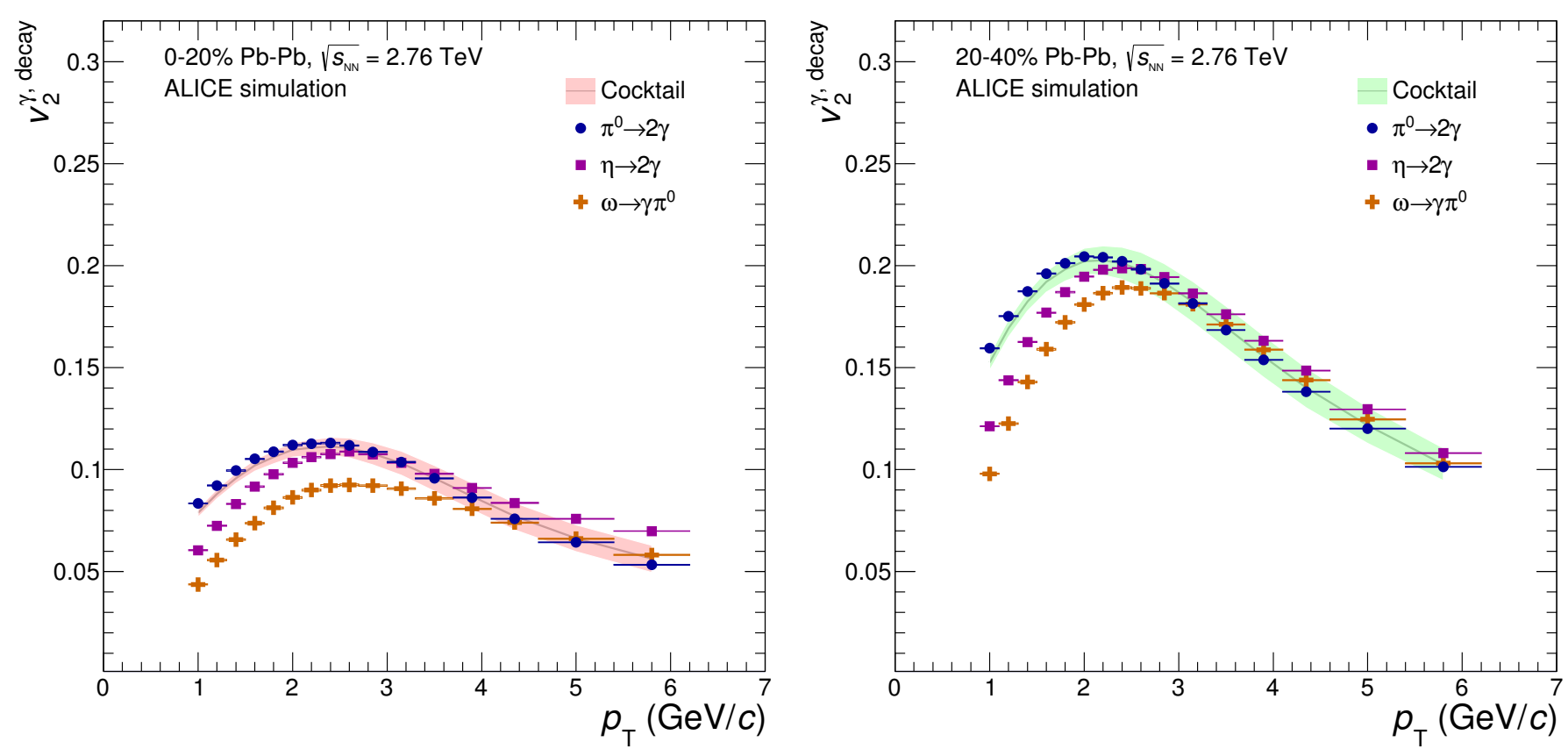

Figure 9.13: (Color online) Elliptic flow of decay photons from $\pi^{0}, \eta, \omega$, and the total cocktail simulation as a function of transverse momentum in the 0-20\% (left) and 20-40\% (right) centrality classes. The band represents the total uncertainty of the total cocktail simulation.

decays are already accounted for in the $\pi^{0}$ contribution. The $K_{s}^{0}$ decay does not contribute significantly to the photon sample measured with the PCM approach. Therefore, we correct the PHOS measurement for this contribution before combining the PHOS and PCM measurements. Here we use the same approach as in the direct-photon spectrum analysis [183], but this time the simulation of the elliptic flow is added. To estimate the elliptic flow of neutral pions, a parametrization has been made of the charged pion flow measured under the same conditions, i.e., charged pions measured in the TPC and reference particles in the V0-A and V0-C detectors $[187,188]$ are used. To estimate the contribution of $\eta$ and $\omega$ mesons, the measured elliptic flow of charged and neutral kaons [187] is scaled, assuming scaling with the transverse kinetic energy $K E_{\mathrm{T}}=m_{\mathrm{T}}-m$. The comparison of different contributions and overall decay photon flow is shown in Fig. 9.13. The $v_{2}$ contributions were added with weights, proportional to the relative decay photon yield of a meson in the total decay yield [183]. The width of the colored band represents the systematic uncertainties of the decay photon elliptic flow $v_{2}^{\gamma \text {,dec }}$. The decay photon flow is mainly determined by the $\pi^{0}$ flow, while other contributions make relatively small corrections: the $\eta$ and $\omega$ contributions slightly reduce the decay photon elliptic flow at $p_{\mathrm{T}}<2 \mathrm{GeV} / c$ and increase it compared to the $\pi^{0}$ contribution at higher $p_{\mathrm{T}}$.

The $v_{2}^{\gamma, \text { inc }}$ measured in two centrality classes together with the $v_{2}^{\gamma, \text { dec }}$ are shown in Fig. 9.14. The elliptic flow coefficients of inclusive photons and decay photons are very similar over the full range $0.9<p_{\mathrm{T}}<$ $6.2 \mathrm{GeV} / c$. As the fraction of direct photon over the inclusive photon yield is relatively small, $\sim 10 \%$ in our $p_{\mathrm{T}}$ range [183], the collective flow of inclusive photons is dominated by the decay photon flow. For both centrality classes the model calculations are overshooting the experimental data. In models based on relativistic hydrodynamics the medium is assumed to be in or close to local thermal equilibrium. An 

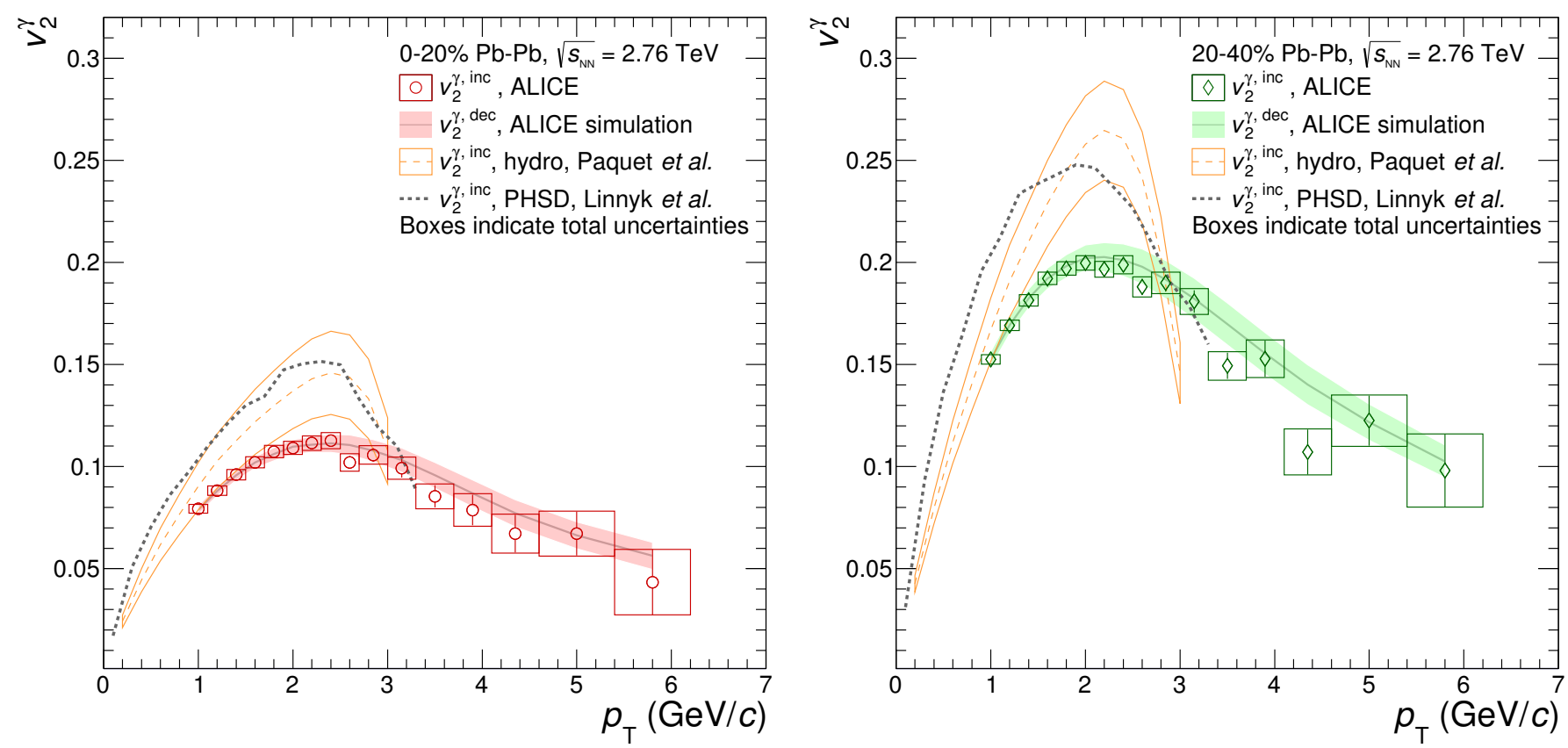

Figure 9.14: (Color online) Elliptic flow of inclusive photons and decay photons, compared to hydrodynamic [192] and transport PHSD [193] model predictions in the 0-20\% (left) and 20-40\% (right) centrality classes. The vertical bars on each data point indicate the statistical uncertainties and the boxes indicate the sizes of the total uncertainties.

equation of state is used to relate thermodynamic quantities like temperature, energy density, and pressure. Photon production is modeled by folding the space-time evolution of a collision with temperature-dependent photon production rates in the QGP and the hadron gas. Another approach is taken, e.g., in the PHSD transport model in which the QGP degrees of freedom are modeled as massive strongly-interacting quasiparticles [189]. For both classes of models the development of a strong early elliptic flow, necessary to reproduce the observed direct-photon flow, gives rise to a large pion elliptic flow at freeze-out and therefore to a large inclusive photon elliptic flow. It is therefore an important test to check whether a model can describe both the inclusive and the direct-photon elliptic flow. The prediction of the hydrodynamic model described in [190] for the inclusive photon $v_{2}$ in the range $1<p_{\mathrm{T}}<3 \mathrm{GeV} / c$ is about $40 \%$ above the data, though the magnitude of the elliptic flow of unidentified hadrons is reproduced within 10-20\% accuracy in this $p_{\mathrm{T}}$ range [191]. The PHSD model [189] also predicts an $\sim 40 \%$ higher inclusive photon flow, even though it reproduces the unidentified hadron flow well. This discrepancy is not resolved.

The direct-photon $v_{2}$ is calculated from the combined PCM and PHOS photon excess $R_{\gamma}$ [183], the combined inclusive $v_{2}$, and the calculated decay photon $v_{2}$. In the propagation of uncertainties, the relatively small significance of the photon excess of about 1-3 standard deviations (depending on the centrality class and $p_{\mathrm{T}}$ interval) requires special attention. This is illustrated for a selected $p_{\mathrm{T}}$ interval in the left panel of Fig. 9.15 which shows the obtained $v_{2}^{\gamma \text {,dir }}$ and its uncertainty as a function of the photon excess $R_{\gamma}$. The Gaussian function in this panel represents the measured value of $R_{\gamma}$ in this $p_{\mathrm{T}}$ interval (dashed line) and its $1 \sigma$ total uncertainty (dark blue shaded area). For $R_{\gamma} \lesssim 1.05$ one loses the sensitivity to $v_{2}^{\gamma \text {,dir }}$ as the uncertainties, 
indicated by the red shaded band, increase drastically. With the current uncertainties on $R_{\gamma}$ we cannot rule out completely that $R_{\gamma} \lesssim 1.05$.
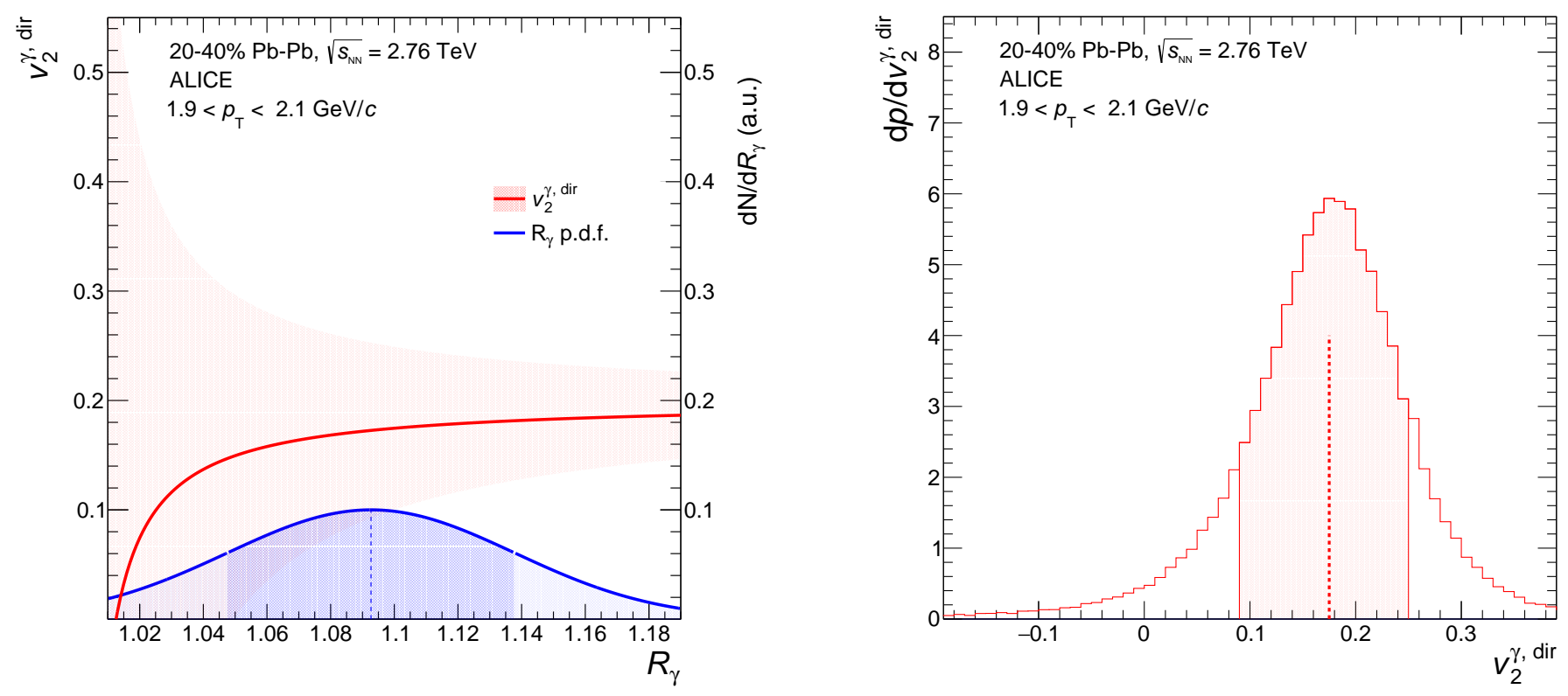

FIGURE 9.15: (Color online) Left: Central value (solid red line) and uncertainty of the direct-photon $v_{2}$ for a selected $p_{\mathrm{T}}$ interval. The upper and lower edges of the red shaded area correspond to the total uncertainty of $v_{2}^{\gamma, \text { dir }}$ as obtained from linear Gaussian propagation of the uncertainties $\sigma\left(v_{2}^{\gamma, \text { inc }}\right)$ and $\sigma\left(v_{2}^{\gamma, \text { dec }}\right)$. The Gaussian (with arbitrary normalization) reflects the measured value of $R_{\gamma}$ in this $p_{\mathrm{T}}$ interval (blue dashed line) and its $\pm 1 \sigma$ uncertainty (dark-blue shaded interval). Right: Posterior distribution of the true value of $v_{2}^{\gamma, \text { dir }}$ for the same interval in the Bayesian approach. Note that the distribution has a non-Gaussian shape, implying that the $\pm 2 \sigma$ interval typically corresponds to a probability of less than $95.45 \%$ as would be the case for a Gaussian.

We address the limited significance of the direct-photon excess by employing a Bayesian approach. The parameters $R_{\gamma, \mathrm{t}}, v_{2}^{\gamma, \text { inc,t }}, v_{2}^{\gamma, \text { dec,t }}$ denoting the true values carry the index "t" and the measured quantities $R_{\gamma, \mathrm{m}}, v_{2}^{\gamma, \mathrm{dec}, \mathrm{m}}, v_{2}^{\gamma, \mathrm{dec}, \mathrm{m}}$ the index "m". Note that $R_{\gamma, \mathrm{t}}$ is restricted to its physically allowed range $\left(R_{\gamma, \mathrm{t}} \geq 1\right)$, while the measured value $R_{\gamma, \mathrm{m}}$ can fluctuate below unity. The posterior distribution of the true parameters can be written as

$$
P(\vec{\vartheta} \mid \vec{m}) \propto P(\vec{m} \mid \vec{\vartheta}) \pi(\vec{\vartheta}), \quad \pi(\vec{\vartheta}) \equiv \pi\left(\vec{R}_{\gamma, \mathrm{t}}\right)=\Theta\left(R_{\gamma, \mathrm{t}, 1}-1, \ldots, R_{\gamma, \mathrm{t}, n}-1\right)
$$

where in $\vec{m}=\left(\vec{R}_{\gamma, \mathrm{m}}, \vec{v}_{2}^{\gamma, \text { inc,m }}, \vec{v}_{2}^{\gamma, \text { dec,m }}\right), \vec{\vartheta}=\left(\vec{R}_{\gamma, \mathrm{t}}, \vec{v}_{2}^{\gamma, \text { inc,t }}, \vec{v}_{2}^{\gamma, \text { dec,t }}\right)$. Here we use the notation introduced in Eq. (9.19): vectors represent sets of measurements in different $p_{\mathrm{T}}$ bins and $n$ is the number of these bins. The function $\pi\left(\vec{R}_{\gamma, \mathrm{t}}\right)$ encodes the prior knowledge about $R_{\gamma}$. The multivariate Heaviside $\Theta$ function corresponds to a constant (improper) prior for $R_{\gamma, \mathrm{t}} \geq 1$. The probability to observe a certain set of measured values given the true values is modeled with multivariate Gaussians $G(\vec{x} ; \vec{\mu}, V)$ (where $\vec{\mu}$ is the vector of mean values and $V$ is the covariance matrix):

$$
P(\vec{m} \mid \vec{\vartheta})=\prod_{x=R_{\gamma}, v_{2}^{\gamma, \text { inc }}, v_{2}^{\gamma, \text { dec }}} G\left(\vec{x}_{\mathrm{m}} ; \vec{x}_{\mathrm{t}}, V_{x}\right)
$$


By sampling the posterior distribution $P(\vec{\vartheta} \mid \vec{m})$, we obtain triplets $\left(R_{\gamma}, v_{2}^{\gamma, \text { inc }}, v_{2}^{\gamma, \text { dec }}\right)$ for each $p_{\mathrm{T}}$ bin from which we calculate $v_{2}^{\gamma \text {,dir }}$ according to Eq. 9.2. An example of the resulting distribution for $v_{2}^{\gamma, \text { dir }}$ is shown in Fig. 9.15 (right panel). The medians of the $v_{2}^{\gamma \text {,dir }}$ distributions are taken as central values. The lower and upper edges of the error bars correspond to values of $v_{2}^{\text {dir }}$ at which the integral of the $v_{2}$ distribution is $15.87 \%$ and $84.13 \%$ of the total integral. In case of a Gaussian distribution this corresponds to $1 \sigma$ uncertainties.

The results for the direct-photon elliptic flow for the two centrality classes, $0-20 \%$ and $20-40 \%$, are shown in Fig. 9.16. The total uncertainties, reflecting the Bayesian posterior distributions, are shown as boxes, and the error bars represent statistical uncertainties. The correlation of $v_{2 \text {,dir }}$ points for different $p_{\mathrm{T}}$ bins as quantified by the correlation matrix is strong at low $p_{\mathrm{T}} \lesssim 2 \mathrm{GeV} / c$ (correlation coefficients typically in the range $0.6-0.75)$ whereas the uncertainties at high $p_{\mathrm{T}}$ are dominated by statistical uncertainties. We compare our results to measurements made at RHIC energies by the PHENIX collaboration [194]. The inclusive photon $v_{2}$ was measured by PHENIX through the reconstruction of $e^{+} e^{-}$pairs from photon conversions and with an electromagnetic calorimeter. The direct-photon elliptic flow in $\mathrm{Au}-\mathrm{Au}$ collisions at RHIC and in $\mathrm{Pb}-\mathrm{Pb}$ collisions at the LHC are found to be compatible within uncertainties. A simple explanation of the large and similar direct-photon elliptic flow for $p_{\mathrm{T}} \lesssim 2 \mathrm{GeV} / c$ at RHIC and the LHC is that the bulk of the thermal direct photons is produced late at temperatures close to the transition temperature $T_{\mathrm{c}}$. This is interesting as naïvely one would expect the $T^{2}$ temperature dependence of the photon emission rate to make the early hot QGP phase after thermalization also the brightest phase.
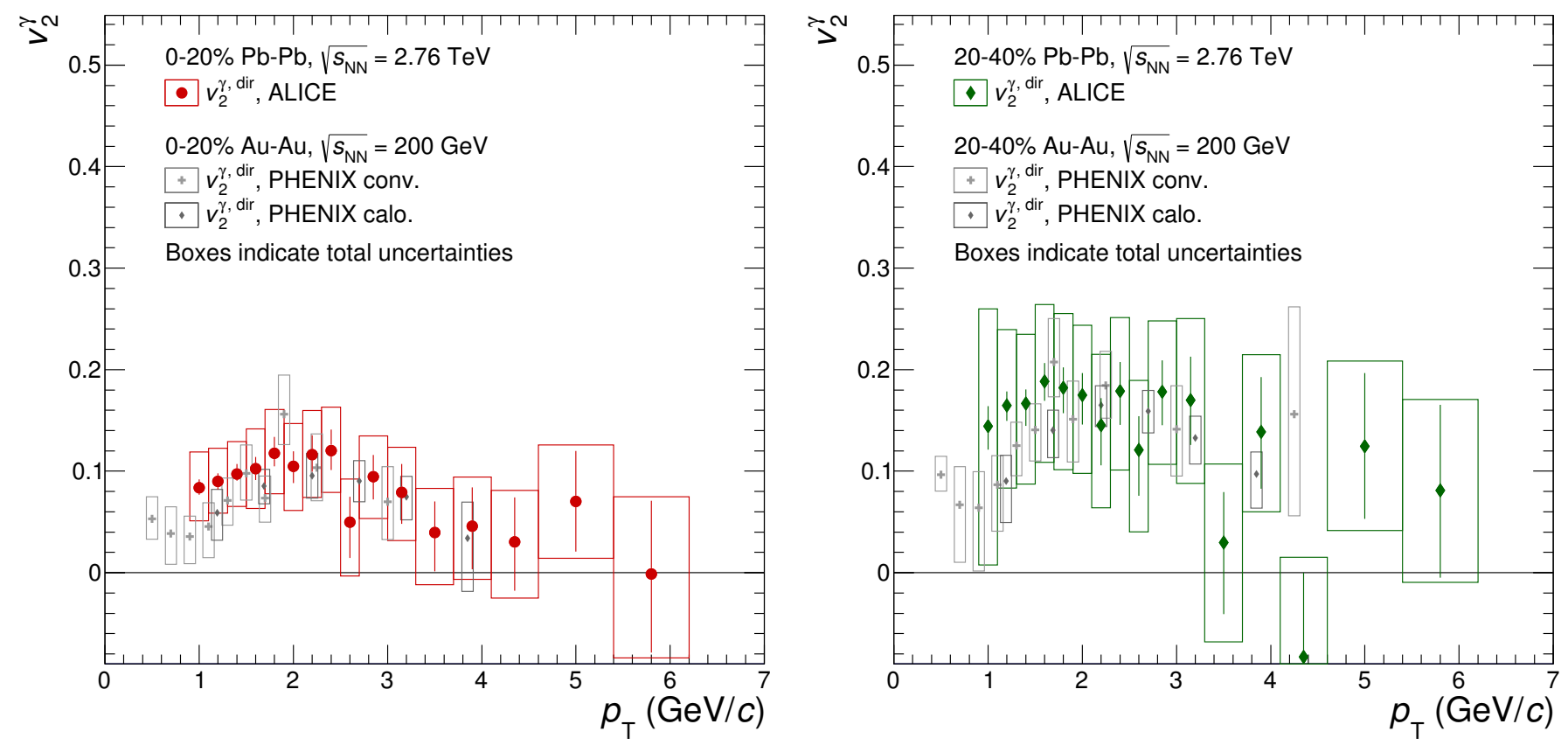

FIGURE 9.16: (Color online) Elliptic flow of direct photons compared with PHENIX results [194] for the 0$20 \%$ (left) and 20-40\% (right) centrality classes. The vertical bars on each data point indicate the statistical uncertainties and the boxes the total uncertainty.

Figure 9.17 compares the measured direct-photon elliptic flow $v_{2}^{\gamma, \text { dir }}$ to the estimated decay photon elliptic 

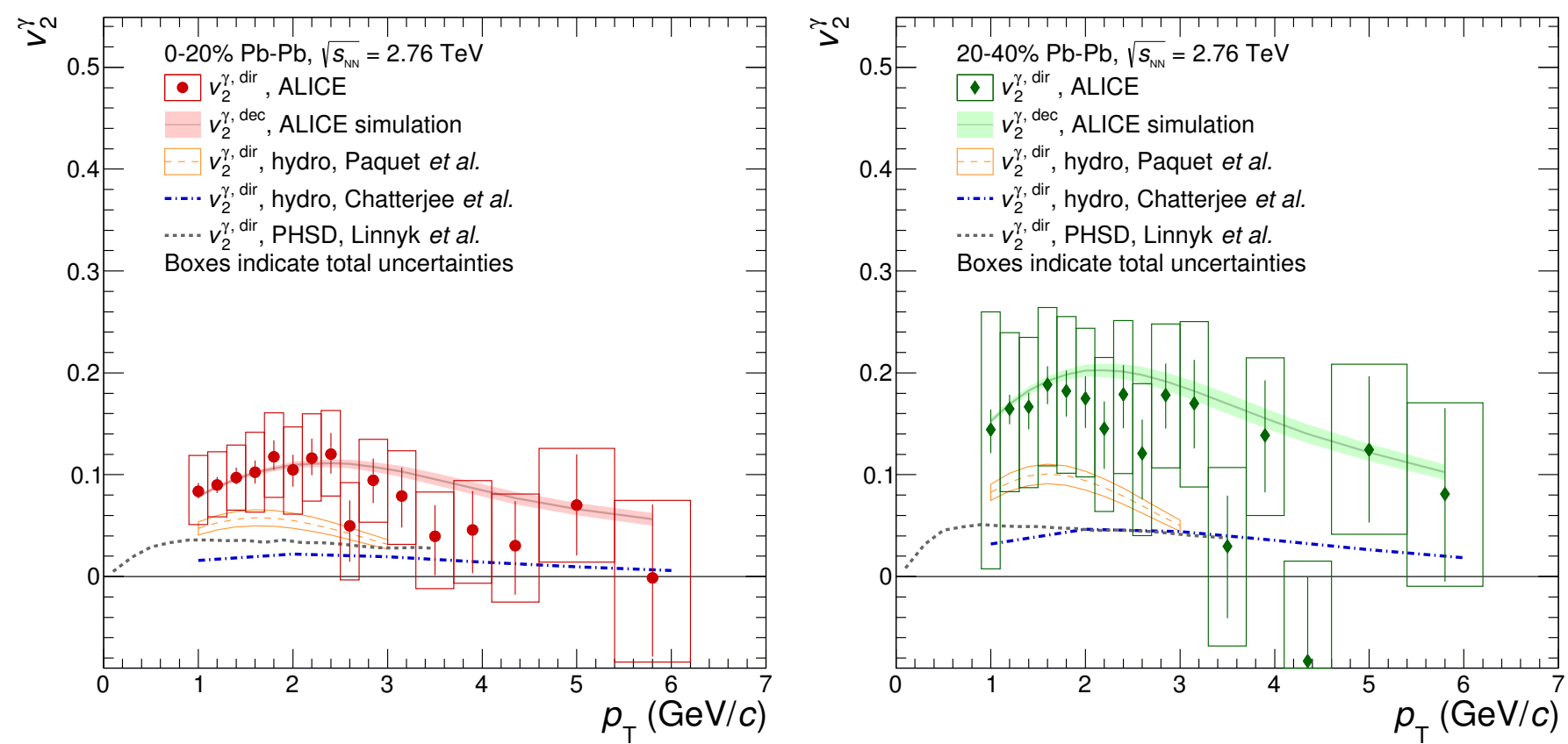

Figure 9.17: (Color online) Elliptic flow of direct photons compared to model calculations in the 0-20\% (left) and $20-40 \%$ (right) centrality classes. The vertical bars on each data point indicate the statistical uncertainties and the boxes the total uncertainty.

flow $v_{2}^{\gamma, \text { dec }}$, marked as cocktail, and to the predictions of several theoretical models. Similarly to measurements at RHIC energies [195], we find that the direct and decay photon elliptic flow are similar. We compare our measurements to state-of-the-art hydrodynamic model calculations $[192,196]$ and the PHSD transport model [189]. The measured direct-photon elliptic flow is systematically higher than theoretical predictions, but is still compatible.

In order to quantify the deviation of the direct-photon $v_{2}$ measurement from a certain hypothesis with a frequentist $p$-value or, equivalently, the corresponding number of standard deviations, we use a Bayesianinspired method [197]. In this approach, the likelihood $L\left(\vec{v}_{2}^{\gamma, \text { inc,m }} \mid \vec{v}_{2}^{\gamma, \text { dir,t }}\right)$ serves as a test statistic and is obtained by integrating over the nuisance parameters $\vec{v}_{2}^{\gamma, \text { dec,t }}$ and $\vec{R}_{\gamma, t}$ using their Bayesian posterior distributions as weights. We focus on the interval $0.9<p_{\mathrm{T}}<2.1 \mathrm{GeV} / c$ in which the contribution of thermal photons is expected to be important. The significance of the deviation from the hypothesis $v_{2}^{\gamma, \text { dir,t }}=0$ for individual $p_{\mathrm{T}}$ bins is in the range $1.8-2.1 \sigma$ for the $0-20 \%$ class and $0.9-1.5 \sigma$ for the $20-40 \%$ class. We also go a step further and estimate the combined significance of the deviation from the hypothesis $v_{2}^{\gamma, \text { dir }} \equiv 0$ for this $p_{\mathrm{T}}$ interval. This tests in addition how well the shape of $v_{2}^{\gamma, \text { inc,m }}$ as a function of $p_{\mathrm{T}}$ agrees with $v_{2}^{\gamma, \text { dec,m }} / R_{\gamma}$, i.e., with the expectation for $v_{2}^{\gamma, \text { dir }} \equiv 0$. We estimate the covariance matrix describing the correlation by characterizing the different sources of systematic uncertainties of $R_{\gamma}$, the inclusive, and the decay photon flow as either fully uncorrelated or fully correlated in $p_{\mathrm{T}}$. Varying the assumptions about the correlation of the data points we obtain significances of typically less than $1 \sigma$ for both centrality classes. While the applied method is essential for a meaningful comparison of the $v_{2}^{\gamma \text {,dir }}$ data with different model predictions, the methods to estimate the covariance matrix can be improved in future analyses. 


\subsection{Discussion}

In summary, we report the first measurement of elliptic flow of inclusive and direct photons as a function of transverse momentum in the range $0.9<p_{\mathrm{T}}<6.2 \mathrm{GeV} / c$ for central and semi-central $\mathrm{Pb}-\mathrm{Pb}$ collisions at $\sqrt{s_{\mathrm{NN}}}=2.76 \mathrm{TeV}$. The elliptic flow of inclusive photons was measured with the scalar product method, independently in the electromagnetic calorimeter PHOS and with the photon conversion method where the reference particles in both cases were measured by the V0-A and V0-C detectors. The combined inclusive photon $v_{2}^{\gamma, \text { inc }}$, together with the calculated decay photon $v_{2}^{\gamma, \text { dec }}$ and the previously measured $R_{\gamma}$ are used to calculate the elliptic flow of direct photons. The measured direct-photon flow $v_{2}^{\gamma, \text { dir }}$ appears to be close to the decay photon flow for both centrality classes, similar to observations at lower collision energies. Moreover, the measured $v_{2}^{\gamma, \text { dir }}$ is similar to the measurements by the PHENIX collaboration at RHIC. The considered hydrodynamic and transport models predict a larger inclusive photon elliptic flow (by approximately 40\%) and a smaller direct-photon elliptic flow than observed. With current uncertainties, however, these models are consistent with the presented direct-photon elliptic flow data. Future measurements using a larger statistics dataset will greatly increase the precision of this measurement and allow us to extend the measurement to higher $p_{\mathrm{T}}$, since the statistical uncertainty is dominating the total uncertainty for $p_{\mathrm{T}}>2.0 \mathrm{GeV} / c$ and $p_{\mathrm{T}}>3.0 \mathrm{GeV} / c$ for the PHOS and PCM inclusive photon flow measurement, respectively. In addition, a larger statistics dataset will also help to constrain the systematic uncertainties on the inclusive and decay photon flow, as well as the measurement of $R_{\gamma}$ over the whole $p_{\mathrm{T}}$ range. A further reduction of the systematic uncertainties is expected from improved detector knowledge. For instance, in case of PCM the largest systematic uncertainty in the measurement of $R_{\gamma}$ is related to modeling the material in which the photons convert. Calibrating regions of the detector with less well known material budget based on regions with very well known material might significantly reduce the overall material budget uncertainty. The $R_{\gamma}$ measurement can be improved further by measuring neutral pion and eta meson spectra in a combined PCM-calorimeter approach in which one decay photon is measured through conversion and the other with a calorimeter. 


\section{Chapter 10}

\section{Direct photon HBT}

\subsection{Introduction}

HBT correlations, as explained in chapter 2, are able to probe the space-time evolution of the QGP by measuring the two particle correlation function $C\left(Q_{\text {inv }}\right)$, as it is sensitive to the size of the emitting source $[17,61,62,198,199]$. As direct photons escape the medium when they are produced, they are not affected by rescattering and hadronization effects, which makes direct photon HBT uniquely suited to access information of the early stage of the collision [200, 201].

In contrast to $\mathrm{Pb}-\mathrm{Pb}$ collisions, it is expected that there is no significant contribution to the HBT signal from a thermal source in pp collisions, as the yield of direct photons in this system is at lower $p_{\mathrm{T}}$ is very small [202]. There could still be a small HBT signal in pp collisions as there are prompt photons produced in the hard interactions. A strong correlation signal would indicate an origin that is unrelated to the HBT effect, but e.g. relates to effects like jet fragmentation. In $\mathrm{Pb}-\mathrm{Pb}$ collisions the magnitude of this effect is expected to be diminished, due to the large particle multiplicities of the bulk.

In this chapter, the results of the direct photon $\mathrm{HBT}$ correlations in both $\mathrm{pp}$ and $\mathrm{Pb}-\mathrm{Pb}$ collisions at a center-of-mass energy of $5.02 \mathrm{TeV}$ will be presented. The results for pp collisions are rather surprising, and lead to experimental limitations for results obtained in $\mathrm{Pb}-\mathrm{Pb}$ collisions. This will now be discussed in detail. 


\subsection{Analysis method}

The HBT correlation of direct photons is measured using

$$
C\left(Q_{\text {inv }}\right)=\frac{A\left(Q_{\text {inv }}\right)}{B\left(Q_{\text {inv }}\right)}
$$

where for massless particles, such as photons, the relative momentum of one of the particles relative to the pair momentum $\left(Q_{\text {inv }}\right)$ is equal to the invariant mass of the two-particle system, i.e. $Q_{\text {inv }}=M_{\mathrm{inv}}$. The terms $A\left(Q_{\text {inv }}\right)$ and $B\left(Q_{\text {inv }}\right)$ are the same-event and mixed-event pair distributions, respectively. The method to obtain $C\left(Q_{\text {inv }}\right)$ has many similarities to obtaining the yield of neutral mesons as presented in an earlier chapter, e.g. $A\left(Q_{\text {inv }}\right)$ is equivalent to the same-event photon pair distribution of the neutral mesons and $B\left(Q_{\text {inv }}\right)$ is equivalent to the photon pair distributions obtained with the event-mixing method (see chapter 6). To obtain the HBT correlation of direct photons, the invariant mass distribution of photon pairs is divided by the distribution obtained by the event-mixing method, and this is done in intervals of pair momentum $k_{\mathrm{T}}=p_{\mathrm{T}, \text { pair }} / 2$.

Assuming a Gaussian source of the emitted photons, the HBT signal can be approximated by fitting $C\left(Q_{\text {inv }}\right)$ with

$$
f\left(Q_{\text {inv }}\right)=1+\lambda \exp \left(-R^{2} Q_{\text {inv }}^{2}\right)
$$

where $\lambda$ is a free parameter of the fit, and $R$ the size of the source [203]. Furthermore, the photons coming from hadronic decays are effectively uncorrelated with each other and with the direct photons, as the decay photons from for example the $\pi^{0}$ meson come from a source with $c \tau=25 \mathrm{~nm}$. This would create a signal with a width on the order of $Q_{\mathrm{inv}}=10 \mathrm{eV}$, and this is unresolvable by the detector. Then, $\lambda$ is proportional to the excess of direct photons $r_{\gamma}$ via

$$
\lambda=\frac{1}{2} r_{\gamma}^{2}, \quad \text { and } \quad r_{\gamma}=\frac{N_{\text {dir }}}{N_{\text {inc }}}
$$

where $N_{\text {dir }}$ and $N_{\text {inc }}$ equal the amount of direct and inclusive photons. As an example, for a direct photon excess of $8 \%, r_{\gamma}=0.08$ defining $\lambda=0.003$. Typical source sizes are $4-8 \mathrm{fm}$. This means that for $Q_{\text {inv }}=0$, $f\left(Q_{\text {inv }}\right)=1+\lambda \simeq 1.003$. This gives a good estimate for the typical HBT-like signal for $Q_{\text {inv }} \sim 0$ in $\mathrm{Pb}-\mathrm{Pb}$ collisions [204].

As the HBT correlation is the strongest for small values of $Q_{\text {inv }}$, which increases with the photon energies and the opening angle between the correlated particles, it is important to use a photon reconstruction method which is able to resolve photons that are close in space, as well as having low energy thresholds. To accommodate this, the photons are reconstructed using the PCM-PHOS reconstruction method, meaning 
that the converted photons that are reconstructed in the central barrel (ITS and TPC) are paired with the photons reconstructed by the PHOS calorimeter. This has the advantage that the magnetic field curves the path of the electrons of the converted photon, making it possible to pair photons with very small opening angles, as well as avoiding correlations from detector effects such as shower overlaps. Furthermore, the standard procedure of only selecting PHOS clusters that do not have a charged track pointing to the cluster removes the correlations between the converted photon and its electron daughters.

All the reconstruction and selection criteria for the photons used in this analysis are exactly the same as in the PCM-PHOS analysis of the neutral meson invariant yield (chapter 6), and their description will be omitted in this section to avoid repetition. In addition, the implementation of the event-mixing method is identical to the one used for the neutral meson invariant yield, i.e. using multiple FIFO buffers storing the last 80 photons with the event characteristics listed in Table 6.1. The analysis uses the minimum bias pp and central and semi-central $\mathrm{Pb}-\mathrm{Pb}$ data recorded in 2017 and 2018, respectively. The datasets are listed in Table 6.3 and Table 6.6.

\section{$10.3 \quad$ Uncertainties}

The analysis presented in this chapter will only include statistical uncertainties, as its magnitude is too large to even determine whether an HBT effect is observed. This also hinders the calculation of the source size of the QGP, for now making the calculation of systematic uncertainties obsolete. The statistical uncertainties of the same-event and event-mixed pair distributions are equal to $\sigma_{\text {stat }}=\sqrt{N}$, where $N$ is the contents of the interval in $Q_{\text {inv }}$. This statistical uncertainty of $C\left(Q_{\text {inv }}\right)$ is calculated assuming uncorrelated uncertainties. This is strictly speaking not true, as both $A\left(Q_{\text {inv }}\right)$ and $B\left(Q_{\text {inv }}\right)$ use the same data, and in particular, $B\left(Q_{\text {inv }}\right)$ uses the same data multiple times. But as the statistical uncertainties of $B\left(Q_{\text {inv }}\right)$ are so small, it is safe to neglect this.

\subsection{Results}

\subsubsection{Photon correlations in pp collisions}

As explained in the previous section, the correlation function $C\left(Q_{\text {inv }}\right)$ is calculated by dividing the sameevent pair distributions by the uncorrelated pair distributions obtained with the event-mixed method. These distributions as measured in pp collisions at $\sqrt{s}=5.02 \mathrm{TeV}$ are shown in Fig. 10.1 for $250<k_{\mathrm{T}}<$ $500 \mathrm{MeV} / c$, for photons reconstructed in experimental data, Monte Carlo data on reconstructed photon level, and Monte Carlo data on generator level. The difference between the Monte Carlo reconstructed and generator level data is that it either includes or excludes the detector part of the simulation. First, in all cases it shows that the uncorrelated background from the event-mixing method is correctly describing the 

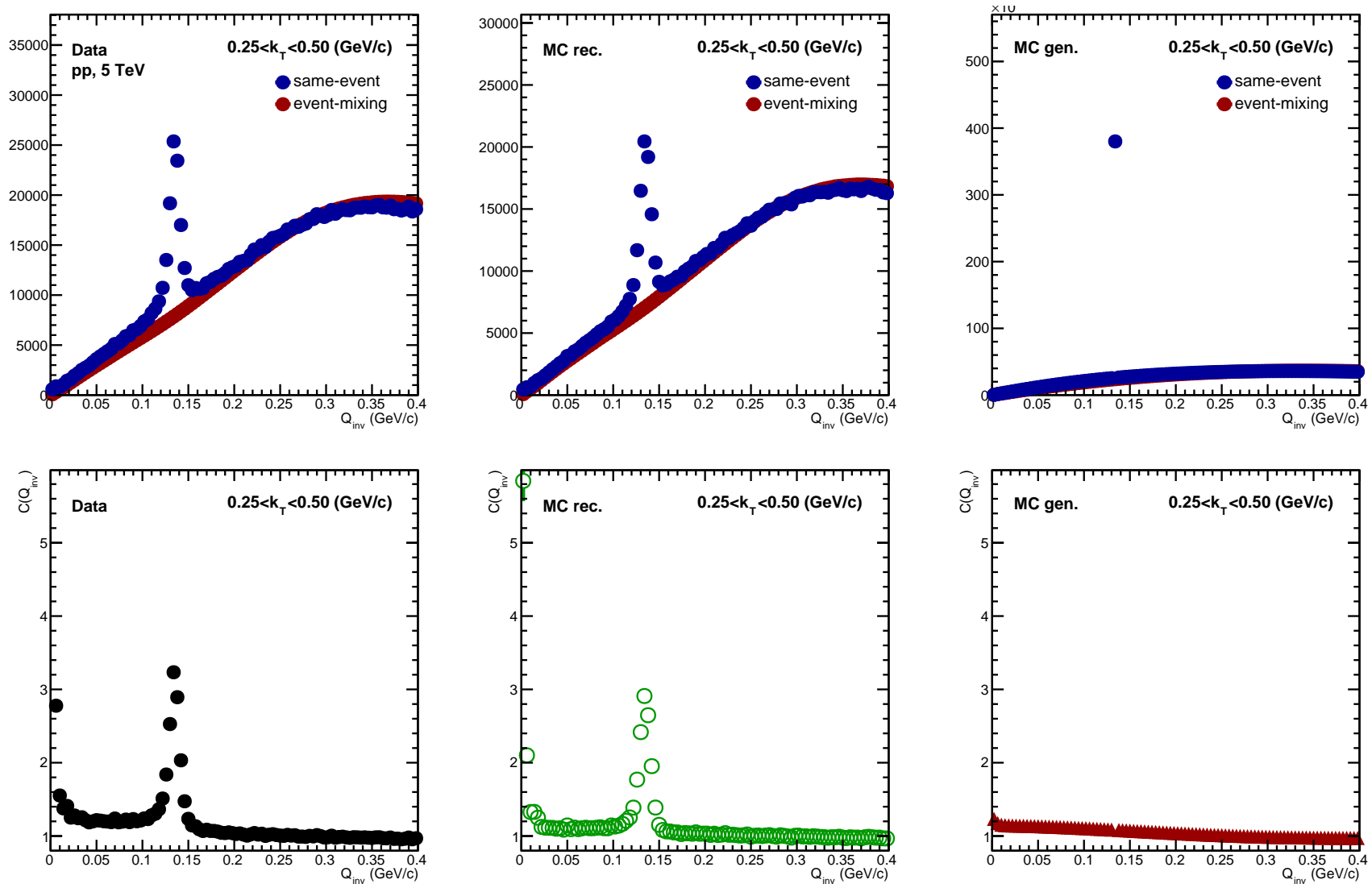

Figure 10.1: Photon pair distributions as function of $Q_{\text {inv }}$ (top) and the correlation function $C\left(Q_{\text {inv }}\right)$ (bottom) for pairs with a relative momentum of $250<k_{\mathrm{T}}<500 \mathrm{MeV} / c$, as measured with PCM-PHOS in pp collisions at $\sqrt{s}=5.02 \mathrm{TeV}$.

same-event pair distributions at higher $Q_{\text {inv }}$. The distributions of the Monte Carlo data on generator level shows the $\pi^{0}$ mass peak as a single bin peak in the same-event distribution, as the reconstructed width is only due to detector effects. Most notably, as shown in the bottom row of Fig. 10.1, there is a strong increase in $C\left(Q_{\text {inv }}\right)$ for $Q_{\text {inv }}<20 \mathrm{MeV} / c$, which is even present on generator level. This indicates a strong correlation or enhancement of same-event pairs with respect to the uncorrelated pairs, most probably due to small angle radiation of additional photons. The more broad enhancement, or slope, that is present for the entire range of $Q_{\mathrm{inv}}$ and that increases with $k_{\mathrm{T}}$, is due to correlations of photons coming from the same parton shower, and this was previously investigated in Chapter 7. Both these enhancements are not HBT effects, and are also much larger in magnitude than what is expected from an HBT signal.

To unfold the detector effects from the data in a simple manner, one can calculate the corrected data by

$$
C\left(Q_{\text {inv }}\right)_{\text {data,corrected }}=C\left(Q_{\text {inv }}\right)_{\text {data }} \frac{C\left(Q_{\text {inv }}\right)_{\mathrm{MC}, \text { generated }}}{C\left(Q_{\text {inv }}\right)_{\mathrm{MC}, \text { reconstructed }}}
$$

The correlation function $C\left(Q_{\text {inv }}\right)$ for data, corrected data, $\mathrm{MC}$ reconstructed data, and $\mathrm{MC}$ generator level data is shown in Fig. 10.2 (left) and Fig. 10.3 (left), for $250<k_{\mathrm{T}}<500 \mathrm{MeV} / c$ and $0.5<$ 
$k_{\mathrm{T}}<1.1 \mathrm{GeV} / c$, respectively. Then, to further explore the differences between data and MC, the ratio $C\left(Q_{\text {inv }}\right)_{\text {data,corrected }} / C\left(Q_{\text {inv }}\right)_{\mathrm{MC}, \text { generated }}$ is calculated and is shown in Fig. 10.2 (right) and Fig. 10.3 (right), for $250<k_{\mathrm{T}}<500 \mathrm{MeV} / c$ and $0.5<k_{\mathrm{T}}<1.0 \mathrm{GeV} / c$, respectively. The main conclusion is that the amplitude of the correlations found in data are about $\sim 10 \%$ stronger with respect to MC, and the strength of the correlation increases with $k_{\mathrm{T}}$. To emphasize, the magnitude of the observed correlations are too large to be an HBT-like effect, as $\lambda \sim 0.003$, which is about 30 times smaller than what is seen here. A large part of this effect is likely related to jet-like correlations as discussed in Chapter 7, which are also for this particular measurement not perfectly described in the simulated data.
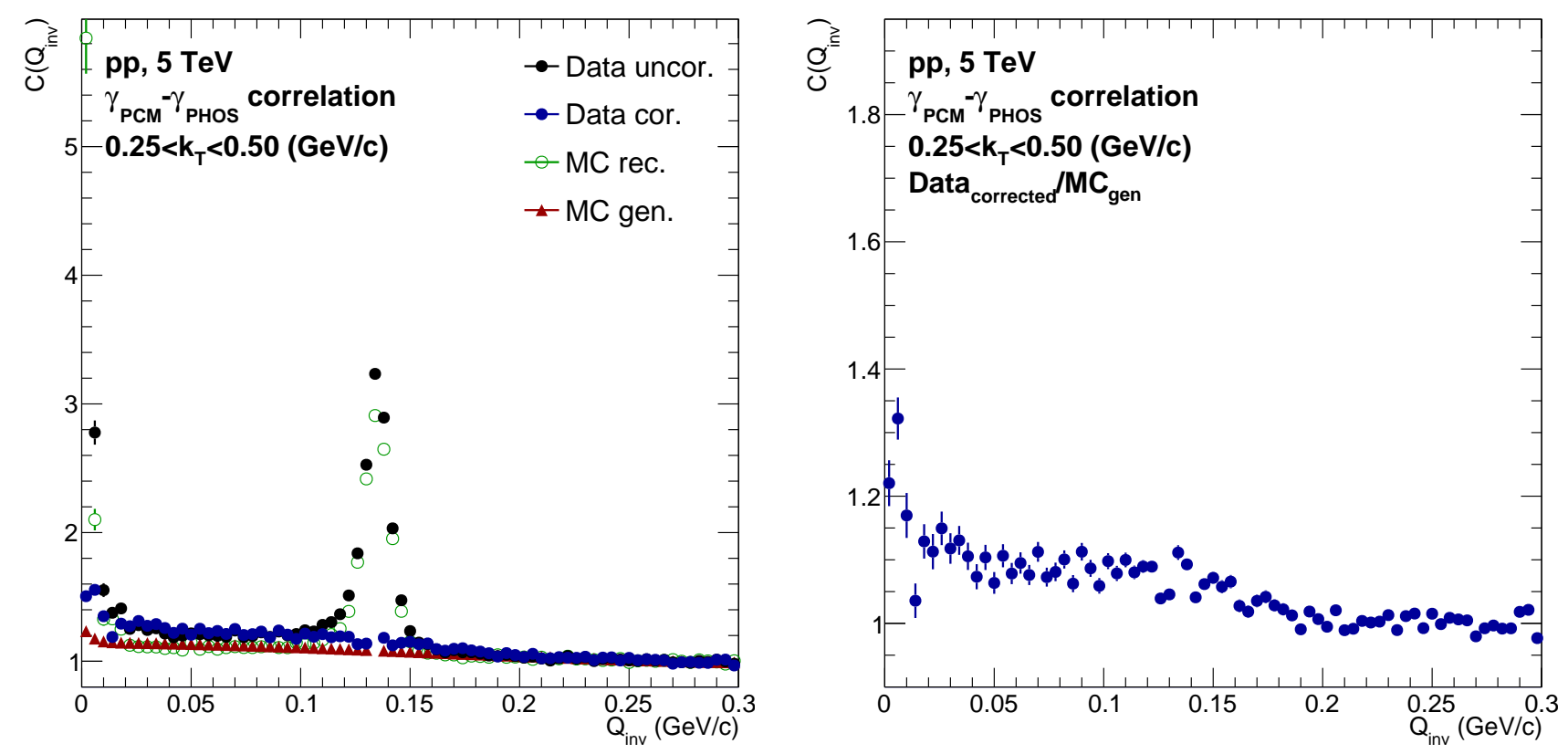

FIGURE 10.2: Photon pair correlations $C\left(Q_{\text {inv }}\right)$ as function of $Q_{\text {inv }}$ for experimental and simulated data (left), and the ratio $C\left(Q_{\text {inv }}\right)_{\text {data,corrected }} / C\left(Q_{\text {inv }}\right)_{\mathrm{MC} \text {,generated }}$ as function of $Q_{\text {inv }}$ (right), both for pairs with a relative momentum of $250<k_{\mathrm{T}}<500 \mathrm{MeV} / c$, as measured with PCM-PHOS in pp collisions at $\sqrt{s}=5.02 \mathrm{TeV}$.

\subsubsection{Photon correlations in $\mathrm{Pb}-\mathrm{Pb}$ collisions}

The same direct photon HBT measurements as presented in the previous section for pp collisions have been performed for central and semi-central $\mathrm{Pb}-\mathrm{Pb}$ collisions. The results for the photon pair distributions as function of $Q_{\text {inv }}$ and the correlation function $C\left(Q_{\text {inv }}\right)$ for pairs with a relative momentum of $250<k_{\mathrm{T}}<$ $500 \mathrm{MeV} / c$ are shown in Fig. 10.4. The main difference is the amount of combinatorial background present in the same-event pair distributions, which makes the $\pi^{0}$ peak hard to distinguish from the rest. The pair distributions obtained with the event-mixing method describes the same-event pair distributions much better, both for experimental and simulated data. The ratio, shown in the bottom row plots, again shows qualitatively the same features as the distribution obtained for pp collisions, although, the amplitude of the correlation function $C\left(Q_{\text {inv }}\right)$ is now much smaller. This is most likely due to the large particle multiplicities, which reduce the importance of jet-related correlations in both signal and background. 

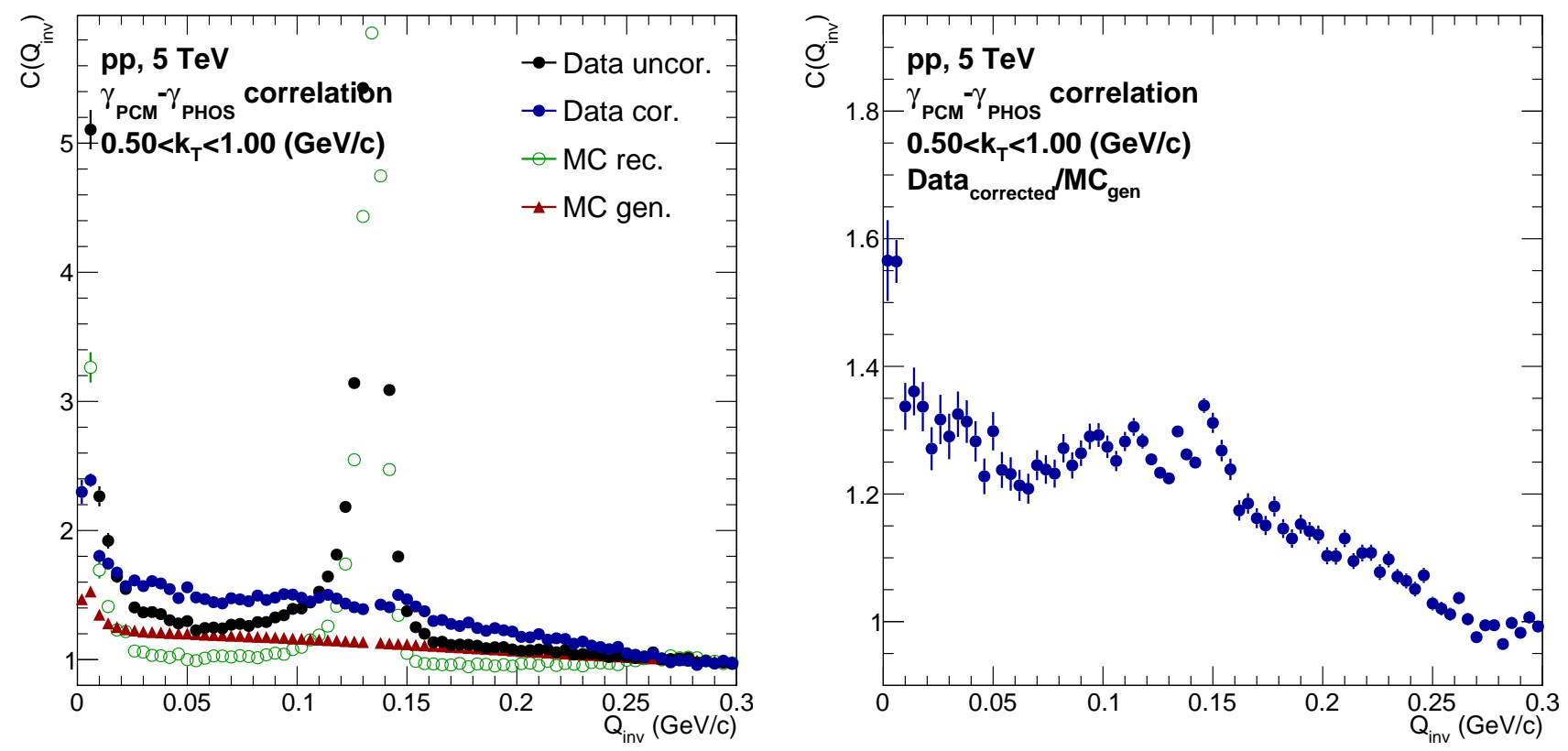

Figure 10.3: Photon pair correlations $C\left(Q_{\text {inv }}\right)$ as function of $Q_{\text {inv }}$ for experimental and simulated data (left), and the ratio $C\left(Q_{\text {inv }}\right)_{\text {data,corrected }} / C\left(Q_{\text {inv }}\right)_{\mathrm{MC} \text {,generated }}$ as function of $Q_{\text {inv }}$ (right), both for pairs with a relative momentum of $0.5<k_{\mathrm{T}}<1.0 \mathrm{GeV} / c$, as measured with PCM-PHOS in pp collisions at $\sqrt{s}=5.02 \mathrm{TeV}$.

In Fig. 10.5 the photon pair correlations $C\left(Q_{\mathrm{inv}}\right)$ as function of $Q_{\mathrm{inv}}$ for experimental and simulated data (left) and the ratio $C\left(Q_{\text {inv }}\right)_{\text {data,corrected }} / C\left(Q_{\text {inv }}\right)_{\mathrm{MC}, \text { generated }}$ as function of $Q_{\text {inv }}$ (right), are shown for central $\mathrm{Pb}-\mathrm{Pb}$ collisions for pairs with a relative momentum of $250<k_{\mathrm{T}}<500 \mathrm{MeV} / c$ and $0.5<k_{\mathrm{T}}<1.0 \mathrm{GeV} / c$, respectively. The same results are shown in Fig. 10.6 for semi-central $\mathrm{Pb}-\mathrm{Pb}$ collisions. There is again a strong enhancement of $C\left(Q_{\mathrm{inv}}\right)<20 \mathrm{MeV} / c$, similar to the results in pp collisions, although also with a diminished magnitude. Furthermore, the ratio plots (right) include as a black line the theoretical prediction for a Gaussian source emitting direct photons with $\lambda=0.003$ and $R=6 \mathrm{fm}$, which would be a typical direct photon HBT-like correlation from a QGP. The results are consistent with this particular choice of parameter values, although the uncertainties are too large to exclude also other values and notably also a null effect.

\subsection{Discussion}

The direct photon HBT effect is studied by calculating the correlation $C\left(Q_{\text {inv }}\right)$ as function of $Q_{\text {inv }}$, where photons candidates are paired to obtain the same-event and mixed-event pair distributions. The results from pp collisions show a large increase in $C\left(Q_{\text {inv }}\right)$ for lower $Q_{\text {inv }}$ and higher $k_{\mathrm{T}}$, which is most likely associated to correlated particle production from parton fragmentations. Furthermore, these correlations are reproduced to a large extent by the MC simulations, and therefore provide information on dynamical photon correlations at small relative momentum rather than an HBT-like effect. 

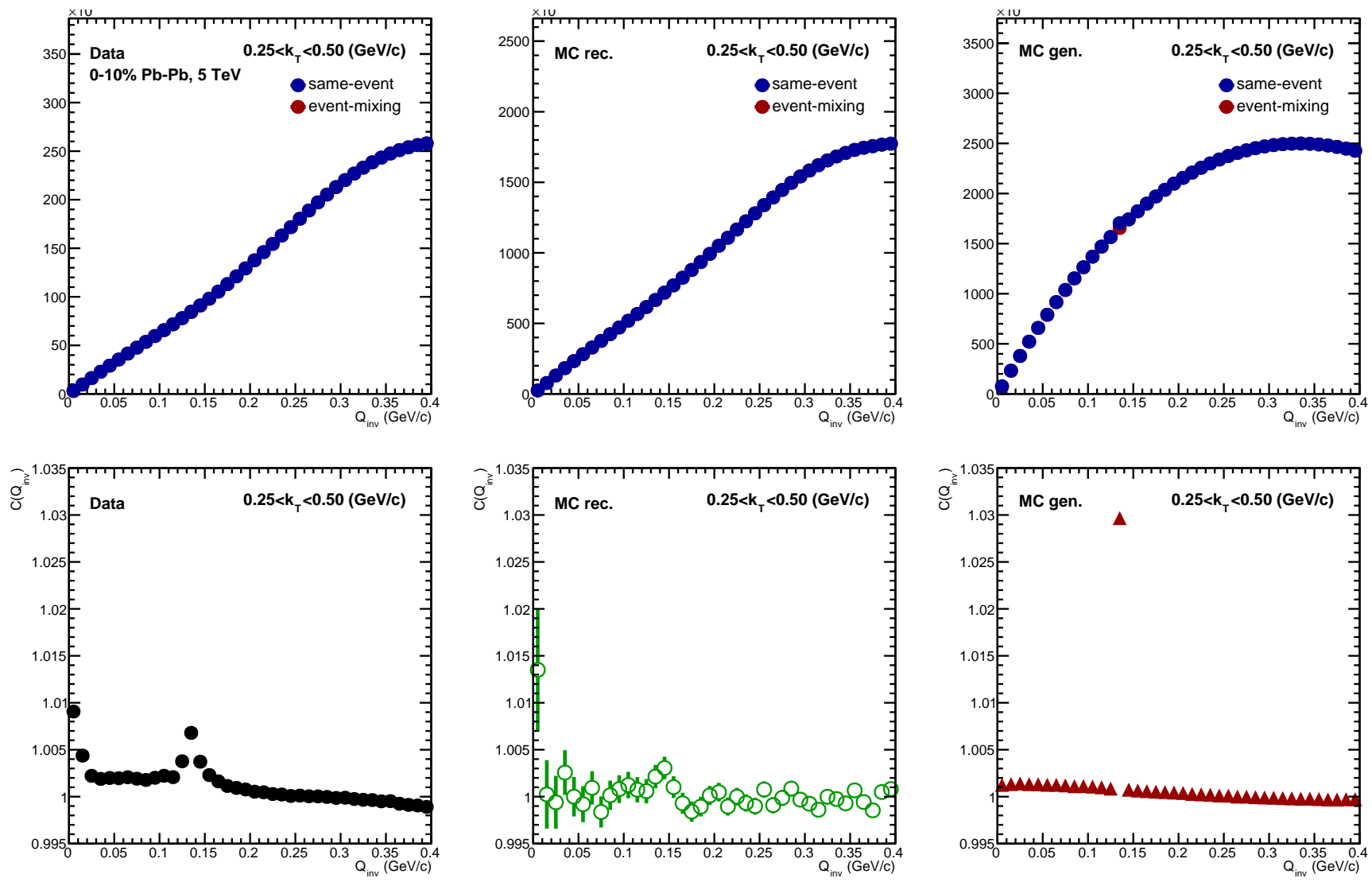

Figure 10.4: Photon pair distributions as function of $Q_{\text {inv }}$ (top) and the correlation function $C\left(Q_{\text {inv }}\right)$ (bottom) for pairs with a relative momentum of $250<k_{\mathrm{T}}<500 \mathrm{MeV} / c$, as measured with PCM-PHOS in central $\mathrm{Pb}-\mathrm{Pb}$ collisions at $\sqrt{s_{N N}}=5.02 \mathrm{TeV}$.

In $\mathrm{Pb}-\mathrm{Pb}$ collisions, the magnitude of the correlation function is much smaller, but still too large to originate from an HBT-like effect. Most likely this signal is again dominated by the same effect as what has been found in the results for pp collisions. However, after dividing out the correlations found in MC simulations, which again are not HBT-like effects, there is a residual correlation that is consistent with the expected signal from a QGP with a size of $6 \mathrm{fm}$ and a direct photon excess $r_{\gamma}=0.08$. The uncertainties, especially from the MC simulation, are too large to reasonably constrain these parameters.

Interestingly, these results are qualitatively consistent with the earlier results found by WA98 experiment [59], which subscribed the enhancement at very low $Q_{\text {inv }}$ possibly to distortions of the photon pairs due to detector effects. It is probable that these effects also play a role for the measurements presented in this chapter, but it seems most important to understand the correlations due to parton fragmentations which are more broad in $Q_{\text {inv }}$. The observation of the jet-related correlation effects is interesting in itself and can possibly shed light on the particle production mechanisms. An understanding of this will be needed to extract a direct photon HBT signal. 

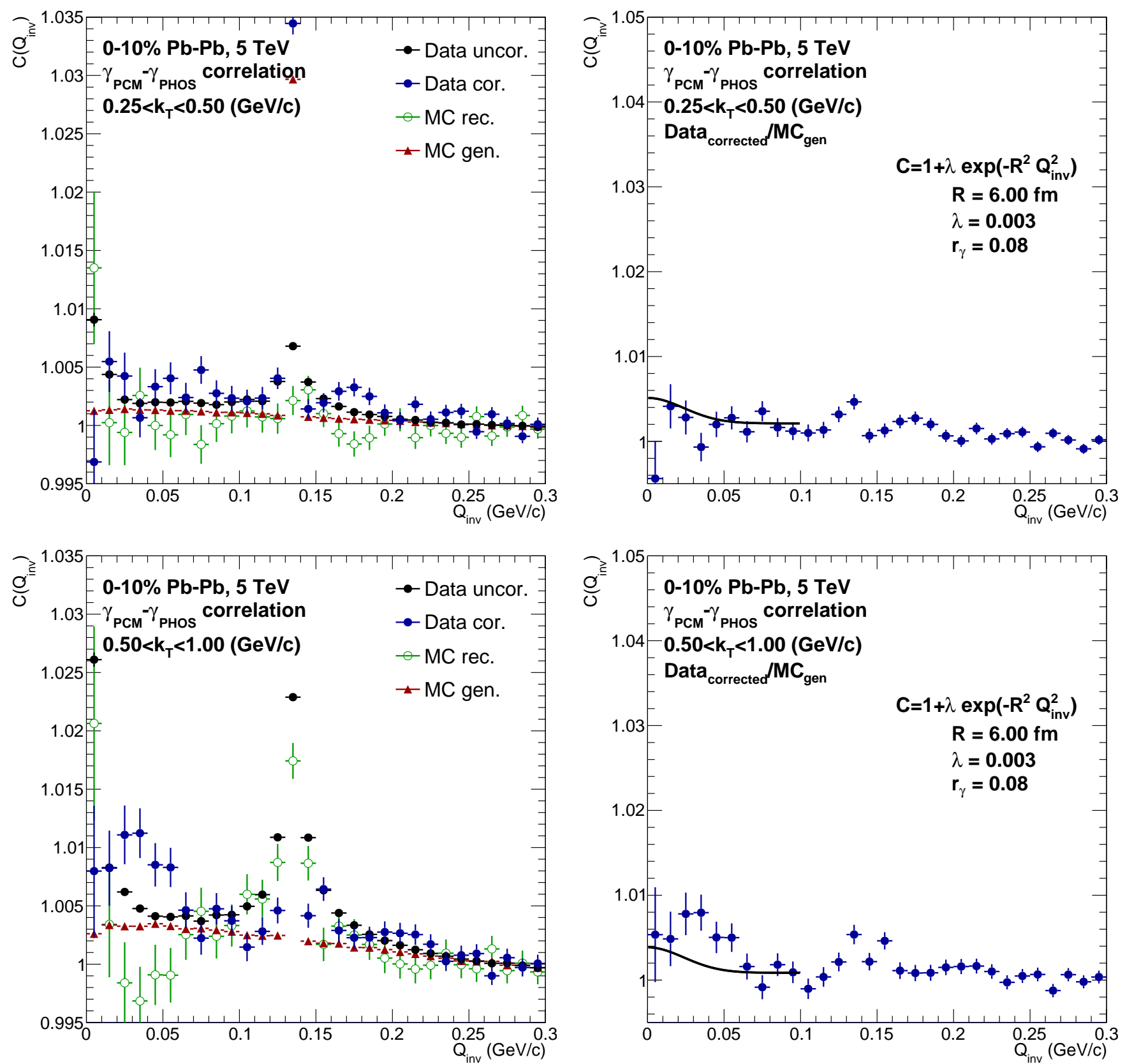

FiguRe 10.5: Photon pair correlations $C\left(Q_{\text {inv }}\right)$ as function of $Q_{\text {inv }}$ for experimental and simulated data (left), and the ratio $C\left(Q_{\text {inv }}\right)_{\text {data,corrected }} / C\left(Q_{\text {inv }}\right)_{\mathrm{MC} \text {,generated }}$ as function of $Q_{\text {inv }}$ (right), both for pairs with a relative momentum of $250<k_{\mathrm{T}}<500 \mathrm{MeV} / c$ (top) and $0.5<k_{\mathrm{T}}<1.0 \mathrm{GeV} / c$ (bottom), as measured with PCM-PHOS in central $\mathrm{Pb}-\mathrm{Pb}$ collisions at $\sqrt{s_{N N}}=5.02 \mathrm{TeV}$. 

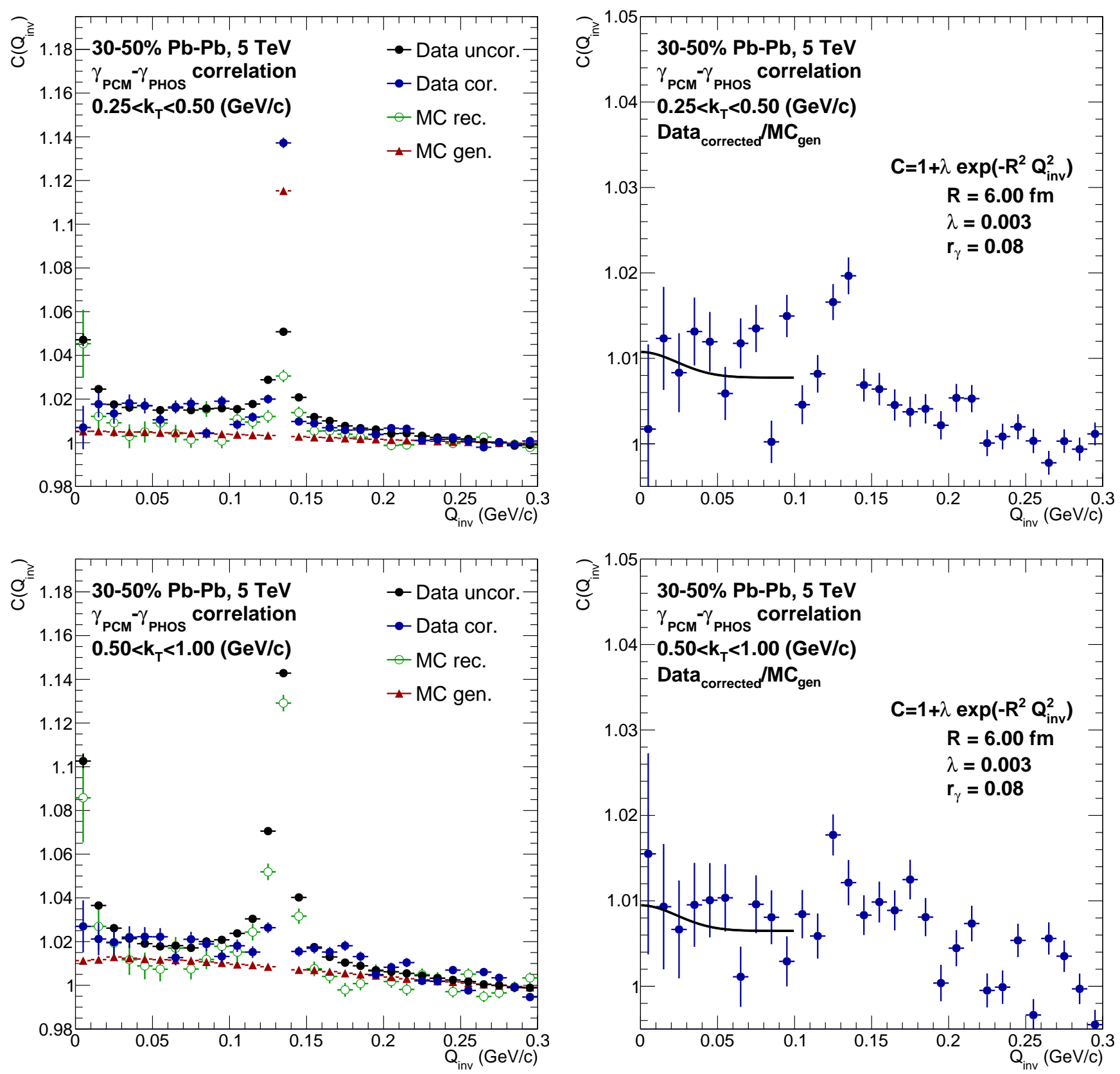

FiguRe 10.6: Photon pair correlations $C\left(Q_{\text {inv }}\right)$ as function of $Q_{\text {inv }}$ for experimental and simulated data (left), and the ratio $C\left(Q_{\text {inv }}\right)_{\text {data,corrected }} / C\left(Q_{\text {inv }}\right)_{\mathrm{MC}, \text { generated }}$ as function of $Q_{\text {inv }}$ (right), both for pairs with a relative momentum of $250<k_{\mathrm{T}}<500 \mathrm{MeV} / c$ (top) and $0.5<k_{\mathrm{T}}<1.0 \mathrm{GeV} / c$ (bottom), as measured with PCM-PHOS in semi-central $\mathrm{Pb}-\mathrm{Pb}$ collisions at $\sqrt{s_{N N}}=5.02 \mathrm{TeV}$. 



\section{Chapter 11}

\section{Photon yield modifications in pp and $\mathrm{Pb}-\mathrm{Pb}$ collisions}

\subsection{Introduction}

The energy of the photons that are produced in $\mathrm{pp}$ and $\mathrm{Pb}-\mathrm{Pb}$ collisions vary event by event and depend on the physical processes that happen in the respective collision. For example, pp collisions that have a highly energetic jet in the final state will produce on average more high energy photons compared to pp collisions with predominantly soft processes. As a consequence, events that contain a high $p_{\mathrm{T}}$ particle in the event should have a different photon spectrum compared to events without a high $-p_{\mathrm{T}}$ particle. This should also be the case in $\mathrm{Pb}-\mathrm{Pb}$ collisions, although there are the hot nuclear matter effects such as energy loss (jet-quenching) and thermal production by the QGP, which will modify the behavior. In addition, if high momentum particles produced in a $\mathrm{Pb}-\mathrm{Pb}$ collisions are largely uncorrelated with the bulk, there should be a smaller relative modification of the photon yield in $\mathrm{Pb}-\mathrm{Pb}$ collisions compared to pp collisions. Also, it is expected that more peripheral $\mathrm{Pb}-\mathrm{Pb}$ collisions are more "pp-like", as the average number of participants at some point equal that of a single pp collision. To this extent it could be interesting to study photon yield modifications due to the presence of a high momentum charged particle in both $\mathrm{pp}$ and $\mathrm{Pb}-\mathrm{Pb}$ collisions. It is to be noted that most of the inclusive photons are produced from hadronic decays, with the $\pi^{0}$ playing the dominant role, meaning that this analysis implicitly studies neutral mesons in addition.

This chapter presents the results on an exploratory study of photon production with and without the presence of a high momentum charged particle, for both $\mathrm{pp}$ and $\mathrm{Pb}-\mathrm{Pb}$ collisions at a center of mass energy of $5.02 \mathrm{TeV}$. The comparison between the results obtained in both collision systems are able to show how the presence of a high $p_{\mathrm{T}}$ particle modifies the photon yield. The analysis method will be discussed first, followed by the results and a discussion. 


\subsection{Analysis method}

The $\mathrm{pp}$ and $\mathrm{Pb}-\mathrm{Pb}$ datasets that are used for the results presented in this chapter are recorded at $\sqrt{s}=$ $5.02 \mathrm{TeV}$ and $\sqrt{s_{N N}}=5.02 \mathrm{TeV}$, respectively, and are the same datasets as used for the neutral meson analysis (chapter 6), with their details listed in Table 6.3 and Table 6.6. The pp dataset LHC15n has been excluded for this exploratory study as LHC17p and LHC17q have a sufficient amount of events. The MC productions LHC18j2 and LHC1818a4 are used to calculate the predictions from PYTHIA and HIJING, respectively. Events are only selected if they satisfy the event selection criteria listed in section 4.3.1.

The photons are reconstructed using the photon conversion method, which is explained in detail in section 5.2. The selection criteria for the converted photons are also the same as for the previous analyses, and are listed in Table 5.3. The charged tracks are reconstructed as explained in section 4.3.3, and the events containing one or multiple primary charged tracks with $p_{\mathrm{T}}>10 \mathrm{GeV} / c$ are labeled as "with trigger", whereas events that do not contain any primary charged tracks above this threshold are labeled as "no trigger". The yield of photons $\left(\mathrm{d} N_{\gamma} / \mathrm{d} p_{\mathrm{T}}\right)$ is measured as function of $p_{\mathrm{T}}$ with $0.2<p_{\mathrm{T}, \gamma}<25 \mathrm{GeV} / c$, for events with and without the trigger, and are normalized to the amount of events in the respective sample.

There are no corrections applied to the measured photon yields, using the assumption that acceptance, efficiency, and purity corrections cancel in the ratio

$$
\left(\frac{\mathrm{d} N_{\gamma} / \mathrm{d} p_{\mathrm{T}}, \text { trigger }}{\mathrm{d} N_{\gamma} / \mathrm{d} p_{\mathrm{T}}, \text { no trigger }}\right)
$$

which is a rather safe assumption in the case where the trigger particle does not greatly influence the overall multiplicity of the event. Even for the large multiplicity variations between central and semi-central collisions the reconstruction efficiency of PCM changes by roughly $5-10 \%$, which is far smaller than the effects that are studied in this analysis. Furthermore, the uncertainties only include statistical uncertainties since this is a purely exploratory study, with $\sigma_{\text {stat }}=\sqrt{N_{\gamma}}$.

\subsection{Results}

In Fig. 11.1 (left) the uncorrected photon yield as function of $p_{\mathrm{T}}$ is shown for pp collisions at $\sqrt{s}=5 \mathrm{TeV}$ with and without trigger, i.e. with and without a charged particle with $p_{\mathrm{T}}>10 \mathrm{GeV} / c$ within the detector acceptance. The experimental data is shown in blue and the simulated MC data in red. Even though the distributions are uncorrected, they clearly indicate an increase of high $-p_{\mathrm{T}}$ photons for events with the trigger, as the spectrum is much harder compared to the spectrum without the trigger. In addition, the experimental data shows a qualitatively similar behavior as the simulated MC data, indicating that PYTHIA correctly reproduces the photon spectrum in the case where a high momentum charged particle is either present or not. In Fig. 11.1 (right), the ratio between the triggered and non-triggered events are 
shown for experimental and simulated MC data. The main observation is that for pp collisions, the ratio changes by 3 orders of magnitude for photons with $0.2<p_{\mathrm{T}, \gamma}<25 \mathrm{GeV} / c$.
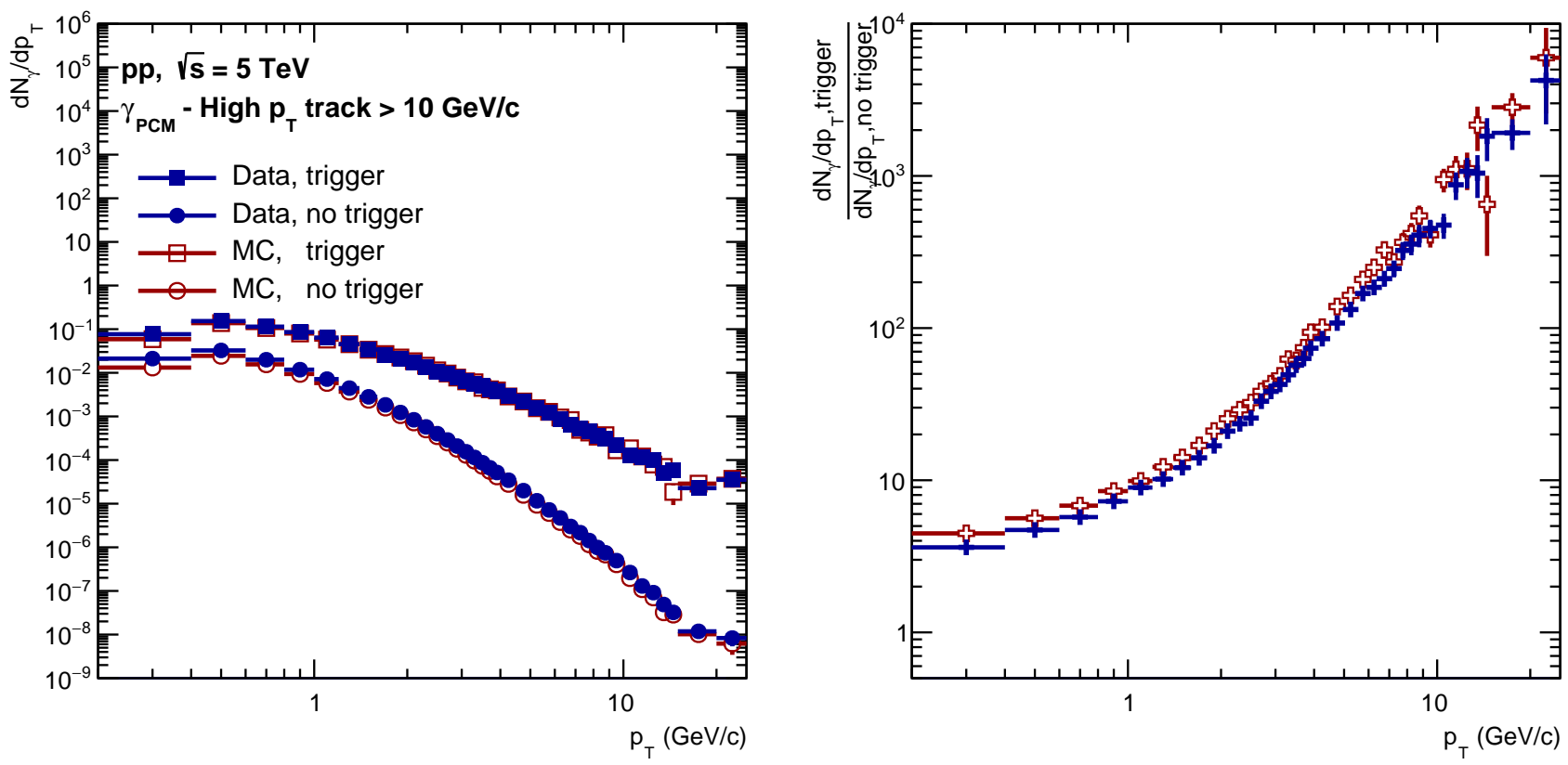

Figure 11.1: The uncorrected yield of photons as function of $p_{\mathrm{T}}$, as measured with PCM in pp collisions at $\sqrt{s}=5 \mathrm{TeV}$ for events with and without a charged particle with $p_{\mathrm{T}}>10 \mathrm{GeV} / c$ within the detector acceptance (left) and the ratio (right). The analysis is performed for experimental (blue) and simulated (red) data.

The same analysis has been performed for $\mathrm{Pb}-\mathrm{Pb}$ collisions at $\sqrt{s_{N N}}=5.02 \mathrm{TeV}$, and the results are shown in Fig. 11.2. It shows the uncorrected photon yield as function of $p_{\mathrm{T}}$ with and without trigger for central (top) and peripheral (bottom) $\mathrm{Pb}-\mathrm{Pb}$ collisions. The experimental data is shown in green and the simulated MC data in red. One of the observations is that, compared to the results obtained in pp collisions, the photon yields with the trigger are modified much less and are closer to the photon yields without trigger, especially for central $\mathrm{Pb}-\mathrm{Pb}$ collisions. Also, the simulated $\mathrm{MC}$ data seems unable to describe the centrality dependence of this observable. For experimental data the ratio changes by 1 order of magnitude for central $\mathrm{Pb}-\mathrm{Pb}$ collisions and by 2 orders of magnitude for peripheral $\mathrm{Pb}-\mathrm{Pb}$ collisions, which is clearly less modification compared to the results in pp collisions. There is still a hardening of the spectrum, but below $\sim 2-3 \mathrm{GeV} / c$ there seems to be almost no modification.

For the next result, the ratio of the yield of photons with and without the trigger for central and semi-central $\mathrm{Pb}-\mathrm{Pb}$ collisions are divided by the same ratio measured in pp collisions. This double ratio is shown in Fig. 11.3, for experimental data in black and simulated $\mathrm{MC}$ in red. It indicates that high photon $p_{\mathrm{T}}$, relative to low photon $p_{\mathrm{T}}$, is more strongly modified for central compared to peripheral $\mathrm{Pb}-\mathrm{Pb}$ collisions when taking the ratio to the result of pp collisions. Also, a stronger overall suppression is observed for the central collisions. This is consistent with the interpretation that peripheral $\mathrm{Pb}-\mathrm{Pb}$ collisions are more $\mathrm{pp}$-like, and that the photon production in central $\mathrm{Pb}-\mathrm{Pb}$ collisions is much more dominated by production from the 

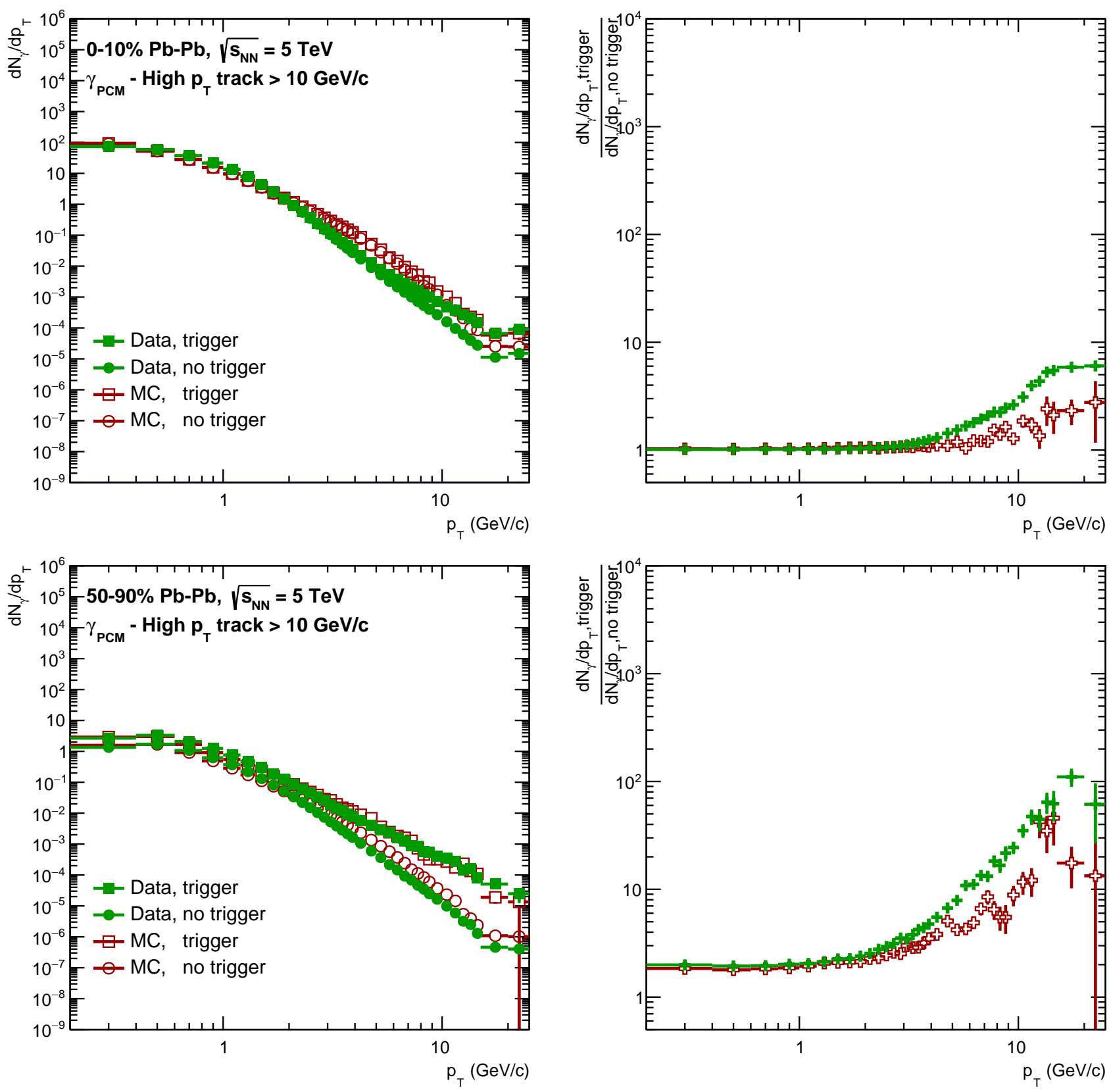

Figure 11.2: The uncorrected yield of photons as function of $p_{\mathrm{T}}$, as measured with PCM in central (top) and peripheral (bottom) $\mathrm{Pb}-\mathrm{Pb}$ collisions at $\sqrt{s_{N N}}=5 \mathrm{TeV}$ for events with and without a charged particle with $p_{\mathrm{T}}>10 \mathrm{GeV} / c$ within the detector acceptance (left) and the ratio (right). The analysis is performed for experimental (green) and simulated (red) data.

bulk. Although the simulated MC data qualitatively describes the data, there is an increasing difference for higher photon $p_{\mathrm{T}}$ which is driven by the $\mathrm{Pb}-\mathrm{Pb}$ results.

Furthermore, it is interesting to test whether the ratios for central and peripheral $\mathrm{Pb}-\mathrm{Pb}$, as shown in Fig. 11.3 (right), can be approximated by superimposing a combination of photon yields $\left(\mathrm{d} N_{\gamma} / \mathrm{d} p_{\mathrm{T}}\right)$ as measured in pp collisions. The superposition of photon yields is defined as 

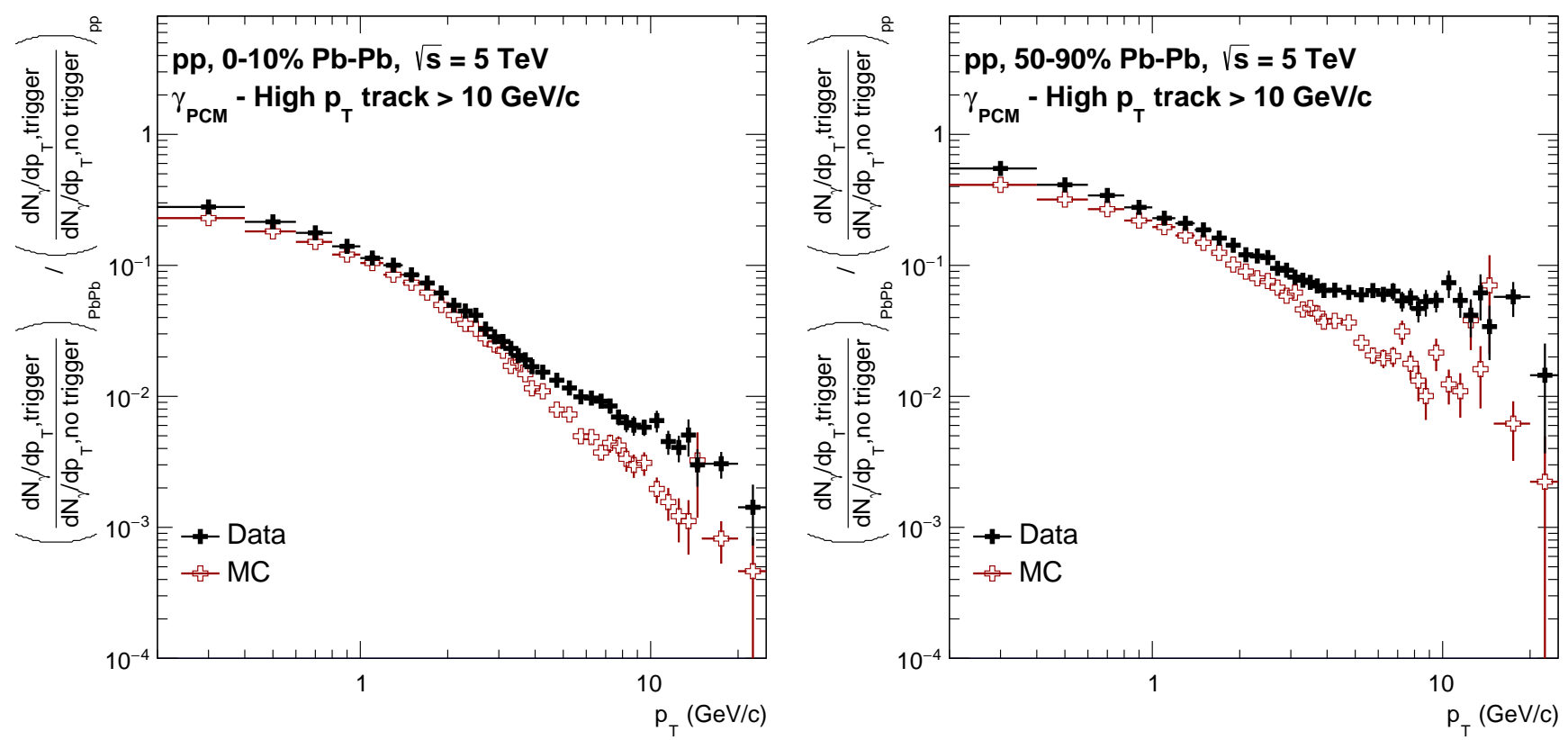

Figure 11.3: The double ratio of the yield of photons with and without trigger, using central (left) and peripheral (right) $\mathrm{Pb}-\mathrm{Pb}$ collisions at $\sqrt{s_{N N}}=5.02 \mathrm{TeV}$, as well as pp collisions at $\sqrt{s}=5.02 \mathrm{TeV}$. The results are calculated for experimental (black) and simulated (red) data using the photon conversion method.

$$
\frac{\mathrm{d} N_{\gamma}}{\mathrm{d} p_{\mathrm{T}} \text { modified }}=\frac{\mathrm{d} N_{\gamma}}{\mathrm{d} p_{\mathrm{T}} \text { trigger }}+A \times \frac{\mathrm{d} N_{\gamma}}{\mathrm{d} p_{\mathrm{T}} \text { no trigger }},
$$

where $A$ is an integer that defines the number of superimposed photon yield spectra without the presence of the trigger particle. It has been chosen to leave $\mathrm{d} N_{\gamma} / \mathrm{d} p_{\mathrm{T}}$, trigger unscaled, i.e. the ratio of the photon yields in $\mathrm{Pb}-\mathrm{Pb}$ collisions will be approximated using a single triggered spectrum summed with $A$ spectra without trigger. The modified ratio then reads

$$
\left(\frac{\mathrm{d} N_{\gamma} / \mathrm{d} p_{\mathrm{T}}, \text { modified }}{(A+1) \times \mathrm{d} N_{\gamma} / \mathrm{d} p_{\mathrm{T}}, \text { no trigger }}\right)_{\mathrm{pp}} .
$$

where $\mathrm{d} N_{\gamma} / \mathrm{d} p_{\mathrm{T}}$, modified is defined in Eq. 11.2. The results for different choices of $A$ are shown in Fig. 11.4, for central (left) and peripheral (right) $\mathrm{Pb}-\mathrm{Pb}$ collisions, using the modified ratio as defined in Eq. 11.3. It turns out that to approximate the photon yield ratio as measured in central $\mathrm{Pb}-\mathrm{Pb}$ collisions, $A$ needs to be higher than 100, as at higher $p_{\mathrm{T}}$ the modified ratio gets too large compared to the ratio as measured in $\mathrm{Pb}-\mathrm{Pb}$ collisions, and $A$ needs to be smaller than 400, as the modified ratio gets too small compared to the ratio as measured in $\mathrm{Pb}-\mathrm{Pb}$ collisions. For $A \sim 250$, the double ratio remains relatively flat with values close to 1 . For peripheral collisions the value of $A \sim 40$ achieves also a flat ratio, although the absolute magnitude is larger. 

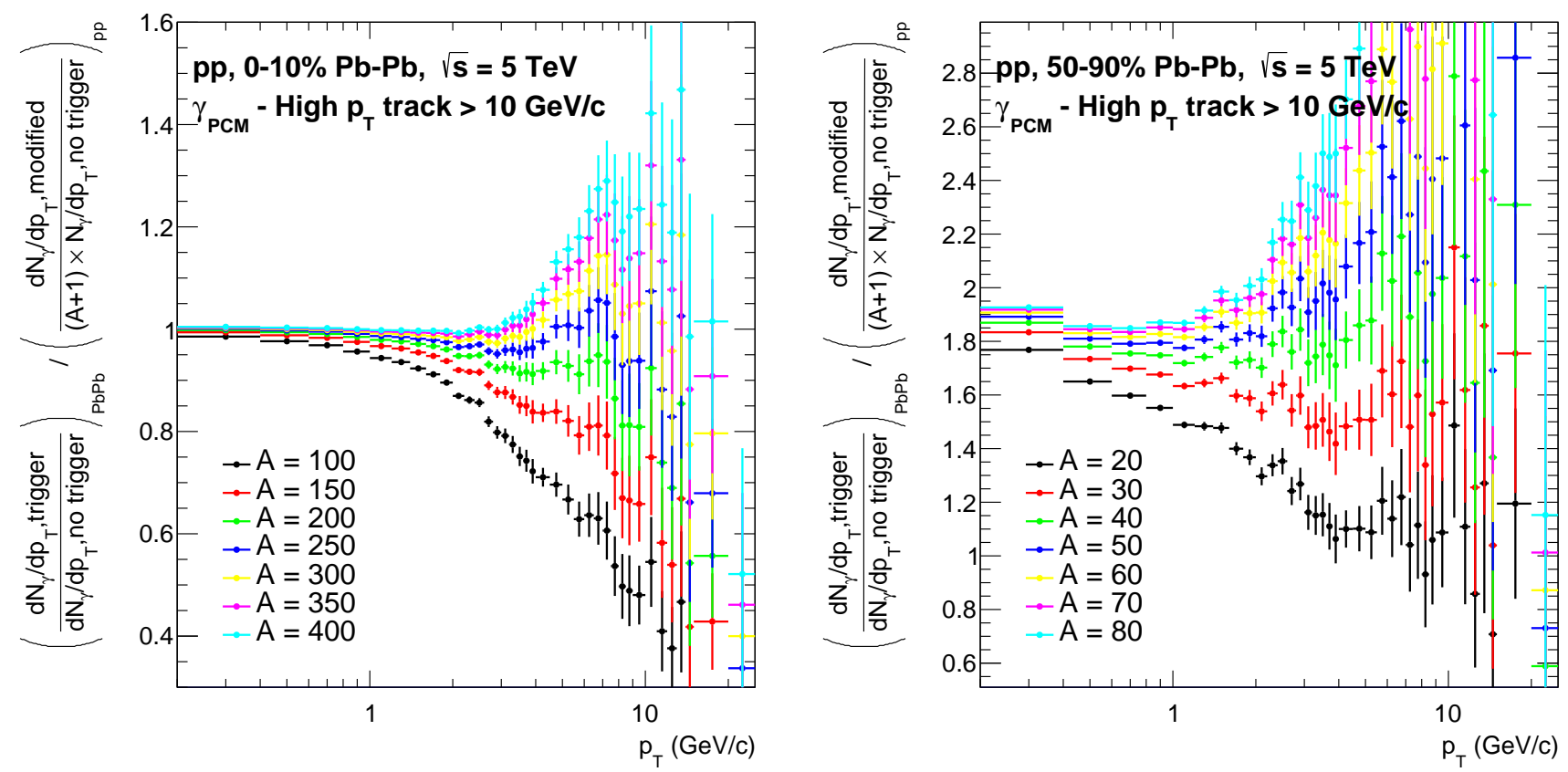

Figure 11.4: The double ratio of the yield of photons with and without trigger, using central (left) and peripheral (right) $\mathrm{Pb}-\mathrm{Pb}$ collisions at $\sqrt{s_{N N}}=5.02 \mathrm{TeV}$, as well as pp collisions at $\sqrt{s}=5.02 \mathrm{TeV}$. The results are calculated using the modified ratio in pp collisions as given in Eq. 11.3. 


\subsection{Discussion}

This chapter presented an exploratory study of the modification of the photon yields due to the presence of a high momentum charged particle. A hardening of the spectrum in triggered events is expected due to the fact that high momentum charged particles are associated with high momentum transfer processes between the constituents of the collision, which on average also produce more high momentum photons. This effect is clearly seen in Fig. 11.1. However, Fig. 11.2 shows that there is much less modification for the photon yields in $\mathrm{Pb}-\mathrm{Pb}$ collisions.

One way to interpret these results is to approximate a pp collision as a single emission source of particles, while the a $\mathrm{Pb}-\mathrm{Pb}$ collision produces a larger collection of such sources. This would imply that the presence of a high momentum particle in a $\mathrm{Pb}-\mathrm{Pb}$ collision only relates back to a single source, while all other sources still produce particles according to the unmodified spectrum. Since peripheral $\mathrm{Pb}-\mathrm{Pb}$ collisions have fewer number of participants compared to central $\mathrm{Pb}-\mathrm{Pb}$ collisions, its modification should be more like a pp collision, which is confirmed in Fig. 11.2. Interestingly, while HIJING [162] simulates events by multiple minijet production and includes a simple jet quenching mechanism, it shows much less modification than the experimental data. This indicates that in data there is an increased correlation between the presence of a high momentum charged particle and the yield of photons, and is not simply a collection of independent particle production sources.

This interpretation is tested more in depth by superimposing multiple photon yields as defined in Eq. 11.2 and Eq. 11.3, with the full result given in Fig. 11.4. In the limit of $A$ going to infinity, the shape of the resulting photon yield will be that of the photon yield without trigger particle. Both for central and peripheral $\mathrm{Pb}-\mathrm{Pb}$ collisions the results show that for the higher values of $A$ the double ratio increases strongly with photon $p_{\mathrm{T}}$, indicating that the triggered source from the $\mathrm{Pb}-\mathrm{Pb}$ collisions produce a much harder spectrum. The other way around, when $A$ is too small, the double ratio decreases strongly with photon $p_{\mathrm{T}}$, now indicating that the modified photon spectrum is too hard.

It turns out that this is an interesting way to study the effects of a high $-p_{\mathrm{T}}$ trigger on the photon yields, and is able to give new interpretations of particle production mechanisms that connects small and large collisions systems. It would be interesting to repeat these measurements for other identified particles, as well as for $\mathrm{p}-\mathrm{Pb}$ collisions. More work is needed to understand this in detail. 



\section{Part IV}

\section{Summary and Outlook}





\section{Chapter 12}

\section{Summary}

We want to know what it all means. What is the universe made of? What is space and time? How do the fundamental forces work? What is the nature of reality? Driven by pure curiosity we are trying to answer these questions, and thereby expanding the boundary of knowledge further and further. While we are learning more about what makes the universe tick, there is one thing we know for sure. We didn't discover everything there is to discover, and there is still a vast amount of knowledge beyond the horizon. What we do know is carefully formulated in theories that get tested by experimental observations, using the process of the scientific method.

According to our current understanding, the universe around us is made of indivisible building blocks that interact with each other via the fundamental forces, which is formulated in the Standard Model of particle physics using an elegant mathematical framework. And even though we recently discovered the Higgs boson, confirming a long-hypothesized piece of the puzzle, there are still many remaining areas of highenergy particle physics that have their own puzzles that are known to be incomplete. One of these areas is heavy-ion physics, which studies the intricate details of nuclear matter under extreme conditions.

Under normal conditions we find that quarks are always tightly bound together by gluons into colorless objects that we call hadrons, such as the proton and neutron. However, there is a temperature at which a phase transition occurs that transforms confined nuclear matter into a deconfined medium of strongly coupled quarks and gluons. This state of matter is referred to as the quark gluon plasma (QGP) and is also the type of matter that filled the universe just microseconds after the big bang. Even though we are unable to probe this primordial matter directly, since as the universe expanded it cooled down and formed the matter that we find around us today, we are able to create the QGP in the laboratory by colliding heavy ions using large scale accelerator experiments.

In a simplified view, the QGP is formed by the overlapping nucleons of two colliding nuclei. In this region the thermodynamic properties of the matter, such as temperature, pressure, and energy density, are sufficient for the phase transition to occur. Then after its creation the QGP will expand, cool down, and reach the 
temperature at which the system hadronizes back into hadronic matter, all within about $10^{-22}$ seconds. It is via the aftermath of the heavy-ion collision that we infer information about this extraordinary state of matter, i.e. by measuring properties of the particles that originate from this collision.

One of the most insightful methods to study the QGP is by measuring, as precise as possible, the properties of all the particles that are produced in the heavy-ion collisions. These measurements provides great constraining power between various theoretical model calculations that can incorporate different particle production mechanisms and underlying assumptions. Additional insights are obtained by making comparisons with similar measurements in proton proton (pp) and proton nucleus (pA) collisions. It was found that the particles that are produced in processes with a high momentum transfer are affected by the presence of the plasma in the form of energy loss by traversing the medium, as well as receiving additional momentum due to its expansion. The plasma turns out to be so extremely hot that it is able to thermally produce quarks and gluons, which result in additional final state particles. Also, two-particle correlations revealed that the QGP is an almost perfect fluid, i.e. a fluid with an extremely low shear viscosity, and that it can be described using relativistic hydrodynamics. While many properties of the QGP are more and more understood, there are still many open questions that are also of interest to the wider field of high energy particle physics.

This thesis presents results on neutral meson measurements to uncover details on light flavor particle production mechanisms in pp, pA and AA collisions, as well as direct photon correlation measurements that are sensitive to the space-time evolution of the QGP. Photons are unique due to the fact that their mean free path in a QGP is much larger than the size of the QGP, such that thermal photons that are emitted leave the medium unaffected. This means that, in contrast to other particles that continue to interact and participate in the expansion, the thermal photons are providing valuable input about the early space-time dynamics of the QGP, and are able to constrain its effective temperature. Experimentally it is very challenging to measure direct photons, as almost all photons are produced by the decay of neutral mesons. This makes it important to study and measure neutral mesons as precise as possible, which in itself can lead to insights into particle production mechanisms. Hence, direct photon measurements are promising in providing answers to open questions.

The analyses that are presented in this work use experimental data collected by the ALICE detector, which is one of the four large experiments at CERN which is optimized to handle the high particles multiplicities produced by heavy-ion collisions, and is installed at the 2 nd interaction point of the $27 \mathrm{~km}$ long Large Hadron Collider. The detector consists of numerous subsystems that all work together to provide information about the production point, energy, momentum, and identity of the charged and neutral particles produced in the collisions. More specifically, in this thesis the central barrel is used to reconstruct photons converting in the detector material, which enables photons measurements down to very low momentum, and the calorimeters EMCal, DCal, and PHOS are used to reconstruct the photons with large efficiencies to higher momentum.

The invariant yield of the $\pi^{0}$ and $\eta$ meson is measured in both pp, $\mathrm{p}-\mathrm{Pb}$, and $\mathrm{Pb}-\mathrm{Pb}$ collisions at $\sqrt{s_{N N}}=$ $5.02 \mathrm{TeV}$, utilizing all the photon reconstruction methods and trigger capabilities the ALICE detector has to 
offer. For the results in pp collisions, it is the first time that an identified particle spectrum has been measured using the full Run 2 dataset. Due to the large amount of statistics, the invariant yield of both mesons are also measured more differentially, i.e. as function of event multiplicity, its event shape, as well as their production inside jets. One of the main findings is that the in-jet $\eta / \pi^{0}$ is heavily suppressed compared to the same ratio found in $\mathrm{MB}$ collisions. The $\mathrm{p}-\mathrm{Pb}$ results present the neutral meson invariant yield in $\mathrm{MB}$ collisions, as well as for the first time its dependence on the event multiplicity, which enables studying the multiplicity dependent nuclear modification factor $Q_{\mathrm{pA}}$. It is found that the results for high event multiplicity has a significant enhancement compared to the $Q_{\mathrm{pA}}$ in $\mathrm{MB}$ and to low event multiplicity, which is consistent with a multiplicity dependent Cronin peak. The $\mathrm{Pb}-\mathrm{Pb}$ results present the invariant yield of the $\pi^{0}$ and $\eta$ meson for the combined Run 2 dataset, achieving an unprecedented precision measurement for these mesons over a wide range of transverse momentum. The $\eta / \pi^{0}$ ratio shows a significant enhancement for central $\mathrm{Pb}-\mathrm{Pb}$ collisions, which is attributed to the radial flow of the plasma. Furthermore, the nuclear modification factor $R_{\mathrm{AA}}$ shows a centrality dependent suppression, where central collisions are more suppressed than peripheral collisions, in line with what is expected. These results are in addition a stepping stone towards the extraction of direct photons, for which the decay of the $\pi^{0}$ and $\eta$ meson are the most dominant backgrounds. For all these results in $\mathrm{pp}, \mathrm{p}-\mathrm{Pb}$, and $\mathrm{Pb}-\mathrm{Pb}$ collisions it is safe to say that the production of neutral mesons has never before been studied under so many different event conditions, posing many constraints on theoretical model calculations.

One of the observations from the neutral meson measurements is that the combinatorial background present in the diphoton invariant mass distributions is not well reproduced by the event-mixing method alone, and requires additional constraints on the background to remove also the correlated background. This thesis presents a dedicated model study that shows that the correlations at lower $M_{\text {inv }}$ are not due to decay kinematics, but rather by correlations present in the parton shower. While it is difficult to capture these effects in data, a modified event-mixing method is proposed that mixes photons only for the case where their originating parton points in a similar direction. These finding are important for both neutral meson and direct photon HBT measurements.

Another model study dives into the correlations between the shape of an event and the probability to find jet objects. In the current literature it is stated that pencil-like events are jet-like, and that sphere-like events are dominated by soft particle production from the underlying event. The results presented in this thesis show that this is indeed the case for $e^{+} e^{-}$collisions, where the event-shape observable $S_{\mathrm{T}}$ can be used to efficiently select di-jet and multi-jet topologies. For pp collisions it is found that this completely breaks down, and selections on $S_{\mathrm{T}}$ are unable to significantly increase the number of jets found in the sample. Rather, it is shown that the average number of multi-parton interactions for the $10 \%$ most spherical-like events are almost 10 times higher than the $10 \%$ most pencil-like events. This insight could pave the way for more detailed studies related to multi-parton interactions.

Furthermore, this thesis presents the first measurement of direct photon elliptic flow at LHC energies at $\sqrt{s_{N N}}=2.76 \mathrm{TeV}$. It presents the results that are obtained using the photon conversion method, for which 
a novel way of correcting for inclusive photon impurities has been developed. After combining the inclusive photon measurement of PCM and PHOS, of which the latter is obtained by a collaborator, the direct photon flow is calculated by using a decay photon cocktail and the previously measured direct photon excess $R_{\gamma}$, using a Bayesian approach. It is found that the flow coefficient $v_{2}$ of direct photons is consistent with the decay photon flow, as well as the results obtained at RHIC. The model calculations underestimate the measured $v_{2}$, and struggle to simultaneously describe the yield and flow of direct photons also at LHC energies. However, the experimental uncertainties are too large to claim that there is a puzzle at LHC energies. A future measurement of this observable with higher precision is one of the most anticipated measurements of the field.

In addition, this thesis presents the first measurement of photon-pair correlations in search of direct photon HBT at the LHC, for both pp and central and peripheral $\mathrm{Pb}-\mathrm{Pb}$ collisions at $\sqrt{s_{N N}}=5.02 \mathrm{TeV}$. The results for pp collisions show a large increase in the correlation function $C\left(Q_{\text {inv }}\right)$ for lower $Q_{\text {inv }}$ and increasing relative pair momentum $k_{\mathrm{T}}$, which most likely is associated to correlated particle production from the parton showers, which is not an HBT-like effect. This effect is also present in the result obtained for $\mathrm{Pb}-\mathrm{Pb}$ results, although smaller in magnitude due to the large particle multiplicities. After correcting for these effects, the results in $\mathrm{Pb}-\mathrm{Pb}$ collisions are consistent with an HBT-like signal that one might expect from a QGP, but the uncertainties are still too large to make conclusions.

Last but not least, this thesis presents the results on an exploratory study of photon production with and without the presence of a high momentum charged particle, for both $\mathrm{pp}$ and $\mathrm{Pb}-\mathrm{Pb}$ collisions at a center of mass energy of $\sqrt{s_{N N}}=5.02 \mathrm{TeV}$. The comparison between the results obtained in both collision systems shows how the presence of a high $p_{\mathrm{T}}$ particle hardens the photon spectra, and indicate that low $p_{\mathrm{T}}$ photon production in $\mathrm{Pb}-\mathrm{Pb}$ collisions is dominated by the bulk. Using the crude assumption that a $\mathrm{Pb}-\mathrm{Pb}$ collision is a superposition of pp-like production sources, it is found that central $\mathrm{Pb}-\mathrm{Pb}$ collisions require about five times as many sources than peripheral $\mathrm{Pb}-\mathrm{Pb}$ collisions.

Altogether, the work presented in this thesis is a journey through the field of relativistic heavy-ion physics, using the photon as the main experimental object. Nearly all the existing experimental observables and probes have been utilized to learn more about all the different collision systems that the ALICE data has to offer. Additional studies have been undertaken that attempt to give additional insights into hot and cold nuclear matter effects. 


\section{Chapter 13}

\section{Outlook}

Exciting times are ahead for the field of heavy-ion physics, and even though this statement is true nearly at every point in time, as technology is driving the amount and precision of the data that is collected by orders, it might now be more true than ever. As this is written, the ALICE detector is being upgraded to be able to accommodate the higher interaction rates available in the Run 3 LHC running schedule [205], which will increase the data taking rate by about a factor 50. In addition, the central barrel will be home to a new ITS with great improvements to tracking precision and vertex reconstruction, and the TPC will be improved by renewed readout. In light of the analyses presented in this thesis this will have, in my view, a strong impact.

First, the measurements that already have proven to give insights in the physics that we study, will, when repeated for the new datasets, give unprecedented constraints and discriminating power for theoretical model calculations. This will advance heavy-ion physics, as well as delivering high precision QCD measurements at lower particle energies, for which the ALICE detector is a highly capable device. One highly anticipated analysis is the search for thermal direct photons in high multiplicity $\mathrm{pp}$ and $\mathrm{p}-\mathrm{Pb}$ events, which could indicate the formation of a QGP droplet in smaller systems. In addition, the data from Run 3 will be capable of finding out whether there is a direct photon puzzle at the LHC, which, whatever the answer might be, will give valuable insights in the space-time dynamics of the QGP.

Secondly, the increased statistics of the newly acquired datasets will enable us to study in great detail various rare processes that are currently out of reach, and that have very promising prospects. An example of this is the case where a back-to-back photon-jet object is created via a hard process in a heavy-ion collision, such that the photon escaped unaffected and the jet is quenched by the plasma. This rare process will have the unique feature that the absolute energy that is lost by the jet traversing the medium is known by measuring the photon energy, and thereby unambiguously probe the plasma with a calibrated probe. A selection of ideas for future measurements are discussed in the next chapter. 
As the ALICE Collaboration is also preparing for beyond Run 3 with a even more improved detector, it would also be good to reflect and think about the calorimetric setup of a future detector. In my view, the calorimetric setup of the ALICE detector has become too much of a patchwork that can be done better with less resources, i.e. there should have been a single $2 \pi$ calorimeter enclosing the nominal interaction point. The current setup requires unnecessary hassle from the analysis point of view, as it is just more difficult to combine photon candidates from multiple detector systems, each with their own area in $\eta$ and $\varphi$. I would therefor advocate for a rebuilt or new calorimeter, with updated hardware and triggering capabilities, that would be installed in one of the next long shutdowns where also again the rest of the ALICE detector is getting major upgrades. The advantages are numerous, from being able to reconstruct full jets in a larger acceptance, to greatly increasing the statistics for photon and jet related studies, all without the hassle of dealing with limited acceptance in $\varphi$.

Also, we as a field can, and in my view should, seek further connections with other fields. One of the most interesting ones, which is already coming to fruition, is the connection between heavy-ion physics and gravitational wave physics. Currently it is hypothesized that the QGP that is created only briefly in heavy-ion collisions is also the state of matter at the core of a neutron star. So the equation of state that is parametrized by measurements at the LHC constrain model calculations of the matter found deep inside these extremely dense stars that are light years away. The gravitation wave detectors are now precise enough to measure the gravitational waves emitted by neutron star mergers, and as such benefit greatly from the input from the heavy-ion physics community. A strengthened collaboration between these two fields could lead to large discoveries, that affects both our understanding of the very small and the very large.

I also firmly believe that we should keep reminding ourselves about the big picture. Think about if our short and mid term goals are in line with the large questions we want to answer about the universe, which in many cases resonate more broadly with society. Science is there to serve society, and it is becoming even more important to have strong connections with the general public, as the acceptance of scientific knowledge sometimes seems to erode. As scientists I believe we have the mandate of advancing the knowledge of humankind, creating new technologies, and sharing our passion to try to answer what it all means. 


\section{Chapter 14}

\section{Ideas for future measurements}

The purpose of this chapter is to collect my ideas for future measurements that are in my view high priority to pursue and are at the same time related to the work presented in this thesis. Some of them could be done on existing data, and others would require substantial detector upgrades or modifications to the LHC running schedule, of which some are already in process. They are now discussed in no particular order.

\section{Direct thermal photons in small systems}

One of the most interesting findings of the last decade within the field of heavy-ion physics is that also the smaller collision systems, i.e. pp or p-Pb collisions, are showing QGP-like behavior. While the potential signatures of the QGP, such as long range correlations, anisotropic flow, and enhanced production of multistrange particles, were thought to be exclusive to the formation of the QGP, the measurements in smaller systems indicated otherwise. Both in $\mathrm{pp}$ and $\mathrm{p}-\mathrm{Pb}$ collisions there is significant elliptic flow as well as the enhancement of strange particle production for high-multiplicity events. However, jet-quenching is so far not observed in the smaller systems, which would be expected if there would be a QGP. Cutting to the main point, it is the question whether there is a QGP droplet produced in small systems, or that these observations have a different origin altogether.

This is where a precise measurement of a direct photons in high multiplicity pp and $\mathrm{p}-\mathrm{Pb}$ collisions would provide unique input. If there is such a thing as the formation of a QGP droplet in small collision systems, it must radiate thermal photons proportionally to the temperature of the system, thereby creating an excess of direct photons over photons coming from hadronic decays at lower transverse momentum $\left(p_{\mathrm{T}}<3 \mathrm{GeV} / c\right.$. The other way around, if the measurement is precise enough to rule out the existence of a significant direct photon excess, the previous observations have to be explained without invoking QGP physics.

So far, the available ALICE datasets are insufficient to get a statistically significant measurement of the direct photon excess $R_{\gamma}$ in pp and $\mathrm{p}-\mathrm{Pb}$ collisions at $5 \mathrm{TeV}$. The other limiting factor is the systematic 
uncertainty of the material budget of the ITS and TPC, which amount to about $5 \%$ uncertainty for the measurement of converted photons. This uncertainty is of similar magnitude as the expected excess in case of a small radiating QGP droplet. As such, it is highly likely that analysis on run 1 and run 2 will give inconclusive results. Fortunately, ALICE is installing incredible detector upgrades for LHC run 3, completely replacing the ITS with a new inner tracker and also improving the readout of the TPC. With these upgrades, improved statistics, and understanding the reduced material budget of the inner detectors, it will be possible to either confirm or debunk the existence of a small QGP droplet to be formed in small systems, which is a high impact measurement for the field of heavy-ion physics, as well as particle physics as a whole.

\section{High precision fragmentation functions of identified particles}

As has been presented in this thesis, the invariant yield of the $\pi^{0}$ and $\eta$ meson in pp collisions deviates from the PYTHIA model prediction and the NLO calculations by $10-100 \%$. It would be valuable to measure the fragmentation functions of these particles with high precision, such that these model calculations are improved, also in terms of their relative uncertainties, to a level where there is discriminating power between the models and the data. This is even more the case for multi-differential measurements of the invariant yield, such as multiplicity and event-shape dependence.

The fragmentation function is obtained by measuring the neutral mesons as function of $z=p_{T, \text { meson }} / p_{T \text {, jet }}$, the momentum fraction of the meson compared to the total jet momentum in which it is reconstructed. There are many similarities to the analysis presented in section 6.4.2, except that the $z$ distribution needs to be corrected for background. This can be done measuring the fragmentation function $z$ in the main signal peak of the meson, as well as in the sidebands near the respective neutral meson and calculating the corrections using the relative counts in the main signal area and the sidebands.

Since this measurement requires reconstructed jets above a $p_{\mathrm{T}}>10 \mathrm{GeV} / c$, which occur in roughly $1 \%$ of minimum bias events, it favors the dataset collected at $13 \mathrm{TeV}$ above $5 \mathrm{TeV}$, as it has about double the statistics in the minimum bias sample. In contrast to other possible measurements, this measurement has less potential to provide a large impact on the field, but it would pave the way for many other measurements to have discriminating power with model calculations. In a rather straightforward way, this analysis can be extended to other identified particles, such as charged pions, kaons, protons, and even multi-strange particles, where there are possible surprises waiting to be discovered. 


\section{Direct photon flow revisited}

Direct photons are unique in probing the QGP, as they are able to escape the medium unaffected due to the fact that their mean free path is larger than the size of the system. This results in a sensitivity to the early stage dynamics of the QGP, when it is extremely hot and most of the flow of the medium still has to develop. As the abundance of direct photons is proportional to the temperature of the system, and their anisotropy is driven by the particles that emit them, the combination of the yield and flow is able to heavily constrain the space-time evolution of the system. These measurements performed at the Relativistic Heavy-Ion Collider led to the direct photon puzzle which remains unsolved.

The puzzle, in short, is the inability of model calculations to describe both the yield and flow of direct photons in heavy-ion collisions at the same time. When models rightly tune the parameters to describe the yield, it significantly underestimates the flow. While increasing the initial temperature of the system, the yield increases, but simultaneously also lowers their elliptic flow as the majority gets emitted at earlier times when the anisotropy of the system is still small.

Chapter 9 presented the first published direct photon elliptic flow measurement in $\mathrm{Pb}-\mathrm{Pb}$ collisions at LHC energies, showing a significant $v_{2}$ for two collision centralities using the previously measured $R_{\gamma}$ in the same dataset. This $v_{2}$ is consistent with the flow of charged particles that hadronize at the end of the evolution of the QGP, but the uncertainties are too large to claim a puzzle at LHC energies.

It would therefore be interesting and also straightforward to repeat both the direct photon yield and flow measurements using the ALICE Pb-Pb data collected at $\sqrt{s_{N N}}=5 \mathrm{TeV}$. More specifically, in these datasets there are triggers used to enhance the statistics for collision centralities $0-10 \%$ and $30-50 \%$, resulting in about 10 times more statistics compared to the dataset collected at $\sqrt{s_{N N}}=2.76 \mathrm{TeV}$. This measurement would significantly improve the precision of the direct photon excess $\left(R_{\gamma}\right)$, which in turn also increases the precision of the direct photon flow measurement as it is highly sensitive to the uncertainty on $R_{\gamma}$. There is a high probability that this measurement can show that the puzzle either does or does not exist at LHC energies, providing important information on the understanding of the QGP physics.

Thinking further ahead, the new ITS that is installed for run 3 together with the large increase in integrated luminosity of the run 3 datasets would dramatically improve both the statistical and systematic uncertainty of the measured $R_{\gamma}$ and $v_{n}$. This can potentially extent the $v_{n}$ measurement to also include $n=3$ and $n=4$, which are important constraints for the hydrodynamic phase of the QGP. 


\section{Photon-jet measurements, the golden probe of the QGP}

The main goal of studying heavy-ion collisions is the characterization of the Quark Gluon Plasma that is created in these collisions. Important properties such as the transport coefficients of this QGP can be inferred by studying the energy that is lost by highly energetic particles traversing the medium. More specifically, experimentally it is possible to measure the energy imbalance of di-jets that are produced from the initial hard scatterings, and show that the sub-leading jet loses significantly more energy than the leading jet. This is explained by the fact that the parton energy loss is proportional to the distance traveled in the medium and thus depends on the di-jet production point inside the medium. This means that with this approach, even if it was one of the first smoking-gun signatures of the QGP, the energy lost by the leading jet is still unknown.

However, the fact that photons have no further interactions with the medium once they are produced and escape unaffected, there is an approach that does not obscure the energy loss of a single parton. This is by measuring $\gamma$-jet production in heavy-ion collisions, and using the energy of the photon as the initial parton energy. Simply put, the energy loss $\Delta E=E_{\gamma}-E_{j e t}$, exploiting the conservation of energy and momentum. This measurement can be done dependent on collision centrality and more importantly intervals of photon energy. Obtaining the $\Delta E$ distributions for photons in the range of $1<E_{\gamma}<100$ would give strong constraints on the energy loss of the parton traversing the medium, especially when comparing to the same observable obtained in pp collisions. Here, ALICE has an advantage because of the low $p_{\mathrm{T}}$ reconstruction capabilities of the detector, in contrast to for example the ATLAS detector, with which a $\gamma$-jet measurement as function of $x_{J, \gamma}=p_{T, j e t} / p_{T<\gamma}$ was performed in the range of $63.1<p_{T, \gamma}<200 \mathrm{GeV}$ [ref 1809.07280]. The ATLAS measurement shows that for central $\mathrm{Pb}-\mathrm{Pb}$ collisions the $x_{J, \gamma}$ distribution shifts to lower values compared to peripheral $\mathrm{Pb}-\mathrm{Pb}$ collisions as well as pp collisions, clearly indicating parton energy loss which also puts strong constraints on model calculations.

In addition to providing the same measurement down to lower photon energies, it is possible to tag the events that contain an heavily quenched $\gamma$-jet, i.e. with large $\Delta E$ or small $x_{J, \gamma}$, and within this sample, measure the direct photon excess within the cone of the jet. The idea behind this is that the electromagnetic interactions of charged constituents within the plasma are largely unknown, and there are several ideas that talk about a wake being generated by a highly energetic probe penetrating the plasma. Furthermore, this would also be a first direct measurement of the direct photons produced from jet-fragmentation in heavy-ion collisions, and can likewise be performed in pp collisions. In this analysis, it would be necessary to simulate the contribution from decay photons, similar to the cocktail simulations used in this thesis. It would be worthwhile to investigate whether the existing ALICE $2018 \mathrm{~Pb}-\mathrm{Pb}$ datasets would already be sufficient for a first measurement. 


\section{Multi-dimensional event properties}

Measuring high energy particle collisions mostly involves calculating a single observable at a time, as function of some other quantity such as particle momentum and for a certain selection of events. Plenty of examples are presented in this thesis, such as the invariant yield of neutral mesons in pp collisions as function of meson $p_{\mathrm{T}}$ for different event multiplicity classes, for different event shapes, and in-jet production. However, using this example, it was previously never verified if pencil-like events correlate with the presence of jets, and was just assumed from earlier studies performed in $e^{+} e^{-}$collisions, as presented in Chapter 8. In this case it turns out that it is invalid to label low sphericity events as jet-like events, showing that measurements can be very capable of making model comparisons, but at the same time can be misleading and lead to wrong conclusions due to previously made assumptions and not using all the available information. This is where a multi-dimensional analysis of event properties comes in.

Here it is proposed to simultaneously study a large number of event properties and information, such as charged and neutral particle multiplicities, the number and energy of the jets contained in the event including their orientation, momentum of the leading and sub-leading particles, mean $p_{\mathrm{T}}$ of the reconstructed particles, shape of the event as calculated using the sphericity observable, flow coefficients, and so on. It is important to preserves the information to which event these properties belong, such that correlations between them can be accessed. In simulated data even more event properties can be stored which can be model specific such as the number of multi-parton interactions in PYTHIA. With $N$ properties there are already $N(N-1)$ two-dimensional correlations to be calculated, increasing rapidly for higher dimensional correlations. Here, the main idea is to perform a wide search for large discrepancies between event property correlations in experimental and simulated data, as well as understanding the correlations that are already simulated qualitatively.

It is only possible to speculate what this analysis is able to find, but could possibly find a multi-dimensional selection of event properties for which there are no equivalent events in simulated data, or the other way around. Technically it would be interesting to investigate using the pattern recognition tools of machine learning for quick and powerful classification of the multi-dimensional variables. Using this analysis scheme, it would be extremely easy to expose the shortcomings of state-of-the-art model calculations. The observations could potentially lead to large discoveries and increased understanding of particle production mechanisms in both small and large systems. 



\section{Appendix A}

\section{Additional neutral meson plots}

\section{A.1 Invariant mass distributions of the neutral mesons in pp collisions}

This appendix lists the invariant mass distributions of all neutral meson reconstruction methods in pp collisions at $\sqrt{s}=5.02 \mathrm{TeV}$, as presented in chapter 6 . 


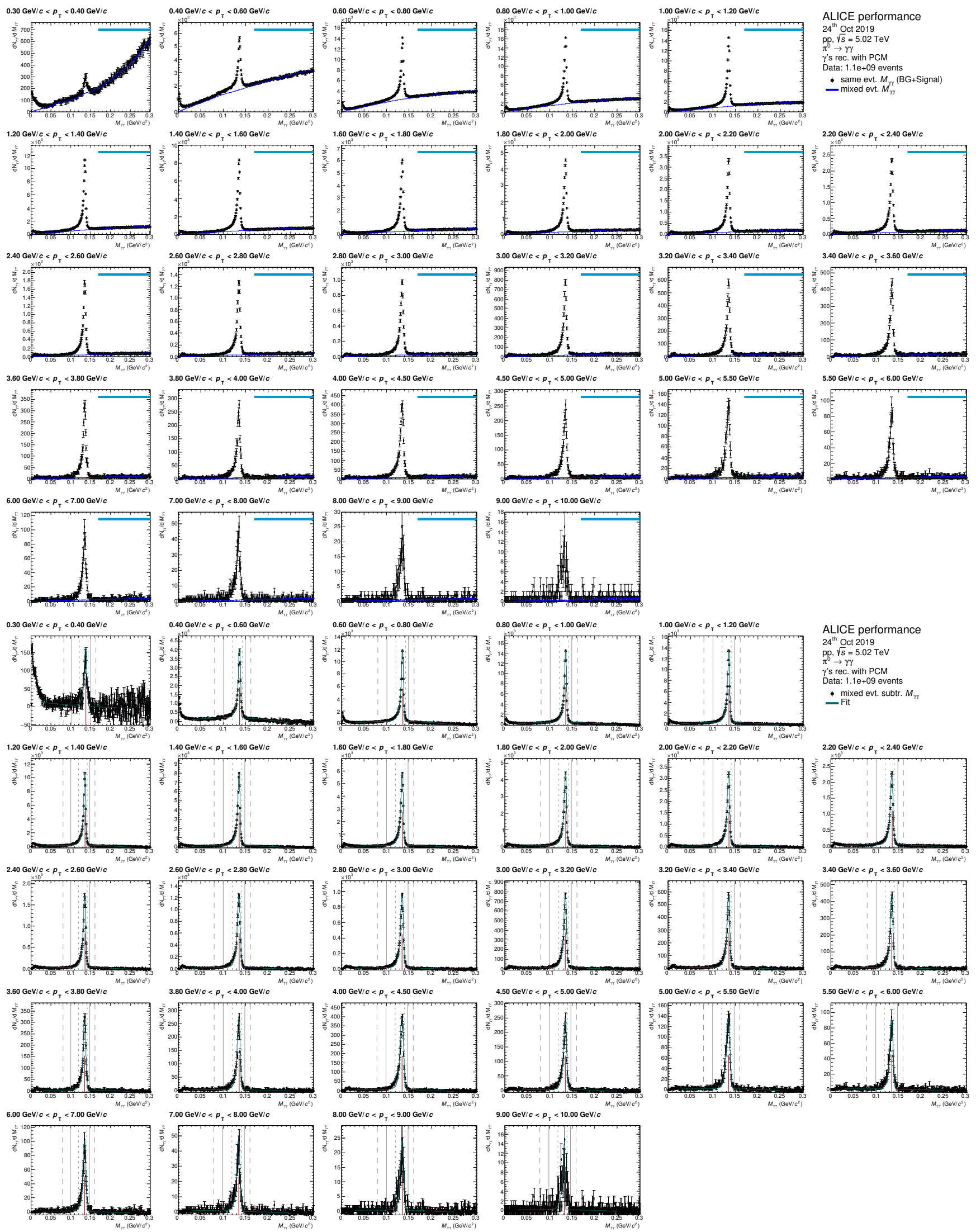

Figure A.1: Invariant mass distribution of photon pairs around the nominal value of the $\pi^{0}$ mass before(top) and after(bottom) subtraction of the combinatorial background, as measured with the ALICE detector in pp collisions at a center-of-mass energy of $\sqrt{s}=5.02 \mathrm{TeV}$. The meson candidates are reconstructed using PCM, covering $0.3<p_{\mathrm{T}}<10.0 \mathrm{GeV} / c$. 

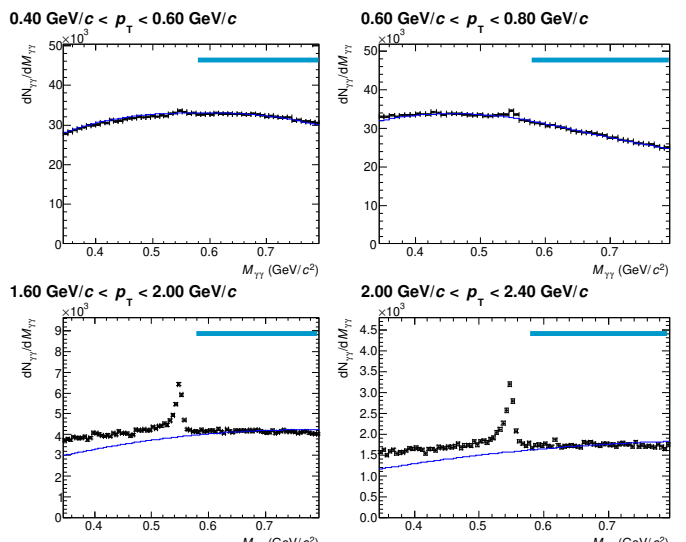

$3.60 \mathrm{GeV} / c<p_{\mathrm{T}}<4.00 \mathrm{GeV} / c$

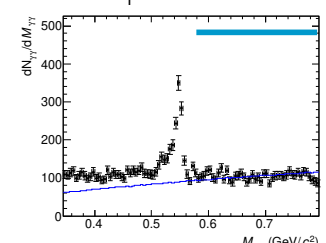

$7.00 \mathrm{GeV} / c<p_{\mathrm{T}}<8.00 \mathrm{GeV} / c$

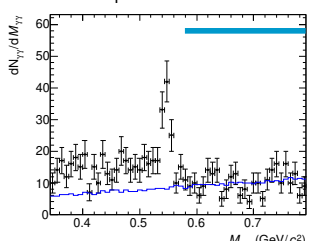

$0.40 \mathrm{GeV} / c<p_{\mathrm{T}}<0.60 \mathrm{GeV} / c$

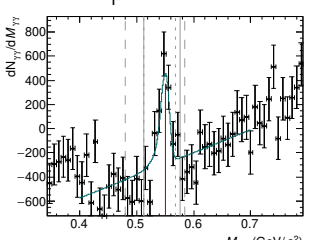

$1.60 \mathrm{GeV} / c<p_{\mathrm{T}}<2.00 \mathrm{GeV} / c$

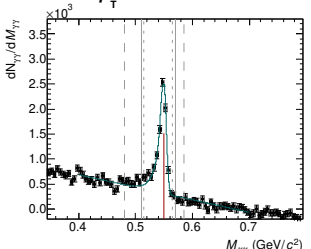

$3.60 \mathrm{GeV} / c<p_{\mathrm{T}}<4.00 \mathrm{GeV} / c$

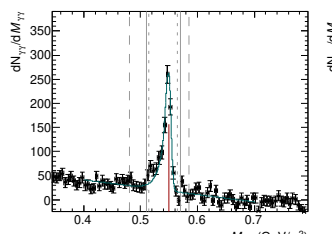

$7.00 \mathrm{GeV} / c<p_{\mathrm{T}}<8.00 \mathrm{GeV} / c^{M_{r y}\left(\mathrm{GeV} / c^{2}\right)}$

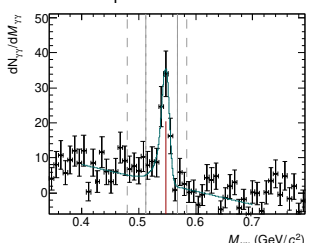

$2.00 \mathrm{GeV} / c<p_{\mathrm{T}}<2.40 \mathrm{GeV} / c$

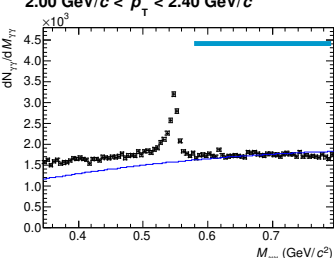

$4.00 \mathrm{GeV} / c<p_{\mathrm{T}}<4.50 \mathrm{GeV} / c$

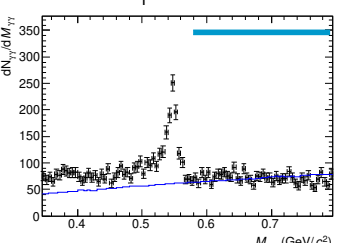

$8.00 \mathrm{GeV} / c<p_{\tau}<10.00 \mathrm{GeV} / c$
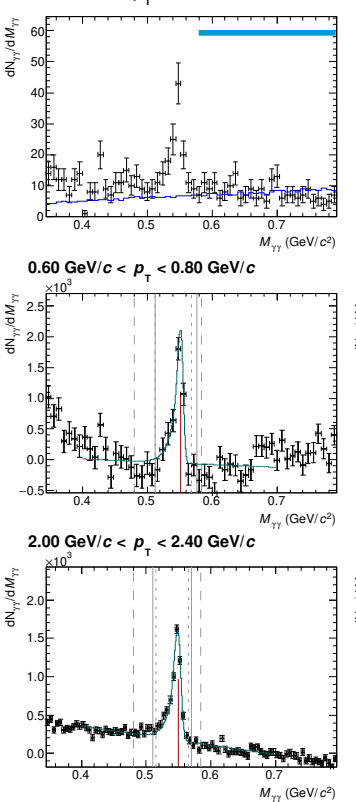

$4.00 \mathrm{GeV} / c<p<4.50 \mathrm{GeV} / c$

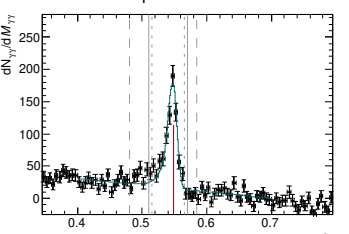

$8.00 \mathrm{GeV} / c<p_{T}<10.00 \mathrm{GeV} / c_{r y}\left(\mathrm{GeV} / \mathrm{c}^{2}\right)$

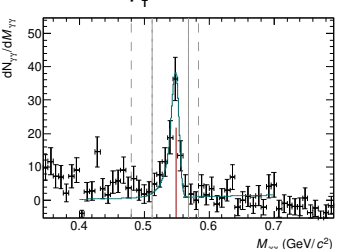

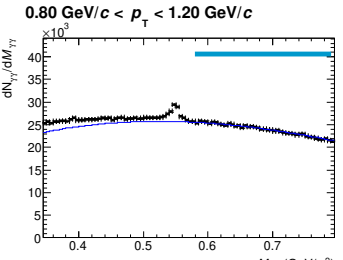

$1.20 \mathrm{GeV} / c<p_{T}<1.60 \mathrm{GeV} / c$
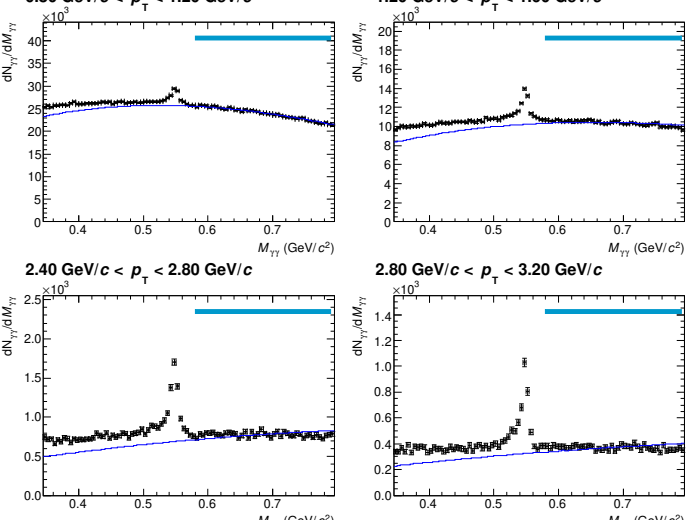

$4.50 \mathrm{GeV} / c<p_{\mathrm{T}}<5.00 \mathrm{GeV} / c$

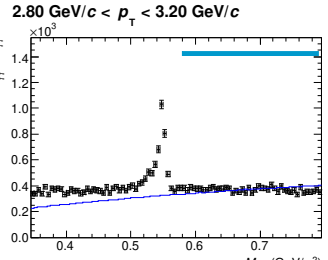

$5.00 \mathrm{GeV} / c<p_{\mathrm{T}}<6.00 \mathrm{GeV} / c$
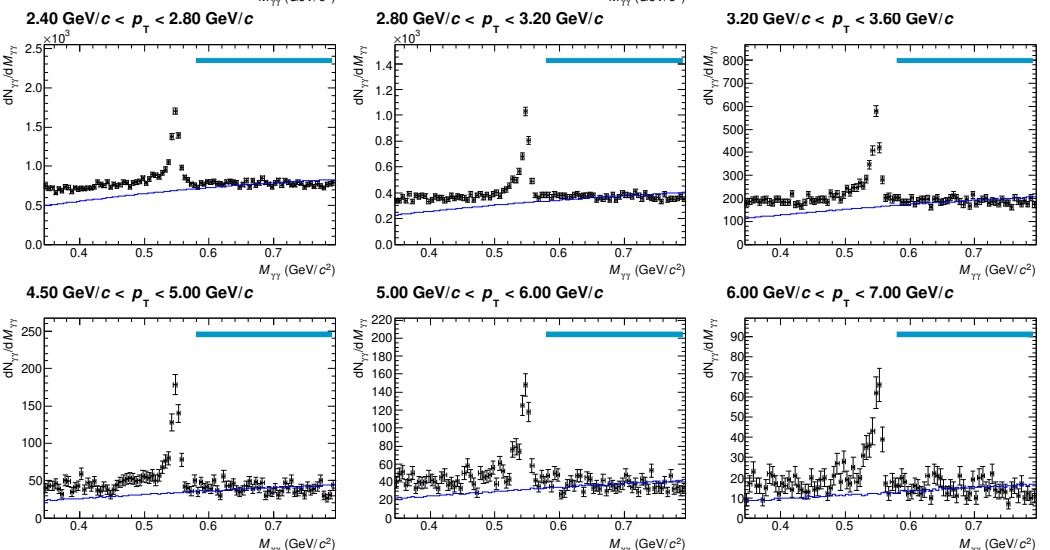

$6.00 \mathrm{GeV} / c<p_{\tau}<7.00 \mathrm{GeV} / c$

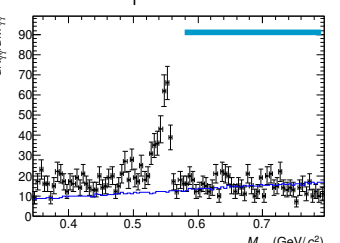

ALICE performance

$24^{\text {th }}$ Oct 2019
$\mathrm{pp}, \sqrt{s}=5.02 \mathrm{TeV}$

$\eta \rightarrow r \gamma$
$\gamma$ 's rec. with PCM

Data: $1.1 \mathrm{e}+09$ events
Dats

- same evt. $M_{r_{\gamma}}$ (BG+Signal)

-
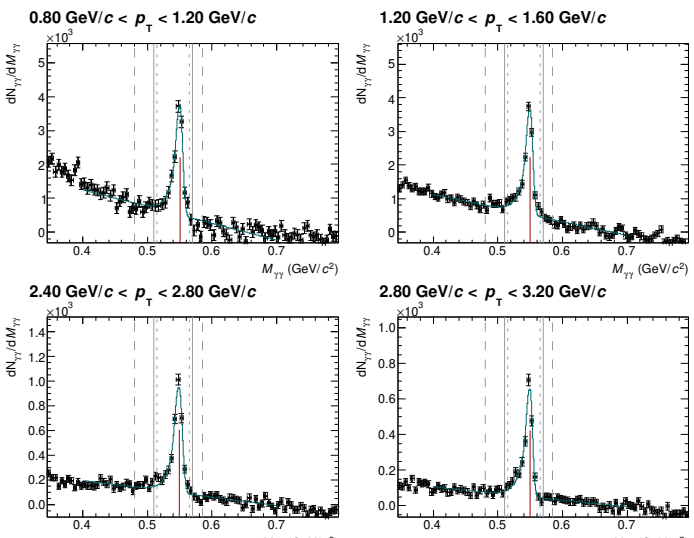

ALICE performance

$24^{\text {th }}$ Oct 2019
$\mathrm{pp}, \sqrt{s}=5.02 \mathrm{TeV}$

$\underset{\eta \rightarrow \gamma}{\mathrm{pp}, \sqrt{s}=5.02 \mathrm{TeV}}$

$\eta \rightarrow \gamma \gamma$
$\gamma$ 'r rec. with PCM
Data: $1.1 \mathrm{e}+09$ events

mixed evt. subtr. $M_{w}$
Fit

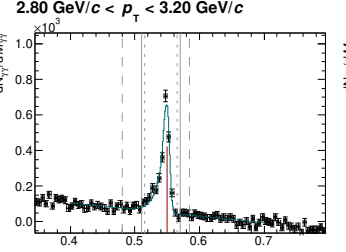

$4.50 \mathrm{GeV} / c<p_{\tau}<5.00 \mathrm{GeV} / c^{M_{r i}(\mathrm{GeV} / \mathrm{c})}$

$5.00 \mathrm{GeV} / c<p_{T}<6.00 \mathrm{GeV} / c^{M_{r r}\left(\mathrm{GeV} / c^{2}\right)}$
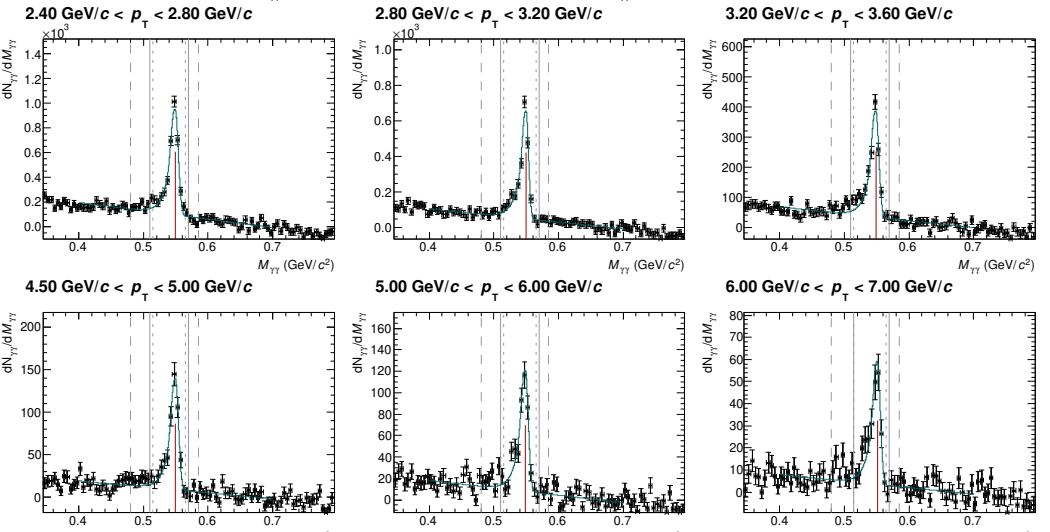

$6.00 \mathrm{GeV} / c<p_{\mathrm{T}}<7.00 \mathrm{GeV} / c M_{r r}\left(\mathrm{GeV} / c^{2}\right)$

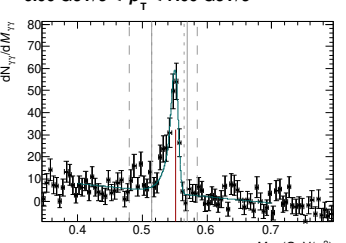

Figure A.2: Invariant mass distribution of photon pairs around the nominal value of the $\eta$ mass before(top) and after(bottom) subtraction of the combinatorial background, as measured with the ALICE detector in pp collisions at a center-of-mass energy of $\sqrt{s}=5.02 \mathrm{TeV}$. The meson candidates are reconstructed using PCM, covering $0.4<p_{\mathrm{T}}<10.0 \mathrm{GeV} / c$. 


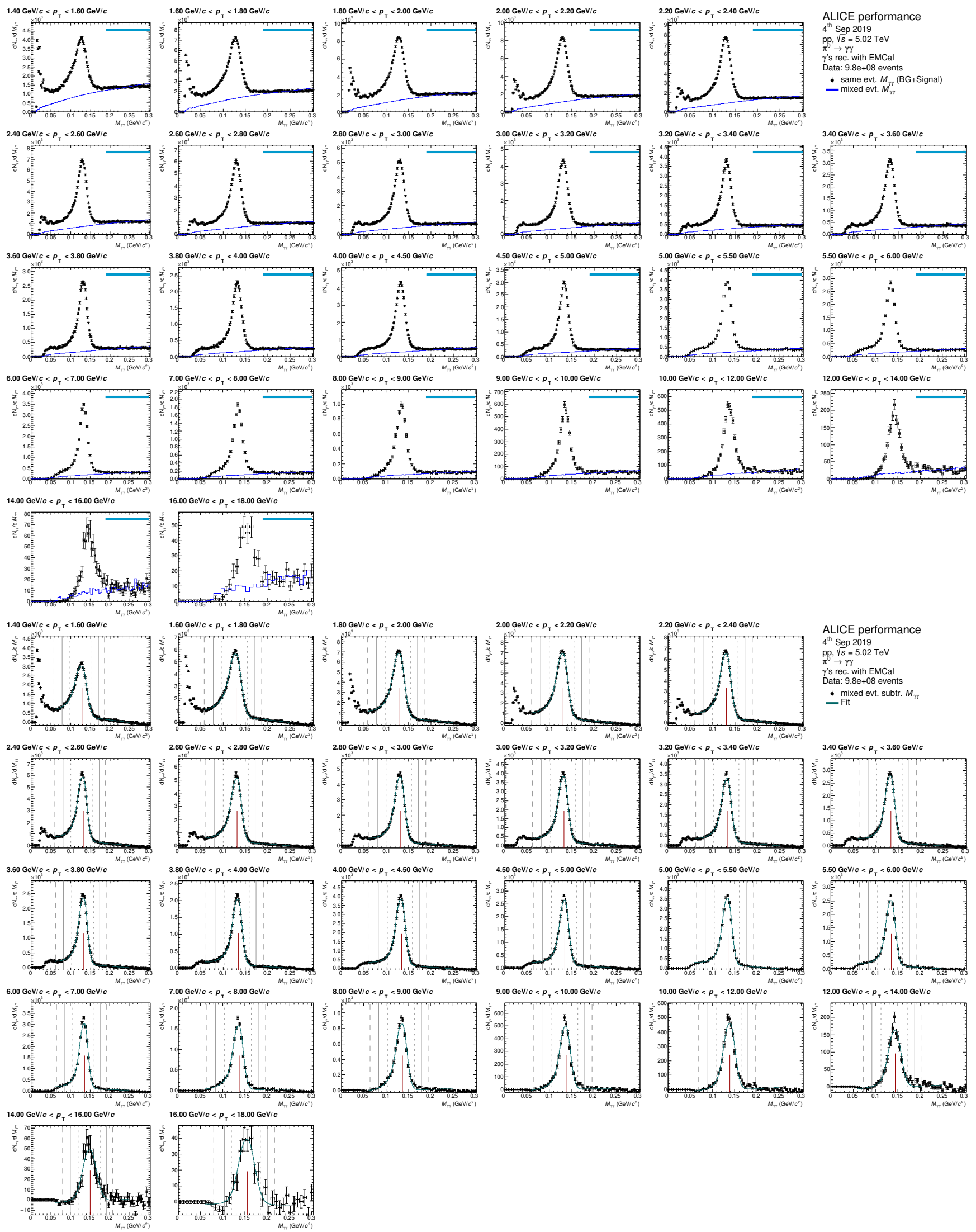

Figure A.3: Invariant mass distribution of photon pairs around the nominal value of the $\pi^{0}$ mass before(top) and after(bottom) subtraction of the combinatorial background, as measured with the ALICE detector in pp collisions at a center-of-mass energy of $\sqrt{s}=5.02 \mathrm{TeV}$. The meson candidates are reconstructed using EMC, covering $1.4<p_{\mathrm{T}}<18.0 \mathrm{GeV} / c$. 

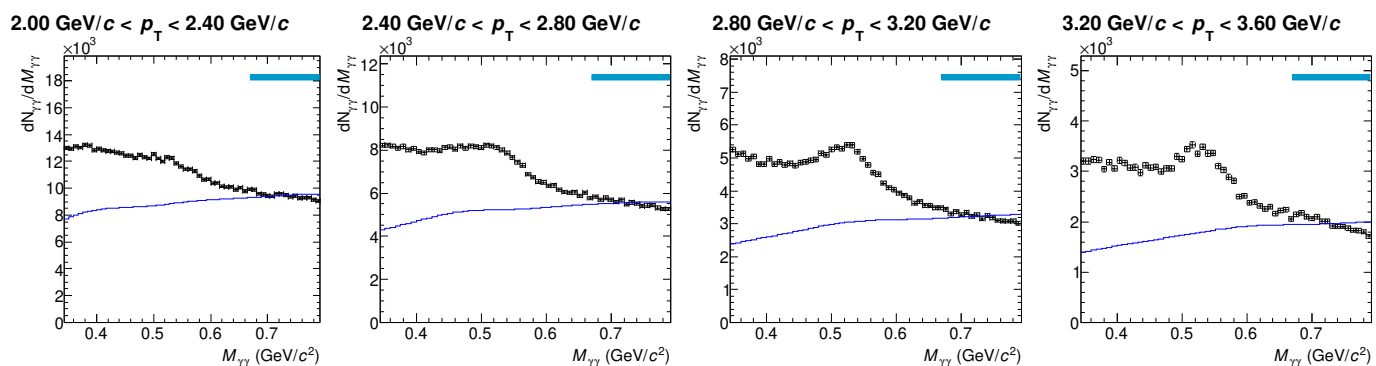

ALICE performance

$4^{\text {th }}$ Sep 2019
pp, $\sqrt{s}=5.02 \mathrm{TeV}$

$\eta \rightarrow \gamma \gamma$
$\gamma$ 's rec. with EMCal

ata: $9.8 \mathrm{e}+08$ events

- same evt. $M_{r_{y}}$ (BG+Signal)
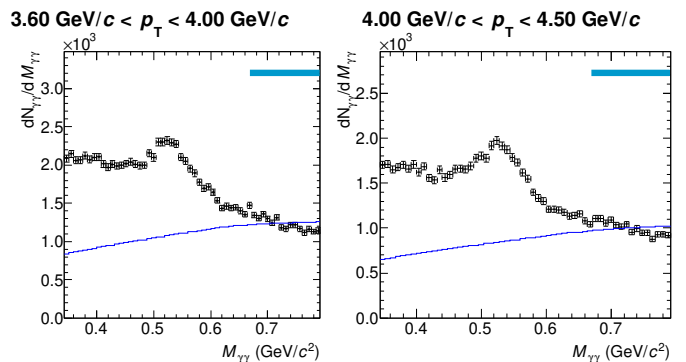

$4.50 \mathrm{GeV} / c<p_{\tau}<5.00 \mathrm{GeV} / c$

$5.00 \mathrm{GeV} / c<p_{\mathrm{T}}<6.00 \mathrm{GeV} / c$

$6.00 \mathrm{GeV} / c<p_{\mathrm{T}}<7.00 \mathrm{GeV} / c$
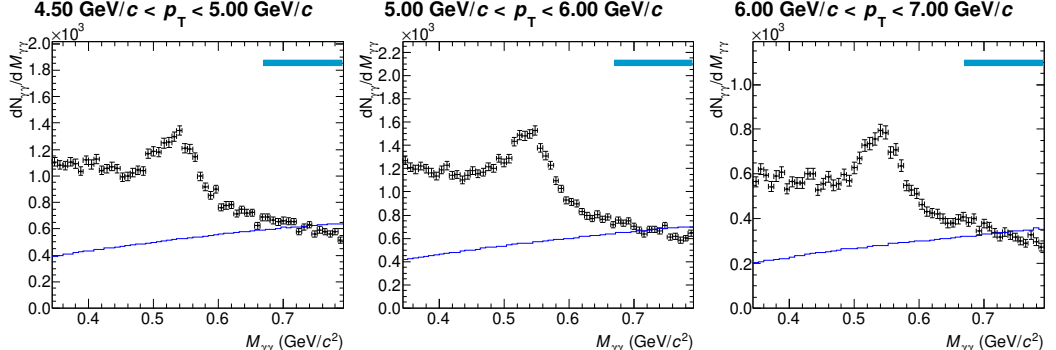

$7.00 \mathrm{GeV} / c<p_{\mathrm{T}}<8.00 \mathrm{GeV} / c$
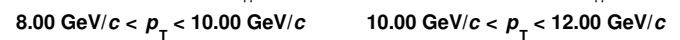

$12.00 \mathrm{GeV} / c<p_{\mathrm{T}}<16.00 \mathrm{GeV} / c$
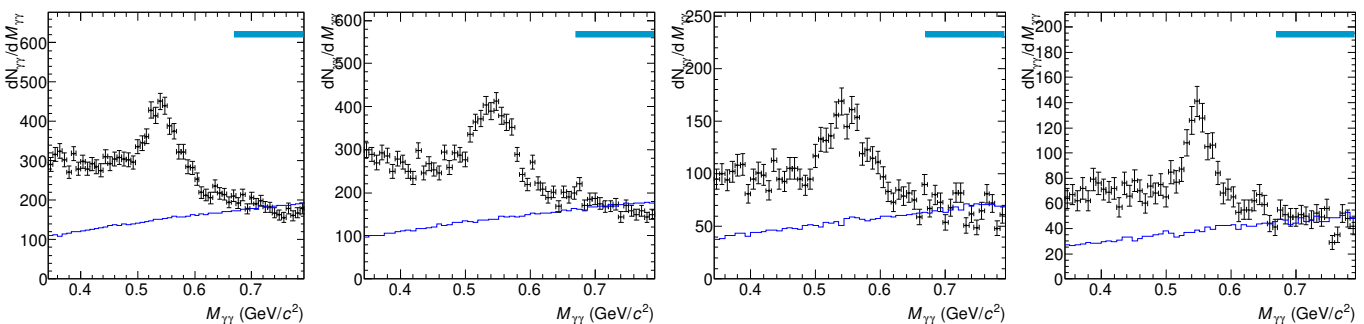

$16.00 \mathrm{GeV} / c<p_{\mathrm{T}}<20.00 \mathrm{GeV} / c$
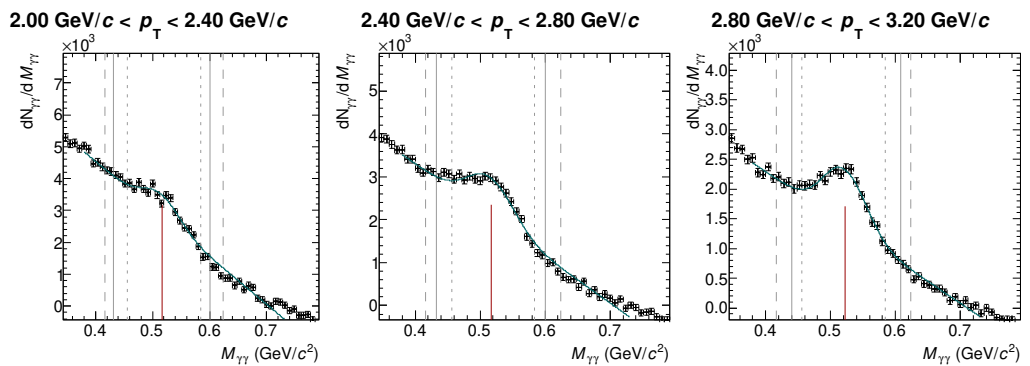

$3.20 \mathrm{GeV} / c<p_{\mathrm{T}}<3.60 \mathrm{GeV} / c$

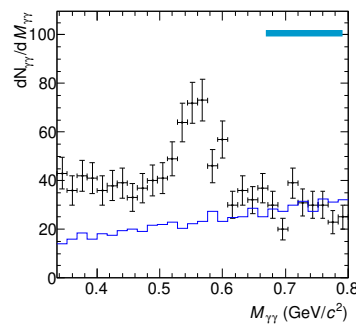

ALICE performance

$4^{\text {th }}$ Sep 2019
$\mathrm{pp}, \sqrt{\mathrm{s}}=5.02 \mathrm{TeV}$

$\eta \rightarrow \gamma \gamma$
$\gamma$ 's rec. with EMCal

. mixed evt. subtr. $M$
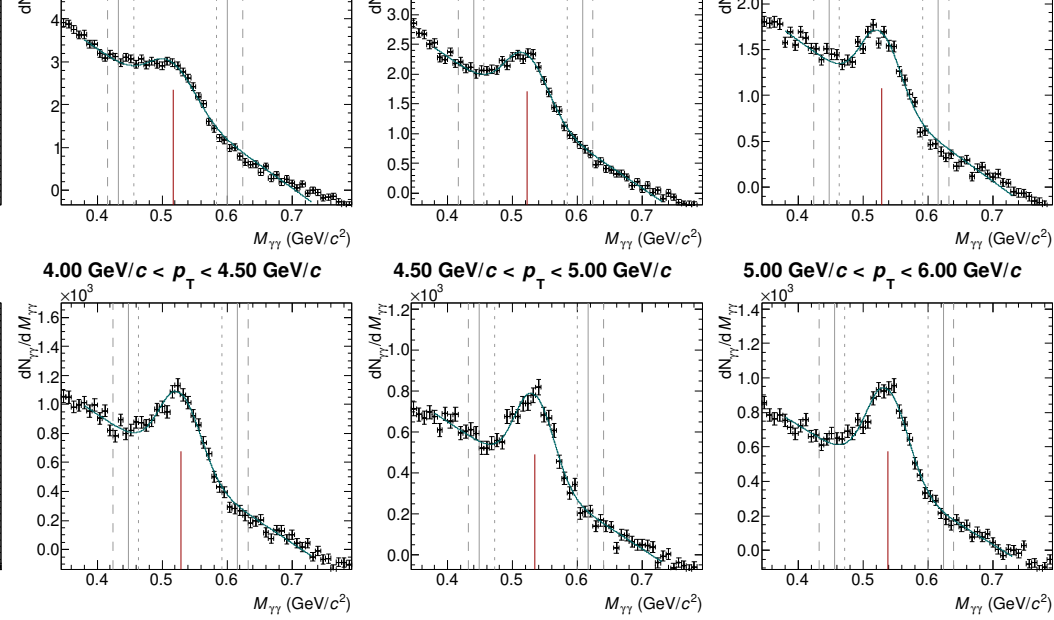

$5.00 \mathrm{GeV} / c<p_{\mathrm{T}}<6.00 \mathrm{GeV} / c$

$6.00 \mathrm{GeV} / c<p_{\mathrm{T}}<7.00 \mathrm{GeV} / c$

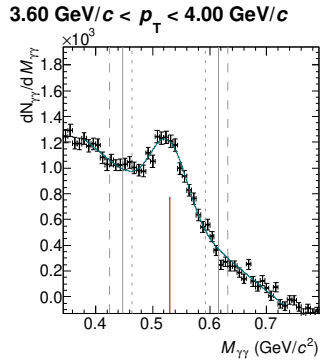

$7.00 \mathrm{GeV} / c<p_{\mathrm{T}}<8.00 \mathrm{GeV} / c$

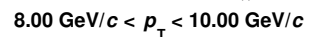

$10.00 \mathrm{GeV} / c<p_{\mathrm{T}}<12.00 \mathrm{GeV} / c$
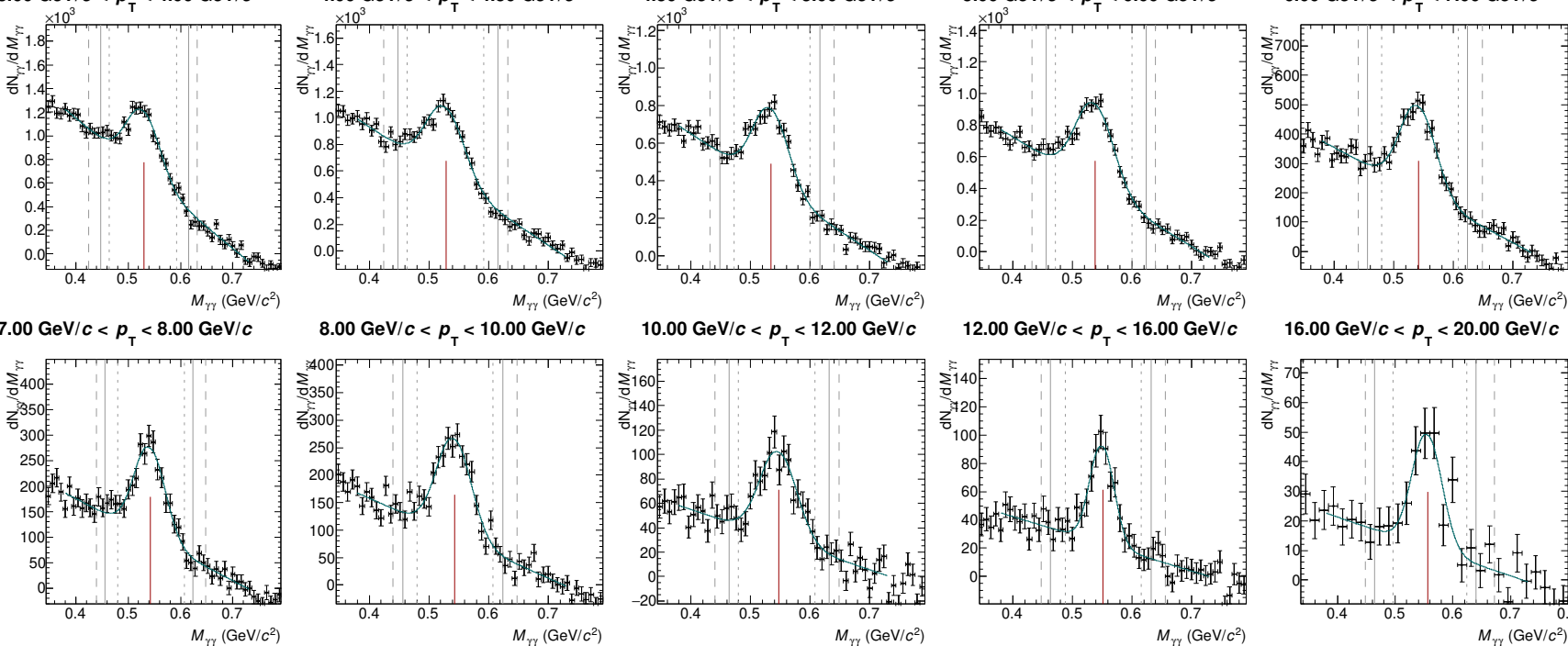

$12.00 \mathrm{GeV} / c<p_{\mathrm{T}}<16.00 \mathrm{GeV} / c$

$16.00 \mathrm{GeV} / c<p_{\mathrm{T}}<20.00 \mathrm{GeV} / c$

FIGURE A.4: Invariant mass distribution of photon pairs around the nominal value of the $\eta$ mass before(top) and after(bottom) subtraction of the combinatorial background, as measured with the ALICE detector in pp collisions at a center-of-mass energy of $\sqrt{s}=5.02 \mathrm{TeV}$. The meson candidates are reconstructed using EMC, covering $2.0<p_{\mathrm{T}}<20.0 \mathrm{GeV} / c$. 


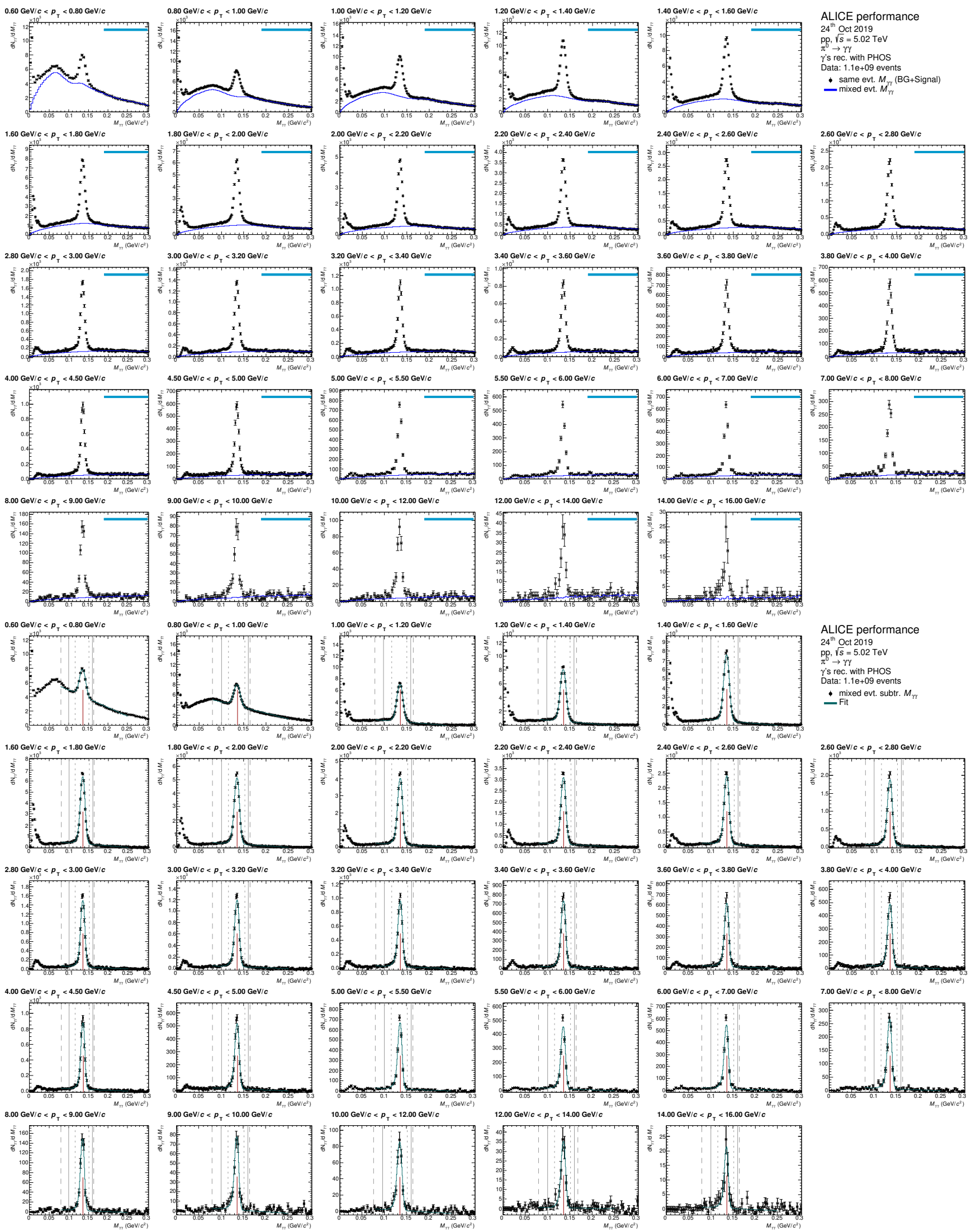

Figure A.5: Invariant mass distribution of photon pairs around the nominal value of the $\pi^{0}$ mass before(top) and after(bottom) subtraction of the combinatorial background, as measured with the ALICE detector in pp collisions at a center-of-mass energy of $\sqrt{s}=5.02 \mathrm{TeV}$. The meson candidates are reconstructed using PHOS, covering $0.6<p_{\mathrm{T}}<16.0 \mathrm{GeV} / c$. 

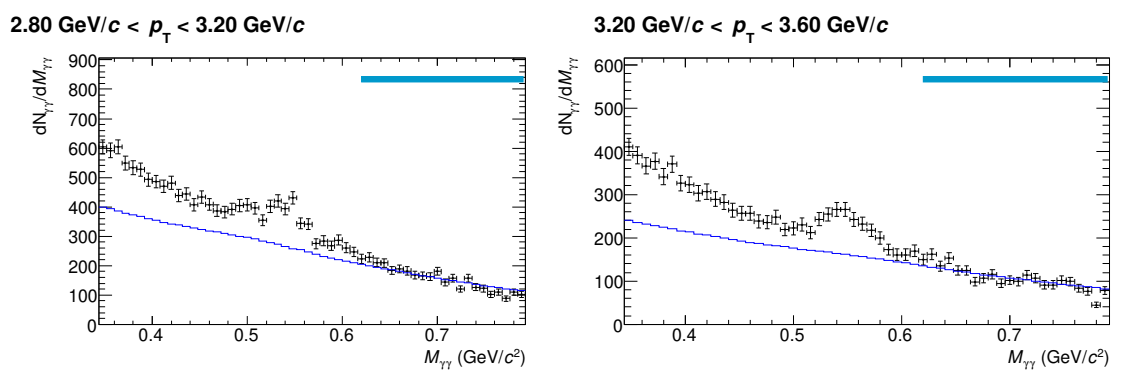

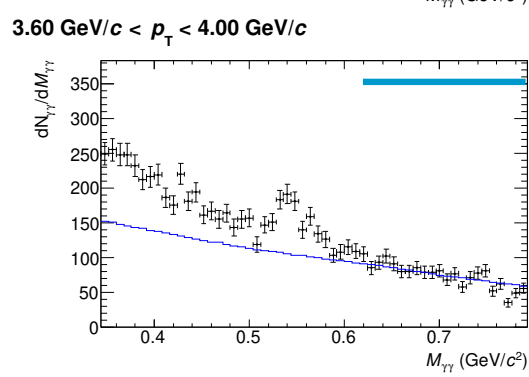

\section{$4.00 \mathrm{GeV} / c<p_{\mathrm{T}}<4.50 \mathrm{GeV} / c$}

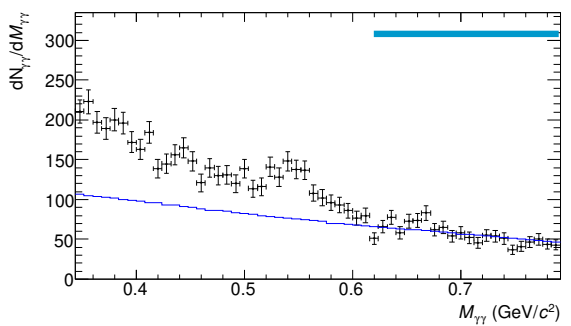

$6.00 \mathrm{GeV} / c<p_{\mathrm{T}}<7.00 \mathrm{GeV} / c$

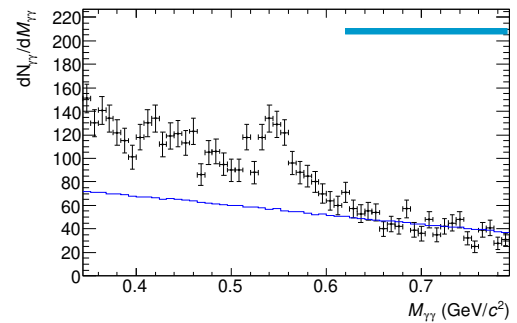

$2.80 \mathrm{GeV} / c<p_{\mathrm{T}}<3.20 \mathrm{GeV} / c$

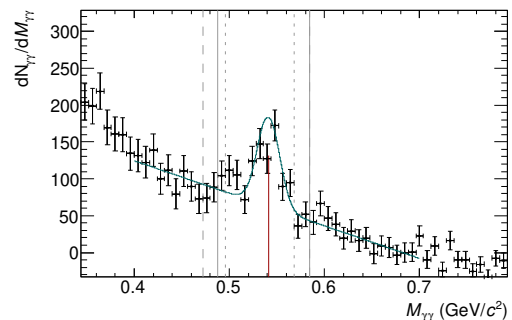

$3.60 \mathrm{GeV} / c<p_{\mathrm{T}}<4.00 \mathrm{GeV} / c$

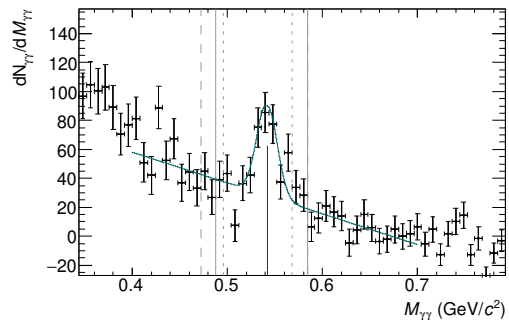

$5.00 \mathrm{GeV} / c<p_{\mathrm{T}}<6.00 \mathrm{GeV} / c$

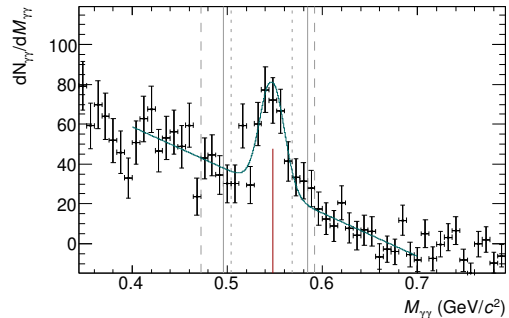

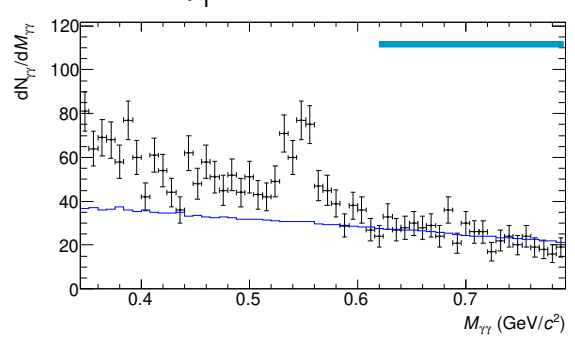

$3.20 \mathrm{GeV} / c<p_{\mathrm{T}}<3.60 \mathrm{GeV} / c$

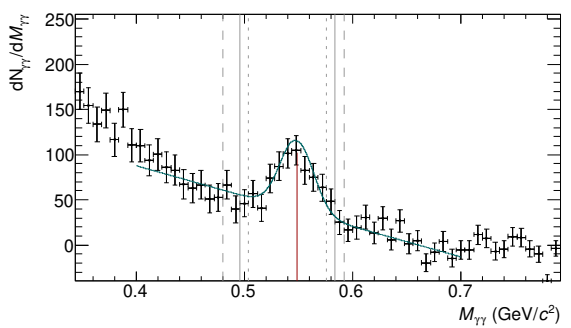

$4.00 \mathrm{GeV} / c<p_{\mathrm{T}}<4.50 \mathrm{GeV} / c$

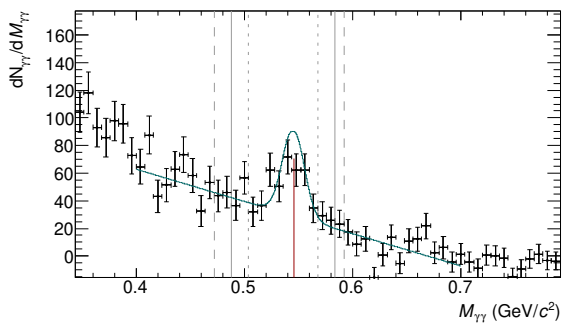

$6.00 \mathrm{GeV} / c<p_{\mathrm{T}}<7.00 \mathrm{GeV} / c$

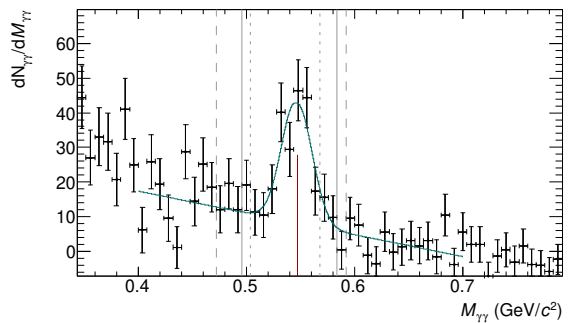

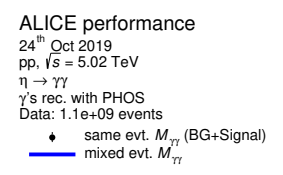

ALICE performance

$24, \sqrt{n}, \sqrt{s}=5.19$
$\mathrm{pp}$

$\gamma$ 's rec. with PHOS

- $\quad$ same evt. $M_{r y}(\mathrm{BG}+$ Signal $)$

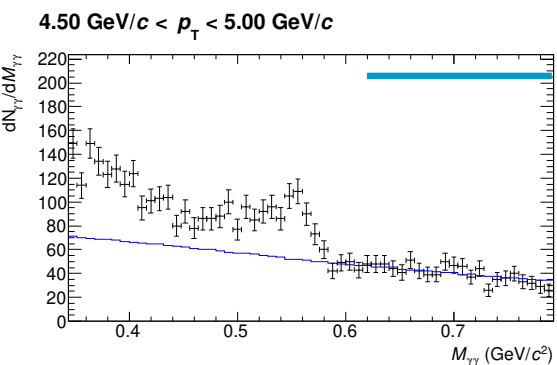

$7.00 \mathrm{GeV} / c<p_{\mathrm{T}}<8.00 \mathrm{GeV} / c$

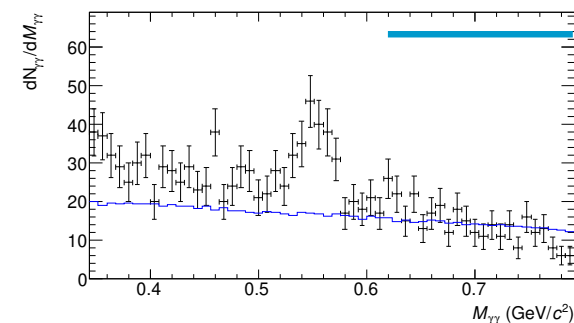

ALICE performance

$24^{\text {th }}$ Oct 2019
$\mathrm{pp}, \sqrt{s}=5.02 \mathrm{TeV}$

$\eta \rightarrow \gamma \gamma$
$\gamma$ 's rec. with PHOS

$+\quad$ mixed evt. subtr. $M_{r}$

$4.50 \mathrm{GeV} / c<p_{\mathrm{T}}<5.00 \mathrm{GeV} / c$

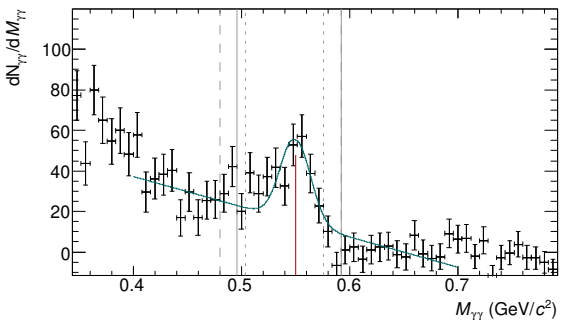

$7.00 \mathrm{GeV} / c<p_{\mathrm{T}}<8.00 \mathrm{GeV} / c$

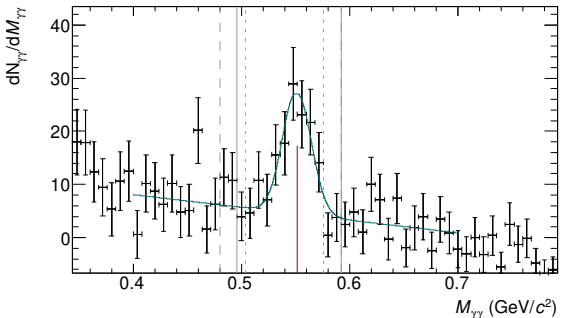

FiguRE A.6: Invariant mass distribution of photon pairs around the nominal value of the $\eta$ mass before(top) and after(bottom) subtraction of the combinatorial background, as measured with the ALICE detector in pp collisions at a center-of-mass energy of $\sqrt{s}=5.02 \mathrm{TeV}$. The meson candidates are reconstructed using PHOS, covering $2.8<p_{\mathrm{T}}<8.0 \mathrm{GeV} / c$. 


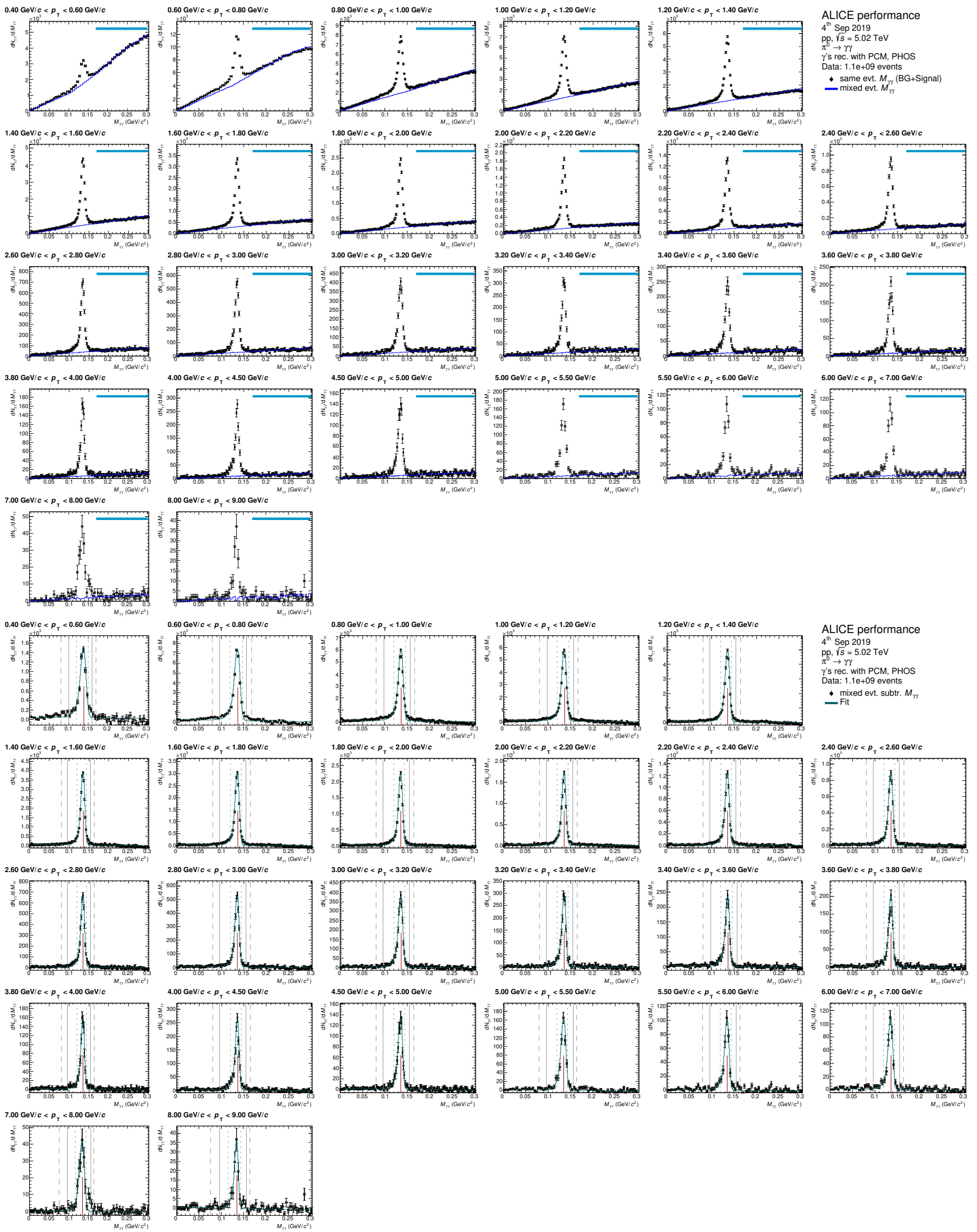

Figure A.7: Invariant mass distribution of photon pairs around the nominal value of the $\pi^{0}$ mass before(top) and after(bottom) subtraction of the combinatorial background, as measured with the ALICE detector in pp collisions at a center-of-mass energy of $\sqrt{s}=5.02 \mathrm{TeV}$. The meson candidates are reconstructed using PCM-PHOS, covering $0.4<p_{\mathrm{T}}<9.0 \mathrm{GeV} / c$. 

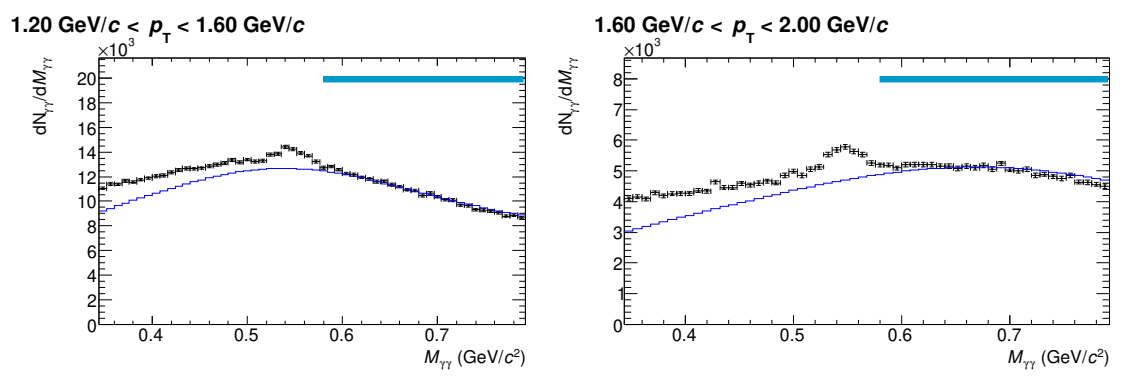

ALICE performance

$4^{\text {th }}$ Sep 2019
pp, $\sqrt{s}=5.02 \mathrm{TeV}$

$\eta \rightarrow \gamma \gamma$
$\gamma$ 's rec. with PCM, PHOS

same evt. $M_{r_{r \prime}}(\mathrm{BG}+\mathrm{Signal})$
mixed evt. $M_{v y}$
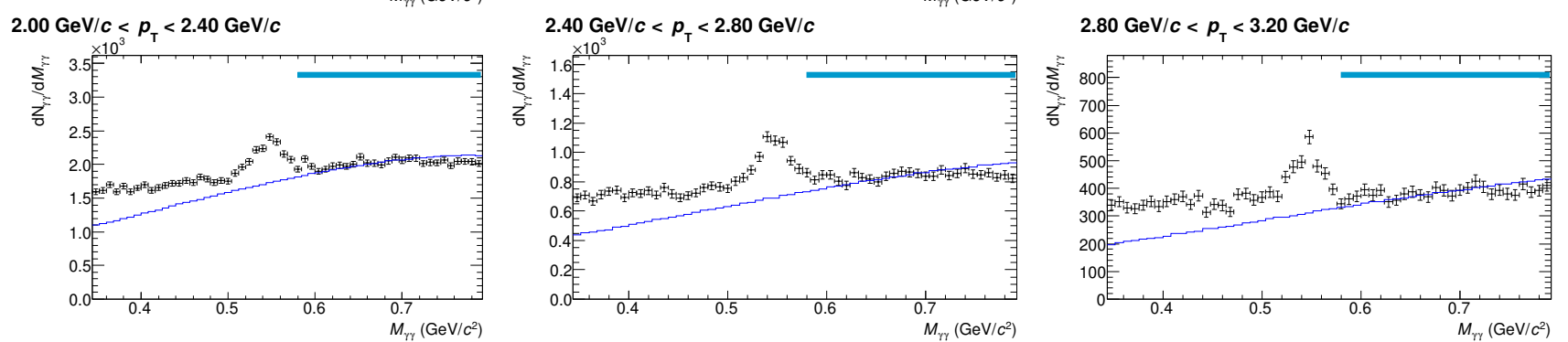

$3.20 \mathrm{GeV} / c<p_{\mathrm{T}}<3.60 \mathrm{GeV} / c$

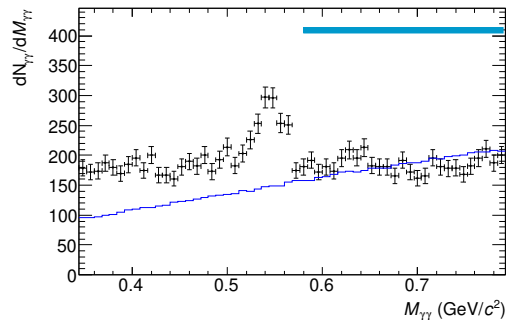

\section{$3.60 \mathrm{GeV} / c<p_{\mathrm{T}}<4.00 \mathrm{GeV} / c$}
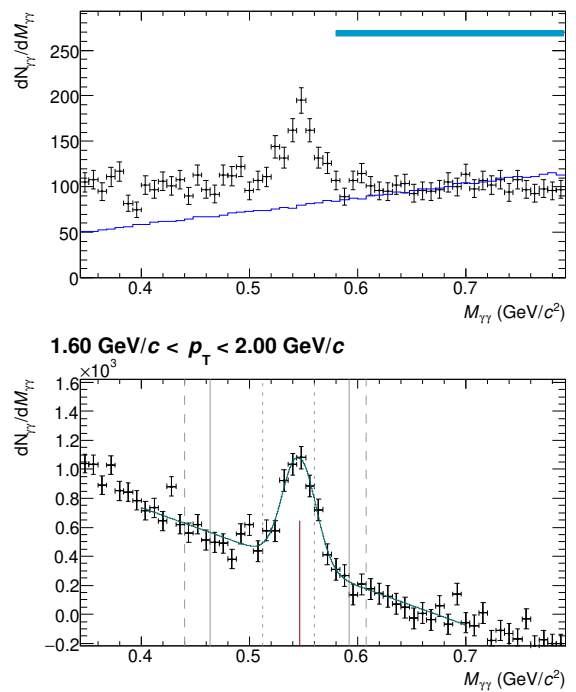

$2.40 \mathrm{GeV} / c<p_{\mathrm{T}}<2.80 \mathrm{GeV} / \mathrm{c}$

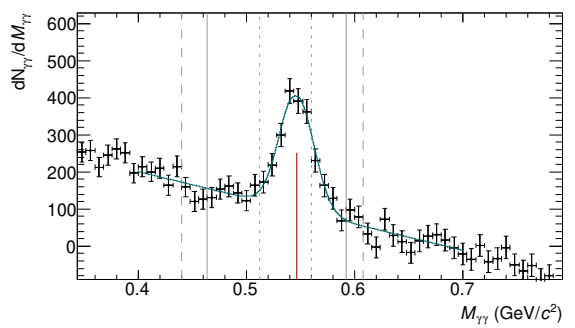

$3.60 \mathrm{GeV} / c<p_{\mathrm{T}}<4.00 \mathrm{GeV} / c$

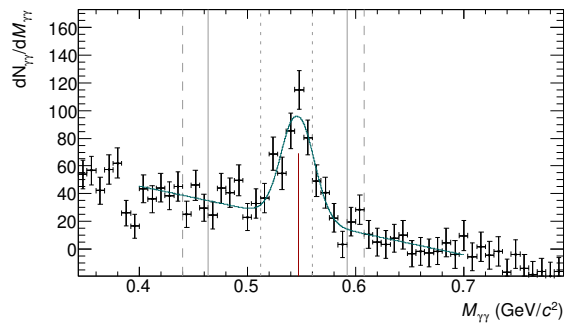

\section{$4.00 \mathrm{GeV} / c<p_{\mathrm{T}}<4.50 \mathrm{GeV} / c$}

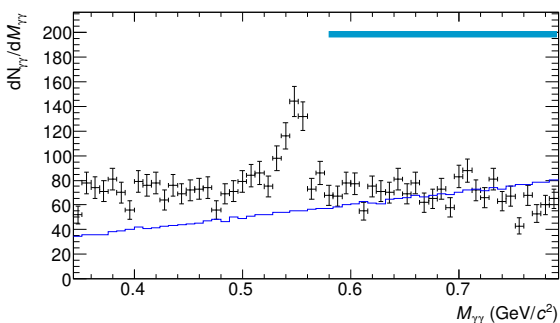

ALICE performance

$4^{\text {th }}$ Sep 2019
$\mathrm{pp}, \sqrt{s}=5.02 \mathrm{TeV}$

$\eta \rightarrow \gamma \gamma$
$\gamma$ 's rec. with PCM, PHOS

- mixed evt. subtr. $M_{r r}$
$2.80 \mathrm{GeV} / c<p_{\mathrm{T}}<3.20 \mathrm{GeV} / c$

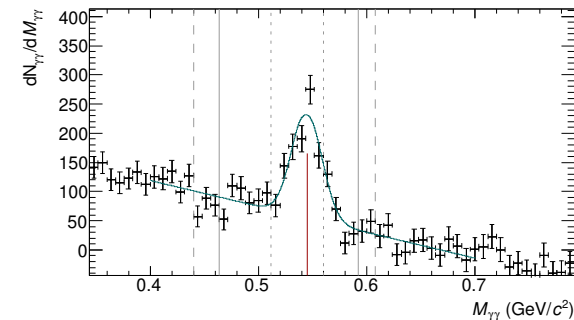

$4.00 \mathrm{GeV} / c<p_{\mathrm{T}}<4.50 \mathrm{GeV} / \mathrm{c}$

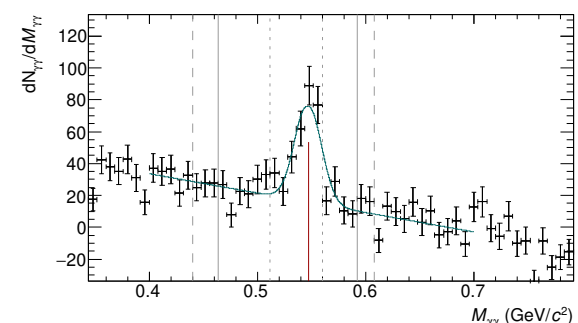

Figure A.8: Invariant mass distribution of photon pairs around the nominal value of the $\eta$ mass before(top) and after(bottom) subtraction of the combinatorial background, as measured with the ALICE detector in pp collisions at a center-of-mass energy of $\sqrt{s}=5.02 \mathrm{TeV}$. The meson candidates are reconstructed using PCM-PHOS, covering $1.2<p_{\mathrm{T}}<4.5 \mathrm{GeV} / c$. 


\section{A.2 Neutral meson raw yield correction in $\mathrm{Pb}-\mathrm{Pb}$ collisions}
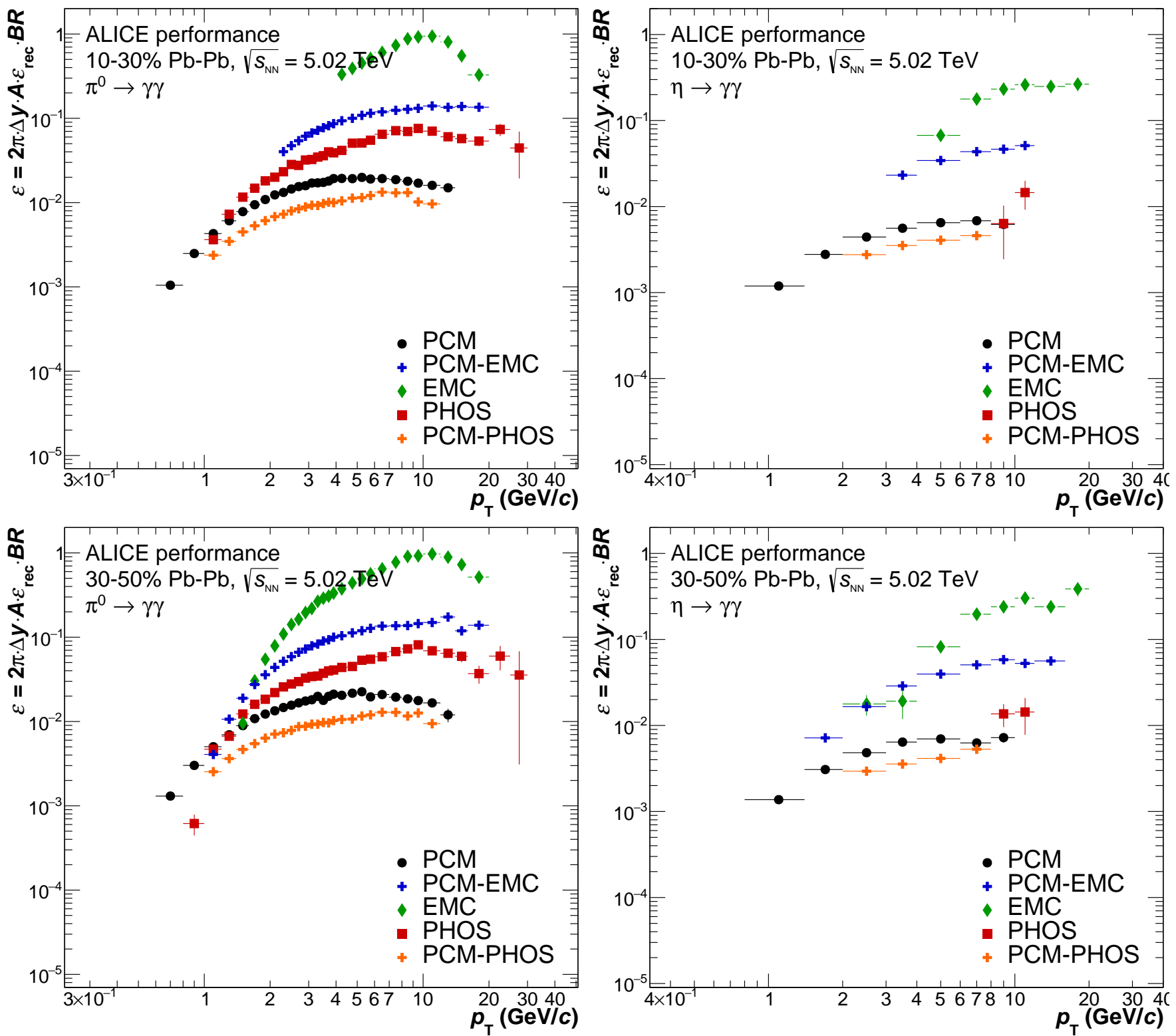

Figure A.9: The acceptance times efficiency for the $\pi^{0}$ (left), and the $\eta$ meson (right), as measured for the $10-30 \%$ (top) and $30-50 \%$ (bottom) V0M multiplicity classes in $\mathrm{Pb}-\mathrm{Pb}$ collisions at $\sqrt{s_{N N}}=5.02 \mathrm{TeV}$. 


\section{A.3 Systematic uncertainties of the neutral meson invariant yield}

This appendix lists the systematic uncertainties of all neutral meson reconstruction methods in pp collisions at $\sqrt{s}=5.02 \mathrm{TeV}$, for the EMCal reconstruction method in $\mathrm{p}-\mathrm{Pb}$ collisions at $\sqrt{s_{N N}}=5.02 \mathrm{TeV}$, and again for all neutral meson reconstruction methods in $\mathrm{Pb}-\mathrm{Pb}$ collisions at $\sqrt{s_{N N}}=5.02 \mathrm{TeV}$, as presented in chapter 6 . 

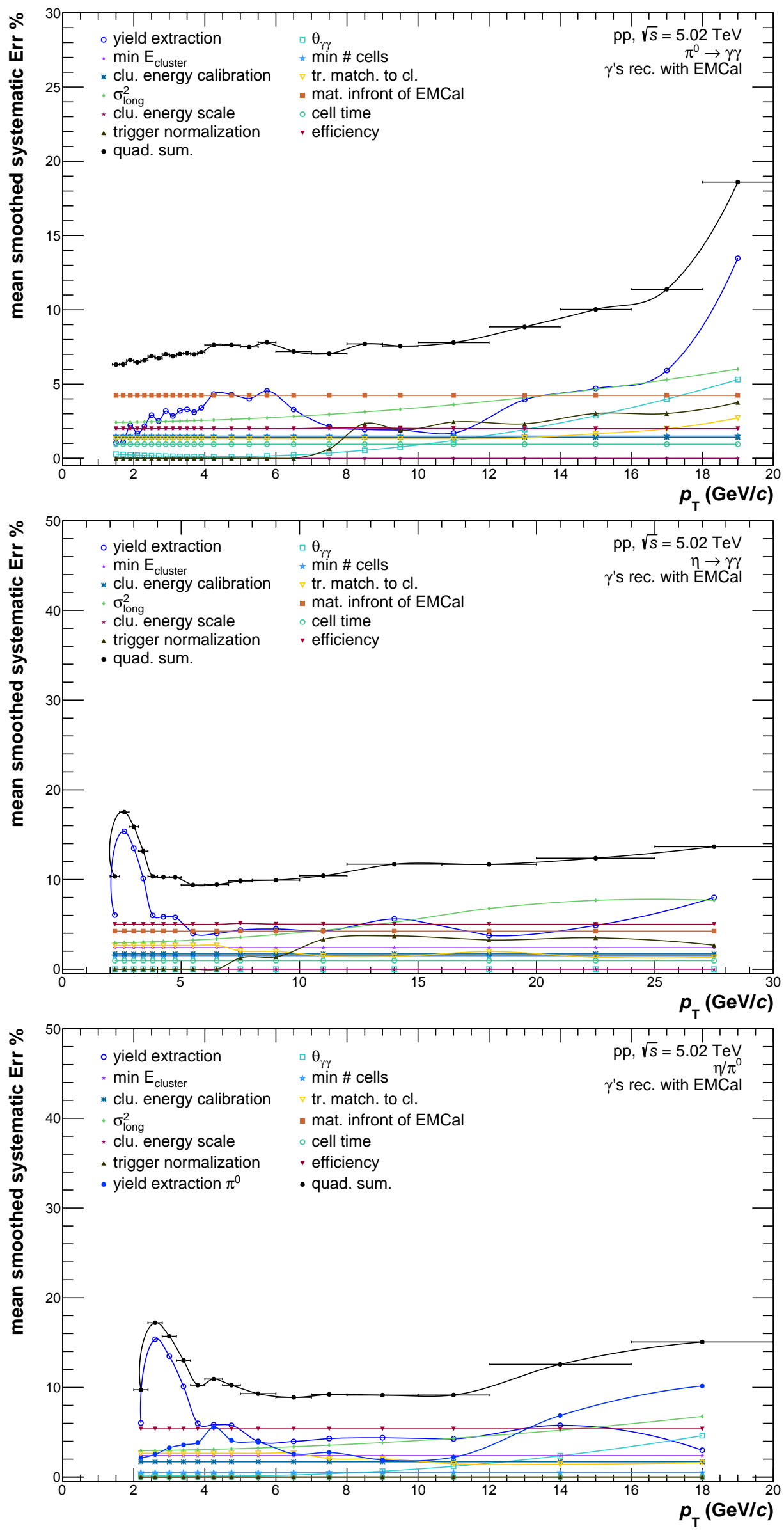

Figure A.10: The systematic uncertainties of the $\pi^{0}$ (top) and $\eta$ (middle) invariant yield, as well as the $\eta / p i^{0}$ ratio (bottom), as measured with EMCal in pp collisions at $\sqrt{s}=5.02 \mathrm{TeV}$. 

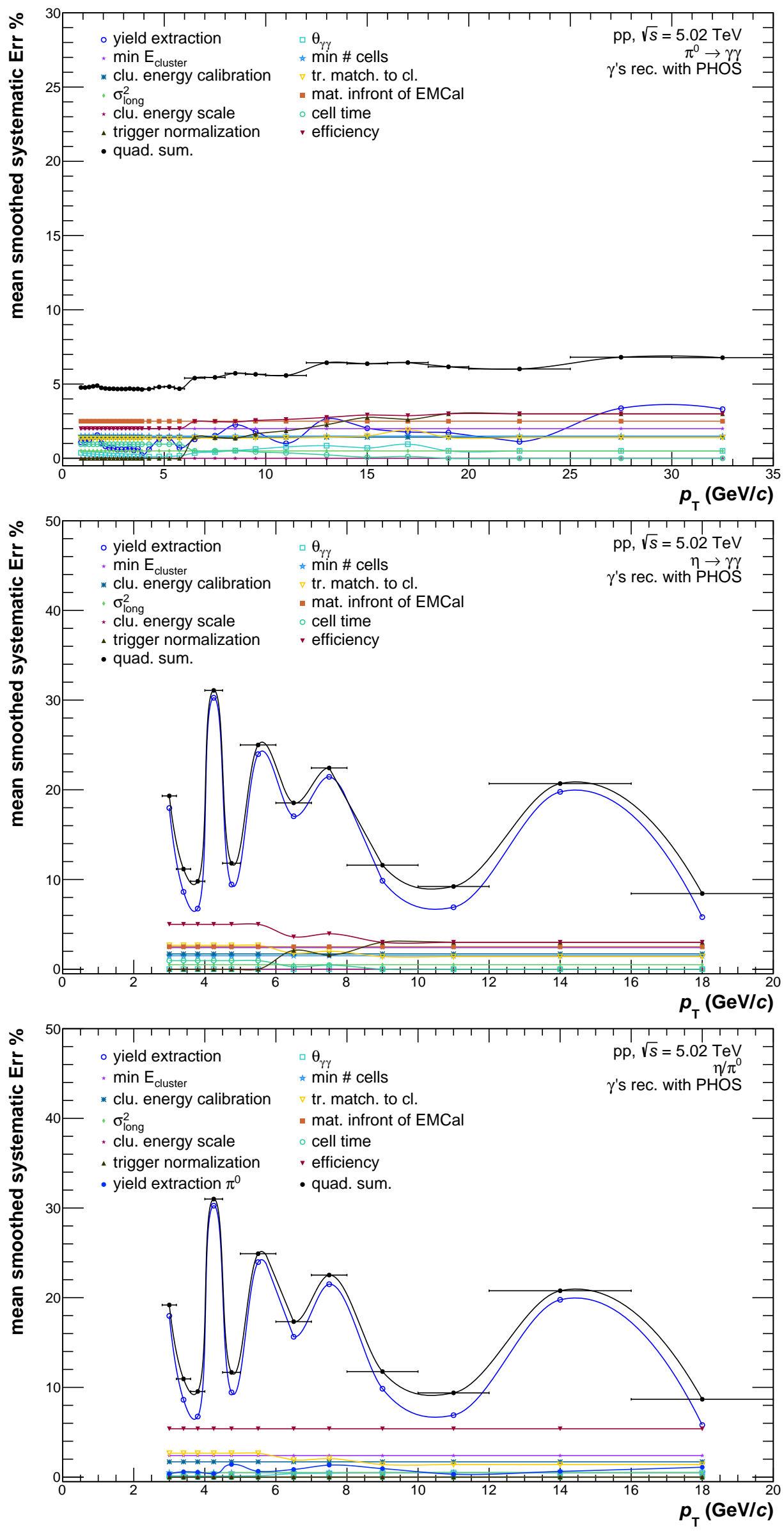

Figure A.11: The systematic uncertainties of the $\pi^{0}$ (top) and $\eta$ (middle) invariant yield, as well as the $\eta / p i^{0}$ ratio (bottom), as measured with PHOS in pp collisions at $\sqrt{s}=5.02 \mathrm{TeV}$. 

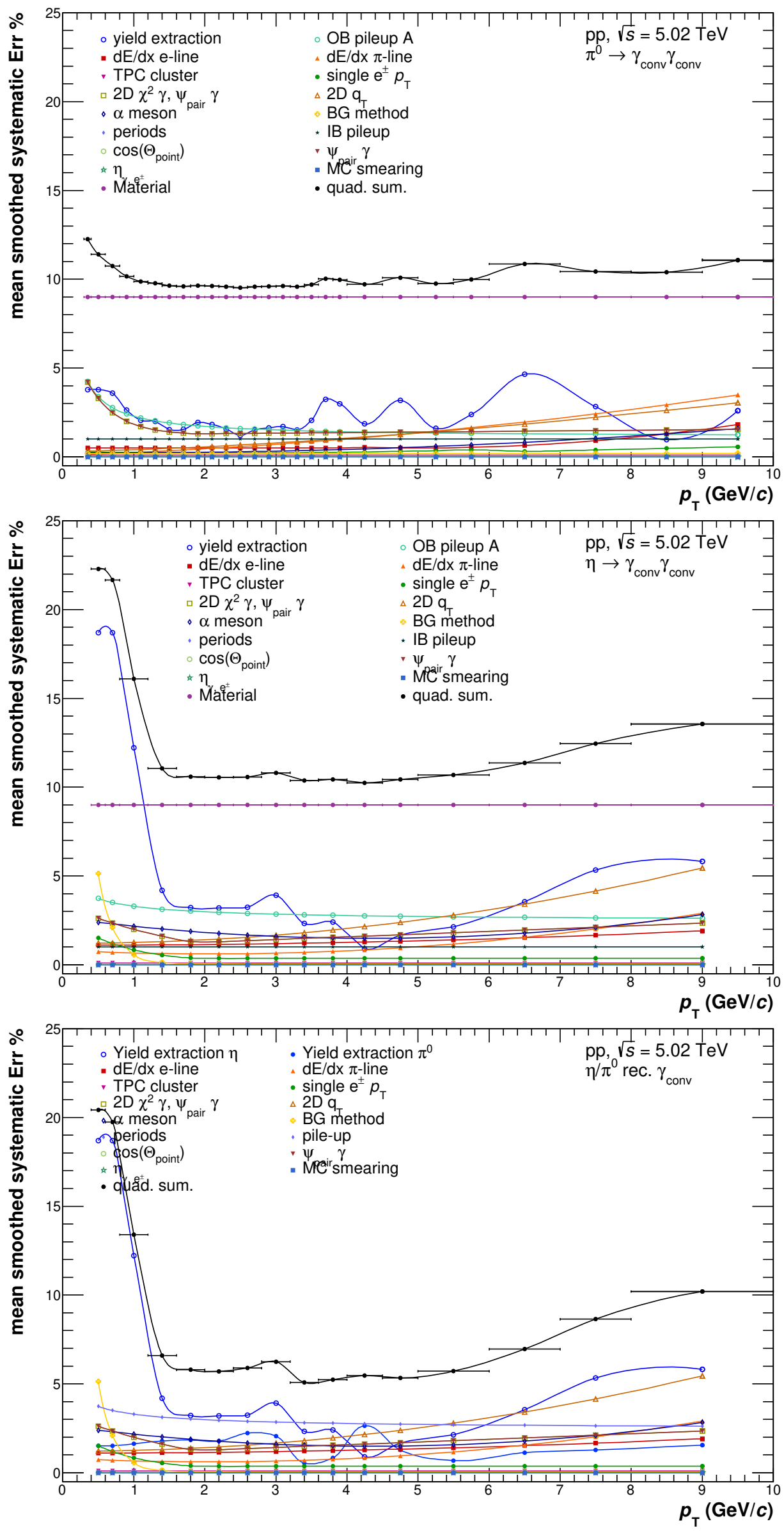

Figure A.12: The systematic uncertainties of the $\pi^{0}$ (top) and $\eta$ (middle) invariant yield, as well as the $\eta / p i^{0}$ ratio (bottom), as measured with PCM in pp collisions at $\sqrt{s}=5.02 \mathrm{TeV}$. 

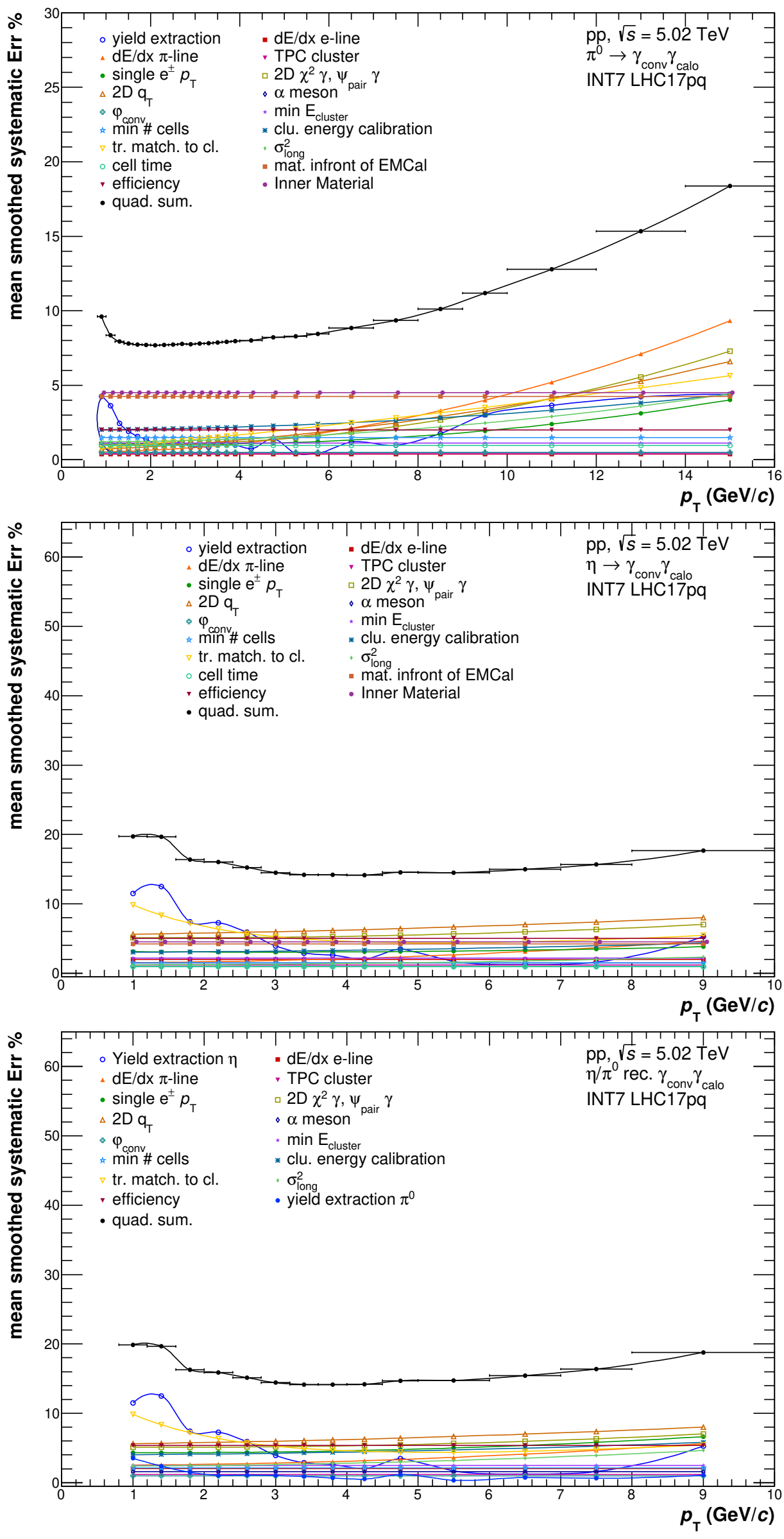

Figure A.13: The systematic uncertainties of the $\pi^{0}$ (top) and $\eta$ (middle) invariant yield, as well as the $\eta / p i^{0}$ ratio (bottom), as measured with PCM-EMC in pp collisions at $\sqrt{s}=5.02 \mathrm{TeV}$. 

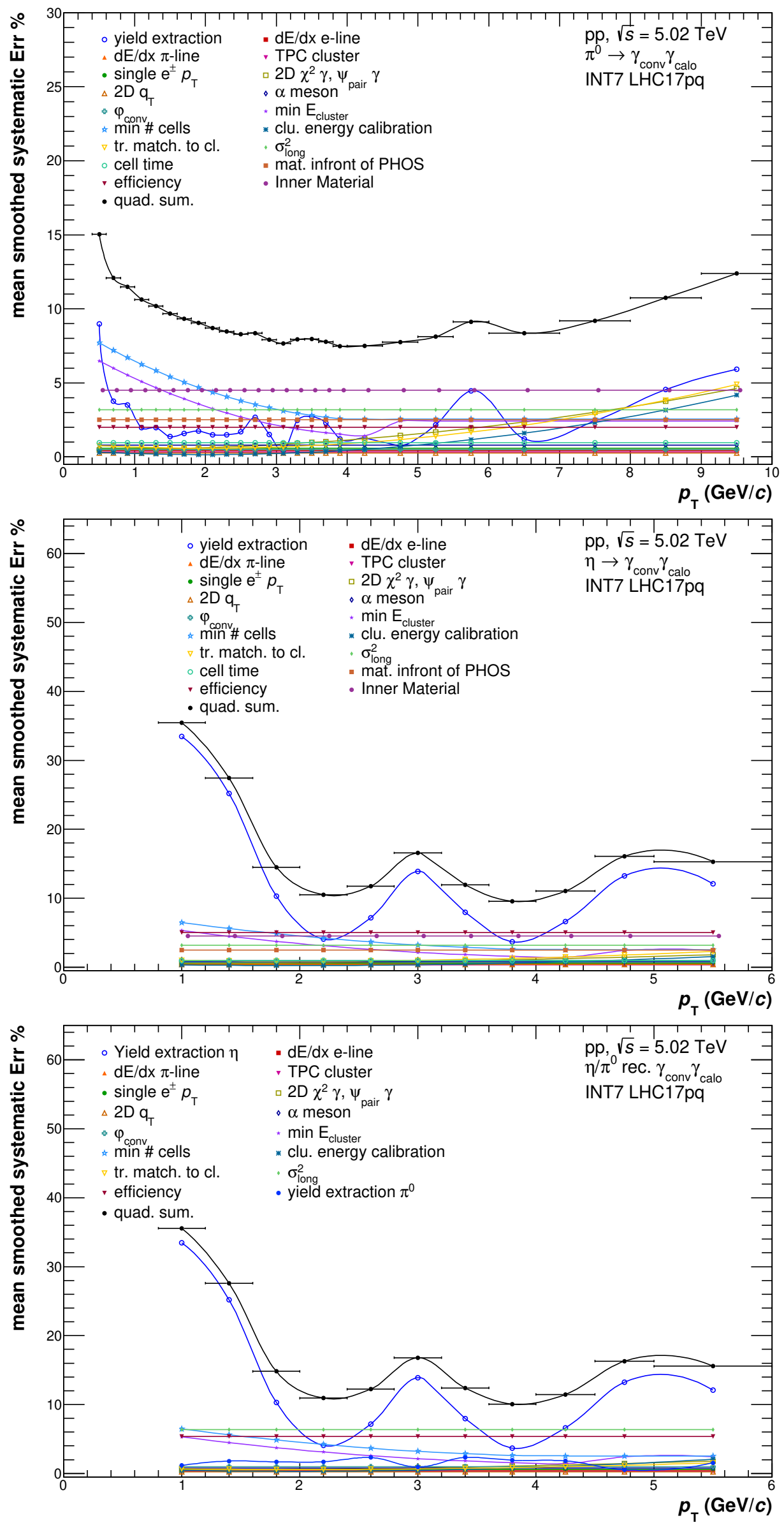

Figure A.14: The systematic uncertainties of the $\pi^{0}$ (top) and $\eta$ (middle) invariant yield, as well as the $\eta / p i^{0}$ ratio (bottom), as measured with PCM-PHOS in pp collisions at $\sqrt{s}=5.02 \mathrm{TeV}$. 

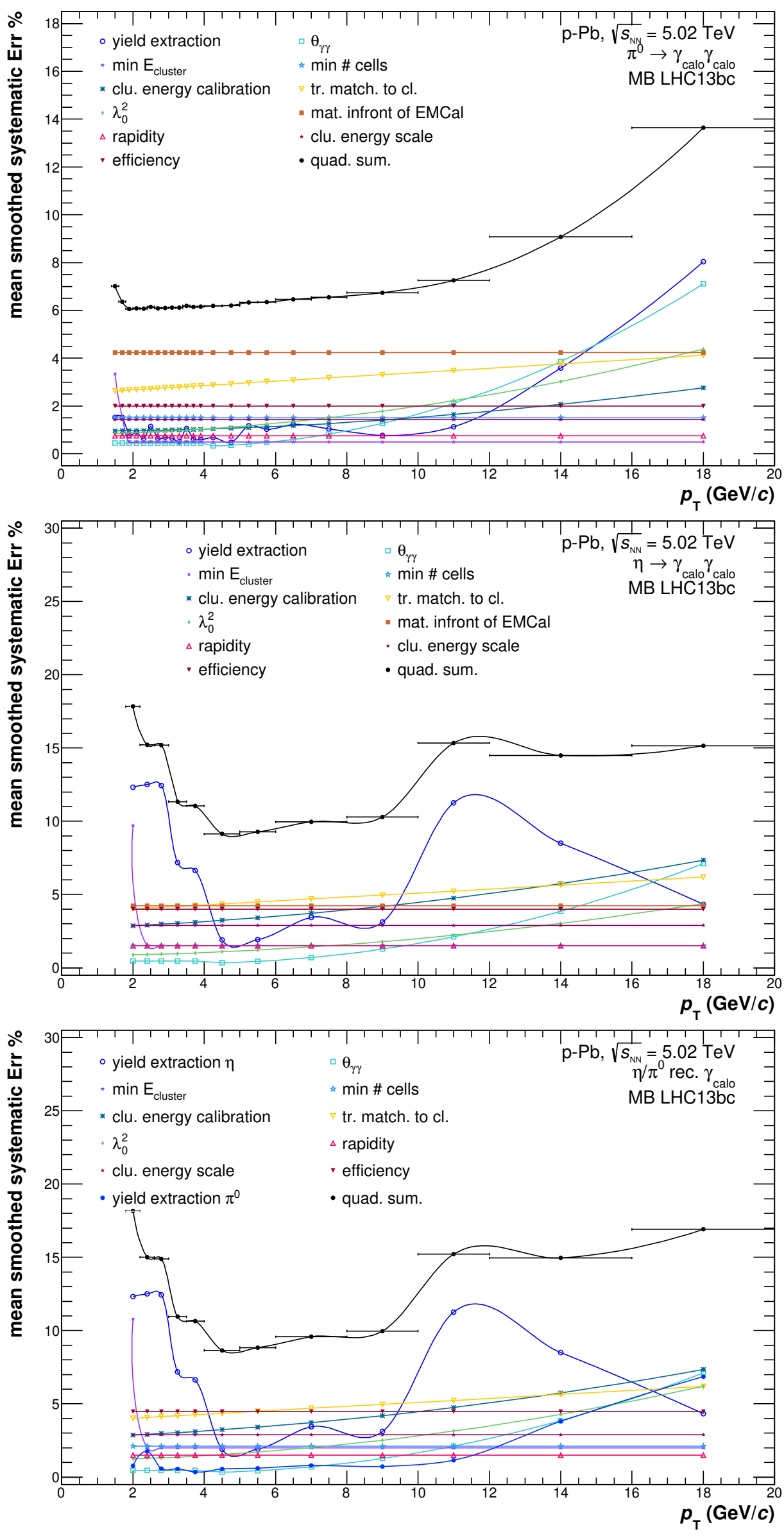

Figure A.15: The systematic uncertainties of the $\pi^{0}$ (top) and $\eta$ (middle) invariant yield, as well as the $\eta / p i^{0}$ ratio (bottom), as measured with EMCal in $\mathrm{p}-\mathrm{Pb}$ collisions at $\sqrt{s_{N N}}=5.02 \mathrm{TeV}$. 

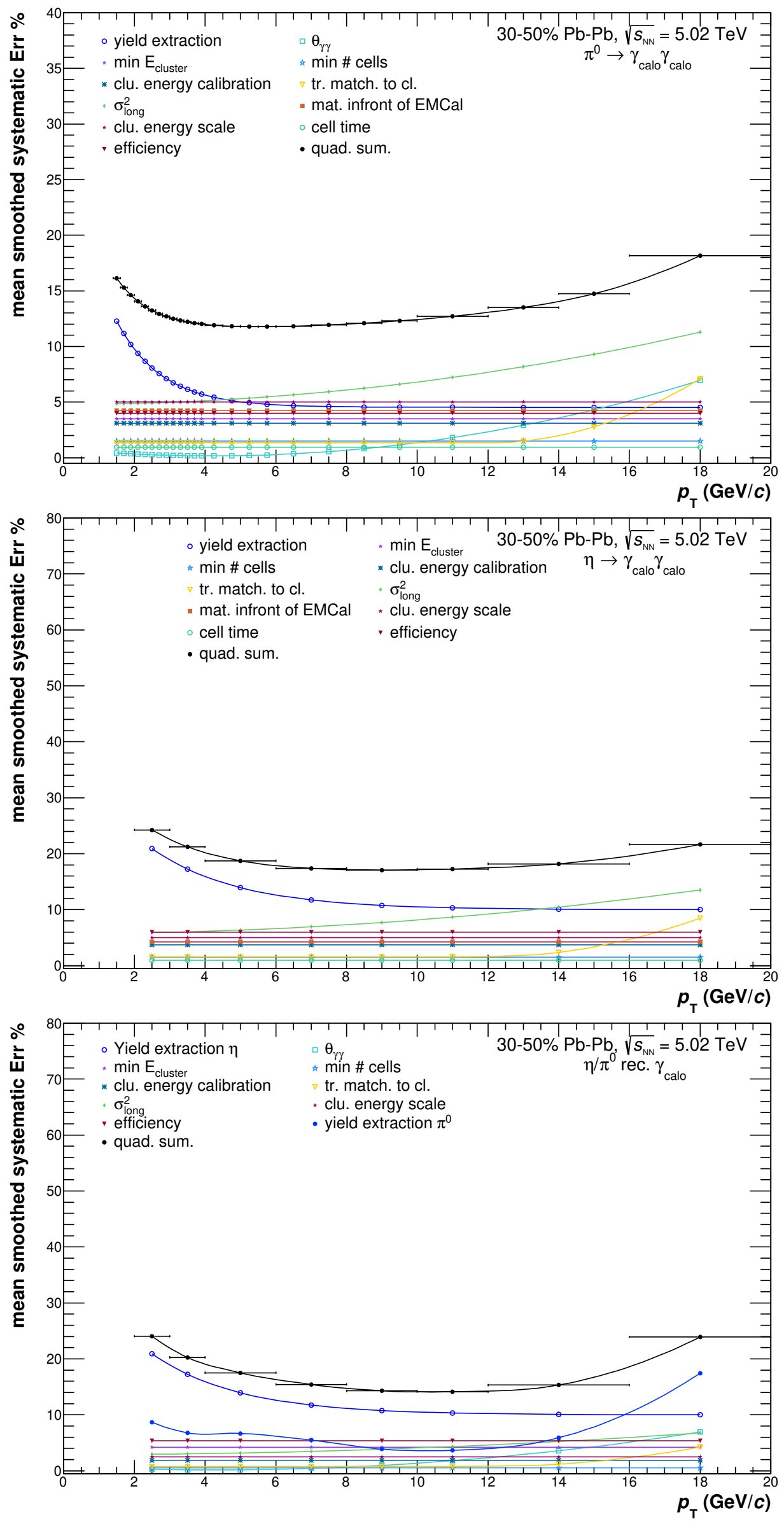

Figure A.16: The systematic uncertainties of the $\pi^{0}$ (top) and $\eta$ (middle) invariant yield, as well as the $\eta / p i^{0}$ ratio (bottom), as measured with EMCal in semi-central $\mathrm{Pb}-\mathrm{Pb}$ collisions at $\sqrt{s}=5.02 \mathrm{TeV}$. 

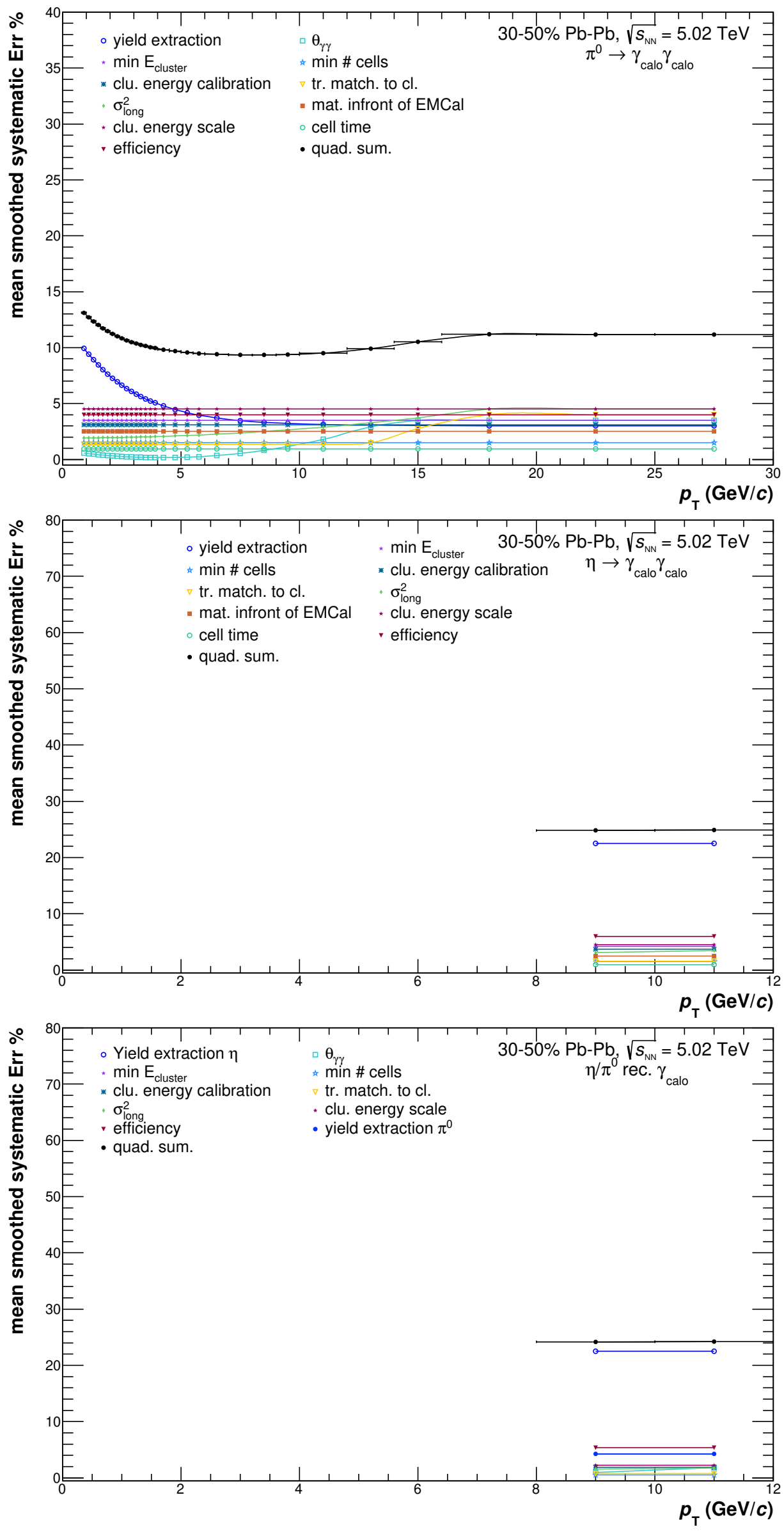

FIguRE A.17: The systematic uncertainties of the $\pi^{0}$ (top) and $\eta$ (middle) invariant yield, as well as the $\eta / p i^{0}$ ratio (bottom), as measured with PHOS in semi-central $\mathrm{Pb}-\mathrm{Pb}$ collisions at $\sqrt{s}=5.02 \mathrm{TeV}$. 

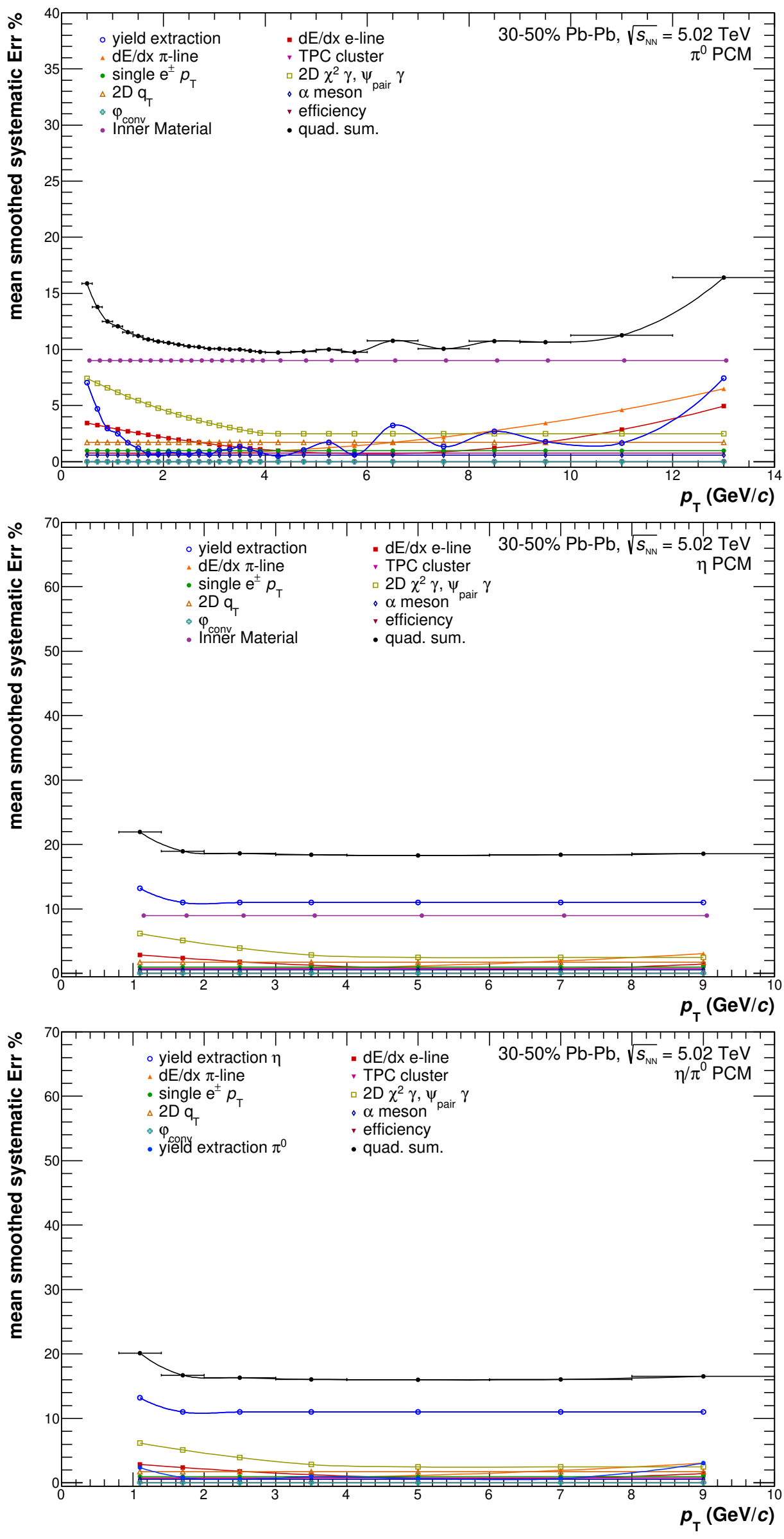

Figure A.18: The systematic uncertainties of the $\pi^{0}$ (top) and $\eta$ (middle) invariant yield, as well as the $\eta / p i^{0}$ ratio (bottom), as measured with PCM in semi-central $\mathrm{Pb}-\mathrm{Pb}$ collisions at $\sqrt{s}=5.02 \mathrm{TeV}$. 

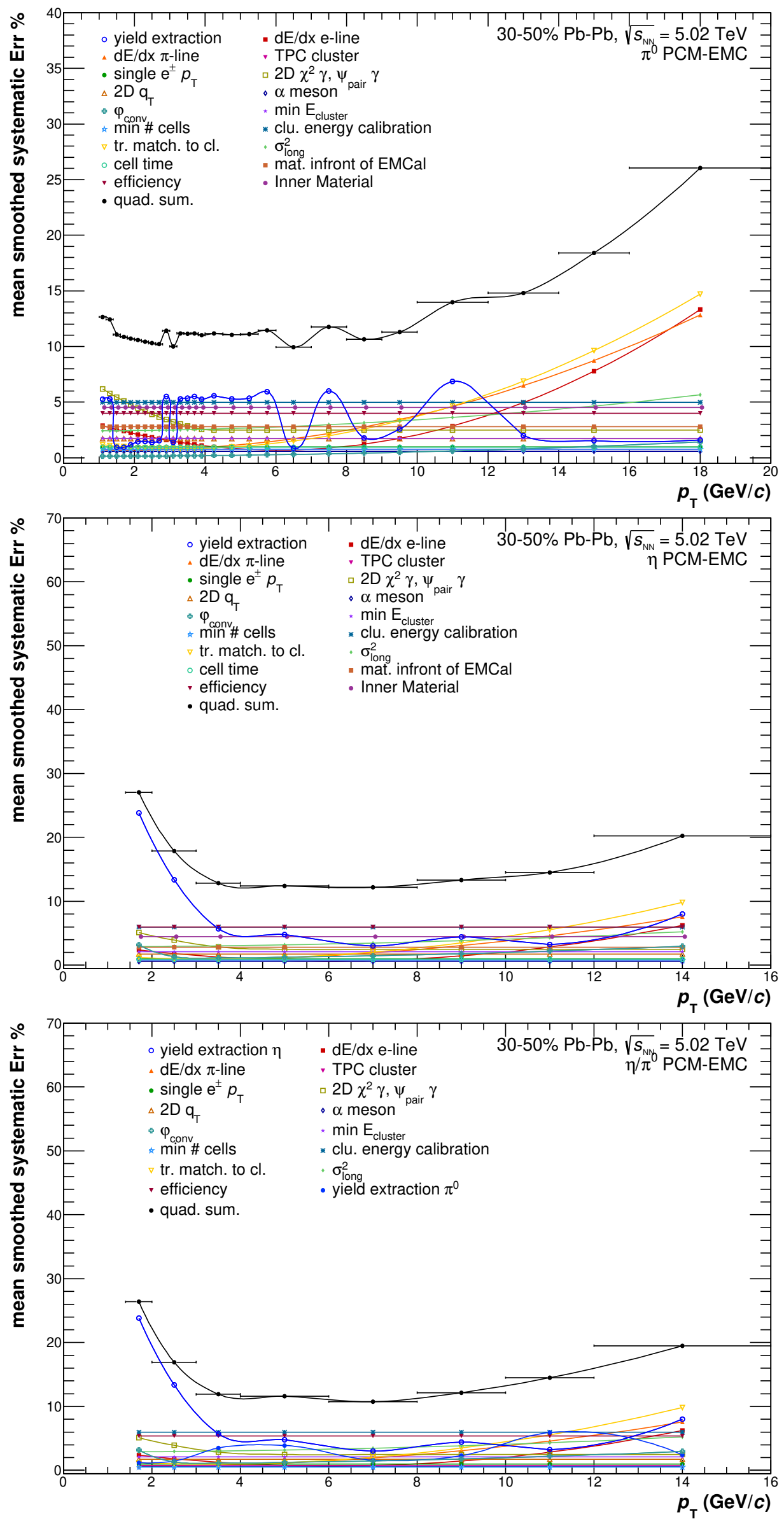

Figure A.19: The systematic uncertainties of the $\pi^{0}$ (top) and $\eta$ (middle) invariant yield, as well as the $\eta / p i^{0}$ ratio (bottom), as measured with PCM-EMC in semi-central $\mathrm{Pb}-\mathrm{Pb}$ collisions at $\sqrt{s}=5.02 \mathrm{TeV}$. 

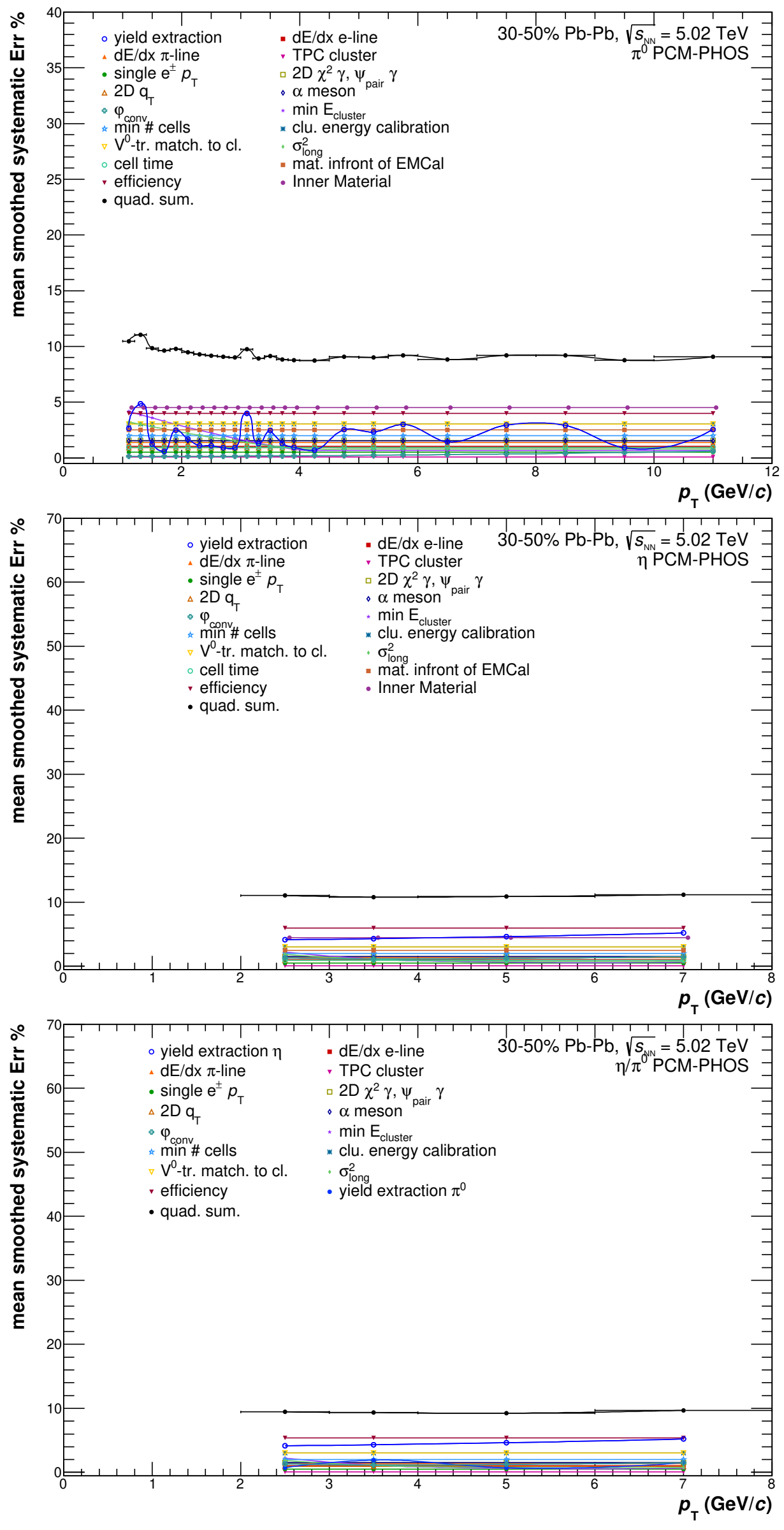

Figure A.20: The systematic uncertainties of the $\pi^{0}$ (top) and $\eta$ (middle) invariant yield, as well as the $\eta / p i^{0}$ ratio (bottom), as measured with PCM-PHOS in $\mathrm{Pb}-\mathrm{Pb}$ collisions at $\sqrt{s}=5.02 \mathrm{TeV}$. 


\section{Bibliography}

[1] Bharat Ratra and Michael S. Vogeley. The Beginning and Evolution of the Universe. Publ. Astron. Soc. Pac., 120:235-265, 2008. doi: 10.1086/529495.

[2] Pratika Dayal and Andrea Ferrara. Early galaxy formation and its large-scale effects. Phys. Rept., 780-782:1-64, 2018. doi: 10.1017/S1743921320001106.

[3] Christopher J. Conselice. The Evolution of Galaxy Structure over Cosmic Time. Ann. Rev. Astron. Astrophys., 52:291-337, 2014. doi: 10.1146/annurev-astro-081913-040037.

[4] M. Thomson. Modern Particle Physics. Cambridge University Press, ISBN-13: 978-1107034266, 2013.

[5] M. Tanabashi et al. Review of Particle Physics. Phys. Rev. D, 98(3):030001, 2018. doi: 10.1103/ PhysRevD.98.030001.

[6] F. Englert and R. Brout. Broken symmetry and the mass of gauge vector mesons. Phys. Rev. Lett., 13: 321-323, Aug 1964. doi: 10.1103/PhysRevLett.13.321. URL https://link.aps.org/doi/10.1103/ PhysRevLett.13.321.

[7] Peter W. Higgs. Broken symmetries and the masses of gauge bosons. Phys. Rev. Lett., 13:508-509, Oct 1964. doi: 10.1103/PhysRevLett.13.508. URL https://link.aps.org/doi/10.1103/PhysRevLett. 13.508.

[8] Georges Aad et al. Observation of a new particle in the search for the Standard Model Higgs boson with the ATLAS detector at the LHC. Phys. Lett. B, 716:1-29, 2012. doi: 10.1016/j.physletb.2012.08.020.

[9] Serguei Chatrchyan et al. Observation of a New Boson at a Mass of $125 \mathrm{GeV}$ with the CMS Experiment at the LHC. Phys. Lett. B, 716:30-61, 2012. doi: 10.1016/j.physletb.2012.08.021.

[10] Antonino Del Popolo. Dark matter and structure formation a review. Astron. Rep., 51:169-196, 2007. doi: 10.1134/S1063772907030018.

[11] R. P. Feynman. Space-time approach to quantum electrodynamics. Phys. Rev., 76:769-789, Sep 1949. doi: 10.1103/PhysRev.76.769. URL https://link.aps.org/doi/10.1103/PhysRev.76.769.

[12] D. J. Gross and F. Wilczek. Asymptotically Free Gauge Theories - I. Phys. Rev. D, 8, 1973. doi: 10.1103/PhysRevD.8.3633. 
[13] Particle Data Group. 9. Quantum Chromodynamics - PDG. , 2019. URL https://pdg.1bl.gov/ 2019/reviews/rpp2019-rev-qcd.pdf.

[14] D J E Callaway and A Rahman. Lattice gauge theory in the microcanonical ensemble. Phys. Rev. D, 28(ANL-HEP-PR-83-04):1506. 37 p, Jan 1983. doi: 10.1103/PhysRevD.28.1506. URL https: //cds.cern.ch/record/144746.

[15] Claudia Ratti. Lattice QCD and heavy ion collisions: a review of recent progress. Rept. Prog. Phys., 81(8):084301, 2018. doi: 10.1088/1361-6633/aabb97.

[16] Szabolcs Borsanyi. Thermodynamics of the QCD transition from lattice. Nucl. Phys. A, 904-905: 270c-277c, 2013. doi: 10.1016/j.nuclphysa.2013.01.072.

[17] Wit Busza, Krishna Rajagopal, and Wilke van der Schee. Heavy Ion Collisions: The Big Picture, and the Big Questions. Ann. Rev. Nucl. Part. Sci., 68:339-376, 2018. doi: 10.1146/ annurev-nucl-101917-020852.

[18] A. Bazavov et al. Equation of state and QCD transition at finite temperature. Phys. Rev. D, 80: 014504, 2009. doi: 10.1103/PhysRevD.80.014504.

[19] Owe Philipsen. The QCD equation of state from the lattice. Prog. Part. Nucl. Phys., 70:55-107, 2013. doi: $10.1016 /$ j.ppnp.2012.09.003.

[20] A. Bazavov et al. Equation of state in ( 2+1 )-flavor QCD. Phys. Rev. D, 90:094503, 2014. doi: 10.1103/PhysRevD.90.094503.

[21] Ulrich W. Heinz. The Strongly coupled quark-gluon plasma created at RHIC. J. Phys. A, 42:214003, 2009. doi: 10.1088/1751-8113/42/21/214003.

[22] Grazyna Odyniec. The RHIC beam energy scan program in STAR and what is next .... Journal of Physics: Conference Series, 455:012037, aug 2013. doi: 10.1088/1742-6596/455/1/012037. URL https://doi.org/10.1088\%2F 1742-6596\%2F455\%2F1\%2F012037.

[23] Jaroslav Adam et al. Enhanced production of multi-strange hadrons in high-multiplicity proton-proton collisions. Nature Phys., 13:535-539, 2017. doi: 10.1038/nphys4111.

[24] Shreyasi Acharya et al. Investigations of Anisotropic Flow Using Multiparticle Azimuthal Correlations in pp, p-Pb, Xe-Xe, and Pb-Pb Collisions at the LHC. Phys. Rev. Lett., 123(14):142301, 2019. doi: 10.1103/PhysRevLett.123.142301.

[25] J. D. Bjorken and E. A. Paschos. Inelastic electron-proton and $\gamma$-proton scattering and the structure of the nucleon. Phys. Rev., 185:1975-1982, Sep 1969. doi: 10.1103/PhysRev.185.1975. URL https: //link.aps.org/doi/10.1103/PhysRev.185.1975.

[26] J. Bernhard. Simulation of the QGP evolution for different times. Taken from presentation, 2020. 
[27] Kari J. Eskola. Initial state of the QGP from perturbative QCD + saturation. Nucl. Phys. A, 702 : 249-258, 2002. doi: 10.1016/S0375-9474(02)00712-1.

[28] Jonah E. Bernhard, J. Scott Moreland, and Steffen A. Bass. Characterization of the initial state and QGP medium from a combined Bayesian analysis of LHC data at 2.76 and $5.02 \mathrm{TeV}$. Nucl. Phys. A, 967:293-296, 2017. doi: 10.1016/j.nuclphysa.2017.05.037.

[29] J. Scott Moreland, Jonah E. Bernhard, and Steffen A. Bass. Bayesian calibration of a hybrid nuclear collision model using p- $\mathrm{Pb}$ and $\mathrm{Pb}-\mathrm{Pb}$ data at energies available at the CERN Large Hadron Collider. Phys. Rev. C, 101(2):024911, 2020. doi: 10.1103/PhysRevC.101.024911.

[30] Larry McLerran and Raju Venugopalan. Computing quark and gluon distribution functions for very large nuclei. Phys. Rev. D, 49:2233-2241, Mar 1994. doi: 10.1103/PhysRevD.49.2233. URL https: //link.aps.org/doi/10.1103/PhysRevD.49.2233.

[31] Michael L. Miller, Klaus Reygers, Stephen J. Sanders, and Peter Steinberg. Glauber modeling in high energy nuclear collisions. Ann. Rev. Nucl. Part. Sci., 57:205-243, 2007. doi: 10.1146/annurev.nucl. 57.090506 .123020 .

[32] Björn Schenke, Prithwish Tribedy, and Raju Venugopalan. Fluctuating Glasma initial conditions and flow in heavy ion collisions. Phys. Rev. Lett., 108:252301, 2012. doi: 10.1103/PhysRevLett.108.252301.

[33] Charles Gale, Sangyong Jeon, Björn Schenke, Prithwish Tribedy, and Raju Venugopalan. Event-byevent anisotropic flow in heavy-ion collisions from combined Yang-Mills and viscous fluid dynamics. Phys. Rev. Lett., 110(1):012302, 2013. doi: 10.1103/PhysRevLett.110.012302.

[34] Chun Shen and Ulrich Heinz. The road to precision: Extraction of the specific shear viscosity of the quark-gluon plasma. Nucl. Phys. News, 25(2):6-11, 2015. doi: 10.1080/10619127.2015.1006502.

[35] Paul Romatschke and Ulrike Romatschke. Viscosity information from relativistic nuclear collisions: How perfect is the fluid observed at rhic? Phys. Rev. Lett., 99:172301, Oct 2007. doi: 10.1103/ PhysRevLett.99.172301. URL https://link.aps.org/doi/10.1103/PhysRevLett.99.172301.

[36] Jonah E. Bernhard, J. Scott Moreland, Steffen A. Bass, Jia Liu, and Ulrich Heinz. Applying Bayesian parameter estimation to relativistic heavy-ion collisions: simultaneous characterization of the initial state and quark-gluon plasma medium. Phys. Rev. C, 94(2):024907, 2016. doi: 10.1103/PhysRevC. 94.024907.

[37] K. Aamodt et al. Two-pion Bose-Einstein correlations in central Pb-Pb collisions at $\sqrt{s_{N N}}=2.76$ TeV. Phys. Lett. B, 696:328-337, 2011. doi: 10.1016/j.physletb.2010.12.053.

[38] Bo Andersson, Sandipan Mohanty, and Fredrik Soderberg. The Lund fragmentation process for a multigluon string according to the area law. Eur. Phys. J. C, 21:631-647, 2001. doi: 10.1007/ s100520100757. 
[39] Nadine Fischer and Torbjörn Sjöstrand. Thermodynamical String Fragmentation. JHEP, 01:140, 2017. doi: 10.1007/JHEP01(2017)140.

[40] Silvia Ferreres-Sol and Torbjörn Sjöstrand. The space-time structure of hadronization in the Lund model. Eur. Phys. J. C, 78(11):983, 2018. doi: 10.1140/epjc/s10052-018-6459-8.

[41] Ralf Rapp. Quark coalescence and charm(onium) in QGP. Eur. Phys. J. C, 43:91-96, 2005. doi: 10.1140/epjc/s2005-02215-0.

[42] Rainer J. Fries, Vincenzo Greco, and Paul Sorensen. Coalescence Models For Hadron Formation From Quark Gluon Plasma. Ann. Rev. Nucl. Part. Sci., 58:177-205, 2008. doi: 10.1146/annurev.nucl.58. 110707.171134.

[43] K.J. Eskola and H. Honkanen. A Perturbative QCD analysis of charged particle distributions in hadronic and nuclear collisions. Nucl. Phys. A, 713:167-187, 2003. doi: 10.1016/S0375-9474(02) 01304-0.

[44] Jonathan M. Butterworth, Guenther Dissertori, and Gavin P. Salam. Hard Processes in ProtonProton Collisions at the Large Hadron Collider. Ann. Rev. Nucl. Part. Sci., 62:387-405, 2012. doi: 10.1146/annurev-nucl-102711-094913.

[45] Gavin P. Salam. QCD in hadron collisions. Nuovo Cim. C, 035(06):155-163, 2012. doi: 10.1393/ncc/ i2012-11362-x.

[46] R. Baier, D. Schiff, and B.G. Zakharov. Energy loss in perturbative QCD. Ann. Rev. Nucl. Part. Sci., 50:37-69, 2000. doi: 10.1146/annurev.nucl.50.1.37.

[47] Xiao-Fang Chen, Tetsufumi Hirano, Enke Wang, Xin-Nian Wang, and Hanzhong Zhang. Suppression of high $p_{T}$ hadrons in $\mathrm{Pb}+\mathrm{Pb}$ Collisions at LHC. Phys. Rev. C, 84:034902, 2011. doi: 10.1103/ PhysRevC.84.034902.

[48] Dmitri Kharzeev, Eugene Levin, and Larry McLerran. Parton saturation and N(part) scaling of semihard processes in QCD. Phys. Lett. B, 561:93-101, 2003. doi: 10.1016/S0370-2693(03)00420-9.

[49] Li Bao-An. Nuclear shadowing effect in relativistic heavy-ion collisions. Nuclear Physics A, 570 (3):797 - 818, 1994. ISSN 0375-9474. doi: https://doi.org/10.1016/0375-9474(94)90084-1. URL http://www. sciencedirect.com/science/article/pii/0375947494900841.

[50] Javier L. Albacete, Nestor Armesto, Alex Kovner, Carlos A. Salgado, and Urs Achim Wiedemann. Energy dependence of the Cronin effect from nonlinear QCD evolution. Phys. Rev. Lett., 92:082001, 2004. doi: 10.1103/PhysRevLett.92.082001.

[51] Panagiota Foka and Magorzata Anna Janik. An overview of experimental results from ultra-relativistic heavy-ion collisions at the cern lhc: Hard probes. Reviews in Physics, 1:172 - 194, 2016. ISSN 2405-4283. doi: https://doi.org/10.1016/j.revip.2016.11.001. URL http://www . sciencedirect.com/ science/article/pii/S2405428316300144. 
[52] Ulrich Heinz and Raimond Snellings. Collective flow and viscosity in relativistic heavy-ion collisions. Ann. Rev. Nucl. Part. Sci., 63:123-151, 2013. doi: 10.1146/annurev-nucl-102212-170540.

[53] S. Voloshin and Y. Zhang. Flow study in relativistic nuclear collisions by Fourier expansion of Azimuthal particle distributions. Z. Phys. C, 70:665-672, 1996. doi: 10.1007/s002880050141.

[54] Zhi Qiu, Chun Shen, and Ulrich Heinz. Hydrodynamic elliptic and triangular flow in $\mathrm{Pb}-\mathrm{Pb}$ collisions at $\sqrt{s}=2.76 \mathrm{ATeV}$. Phys. Lett. B, 707:151-155, 2012. doi: 10.1016/j.physletb.2011.12.041.

[55] C. Aidala et al. Creation of quark-gluon plasma droplets with three distinct geometries. Nature Phys., 15(3):214-220, 2019. doi: 10.1038/s41567-018-0360-0.

[56] U. Gursoy, D.E. Kharzeev, E. Marcus, K. Rajagopal, and C. Shen. Charge-dependent flow induced by electromagnetic fields in heavy ion collisions. In 28th International Conference on Ultrarelativistic Nucleus-Nucleus Collisions, 22020.

[57] R. Hanbury Brown. A Test of a New Type of Stellar Interferometer on Sirius. Nature, 178(4541): 1046-1048, November 1956. doi: 10.1038/1781046a0.

[58] Scott Pratt. Pion interferometry for exploding sources. Phys. Rev. Lett., 53:1219-1221, Sep 1984. doi: 10.1103/PhysRevLett.53.1219. URL https://link.aps.org/doi/10.1103/PhysRevLett.53.1219.

[59] M.M. Aggarwal et al. Interferometry of direct photons in central Pb-208+Pb-208 collisions at 158-AGeV. Phys. Rev. Lett., 93:022301, 2004. doi: 10.1103/PhysRevLett.93.022301.

[60] Steffen A. Bass, Berndt Muller, and Dinesh K. Srivastava. Photon interferometry of Au $+\mathrm{Au}$ collisions at the BNL Relativistic Heavy-Ion Collider. Phys. Rev. Lett., 93:162301, 2004. doi: 10.1103/PhysRevLett.93.162301.

[61] Michael Annan Lisa, Scott Pratt, Ron Soltz, and Urs Wiedemann. Femtoscopy in relativistic heavy ion collisions. Ann. Rev. Nucl. Part. Sci., 55:357-402, 2005. doi: 10.1146/annurev.nucl.55.090704.151533.

[62] Derek Teaney. Effect of shear viscosity on spectra, elliptic flow, and hanbury brown-twiss radii. Phys. Rev. C, 68:034913, Sep 2003. doi: 10.1103/PhysRevC.68.034913. URL https://link.aps.org/doi/ 10.1103/PhysRevC.68.034913.

[63] Steven E. Koonin. Proton pictures of high-energy nuclear collisions. Physics Letters B, 70(1):43 47, 1977. ISSN 0370-2693. doi: https://doi.org/10.1016/0370-2693(77)90340-9. URL http://www . sciencedirect.com/science/article/pii/0370269377903409.

[64] Scott Pratt. Resolving the HBT Puzzle in Relativistic Heavy Ion Collision. Phys. Rev. Lett., 102: 232301, 2009. doi: 10.1103/PhysRevLett.102.232301.

[65] Albert M Sirunyan et al. Bose-Einstein correlations in $p p, p \mathrm{~Pb}$, and $\mathrm{PbPb}$ collisions at $\sqrt{s_{N N}}=0.9-7$ TeV. Phys. Rev. C, 97(6):064912, 2018. doi: 10.1103/PhysRevC.97.064912. 
[66] Shreyasi Acharya et al. Pion-kaon femtoscopy and the lifetime of the hadronic phase in $\mathrm{Pb}-\mathrm{Pb}$ collisions at $\sqrt{s_{\mathrm{NN}}}=2.76 \mathrm{TeV}$. Phys. Lett. B, 813:136030, 2021. doi: 10.1016/j.physletb.2020.136030.

[67] P.V. Ruuskanen. Electromagnetic probes of quark - gluon plasma in relativistic heavy ion collisions. Nucl. Phys. A, 544:169-182, 1992. doi: 10.1016/0375-9474(92)90572-2.

[68] Simon Turbide, Charles Gale, Evan Frodermann, and Ulrich Heinz. Electromagnetic radiation from nuclear collisions at RHIC energies. Phys. Rev. C, 77:024909, 2008. doi: 10.1103/PhysRevC.77.024909.

[69] Jean-Franois Paquet, Chun Shen, Gabriel S. Denicol, Matthew Luzum, Björn Schenke, Sangyong Jeon, and Charles Gale. Production of photons in relativistic heavy-ion collisions. Phys. Rev. C, 93 (4):044906, 2016. doi: 10.1103/PhysRevC.93.044906.

[70] Jean-Franois Paquet. Overview of electromagnetic probe production in ultra-relativistic heavy ion collisions. J. Phys. Conf. Ser., 832(1):012035, 2017. doi: 10.1088/1742-6596/832/1/012035.

[71] Chun Shen. Electromagnetic Radiation from QCD Matter: Theory Overview. Nucl. Phys. A, 956: 184-191, 2016. doi: 10.1016/j.nuclphysa.2016.02.033.

[72] Gabor David. Direct real photons in relativistic heavy ion collisions. Rept. Prog. Phys., 83(4):046301, 2020. doi: 10.1088/1361-6633/ab6f57.

[73] Peter Brockway Arnold, Guy D. Moore, and Laurence G. Yaffe. Photon emission from quark gluon plasma: Complete leading order results. JHEP, 12:009, 2001. doi: 10.1088/1126-6708/2001/12/009.

[74] Chun Shen, Ulrich W Heinz, Jean-Francois Paquet, and Charles Gale. Thermal photons as a quarkgluon plasma thermometer reexamined. Phys. Rev. C, 89(4):044910, 2014. doi: 10.1103/PhysRevC. 89.044910 .

[75] R. Rapp and H. van Hees. Thermal Electromagnetic Radiation in Heavy-Ion Collisions. Eur. Phys. J. A, 52(8):257, 2016. doi: 10.1140/epja/i2016-16257-0.

[76] Jean-Franois Paquet. Probing the space-time evolution of heavy ion collisions with photons and dileptons. Nucl. Phys. A, 967:184-191, 2017. doi: 10.1016/j.nuclphysa.2017.06.003.

[77] A. Adare et al. Enhanced production of direct photons in $\mathrm{Au}+\mathrm{Au}$ collisions at $\sqrt{s_{N N}}=200 \mathrm{GeV}$ and implications for the initial temperature. Phys. Rev. Lett., 104:132301, Mar 2010. doi: 10.1103/ PhysRevLett.104.132301. URL https://link.aps.org/doi/10.1103/PhysRevLett.104.132301.

[78] A. Adare et al. Centrality dependence of low-momentum direct-photon production in $\mathrm{Au}+\mathrm{Au}$ collisions at $\sqrt{s_{N N}}=200 \mathrm{GeV}$. Phys. Rev. C, 91:064904, Jun 2015. doi: 10.1103/PhysRevC.91.064904. URL https://link.aps.org/doi/10.1103/PhysRevC.91.064904.

[79] A. Adare et al. Observation of direct-photon collective flow in $\mathrm{Au}+\mathrm{Au}$ collisions at $\sqrt{s_{N N}}=200 \mathrm{GeV}$. Phys. Rev. Lett., 109:122302, Sep 2012. doi: 10.1103/PhysRevLett.109.122302. URL https://link. aps.org/doi/10.1103/PhysRevLett.109.122302. 
[80] L. Adamczyk et al. Direct virtual photon production in $\mathrm{Au}+\mathrm{Au}$ collisions at $\sqrt{s_{N N}}=200 \mathrm{GeV}$. Phys. Lett. B, 770:451-458, 2017. doi: 10.1016/j.physletb.2017.04.050.

[81] W. Vogelsang and M.R. Whalley. A Compilation of data on single and double prompt photon production in hadron hadron interactions. J. Phys. G, 23:A1-A69, 1997. doi: 10.1088/0954-3899/23/7A/001.

[82] Serguei Chatrchyan et al. Measurement of isolated photon production in $p p$ and $\mathrm{PbPb}$ collisions at $\sqrt{s_{N N}}=2.76 \mathrm{TeV}$. Phys. Lett. B, 710:256-277, 2012. doi: 10.1016/j.physletb.2012.02.077.

[83] Shreyasi Acharya et al. Measurement of the inclusive isolated photon production cross section in pp collisions at $\sqrt{s}=7$ TeV. Eur. Phys. J. C, 79(11):896, 2019. doi: 10.1140/epjc/s10052-019-7389-9.

[84] Morad Aaboud et al. Measurement of the ratio of cross sections for inclusive isolated-photon production in $p p$ collisions at $\sqrt{s}=13$ and $8 \mathrm{TeV}$ with the ATLAS detector. JHEP, 04:093, 2019. doi: 10.1007/JHEP04(2019)093.

[85] Morad Aaboud et al. Measurement of prompt photon production in $\sqrt{s_{\mathrm{NN}}}=8.16 \mathrm{TeV} p+\mathrm{Pb}$ collisions with ATLAS. Phys. Lett. B, 796:230-252, 2019. doi: 10.1016/j.physletb.2019.07.031.

[86] Akihiko Monnai. Thermal photon $v_{2}$ with slow quark chemical equilibration. Phys. Rev., C90(2): 021901, 2014. doi: 10.1103/PhysRevC.90.021901.

[87] Shreyasi Acharya et al. Direct photon elliptic flow in Pb-Pb collisions at $\sqrt{s_{\mathrm{NN}}}=2.76 \mathrm{TeV}$. Phys. Lett. B, 789:308-322, 2019. doi: 10.1016/j.physletb.2018.11.039.

[88] S.S. Adler et al. Elliptic flow of identified hadrons in Au+Au collisions at $\mathrm{s}(\mathrm{NN})^{* *}(1 / 2)=200-\mathrm{GeV}$. Phys. Rev. Lett., 91:182301, 2003. doi: 10.1103/PhysRevLett.91.182301.

[89] Fu-Ming Liu, Sheng-Xu Liu, and Klaus Werner. Another solution to the puzzle of the direct photon elliptic flow. , 122015.

[90] Charles Gale. Direct photon production in relativistic heavy-ion collisions - a theory update. PoS, High-pT2017:023, 2019. doi: 10.22323/1.320.0023.

[91] Moritz Greif, Florian Senzel, Heiner Kremer, Kai Zhou, Carsten Greiner, and Zhe Xu. Nonequilibrium photon production in partonic transport simulations. Phys. Rev. C, 95(5):054903, 2017. doi: 10.1103/ PhysRevC.95.054903.

[92] Jurgen Berges, Klaus Reygers, Naoto Tanji, and Raju Venugopalan. Parametric estimate of the relative photon yields from the glasma and the quark-gluon plasma in heavy-ion collisions. Phys. Rev. C, 95(5):054904, 2017. doi: 10.1103/PhysRevC.95.054904.

[93] L. Oliva, M. Ruggieri, S. Plumari, F. Scardina, G.X. Peng, and V. Greco. Photons from the Early Stages of Relativistic Heavy Ion Collisions. Phys. Rev. C, 96(1):014914, 2017. doi: 10.1103/PhysRevC. 96.014914 . 
[94] CERN. The Large Hadron Collider - picture. , 2018. URL https://cds.cern.ch/images/ CERN-PHOTO-201802-030-10/file?size=large.

[95] UA1 Collaboration. Experimental observation of isolated large transverse energy electrons with associated missing energy at $\mathrm{s}=540$ gev. Physics Letters B, 122(1):103 - 116, 1983 . ISSN 03702693. doi: https://doi.org/10.1016/0370-2693(83)91177-2. URL http://www.sciencedirect.com/ science/article/pii/0370269383911772.

[96] UA2 Collaboration. Observation of single isolated electrons of high transverse momentum in events with missing transverse energy at the cern pp collider. Physics Letters B, 122(5):476 - 485, 1983. ISSN 0370-2693. doi: https://doi.org/10.1016/0370-2693(83)91605-2. URL http://www. sciencedirect. com/science/article/pii/0370269383916052.

[97] Lyndon Evans and Philip Bryant. LHC machine. Journal of Instrumentation, 3(08):S08001-S08001, aug 2008. doi: 10.1088/1748-0221/3/08/s08001. URL https://doi.org/10.1088\%2F1748-0221\% $2 \mathrm{~F} 3 \% 2 \mathrm{~F} 08 \% 2 \mathrm{Fs} 08001$.

[98] The ATLAS Collaboration. The ATLAS experiment at the CERN large hadron collider. Journal of Instrumentation, 3(08):S08003-S08003, aug 2008. doi: 10.1088/1748-0221/3/08/s08003. URL https://doi.org/10.1088\%2F1748-0221\%2F3\%2F08\%2Fs08003.

[99] The CMS Collaboration. The CMS experiment at the CERN LHC. Journal of Instrumentation, 3 (08):S08004-S08004, aug 2008. doi: 10.1088/1748-0221/3/08/s08004. URL https://doi.org/10. $1088 \% 2 \mathrm{~F} 1748-0221 \% 2 \mathrm{~F} 3 \% 2 \mathrm{~F} 08 \% 2 \mathrm{Fs} 08004$.

[100] K. Aamodt et al. The ALICE experiment at the CERN LHC. JINST, 3:S08002, 2008. doi: 10.1088/ $1748-0221 / 3 / 08 / \mathrm{S} 08002$.

[101] Jr. Alves, A.Augusto et al. The LHCb Detector at the LHC. JINST, 3:S08005, 2008. doi: 10.1088/ 1748-0221/3/08/S08005.

[102] ALICE Collaboration, F Carminati, P Foka, P Giubellino, A Morsch, G Paic, J-P Revol, K Safarík, Y Schutz, and U A Wiedemann (editors). ALICE: Physics performance report, volume i. Journal of Physics G: Nuclear and Particle Physics, 30(11):1517-1763, oct 2004. doi: 10.1088/0954-3899/30/11/ 001. URL https://doi.org/10.1088\%2F0954-3899\%2F30\%2F $11 \% 2 \mathrm{~F} 001$.

[103] ALICE Collaboration. ALICE: Physics performance report, volume II. Journal of Physics G: Nuclear and Particle Physics, 32(10):1295-2040, sep 2006. doi: 10.1088/0954-3899/32/10/001. URL https: //doi.org/10. 1088\%2F0954-3899\%2F32\%2F10\%2F001.

[104] B. Abelev et al. Performance of the ALICE Experiment at the CERN LHC. Int. J. Mod. Phys., A29: 1430044, 2014. doi: 10.1142/S0217751X14300440.

[105] K Aamodt et al. Alignment of the ALICE Inner Tracking System with cosmic-ray tracks. JINST, 5: P03003, 2010. doi: 10.1088/1748-0221/5/03/P03003. 
[106] J. Alme et al. The alice tpc, a large 3-dimensional tracking device with fast readout for ultra-high multiplicity events. Nuclear Instruments and Methods in Physics Research Section A: Accelerators, Spectrometers, Detectors and Associated Equipment, 622(1):316367, Oct 2010. ISSN 0168-9002. doi: 10.1016/j.nima.2010.04.042. URL http://dx.doi.org/10.1016/j.nima.2010.04.042.

[107] U. Abeysekara et al. ALICE EMCal Physics Performance Report. , 82010.

[108] Shreyasi Acharya et al. Calibration of the photon spectrometer PHOS of the ALICE experiment. JINST, 14(05):P05025, 2019. doi: 10.1088/1748-0221/14/05/P05025.

[109] P Cortese et al. ALICE technical design report on forward detectors: FMD, T0 and V0, CERNLHCC-2004-025. , 2004.

[110] E. Abbas et al. Performance of the ALICE VZERO system. JINST, 8:P10016, 2013. doi: 10.1088/ 1748-0221/8/10/P10016.

[111] B. Abelev et al. Centrality determination of $\mathrm{Pb}-\mathrm{Pb}$ collisions at $\sqrt{s_{N N}}=2.76 \mathrm{TeV}$ with ALICE. Phys. Rev., C88(4):044909, 2013. doi: 10.1103/PhysRevC.88.044909.

[112] Jaroslav Adam et al. Centrality dependence of particle production in p-Pb collisions at $\sqrt{s_{\mathrm{NN}}}=5.02$ TeV. Phys. Rev. C, 91(6):064905, 2015. doi: 10.1103/PhysRevC.91.064905.

[113] Sruthy Jyothi Das, Giuliano Giacalone, Pierre-Amaury Monard, and Jean-Yves Ollitrault. Relating centrality to impact parameter in nucleus-nucleus collisions. Phys. Rev. C, 97(1):014905, 2018. doi: 10.1103/PhysRevC.97.014905.

[114] ALICE Collaboration. The ALICE definition of primary particles. , Jun 2017. URL https://cds . cern.ch/record/2270008.

[115] Yan Pei, Swarnendu Biswas, Donald S. Fussell, and Keshav Pingali. An elementary introduction to kalman filtering. Communications of the ACM, 62(11):122133, Oct 2019. ISSN 1557-7317. doi: 10.1145/3363294. URL http://dx.doi.org/10.1145/3363294.

[116] B. Andersson, G. Gustafson, G. Ingelman, and T. Sjöstrand. Parton fragmentation and string dynamics. Physics Reports, 97(2):31 - 145, 1983. ISSN 0370-1573. doi: https://doi. org/10.1016/0370-1573(83)90080-7. URL http://www.sciencedirect.com/science/article/pii/ 0370157383900807.

[117] Jose Guilherme Milhano. Theoretical overview of jet quenching. Nucl. Phys. A, 904-905:122c-129c, 2013. doi: 10.1016/j.nuclphysa.2013.01.053.

[118] Karen M. Burke, Alessandro Buzzatti, Ningbo Chang, Charles Gale, Miklos Gyulassy, Ulrich Heinz, Sangyong Jeon, Abhijit Majumder, Berndt Müller, Guang-You Qin, Björn Schenke, Chun Shen, XinNian Wang, Jiechen Xu, Clint Young, and Hanzhong Zhang. Extracting the jet transport coefficient from jet quenching in high-energy heavy-ion collisions. Phys. Rev. C, 90:014909, Jul 2014. doi: 10.1103/PhysRevC.90.014909. URL https://link.aps.org/doi/10.1103/PhysRevC.90.014909. 
[119] Stephen D. Ellis and Davison E. Soper. Successive combination jet algorithm for hadron collisions. Phys. Rev. D, 48:3160-3166, 1993. doi: 10.1103/PhysRevD.48.3160.

[120] Matteo Cacciari, Gavin P. Salam, and Gregory Soyez. The anti- $k_{t}$ jet clustering algorithm. JHEP, 04:063, 2008. doi: 10.1088/1126-6708/2008/04/063.

[121] Matteo Cacciari, Gavin P. Salam, and Gregory Soyez. FastJet User Manual. Eur. Phys. J. C, 72 : 1896, 2012. doi: 10.1140/epjc/s10052-012-1896-2.

[122] Eleazar Cuautle, Raul Jimenez, Ivonne Maldonado, Antonio Ortiz, Guy Paic, and Edgar Perez. Disentangling the soft and hard components of the pp collisions using the sphero(i)city approach. , 4 2014.

[123] Shreyasi Acharya et al. Charged-particle production as a function of multiplicity and transverse spherocity in pp collisions at $\sqrt{s}=5.02$ and $13 \mathrm{TeV}$. Eur. Phys. J. C, 79(10):857, 2019. doi: 10.1140/epjc/s10052-019-7350-y.

[124] Andrew J. Larkoski, Duff Neill, and Jesse Thaler. Jet Shapes with the Broadening Axis. JHEP, 04: 017, 2014. doi: 10.1007/JHEP04(2014)017.

[125] Roger Barlow. Systematic errors: facts and fictions, 2002.

[126] J. Podolanski and R. Armenteros. Iii. analysis of v-events. The London, Edinburgh, and Dublin Philosophical Magazine and Journal of Science, 45(360):13-30, 1954. doi: 10.1080/14786440108520416. URL https://doi.org/10.1080/14786440108520416.

[127] Shreyasi Acharya et al. $\pi^{0}$ and $\eta$ meson production in proton-proton collisions at $\sqrt{s}=8 \mathrm{TeV}$. Eur. Phys. J. C, 78(3):263, 2018. doi: 10.1140/epjc/s10052-018-5612-8.

[128] Shreyasi Acharya et al. Neutral pion and $\eta$ meson production at mid-rapidity in $\mathrm{Pb}-\mathrm{Pb}$ collisions at $\sqrt{s_{N N}}=2.76$ TeV. Phys. Rev. C, 98(4):044901, 2018. doi: 10.1103/PhysRevC.98.044901.

[129] M. Bourquin and J.-M. Gaillard. A Simple Phenomenological Description of Hadron Production. Nucl. Phys. B, 114:334-364, 1976. doi: 10.1016/0550-3213(76)90592-7.

[130] S. Afanasiev, C. Aidala, N. Ajitanand, Yuka Akiba, H. Al-Bataineh, Jordy Alexander, Kazuya Aoki, L. Aphecetche, R. Armendariz, S. Aronson, J. Asai, E. Atomssa, R. Averbeck, Terry Awes, Bob Azmoun, Vladimir Babintsev, Mei Bai, G. Baksay, and L. Zolin. Measurement of neutral mesons in $\mathrm{p}+\mathrm{p}$ collisions at $\mathrm{s}=200 \mathrm{gev}$ and scaling properties of hadron production. Phys. Rev. D, 83, 032011. doi: 10.1103/PhysRevD.83.052004.

[131] B. Abelev et al. Neutral pion and $\eta$ meson production in proton-proton collisions at $\sqrt{s}=0.9 \mathrm{TeV}$ and $\sqrt{s}=7 \mathrm{TeV}$. Phys. Lett., B717:162-172, 2012. doi: 10.1016/j.physletb.2012.09.015.

[132] B. Abelev et al. Neutral pion production at midrapidity in pp and $\mathrm{Pb}-\mathrm{Pb}$ collisions at $\sqrt{s_{\mathrm{NN}}}=$ 2.76 TeV. Eur. Phys. J., C74(10):3108, 2014. doi: 10.1140/epjc/s10052-014-3108-8. 
[133] Shreyasi Acharya et al. Neutral pion and $\eta$ meson production in p-Pb collisions at $\sqrt{s_{\mathrm{NN}}}=5.02 \mathrm{TeV}$. Eur. Phys. J. C, 78(8):624, 2018. doi: 10.1140/epjc/s10052-018-6013-8.

[134] B. Abelev, J. Adams, M.M. Aggarwal, Zubayer Ahammed, J. Amonett, B.D. Anderson, M. Anderson, D. Arkhipkin, G.S. Averichev, Y. Bai, J. Balewski, O. Barannikova, Lee Barnby, Jerome Baudot, S. Bekele, Victoria Belaga, A. Bellingeri-Laurikainen, Rene Bellwied, and F. Benedosso. Strange particle production in $\mathrm{p}+\mathrm{p}$ collisions at $\mathrm{s}=200$ gev. -, 062007.

[135] B. Abelev et al. Production of charged pions, kaons and protons at large transverse momenta in pp and $\mathrm{Pb}-\mathrm{Pb}$ collisions at $\sqrt{s_{\mathrm{NN}}}=2.76 \mathrm{TeV}$. Phys. Lett. B, 736:196-207, 2014. doi: 10.1016/j.physletb. 2014.07.011.

[136] Shreyasi Acharya et al. Multiplicity dependence of light-flavor hadron production in pp collisions at $\sqrt{s}=7$ TeV. Phys. Rev. C, 99(2):024906, 2019. doi: 10.1103/PhysRevC.99.024906.

[137] Shreyasi Acharya et al. Production of charged pions, kaons, and (anti-)protons in $\mathrm{Pb}-\mathrm{Pb}$ and inelastic $p p$ collisions at $\sqrt{s_{N N}}=5.02 \mathrm{TeV}$. Phys. Rev. C, 101(4):044907, 2020. doi: 10.1103/PhysRevC.101. 044907 .

[138] Particle Data Group. Neutral pion - PDG. ,2014. URL https://pdg.lbl.gov/2014/listings/ rpp2014-list-pi-zero.pdf.

[139] Particle Data Group. Eta meson - PDG. , 2019. URL https://pdg.lbl.gov/2019/tables/ rpp2019-tab-mesons-light.pdf.

[140] G.D. Lafferty and T.R. Wyatt. Where to stick your data points: The treatment of measurements within wide bins. Nucl. Instrum. Meth. A, 355:541-547, 1995. doi: 10.1016/0168-9002(94)01112-5.

[141] B Abelev et al. Upgrade of the ALICE Experiment: Letter Of Intent. J. Phys. G, 41:087001, 2014. doi: $10.1088 / 0954-3899 / 41 / 8 / 087001$.

[142] Andrea Valassi and Roberto Chierici. Information and treatment of unknown correlations in the combination of measurements using the BLUE method. Eur. Phys. J. C, 74:2717, 2014. doi: 10.1140/ epjc/s10052-014-2717-6.

[143] Luca Lista. Combination of measurements and the BLUE method. EPJ Web Conf., 137:11006, 2017. doi: 10.1051/epjconf/201713711006.

[144] A.A. Bylinkin and A.A. Rostovtsev. Role of quarks in hadroproduction in high energy collisions. Nucl. Phys. B, 888:65-74, 2014. doi: 10.1016/j.nuclphysb.2014.09.010.

[145] A.A. Bylinkin and M.G. Ryskin. Secondary hadron distributions in two component model. Phys. Rev. D, 90(1):017501, 2014. doi: 10.1103/PhysRevD.90.017501.

[146] Torbjorn Sjostrand, Stephen Mrenna, and Peter Z. Skands. PYTHIA 6.4 Physics and Manual. JHEP, 05:026, 2006. doi: 10.1088/1126-6708/2006/05/026. 
[147] Tim Adye. Unfolding algorithms and tests using RooUnfold. In PHYSTAT 2011, pages 313-318, Geneva, 2011. CERN. doi: 10.5170/CERN-2011-006.313.

[148] G. D'Agostini. A Multidimensional unfolding method based on Bayes' theorem. Nucl. Instrum. Meth. A, 362:487-498, 1995. doi: 10.1016/0168-9002(95)00274-X.

[149] Cheuk-Yin Wong and Grzegorz Wilk. Tsallis fits to $p_{T}$ spectra and multiple hard scattering in pp collisions at the LHC. Phys. Rev. D, 87(11):114007, 2013. doi: 10.1103/PhysRevD.87.114007.

[150] H. Zheng and Lilin Zhu. Can Tsallis Distribution Fit All the Particle Spectra Produced at RHIC and LHC? Adv. High Energy Phys., 2015:180491, 2015. doi: 10.1155/2015/180491.

[151] Kari J. Eskola, Petja Paakkinen, Hannu Paukkunen, and Carlos A. Salgado. EPPS16: Nuclear parton distributions with LHC data. Eur. Phys. J. C, 77(3):163, 2017. doi: 10.1140/epjc/s10052-017-4725-9.

[152] Daniel de Florian, R. Sassot, Manuel Epele, Roger J. Hernndez-Pinto, and Marco Stratmann. Partonto-Pion Fragmentation Reloaded. Phys. Rev. D, 91(1):014035, 2015. doi: 10.1103/PhysRevD.91. 014035 .

[153] D. de Florian, M. Epele, R.J. Hernandez-Pinto, R. Sassot, and M. Stratmann. Parton-to-Kaon Fragmentation Revisited. Phys. Rev. D, 95(9):094019, 2017. doi: 10.1103/PhysRevD.95.094019.

[154] K. Kovarik et al. nCTEQ15 - Global analysis of nuclear parton distributions with uncertainties in the CTEQ framework. Phys. Rev. D, 93(8):085037, 2016. doi: 10.1103/PhysRevD.93.085037.

[155] Christine A. Aidala, Frank Ellinghaus, Rodolfo Sassot, Joseph P. Seele, and Marco Stratmann. Global Analysis of Fragmentation Functions for Eta Mesons. Phys. Rev. D, 83:034002, 2011. doi: 10.1103/ PhysRevD.83.034002.

[156] K. Werner, B. Guiot, Iu. Karpenko, and T. Pierog. Analysing radial flow features in p-Pb and p-p collisions at several $\mathrm{TeV}$ by studying identified particle production in EPOS3. Phys. Rev. C, 89(6): 064903, 2014. doi: 10.1103/PhysRevC.89.064903.

[157] Iurii Karpenko, Joerg Aichelin, Pol Bernard Gossiaux, Martin Rohrmoser, and Klaus Werner. Jetfluid interaction in the EPOS3-Jet framework. In 18th International Conference on Strangeness in Quark Matter, 112019.

[158] Chun Shen, Zhi Qiu, Huichao Song, Jonah Bernhard, Steffen Bass, and Ulrich Heinz. The iEBEVISHNU code package for relativistic heavy-ion collisions. Comput. Phys. Commun., 199:61-85, 2016. doi: 10.1016/j.cpc.2015.08.039.

[159] Wenbin Zhao, Lilin Zhu, Hua Zheng, Che Ming Ko, and Huichao Song. Spectra and flow of light nuclei in relativistic heavy ion collisions at energies available at the BNL Relativistic Heavy Ion Collider and at the CERN Large Hadron Collider. Phys. Rev. C, 98(5):054905, 2018. doi: 10.1103/PhysRevC.98. 054905 . 
[160] Stefan Roesler, Ralph Engel, and Johannes Ranft. The Monte Carlo event generator DPMJETIII. In International Conference on Advanced Monte Carlo for Radiation Physics, Particle Transport Simulation and Applications (MC 2000), pages 1033-1038, 12 2000. doi: 10.1007/978-3-642-18211-2 $-166$.

[161] P.D.B. Collins. An Introduction to Regge Theory and High-Energy Physics. Cambridge Monographs on Mathematical Physics. Cambridge Univ. Press, Cambridge, UK, 5 2009. ISBN 978-0-521-11035-8. doi: $10.1017 /$ CBO9780511897603.

[162] Xin-Nian Wang and Miklos Gyulassy. hijing: A monte carlo model for multiple jet production in pp, pA, and AA collisions. Phys. Rev. D, 44:3501-3516, Dec 1991. doi: 10.1103/PhysRevD.44.3501. URL https://link.aps.org/doi/10.1103/PhysRevD.44.3501.

[163] K. Aamodt et al. Femtoscopy of pp collisions at $\sqrt{s}=0.9$ and 7 tev at the lhe with two-pion boseeinstein correlations. Phys. Rev. D, 84:112004, Dec 2011. doi: 10.1103/PhysRevD.84.112004. URL https://link.aps.org/doi/10.1103/PhysRevD.84.112004.

[164] Torbjörn Sjöstrand et al. A brief introduction to PYTHIA 8.1. Comput. Phys.Commun, 178, 2008. doi: $10.1016 /$ j.cpc.2008.01.036.

[165] B. Abelev et al. Neutral pion and $\eta$ meson production in proton-proton collisions at $\sqrt{s}=0.9 \mathrm{TeV}$ and $\sqrt{s}=7$ TeV. Phys. Lett. B, 717, 2012. doi: 10.1016/j.physletb.2012.09.015.

[166] S. Acharya et al. $\pi^{0}$ and $\eta$ production in proton-proton collisions at $\sqrt{s_{\mathrm{NN}}}=8 \mathrm{TeV}$. EPJC, 78, 2018. doi: $10.1140 /$ epjc/s10052-018-5612-8.

[167] S. Acharya et al. Neutral pion and $\eta$ production at mid-rapidity in $\mathrm{PbPb}$ collisions at $\sqrt{s_{\mathrm{NN}}}=2.76$ TeV. Phys. Rev. C, 98, 2018. doi: 10.1103/PhysRevC.98.044901.

[168] S. Acharya et al. Neutral pion and $\eta$ meson production in $\mathrm{pPb}$ collisions at $\sqrt{s_{\mathrm{NN}}}=5.02 \mathrm{TeV}$. EPJC, 78, 2018. doi: 10.1140/epjc/s10052-018-6013-8.

[169] B. Abelev et al. Inclusive $\pi^{0}, \eta$, and direct photon production at high transverse momentum in $\mathrm{p}+\mathrm{p}$ and d+au collisions at 200 gev. Physical Review C, 81(6), Jun 2010. ISSN 1089-490X. doi: 10.1103/physrevc.81.064904. URL http://dx.doi.org/10.1103/PhysRevC.81.064904.

[170] B. Abelev et al. Transverse sphericity of primary charged particles in minimum bias protonproton collisions at $\sqrt{\mathbf{s}}=\mathbf{0 . 9}, \mathbf{2 . 7 6}$ and $\mathbf{7} \mathrm{TeV}$. The European Physical Journal C, 72(9), Sep 2012. ISSN 1434-6052. doi: 10.1140/epjc/s10052-012-2124-9. URL http://dx.doi.org/10.1140/epjc/ s10052-012-2124-9.

[171] S. Acharya et al. Event-shape and multiplicity dependence of freeze-out radii in pp collisions at $\sqrt{s}=7$ tev. Journal of High Energy Physics, 2019(9), Sep 2019. ISSN 1029-8479. doi: 10.1007/ jhep09(2019)108. URL http://dx.doi.org/10.1007/JHEP09(2019) 108. 
[172] R.Keith Ellis, D.A. Ross, and A.E. Terrano. The Perturbative Calculation of Jet Structure in e+ eAnnihilation. Nucl. Phys. B, 178:421-456, 1981. doi: 10.1016/0550-3213(81)90165-6.

[173] K. Abe et al. Measurement of alpha-s (M(Z)**2) from hadronic event observables at the Z0 resonance. Phys. Rev. D, 51:962-984, 1995. doi: 10.1103/PhysRevD.51.962.

[174] J. Abdallah et al. A Study of the energy evolution of event shape distributions and their means with the DELPHI detector at LEP. Eur. Phys. J. C, 29:285-312, 2003. doi: 10.1140/epjc/s2003-01198-0.

[175] P. Achard et al. Studies of hadronic event structure in $e^{+} e^{-}$annihilation from 30-GeV to 209-GeV with the L3 detector. Phys. Rept., 399:71-174, 2004. doi: 10.1016/j.physrep.2004.07.002.

[176] Stefan Weinzierl. Event shapes and jet rates in electron-positron annihilation at NNLO. JHEP, 06: 041, 2009. doi: 10.1088/1126-6708/2009/06/041.

[177] Mrinal Dasgupta and Gavin P. Salam. Event shapes in e+ e- annihilation and deep inelastic scattering. J. Phys. G, 30:R143, 2004. doi: 10.1088/0954-3899/30/5/R01.

[178] G. Abbiendi et al. Measurement of event shape distributions and moments in e+ e- - $i$ hadrons at $91-\mathrm{GeV}$ - 209-GeV and a determination of alpha(s). Eur. Phys. J. C, 40:287-316, 2005. doi: 10.1140/epjc/s2005-02120-6.

[179] Stefan Kluth. Tests of Quantum Chromo Dynamics at e+ e- Colliders. Rept. Prog. Phys., 69:17711846, 2006. doi: 10.1088/0034-4885/69/6/R04.

[180] A. Gehrmann-De Ridder, T. Gehrmann, E.W.N. Glover, and G. Heinrich. Second-order QCD corrections to the thrust distribution. Phys. Rev. Lett., 99:132002, 2007. doi: 10.1103/PhysRevLett.99. 132002 .

[181] V. Khachatryan et al. First measurement of hadronic event shapes in pp collisions at s=7tev. Physics Letters B, 699(1):48 - 67, 2011. ISSN 0370-2693. doi: https://doi.org/10.1016/j.physletb.2011.03.060. URL http://www.sciencedirect.com/science/article/pii/S0370269311003455.

[182] Andrea Banfi, Gavin P. Salam, and Giulia Zanderighi. Phenomenology of event shapes at hadron colliders. JHEP, 06:038, 2010. doi: 10.1007/JHEP06(2010)038.

[183] Jaroslav Adam et al. Direct photon production in $\mathrm{Pb}-\mathrm{Pb}$ collisions at $\sqrt{s_{\mathrm{NN}}}=2.76 \mathrm{TeV}$. Phys. Lett., B754:235-248, 2016. doi: 10.1016/j.physletb.2016.01.020.

[184] R Brun, F Bruyant, M Maire, A C McPherson, and P Zanarini. GEANT 3: user's guide Geant 3.10, Geant 3.11; rev. version. CERN, Geneva, 1987. URL https://cds.cern.ch/record/1119728.

[185] S. Agostinelli et al. GEANT4-a simulation toolkit. Nucl. Instrum. Meth. A, 506:250-303, 2003. doi: 10.1016/S0168-9002(03)01368-8. 
[186] C. Adler et al. Elliptic flow from two and four particle correlations in Au+Au collisions at s(NN)**(1/2) =130-GeV. Phys. Rev., C66:034904, 2002. doi: 10.1103/PhysRevC.66.034904.

[187] B. Abelev et al. Elliptic flow of identified hadrons in Pb-Pb collisions at $\sqrt{s_{\mathrm{NN}}}=2.76 \mathrm{TeV}$. JHEP, 06:190, 2015. doi: 10.1007/JHEP06(2015)190.

[188] B. Abelev et al. Anisotropic flow of charged hadrons, pions and (anti-)protons measured at high transverse momentum in $\mathrm{Pb}-\mathrm{Pb}$ collisions at $\sqrt{s_{N N}}=2.76 \mathrm{TeV}$. Phys. Lett., B719:18-28, 2013. doi: 10.1016/j.physletb.2012.12.066.

[189] O. Linnyk, V. Konchakovski, T. Steinert, W. Cassing, and E. L. Bratkovskaya. Hadronic and partonic sources of direct photons in relativistic heavy-ion collisions. Phys. Rev., C92(5):054914, 2015. doi: 10.1103/PhysRevC.92.054914.

[190] Jean-Franois Paquet, Chun Shen, Gabriel S. Denicol, Matthew Luzum, Björn Schenke, Sangyong Jeon, and Charles Gale. The production of photons in relativistic heavy-ion collisions. Phys. Rev. C, 93: 044906, 2016. doi: 10.1103/PhysRevC.93.044906.

[191] Sangwook Ryu, Jean-Franois Paquet, Chun Shen, Gabriel Denicol, Björn Schenke, Sangyong Jeon, and Charles Gale. Effects of bulk viscosity and hadronic rescattering in heavy ion collisions at energies available at the BNL Relativistic Heavy Ion Collider and at the CERN Large Hadron Collider. Phys. Rev., C97(3):034910, 2018. doi: 10.1103/PhysRevC.97.034910.

[192] Charles Gale, Yoshimasa Hidaka, Sangyong Jeon, Shu Lin, Jean-Franois Paquet, Robert D. Pisarski, Daisuke Satow, Vladimir V. Skokov, and Gojko Vujanovic. Production and Elliptic Flow of Dileptons and Photons in a Matrix Model of the Quark-Gluon Plasma. Phys. Rev. Lett., 114:072301, 2015. doi: 10.1103/PhysRevLett.114.072301.

[193] O. Linnyk, W. Cassing, and E. L. Bratkovskaya. Centrality dependence of the direct photon yield and elliptic flow in heavy-ion collisions at $\sqrt{s_{N N}}=200 \mathrm{GeV}$. Phys. Rev., C89(3):034908, 2014. doi: 10.1103/PhysRevC.89.034908.

[194] A. Adare et al. Azimuthally anisotropic emission of low-momentum direct photons in $\mathrm{Au}+\mathrm{Au}$ collisions at $\sqrt{s_{N N}}=200 \mathrm{GeV}$. , 2015 .

[195] A. Adare et al. Observation of direct-photon collective flow in $\sqrt{s_{N N}}=200 \mathrm{GeV} \mathrm{Au}+\mathrm{Au}$ collisions. Phys. Rev. Lett., 109:122302, 2012. doi: 10.1103/PhysRevLett.109.122302.

[196] Rupa Chatterjee, Pingal Dasgupta, and Dinesh K. Srivastava. Anisotropic flow of thermal photons at energies available at the BNL Relativistic Heavy Ion Collider and at the CERN Large Hadron Collider. Phys. Rev., C96(1):014911, 2017. doi: 10.1103/PhysRevC.96.014911.

[197] Robert D. Cousins and Virgil L. Highland. Incorporating systematic uncertainties into an upper limit. Nucl. Instrum. Meth., A320:331-335, 1992. doi: 10.1016/0168-9002(92)90794-5. 
[198] A. Timmermann, M. Plümer, L. Razumov, and R. M. Weiner. Photon interferometry of quark-gluon dynamics reexamined. Phys. Rev. C, 50:3060-3063, Dec 1994. doi: 10.1103/PhysRevC.50.3060. URL https://link.aps.org/doi/10.1103/PhysRevC.50.3060.

[199] Ulrich W. Heinz. Hanbury-Brown/Twiss interferometry for relativistic heavy ion collisions: Theoretical aspects. In International Summer School on Correlations and Clustering Phenomena in Subatomic Physics, pages 137-177, 81996.

[200] D. Peressounko. Hanbury brown-twiss interferometry of direct photons in heavy ion collisions. Phys. Rev. C, 67:014905, Jan 2003. doi: 10.1103/PhysRevC.67.014905. URL https://link.aps.org/doi/ 10.1103/PhysRevC.67.014905.

[201] Rupa Chatterjee, Lusaka Bhattacharya, and Dinesh K. Srivastava. Electromagnetic probes. Lecture Notes in Physics, page 219264, 2009. ISSN 1616-6361. doi: 10.1007/978-3-642-02286-9_7. URL http://dx.doi.org/10.1007/978-3-642-02286-9_7.

[202] Nicolas Schmidt. Direct photon production and flow at low transverse momenta in pp, p-Pb and Pb-Pb collisions. PoS, HardProbes2018:183, 2018. doi: 10.22323/1.345.0183.

[203] Evan Frodermann and Ulrich Heinz. Photon HBT interferometry for non-central heavy-ion collisions. Phys. Rev. C, 80:044903, 2009. doi: 10.1103/PhysRevC.80.044903.

[204] Oscar Garcia-Montero, Nicole Lher, Aleksas Mazeliauskas, Jrgen Berges, and Klaus Reygers. Probing the evolution of heavy-ion collisions using direct photon interferometry. Physical Review C, 102(2), Aug 2020. ISSN 2469-9993. doi: 10.1103/physrevc.102.024915. URL http://dx.doi.org/10.1103/ PhysRevC.102.024915.

[205] S. Acharya et al. ALICE upgrade physics performance studies for 2018 Report on HL/HE-LHC physics. -, Feb 2019. URL https://cds.cern.ch/record/2661798. 


\section{Samenvatting in het nederlands}

We willen weten wat het allemaal betekent. Waaruit bestaat het universum? Wat is ruimte en tijd? Hoe werken de fundamentele krachten? Gedreven door pure nieuwsgierigheid proberen wij deze vragen te beantwoorden, waardoor de grens van kennis beetje bij beetje uitgebreid wordt. Terwijl we meer leren over hoe het universum in elkaar steekt, is er een ding dat we zeker weten. We hebben niet alles ontdekt wat we kunnen ontdekken, en er is nog steeds een grote hoeveelheid kennis dat ons te wachten staat. Wat we wel weten is nauwkeurig geformuleerd in theorieën die getest worden door experimentele observaties, met de wetenschappelijke methode als basis.

Voor zover wij het weten is het universum om ons heen opgebouwd uit ondeelbare bouwstenen die via de fundamentele krachten met elkaar interacteren, welke geformuleerd is in het standaardmodel van de deeltjesfysica door middel van een elegant wiskundig raamwerk. En ook al hebben we reeds het Higgs deeltje gevonden, wat al lang een voorspelling was van de theorie, zijn er nog veel overgebleven gebieden binnen de hoge-energie fysica die hun eigen puzzels hebben waarvan we weten dat ze incompleet zijn. Een van deze gebieden is zware-ionen fysica, dat de gecompliceerde details van nucleaire materie onder extreme condities bestudeerd.

Onder normale omstandigheden worden quarks altijd sterk met elkaar verbonden door gluonen in wat we kleurloze objecten of hadronen noemen, zoals het proton of neutron. Echter, er is een temperatuur waarbij er een fasetransitie optreedt die de bouwstenen van nucleaire materie bevrijdt zodat er een ongebonden medium van sterk gekoppelde quarks en gluonen ontstaat. Deze toestand van materie wordt het quark gluon plasma (QGP) genoemd en was ook de toestand van de materie dat het universum vulde net na de oerknal. En ook al kunnen we deze oersoep niet direct waarnemen, omdat het universum na de oerknal is uitgedeid en afgekoelt, waardoor er de materie gevormd is die we om ons heen zien, kunnen we toch een QGP maken in het laboratorium door zware ionen te laten botsen in grote deeltjesversneller experimenten.

In een versimpeld beeld wordt het QGP gevormd door de overlappende nucleonen van de twee botsende kernen. In deze regio zijn de thermodynamische grootheden, zoals temperatuur, druk, en energiedichtheid, voldoende om een faseovergang te veroorzaken. Nadat het QGP gemaakt is expandeert het, koelt het af, en bereikt het een temperatuur waarbij het systeem weer overschakelt naar hadronische materie, allemaal binnen $10^{-22} \mathrm{~s}$. Het is door de nasleep van deze zware-ionen botsing, dus door het meten van de eigenschappen van alle deeltjes die in de botsing geproduceerd worden, dat we informatie kunnen afleiden over deze uitzonderlijke toestand van materie.

Een van de meest inzichtgevende methodes om het QGP te bestuderen is door zo precies mogelijk de eigenschappen te meten van de deeltjes die geproduceerd worden in de zware-ionen botsingen. Deze metingen geven een goed onderscheidingsvermogen tussen verschillende theoretische model berekeningen, die ieder andere deeltjes productie mechanismes en onderliggen aannames bevatten. Bijkomende inzichten kunnen verkregen worden door het vergelijk met pp en pA botsingen te maken. Zo was het ontdekt dat de deeltjes 
die in harde processen geproduceerd beïnvloed kunnen worden door de aanwezigheid van het plasma, dat zich dan uit in de vorm van energieverlies als het het plasma doorkruist, als mede dat het impuls kan toenemen door de expansie van het medium. Het is gebleken dat het plasma zo extreem heet is dat het thermisch quarks en gluonen kan produceren, wat resulteert in additionele deeltjes. Ook hebben de tweedeeltjes correlaties laten zien dat het QGP een bijna perfecte vloeistof is, dus een vloeistof met een extreem lage viscositeit, dat zich laat beschrijven door relativistische hydrodynamica. Terwijl er veel eigenschappen van het QGP meer en meer begrepen worden, zijn er nog steeds veel open vraagstukken die ook van belang zijn voor het bredere onderzoeksveld van de hoge-energie fysica.

Deze thesis presenteert de resultaten van neutrale mesonen metingen in pp, pA, en AA botsingen die details van deeltjes productie blootleggen, als mede de resultaten van directe foton correlatie metingen die gevoelig zijn voor de ruimte-tijd evolutie van het QGP. Fotonen zijn uniek omdat ze een gemiddelde vrije weglengte in het QGP hebben die veel groter is dan de grootte van het systeem, zodat de thermisch geproduceerde fotonen onaangetast het systeem verlaten. Dit betekent, in contrast met dat andere deeltjes continu met het systeem interacteren en deelnemen in de expansie, dat thermische fotonen waardevolle input geven over de vroege-tijds dynamica van het QGP, en kunnen de effectieve temperatuur afbakenen. Experimenteel is het zeer uitdagend om directe fotonen te meten, omdat bijna alle fotonen geproduceerd worden in het verval van neutrale mesonen. Dit maakt het belangrijk om neutrale mesonen zo nauwkeurig mogelijk te bestuderen en te meten, wat in zichzelf kan leiden tot inzichten in deeltjes productie mechanismen voor alle botsing systemen evenals het afbakenen van model calculaties.

De analyses die gepresenteerd worden in dit werk gebruiken experimentele data die met de ALICE detector verzameld zijn, wat een van de vier grote detector experimenten is op CERN dat geoptimaliseerd is om hoge deeltjes multipliciteiten te hanteren dat geproduceerd wordt in zware-ionen botsingen, en is geïnstalleerd in het tweede interactiepunt van de $27 \mathrm{~km}$ lange Large Hadron Collider (LHC). De detector bestaat uit vele subsystemen die allemaal samenwerken om informatie te verzamelen over het productiepunt, energie, impuls, en identiteit van de geladen en neutrale deeltjes die geproduceerd worden in de botsingen. Voor deze thesis wordt het centrale gedeelte van de detector gebruikt om fotonen te reconstrueren die converteren in de detector materiaal, wat zorgt voor foton metingen tot zeer lage impuls, evenals dat de EMCal, DCal, en PHOS calorimeters gebruikt worden om fotonen te meten met hoge efficiëntie en hoge impulsen.

De invariante productie van $\pi^{0}$ en $\eta$ mesonen is gemeten in pp, p- $\mathrm{Pb}$, en $\mathrm{Pb}-\mathrm{Pb}$ botsingen bij $\sqrt{s_{N N}}=$ $5.02 \mathrm{TeV}$, waarbij alle foton reconstructie methodes en trigger mogelijkheden van de ALICE detector gebruikt worden. Voor de resultaten van pp botsingen is het de eerste keer dat een geïdentificeerde deeltjes spectrum is gemeten voor de volledige Run 2 dataset. Door de grote hoeveelheid statistiek wordt de invariantie productie van beide mesonen meer differentieel gemeten, zoals als functie van event multipliciteit, vorm van het event, en de productie in jets. Een van de hoofdzakelijke bevindingen is dat de in-jet $\eta / \pi^{0}$ ratio zwaar gedempt is ten opzichte van dezelfde ratio in $\mathrm{MB}$ botsingen. De $\mathrm{p}-\mathrm{Pb}$ resultaten presenteren de neutrale mesonen productie in MB botsingen, als ook voor het eerste de afhankelijkheid naar event multipliciteit, waardoor multipliciteit afhankelijke nucleaire modificatiefactor $Q_{\mathrm{pA}}$ bestudeerd kan worden. 
Hier is het gevonden dat de $Q_{\mathrm{pA}}$ voor hoge multipliciteiten botsingen significant groter is vergeleken met de resultaten in lage multipliciteiten inclusieve botsingen, wat consistent is met een multipliciteit afhankelijke Cronin piek. De $\mathrm{Pb}-\mathrm{Pb}$ resultaten presenteren een invariante productie van $\pi^{0}$ en $\eta$ mesonen voor de gecombineerde Run 2 dataset, wat een ongeëvenaarde precisie meting oplevert voor deze mesonen over een groot interval van transverse impuls. De $\eta / \pi^{0}$ ratio is significant hoger voor centrale $\mathrm{Pb}-\mathrm{Pb}$ botsingen, wat toegekend wordt aan de radiale flow van het plasma. De nucleaire modificatiefactor $R_{\mathrm{AA}}$ laat een centraliteit afhankelijke demping zien, waar centrale botsingen meer gedempt zijn dan perifere botsingen, wat in lijn is met de verwachting. Deze resultaten zijn een stap richting de meting van directe fotonen, voor welke het verval van $\pi^{0}$ en $\eta$ mesonen de meest voornamelijke achtergrond is. Voor al deze resultaten in pp, $\mathrm{p}-\mathrm{Pb}$, en $\mathrm{Pb}-\mathrm{Pb}$ botsingen is het goed om te benadrukken dat de productie van neutrale mesonen nog niet eerder voor zoveel verschillende event condities bestudeerd is, wat de theoretische modellen goed kan afbakenen.

Een van de observaties van de neutrale mesonen metingen is dat de combinatorische achtergrond van de foton paar distributies niet goed gereproduceerd wordt door enkel de event-mixing methode, waardoor er additionele stappen nodig zijn om ook de gecorreleerde achtergrond te verwijderen. Deze thesis presenteert een model studie dat laat zien dat de correlaties voor lagere $M_{\text {inv }}$ niet door verval kinematica komt, maar door correlaties in de parton regen. Een gemodificeerde event-mixing methode wordt voorgesteld waarbij fotonen enkel gecombineerd worden als de oorspronkelijke partonen bij elkaar in de buurt zitten. Deze bevindingen zijn belangrijk voor beide de neutrale mesonen en directe foton HBT metingen.

Een andere model studie duikt in de correlaties tussen de vorm van het event en de waarschijnlijkheid om een jet te vinden. In de huidige literatuur wordt vaak de connectie gelegd dat potlood-achtige events jets bevatten, en dat bolvormige-events gedomineerd worden door zachte deeltjes productie door het onderliggende event. De resultaten laten zien dat dit inderdaad het geval is voor $e^{+} e^{-}$botsingen, waar de event-vorm observabele $S_{\mathrm{T}}$ gebruikt kan worden om efficiënt een bepaalde jet topologie te selecteren. Voor pp botsingen klopt dit niet, en selecties op $S_{\mathrm{T}}$ zorgen onder geen enkele omstandigheid voor een significante verandering in de samenstelling van de hoeveelheid jets in het sample. Echter, de resultaten laten zien dat de gemiddelde hoeveelheid parton parton interacties voor de 10\% meest bolvormige events bijna 10 keer hoger zijn ten opzichte van de $10 \%$ meest potlood-achtige events. Dit inzicht kan een nieuw pad inslaan voor het gedetailleerd bestuderen van parton parton interacties.

Tevens presenteert deze thesis de eerste meting van directe foton elliptische flow bij LHC energieën van $\sqrt{s_{N N}}=2.76 \mathrm{TeV}$. Voor dit werk worden de fotonen gemeten via de foton conversie methode (PCM), waarvoor een nieuwe methode ontwikkeld is voor het corrigeren van foton onzuiverheden. Na het combineren van de inclusieve foton metingen van PCM en PHOS, van welke de laatste is gedaan door een collega, wordt de directe foton flow berekent door het gebruik van een foton cocktail en de eerder gemeten $R_{\gamma}$. Het is vernomen dat de flow coefficient $v_{2}$ consistent is met de vervalsfoton flow, evenals de resultaten verkregen bij RHIC. De model calculaties onderschatten de gemeten $v_{2}$, en het blijkt lastig om beide de productie en flow van de directe fotonen te beschrijven. De experimentele onzekerheden zijn te groot om een direct foton 
puzzel te claimen bij LHC energieën. Een toekomstige meting van deze observabele met hogere precisie is een van de meest geanticipeerde metingen van het onderzoeksveld.

Ook presenteert deze thesis de eerste meting van directe foton HBT bij de LHC, voor beide pp en $\mathrm{Pb}$ $\mathrm{Pb}$ botsingen van $\sqrt{s_{N N}}=5.02 \mathrm{TeV}$. De resultaten voor pp botsingen laten een grote toename in de correlatie functie $C\left(Q_{\text {inv }}\right)$ zien voor lagere $Q_{\text {inv }}$ en hogere relatieve paar impuls $k_{\mathrm{T}}$, wat waarschijnlijk geassocieerd is met de gecorreleerde deeltjes productie in parton regens, wat geen HBT-achtig effect is. Dit effect is ook te zien in de resultaten in $\mathrm{Pb}-\mathrm{Pb}$ botsingen, enkel is het kleiner door de hoge event multipliciteit. $\mathrm{Na}$ het corrigeren voor dit effect zijn de resultaten in $\mathrm{Pb}-\mathrm{Pb}$ botsingen consistent met een HBT-achtig signaal dat we zouden verwachten van een QGP, alleen zijn de onzekerheden nog te groot om conclusies aan te verbinden.

Tenslotte wordt er een verkennende studie gepresenteerd over foton productie met en zonder de aanwezigheid van een geladen deeltje met hoog impuls, in beide $\mathrm{pp}$ en $\mathrm{Pb}-\mathrm{Pb}$ botsingen met een botsingsenergie van $\sqrt{s_{N N}}=5.02 \mathrm{TeV}$. Het vergelijk tussen de resultaten die verkregen zijn in deze systemen laten zien hoe de aanwezigheid van een dergelijk deeltje het spectrum verhard, met een indicatie dat lage impuls fotonen productie in $\mathrm{Pb}-\mathrm{Pb}$ botsingen gedomineerd is door de bulk. Als we de brede aanname maken dat een $\mathrm{Pb}-$ $\mathrm{Pb}$ botsing een superpositie is van pp-achtige productie bronnen, vinden we dat centrale $\mathrm{Pb}-\mathrm{Pb}$ botsingen ongeveer vijf keer meer bronnen nodig hebben vergeleken met perifere $\mathrm{Pb}-\mathrm{Pb}$ botsingen.

In samenvatting is dit werk een reis door het veld van zware-ionen fysica, waarbij het foton het centrale experimentele object is. Bijna alle bestaande experimentele observabelen zijn gebruikt om meer te leren over de verschillende botsing systemen die beschikbaar zijn in de ALICE data. Meerdere studies zijn ondernomen die inzichten geven in hete en koude nucleaire materie effecten. 


\section{List of publications}

Impact of residual contamination on inclusive and direct photon flow

J. Phys. G: Nucl. Part. Phys. 44 (2017) 025106

https://arxiv.org/abs/1606.06077

Neutral pion and $\eta$ meson production in p-Pb collisions at $\sqrt{s_{\mathrm{NN}}}=5.02 \mathrm{TeV}$

Eur. Phys. J. C (2018) 78: 624

https://arxiv.org/abs/1801.07051

Direct photon elliptic flow in $\mathbf{P b}-\mathbf{P b}$ collisions at $\sqrt{s_{\mathrm{NN}}}=5.02 \mathrm{TeV}$

Phys. Lett. B 789 (2019) 308

https://arxiv.org/abs/1805.04403

Report on the ECFA Early-Career Researchers Debate on the 2020 European Strategy Update for Particle Physics

CERN-OPEN-2020-006

https://arxiv.org/abs/2002.02837

Photon pair distributions and event-mixing at low invariant mass

Submitted to European Physical Journal C

https://arxiv.org/abs/2101.11004

Event shapes and jets in $e^{+} e^{-}$and pp collisions

Submitted to Nuclear Physics A

https://arxiv.org/abs/2101.12367 



\section{Acknowledgements}

In a way, life is a continuous chain of events that shapes the path we walk. Everyone has his own story that gets enriched by the people that you meet along the way. In these acknowledgements I will take the opportunity to thank the people that are of special importance to me while obtaining the $\mathrm{PhD}$ degree. Since this is the chapter that is arguably the most read chapter, I will also make it just a tiny bit larger.

I would like to first thank the particle physics community as a whole, with in particular the colleagues of the ALICE collaboration, University of Utrecht, and Nikhef. It is an understated privilege to be part of a diverse group of people from all the corners of the world that share an honest curiosity of finding things out. This simple concept continues to motivate me almost endlessly, and for me is a solid reason to try my best and help the community to thrive. It has been an absolute pleasure to participate at the forefront of science.

Raimond. It was already back in 2013 that I attended your presentation in Utrecht, which was about the experimental physics masters program and the ALICE experiment at the LHC. I would like to specially thank you for this defining moment, as well as for leading our wonderful group at Utrecht and Nikhef, countless laughs at the coffee corner, and for introducing me rather informally to Helen while we all were slightly tipsy at Quark Matter.

Thomas. After the CERN summer student program, where I started my first photon analyses, I decided to do the master thesis with you and to continue working on neutral meson and direct photon flow measurements. Nearing the end of the master thesis we went to this pizza place close to CERN, where you asked me if I wanted to do a PhD with you. This was the perfect opportunity for me, and we turned out to be a very good team. I would like to thank you for giving me almost absolute freedom in pursuing the analyses that interested me, making me think more and deeper about the observations than what I would naturally do, and giving many clever insights and ideas for all the photon related studies that we performed. We also had our fair share of adventure with all our students, and I admire that you always have time and energy for them. Also, I will never forget that you plugged the sound speakers into your phone during the ALICE summer BBQ at point 2, put on 90s dance music, creating an explosion of dancing physicists. That somehow blew my mind.

My colleagues at Utrecht University and Nikhef. Luuk, we had a lot of adventures together. Like the time we went to Ghent for the EPS-HEP conference, where we noticed that many of the books in our apartment were about a very particular subject, and the writer was the same man as the owner of the place (intentionally cryptic). Apart from being conference buddies we also went biking together, and I still dont really understand how you can go so crazy fast for so long. Lennart, I enjoyed our discussions a lot, which were mostly about politics and the news. We also started to change the way conferences are organized. 
This gave us chances to help organize them, and it is safe to say that the success of these conferences were one of the most rewarding achievements of the $\mathrm{PhD}$. Also, for the last years, Luuk, Lennart, and me have a Whatsapp group called koffieslurpers. We pretend that is all we do all day at the University. The amount of almost undrinkable coffee that we drank is really a lot. Jacopo, we just quickly became good friends, having whiskey/art painting sessions, long discussions about the organization of science, and countless laughs. Thanks for running into my life. Henrique, we had a crazy adventure to the Quark Matter conference in Wuhan, which includes near-death experiences in the taxi and discovering the local food. This obviously resulted in becoming good friends. Marta, it just made me happy that you joined our group. It was a great experience to teach particle physics together and to have drinks in the office that were slightly early. Alessandro, you are just crazy. Not sure I should thank you for that, but you surely add something that is otherwise not present. I would also like to thank Auro, Zhanna, Davide, Barbara, Cristina, Marco, Panos, and Paul, for all the fun moments that we shared. Having you all around in Utrecht and Nikhef truly enriched my life.

My close colleagues at CERN. Nico, during the PhD you became my day-to-day office mate, also while I was not at CERN. We shared all the analysis chaos of our physics working group, and also produced a ton of work together. While we didnt really get to the point of going to a conference together, we did go downhill mountain biking, which turned out to be one of the best holidays of my life. Florian, we share many interests, but one of the most memorable moments is for sure that we shorted the stock market during the dip. What a ride. Friederike, you were a great mentor when I started out in ALICE, and are a very good friend. You taught me so many things and I'm proud that we made a significant impact on the physics output of the working group. I owe you so much. Constantin, you were the mentor I really needed at times. Thanks for that, and also for the first paper we wrote together. Your work ethic is certainly one of the reasons why I started to like academia so much. Norbert, thanks for inviting me to Japan and giving me the opportunity to teach the local students in the ways of analysis. As you probably would have guessed, I miss the sushi place.

I would like to thank all the students that I had the pleasure to supervise for their own thesis. For every one of you it felt like we worked together and explored unknown territory. You all made my days so much better and more interesting. Lizette, Samuel, Ngozi, Toon, Remco, Tijmen, Wessel, Jasper, Jonathan, Rick, Hidde, Masahiro, Hanseo, Sophie, Gijs, Jonno, Cas, and Jurre, it was great to have you all working in my office, and together perform exciting analyses.

Rudi. We have been friends for longer than I can even remember. We share and have shared so many hobbies, always challenging each other to become better. Most importantly, we just focused on doing memorable things together. I believe that our friendship shaped us the way we are, and made us successful in what we find important in life. You are very dear to me, and I can't wait to learn what the future holds for the both of us.

Peter. Together we discovered the world of physics. From the moment we started our bachelor degree in Eindhoven we have been inspired by physics and mathematics, both sharing the same kind of energy 
and enthusiasm towards really understanding what we are studying. Instead of taking a summer holiday we decided to work through an entire book of mathematical proofs, and reading the legendary Feynman lectures. We even managed to get money to measure entangled photons and perform the bell tests. You really amplified my interest in physics, and I couldn't have imagined we both would end up doing a $\mathrm{PhD}$ in Utrecht, which led to over a hundred walks through the botanical gardens and a priceless friendship.

Vera. Ever since high school we have been like brother and sister, always there for each other, having fun, and catching up on what is happening in each other's lives. Thank you for always taking interest in what I'm doing, as well as all the good times we have together.

Annemieke. You have been such an important person to me over the last few years. Your intelligence, strive for excellence, and ways to enjoy life has changed me as a person. Being with you also meant that I could completely forget about physics, and at the same time be incredibly productive. We shared our lives on such a deep level that a part of this thesis is also yours.

Dad and Rick. The three of us have endured a lot together which created this unbreakable bond that we have. Literally no day goes by without having spoken to both of you, no matter where we are or what we are doing. The unconditional support, advice, adventures, and time that we share is such a huge foundation to everything else. It made it easy to take a leap of faith in trying to be a successful scientist, knowing that you two will always have my back and will always be proud, no matter what. I remember the most simple piece of advice when I was pondering whether to pursue a career in academia or industry. Dad, you asked me: ẅhat would you do if you had infinite money?; to which I answered, research: So here I am, doing what I like the most.

Finally, I would like to thank my new colleagues at the Yale Relativistic Heavy Ions Group, with in particular Hannah, Laura, Caitie, and Raghav, for their warm welcome. I'm looking forward to continuing the journey of finding things out! 